\title{
Ruthenium- and Cobalt-Catalyzed Chelation-Assisted C-H Functionalizations
}

\author{
Dissertation \\ for the award of the degree \\ "Doctor rerum naturalium"(Dr. rer. nat.) \\ of the Georg-August-Univers ität Göttingen
}

within the doctoral program of chemistry

of the Georg-August University School of Science (GAUSS)

submitted by

Jie Li

from Xiang Tan (China)

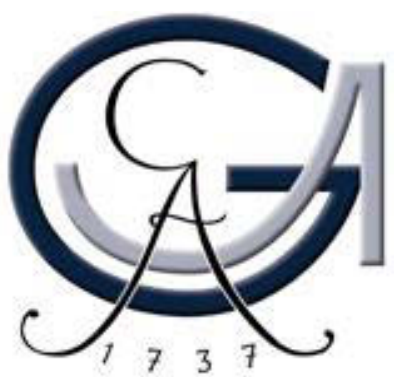

Göttingen, 2015 


\section{Thesis Committee}

Prof. Dr. L. Ackermann, Institute of Organic and Biomolecular Chemistry

Prof. Dr. K. Koszinowski, Institute of Organic and Biomolecular Chemistry

\section{Membe rs of the Examination Board}

Reviewer: Prof. Dr. L. Ackermann, Institute of Organic and Biomolecular Chemistry

Prof. Dr. K. Koszinowski, Institute of Organic and Biomolecular Chemistry

\section{Further me mbers of the Examination Board}

Prof. Dr. C. Höbartner, Institute of Organic and Biomolecular Chemistry

Prof. Dr. U. Diederichsen, Institute of Organic and Biomolecular Chemistry

Dr. A. Breder, Institute of Organic and Biomolecular Chemistry

Prof. Dr. D. Stalke, Institute of Inorganic Chemistry

Date of the oral examination: July $3^{\text {rd }}, 2015$ 


\section{Contents}

1 INTRODUCTION

1.1 Transition-Metal-Catalyzed C-H Functionalizations .............................................................

1.2 Carboxylate-Assisted Ruthenium-Catalyzed C-H Functionalizations ...................................

1.2.1 Carboxylate-Assisted Ruthenium-Catalyzed Oxidative Alkyne Annulations and Oxidative

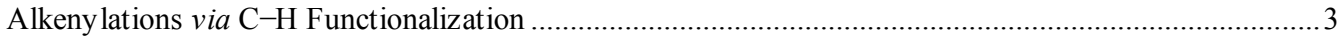

1.2.2 Carboxy late-Assisted Ruthenium-Catalyzed Hydroary lations via $\mathrm{C}-\mathrm{H}$ Bond Functionalization...7

1.3 Cobalt-Catalyzed C-H Bond Functionalizations..................................................................

1.3.1 Cobalt-Catalyzed C-H Bond Functionalizations in Aldehy des.................................................. 12

1.3.2 Low-Valent Cobalt-Catalyzed Chelation-Assisted C-H Bond Functionalizations ....................... 13

1.3.3 High-Valent Cobalt-Cataly zed C-H Bond Functionalizations..................................................25

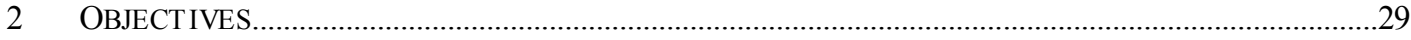

3 RUTHENIUM(II)-CATALYZED OXIDATIVE ALKYNE ANNULATION BY C-H BOND ACTIVAT ION ON

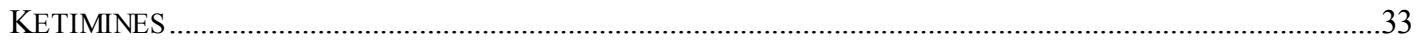

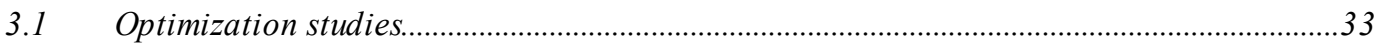

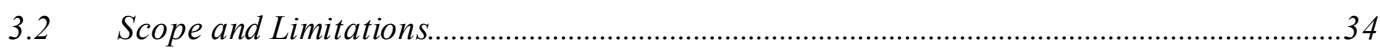

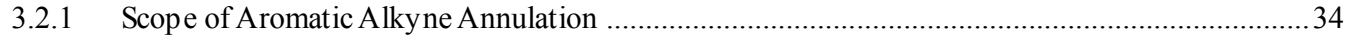

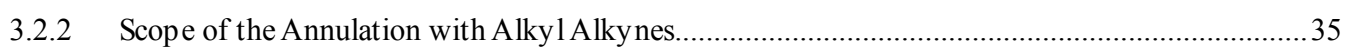

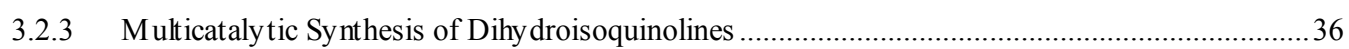

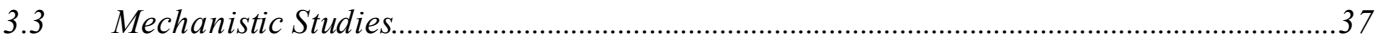

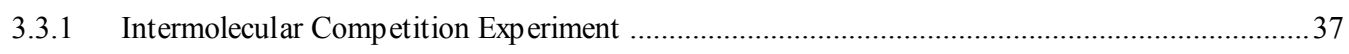

3.3.2 Reaction in the Presence of Isotopically Labeled Solvent ...........................................................38

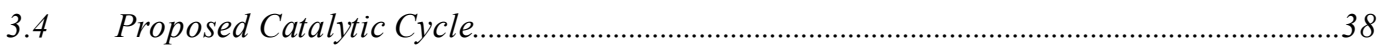

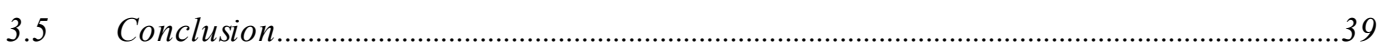

4 AMIDINES FOR VERSATILE RUTHENIUM(II)-CATALYZED OXIDATIVE C-H BOND ACT IVATION WITH INTERNAL ALKYNES AND ACRYLAT ES............................................................................................4

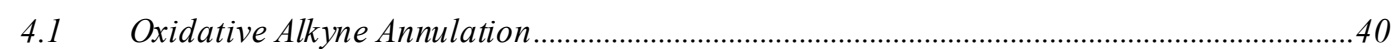

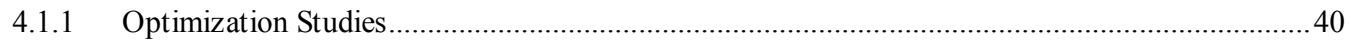

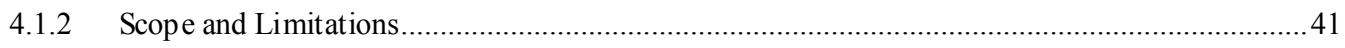

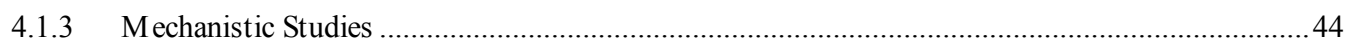

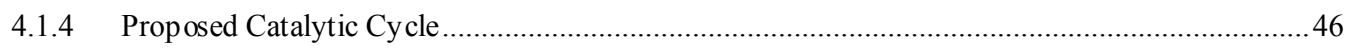

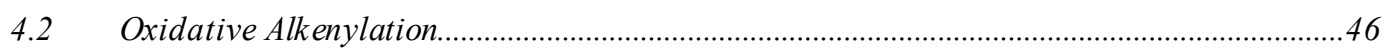

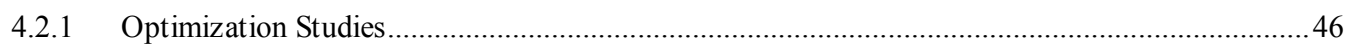

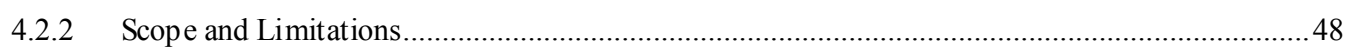

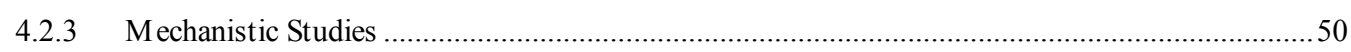

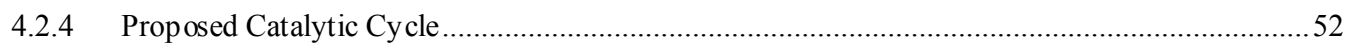

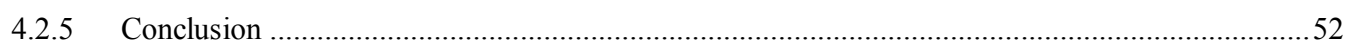

5 RUTHENIUM(II)-CATALYZED C-H BOND HYDROARYLATION AND OXIDATIVE ANNULAT IONWITH

A,B-UNSAT URAT ED KETONES VIA MONODENTATE DIRECTING GROUP ...................................................54

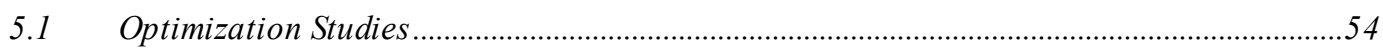

5.2 Scope of the Alkylation with $\alpha, \beta$-unsaturated Ketones............................................................56

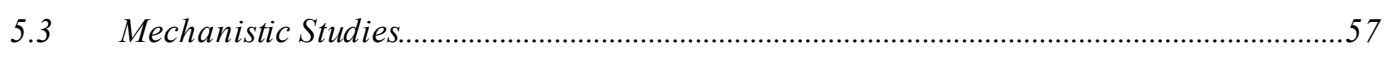

5.3.1 Comparison of the Directing Group Power ...............................................................................5 
5.3.2 Reaction in the Presence of Isotopically Labeled Solvent ..............................................................58

$5.4 \quad$ Scope of the Oxidative Annulation with $\alpha, \beta$-Unsaturated Ketone .........................................59

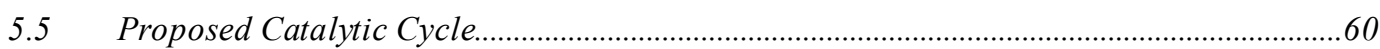

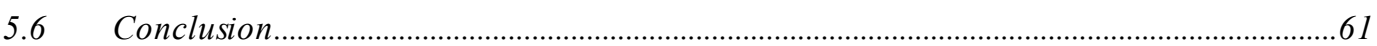

6 COBALT-CATALYZED C-H ARYLATION WITH WEAKLY-COORDINAT ING AMIDES AND TET RAZOLES: EXPEDIENT ROUTE TOANGIOTENSIN-II-RECEPTOR BLOCKERS..................................................................62

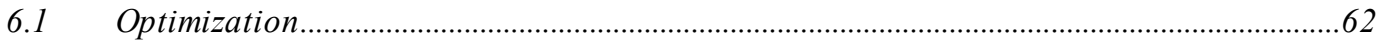

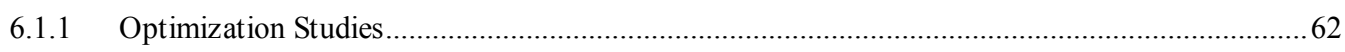

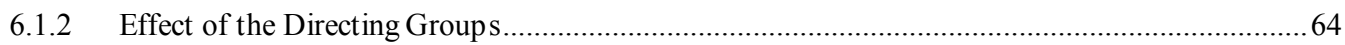

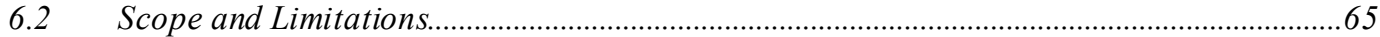

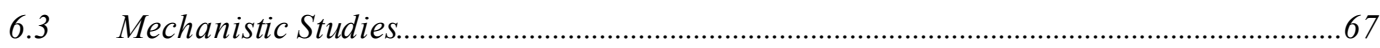

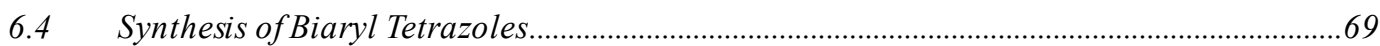

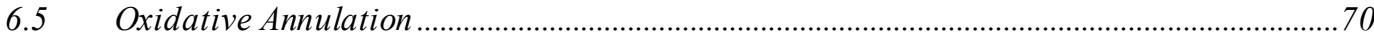

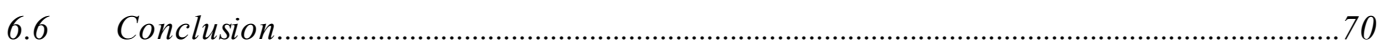

7 COBALT (III)-CATALYZED C-H BOND CYANATION OF ARENES AND HETEROARENES ......................72

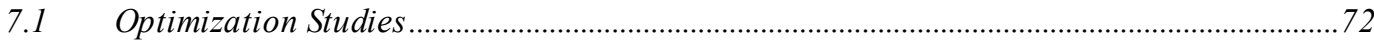

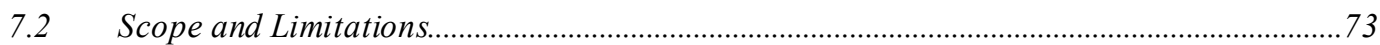

7.2.1 Substrate Scope of Cobalt-Catalyzed C-H Bond Cyanation..................................................... 73

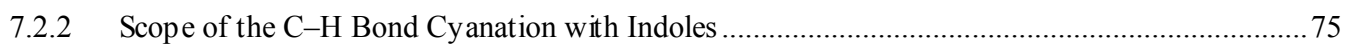

7.2.3 Scope of the $\mathrm{C}-\mathrm{H}$ Bond Cyanation with Heteroarenes.............................................................. 76

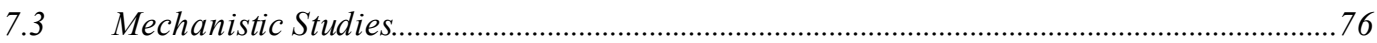

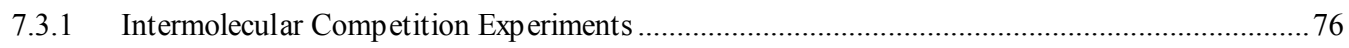

7.3.2 Reactions with Isotopically Labelled Reagents ........................................................................ 77

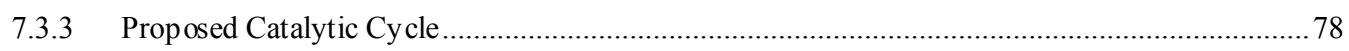

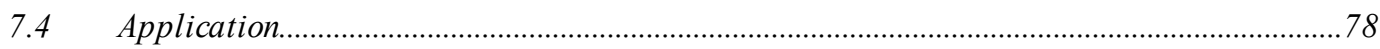

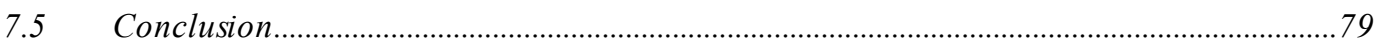

8 COBALT(III)-CATALYZED ARYL- AND ALKENYL-C-H BOND AMINOCARBONYLATIONWITH

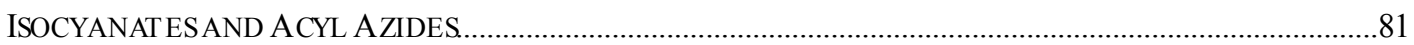

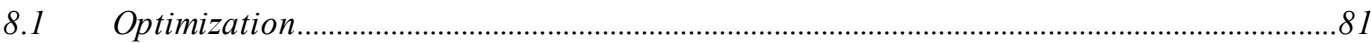

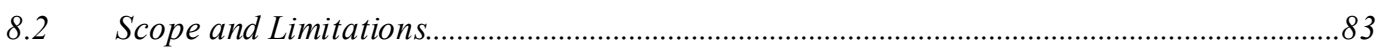

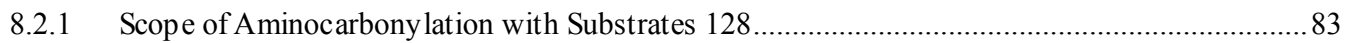

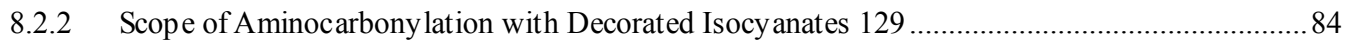

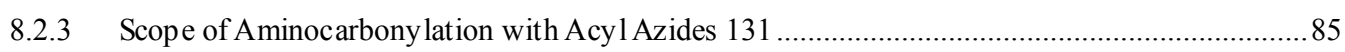

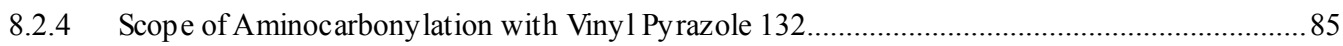

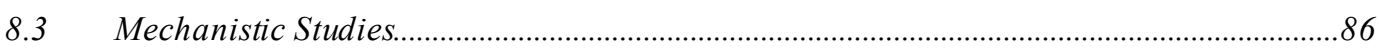

8.3.1 H/D Exchange Experiments and Kinetic Isotope Experiments ...................................................86

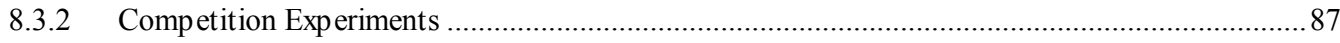

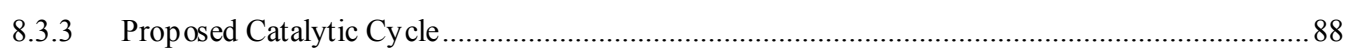

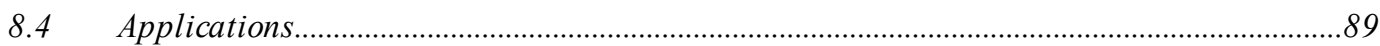

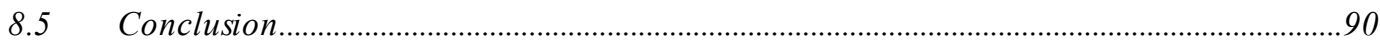

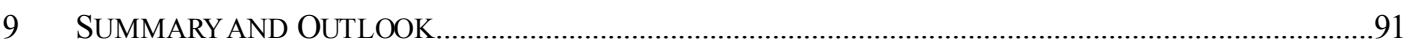

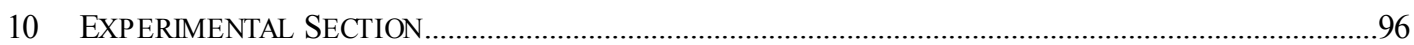

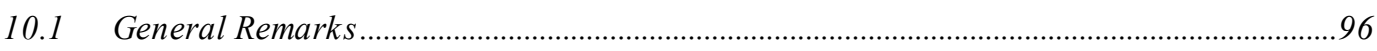

10.2 Synthesis of the Starting Materials .....................................................................................98 
10.3 General Procedures...

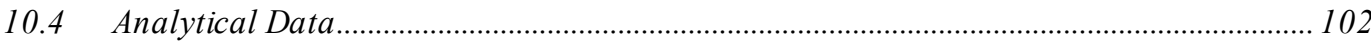

10.4.1 Analytical Data for the Product of Ruthenium(II)-Catalyzed Oxidative Alkyne Annulation with Ketimines.

10.4.2 Analytical Data for the Products of the Ruthenium(II)-Catalyzed Oxidative C-H Activation with Internal Alkynes and Acry lates...

10.4.3 Analytical Data for the Products of Ruthenium(II)-Catalyzed C-H Bond Hydroary lation and Oxidative Annulation with $\alpha, \beta$-Unsaturated Ketones via Monodentate Coordination..

10.4.4 Analytically Data for the Products of Cobalt-Catalyzed Direct Ary lation of Aromatic Amides 181

10.4.5 Analytical Data for the Products of Cobalt(III)-Catalyzed C-H Bond Cyanation of (Hetero)Arenes .212

10.4.6 Analytical Data for the Products of Cobalt(III)-Catalyzed Aryl and Alkenyl C-H Bond Aminocarbonylation with Isocy anates and Acyl Azides. 


\section{Abbreviations}

\begin{tabular}{|c|c|c|c|}
\hline $\mathrm{Ac}$ & acetyl & $J$ & coupling constant \\
\hline Ad & adamantly & KIE & kinetic isotope effect \\
\hline Alk & alkyl & $\mathrm{L}$ & ligand \\
\hline AMLA & amb iphilic metal-ligand activation & $m$ & meta \\
\hline aq. & aqueous & $\mathrm{m}$ & mu ltip let \\
\hline Ar & aryl & M & molar, metal \\
\hline ARBs & angiotens in-II-receptor blockers & {$[\mathrm{M}]^{+}$} & mo lecular ion peak \\
\hline atm & atmospheric pressure & $\mathrm{Me}$ & methyl \\
\hline $\mathrm{Bn}$ & benzyl & Mes & mesityl \\
\hline $\mathrm{Bu}$ & butyl & $\mathrm{mg}$ & milligram \\
\hline calc. & calculated & $\mathrm{MHz}$ & megahertz \\
\hline CAN & ceric ammonium $n$ itrate & $\min$ & minute \\
\hline cat. & catalyst & $\mathrm{mL}$ & milliliter \\
\hline CMD & concerted-metalation-deprotonation & mmol & millimol \\
\hline conv. & conversion & M. p. & melting point \\
\hline $\mathrm{Cp} *$ & pentamethylcyclopentadienyl & MPV & membrane pump vacuum \\
\hline Cy & cyclohexyl & MS & mass spectrometry \\
\hline$d$ & doublet & $m / z$ & mass-to-charge ratio \\
\hline DCE & 1,2-dich loroethane & MVK & methyl v inyl ketone \\
\hline $\mathrm{dd}$ & doublet of doublet & $n$ & normal \\
\hline DG & directing group & $\mathrm{NHC}$ & $N$-heterocyclic carbene \\
\hline DMA & $N, N$-dimethylacetamide & NMP & $N$-methylpyrrolidinone \\
\hline DME & dimethoxyethane & $\mathrm{Ph}$ & phenyl \\
\hline $\mathrm{DMF}$ & $N, N$-dimethylformamide & Piv & pivaloyl \\
\hline DMSO & dimethyl sulfo xide & PMB & para-metho xybenzyl \\
\hline DMPU & $N, N$ '-dimethyl- $N, N$ '-propylene urea & PMP & para-metho xyphenyl \\
\hline $\mathrm{dt}$ & doublet of triplet & py & pyridyl \\
\hline Ed. & editor & $\mathrm{PyO}$ & 2-aminopyridine-1-oxide \\
\hline equiv & equivalent & pym & pyrimidyl \\
\hline ESI & electros pray ionization & Pyr & pyrrole \\
\hline Et & ethyl & Q & quinoline \\
\hline FG & functional group & $r$ & removable \\
\hline g & gram & $\mathrm{R}_{\mathrm{L}}$ & $\mathrm{R}_{\text {Large }}$ \\
\hline $\mathrm{GC}$ & gas chromatography & $\mathrm{R}_{\mathrm{S}}$ & $\mathrm{R}_{\text {Small }}$ \\
\hline $\mathrm{h}$ & hour & $\delta$ & chemical shift \\
\hline Het & hetero(aryl) & $T$ & temperature \\
\hline HPLC & $\begin{array}{l}\text { high performance liquid } \\
\text { chromatography }\end{array}$ & TBS & tert-butyldimethylsilyl \\
\hline HRMS & high resolution mass spectrometry & THF & Tetrahydrofuran \\
\hline $\mathrm{Hz}$ & Hertz & $\mathrm{TM}$ & transition metal \\
\hline$i$ & iso & Ts & para-toluenesulfonyl \\
\hline IES & intramolecular electrophilic & $\mathrm{X}$ & (pseudo)halide \\
\hline
\end{tabular}


substitution

IPr 1,3-bis(2,6-d iis opropylphenyl)

IR

infrared spectros copy
XPhos 2-dicyc lohexylphosphino-2',4',6'-triiso propylbiphenyl 


\section{Introduction}

\subsection{Transition-Metal-Catalyzed C-H Functionalizations}

A long-standing challenge in synthetic organic chemistry is the development of methods for the direct conversion of unactivated carbon-hydrogen bonds into carbon-carbon, carbon-nitrogen, carbon-halogen, or carbon-oxygen bonds among others. Mild and selective transformations of this type will undoubtedly obta in a wide range of potential applications in varies applied fields. During the last few decades of the previous century, a new family of $\mathrm{C}-\mathrm{C}$ bond forming reactions based on transition-metal catalysts has emerged as a powerful tool and experienced a remarkable progress which played a vital role in the synthesis of pharmaceuticals, natural products, agrochemicals, polymers and feedstock commodity chemicals. ${ }^{1}$ Among them, transition-metal-catalyzed cross-coupling reactions are arguably the most prominent approach to the construction of $\mathrm{C}-\mathrm{C}$ bonds (Scheme 1.1). ${ }^{1 \mathrm{a} 2}$

\begin{tabular}{|c|c|c|c|c|c|c|}
\hline$R^{1}-X$ & & $M-R^{2}$ & ], - - & & & $R^{1}-R^{2}$ \\
\hline $\begin{array}{l}R^{1}, R^{2} \\
X\end{array}$ & $\begin{array}{l}= \\
=\end{array}$ & $\begin{array}{l}\text { aryl, vinyl, alkyl } \\
\text { I, OTf, Br }\end{array}$ & & & $\begin{array}{l}\mathrm{B} \\
\mathrm{Sn} \\
\mathrm{Si} \\
\mathrm{Zn} \\
\mathrm{Mg}\end{array}$ & $\begin{array}{l}\text { Suzuki } \\
\text { Stille } \\
\text { Hiyama } \\
\text { Negishi } \\
\text { Kumada, etc }\end{array}$ \\
\hline
\end{tabular}

Scheme 1.1. Transition-metal-catalyzed cross-coupling reactions.

However, the formation of such bonds relies on prefunctionalized starting materials, such as organoboron, organozinc or organotin compounds, which add costly chemical steps to the overall synthes is. Circumventing the disadvantages of the traditional approaches will not only improve atom economy, ${ }^{3}$ but also increase the overall efficiency of multistep synthetic sequences. In recent decades, transition-metal-catalyzed $\mathrm{C}-\mathrm{H}$ bond functionalizations became

\footnotetext{
${ }^{1}$ a) Transition Metals for Organic Synthesis (Eds.: M. Beller and C. Bol m), $2^{\text {nd }}$ ed., Wiley-VCH, Weinhei m, 2004; b) Metal-Catalyzed Cross-Coupling Reactions and More; (Eds.: A. de Meijere, S. Bräse, M. Oestreich), Wiley-VCH, Weinheim, 2014.

2 a) Metal-Catalyzed Cross-Coupling Reactions, (Eds: A. de Meijere and F. Diederich), $2^{\text {nd }}$ ed. Wiley-VCH, Weinheim, 2004. For selected reviews on $C-C$ bond forma tion via traditional cross-coupling rea ctions, see: $b) C$. $C$. C. J. Seechum, M. O. Kitching, T. J. Cola cot, V. Snieckus, Angew. Chem. Int. Ed. 2012, 51, 5062-5085; c) H. Li, C. C. C. J. Seechum, T. J. Cola cot, ACS Catal. 2012, 2, 1147-1164; d) B. M. Rosen, K. W. Quasdorf, D. A. Wilson, N. Zhang, A. M. Resmerita, N. K. Garg, V. Percec, Chem. Rev. 2011, 111, 1346-1416; e) G. Cahiez, A. Moyeux, Chem. Rev. 2010, 110, 1435-1462; f) A. Roglans, A. Pla -Quintana, M. Moreno-Mañas, Chem. Rev. 2006, 106, 4622-4643; g) J. P. Corbet, G. Mignani, Chem. Rev. 2006, 106, 2651-2710, and references cited the rein.

3 B. M. Trost, Acc. Chem. Res. 2002, 35, 695-705.
} 
an attractive strategy to streamline chemical synthesis. ${ }^{4}$ Due to its high atom- and step-economy, intensive research efforts have led to the remarkable progress for challenging $\mathrm{C}-\mathrm{H}$ bond functionalization. ${ }^{5}$

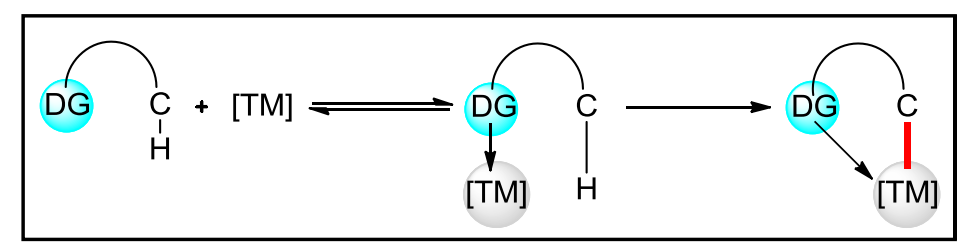

Scheme 1.2. Regioselective intermolecular cleavage of $\mathrm{C}-\mathrm{H}$ bonds through the use of a directing

$$
\text { group (DG). }
$$

Direct $\mathrm{C}-\mathrm{H}$ bond functionalizations are limited by mainly two challenging issues: (i) The requirement to control site-selectivity of the $\mathrm{C}-\mathrm{H}$ functionalization in a molecule that contains various potentially reactive $\mathrm{C}-\mathrm{H}$ bond; and (ii) the requirement to achieve selective functionalization of a single $\mathrm{C}-\mathrm{H}$ bond within a complex molecule. The most common strategy involves the use of substrates that contain a directing group (DG). These directing groups coordinate to the metal center and selectively bring the metal to the proximity of a $\mathrm{C}-\mathrm{H}$ bond (Scheme 1.2) ${ }^{6}$ and subsequently allow its activation and cleavage. Many transition metals, including, ruthenium, rhodium, palladium and cobalt, undergo stoichiometric cyclometalations. $^{5,6 \mathrm{~b}, 7}$ By far, a large remarkable progress in organometallic chemistry has set the stage for the development of increasingly viable metal catalysts for $\mathrm{C}-\mathrm{H}$ bond

\footnotetext{
${ }^{4}$ a) Handbook of $C-H$ Transformations (Ed: G. Dyker), Wiley-VCH, Weinheim, 2005; b) F.Kakiuchi, N. Cha tani, Adv. Synth. Catal. 2003, 345, 1077-1101; c) M. Mi ura, M. Nomura, Top. Curr. Chem. 2002, 219, 212-237; d) V. Ritleng, C. Si rin, M. Pfeffer, Chem. Rev. 2002, 102, 1731-1770; e) L. Adkermann, Synlett 2007, 4, 507-526.

${ }^{5}$ a) Modern Arylation Methods, (Ed: L. Ackermann), Wiley-VCH, Weinheim, 2009; For recent representative general reviews on $\mathrm{C}-\mathrm{H}$ bond functionalizations, see: b) J. Mo, L. Wang, Y. Lu, X. Cui, Synthesis 2015, 47, 439-459; c) G. Qiu, J. Wu, Org. Chem. Front. 2015, 2, 169-178; d) J. Yang, Org. Biomol. Chem. 2015, 13, 1930-1941; e) L. Ackermann, Org. Process Res. Dev. 2015, 18, 260-269; f) F. Zhang, D. R. Spring, Chem. Soc. Rev. 2014, 43, 6906-6919; g) A. F. M. Noisier, M. A. Brimble, Chem. Rev. 2014, 114, 8775-8806; h) V. S. Thi runa vukkarasu, S.I. Kozhushkov, L. Ackermann, Chem. Commun. 2014, 50, 29-39; i) L. Ackermann, Acc. Chem. Res. 2014, 47, 281-295; j) K. Gao, N. Yoshikai, Acc. Chem. Res. 2014, 47, 1208-1219; k) L. Ackermann, J.Org. Chem. 2014, 79, 8948-8954; I) J. J. Mousseau, A. B. Charrette, Acc. Chem. Res. 2013, 46, 412-424; m) K. M. Engle, T. S. Mei, M. Wasa, J. Q. Yu, Acc. Chem. Res. 2012, 45, 788-802; n) S. R. Neufeldt, M. S. Sanford, Acc. Chem. Res. 2012, 45, 936-946; o) J. Wencel-Delord, T. Droege, F. Glorius, Chem. Soc. Rev. 2011, 40, 4740-4761; p) C. S. Yeung, V. M. Dong, Chem. Rev. 2011, 111, 1215-1292; q) L. Ackermann, Chem. Rev. 2011, 111, 1315-1345; r) M. C. Willis, Chem. Rev. 2010, 110, 725-748; s) D. A. Colby, R. G. Bergman, J. A. Ellman, Chem. Rev. 2010, 110, 624-655; t) D. Alberico, M. E. Scott, M. Lautens, Chem. Rev. 2007, 107, 174-238; u) P. Sehnal, R. J. K. Taylor, I. J. S. Fairlamb, Chem. Rev. 2010, 110, 824-889, and the references the rein.

${ }^{6}$ a) L. Ackermann, R. Vicente, A. R. Kapdi, Angew. Chem. Int. Ed. 2009, 48, 9792-9826; b) L. Ackermann, Top. Organoment. Chem. 2007, 24, 35-60; c) I. Omae, Coord. Chem. Rev. 2004, 248, 995-1023.

7 a) N. Kuhl, N. Schroeder, F. Glorius, Adv. Synth. Catal. 2014, 356, 1443-1460; b) S. D. Sarkar, W. Liu, S. I. Kozhushkov, L. Ackermann, Adv. Synth. Catal. 2014, 356, 1461-1479; c) S. I. Kozhushkov, L. Ackermann, Chem. Sci. 2013, 4, 886-896; d) T. S. Mei, L. Kou, S. Ma, K. M. Engle, J. Q. Yu, Synthesis 2012, 44, 1778-1791, and refe rences cited the rein.
} 
functionalization reactions. ${ }^{5-7}$

\subsection{Carboxylate-Assisted Ruthenium-Catalyzed C-H Functionalizations}

Looking at the development in the area of utilizing unreactive $\mathrm{C}-\mathrm{H}$ bonds in chemical synthesis via transition metal catalys is, one can easily realize that the majority of achievements in $\mathrm{C}-\mathrm{H}$ functionalization reactions rely on noble metal catalysts, typically based on palladium, rhodium or ruthenium..$^{5 b-5 i, 51-5 u, 8}$ The pivotal metalation step of $\mathrm{C}-\mathrm{H}$ bond activation reactions, was proposed to proceeded by oxidative addition, electrophilic substitution, $\sigma$-bond metathesis or 1,2-addition. ${ }^{5 \mathrm{i}, 5 \mathrm{q}} \mathrm{A}$ few early reports indicated another potential possibility, which suggests that the reactions proceed via base-assisted metalation. ${ }^{5 \mathrm{i}, 5 \mathrm{q}}$ An early stoichiometric cyclometalation reactions of ruthenium was disclosed by Davies and coworkers, and revealed the beneficial effect of NaOAc. ${ }^{9}$ However, the first ruthenium(II)-catalyzed $\mathrm{C}-\mathrm{H}$ bond functionalization with carboxylate assistance was reported by Ackermann in 2008 (Scheme 1.3). ${ }^{10}$ Since then, the use of various carboxylates as cocatalytic additives for ruthenium catalysis became popular and widespread.

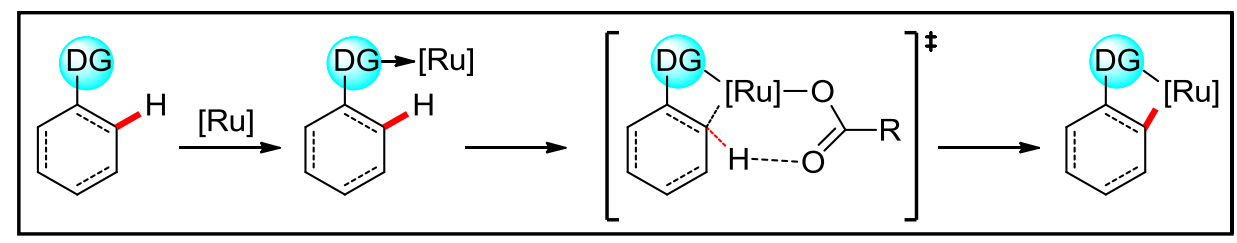

Scheme 1.3. Proposed rationalization for base-assisted $\mathrm{C}-\mathrm{H}$ ruthenation.

\subsubsection{Carboxylate-Assisted Ruthenium-Catalyzed Oxidative Alkyne Annulations and Oxidative Alkenylations via $\mathrm{C}-\mathrm{H}$ Functionalization}

Since Ackermann and coworkers suggested that ruthenium-catalyzed direct arylation involves reversible $\mathrm{C}-\mathrm{H}$ bond activations via carboxylate-assisted and subsequent deprotonative ruthenations, carboxylates were also explored as cocatalytic additives for ruthenium-catalyzed oxidative $\mathrm{C}-\mathrm{H}$ bond functionalizations ${ }^{11}$. The Ackermann group developed ruthenium

\footnotetext{
${ }^{8}$ Recent examples for C-H functionalizations. Pd: a) K. S. L. Chan, H.-Y. Fu, J.-Q. Yu, J. Am. Chem. Soc. 2015, 137, 2042-2046. Rh: b) S. Yu, S. Lu, Y. Lan, B. Wan, X. Li, J. Am. Chem. Soc. 2015, 137, 1623-1631; c) G. Zhang, H. Yu, G. Qin, H. Huang, Chem. Commun. 2014, 50, 4331-4334. Ru: d) S. Wa rratz, C. Komhaass, A. Caja ra ville, B. Niepoetter, D. Stalke, L. Ackermann, Angew. Chem. Int. Ed. 2015, 54, 5513-5517; e) F. Yang, K. Rauch, K. Kettelhoit, L. Ackermann, Angew. Chem. Int. Ed. 2014, 53, 11285-11288.

9 D. L. Davies, O. Al-Duaij, J. Fa wcett, M. Giardiello, S. T. Hilton, D. R. Russell, Dalton Trans. 2003, 4132-4138.

${ }^{10}$ L. Ackermann, R. Vicente, A. Al tha mmer, Org. Lett. 2008, 10, 2299-2302.

11 a) H. Weissman, X. Song, D. Milstein, J. Am. Chem. Soc. 2001, 123, 337-338; b) T. Ueyama, S. Mochida, T.
} 
catalytic systems for oxidative alkyne annulations, with the assistance of various directing groups. As a consequence, the first ruthenium-catalyzed oxidative annulations of alkynes through $\mathrm{C}-\mathrm{H}$ and $\mathrm{N}-\mathrm{H}$ bond cleavages for the synthesis of bioactive isoquinolones were reported in $2011 .^{12}$<smiles>[R]c1c([R])n([R])c(=O)c2ccccc12</smiles>

Ackermann (2011)<smiles>[R]c1c([R])c([R])n([R7])c(=O)c1[R]</smiles>

Ackermann (2011)<smiles>[R]c1c([R])n(-c2ncccn2)c2ccccc12</smiles>

Ackermann (2012)<smiles></smiles>

Ackermann (2012)

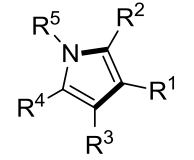

Ackermann (2013)
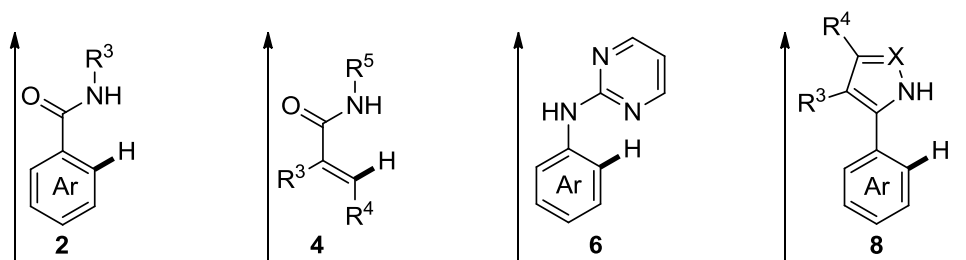

11

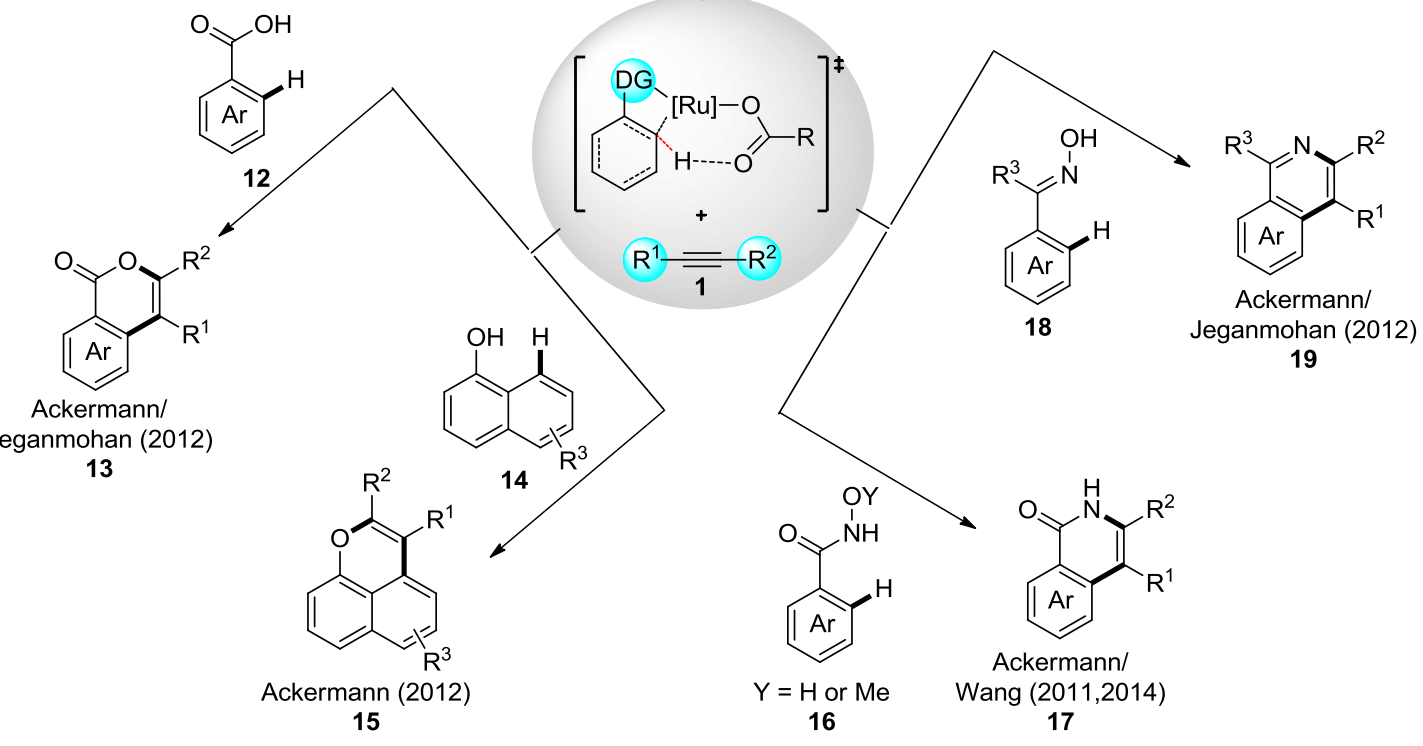

Scheme 1.4. The synthesis of heterocycles through alkyne annulations via ruthenium-catalyzed

$$
\mathrm{C}-\mathrm{H} \text { bond cleavage. }
$$

Further mechanistic studies proposed the ruthenium-catalyzed oxidative annulations to proceed by an initial carboruthenation via acetate-assisted $\mathrm{C}-\mathrm{H}$ bond cleavage, followed by migratory insertion, reductive elimination and reoxidation of the ruthenium( 0$)$ species. $^{5 i, 12}$ Thereafter, additional evidence was provided, through synthesis and isolation of key intermediates. ${ }^{8 f, 13}$ Notably, during the last few years, the scope of alkyne annulations by

Fukutani, K. Hirano, T. Satoh, M. Miura, Org. Lett. 2011, 13, 706-708.

12 L. Ackermann, A. V. Lygin, N. Hofmann, Angew. Chem. Int. Ed. 2011, 50, 6379-6382.

13 a) B. L, H. Feng, N. Wang, J. Ma, H. Song, S. Xu, B. Wang, Chem. Eur. J. 2012, 18, 12873-12879; b) B. L, T. 
$\mathrm{C}-\mathrm{H} / \mathrm{Het}-\mathrm{H}$ bond functionalization have witnessed significant progress in the following aspects: oxidative alkyne annulations through (i) $\mathrm{C}-\mathrm{H} / \mathrm{N}-\mathrm{H}$ bonds cleavages, ${ }^{12,14}$ $\mathrm{C}-\mathrm{H} / \mathrm{O}-\mathrm{H}$ bonds cleavages; ${ }^{15}$ (iii) $\mathrm{C}-\mathrm{H} / \mathrm{N}-\mathrm{O}$ bonds cleavages. ${ }^{16}$ Therefore, ruthenium(II)-catalyzed annulations of alkynes are among the most important approaches for the preparation of heterocyclic molecules (Scheme 1.4).

In the meantime, carboxylate-assisted ruthenium-catalyzed direct oxidative alkenylations were also developed in recent years, although the first ruthenium-catalyzed oxidative alkenylation can be traced back to 2001, as reported by Milstein and coworkers. They employed molecular oxygen as the terminal oxidant, and obtained a rather narrow and limited scope under these harsh reaction conditions. ${ }^{11 a}$ A carboxylate-assisted procedure for the successful ruthenium-catalyzed direct alkenylation of heteroaromatic ${ }^{1 \mathrm{~b}}$ and $\operatorname{aromatic}^{17}$ acids was reported in 2011 by Miura and coworkers as well as by Ackermann and coworkers. Further mechanistic studies showed the importance of acetates to be dominated for efficient $\mathrm{C}-\mathrm{H}$ bond metalations. ${ }^{17}$ Thereafter, the groups of Miura and Ackermann independently revealed the powerful ruthenium-catalyzed direct oxidative alkenylation of $N, N-\mathrm{di}^{18}$ and $\mathrm{N}$-monoa kylated benzamides $2 .^{19}$

The direct alkenylation of acrylamides 4 with alkenes was reported by Zhang and Loh. ${ }^{20}$ Similarly, Wang and coworkers described an alkenylation reaction of $N$-methoxybenzamides 2 bearing an internal oxidizing directing group. ${ }^{21}$

Roisnel, C. Darcel, P. H. Dixneuf, Dalton Trans. 2012, 41, 10934-10937.

${ }^{14}$ a) L. Ackermann, A. V. Lygin, N. Hofmann, Org. Lett. 2011, 13, 3278-3281; b) L. Ackermann, A. V. Lygin, Org. Lett. 2012, 14, 764-767; c) L. Ackermann, L. Wang, A. V. Lygin, Chem. Sci. 2012, 3, 177-180; d) W. Ma, K. Graczyk, L. Ackermann, Org. Lett. 2012, 14, 6318-6321; e) L. Wang, L. Ackermann, Org. Lett. 2013, 15, 176-179.

15 a) M. Deponti, S. I. Kozhushkov, D. S. Yufit, L. Ackermann, Org. Biomol. Chem. 2013, 11, 142-148, and references cited the rein; b) L. Ackermann, J. Pospech, K. Graczyk, K. Rauch, Org. Lett. 2012, 14, 930-933; c) R. K. Chi nnagolla, M. Jeganmohan, Chem. Commun. 2012, 48, 2030-2032; d) V. S. Thi runa vuarasu, M. Dona ti, L. Ackermann, Org. Lett. 2012, 14, 4210-4213.

16 a) L. Ackermann, S. Fenner, Org. Lett. 2011, 13, 6548-6551; b) B. L, H. Feng, S. Xu, B. Wang, Chem. Eur. J. 2011, 17, 12573-12577; c) K. Parthasara thy, N. Senthilkumar, J. Ja yakumar, C.-H. Cheng, Org. Lett. 2012, 14, 3478-3481; d) C. Kornhaass, J. L, L. Ackermann, J. Org. Chem. 2012, 77, 9190-9198; e) R.K. Chinnagolla, S. Pimparkar, M. Jeganmohan, Org. Lett. 2012, 14, 3032-3035; f) F. Yang, L. Ackermann, J. Org. Chem. 2014, 79, 12070-12082.

17 L. Ackermann, J. Pospech, Org. Lett. 2011, 13, 4153-4155.

18 Y. Hashimoto, T. Ortloff, K. Hirano, T. Satoh, C. Bolm, M. Miura, Chem. Lett. 2012, 41, 151-153.

19 L. Ackermann, L. Wang, R. Wolfram, A. V. Lygin, Org. Lett. 2012, 14, 728-731.

20 J. Zhang, T.-P. Loh, Chem. Commun. 2012, 48, 11232-11234.

${ }^{21}$ B. Li, J. Ma, N. Wang, H. Feng, S. Xu, B. Wang, Org. Lett. 2012, 14, 736-739. 
<smiles>[R]CC1OC(=O)c2ccccc21</smiles>

Ackermann (2011)

21<smiles>[R]C=Cc1ccccc1OC([R12])=O</smiles>

Ackermann/ Jeganmohan (2012/2013)

23

\section{$\mathrm{R}^{1}$}<smiles>[R]C=Cc1ccccc1Oc1ccccn1</smiles>

Ackermann (2013)

25

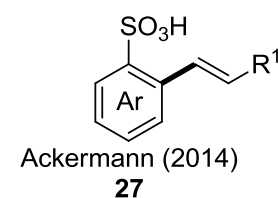

$=r$<smiles>[Z][Y]1:[Z]cnc1</smiles><smiles>[R]C=Cc1ccccc1I</smiles>

Dixneuf/Miura (2011/2012) Ackermann (2015) $\underbrace{\mathrm{Ar} \|_{22}^{\mathrm{O}}}_{12}$

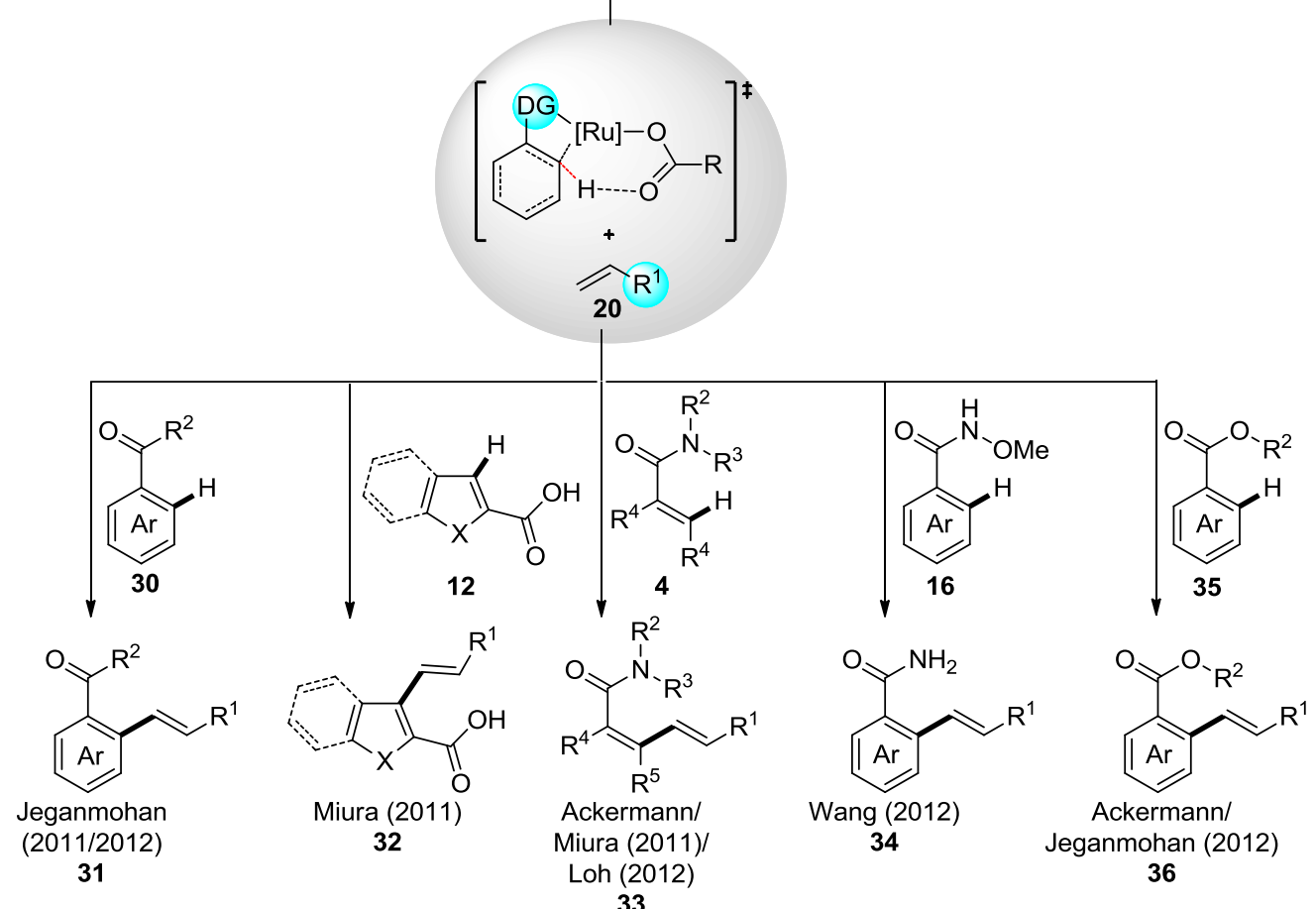

Scheme 1.5. Carboxylate-assisted ruthenium-catalyzed oxidative $\mathrm{C}-\mathrm{H}$ bond alkenylations.

Based on these contributions, the following research for the mentioned ruthenium(II) catalytic system came to extend the scope of directing groups, to include ester (35), ${ }^{22-23}$ ketone (30), aldehyde, ${ }^{25}$ carbamate (22), ${ }^{26-27}$ 2-pyridyloxy (24), ${ }^{28}$ sulfonic acid (26), ${ }^{29}$ oxazole $(\mathbf{2 8}),{ }^{30}$

\footnotetext{
${ }^{22}$ K. Graczyk, W. Ma, L. Ackermann, Org. Lett. 2012, 14, 4110-4113.

${ }^{23}$ K. Padala, S. Pimparka r, P. Ma dasamy, M. Jeganmohan, Chem. Commun. 2012, 48, 7140-7142.

${ }^{24}$ K. Padala. M. Jeganmhan, Org. Lett. 2011, 13, 6144-6147.

25 K. Padala. M. Jeganmhan, Org. Lett. 2012, 14, 1134-1137.

26 J. L. C. Kornhaass, L. Ackermann, Chem. Commun. 2012, 48, 11343-11345.

27 M. C. Reddy, M. Jeganmohan, Eur. J. Org. Chem. 2013, 1150-1157.

${ }^{28}$ W. Ma, L. Ackermann, Chem. Eur. J. 2013, 19, 1150-1157.

${ }^{29}$ W. Ma, R. Mei, G. Tenti, L. Ackermann, Chem. Eur. J. 2014, 20, 15248-15251.

${ }^{30}$ B. Li, K. Devaraj, C. Da rcel, P. Dixne uf, Green Chem. 2012, 14, 2706-2709.
} 
triazole, ${ }^{31}$ among others. (Scheme 1.5$) .{ }^{32}$

\subsubsection{Carboxylate-Assisted Ruthenium-Catalyzed Hydroarylations via $\mathbf{C}-\mathbf{H}$ Bond Functionalization}

As discussed above, the carboxylate-assisted ruthenium-catalyzed oxidative $\mathrm{C}-\mathrm{H}$ bond functionalizations can be used as one of the reliable methods for $\mathrm{C}-\mathrm{C}$ bond formation. Meanwhile, the developments of ruthenium-catalyzed hydroarylation reactions in an atomand step-economical way, under mild reaction conditions were also achieved. Pioneering finding by Lewis, indicated the first ortho-hydroarylation of alkenes with phenol. ${ }^{33}$ A further breakthrough was made in 1993 by Murai and coworkers, when they reported on the ruthenium(0)-catalyzed direct hydroarylation of alkenes $\mathbf{2 0}$ via chelation-assisted $\mathrm{C}-\mathrm{H}$ bond activation in aromatic ketones 30 (Scheme 1.6). ${ }^{34}$ The reaction can be considered to constitute an ideal pathway, not only in terms of atom- and step-economy, but also because of its high site-selectivity. A series of hydroarylation reactions were reported by Murai and coworkers in the following years, ${ }^{35-36}$ including a direct alkylation with decorated vinylsilanes 20, as reported by Trost. ${ }^{37}$

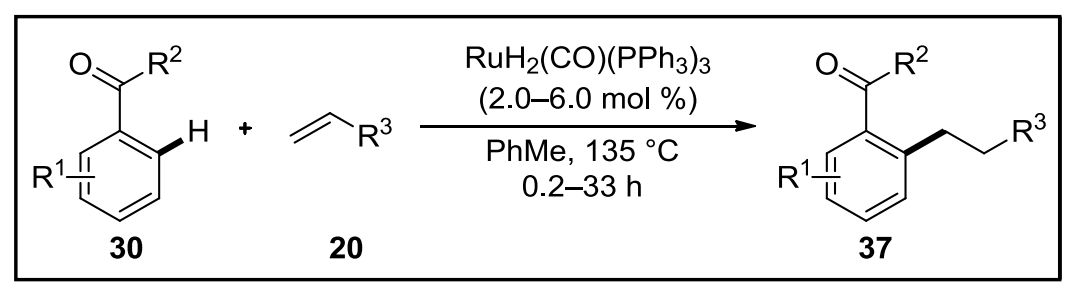

Scheme 1.6. Ruthenium(0)-catalyzed direct hydroarylation by Murai.

Subsequently, progress was made by Genet, Darses and coworkers utilizing a more flexible and practical $\left[\mathrm{RuCl}_{2}(p \text {-cymene })\right]_{2}$ precursor, in association with sodium formiate and a

\footnotetext{
${ }^{31}$ C. Tirler, L. Ackermann, Tetrahedron 2015, DOI:10.1016/j.tet.2015.02.033.

32 a) Y. Hashimoto, T. Ueya ma, T. Fukutani, K. Hirano, T. Satoh, M. Miura, Chem. Lett. 2011, 40, 1165-1166; b) P. B. Arockiam, C. Fischmeister, C. Bruneau, P. H. Dixne uf, Green Chem. 2011, 13, 3075-3078; c) L.-Q. Zhang, S. Yang, X. Huang, J. You, F. Sodi rectng, Chem. Commun. 2013, 49, 8830-8832; d) B. L, J. Ma, W. Xie, H. Song, S. Xu, B. Wang, J. Org. Chem. 2013, 78, 9345-9353.

${ }^{33}$ L. N. Lewis, J. F. Smith, J. Am. Chem. Soc. 1986, 108, 2728-2735.

${ }^{34}$ S. Murai, F. Kakiuchi, S. Sekine, Y. Tanaka, A. Kama tani, M. Sonoda, N. Chatani, Nature 1993, 366, 529-531.

35 a) M. Sonoda, F. Ka kiuchi, A. Kamatani, N. Chatani, S. Murai, Chem. Lett. 1996, 109-110; b) F. Kakiuchi, M. Yamauchi, N. Chatani, S. Murai, Chem. Lett. 1996, 111-112; c) T. Sa to, F. Kakiuchi, N. Chatani, S. Murai, Chem. Lett. 1998, 893-894; d) F. Kakiuchi, T. Sato, M. Yanauchi, N. Chatani, S. Murai, Chem. Lett. 1999, 19-20.

${ }^{36}$ a) F. Kakiuchi, T. Sato, K. Igi, N. Chatani, S. Murai, Chem. Lett, 2001, 386-387. Selected reviews: b) J. R. Andreatta, B. A. McKeown, T. B. Gunnoe, J. Organomet. Chem. 2011, 696, 305-315; c) F. Kakiuchi, Top. Organomet. Chem. 2007, 24, 1-33.

37 B. M. Trost, K. Imi, I. W. Davies, J. Am. Chem. Soc. 1995, 117, 5371-5372.
} 
phosphine ligand (Scheme 1.7)..$^{38}$

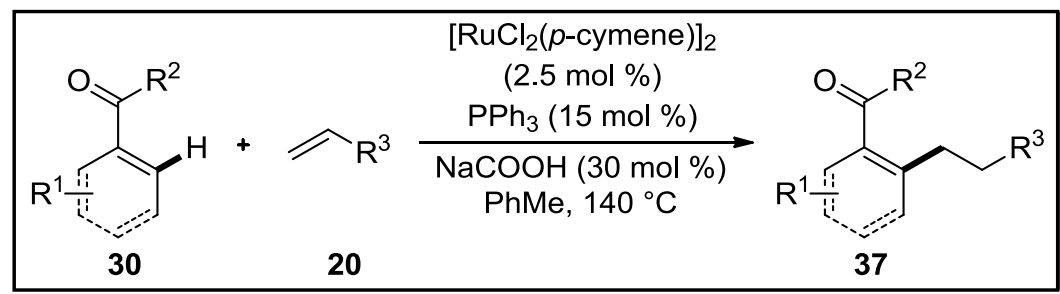

Scheme 1.7. In-situ ruthenium(0) catalyst for hydroarylation by Genet and Darses.

Meanwhile, the Ackermann group disclosed a ruthenium-based catalytic system, consisting of $\left[\mathrm{RuCl}_{2}(\mathrm{cod})\right]_{\mathrm{n}}$ and XPhos, that promoted the addition of arenes to various methylenecyclopropanes $38 .^{39}$

Nevertheless, among the many strategies available, carboxylate-assisted ruthenium(II)-catalyzed $\mathrm{C}-\mathrm{H}$ bond functionalizations were successfully utilized in the hydroarylations by Ackermann and coworkers in 2013..$^{40-41}$ The reaction unveiled a highly efficient and broadly applicable ruthenium(II)biscarboxylate catalyst for additions of $\mathrm{C}-\mathrm{H}$ bonds to methylenecyclopanes and even unactivated alkenes (Scheme 1.8). Very recently, a highly efficient ruthenium-catalyzed $\alpha$-alkylation of a $\mathrm{C}\left(\mathrm{sp}^{3}\right)-\mathrm{H}$ bonds in pyrrolidines was also reported by Ackermann and coworkers. ${ }^{42}$

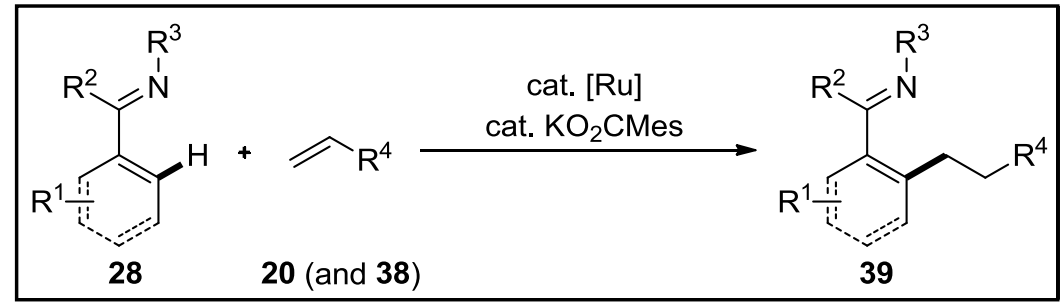

Scheme 1.8. Carboxylate-assisted ruthenium(II)-catalyzed direct hydroarylation.

Despite these notable advances, the hydroarylation with olefins bearing functional groups remained challenging, especially those of more important families of acceptors, such as $\alpha, \beta$-unsaturated acceptors. Moreover, sporadic early reports were limited to the use of rather

\footnotetext{
38 a) R. Martinez, R. Chevalier, S. Darses, J. P. Genet, Angew. Chem. Int. Ed. 2006, 45, 8232-8235; b) R. Martinez, J. P. Genet, S. Darses, Chem. Commun. 2008, 3855-3857; c) R. Martinez, M. O. Simon, R. Chevalier, C. Pautigny, J. P. Genet, S. Darses, J. Am. Chem. Soc. 2009, 131, 7887-7895; d) M.-O. Simon, R. Ma rtinez, J.-P. Genet, S. Darses, Adv. Synth. Catal. 2009, 351, 153-157; e) M. O. Simon, R. Martinez, J. P. Genet, S. Darses, J. Org. Chem. 2010, 75, 208-210; f) M.-O. Simon, S. Darses, J. Org. Chem. 2013, 78, 9981-9985.

${ }_{39}$ a) S. I. Kozhushkov, D. S. Yufit, L. Ackermann, Org. Lett. 2008, 10, 3409-3412; b) L. Ackermann, S. I. Kozhushkov, D. S. Yufit, Chem. Eur. J. 2012, 18, 12068-12077.

40 M. Schinkel, I. Ma rek, L. Ackermann, Angew. Chem. Int. Ed. 2013, 52, 3977-3980.

${ }^{41}$ M. Schinkel, J. Wallbaum, S.I. Kozhushkov, I. Ma rek, L. Ackermann, Org. Lett. 2013, 15, 4482-4484.

${ }^{42}$ M. Schinkel, L. Wang, K. Bielefeld, L. Ackermann, Org. Lett. 2014, 16, 1876-1879.
} 
expensive $^{43}$ rhodium $^{44}$ or rhenium ${ }^{45}$ catalysts. Until the introduction of bidentate-chelation assistance, Chatani and coworkers described a new ruthenium-catalyzed $\mathrm{C}-\mathrm{H}$ hydroarylation of aromatic amides (40) with a wide range of $\alpha, \beta$-unsaturated ketones (41) via bidentate-chelation assistance (Scheme 1.9). ${ }^{46}$

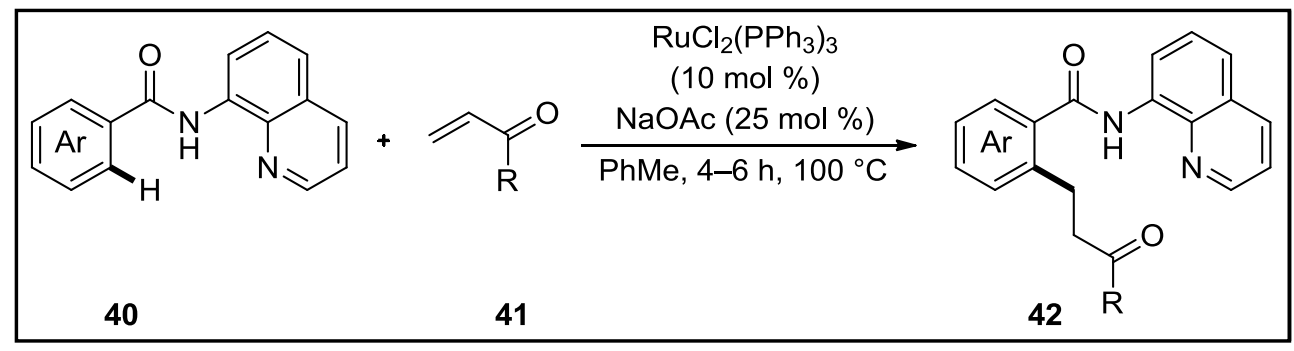

Scheme 1.9. Ruthenium(II)-catalyzed direct hydroarylation of aromatic amides 40 with $\alpha, \beta$-unsaturated ketones 41 .

In spite of the significant progress achieved in the last two decades in the area of ruthenium-catalyzed direct hydroarylation, a great deal of work, such as improving the tolerance of a range of functional groups, still has to be done in this field of catalysis.

\subsection{Cobalt-Catalyzed C-H Bond Functionalizations}

Over the last few decades, most of the remarkable advances in transition metal-catalyzed $\mathrm{C}-\mathrm{H}$ bond functionalization were achieved employing the expensive ${ }^{43}$ second-row transition metals. The development of catalysts based on the naturally more abundant first-row transition metals and complexes, which would enable $\mathrm{C}-\mathrm{H}$ bond functionalization to be accomplished under mild reaction conditions, would present a more activation strategy. Consequently, the use of $3 \mathrm{~d}$ transition metal catalysts has witnessed considerable recent attention. $^{47}$

Among the first-row transition metals, the notable power of cobalt salts as effective catalysts

\footnotetext{
${ }^{43}$ The price of transition metals: see a) http://www.platinum.matthey.com/; b) http://www.chemicool.com/ (20.05.2015).

44 a) S. G. Lim, J. A. Ahn, C. H. Jun, Org. Lett. 2004, 6, 4687-4690; b) L. Yang, C. Correia, C. Li, Org. Biomol. Chem. 2011, 9, 7176-7179; c) L. Yang, B. Qian, H. Huang, Chem. Eur. J. 2012, 18, 9511-9515.

${ }^{45}$ Y. Kuninobu, Y. Nishina, K. Okaguchi, M. Shouho, K. Takai, Bull. Chem. Soc. Jpn. 2008, 81, 1393-1401.

${ }^{46}$ G. Rouquet, N. Chatani, Chem. Sci. 2013, 4, 2201-2208.

47 a) B. Su, Z.-C. Cao, Z.-J. Shi, Acc. Chem. Res. 2015, 48, 886-896; b) E. Nakamura, T. Hatakeyama, S. Ito, K. Ishizuka, L. Ilies, M. Nakamura, Org. React. 2014, 83, 1-209; c) J. Yamaguchi, K. Muto, K. I ta mi, Eur. J. Org. Chem. 2013, 19-30; d) N. Yoshikai, Synlett 2011, 1047-1051; e) Y. Nakao, Chem. Rec. 2011, 11, 242-251; f) E. Nakamura, N. Yoshikai, J. Org. Chem. 2010, 75, 6061-6067; f) A. Kulka mi, O. Daugulis, Synthesis 2009, 4087-4109.
} 
for homocouplings of Grignard reagents was pioneered by Kharasch and Fields in $1941{ }^{48}$ The first example of cobalt used in chelation-assisted $\mathrm{C}-\mathrm{H}$ functionalization was developed in 1955 by Murahashi who revealed an ortho-carbonylation reaction of aldimine 43 using dicobalt octacarbonyl as the catalyst, giving phthalimidine 44 again under rather harsh reaction conditions, however under high temperature and pressure (Scheme 1.10a). ${ }^{49}$ Later, Murahashi and Horiie showed that azobenzene 45 could undergo a similar direct metalation furnishing the indazolone 46 (Scheme 1.10b). ${ }^{50}$ In the next few decades, the application of cobalt in chelation-assisted $\mathrm{C}-\mathrm{H}$ bond functionalization was stagnant until 1994, when a $\mathrm{Co}(\mathrm{H})\left(\mathrm{N}_{2}\right)\left(\mathrm{PPh}_{3}\right)_{3}$-catalyzed ortho-alkenylation of azobenzene derivative 47 with tolane (1) was reported (Scheme 1.10c). ${ }^{51}$ Meanwhile, Kle in and coworkers described the first example of a well-defined stoichiometric cyclometalation, employing azobenzene and $\mathrm{Co}(\mathrm{Me})\left(\mathrm{PMe}_{3}\right)_{4}{ }^{52}$

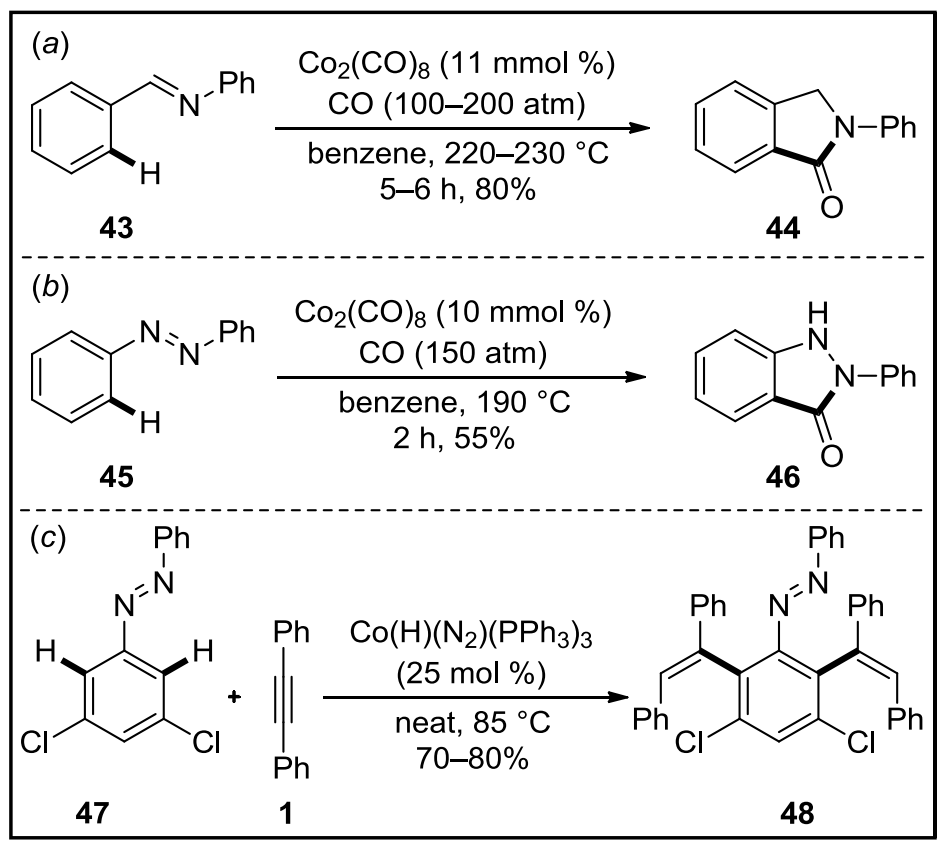

Scheme 1.10. Cobalt-mediated chelation-assisted $\mathrm{C}-\mathrm{H}$ bond functionalizations reported before 1995 .

Further research provided a series of aromatic and olefinic substrates bearing various

\footnotetext{
48 M. S. Kha rasch, E. K. Fields, J. Am. Chem. Soc. 1941, 63, 2316-2320.

49 S. Murahashi, J. Am. Chem. Soc. 1955, 77, 6403-6404.

50 S. Murahashi, S. Horiie, J. Am. Chem. Soc. 1956, 78, 4816-4817.

${ }^{51}$ G. Halbritter, F. Knoch, A. Wolski, H. Kisch, Angew. Chem. Int. Ed. 1994, 33, 1603-1605.

52 H.-F. Klein, M. Helwig, U. Koch, U. Flörke, H.-J. Haupt, Z. Naturforsch. B: Chem. Sci. 1993, 48, 778-784.
} 
directing groups containing nitrogen,${ }^{52-53}$ oxygen,${ }^{54}$ sulfur ${ }^{55}$ and phosphorus ${ }^{56-57,58}$ atoms, which could assist the stoichimetric oxidative addition of the ortho $\mathrm{C}-\mathrm{H}$ bond to the cobalt complex, with concomitant reductive elimination of methane. In particular, both the five- and six-membered cobaltocycles can be formed with phosphorus chelating ligands via $\mathrm{C}-\mathrm{H}$ bond activation, including a $\mathrm{C}\left(\mathrm{sp}^{3}\right)-\mathrm{H}$ bond (Scheme 1.11). ${ }^{54}$ All of this knowledge implied that complexes of cobalt could potentially allow for mild $\mathrm{C}-\mathrm{H}$ bond functionalization although still facing many challenges.

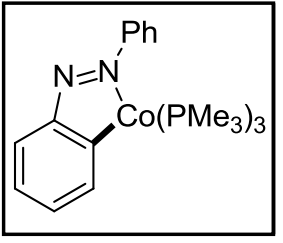

49

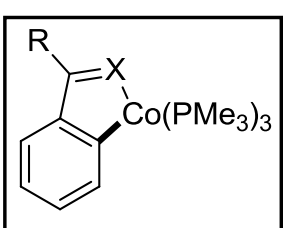

51 $\mathrm{X}=\mathrm{NH}, \mathrm{O}, \mathrm{S}$<smiles>c1ccc(N=Nc2ccccc2)cc1</smiles>

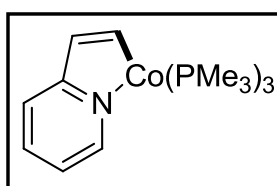

53

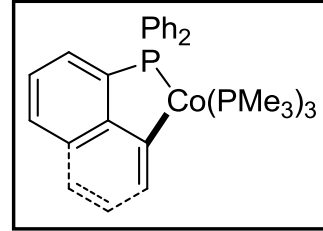

55<smiles>[X]C(=C)c1ccccc1</smiles>

\begin{tabular}{l|l}
50 & 52
\end{tabular}

52<smiles>Cc1ccc2c(P)cccc2c1</smiles>

54

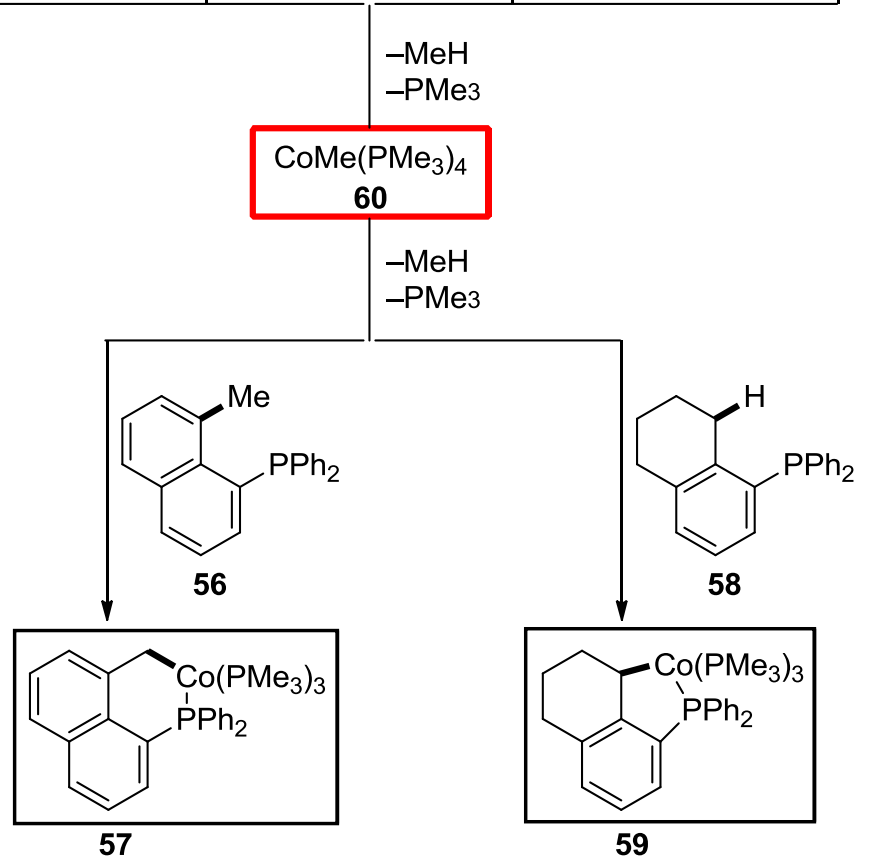

Scheme 1.11. Stoichiometric cyclometalation with cobalt complex 60.

\footnotetext{
53 H.-F. Kein, S. Ca madanli, R. Beck, D. Le ukel, U. Flörke, Angew. Chem. Int. Ed. 2005, 44, 975-977.

54 S. Ca madanli, R. Beck, U. Flörke, H.-F. Klein, Dalton Trans. 2008, 5701-5704.

${ }^{55}$ R. Beck, H. Sun, X. L, S. Camadanli, H.-F. Kein, Eur. J. Inorg. Chem. 2008, 3253-3257.

${ }^{56}$ H.-F. Klein, S. Schneider, M. He, U. Flörke, H.-J. Ha upt, Eur. J. Inorg. Chem. 2000, 2295-2301.

57 H.-F. Klein, R. Beck, U. Flörke, H.-J. Haupt, Eur. J. Inorg. Chem. 2003, 1380-1387.

${ }^{58}$ R. Beck, H. Sun, X. L, H.-F. Klein, Z. Anorg. Allg. Chem. 2009, 635, 99-105.
} 


\subsubsection{Cobalt-Catalyzed C-H Bond Functionalizations in Aldehydes}

Based on early contributions, functionalization of the aldehyde $\mathrm{C}-\mathrm{H}$ bond was first reported by Brookhart and coworkers in 1997. The $\mathrm{Cp} * \mathrm{Co}\left(\mathrm{CH}_{2}=\mathrm{CHSiMe}_{3}\right)_{2}$ (61) catalyst succeeded in the hydroacylation of olefins (Scheme 1.12). ${ }^{59-60}$ Further research described clear evidence for the oxidative addition of $\mathrm{C}\left(\mathrm{sp}^{2}\right)-\mathrm{H}$ bonds to the $\mathrm{Cp} * \mathrm{Co}(\mathrm{I})$ moiety. ${ }^{61}$

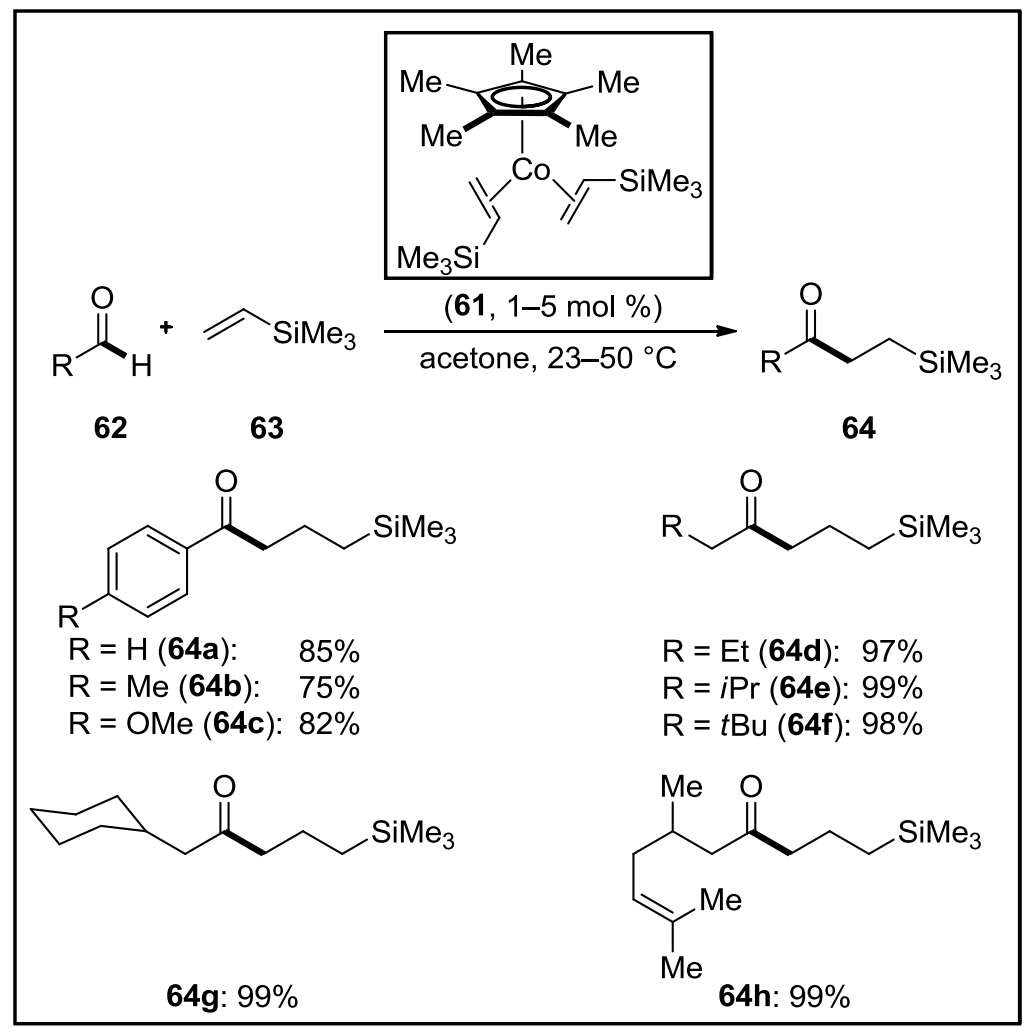

Scheme 1.12. Cobalt-catalyzed formyl C-H functionalization in aldehydes 62 reported before 2014.

Recent progress in the formyl-H bond functionalization of aldehydes was made in 2014 by Dong and Yoshikai. Dong and coworkers described a cobalt-catalyzed hydroacylation of 1,3-dienes 65 with aldehydes $\mathbf{6 2}$, and proposed an oxidative cyclization mechanism that involved a cobaltacycle intermediate, which predetermined the regio- and stereoselectivity of the transformation (Scheme 1.13a). ${ }^{62}$ Taking in consideration this recent work on cobalt and the previously reported contributions on enantioselective Rh-catalyzed transformations, ${ }^{63}$

\footnotetext{
59 C. P. Lenges, M. Brookhart, J. Am. Chem. Soc. 1997, 119, 3165-3166.

60 C. P. Lenges, P. S. White, M. Brookhart, J. Am. Chem. Soc. 1998, 120, 6965-6979.

61 C. P. Lenges, M. Brookha rt, B. E. Grant, J. Organomet. Chem. 1997, 528, 199-203.

62 Q.-A. Chen, D. K. Kim, V. M. Dong, J. Am. Chem. Soc. 2014, 136, 3772-3775.

63 a) D. H. Phan, K. G. M. Kou, V. M. Dong, J. Am. Chem. Soc. 2010, 132, 16354-16355; b) M. M. Coulter, K. G. M. Kou, B. Galligan, V. M. Dong, J. Am. Chem. Soc. 2010, 132, 16330-16333; c) D. H. T. Phan, B. Kim, V. M. Dong, J.
} 
Yoshikai and coworkers reported the first chiral cobalt-diphosphine catalytic system, which allowed for intramolecular hydroacylation of 2-acylbenzaldehydes $\mathbf{6 7}$ and 2-alkenylbenzaldehydes 69 , to generate phthalides 68 or indanones 70 derivatives, respectively (Scheme 1.13b). ${ }^{64}$

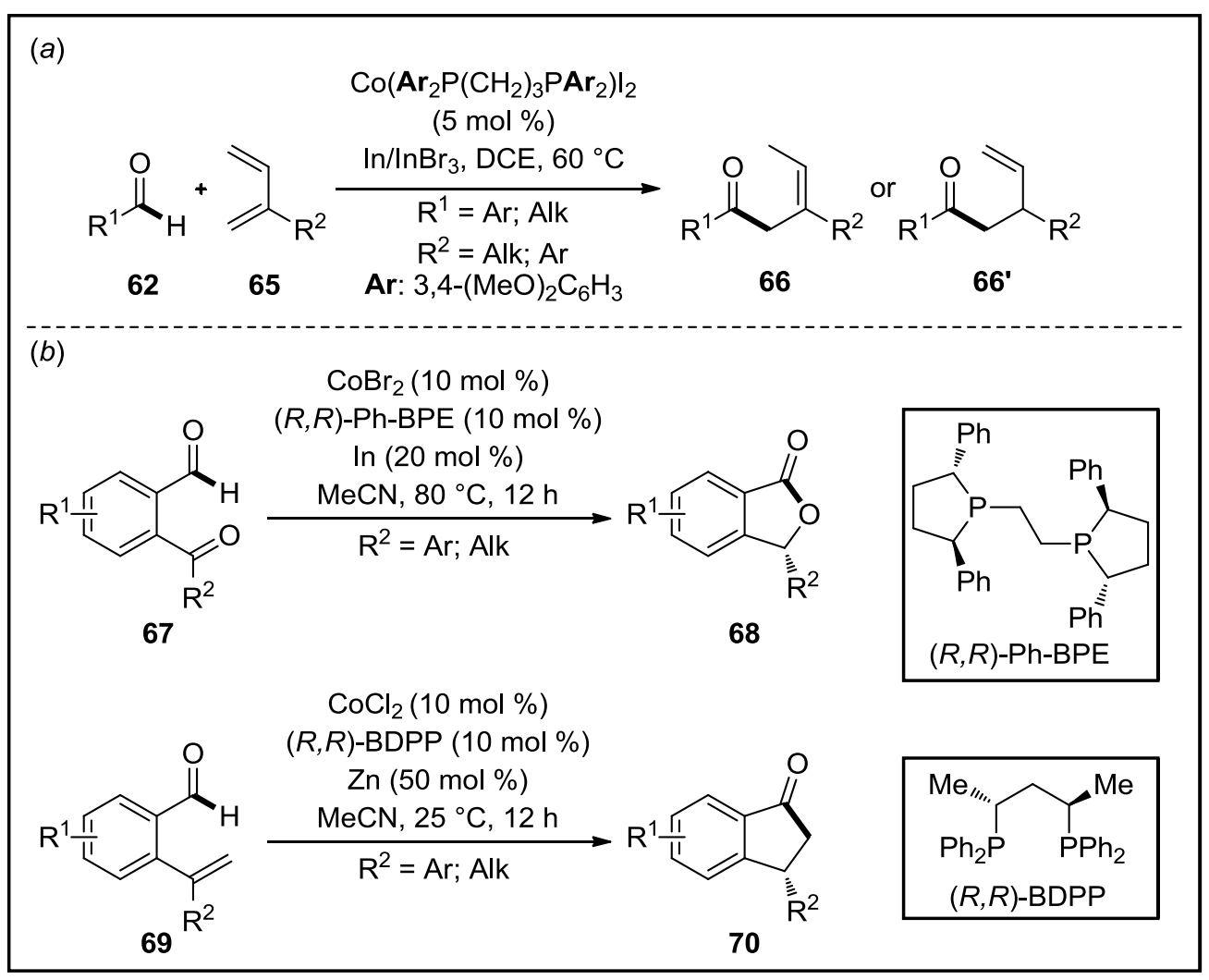

Scheme 1.13. a) Cobalt-catalyzed hydroacylation by oxidative cyclization. b) Cobalt-catalyzed enantioselective intramolecular hydroacylation.

\subsubsection{Low-Valent Cobalt-Catalyzed Chelation-Assisted C-H Bond Functionalizations}

The chelation-assisted direct conversion of $\mathrm{C}-\mathrm{H}$ bonds into $\mathrm{C}-\mathrm{C}$ bonds using low-valent cobalt catalysts was studied independently by the research groups of Yoshikai, ${ }^{65}$ Nakamura, ${ }^{66}$

Am. Chem. Soc. 2009, 131, 15608-15609; d) K. Kundu, J. V. McCullagh, A. T. Jr. Morehead, J. Am. Chem. Soc. 2005, 127, 16042-16043.

${ }^{64}$ J. Yang, N. Yoshikai, J. Am. Chem. Soc. 2014, 136, 16748-16751.

65 Selected representa tive examples: a) W. Xu, N. Yoshikai, Angew. Chem. Int. Ed. 2014, 53, 14166-14170; b) Z. Ding, N. Yoshikai, Angew. Chem. Int. Ed. 2013, 52, 8574-8578; c) K. Gao, N. Yoshikai, J. Am. Chem. Soc. 2013, 135, 9279-9282; d) Z. Ding, N. Yoshikai, Angew. Chem. Int. Ed. 2012, 51, 4698-4701; e) P.-S. Lee, T. Fuji ta, N. Yoshikai, J. Am. Chem. Soc. 2011, 133, 17283-17295; f) K. Gao, P.-S. Lee, T. Fujita, N. Yoshikai, J. Am. Chem. Soc. 2010, 132, 12249-12251.

${ }^{66}$ a) Q. Chen, L. Ilies, N. Yoshikai, E. Naka mura, Org. Lett. 2011, 13, 3232-3234; b) L. Ilies, Q. Chen, X. Zeng, E. Nakamura, J. Am. Chem. Soc. 2011, 133, 5221-5223; c) Q. Chen, L.llies, E. Nakamura, J. Am. Chem. Soc. 2011, 133, 428-429. 
and Ackermann. ${ }^{67-68}$

\subsubsection{Low-Valent Cobalt-Catalyzed C-H Bond Functionalizations}

According to the two different types of developed cobalt-catalyzed $\mathrm{C}-\mathrm{H}$ bond functionalization, the subsequent discussion in this section will be divided in two topics:

\section{(a) Cobalt-catalyzed hydroarylation of alkynes and ole fins}

Based on Kisch's report on the first cobalt(I)-catalyzed ortho-dialkenylation reactions, ${ }^{51}$ Yoshikai and coworkers devised a ternary catalytic system consisted of $\mathrm{CoBr}_{2}$, phosphine ligand $\left(\mathrm{PMePh}_{2}\right)$ and a reductant $(\mathrm{MeMgCl})$, which catalyzed the hydroarylation reaction between 2-arylpyridines $\mathbf{2 8}$ and oct-4-yne $\mathbf{1}$ to yield the desired hydroarylated product $\mathbf{7 1}$ (Scheme 1.14a). ${ }^{65 f}$ The scope of cobalt-catalysis was further expanded to use pyrimidin-2-yl as a removable directing group. ${ }^{69}$ Remarkably, the C2-selective alkenylation of $\mathrm{N}$-pyrimidylindoles 7 with internal alkynes $\mathbf{1}$ was catalyzed by low-valent cobalt at ambient temperature (Scheme 1.14b). ${ }^{65 \mathrm{~d}}$

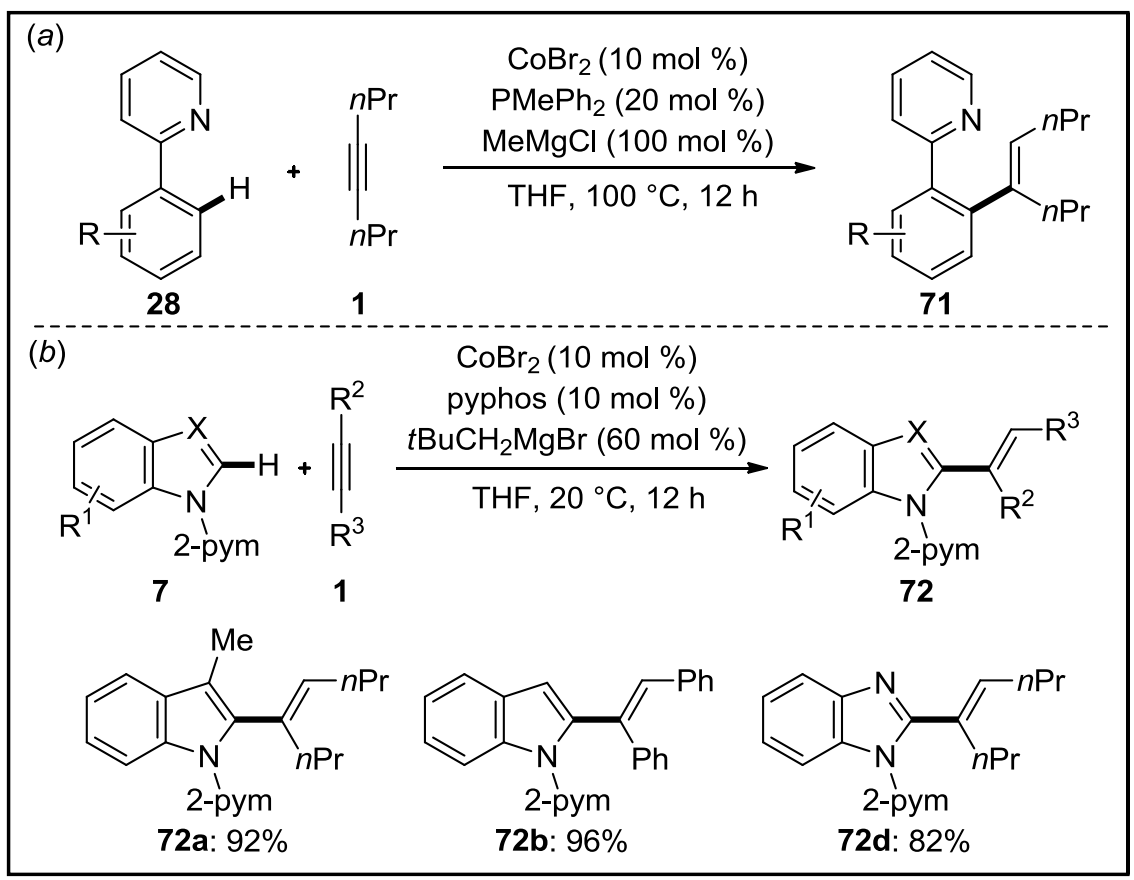

Scheme 1.14. Cobalt-catalyzed hydroarylation via $\mathrm{C}-\mathrm{H}$ bond activation in 2-arylpyridines 28 and $N$-(pyrimidin-2-yl) indoles 7.

\footnotetext{
${ }^{67}$ a) B. Punji, W. Song, G. Shevchenko, L. Ackermann, Chem. E. J. 2013, 19, 10605-10610; b) W. Song, L. Ackermann, Angew. Chem. Int. Ed. 2012, 51, 8251-8254.

${ }^{68}$ See also: a) L. Grigorjeva, O. Daugulis, Org. Lett. 2014, 16, 4688-4690; b) L. Griorje va, O. Daugulis, Org. Lett. 2014, 16, 4684-4687; c) L. Grigorjeva, O. Daugulis, Angew. Chem. Int. Ed. 2014, 53, 10209-10212.

69 L. Ackermann, A. V. Lygin, Org. Lett. 2012, 14, 764-767.
} 
Schiff bases (73 and $\mathbf{4 3})$ as directing groups behaved in a similar fashion to phenylpyridine (28). The additions of aryl ketimines 73 and aldimines 43 to internal alkynes were achieved by a catalytic system of $\mathrm{CoBr}_{2}$, phosphine ligand, Grignard reagent and pyridine. ${ }^{67 e}$ Under acidic reaction conditions, the diarylacetylenes $\mathbf{1}$ afforded the corresponding ketones $\mathbf{7 4}$ (Scheme 1.15a) and aldehydes 75 (Scheme 1.15b), while the products of dialkyl- and alkylarylacetylenes underwent cyclization to give benzofulvene derivatives. ${ }^{65 e, 70}$

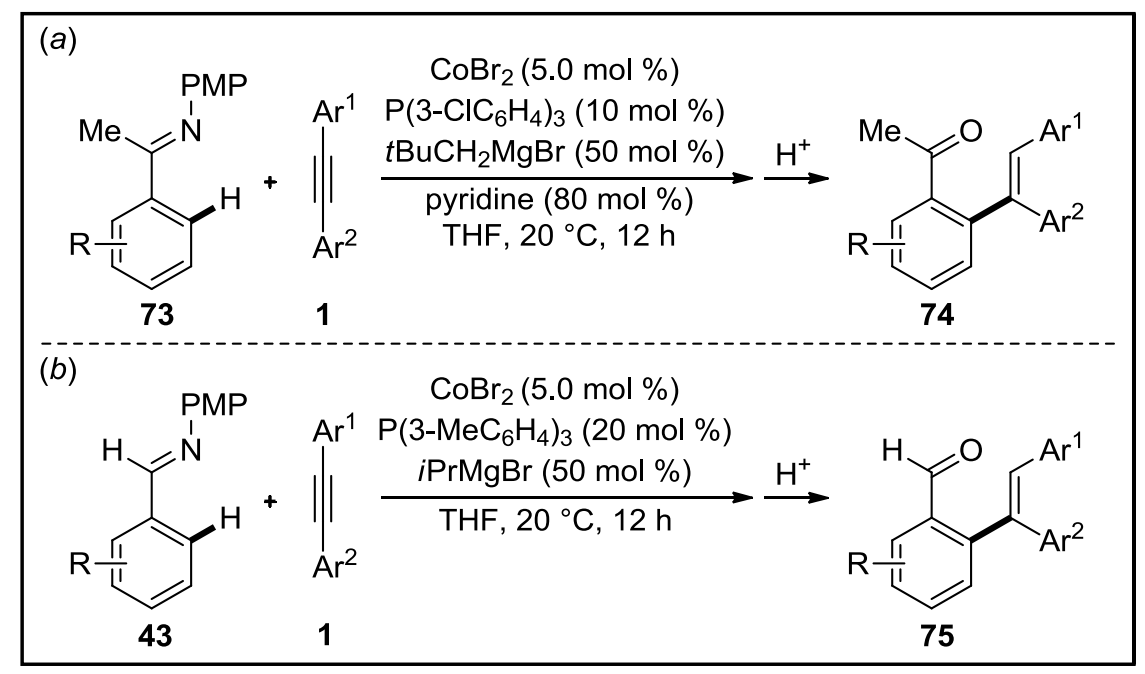

Scheme 1.15. Cobalt-catalyzed hydroarylations with aryl ketimines 73 and aldimines 43 via $\mathrm{C}-\mathrm{H}$ activation.

The proposed catalytic cycle of these novel cobalt-catalyzed direct hydroarylations began with the reduced form of the active cobalt catalyst, which was generated from the cobalt(II) precatalyst and an excess of $t \mathrm{BuCH}_{2} \mathrm{MgBr}$. Precoordination of the alkyne $\mathbf{1}$ to the active cobalt species was followed by oxidative addition of the ortho $\mathrm{C}-\mathrm{H}$ bond in $\mathbf{7 3}$ to the cobalt complex A. Intramolecular hydrocobaltation in complex $\mathbf{B}$ and subsequent reductive elimination in the intermediate $\mathbf{C}$ furnished the desired product and regenerated the cobalt catalyst (Scheme 1.16). ${ }^{5 \mathrm{j}, 65 \mathrm{e}}$ In the case of unsymmertrical alkynes $\mathbf{1}$, the product via $\mathrm{C}-\mathrm{C}$ bond formation at the less hindered acetylenic carbon was formed predominantly. Such a regioselectivity was rationalized in terms of significant steric interactions on the cobalt center upon the transformation $\mathbf{B} \rightarrow \mathbf{C}$.

\footnotetext{
70 T. Ya makawa, N. Yoshikai, Tetrahedron 2013, 69, 4459-4465.
} 


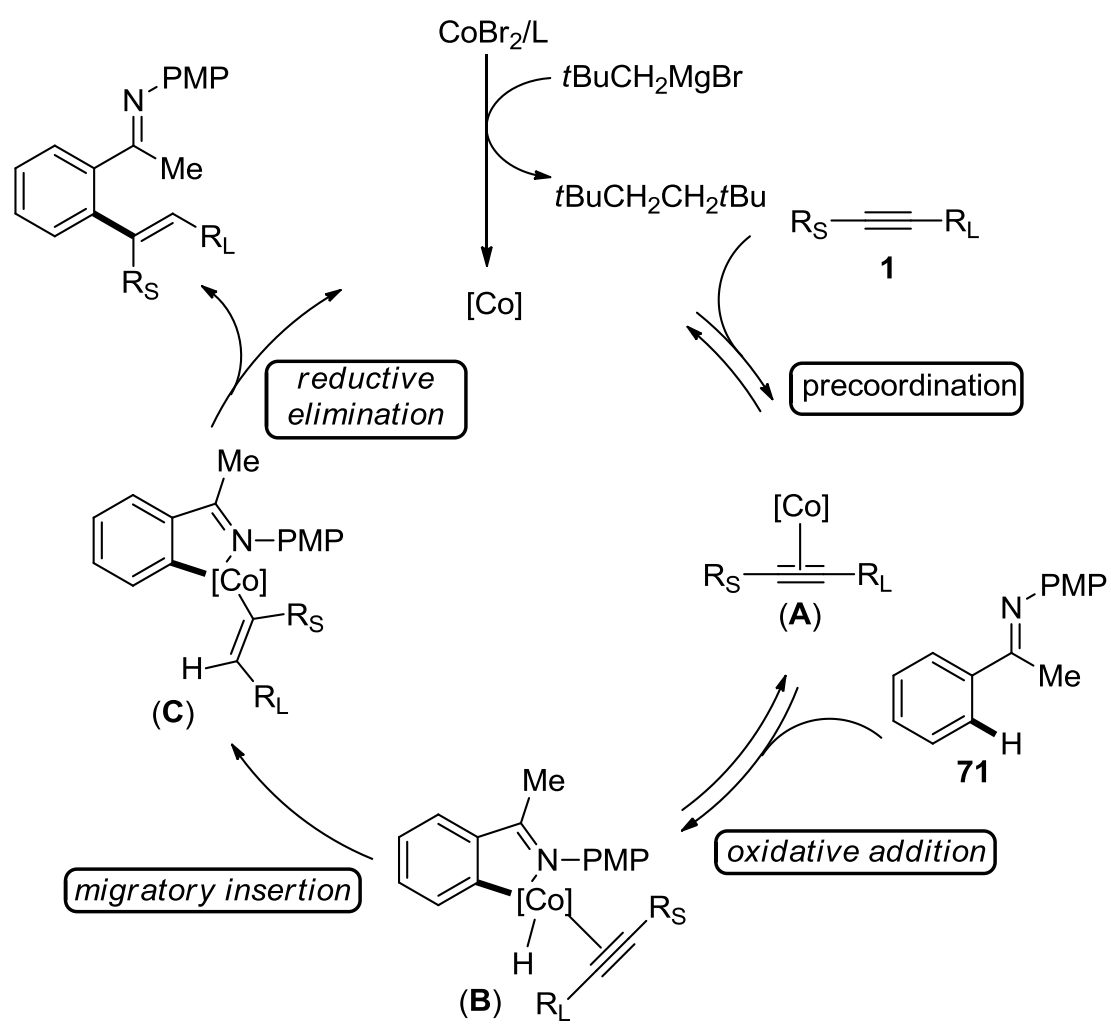

Scheme 1.16. Plausible catalytic cycle for the cobalt-catalyzed hydroarylation with internal

alkynes 1.

Notably, the hydroarylation proceeded not only on aryl imines but also on olefins via alkenyl $\mathrm{C}-\mathrm{H}$ activation. Thus, dihydropyridine derivatives $\mathbf{7 6}$ were generated by annulations of $\alpha, \beta$-unsaturated imines (Scheme 1.17). ${ }^{71}$

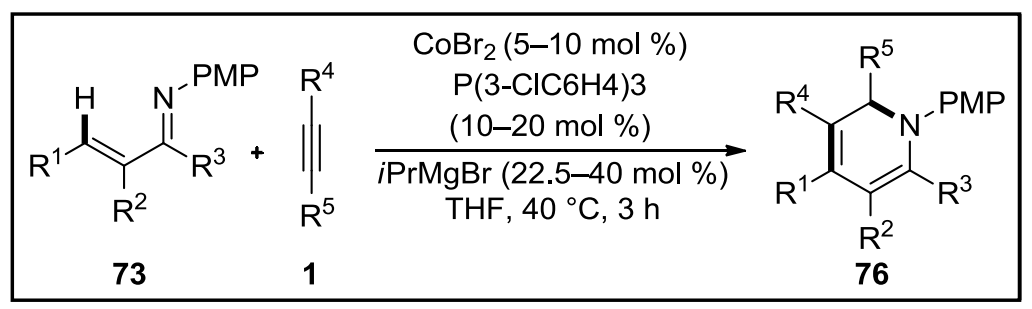

Scheme 1.17. Annulation of alkynes 1 with $\alpha, \beta$-unsaturated imines 73.

Utilizing the differences in bond acidities of diversely positioned $\mathrm{C}-\mathrm{H}$ bonds is another viable approach to perform site-selective $\mathrm{C}-\mathrm{H}$ bond functionalizations in heteroaromatic compounds. Thus, Yoshikai and coworkers developed a cobalt-based catalytic system for the syn-additions of (benzo)azoles $\mathbf{7 7}$ or $\mathbf{7 9}$ to internal alkynes via $\mathrm{C}-\mathrm{H}$ bond functionalization with high chemo-, regio- and stereoselectivities under mild conditions (Scheme 1.18). ${ }^{72}$

${ }^{71}$ T. Ya makawa, N. Yoshikai, Org. Lett. 2013, 15, 196-199.

72 a) Z. Ding, N. Yoshikai, Org. Lett. 2010, 12, 4180-4183; b) Z. Ding, N. Yoshikai, Synthesis 2011, 2561-2566. 


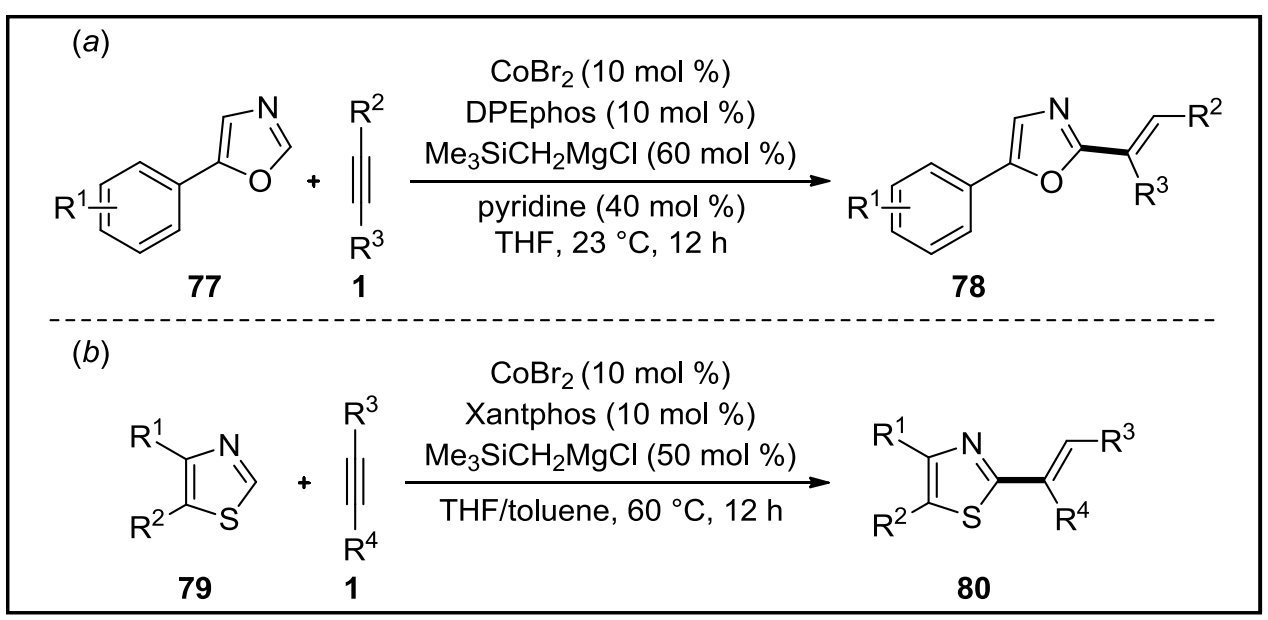

Scheme 1.18. Alkenylations of azoles 77 and 79 with alkynes 1.

Considering the remarkabe high efficacy of alkyne hydroarylation, Yoshikai and coworkers next became intrigued by the development of analogous novel reactions for styrenes. So far, the low-va lent cobalt have already successfully catalyzed pyridinyl- $(\mathbf{2 8}){ }^{73}$ benzamide- $(2)^{66 b}$ and imine-assisted $(\mathbf{7 3})^{65 \mathrm{a}-65 \mathrm{~b}, 74} \mathrm{C}-\mathrm{H}$ bond hydroarylation with good regioselectivity (Scheme $1.19)$.

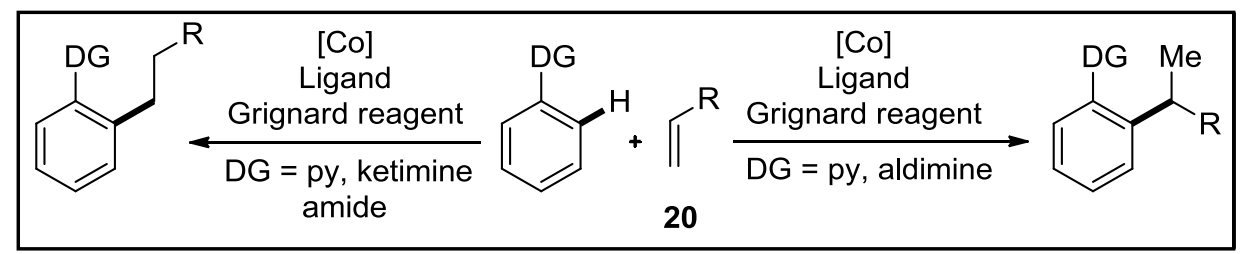

Scheme 1.19. Various directing groups assisted hydroarylations of olefins.

The first example of intramolecular cobalt catalysis, namely the cobalt/NHC-catalyzed intramolecular hydroarylation of alkene moieties after $\mathrm{C}-\mathrm{H}$ bond activation on $\mathrm{C} 2$ position of indole fragments, was investigated by Yoshikai's group (Scheme 1.20). ${ }^{65 b}$ The reaction allowed for the direct transformation of indole derivatives $\mathbf{4 3}$ into dihydropyrroloindoles $\mathbf{8 1}$ or tetrahydropyridoindoles $\mathbf{8 2}$ under mild conditions. Interestingly, the size of the formed cycle was not only dependent on the length of the olefin tether, but also controlled by the steric properties of the NHC ligand. Thus, the cobalt-IPr catalyst promoted the regioselective intramolecular cyclizations of olefins to tetrahydropyridoindole 82, whereas the cobalt-SIMes analogue switched regioselectivity toward the formation of dihydropyrroloindole $\mathbf{8 1}$.

\footnotetext{
73 K. Gao, N. Yoshikai, J. Am. Chem. Soc. 2011, 133, 400-402.

74 a) K. Gao, N. Yoshikai, Angew. Chem. Int. Ed. 2011, 50, 6888-6892; b) P.-S. Lee, N. Yoshikai, Angew. Chem. Int. Ed. 2013, 52, 1240-1244; c) T. Yamakawa, N. Yoshikai, Chem. Asian. J. 2014, 9, 1242-1246.
} 


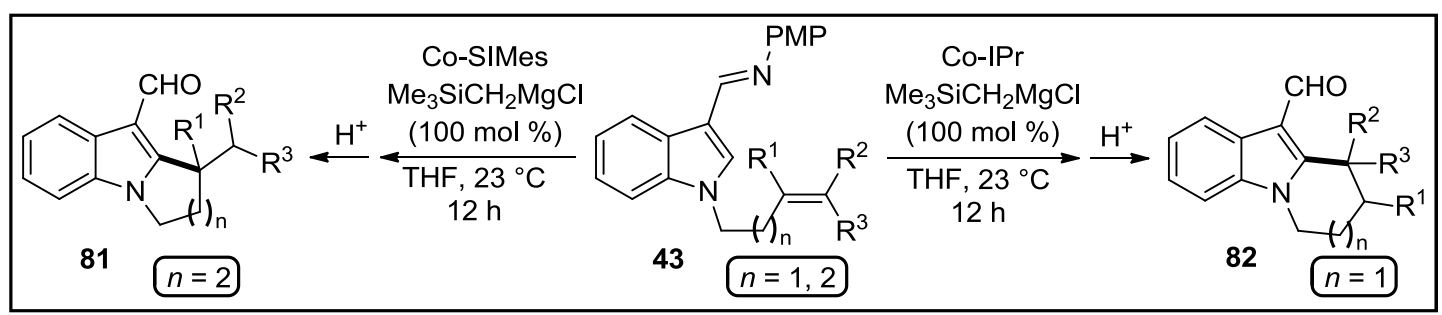

Scheme 1.20. Cobalt/NHC-catalyzed intramolecular hydroarylation leading to dihydropyrroloindoles $\mathbf{8 1}$ or tetrahydropyridoindoles $\mathbf{8 2}$.

\section{(b) Cobalt-catalyzed coupling reactions with organic ele ctrophiles}

In recent years, some examples of cobalt-catalyzed $\mathrm{C}-\mathrm{H}$ coupling reactions with organic electrophiles have been reported. Cobalt-catalyzed ortho-alkylation of benzamides 2 with primary alkyl chlorides $\mathbf{8 1}$ was reported by Nakamura and coworkers (Scheme 1.21a), ${ }^{660}$

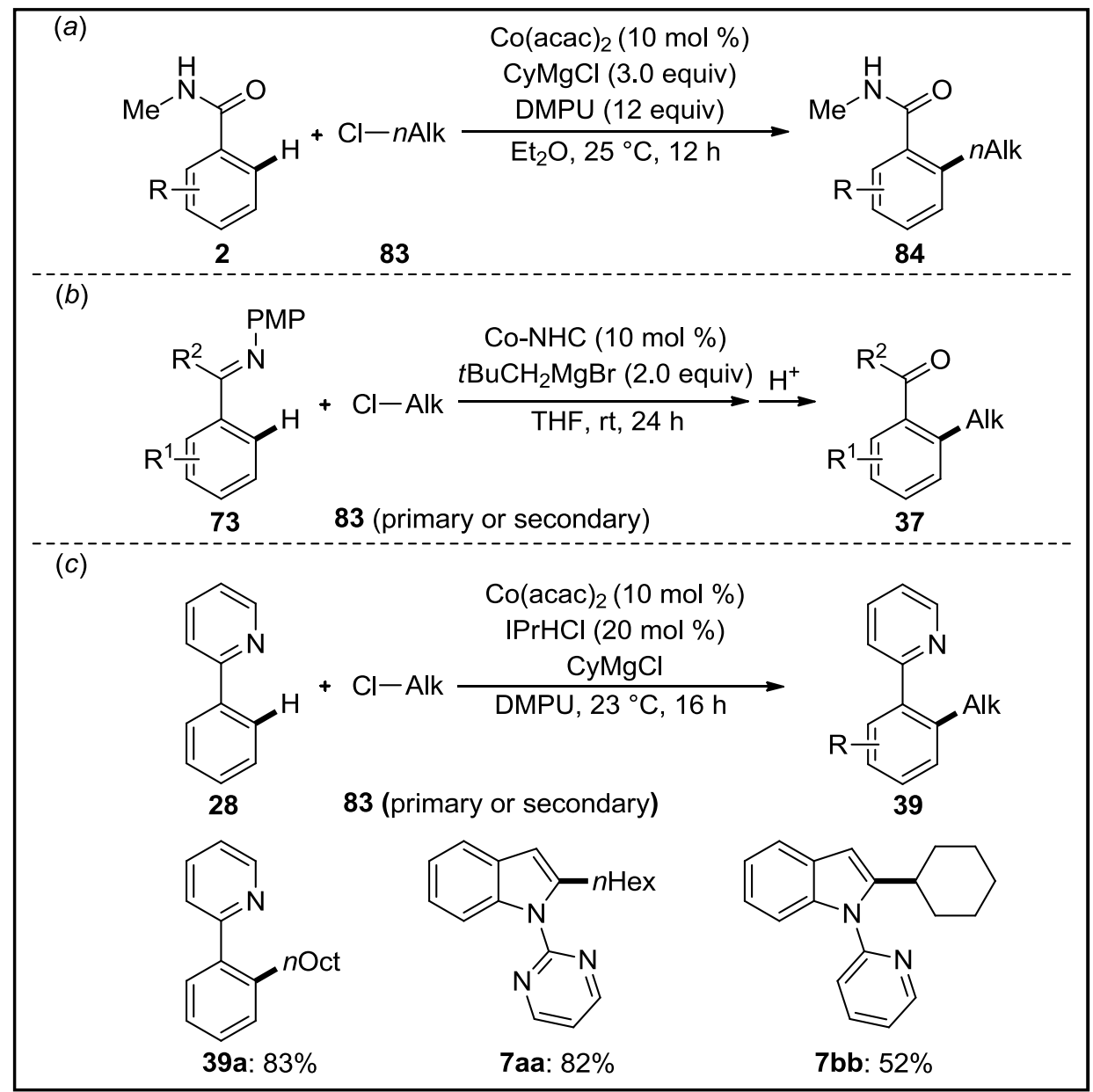

Scheme 1.21. Cobalt-catalyzed $\mathrm{C}-\mathrm{H}$ alkylation with diverse directing groups.

The reaction could also be performed with ketimine- (73), ${ }^{65 \mathrm{c}}$ pyridyl- and pyrimidyl-assistance (28), ${ }^{67 a}$ thus achieving direct alkylation with a broad range of primary and secondary alkyl chlorides and bromides through $\mathrm{C}-\mathrm{H}$ bond activation (Schemes 
1.21b-1.21c). In addition, oxidative ortho-alkylation of benzamides 2 and 2-arylpyridines 28 with alkyl Grignard reagents was also reported by Nakamura and coworkers. ${ }^{66 a}$

The cobalt-catalyzed functionalization of $\mathrm{C}-\mathrm{H}$ bonds could be applied to challenging direct arylation. Ackermann and coworkers initially developed a cobalt-IMes catalytic system for direct $\mathrm{C}-\mathrm{H}$ arylations of arenes with the electronically deactivated aryl sulfamates $\mathbf{8 5}$ and aryl carbamates $\mathbf{8 6}^{67 \mathrm{~b}}$ These direct functionalizations chemoselectively delivered the monoarylated products 87 (Scheme 1.22a). The versatile cobalt catalyst was not limited to 2-arylpyridines $\mathbf{2 8}$ but also set the stage for the synthesis of potentially bioactive $N$-substituted indoles 7.

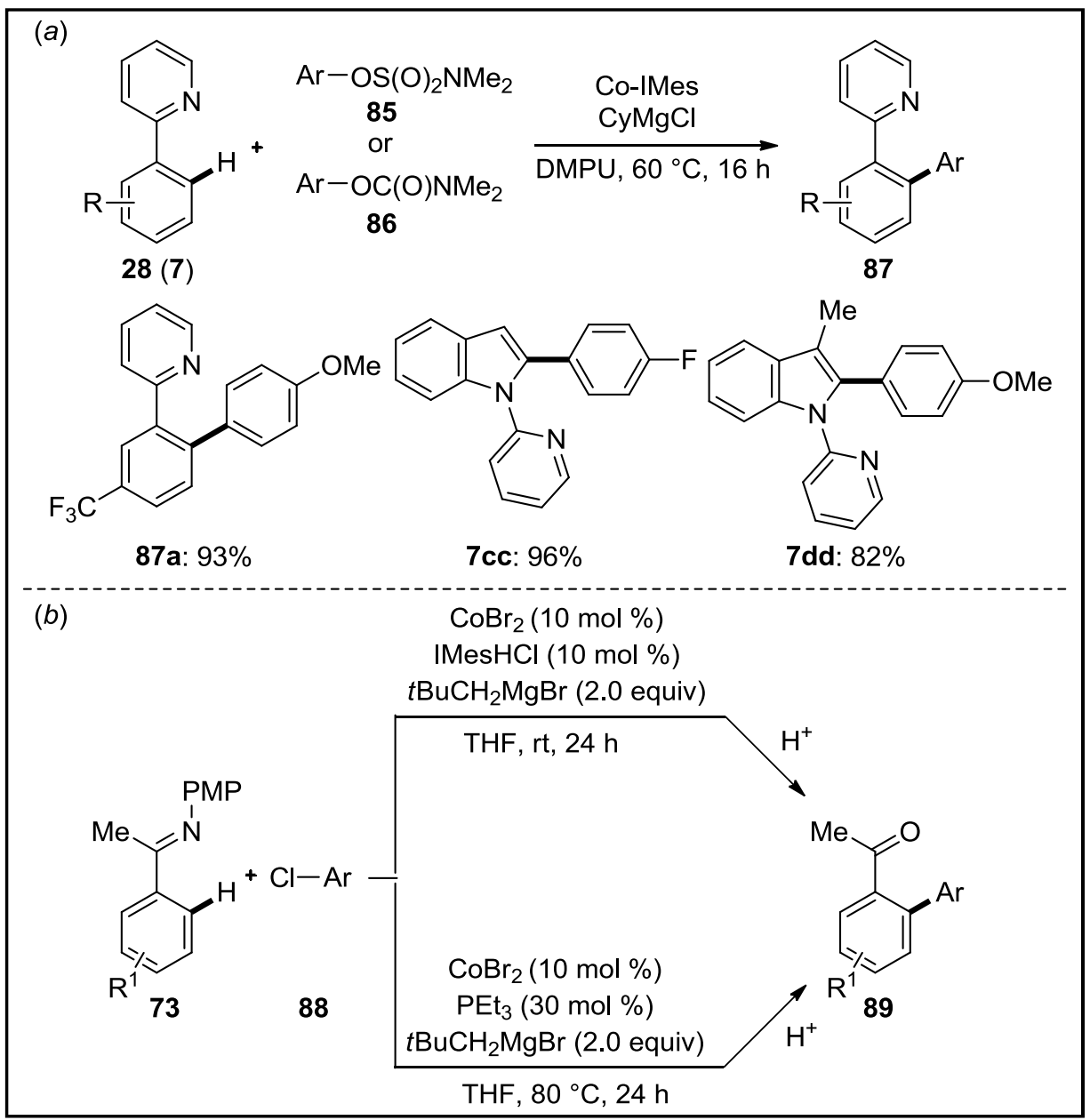

Scheme 1.22. Cobalt/NHC-catalyzed $\mathrm{C}-\mathrm{H}$ bond arylations with diverse directing groups.

Aryl chlorides $\mathbf{8 8}$ are the ideal electrophilic aryl halides for direct arylation reactions, since they are cost-effective, whilst being widely available. Ackermann and Yoshikai explored a cobalt-based catalytic system that allowed the direct arylation of aryl pyridines $\mathbf{2 8}$ and ketimines $\mathbf{7 3}$ with decorated chlorobenzene at ambient or elevated temperatures in the 
presence of NHC or phosphine ligands, respectively. After hydrolys is, biarylketones $\mathbf{8 9}$ were obtained (Scheme 1.22b). ${ }^{67 a, 75}$ In contrast, oxidative arylation of 2-arylpyridines with aryl Grignard reagents was reported by Shi and coworkers. ${ }^{76}$

Meanwhile, the cobalt-IMes catalyst was not restricted to the synthesis of biaryl compounds, but also enabled effective $\mathrm{C}-\mathrm{H}$ bond benzylation reactions on indole $7 \mathbf{a}$ with benzyl phosphates 90 as electrophiles at ambient temperature (Scheme 1.23). ${ }^{67 \mathrm{~b}}$

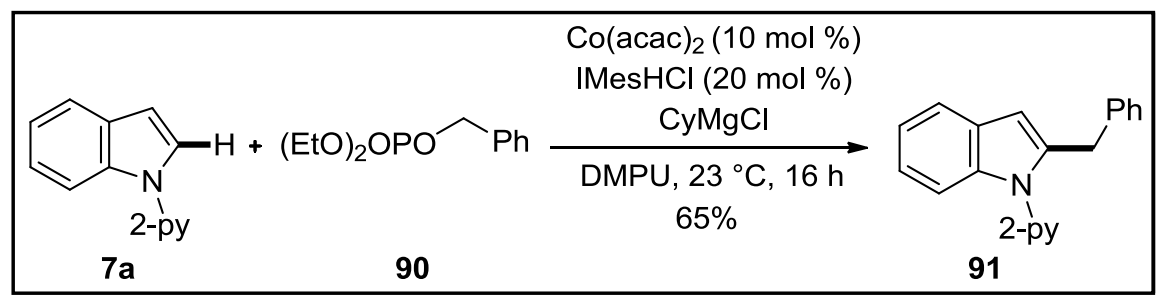

Scheme 1.23. Cobalt-catalyzed C-H benzy lation of indole 7.

The general consensus is that the cobalt-catalyzed $\mathrm{C}-\mathrm{H}$ alkylation involves a radical intermediate. Thus, a proposed catalytic cycle is shown below (Scheme 1.24). ${ }^{5 \mathrm{j}-5 \mathrm{k}}$

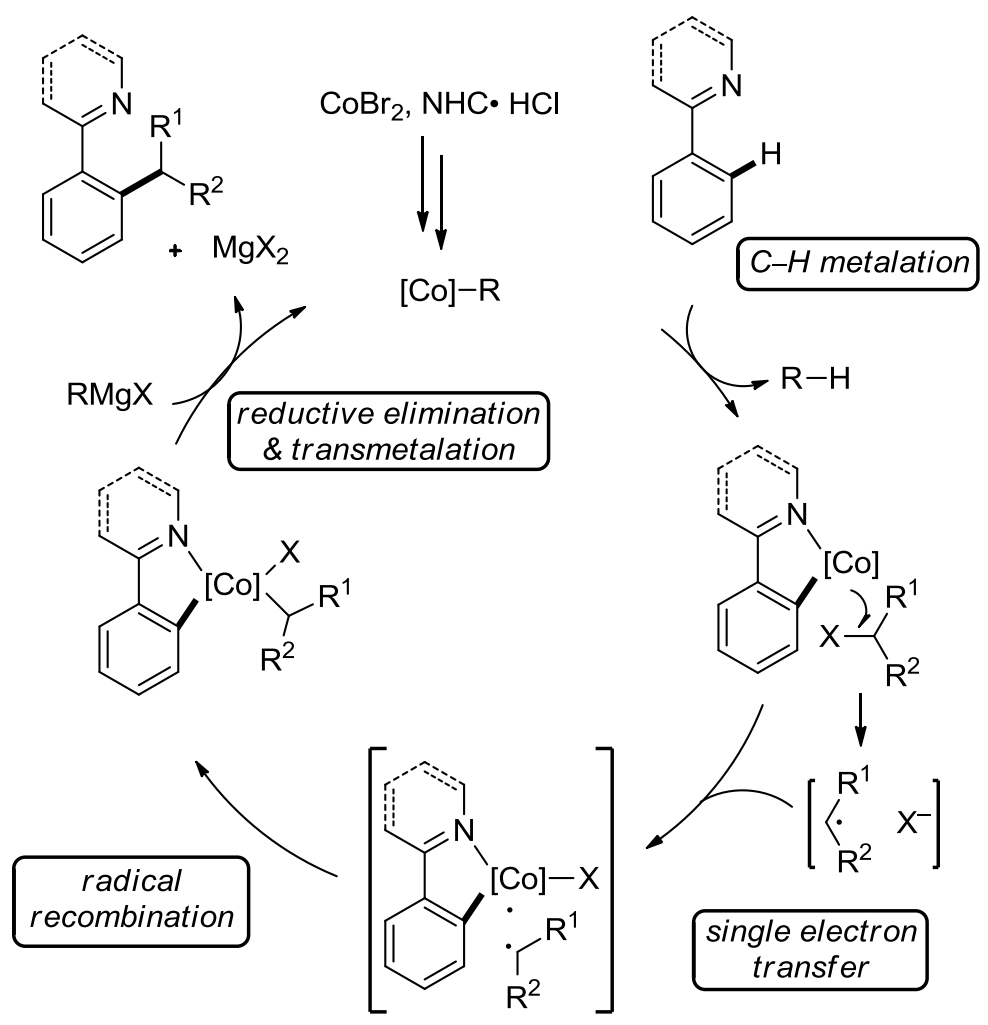

Scheme 1.24. Proposed catalytic cycle for the cobalt-catalyzed $\mathrm{C}-\mathrm{H}$ bond functionalization.

The cycle is initiated by a $\mathrm{C}-\mathrm{H}$ cyclocobaltation and subsequent single electron transfer (SET)

75 K. Gao, P.-S. Lee,C. Long, N. Yoshikai, Org. Lett. 2011, 14, 4234-4237.
76 B. L, Z.-H. Wu, Y.-F. Gu, C.-L. Sun, B.-Q. Wang, Z.-J. Shi, Angew. Chem. Int. Ed. 2011, 50, 1109-1113. 
from the cobalt center to the alkyl halide 83. The desired product resulted from radical $\mathrm{C}-\mathrm{C}$ coupling followed by reductive elimination, and the alkyl cobalt species was regenerated after the transmetalation of cobalt halide with the Grignard reagent.

\subsubsection{Cobalt-Catalyze d Oxidative C-H Bond Functionalizations}

Since Daugulis initially devised the 2-aminoquinoline and picolinamide directing groups for the palladium-catalyzed arylation of $\mathrm{C}\left(\mathrm{sp}^{3}\right)-\mathrm{H}$ bonds in $2005,{ }^{77}$ the bidentate-type directing groups have quickly emerged as a new tool in exploring $\mathrm{C}-\mathrm{H}$ activation reactions. ${ }^{78}$ Interestingly, another remarkable progress was made by Daugulis and coworkers, by reporting an approach for cobalt-catalyzed direct oxidative alkyne annulation via aminoquinoline $\mathbf{4 0}$ and picolinamide 92 as bidentate-chelation assistance. The reaction was successful with both terminal and internal alkynes. This approach provided expedient access to diversely decorated isoquinolin-1-ones $\mathbf{9 3}$ with ample scope of substrates, such as heteroarenes and vinyl amide 40 (Scheme 1.25a). Furthermore, it allowed the subsequent removal of the directing groups under simple reaction conditions (Scheme 1.25b). ${ }^{68 \mathrm{c}}$

\footnotetext{
77 V. G. Zaitsev, D. Shabashov, O. Da ugulis, J. Am. Chem. Soc. 2005, 127, 13154-13155.

${ }^{78}$ Recent reviews on $\mathrm{C}-\mathrm{H}$ activation with bidentate directing groups: a) O. Daugulis, J. Roane, L. D. Tran, Acc. Chem. Res. 2015, 48, 1053-1064; b) L. C. M. Castro, N. Chatani, Chem. Lett. 2015, 44, 410-421; c) M. Corbet, F. De Campo, Angew. Chem. Int. Ed. 2013, 52, 9896-9898; d) G. Rouquet, N. Chatani, Angew. Chem. Int. Ed. 2013, 52, 11942-11959. Selected recent examples demonstrating the power of bidenta te directing groups: e) Q. Gu, H. H. Al Mamari, K. Graczyk, E. Diers, L. Ackermann, Angew. Chem. Int. Ed. 2014, 53, 3868-3871; f) W. Song, S. La ckner, L. Ackermann, Angew. Chem. Int. Ed. 2014, 53, 2477-2480; g) Y. Aihara, N. Chatani, J. Am. Chem. Soc. 2014, 136, 898-901.
} 


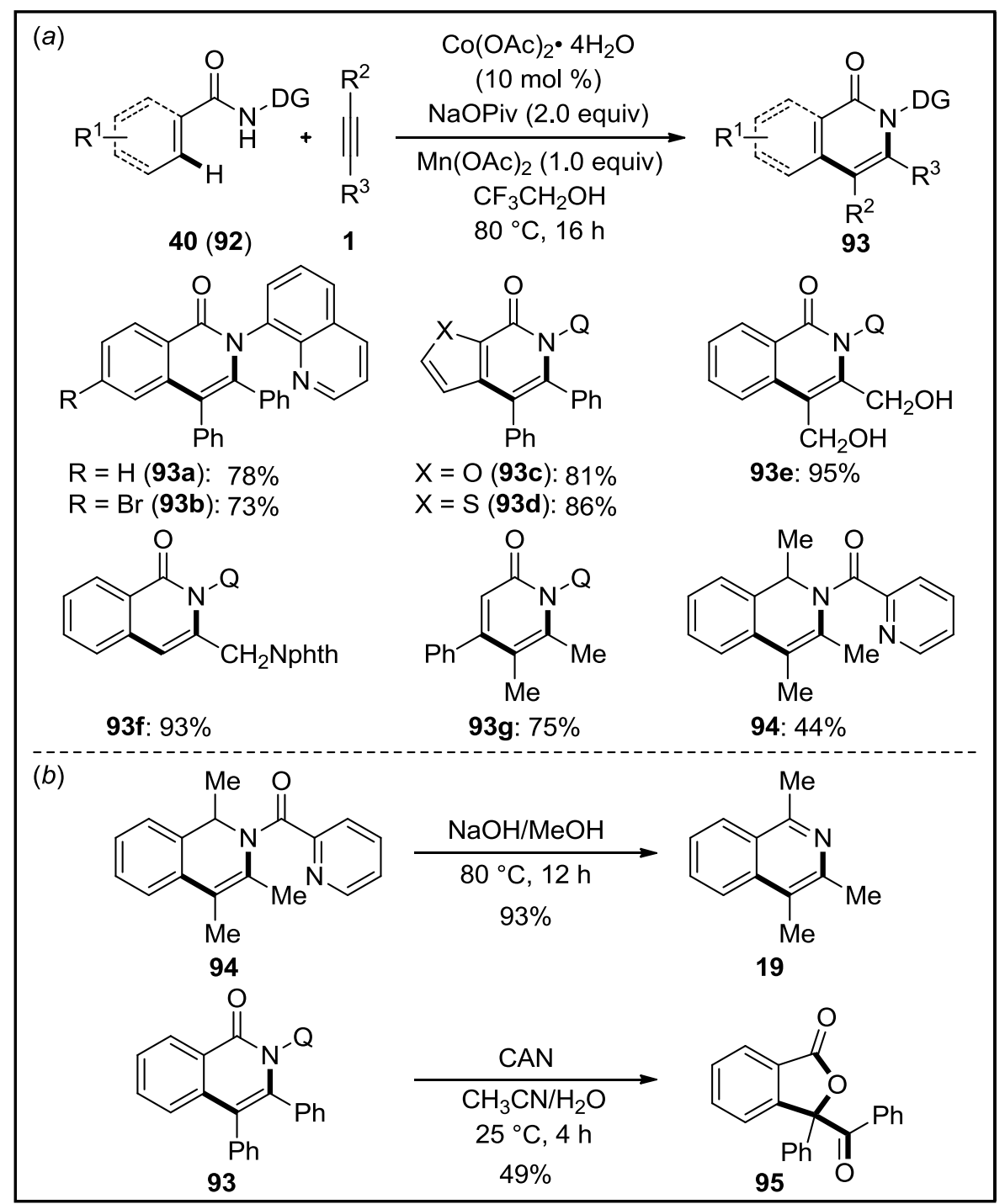

Scheme 1.25. (a) Substrate scope for cobalt-catalyzed C-H annulations. (b) Directing group removal.

After the successful development of cobalt-catalyzed oxidative cyclizations with alkyne 1, further studies indicated that the cobalt catalytic system was not restricted to alkynes, but also exhibited notable power in $\mathrm{C}\left(\mathrm{sp}^{2}\right)-\mathrm{H}$ bond coupling with alkenes. Upon further investigations of the reaction of $\mathrm{N}$-(quinolin-8-yl)benzamide (40) and styrene (20), employing $\mathrm{Co}(\mathrm{acac})_{2}$ as a catalyst, $\mathrm{Mn}(\mathrm{OAc})_{3} \cdot 2 \mathrm{H}_{2} \mathrm{O}$ as a co-catalyst, and oxygen from air as a terminal oxidant, a remarkable scope of aminoquinoline-protected amides of benzoic, heteroaromatic and acrylic acids furnished products 96 in good yields (Scheme 1.26). ${ }^{68 b}$ 


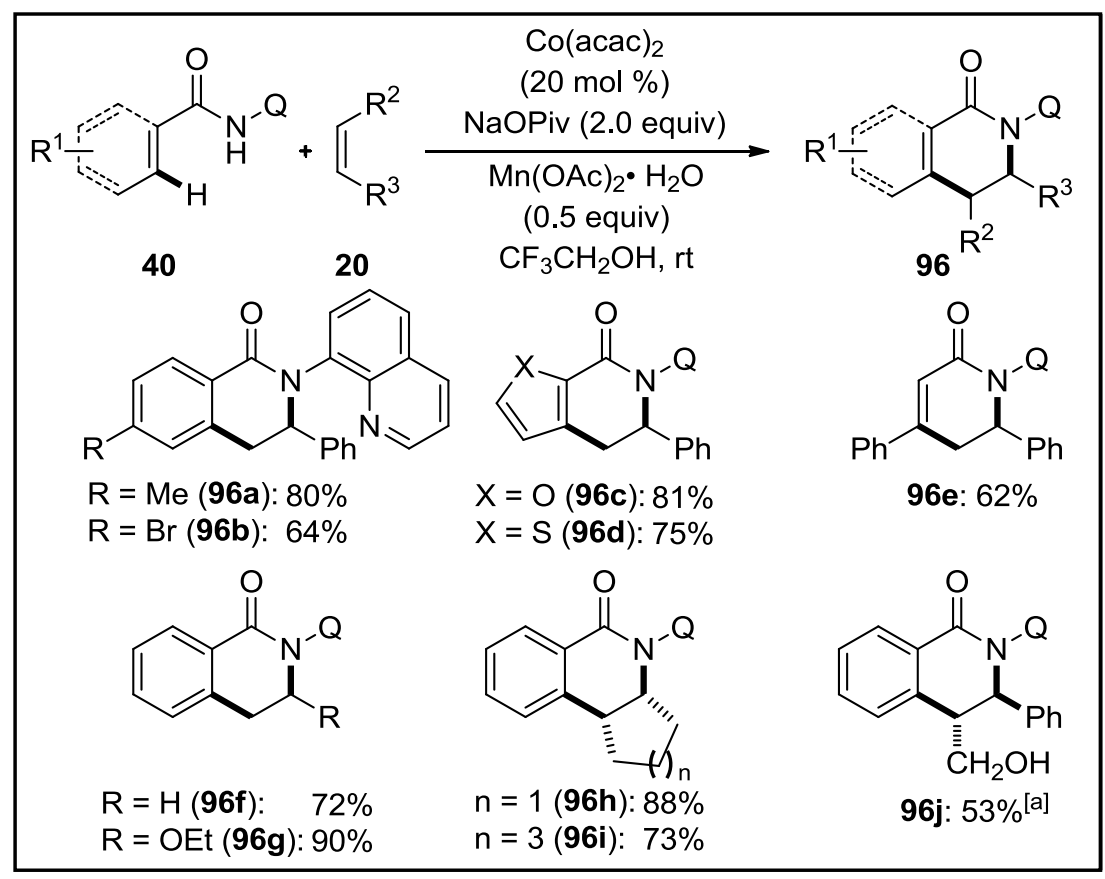

Scheme 1.26. Substrate scope for cobalt-catalyzed alkene annulations through $\mathrm{C}-\mathrm{H}$ bond

$$
\text { activation. [a] } \mathrm{Co}(\mathrm{acac})_{2}(50 \mathrm{~mol} \%), 80^{\circ} \mathrm{C} \text {. }
$$

Based on the high catalytic efficacy of the cobalt catalyst in oxidative annulations of alkynes $\mathbf{1}$ and alkenes 20, Daugulis and coworkers thereafter developed a cobalt-catalyzed direct carbonylation of benzoic- and acrylic acid-derivatives amides $\mathbf{4 0}$ with carbon monoxide through bidentate-chelation assistance. The entire scope was completed at ambient temperature and afforded the desired phthal- and succinimides 97 in good yields (Scheme $1.27 a){ }^{68 a}$

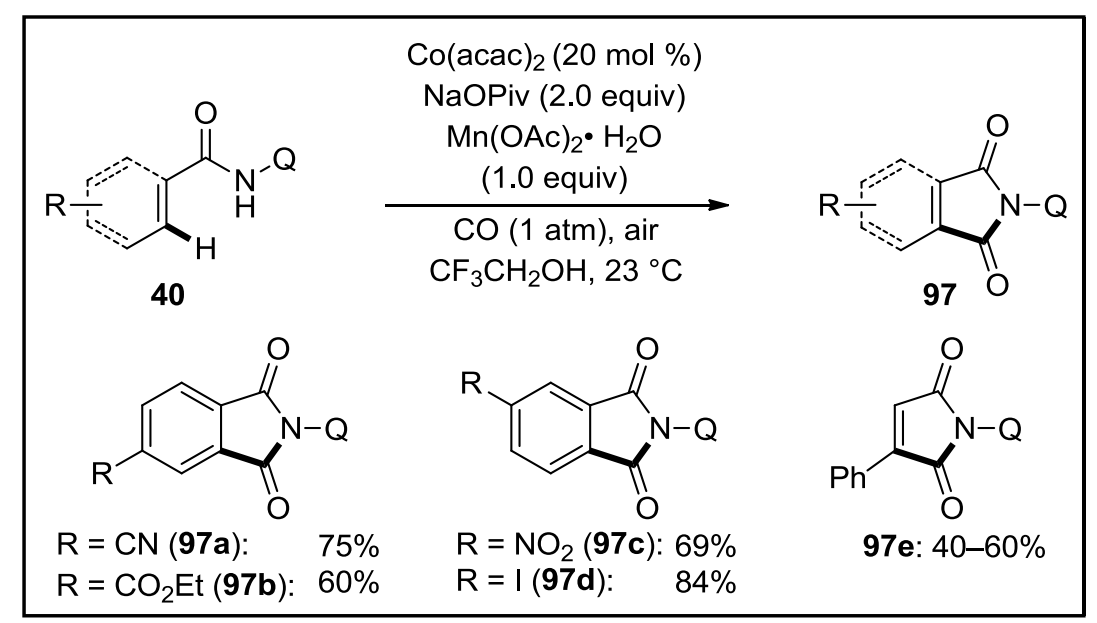

Scheme 1.27. Carbonylation of aminoquinoline-derived amides 40.

A wide range of functional groups, such as halogen, nitrile, ester and cyano substituents, can 
be tolerated under the optimizaed reaction conditions. Intramolecular competition experiments with meta-substituted arenes exhibited high levels of site-selectivity (Scheme 1.27). ${ }^{68 \mathrm{a}}$ The directing group can be easily removed by treatment with methanolic ammonia, and the desired phthalimide 97 were obtained in high yie ld.

Meanwhile, very recently Song and coworkers developed the cobalt-catalyzed $\mathrm{C}-\mathrm{H}$ bond alkoxylation with a cohols in benzamides 98 derived from 2-aminopyridine-1-oxide through a $\mathrm{N}, \mathrm{O}$-bidentate-type directing groups. ${ }^{79}$ The reaction proceeded under mild conditions using $\mathrm{Co}(\mathrm{OAc})_{2} \cdot 4 \mathrm{H}_{2} \mathrm{O}$ as the catalyst and with a wide range of substituted alcohols as well as of benzamides, heteroarenes and substituted vinyl amides 98 decorated with a variety of functional groups (Scheme 1.28a). ${ }^{79}$

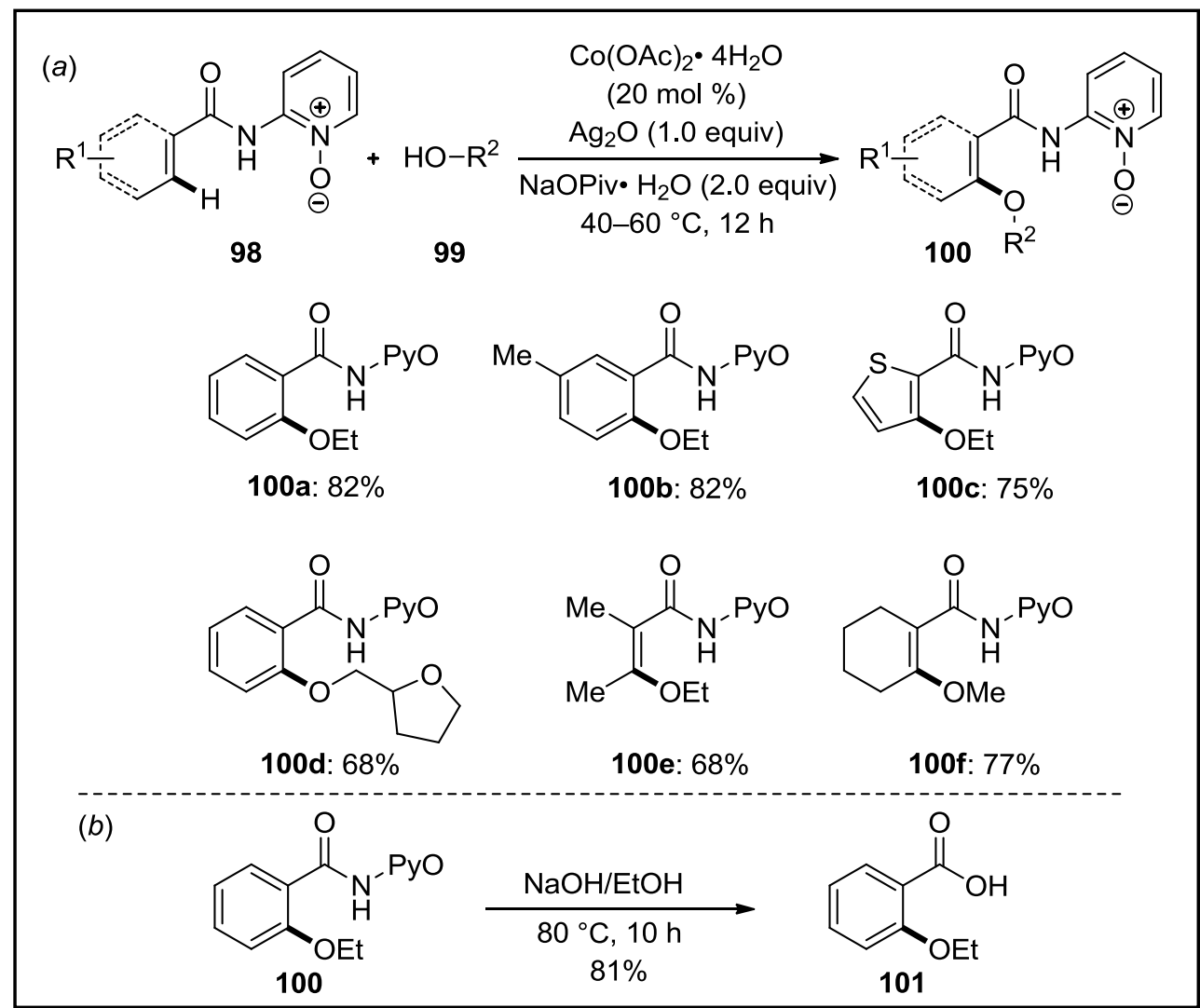

Scheme 1.28. (a) Substrate scope for cobalt-catalyzed C-H alkoxylation. (b) Directing group removal

In addition, the reaction showed a high site-selectivity when meta-substituted substrates were employed. The mechanistic studies revealed that a radical pathway was involved. Further,

\footnotetext{
79 L.-B. Zhang, X.-Q. Hao, S.-K. Zhang, Z.-J. Lu, X.-X. Zheng, J.-F. Gong, J.-L. Niu, M.-P. Song, Angew. Chem. Int. Ed. 2015, 54, 272-275.
} 
kinetic isotope effect (KIE) studies suggested the $\mathrm{C}-\mathrm{H}$ bond activation not to be the rate-limiting step. The 2-aminopyridine-1-oxide directing group can easily be removed affording benzoic acid 101 (Scheme 1.28b).

\subsubsection{High-Valent Cobalt-Catalyze d C-H Bond Functionalizations}

In recent years, $\mathrm{Cp} * \mathrm{Rh}(\mathrm{III})$ complexes have been involved in the rapidly progregressing step-economical ${ }^{80} \mathrm{C}-\mathrm{H}$ bonds functionalizations. ${ }^{5 \mathrm{~b}, 7 \mathrm{a}}$ However, a number of analogous $\mathrm{Cp} * \mathrm{Co}(\mathrm{III})$ complexes have been prepared and characterized up to now (Scheme 1.29). ${ }^{81}$

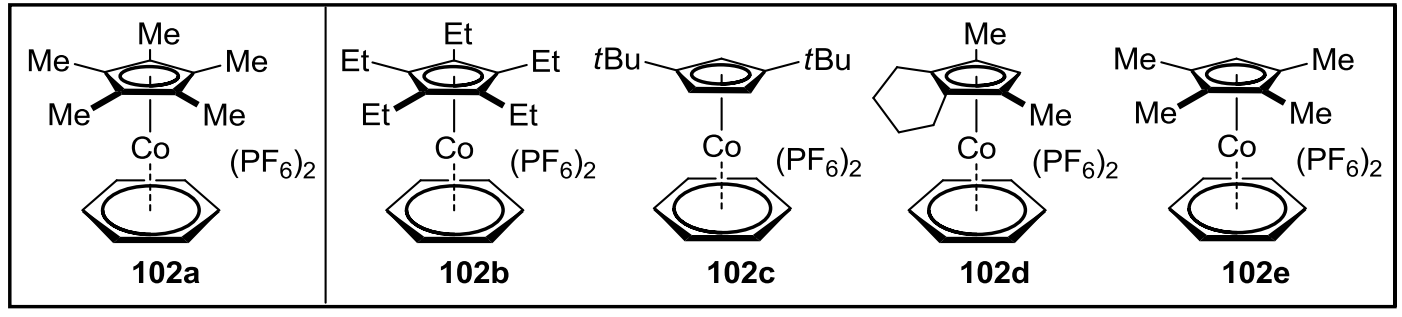

Scheme 1.29. Selected synthesized cationic high-valent cobalt(III) complexes.

Kanai and coworkers chose cobalt, which is isoe lectronic to rhodium, but less expensive and more abundant, and tried to emulate the reactivity of $\mathrm{Cp} * \mathrm{Rh}(\mathrm{III})$. In 2013, they reported on the application of a variety of $\mathrm{Cp} * \mathrm{Co}(\mathrm{III})$ complexes (Scheme 1.29) for synthetic organic transformations, and the $\left[\mathrm{Cp}^{*} \mathrm{Co}(\right.$ benzene $\left.)\right]\left(\mathrm{PF}_{6}\right)_{2}$ complex (102a) appeared to be successful in promoting the addition of 2-arylpyridines $\mathbf{2 8}$ onto multiple bonds in imines 103 (Scheme 1.30a) as well as in $\alpha, \beta$-unsaturated enones 104, and 1-pyrrolylenones 105 (Scheme 1.30b). ${ }^{82}$

\footnotetext{
80 P. A. Wender, V. A. Verma, T. J. Paxton, T. H. Pillow, Acc. Chem. Res. 2008, 41, 40-49.

81 a) E. O. Fischer, R. D. Fisher, Naturforsch. B 1961, 16, 556-557; b) G. Fairhurst, C. White, J. Chem. Soc., Dalton Trans. 1979, 1531-1538; c) U. Koelle, B. Fuss, M. V. Rajasekharan, B. L. Ramakrishna, J. H. Ammeter, M. C. Boehm, J. Am. Chem. Soc, 1984, 106, 4252-4160.

82 T. Yoshino, H. Ikemoto, S. Matsunaga, M. Kanai, Angew. Chem. Int. Ed. 2013, 52, 2207-2211.
} 


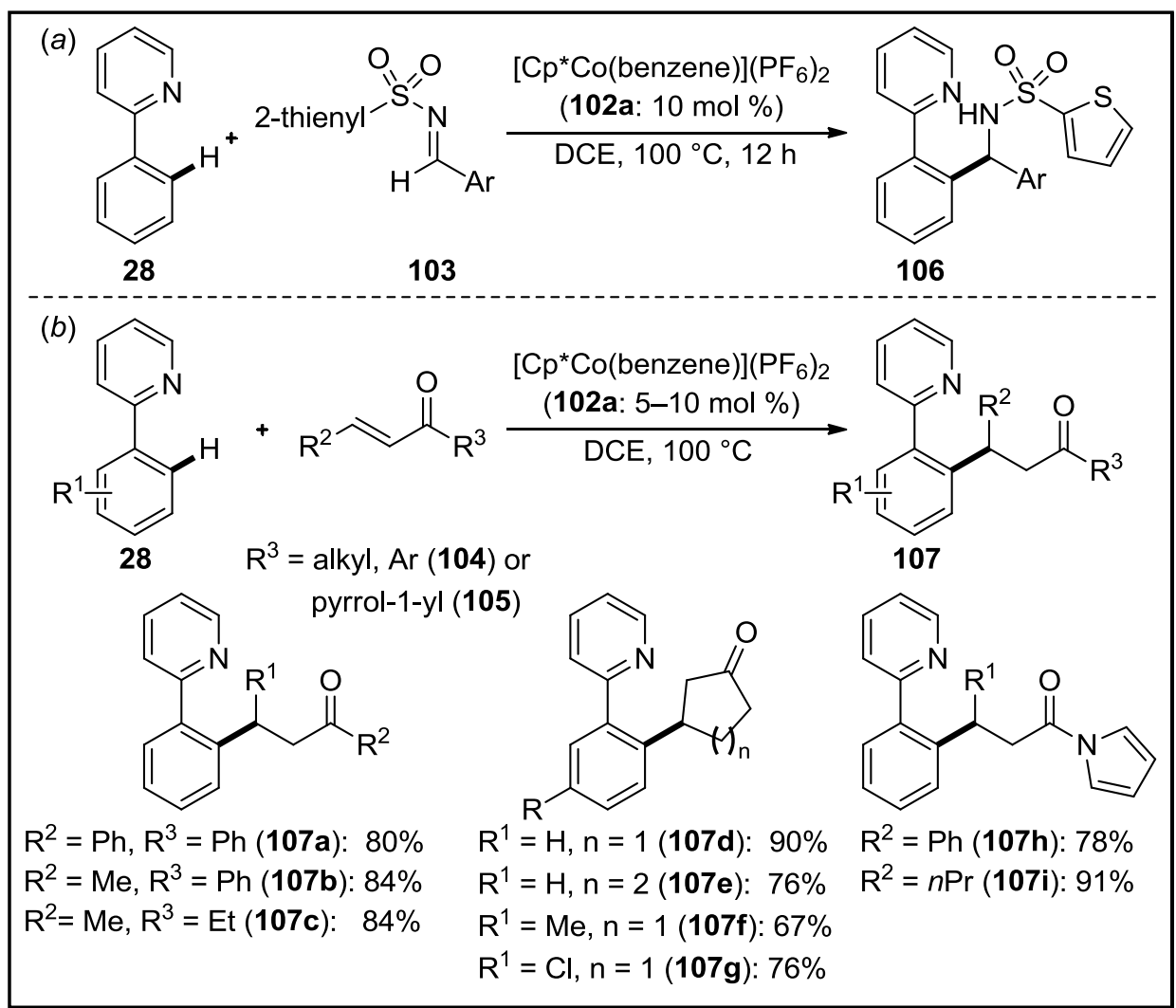

Scheme 1.30. Cobalt(III)-catalyzed addition of 2-aryl pyridines to imines 103, $\alpha, \beta$-unsaturated enones 104 and 105.

Taking into consideration the important biological activity of indole and its derivatives, their modification and functionalization have extensively been studied inter alia by Kanai group applying the high-valent $\left[\mathrm{Cp} * \mathrm{Co}(\right.$ benzene $\left.)\left(\mathrm{PF}_{6}\right)_{2}\right]$ complex (102a). The catalyst efficiently promoted the C2-selective hydroindolation of imines $\mathbf{1 0 3}$ with broad scope (Scheme 1.31). ${ }^{83}$ The pyrimid-2-yl directing group can be easily removed upon treatment with $\mathrm{NaOEt}$, yie lding the NH-free indole derivative in a high yield. ${ }^{69}$

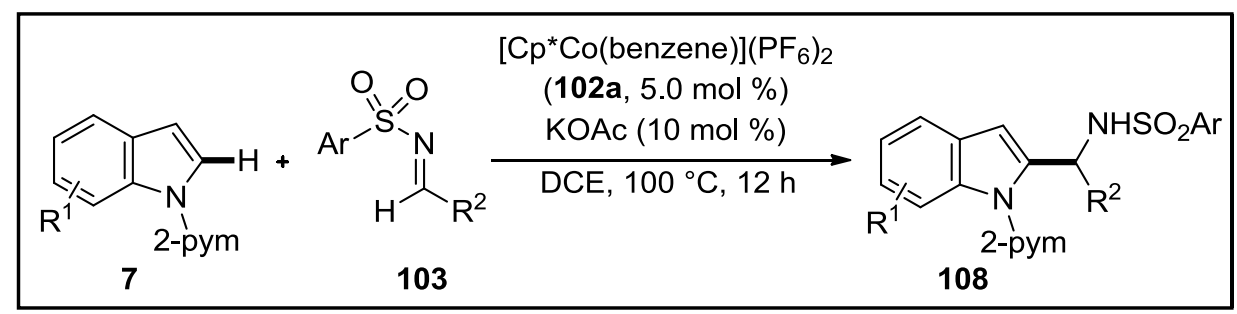

Scheme 1.31. Cobalt(III)-catalyzed addition of $N$-(pyrimidin-2-yl)indoles 7 to imines $\mathbf{1 0 2}$.

${ }^{83}$ T. Yoshino, H. Ikemnto, S. Matsunaga, M. Kanai, Chem. Eur. J. 2013, 19, 9142-9146. 


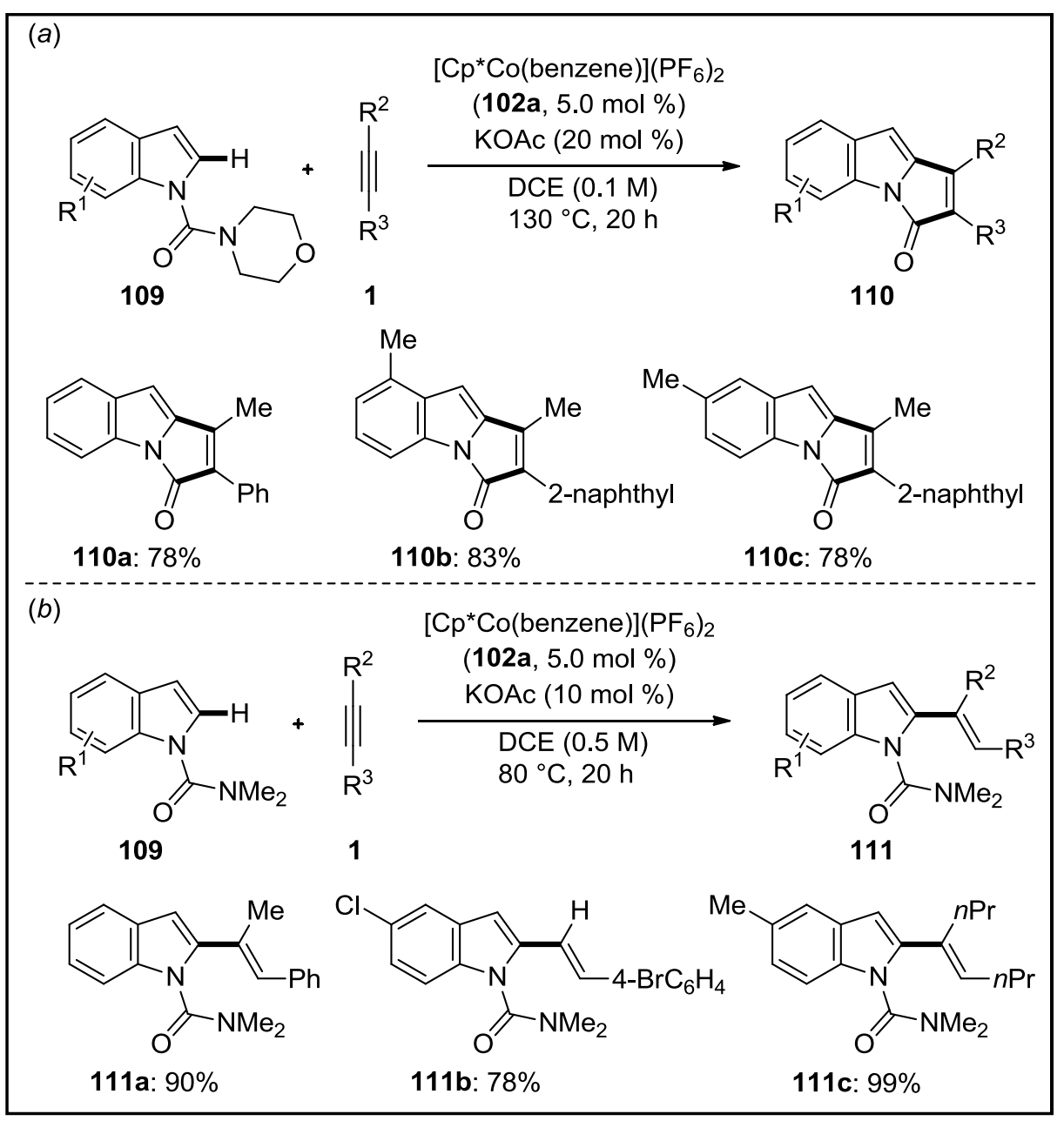

Scheme 1.32. Cobalt(III)-catalyzed annulations and alkenlations of indoles 109 via $\mathrm{C}-\mathrm{H}$ bond alkenylation.

Further studies with $\mathrm{Cp} * \mathrm{Co}(\mathrm{III})$ complexes were extended to catalyze the annulations and alkenylations of $\mathrm{N}$-carbamoylindoles 109 via direct $\mathrm{C}-\mathrm{H}$ activation in reactions with internal alkynes 1 to afford pyrroloindolones 110. The results demonstrated that the $\mathrm{Cp} * \mathrm{Co}(\mathrm{III})$ complexes possess the characteristic reactivity similar to those of the $\mathrm{Cp} * \mathrm{Rh}(\mathrm{III})$ system (Scheme 1.32). ${ }^{84}$ Furthermore, intensive mechanistic studies revealed the difference in the mode of catalytic activity between the $\mathrm{Cp} * \mathrm{Co}(\mathrm{III})$ and $\mathrm{Cp} * \mathrm{Rh}(\mathrm{III})$ complexes, thus highlighted the unique nucleophilic activity of the latter. ${ }^{84}$

Besides their contributions on developing catalytically efficient $\mathrm{Cp} * \mathrm{Co}$ (III) complexes, recently the Kanai group successfully applied the air-stable $\left[\mathrm{Cp}^{*} \mathrm{CoI}_{2}(\mathrm{CO})\right]$ complex 112, prepared by $\mathrm{Li}$ and $\mathrm{J}$ in as early as $2004,{ }^{85}$ for the $\mathrm{C} 2$-selective $\mathrm{C}-\mathrm{H}$ bond amidation in

\footnotetext{
${ }^{84}$ H. Ikemoto, T. Yoshino, K. Saka ta, S. Matsunaga, M. Kanai, J. Am. Chem. Soc. 2014, 136, 5424-5431.

85 W. Li, L. Weng, G. Jin, Inorg. Chem. Commun. 2004, 7, 1174-1177.
} 
indoles 7 (Scheme 1.33). ${ }^{86}$

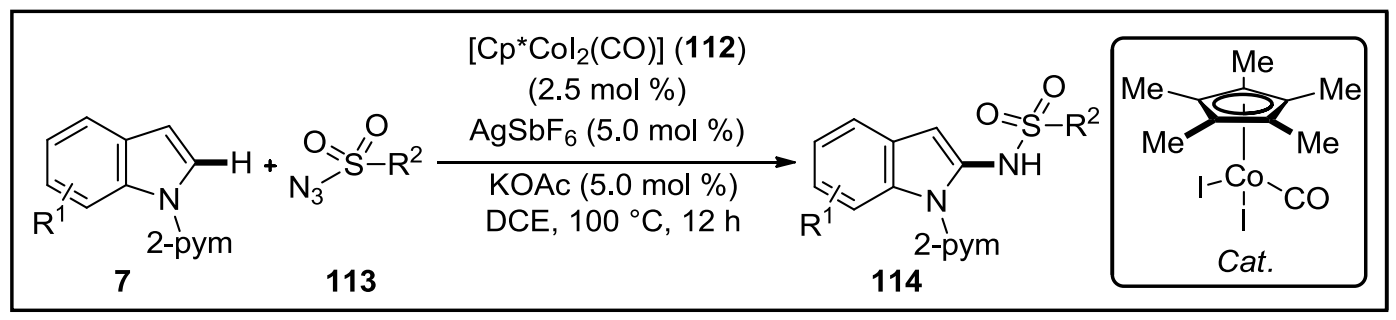

Scheme 1.33. Cobalt(III)-catalyzed $\mathrm{C}-\mathrm{H}$ bond amidation of indoles 7.

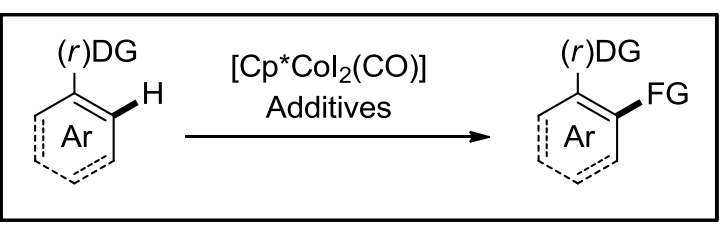

Scheme 1.34. $\mathrm{Cp}^{*} \mathrm{CoI}_{2}(\mathrm{CO})$ catalyzed $\mathrm{C}-\mathrm{H}$ bond functionalizations.

The use of the $\left[\mathrm{Cp}^{*} \mathrm{CoI}_{2}(\mathrm{CO})\right]$ complex (112) attracts an increasing attention in the past several months (Scheme 1.34). Thus, more examples of $\mathrm{C}-\mathrm{H}$ bond amidation were reported using acetoxycarbamates as convenient nitrogen sources, as reported by the Chang group. ${ }^{87}$ Likew ise the direct $\mathrm{C}-\mathrm{H}$ phosphoramidation was developed by the Kanai group. ${ }^{88}$ Moreover, the cobalt(III)-catalyzed direct $\mathrm{C}-\mathrm{H}$ cyanations, halogenations, allylations were developed by the Ackermann, ${ }^{89}$ Glorius, ${ }^{90}$ and Chang groups. ${ }^{91}$ Very recently, Glorius and coworkers provided a reactive modular route towards a new class of conjugated polycyclic hydrocarbons using diazo compounds carbine precursors. ${ }^{92}$ The Kanai group also reported on Cp*Co(III)-catalyzed oxidative alkenylation of bezamides 2 and acetanilides with ethyl acrylate. $^{93}$

\footnotetext{
86 B. Sun, T. Yoshino, S. Ma tsunaga, M. Kanai, Adv. Synth. Catal. 2014, 356, 1491-1495.

87 P. Patel, S. Chang, ACS Catal. 2015, 5, 853-858.

88 B. Sun, T. Yoshino, S. Ma tsunaga, M. Kanai, Chem. Commun. 2015, 51, 4659-4661.

89 J. L. L. Adkermann, Angew. Chem. Int. Ed. 2015, 54, 3635-3638.

90 D.-G. Yu, T. Gensch, F. de Azambuja, S. Vásquez-Céspedes, F. Glorius, J. Am. Chem. Soc. 2014, 136, 17722-17725.

91 A. B. Pawar, S. Chang, Org. Lett. 2015, 17, 660-663.

92 D. Zhao, J. H. Kim, L. Stegemann, C. A. Strassert, F. Glorius, Angew. Chem. Int. Ed. 2015, 54, 4508-4511.

93 Y. Suzuki, B. Sun, T. Yoshino, M. Kanai, S. Matsuna ga, Tetrahedron, 2015, DOI : 10.1016/j.tet.2015.02.032.
} 


\section{Objectives}

Over the last decade, remarkable advances in transition-metal-catalyzed $\mathrm{C}-\mathrm{H}$ bond functionalization have been achieved, which provided a more straightforward pathway for the development of chemo- and site-selective syntheses of valuable organic molecules. As a consequence, the center of gravity in researches of Prof. Dr. Lutz Ackermann and coworkers mainly focused on the development of synthetically useful transition-metal catalyzed $\mathrm{C}-\mathrm{H}$ bond functionalizations. Within this context, major efforts were made to develop further applications of ruthenium and cobalt complexes in catalyzed $\mathrm{C}-\mathrm{H}$ activation.

Isoquinolines are key structural motifs of various heterocyclic compounds with diverse bioactivities. The new efficient methods for the selective preparation of these heterocycles are in strong demand. As a consequence, the method to synthesize decorated isoquinolines through annulations of alkynes with ortho-halosubstituted aromatic imines under transition netal catalysis has been reported. ${ }^{94}$ However, a more step- and atom-economical approach by the transition-metal-catalyzed oxidative $\mathrm{C}-\mathrm{H}$ functionalization has been recognized as an increasingly viable tool for the preparation of substituted heterocycles. Therefore, a new protocol for ruthenium-catalyzed oxidative alkyne annulations by $\mathrm{C}-\mathrm{H}$ bond activation of ketimines $\mathbf{7 3}$ was the prime focus of the first project (Scheme 2.1).

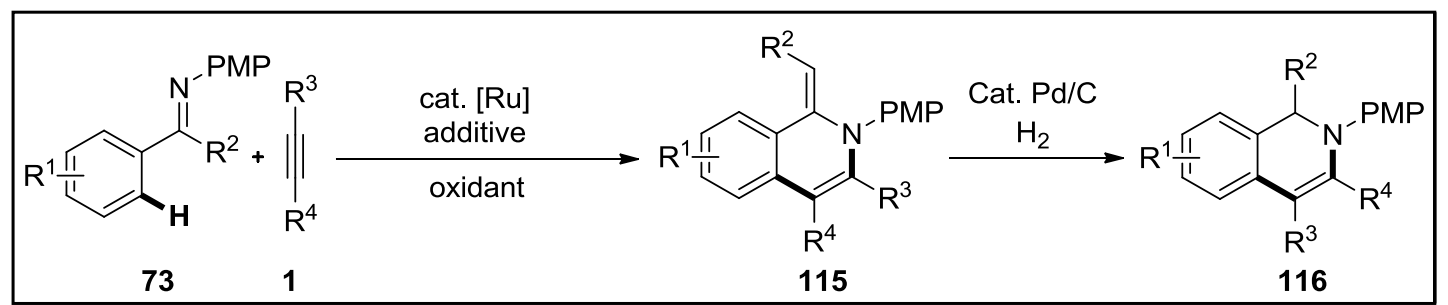

Scheme 2.1. Oxidative $\mathrm{C}-\mathrm{H}$ bond functionalizations with decorated ketimines $\mathbf{7 3}$ and alkynes $\mathbf{1 .}$

As was mentioned above, we were going to manage the extension of isoquinolines to decorated 1-aminoisoquinolines 118 and isoindolines 119. One of the reasons for the significance of these heterocycles is that they constitute important structural motifs of various compounds with activities of relevance to biology or medicinal chemistry. For example, the aminoisoquinoline moiety was found in nonbenzamidine factor VIIa inhibitors against nonthromboembolic cardiovascular disease (Scheme 2.2 and Scheme 2.3).

${ }_{94}$ R. C. La rock, Top. Organomet. Chem. 2005, 14, 147-182. 


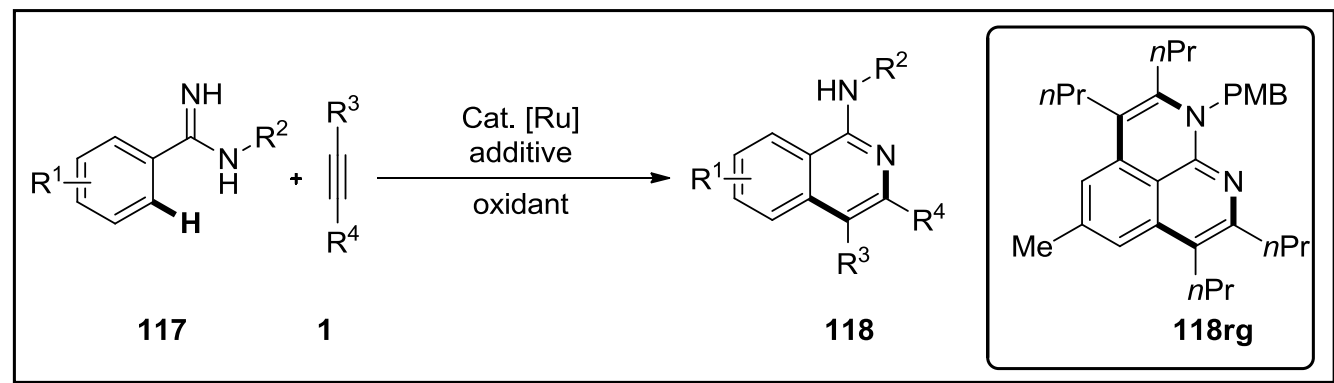

Scheme 2.2. Oxidative annulations of alkynes 1 with decorated amidines 117 through $\mathrm{C}-\mathrm{H}$ activation.

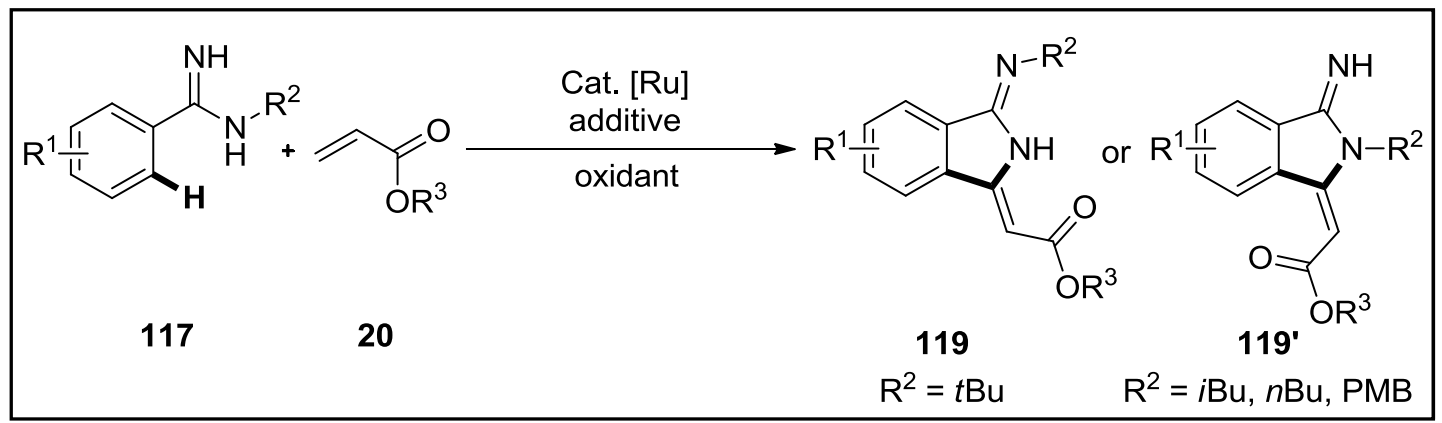

Scheme 2.3. Oxidative alkenylation of decorated amidines $\mathbf{1 1 7}$ with acrylated $\mathbf{2 0}$ through $\mathrm{C}-\mathrm{H}$ bond activation.

The synthetic utility of the transition-metal-catalyzed hydroarylation via $\mathrm{C}-\mathrm{H}$ bond activation was illustrated by Murai. ${ }^{34}$ Despite the remarkable progress achieved during the last decade, $\alpha, \beta$-unsaturated acceptors still could not be utilized for such alkylations until 8 -aminoquinoline was introduced as a removable bidentate directing group by Chatani. ${ }^{46} \mathrm{We}$ planned to establish ruthenium-catalyzed direct hydroarylation of $\alpha, \beta$-unsaturated ketones $\mathbf{4 1}$ with aromatic amides $\mathbf{2}$ via monodentate coordination in an environmentally friendly medium (Scheme 2.4).

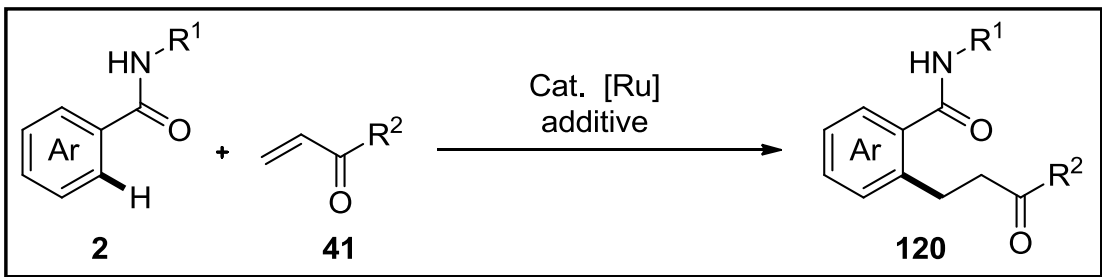

Scheme 2.4. Hydroarylation of $\alpha, \beta$-unsaturated ketones 41 with mono-dentate amides 2.

Meanwhile, oxidative annulations of $\alpha, \beta$-unsaturated ketones 41 with acetanilides 121 to synthesize decorated quinolines $\mathbf{1 2 2}$ were also envisioned (Scheme 2.5). 


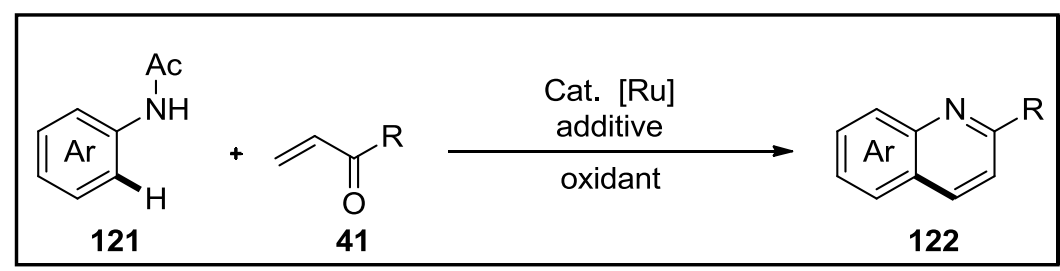

Scheme 2.5. Oxidative annulations of $\alpha, \beta$-unsaturated ketones 41 with acetanilides 121 .

Biaryls constitute the key structural motifs of biologically active and naturally occurring compounds, for example, in angiotensin-II-receptor blockers (ARBs). ${ }^{5 e}$ The synthesis of these structural moiety mostly relied on palladium(0)-catalyzed cross-coupling reactions. Recently, the development of catalysts based on the naturally more abundant and less expensive first-row transition metals and their complexes has witnessed considerable growth. ${ }^{47}$ Among them, inexpensive cobalt catalysts have been recognized as increasingly viable tools for $\mathrm{C}-\mathrm{H}$ bond activations during the last years. Consequently, the use of cobalt complexes were another focus in this context to develop a new step- and atom-economic strategy for the synthesis of ARBs $\mathbf{1 2 4}$ and derivatives, as shown in Scheme 2.6.

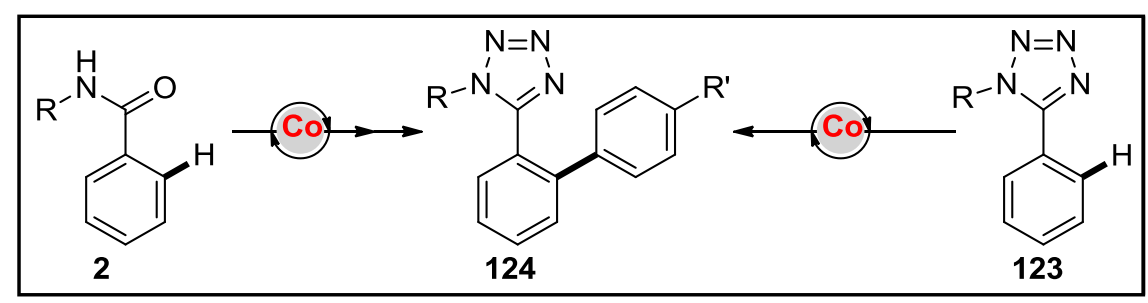

Scheme 2.6. Strategy for the synthesis of ARBs and derivatives.

Generally, many cobalt catalytic systems required Grignard reagents as the base and the reductant to generate the reactive low-valent cobalt catalysts. Hence, most of the functional groups could not be tolerated under these reaction conditions. In order to avoid these disadvantages, the research groups of Kanai, Ackermann, Daugulis and Song developed milder reaction conditions with cobalt catalysts, inc luding high-valent $\mathrm{Cp} * \mathrm{Co}(\mathrm{III})$ derivatives. Based on this progress, we set out to develop an unprecedented cobalt(III)-catalyzed $\mathrm{C}-\mathrm{H}$ cyanation of arenes $\mathbf{2 8}$ and heteroarenes $\mathbf{7}$ as the fifth project. (Scheme 2.7). 


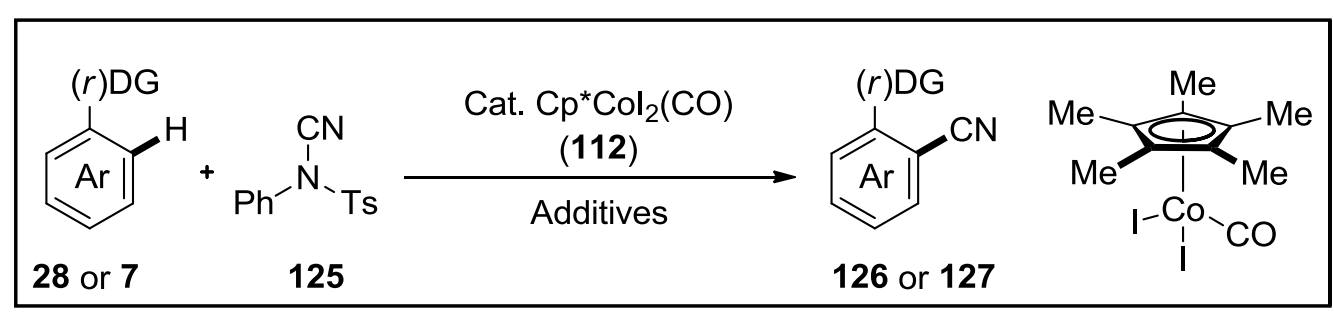

Scheme 2.7. Cobalt(III)-catalyzed C-H cyanation.

In the last few years, transition-metal-catalyzed direct insertion of isocyanates into $\mathrm{C}-\mathrm{H}$ bonds is in great demand, because this approach efficiently provides synthetically valuable amides. However, acyl azides were only found to work as amino sources in the iridium- and ruthenium-catalyzed amidation reactions. Moreover, further application of acyl azides was strongly limited by the difficulty in controlling the dual reactivity of acyl azides, as the reaction results in $\mathrm{C}-\mathrm{C}$ and $\mathrm{C}-\mathrm{N}$ bond formations and often affords a mixture of products. For this reason, it was our goal to develop the first cobalt(III)-catalyzed $\mathrm{C}-\mathrm{H}$ aminocarbonylation with isocyanates $\mathbf{1 2 9}$ and acyl azides $\mathbf{1 3 1}$ (Scheme 2.8).

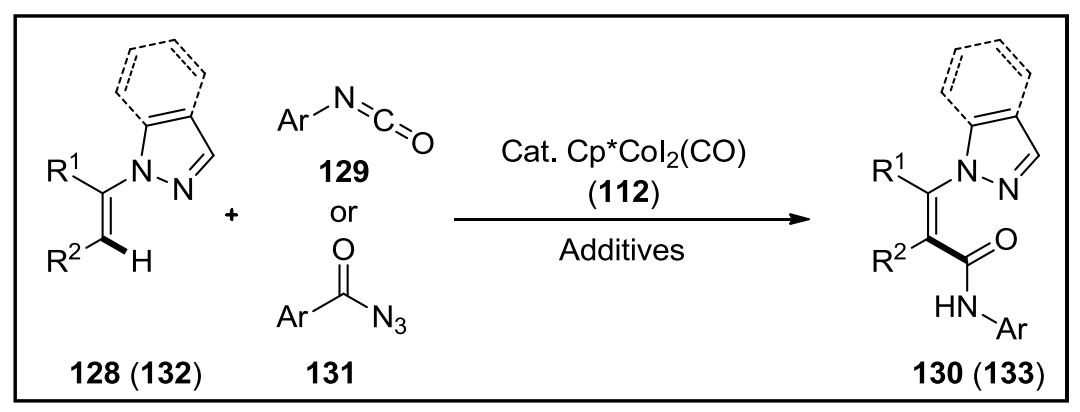

Scheme 2.8. Cobalt(III)-catalyzed aminocarbonylation of C-H bonds. 


\section{Ruthenium(II)-Catalyzed Oxidative Alkyne Annulation by C-H Bond Activation on Ketimines}

Isoquinolines are key structural moieties of various practically useful compounds. One of the most efficient methods for their preparation has been so far the transition-metal-catalyzed oxidative annulation of alkynes with ortho-halo-substituted aromatic imines with alkynes to furnish these heterocycles. ${ }^{95}$ Herein, we established a more straightforward approach to decorated isoquinolines 115 through ruthenium(II)-catalyzed oxidative alkyne annulations with easily accessible ketimines $\mathbf{7 3}$ as the starting materials. The reaction provided an expedient access to exo-methylene-1,2-dihydroisoquinolines $\mathbf{1 1 6}{ }^{96}$

\subsection{Optimization studies}

At the outset of our studies, we explored various reaction conditions for the envisioned ruthenium(II)-catalyzed annulations of tolane (1a) with ketimine 73a, along with $\mathrm{Cu}(\mathrm{OAc})_{2} \cdot \mathrm{H}_{2} \mathrm{O}$ as the oxidant (Table 3.1). Preliminary experiments indicated DCE to be the solvent of choice, while significantly lower yields were obtained with $t-\mathrm{AmOH}, \mathrm{MeOH}, \mathrm{H}_{2} \mathrm{O}$, DMF or toluene. Furthermore, $\mathrm{KPF}_{6}$ and $\mathrm{MesCO}_{2} \mathrm{~K}$ proved to be suitable as cocatalytic additives. Yet, the most effective catalys is was achieved with $\mathrm{AgSbF}_{6}$ (Table 3.1, entries 1-4), which is likely due to the in situ formation of a cationic ruthenium catalyst. It is worth noting that the $\mathrm{C}-\mathrm{H}$ bond functionalization proceeded efficiently under an ambient atmosphere of air, which highlights the user-friendly system (entry 5). While $\mathrm{CuBr}_{2}$ as the oxidant shut down the oxidative alkyne annulation completely (entry 6), interestingly, the catalytic activity was restored through the addition of metal acetates, indicating carboxylate assistance to be of major importance for the $\mathrm{C}-\mathrm{H}$ bond functionalization (entries 7-9). ${ }^{5 \mathrm{q}, 97}$ Additionally, we found that the ruthenium(II) catalyst outcompeted typical rhodium or palladium complexes (Table 3.1, entries 5, 10, and 11).

Table 3.1. Optimization of the oxidative annulations with ketimine 73a. ${ }^{[a]}$

\footnotetext{
95 R. C. La rock, Top. Organomet. Chem. 2005, 14, 147-182.

96 J. Li, L. Adkermann, Tetrahedron 2014, 70, 3342-3348.

97 L. Ackermann, Chem. Commun. 2010, 46, 4866-4877.
} 


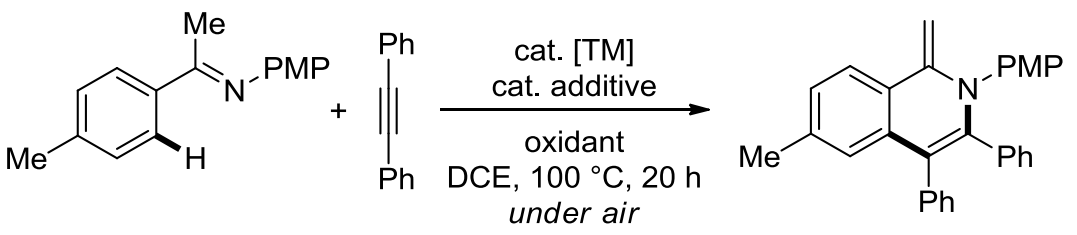

$73 a$

1a

$115 a$

\begin{tabular}{|c|c|c|c|c|}
\hline Entry & Catalyst & Additive & Oxidant & Yield $[\%]^{[\mathrm{b}]}$ \\
\hline 1 & -- & -- & $\mathrm{Cu}(\mathrm{OAc})_{2} \cdot \mathrm{H}_{2} \mathrm{O}$ & 0 \\
\hline 2 & {$\left[\mathrm{RuCl}_{2}(p \text {-cymene })\right]_{2}$} & $\mathrm{KPF}_{6}$ & $\mathrm{Cu}(\mathrm{OAc})_{2} \cdot \mathrm{H}_{2} \mathrm{O}$ & $51^{[\mathrm{c}]}$ \\
\hline 3 & {$\left[\mathrm{RuCl}_{2}(p \text {-cymene })\right]_{2}$} & $\mathrm{MesO}_{2} \mathrm{~K}$ & $\mathrm{Cu}(\mathrm{OAc})_{2} \cdot \mathrm{H}_{2} \mathrm{O}$ & $61^{[\mathrm{c}]}$ \\
\hline 4 & {$\left[\mathrm{RuCl}_{2}(p \text {-cymene })\right]_{2}$} & $\mathrm{AgSbF}_{6}$ & $\mathrm{Cu}(\mathrm{OAc})_{2} \cdot \mathrm{H}_{2} \mathrm{O}$ & $67^{[\mathrm{c}]}$ \\
\hline 5 & {$\left[\mathrm{RuCl}_{2}(\text { p-cym ene })\right]_{2}$} & $\operatorname{AgSbF}_{6}$ & $\mathrm{Cu}(\mathrm{OAc})_{2} \cdot \mathrm{H}_{2} \mathrm{O}$ & 71 \\
\hline 6 & {$\left[\mathrm{RuCl}_{2}(p \text {-cymene })\right]_{2}$} & $\mathrm{AgSbF}_{6}$ & $\mathrm{CuBr}_{2}$ & 0 \\
\hline 7 & {$\left[\mathrm{RuCl}_{2}(p \text {-cymene })\right]_{2}$} & $\mathrm{AgSbF}_{6}$ & $\mathrm{CuBr}_{2} / \mathrm{NaOAc}$ & $54^{[\mathrm{d}]}$ \\
\hline 8 & {$\left[\mathrm{RuCl}_{2}(p \text {-cymene })\right]_{2}$} & $\mathrm{AgSbF}_{6}$ & $\mathrm{CuBr}_{2} / \mathrm{KOAc}$ & $53^{[\mathrm{d}]}$ \\
\hline 9 & {$\left[\mathrm{RuCl}_{2}(p \text {-cymene })\right]_{2}$} & $\mathrm{AgSbF}_{6}$ & $\mathrm{CuBr}_{2} / \mathrm{CsOAc}$ & $37^{[\mathrm{d}]}$ \\
\hline 10 & {$\left[\mathrm{RhCpCl}_{2}\right]_{2}$} & $\mathrm{AgSbF}_{6}$ & $\mathrm{Cu}(\mathrm{OAc})_{2} \cdot \mathrm{H}_{2} \mathrm{O}$ & 46 \\
\hline 11 & $\mathrm{PdCl}_{2}\left(\mathrm{PPh}_{3}\right)_{2}$ & $\mathrm{AgSbF}_{6}$ & $\mathrm{Cu}(\mathrm{OAc})_{2} \cdot \mathrm{H}_{2} \mathrm{O}$ & 0 \\
\hline
\end{tabular}

[a] General reaction conditions: 73a $(0.50 \mathrm{mmol}), \mathbf{1 a}(1.00 \mathrm{mmol})$, catalyst $(5.0 \mathrm{~mol} \%)$, additive (20-30 mol \%), oxidant (0.50 mmol), DCE (2.0 mL), under ambient air, $100 \mathbb{C}, 20 \mathrm{~h}$. [b] Isolated yields. [c] Under an atmosphere of $\mathrm{N}_{2}$. [d] MOAc (1.0 mmol).

\subsection{Scope and Limitations}

\subsubsection{Scope of Aromatic Alkyne Annulation}

With the optimized reaction conditions in hand, we explored its scope and limitations in the oxidative $\mathrm{C}-\mathrm{H}$ functionalization with differently substituted ketimines $\mathbf{7 3}$ and alkynes $\mathbf{1}$ (Scheme 3.1). The oxidative annulation efficiently occurred with acetophenonimines $\mathbf{7 3}$ bearing substituents on the $N$-aryl moiety. Notably, transformations of electron-rich-methylsubstituted ketimines 73a and 73d proceeded very smoothly (115aa, 115da), whereas substrate $73 \mathrm{c}$ with the electron-deficient fluoro group provided only a negligible conversion under the optimized conditions. Furthermore, a more sterically hindered 
substrate 73e bearing an ortho-methyl substituent was also converted, albeit with a relatively low is olated yield of product 115ea. Intramolecular competition experiments with substrate 73f bearing a meta-methyl group was controlled by steric interaction to deliver the product 115fa, which can be rationalized in terms of significant steric interactions. Compound 115ba' was obtained in low yield when (E)-N-[1-(2-bromophenyl)ethylidene]-4-methoxyaniline (73g) was employed. Meanwhile, substituted tolane derivatives $\mathbf{1 b}-\mathbf{1 d}$ were found to be viable substrates as well, thereby delivering the desired products $115 \mathbf{a b}-\mathbf{1 1 5} \mathbf{a d}$, respectively. Yields of the desired products can be affected by different electronic factors in the substituted alkynes. Thus, compounds $\mathbf{1 b}$ and 1c with electron-rich methyl and methoxyl groups delivered the target products in relatively better yields.
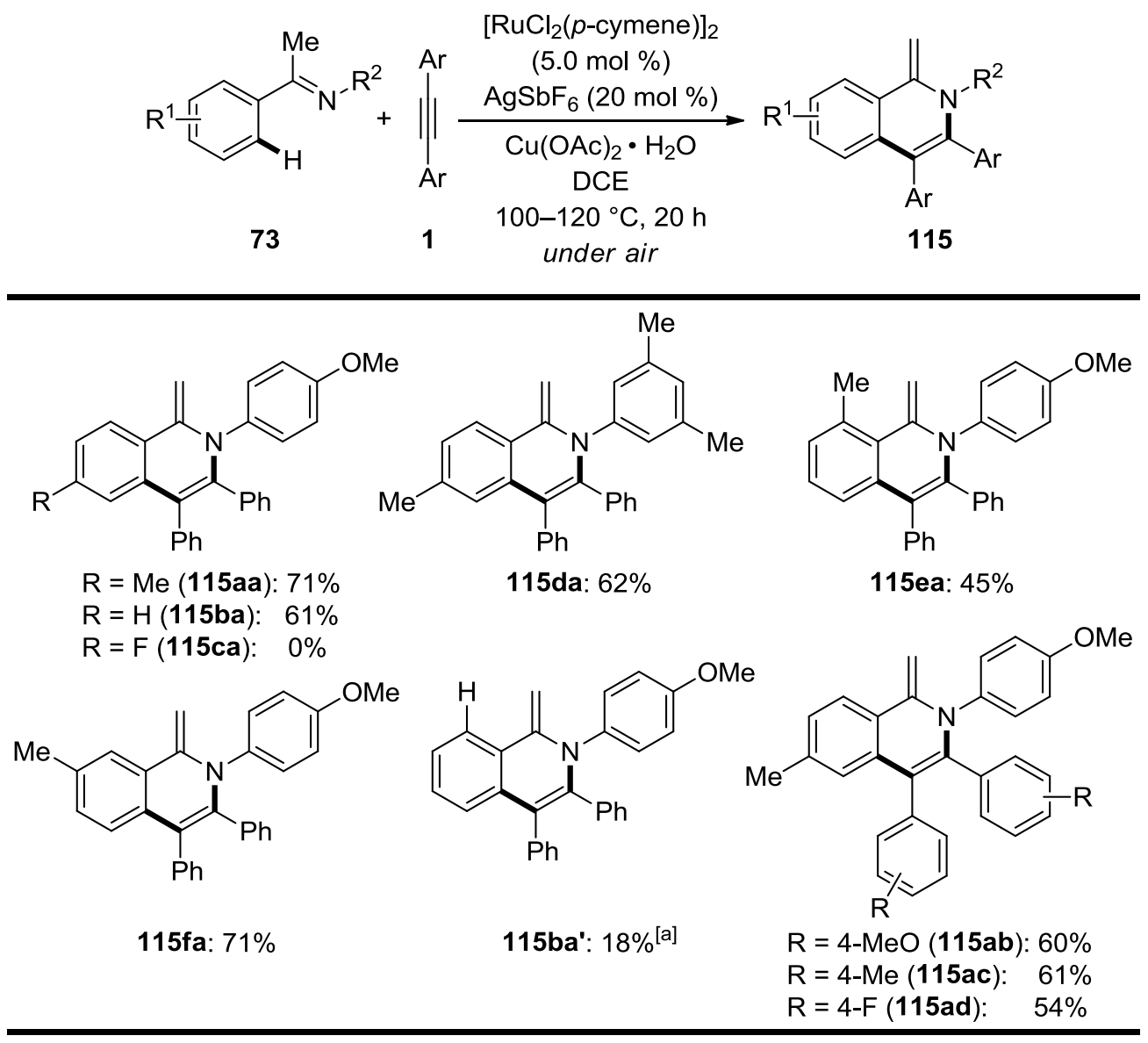

Scheme 3.1. Oxidative $\mathrm{C}-\mathrm{H}$ functionalization of aromatic ketimines 73. [a]

(E)- $N$-[1-(2-bromophenyl)ethylidene]-4-methoxyaniline $(\mathbf{7 3 g}, 0.5 \mathrm{mmol})$ was used.

\subsubsection{Scope of the Annulation with Alkyl Alkynes}

Fortunately, the optimized catalytic system was not restricted to the use of diarylalkynes $\mathbf{1}$ as 
starting materials (Scheme 3.1). We initially tested dialkylsubstituted substrate 1e, which delivered the desired product $\mathbf{1 1 5} \mathbf{a e}$ in acceptable yield (Scheme 3.2). Here, we particularly focused our efforts on the use of unsymmetrical substrates $\mathbf{1}$ to probe the challenging regiocontrol in the oxidative annulation. We were delighted to observe that the $\mathrm{C}-\mathrm{H}$ bond functionalizations proceeded with perfect regioselectivities, placing the aromatic substituent proximal to the nitrogen atom, which can be ascribed to the steric hindrance of the alkyl groups. Furthermore, the ruthenium catalyst displayed high chemoselectivity in which oxidative annulations of alkynes $\mathbf{1} \mathbf{j}$ and $\mathbf{1 k}$ bearing an ester or a ketone group solely took place through chelation assistance by the ketimine moiety to furnish products 115aj and 115ak in moderate yie lds (Scheme 3.2).

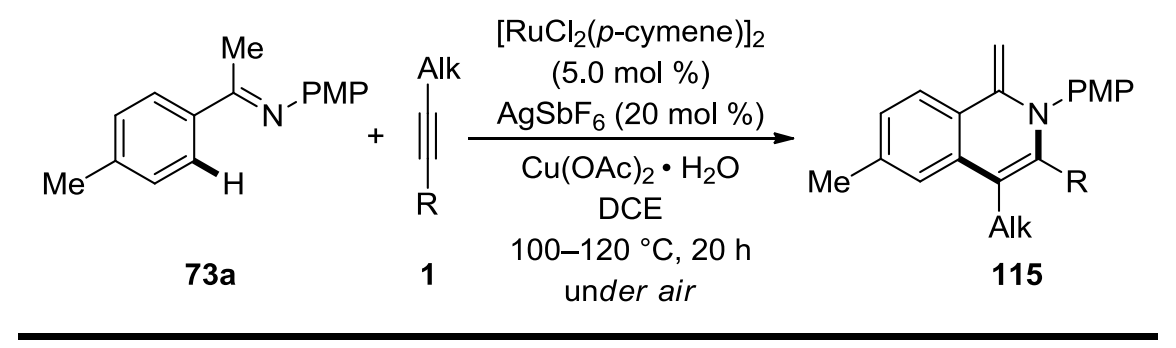<smiles>C=C1c2ccc(C)cc2C(C(C)C)=C(C(C)C)N1P(N)P</smiles>

115ae: $44 \%$<smiles>[R]c1ccc(C2=C(CCCC)c3cc(C)ccc3C(=C)N2P(N)P)cc1</smiles>

$\mathrm{R}=\mathrm{OMe}(\mathbf{1 1 5 a h}): 59 \%$

$\mathrm{R}=\mathrm{F}(115 \mathrm{ai}): \quad 72 \%$<smiles>C=C1c2ccc(C)cc2C(C#N)=C(c2ccccc2)N1P(#N)N</smiles>

Alk $=$ Me (115af): $71 \%$ Alk = Et (115ag): $63 \%$

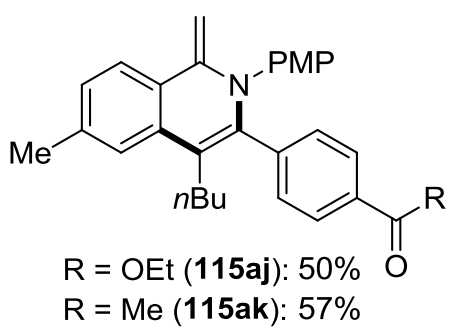

Scheme 3.2. Oxidative C-H functionalization with alkylalkynes $\mathbf{1}$.

\subsubsection{Multicatalytic Synthesis of Dihydroisoquinolines}

Considering that the 2-substituted isoquinoline moiety plays an important role in various heterocyclic compounds with different bioactivities as key structural motifs, we subsequently devised a two-step reaction sequence consisting of the initial ruthenium(II)-catalyzed $\mathrm{C}-\mathrm{H}$ bond functionalization followed by the palladium-catalyzed hydrogenation in a one-pot 
fashion (Scheme 3.3). The multicatalytic approach set the stage for the efficient preparation of the decorated products 116, again occurring with excellent regio- and chemo-selectivities (116af, 116fa). It is noteworthy that a more sterically congested ketimine $\mathbf{7 3 h}$ gave the ethyl-substituted dihydroisoquinoline $\mathbf{1 1 6 h a}$ in a comparable yield.

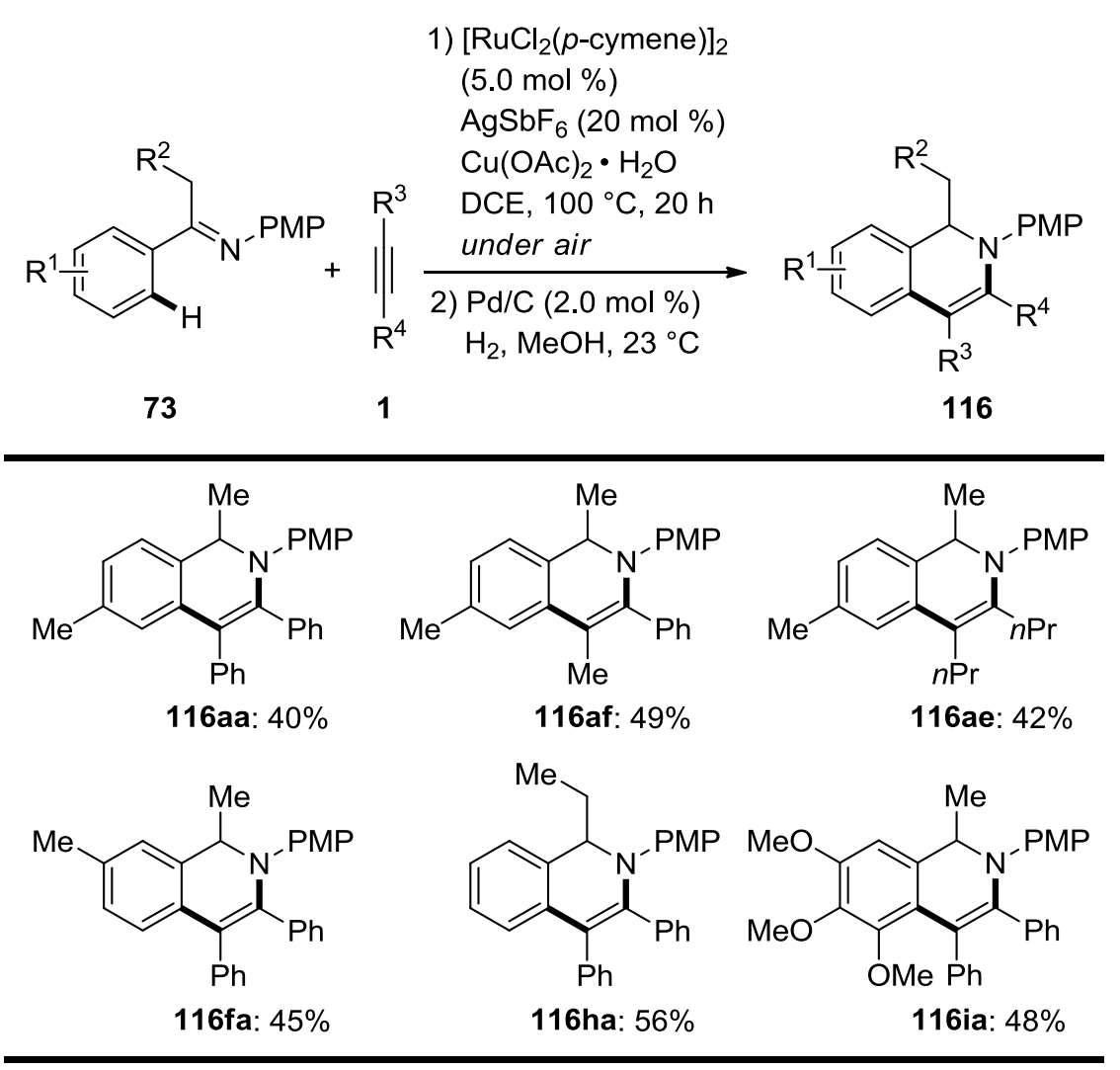

Scheme 3.3. Multicatalytic synthesis of 1,2-dihydroisoquinolines $\mathbf{1 1 6 .}$

\subsection{Mechanistic Studies}

\subsubsection{Inte rmolecular Competition Experiment}

Given the unique selectivity and outstanding efficacy of our ruthenium(II)-catalyzed $\mathrm{C}-\mathrm{H}$ bond functionalization approach, we became interested in exploring its mode of action. Hence, keeping this purpose in mind, a competition experiment between arylalkyne 1a and alkyla kyne 11 was performed and revealed the former to be solely converted (Scheme 3.4). 


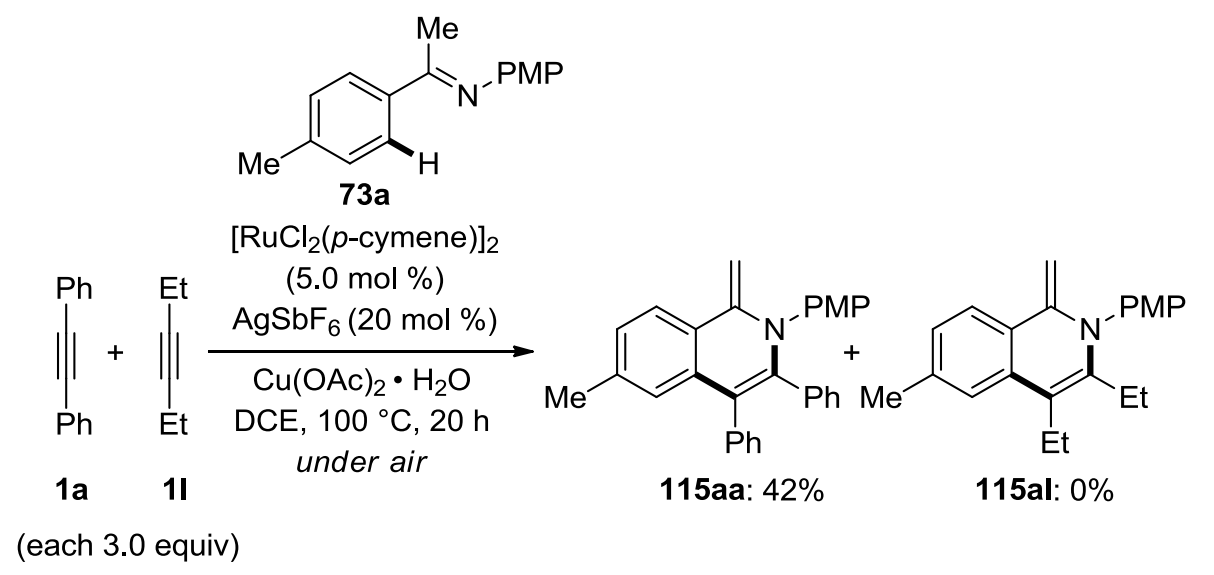

Scheme 3.4. Competition experiment between alkynes 1a and $\mathbf{1 1}$.

\subsubsection{Reaction in the Presence of Isotopically Labeled Solvent}

Furthermore, significant H/D exchange was observed in the presence of the deuterated cosolvent $\left[\mathrm{D}_{4}\right]-\mathrm{MeOH}$, which can be rationalized in terms of a reversible carboxylate-assisted $\mathrm{C}-\mathrm{H}$ bond metalation step by the ruthenium(II) complex (Scheme 3.5).

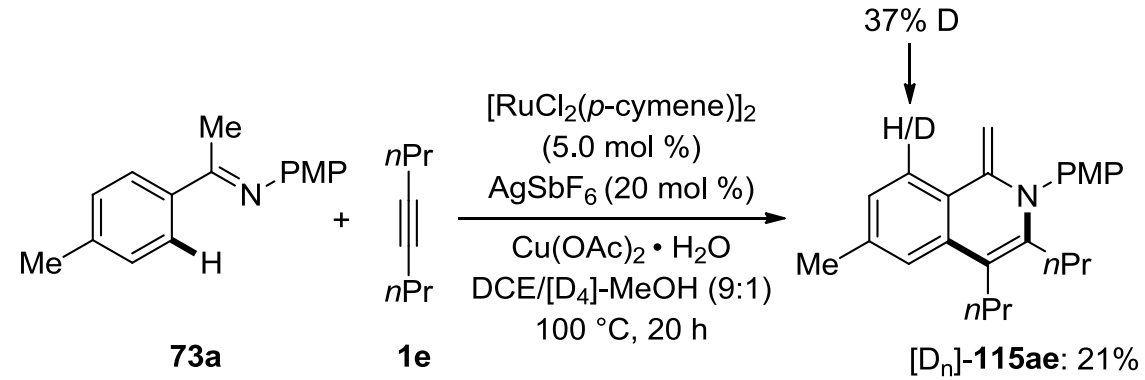

Scheme 3.5. C-H bond functionalization in the presence of deuterated cosolvent $\left[\mathrm{D}_{4}\right]-\mathrm{MeOH}$.

\subsection{Proposed Catalytic Cycle}

Based on these mechanistic studies, we propose the following catalytic cycle for the ruthenium(II)-catalyzed oxidative annulation, which commences with a reversible chelation-assisted $\mathrm{C}\left(\mathrm{sp}^{2}\right)-\mathrm{H}$ bond ruthenation (Scheme 3.6) with a loss of one molecular acetic acid thereby affording intermediate B. Subsequently, a two-step sequence started from coordination of alkyne $\mathbf{1}$ and followed by regioselective migratory insertion ${ }^{5 \mathrm{i}}$ along with

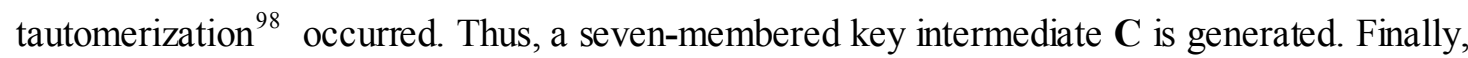
the desired product $\mathbf{1 1 5}$ was furnished via reductive elimination, while the catalytically active species 134 was regenerated through oxidation by $\mathrm{Cu}(\mathrm{OAc})_{2}$.

\footnotetext{
${ }^{98}$ Y. Boutadla, O. Al-Duaij, D. L. Da vies, G. A. Griffith, K. Singh, Organometallics 2009, 28, 433-440.
} 

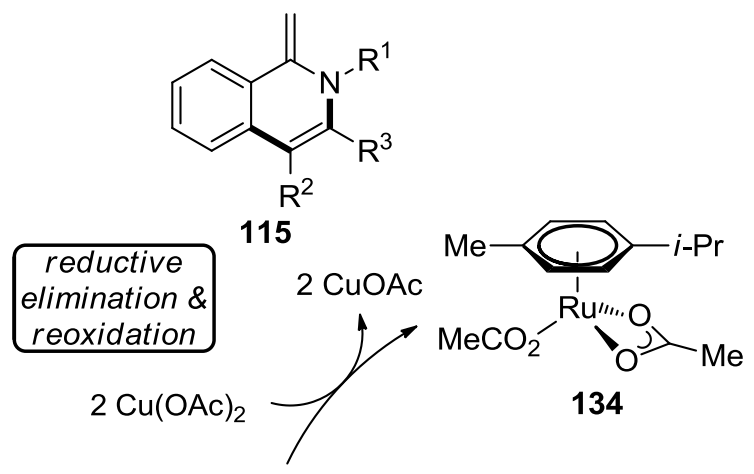<smiles>[R]N=C(C)c1ccccc1</smiles>

73
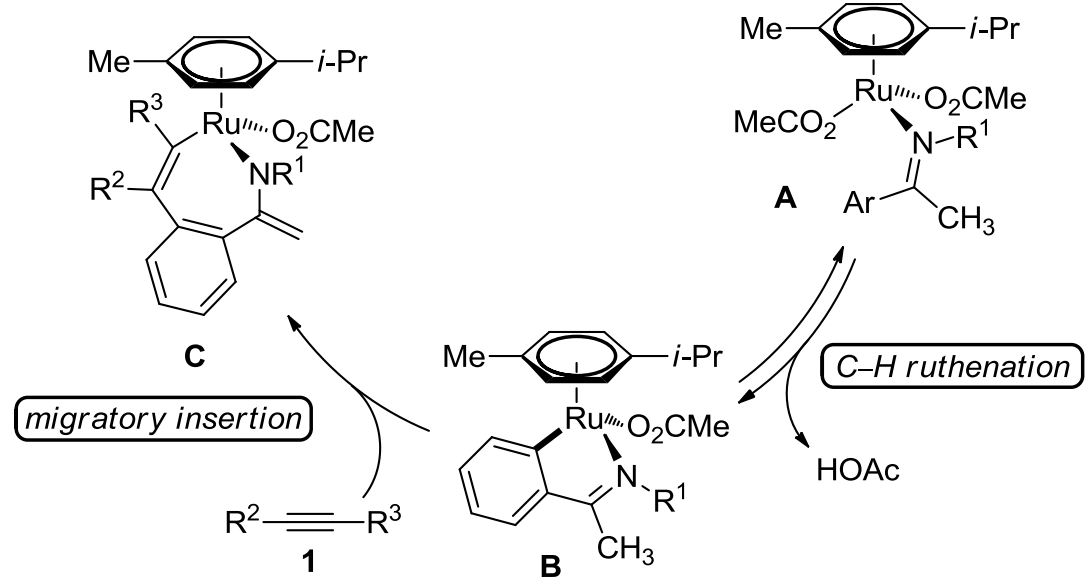

Scheme 3.6. Proposed catalytic cycle.

\subsection{Conclusion}

In summary, we have reported on a novel ruthenium(II)-catalyzed oxidative alkyne annulation to furnish exo-methylene-1,2-dihydroisoquinolines by $\mathrm{C}-\mathrm{H}$ activation on ketimines. Particularly, carboxylate-assisted ruthenium(II) catalys is proved to be decisive for the success of the synthesis of diversely decorated products in high yields. Compounds $\mathbf{1 1 5}$ can easily be transformed into heterocycles $\mathbf{1 1 6}$ via palladium-catalyzed hydrogenation in a one-pot fashion. The ruthenium(II)-catalyzed $\mathrm{C}-\mathrm{H}$ bond functionalizations proceeded with excellent chemo-, site-, and regio-selectivities under an ambient atmosphere of air. Mechanistic studies were indicative of a reversible $\mathrm{C}-\mathrm{H}$ bond metalation step. 


\section{Amidines for Versatile Ruthenium(II)-Catalyzed Oxidative C-H Bond Activation with Internal Alkynes and Acrylates}

Aminoisoquinolines and isoindolines are among the most abundant heterocycles and represent indispensable structural motifs in bioactive compounds (see Chapter 1.2). ${ }^{99}$ With the successful utilization of ruthenium(II) catalysts for oxidative $\mathrm{C}-\mathrm{H}$ bond activations, we were interested to establish the first ruthenium-catalyzed oxidative $\mathrm{C}-\mathrm{H}$ bond functionalizations with benzamidines $\mathbf{1 1 7} .^{100}$

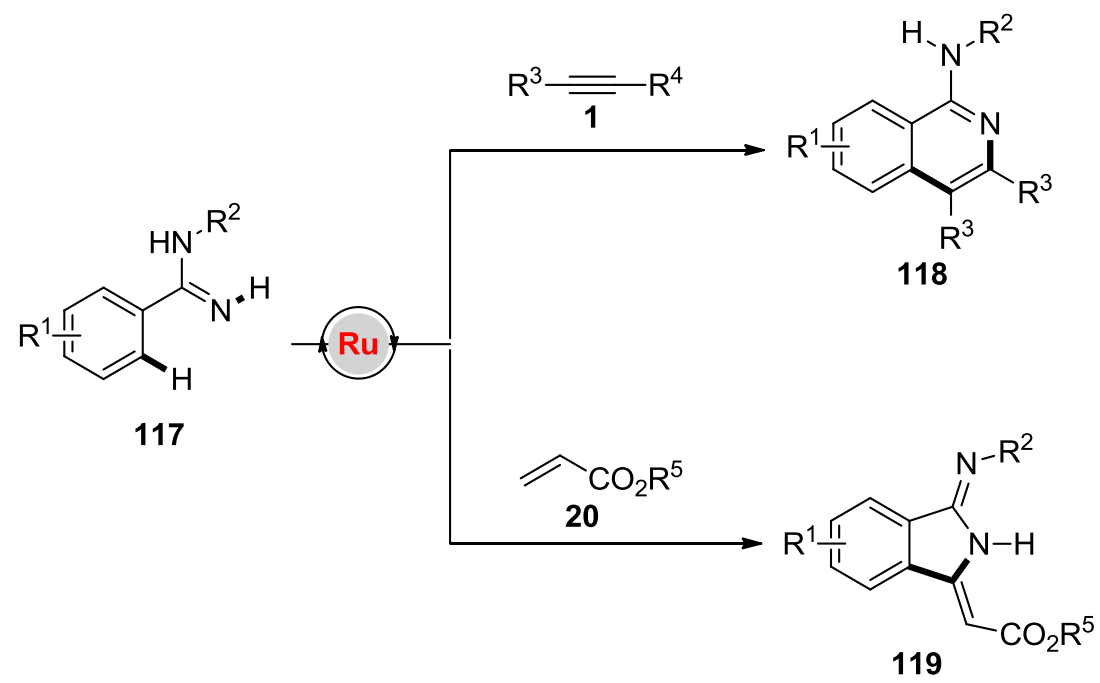

Scheme 4.1. Ruthenium(II)-catalyzed C-H bond activation on amidines 117.

\subsection{Oxidative Alkyne Annulation}

\subsubsection{Optimization Studies}

We initiated our studies by testing different reaction conditions for the desired oxidative annulation of tolane (1a) with benzamidine 117a. Preliminary experiments proved $\left[\mathrm{RuCl}_{2}(p \text {-cymene })\right]_{2}$ as the ideal metal complex and $\mathrm{KPF}_{6}$ as the cocatalytic additive of choice, while significantly lower yields were obtained with $\mathrm{AgSbF}_{6}$ or AgOAc. Among a set of representative solvents, DME provided optimal results (Table 4.1, entries 1-5 and 8). However, it is worth noting that inexpensive, non-toxic $\mathrm{H}_{2} \mathrm{O}^{101,50}$ was found to be a viable reaction medium as well (entries 6 and 7).

\footnotetext{
99 a) R. Alajarin, C. Burgoes, in Modern Heterocyclic Chemistry (Eds.: J. Alva rez-Builla, J. J. Vaque ro, J. Ba rluenga), Wiley-VCH, Weinheim, 2011, 1527-1629; b) N. L. Subasinghe, J. Lanter, T. Markotan, E. Opas, S. McKenney, C. Crysler, C. Hou, J. O'Neill, D. Johnson, Z. Sui, Bioorg. Med. Chem. Lett. 2013, 23, 1063-1069.

100 J. L. M. John, L. Ackermann, Chem. Eur. J. 2014, 20, 5403-5408.

101 B. Li, P. H. Dixneuf, Chem. Soc. Rev. 2013, 42, 5744-6337.
} 
Table 4.1. Optimization of oxidative annulation with benzamidine $117 \mathbf{a}^{[\mathrm{a}]}$

$\mathrm{Cu}(\mathrm{OAc})_{2} \cdot \mathrm{H}_{2} \mathrm{O}$
solvent, $T^{\circ} \mathrm{C}, 22 \mathrm{~h}$

[a] Reaction conditions: 117a $(0.5 \mathrm{mmol}), \mathbf{1 a}(0.6 \mathrm{mmol}), \mathrm{Cu}(\mathrm{OAc})_{2} \cdot \mathrm{H}_{2} \mathrm{O}(1.0 \mathrm{mmol})$, $\left[\mathrm{RuCl}_{2}(p \text {-cymene })\right]_{2}(5.0 \mathrm{~mol} \%), \mathrm{KPF}_{6}(30 \mathrm{~mol} \%)$, solvent $(2.0 \mathrm{~mL})$. [b] Yields of isolated products. [c] 2.0 equiv of alkyne $\mathbf{1 a}$.

\subsubsection{Scope and Limitations}

\subsubsection{Scope of Annulations with Alkynes}

Thereafter, with the optimized catalytic system in hand, we probed its scope and limitations in the oxidative annulation of differently decorated alkynes 1 (Scheme 4.2). We were delighted to find that arylalkynes $\mathbf{1 b}-\mathbf{1} \mathbf{d}$ and $\mathbf{1} \mathbf{m}$ bearing different functional groups were well tolerated by the ruthenium catalyst, though slightly lower yields were obtained when the electron-rich (1b) or electron-deficient diarylalkynes $\mathbf{1 d}$ and $\mathbf{1} \mathbf{m}$ as well as alkyne with sulfur-containing heteroaromatic moieties in substrate $\mathbf{1 n}$ were employed. The catalytic system was not limited to the use of diarylalkynes, but also allowed for the efficient conversion of the more challenging dialkylalkynes 1e and 1l. Unsymmetrically substituted alkynes $\mathbf{1 h}$ and $\mathbf{1 f}$ were 
converted with high regioselectivity, furnishing solely the products 118ah and 118af, respectively, with the aromatic substitutents in the neighbouring position to the nitrogen atom.<smiles></smiles>

$117 a$

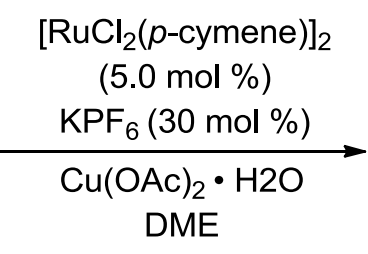

$120-140{ }^{\circ} \mathrm{C}, 22 \mathrm{~h}$

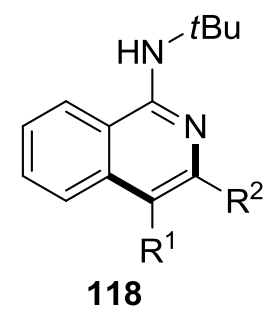

118<smiles>[R]c1ccc(-c2nc(NC(C)(C)C)c3ccccc3c2-c2ccc([R])cc2)cc1</smiles><smiles>CC(C)(C)Nc1nc(-c2cccs2)c(-c2cccs2)c2ccccc12</smiles><smiles>CC(C)(C)Nc1nc([Al])c([Al])c2ccccc12</smiles>

$\mathrm{R}=\mathrm{OMe}(118 \mathrm{ab}): 64 \%$

$\mathrm{R}=\mathrm{Me}(118 \mathrm{ac}): \quad 90 \%$

$\mathrm{R}=\mathrm{H}(118 \mathrm{aa}): \quad 80 \%$

$\mathrm{R}=\mathrm{F}$ (118ad): $\quad 68 \%$

$\mathrm{R}=\mathrm{Cl}$ (118am): $\quad 36 \%$<smiles>CCCCc1c(-c2ccc(OC)cc2)nc(NC(C)(C)C)c2ccccc12</smiles>

118ah: $46 \%$ 118an: $75 \%$

Alk $=n \operatorname{Pr}(118 \mathrm{ae}): 78 \%$

Alk = Et (118al): $\quad 84 \%$<smiles>Cc1c(-c2ccccc2)nc(NC(C)(C)C)c2ccccc12</smiles>

118af: $72 \%$

Scheme 4.2. Ruthenium(II)-catalyzed oxidative annulation with alkynes $\mathbf{1 .}$

\subsubsection{Scope of Annulations with Subs tituted Amidines}

Next, the tolerance of various electrophilic functional groups was investigated under this catalytic system. Substituted benzamidines $\mathbf{1 1 7 b}-\mathbf{1 1 7 h}$ bearing fluoro, chloro, bromo, iodio, or nitro substituents were well tolerated by the robust ruthenium(II) catalyst (Scheme 4.3).

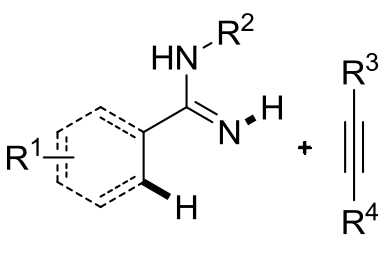

117

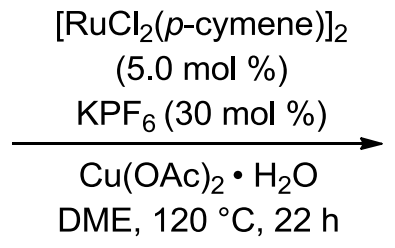

DME, $120^{\circ} \mathrm{C}, 22 \mathrm{~h}$<smiles>[R]Nc1nc([R])c([R])c2c[R14]([R1])ccc12</smiles>

118 


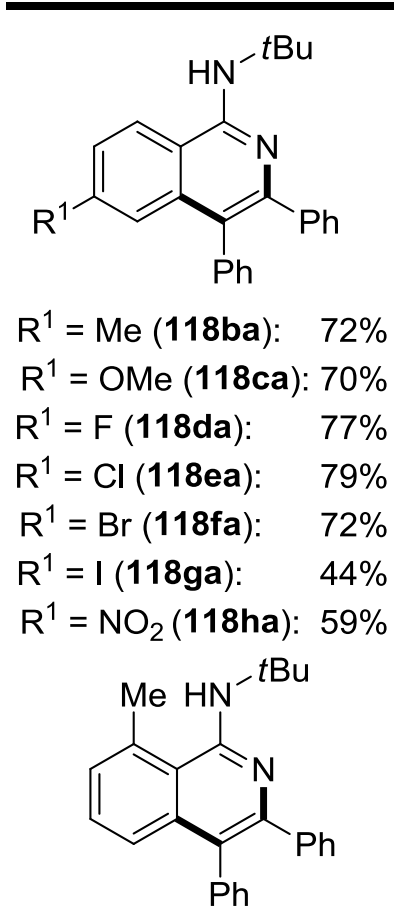

118la: $45 \%$ (in $\mathrm{H}_{2} \mathrm{O}$ )<smiles>[R]c1ccc2c(-c3ccccc3)c(-c3ccccc3)nc(NC(C)(C)C)c2c1</smiles>

$R^{1}=\operatorname{Me~(118ia):~} 62 \%$

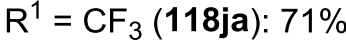<smiles>[R]Nc1nc(-c2ccccc2)c(-c2ccccc2)c2cc(C)ccc12</smiles>

$\mathrm{R}^{1}=i \mathrm{Bu}$ (118ma): $60 \%$ $\mathrm{R}^{1}=n \mathrm{Bu}(118 \mathrm{na}): 48 \%$<smiles>[R]c1nc(NC(C)(C)C)c2ccc3c(c2c1[R])OCO3</smiles>

$\mathrm{R}=n \operatorname{Pr}($ 118ke): $60 \%$

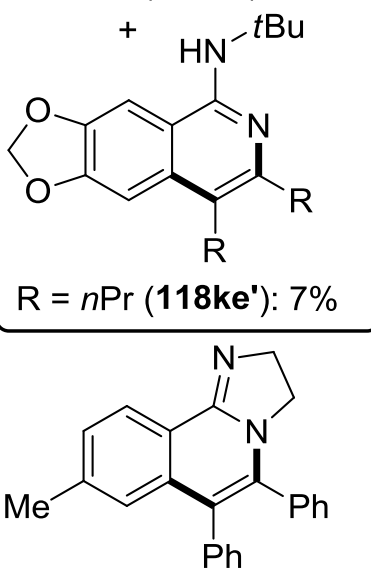

1180a: $62 \%$<smiles>CC(C)c1nc(NC(C)(C)C)c2c([nH]c3ccccc32)c1C(C)C</smiles>

118pe: $42 \%$<smiles>CCCc1c(C(C)C)nc(NC(C)(C)C)c2sccc12</smiles>

118qe: $57 \%\left(\right.$ in $\mathrm{H}_{2} \mathrm{O}$ )

Scheme 4.3. Scope of annulation with substituted amidines 117.

Intramolecular competition experiments with substrates $117 \mathbf{i}$ and $\mathbf{1 1 7} \mathbf{j}$ bearing meta-methyl or meta-trifluoromethyl substituents were controlled by steric interactions to deliver the sole products $118 \mathbf{i a}$ and $118 \mathbf{j a}$, respectively. In contrast, the functionalization occurred predominantly at the more sterically congested $\mathrm{C}-\mathrm{H}$ bond when there is a secondary directing-group installed on the amidine $\mathbf{1 1 7 k}$. Substrate $117 \mathrm{l}$ bearing a sterically hindering ortho-substituent gave the desired product 117la in a slightly reduced yield, as was also observed when using substrates $\mathbf{1 1 7} \mathbf{n}$ with a less bulky $N$-substitution pattern. It is worth noting that cyclic amidine $\mathbf{1 1 7 0}$ also proved to be viable starting materials and underwent the efficient conversion into product 1180a. Moreover, indole and thiophene derivatives $\mathbf{1 1 7 p}$ and 117q gave the desired products of the $\mathrm{C}-\mathrm{H} / \mathrm{N}-\mathrm{H}$ bonds functionalization as well, thus delivering $\gamma$-carboline 118pe and annulated azabenzothiophene 118qe.

Interestingly, a cascade twofold $\mathrm{C}-\mathrm{H} / \mathrm{N}-\mathrm{H}$ bonds functionalization occurred when utilizing 
the para-methoxybenzyl (PMB)-substituted benzamidine 117r and dialkylalkyne $1 \mathbf{e}$ as the starting materials, thereby highlighting the ability of the 1-aminoisoquinolines $\mathbf{1 1 8}$ themselves to serve as useful substrates for directed $\mathrm{C}-\mathrm{H}$ bond transformations. In contrast, the arylalkyne 1a uniquely furnished the product 118ra through a single $\mathrm{C}-\mathrm{H} / \mathrm{N}-\mathrm{H}$ bonds functionalization (Scheme 4.4).

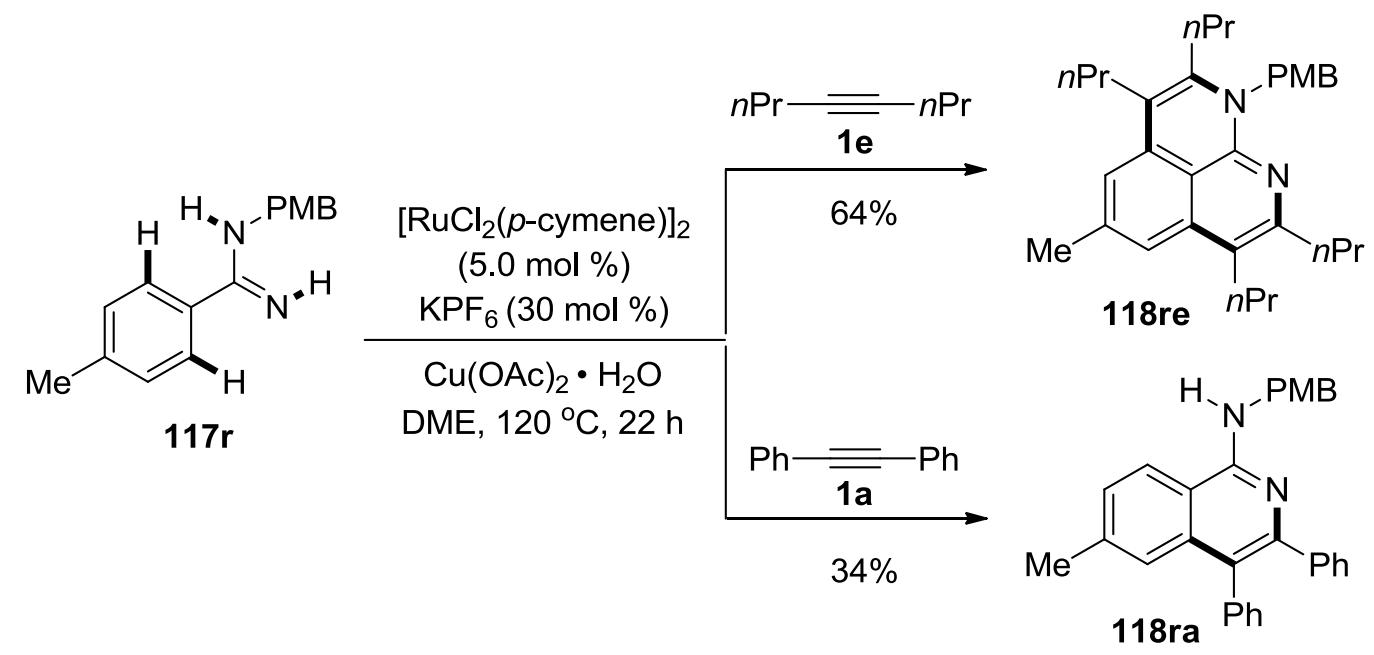

Scheme 4.4. Cascade twofold vs single $\mathrm{C}-\mathrm{H} / \mathrm{N}-\mathrm{H}$ bonds functionalizations.

\subsubsection{Mechanistic Studies}

\subsubsection{Intermolecular Competition Experiments}

Given the remarkable activity of the alkyne-annulation catalyst, we performed mechanistic studies to delineate its mode of action. To this end, intermolecular competition experiments revealed electron-rich benzamidine $\mathbf{1 1 7 c}$ to be preferentially converted (Scheme 4.5).

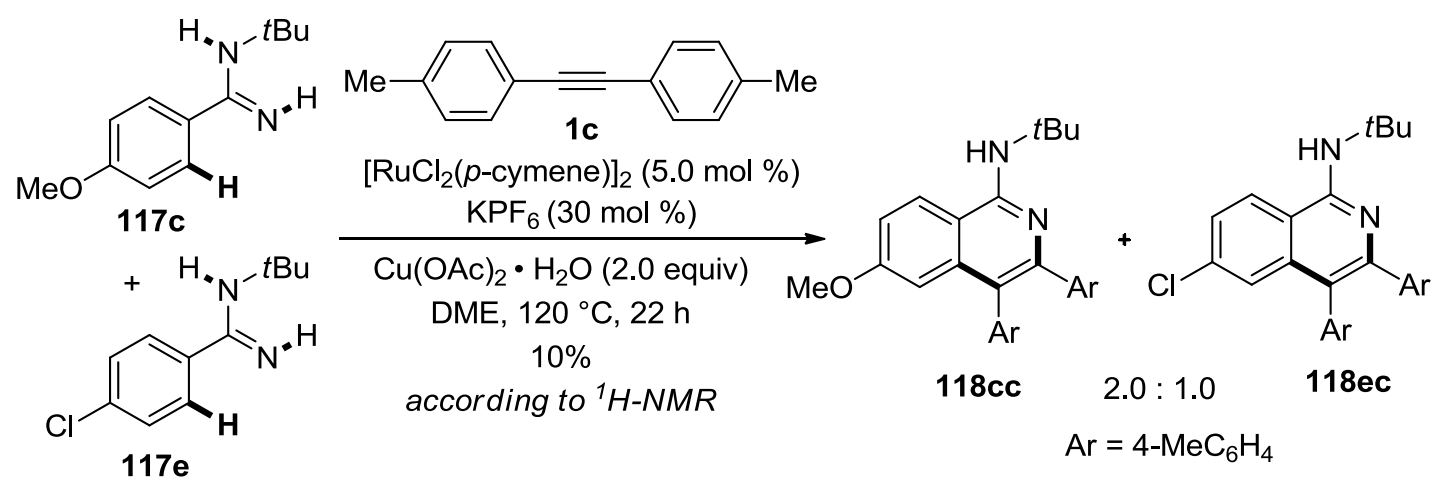

(2.0 equiv each)

Scheme 4.5. Intermolecular competition experiment with benzamidines $117 \mathrm{c}$ and $117 \mathrm{e}$.

This observation is in good agreement with the in situ generated cationic ruthenium(II) 
complex operating by an electrophilic-type activation mode.

Additionally, a competition experiment between diarylalkyne 1d and dialkylalkyne 1e highlighted that aromatic alkynes outperformed the corresponding alkyl-substituted substrate (Scheme 4.6).

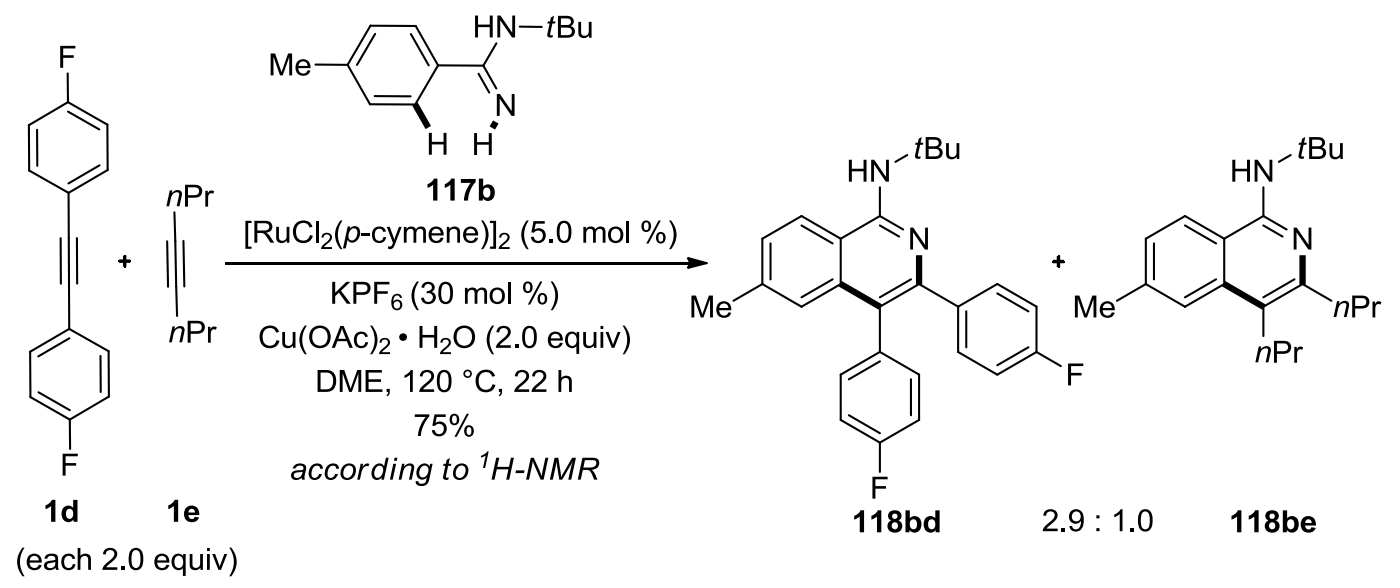

Scheme 4.6. Intermolecular competition experiment between alkynes 1 .

\subsubsection{Reactions in the Presence of Is otopically Labeled Solvent}

Subsequently, we explored the catalytic $\mathrm{C}-\mathrm{H}$ bond functionalization on benzamidine $\mathbf{1 1 7} \mathbf{b}$ in the presence of isotopically labeled water $\left(\mathrm{D}_{2} \mathrm{O}\right)$ as the cosolvent. These studies demonstrated a significant H/D exchange in the ortho-positions of both the reisolated starting material 117b (Scheme 4.7a) and the product 118, albeit with a considerably reduced deuterium incorporation when performing the reaction in the presence of alkyne 1e (Scheme 4.7b).

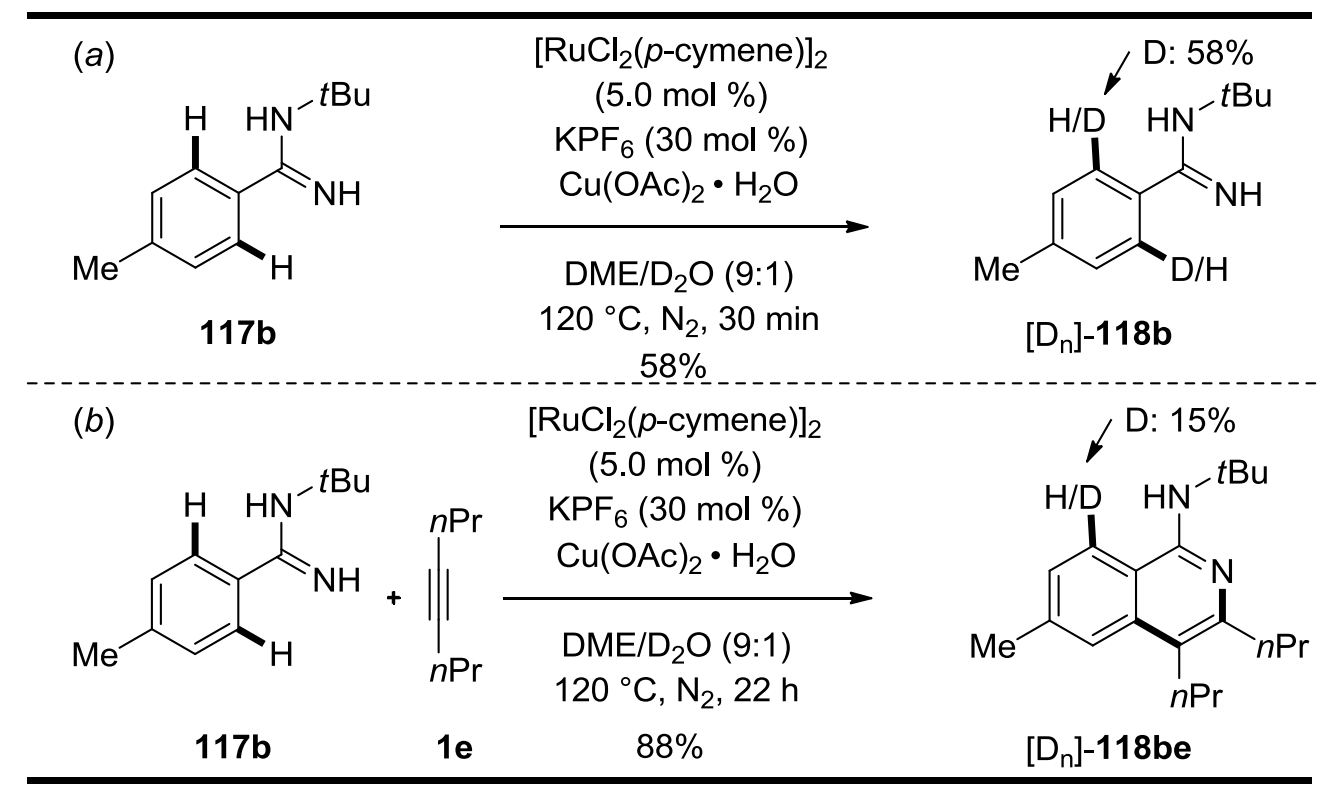

Scheme 4.7. H/D exchange reactions: (a) In the absence of 1. (b) In the presence of 1e. 


\subsubsection{Proposed Catalytic Cycle}

Given our mechanistic studies and the literature precedents, ${ }^{5 i}$ we proposed a plausible catalytic cycle which involves an initial reversible $\mathrm{C}-\mathrm{H}$ bond activation in substrate 117, along with subsequent migratory insertion of alkyne $\mathbf{1}$ into intermediate $\mathbf{A}$ (Scheme 4.8). Thereafter, reductive elimination in ruthenacycle $\mathbf{B}$ delivered the desired product 118, while reoxidation regenerated the catalytically active ruthenium(II) complex.

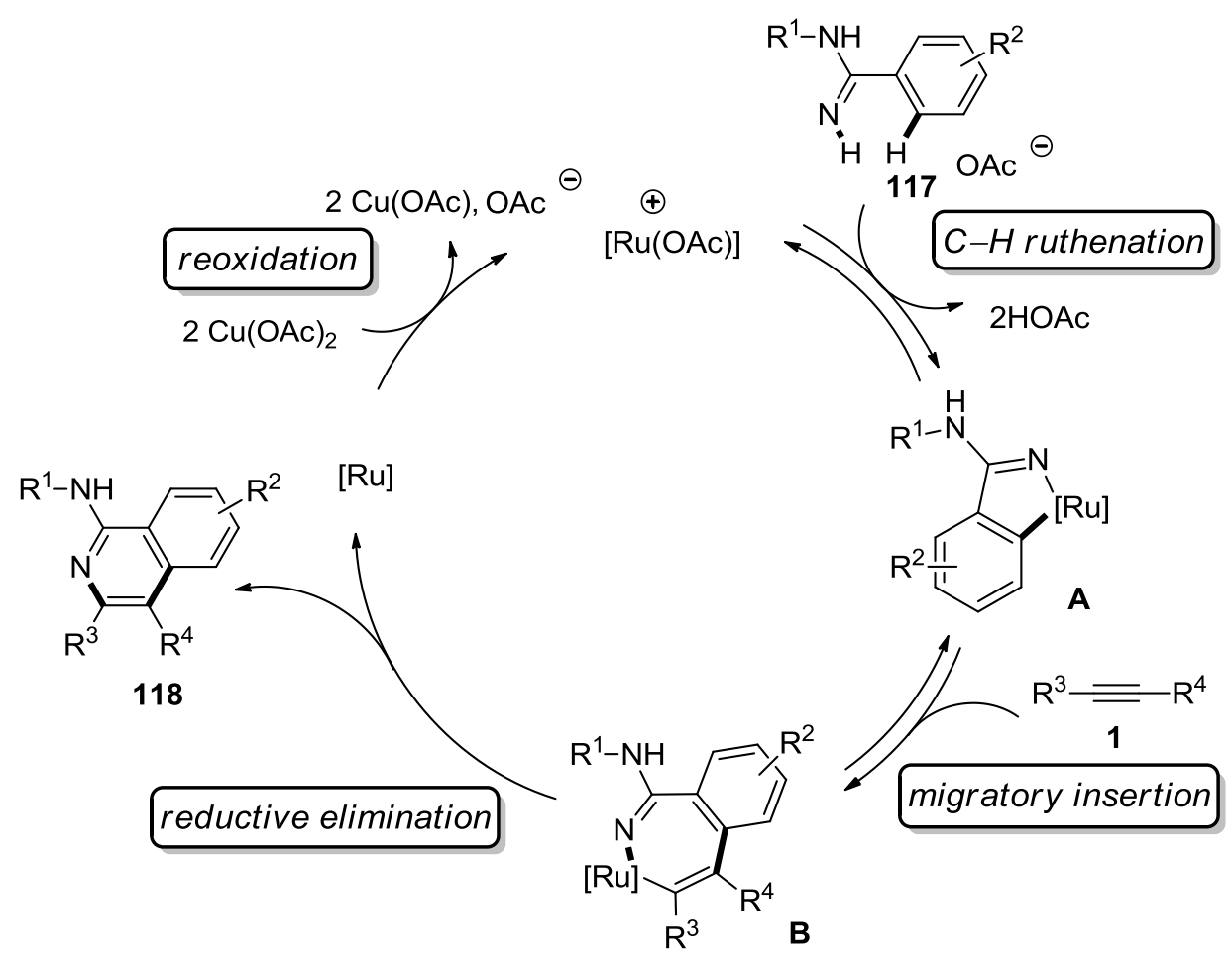

Scheme 4.8. Plausible mode of the catalyst action.

\subsection{Oxidative Alkenylation}

\subsubsection{Optimization Studies}

Taking into consideration the high catalytic efficacy of the ruthenium(II)-catalyzed oxidative annulation of alkynes $\mathbf{1}$, we became intrigued by developing novel oxidative twofold $\mathrm{C}-\mathrm{H}$ bond functionalizations with alkenes $\mathbf{2 0}$. Therefore, we initially studied the effect exerted by representative additives and solvents on the cross-dehydrogenative coupling of alkene 20a with amidine 117b (Table 4.2). Apparently, employing the catalytic system previously optimized for the oxidative alkyne annulation catalytic system delivered 3-alkylydene-1-iminoisoindoline 119ba in unsatisfactorily low yield (entry 1). This was caused by the formation of an undesired by-product stemming from $N$-deprotection, which 
could be isolated in $43 \%$ yield.

Table 4.2. Optimization of oxidative annulation with alkene $20 \mathrm{a} .{ }^{[\mathrm{a}]}$

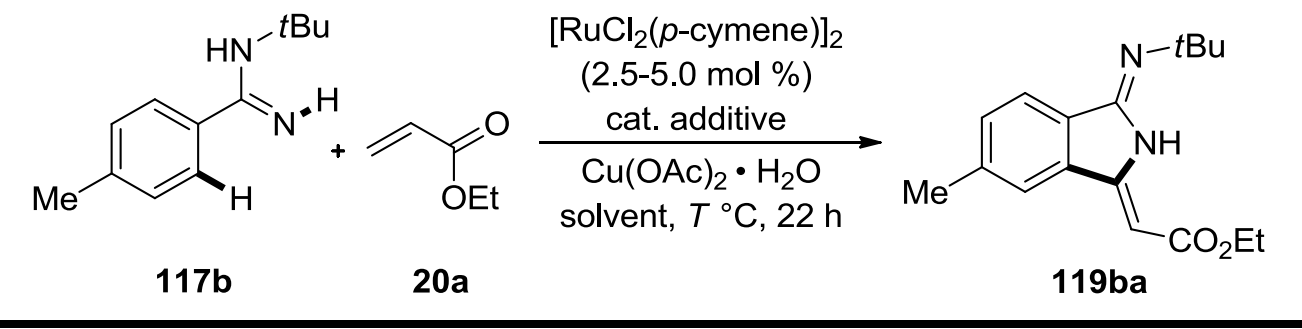

\begin{tabular}{|c|c|c|c|c|}
\hline Entry & Additive ([equiv]) & $T[\mathbb{C}]$ & Solvent & Yield $[\%]^{[b]}$ \\
\hline 1 & $\mathrm{KPF}_{6}(0.3)$ & 120 & DME & $18^{[\mathrm{c}]}$ \\
\hline 2 & $\mathrm{AgSbF}_{6}(0.2)$ & 120 & DME & 41 \\
\hline 3 & $\mathrm{KPF}_{6}(0.3)$ & 120 & DCE & 63 \\
\hline 4 & $\operatorname{AgSbF}_{6}(0.2)$ & 120 & DCE & 66 \\
\hline 5 & $\mathrm{AgPF}_{6}(0.2)$ & 120 & DCE & 13 \\
\hline 6 & $\mathrm{CF}_{3} \mathrm{CO}_{2} \mathrm{Ag}(0.2)$ & 120 & DCE & 59 \\
\hline 7 & $\mathrm{AgOAc}(0.3)$ & 120 & DCE & 70 \\
\hline 8 & $\mathrm{AgOAc}(0.5)$ & 120 & DCE & 75 \\
\hline 9 & $\operatorname{AgOAc}(0.5)$ & 100 & $D C E$ & $73^{[d]}$ \\
\hline 10 & $\mathrm{AgOAc}(0.5)$ & 80 & DCE & $69^{[\mathrm{d}]}$ \\
\hline 11 & $\mathrm{AgOAc}(0.5)$ & 60 & DCE & $64^{[\mathrm{d}]}$ \\
\hline
\end{tabular}

[a] Reaction conditions: $\mathbf{1 1 7 b}(0.5 \mathrm{mmol}), \mathbf{2 0 a}$ (1.5 equiv), $\mathrm{Cu}(\mathrm{OAc})_{2} \cdot \mathrm{H}_{2} \mathrm{O}$ (2.0 equiv), $\left[\mathrm{RuCl}_{2}(p \text {-cymene })\right]_{2}(5.0 \mathrm{~mol} \%)$, cat. additive, solvent $(2.0 \mathrm{~mL}), 18-22 \mathrm{~h}$. [b] Yields of isolated products. [c] Along with $43 \%$ of the corresponding $N$-deprotected product. [d]

$$
\left[\mathrm{RuCl}_{2}(p \text {-cymene })\right]_{2}(2.5 \mathrm{~mol} \%) \text {. }
$$

However, we were pleased to observe that the efficacy of the oxidative alkenylation process was remarkably improved when using $\mathrm{AgSbF}_{6}$ as the additive in DCE as the solvent (entries 2 and 3). Among a series of silver(I) salts (entries 4-8), $50 \mathrm{~mol} \%$ of AgOAc gave the product in optimal yields (entries 8 and 9). This allowed for efficient catalysis to occur at a reduced catalyst loading and at reaction temperatures as low as $60 \mathrm{C} .{ }^{50}$ Various oxidants other than $\mathrm{Cu}(\mathrm{OAc})_{2} \cdot \mathrm{H}_{2} \mathrm{O}$, such as $\mathrm{CuBr}_{2}, \mathrm{~K}_{2} \mathrm{~S}_{2} \mathrm{O}_{8}$, or $\mathrm{PhI}(\mathrm{OAc})_{2}$, did not furnish the desired product $119 \mathrm{ba}$. 


\subsubsection{Scope and Limitations}

\subsubsection{Scope of $\mathrm{N}$-Subs tituents on Amidines}

In order to test the range of our optimized catalytic system for the oxidative alkenylation, we initially evaluated the influence exerted by the different steric bulk of the $N$-substituent in benzamidines 117 (Scheme 4.9). Among the less sterically hindered iso- or $n$-butyl-substituted substrates, amidines $117 \mathbf{m}$ and $117 \mathbf{n}$ (Scheme 4.9) delivered inferior results as compared to the tert-butyl analogue $\mathbf{1 1 7} \mathbf{b}$ (Table 4.2). It is worth noting that detailed spectroscopic studies on the product's connectivity revealed that the reduced steric bulk of the $N$-substituent strongly influenced the chemo- and diastereoselectivity of the isoindoline formation in that the substituted nitrogen atom of the amidine underwent the $\mathrm{C}-\mathrm{N}$ bond formation. Furthermore, the PMB-substituted benzamidine $117 \mathbf{r}$ gave the desired products 119 'ra-119'uc in high yields as well.<smiles></smiles>

117

20
$\left[\mathrm{RuCl}_{2}(p \text {-cymene })\right]_{2}$ $(2.5 \mathrm{~mol} \%)$ $\underset{\mathrm{Cu}(\mathrm{OAc})_{2} \cdot \mathrm{H}_{2} \mathrm{O}}{\stackrel{\mathrm{AgOAc}(50 \mathrm{~mol} \%)}{\longrightarrow}}$ DME, $100^{\circ} \mathrm{C}, 22 \mathrm{~h}$<smiles>[R]OC(=O)/C=C1\c2cc(C)ccc2C(=N)N1[R]</smiles>

$119^{\prime}$

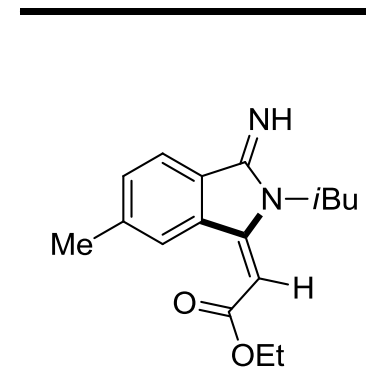

119'ma: $77 \%$<smiles>[R]C(=O)/C=C1\c2cc(C)ccc2C(=N)N1Cc1ccc(OC)cc1</smiles>

$\mathrm{R}=$ OEt (119'ra): $74 \%$

$\mathrm{R}=\mathrm{OBu}\left(119{ }^{\prime} \mathrm{rb}\right): 74 \%$

$\mathrm{R}=\mathrm{OBn}$ (119'rc): $69 \%$

Scheme 4.9. Reactivity of benzamidines 117 with less bulky $N$-substituents.

\subsubsection{Scope of the Oxidative Alkenylation with Aromatic Amidines and Acrylates}

Thereafter, we examined the scope of the ruthenium(II)-catalyzed cross-dehydrogenative alkenylation with different substituted benzamidines 117 and alkenes 20 (Scheme 4.10a). Remarkably, the ruthenium(II) catalyst turned out to be widely applicable and tolerated various electrophilic functional groups, such as fluoro, chloro, bromo, and nitro substituents, 
albeit with a low yield of the desired product $119 \mathrm{ha}$ in the latter case. The $\mathrm{C}-\mathrm{H}$ bond functionalizations occurred efficiently with para- and more sterically hindered ortho-substituted amidines $\mathbf{1 1 7 b}, \mathbf{1 1 7} \mathbf{c}$ and $\mathbf{1 1 7 1}$.

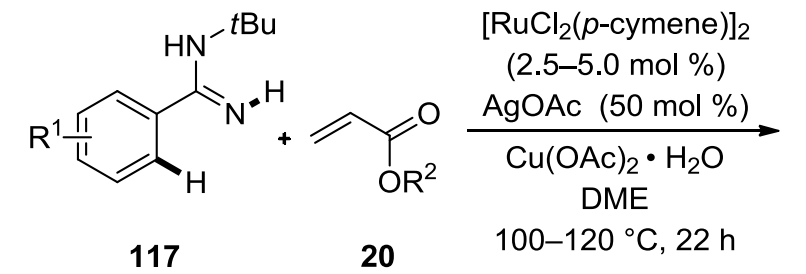<smiles>[R1][R]C=C1N/C(=N\C([R])C)c2cc[R1]([H])cc21</smiles><smiles>[R]C(=O)/C=C1\N/C(=N\CC)c2ccc(C)cc21</smiles>

$R=$ OEt (119ba): $73 \%$ $\mathrm{R}=\mathrm{OBu}(119 \mathrm{bb}): 64 \%$<smiles>[R]c1ccc2c(c1)/C(=N/C(=O)OC)N/C2=N/C(=O)/C=C/C(=O)OCC</smiles>
$\begin{array}{ll}R=H(119 a a): & 58 \% \\ R=O M e(119 c a): & 84 \% \\ R=F(119 \text { da }): & 72 \% \\ R=B r(119 f a): & 64 \% \\ R=\mathrm{NO}_{2} \text { (119ha): } & 26 \%\end{array}$<smiles>CCCCOC(=O)/C=C1\N/C(=N\CC(C)C)c2ccc(OC)cc21</smiles>
$\mathrm{R}=\mathrm{OBn}(119 \mathrm{bc}): 68 \%$<smiles>CCOC(=O)/C=C1\N/C(=N\C(C)C)c2cccc(O)c21</smiles>

119la: $64 \%$<smiles>[R]c1ccc2c(c1)/C(=N/C(C)C)N/C2=C\C(=O)OCC</smiles>

$\mathrm{R}=\mathrm{Et}(\mathbf{1 1 9 e a )}): 70 \%$

$\mathrm{R}=\mathrm{Bu}(119 \mathrm{eb}): 73 \%$

$\mathrm{R}=\mathrm{Me}$ (119ia): $80 \%$

$\mathrm{R}=\mathrm{CF}_{3}$ (119ja): $50 \%$

$\mathrm{R}=\mathrm{Bn}(\mathbf{1 1 9 e c}): 71 \%$

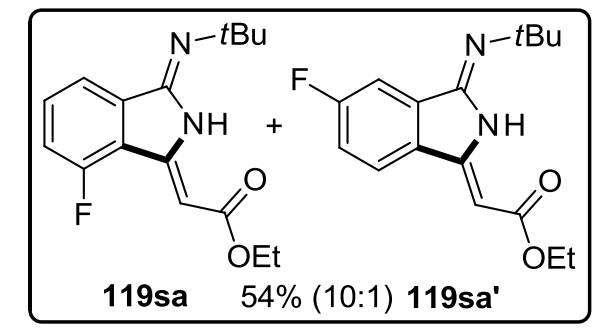<smiles>CCOC(=O)/C=C1\N/C(=N\C(C)C)c2ccc3c(c21)OCO3</smiles>

119ka: $68 \%$

Scheme 4.10. Scope of oxidative synthesis of 1-iminoisoindolines 119.

Intramolecular competition experiments with meta-substituted amidines $117 \mathbf{i}$ and $\mathbf{1 1 7} \mathbf{j}$ were also tested and disclosed the formation of products $119 \mathrm{ia}$ and $119 \mathrm{ja}$ to be controlled predominantly by steric interactions. Yet, when using substrates having heteroatom substituents with a secondary directing group chelation effect ${ }^{102}$ (substrate 117s) or the fluoro substituent possessing the ortho-orienting effect (substrate 117k), ${ }^{103}$ sterically more hindered

102 D. Balcells, E. Clot, O. Eisenstein, Chem. Rev. 2010, 110, 749-823.

103 E. Clot, O. Eisenstein, N. Jasim, S. A. Ma cgregor, J. E. McGrady, N. Perutz, Acc. Chem. Res. 2011, 44, 333-348. 
compounds 119sa and 119ka were obtained as the major or the sole product, respectively (Scheme 4.10). The results of $119 \mathrm{~s}$ a revealed a considerable secondary directing group effect exerted by fluoro and methylenedioxy present in benzamidines $117 \mathrm{~s}$ and $117 \mathrm{k}$, respectively (Scheme 4.10).

\subsubsection{Mechanistic Studies}

\subsubsection{Intermolecular Competition Experiments}

To understand the working mode of our novel 1-imino-isoindoline (119) synthes, we performed intermolecular competition experiments between differently substituted benzamidines $\mathbf{1 1 7}$. These studies revealed that the electron-rich substrate $\mathbf{1 1 7 b}$ is inherently more reactive (Scheme 4.11), which coincides with the observation made within the ruthenium-catalyzed oxidative alkyne annulation (see above).<smiles>Cc1ccc(C(=N)NC(C)(C)C)cc1</smiles>

$117 \mathrm{~b}$<smiles>CC(C)(C)NC(=N)c1ccc(F)cc1</smiles>

$117 \mathrm{~d}$

(each 2.0 equiv)

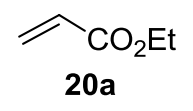

119ba: $15 \%$ 119da: $8 \%$<smiles>CCOC(=O)C=c1[nH]c(=NCC(C)C)c2cc(F)ccc2c1=CC(=O)OCC</smiles>

Scheme 4.11. Intermolecular competition experiment.

\subsubsection{Expe riment in the Presence of Is otopically Labe le d Solvent}

In addition, we performed reactions with the isotopically labeled cosolvent $\left[\mathrm{D}_{4}\right]-\mathrm{MeOH}$. These experiments revealed the high efficiency of the optimized catalytic system with cationic ruthenium(II) catalyst by giving a remarkable conversion of the substrate $117 \mathbf{c}$ within only $15 \mathrm{~min}$. Obvious evidence of H/D scrambling on both of the recycled starting material $\left[D_{n}\right]-117 c$ and desired product $\left[D_{n}\right]-119 c a$ was gathered (Scheme 4.12), which unraveled that the ruthenium(II)-catalyzed alkenylation is undergoing a reversible $\mathrm{C}-\mathrm{H}$ bond metalation step. Notably, an important intermediate $135 \mathrm{ca}$ was also isolated from the reaction mixture, thus indicating the reaction sequence to comprise an initial oxidative alkenylation followed by 
subsequent intramolecular aza-Michael reaction. Both products $119 \mathrm{ca}$ and $135 \mathrm{ca}$ showed deuterium incorporation in the $\alpha$-position to the carbonyl group, which is likely owing to the rapid $\mathrm{H} / \mathrm{D}$ exchange on the $\mathrm{N}-\mathrm{H}$-acidic amidine group prior to the intramolecular conjugate addition.

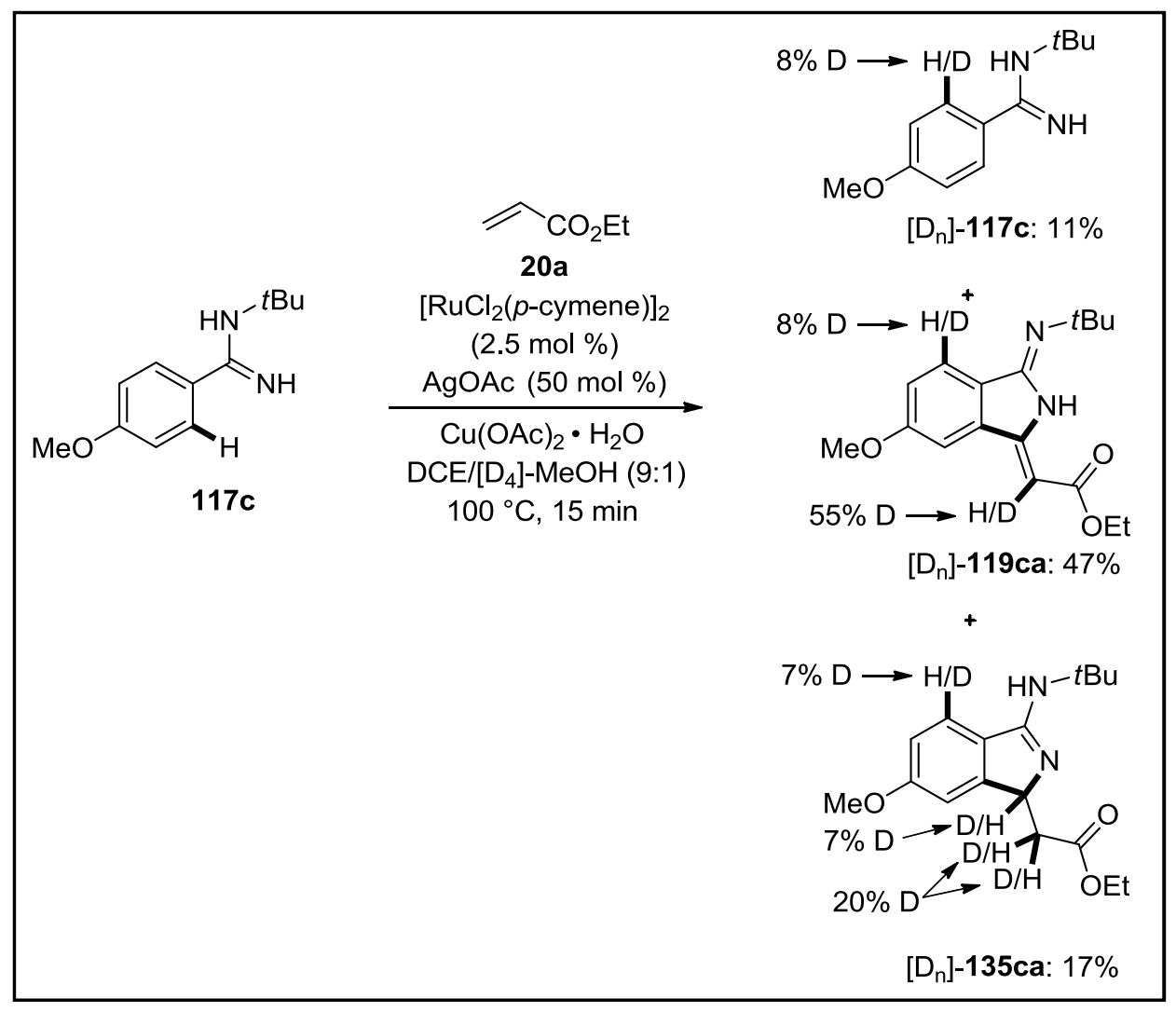

Scheme 4.12. $\mathrm{C}-\mathrm{H}$ bond activation in the presence of $\left[\mathrm{D}_{4}\right]-\mathrm{MeOH}$.

The intermediate $135 \mathrm{ca}$ was obtained as a sole product by performing the cyclization without copper(II) acetate. Moreover, we established that the dehydrogenative formation of product $119 \mathrm{ca}$ from $135 \mathrm{ca}$ was indeed viable in the absence of the ruthenium catalyst, thus solely being mediated by the copper(II) oxidant (Scheme 4.13).

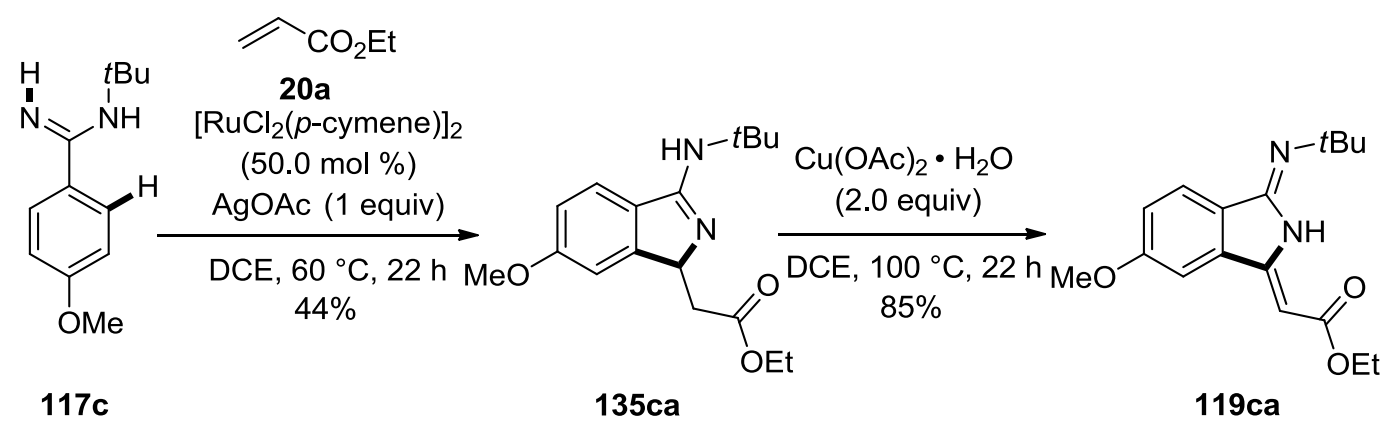

Scheme 4.13. Synthesis of intermediate $135 \mathrm{ca}$ and copper(II)-mediated oxidative alkene formation. 


\subsubsection{Proposed Cataly tic Cycle}

Based on our mechanistic studies, we propose the initial $\mathrm{C}-\mathrm{H}$ bond activation to involve a reversible acetate-assisted cycloruthenation of amidine $\mathbf{1 1 7}$ with the cationic species to form complex 136 (Scheme 4.14), which then undergoes a migratory insertion of a lkene 20 to give the intermediate 137. Subsequently, acetate-assisted $\beta$-hydride elimination gives rise to the product of oxidative alkenylation 138, while reductive elimination and reoxidation by $\mathrm{Cu}(\mathrm{OAc})_{2} \cdot \mathrm{H}_{2} \mathrm{O}$ regenerates the catalytically active ruthenium(II) complex. The desired product $\mathbf{1 1 9}$ is yielded through an intramolecular aza-Michael addition of compound 138, followed by dehydrogenation of the obtained intermediate 135 .

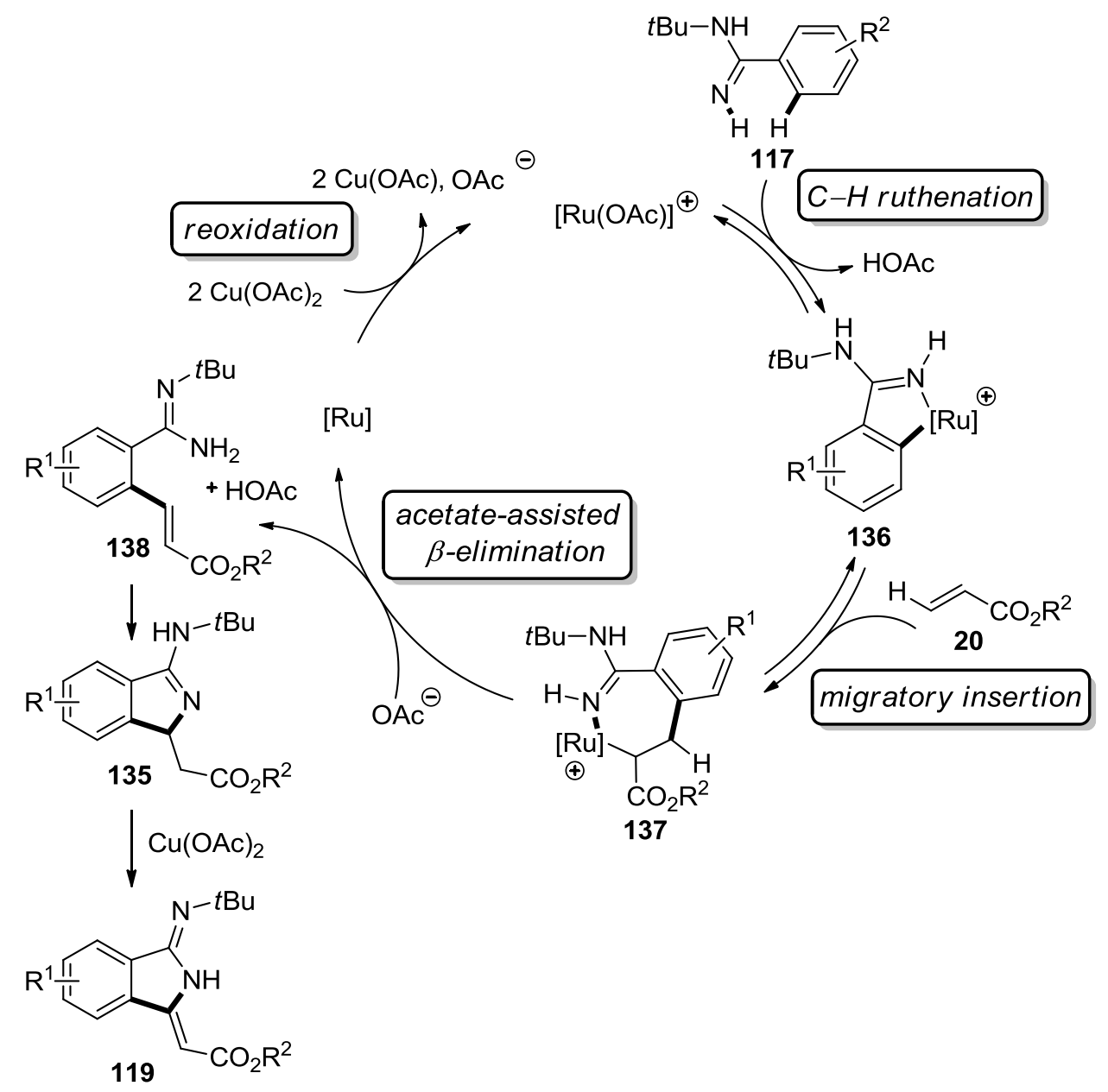

Scheme 4.14. Proposed catalytic cycle.

\subsubsection{Conclusion}

In summary, we have reported on the unprecedented ruthenium(II)-catalyzed oxidative $\mathrm{C}-\mathrm{H}$ bond functionalization on easily accessible aryl amidines $\mathbf{1 1 7}$ with alkynes $\mathbf{1}$ and alkenes $\mathbf{2 0}$. 
Thus, in situ formed cationic ruthenium(II) complexes allowed for alkyne annulations by $\mathrm{C}-\mathrm{H} / \mathrm{N}-\mathrm{H}$ bonds functionalizations to give diversely substituted 1 -aminoisoquinolines $\mathbf{1 1 8}$, which served as viable substrates for catalyzed $\mathrm{C}-\mathrm{H}$ bond activation reactions themselves. Furthermore, novel oxidative alkenylations of benzamidines proved to be viable with versatile cationic ruthenium(II) complexes and, thereby, provided access to structurally diverse iminoisoindolines 119. Detailed mechanistic studies indicated the reversibility of the key $\mathrm{C}-\mathrm{H}$ bond activation step in both cases. 


\section{Ruthenium(II)-Catalyzed C-H Bond Hydroarylation and Oxidative Annulation with $\alpha, \beta$-Unsaturated Ketones via Monodentate Directing Group}

Early development of ruthenium-catalyzed $\mathrm{C}-\mathrm{H}$ bond hydroarylation was started by Lewis and Smith, $^{33}$ as well as Murai and coworkers who published a report on the "ruthenium-catalyzed ortho-C-H bond hydroarylation of aromatic ketones with olefins via a chelation assisted strategy" in $1993 .{ }^{46}$ Advances were subsequently made with the more flexible and practical ruthenium complex $\left[\mathrm{RuCl}_{2}(p \text {-cymene })\right]_{2}$. However, despite of a few examples in the literature which were rather rare and limited to the use expensive rhodium ${ }^{44}$ or rhenium ${ }^{45}$ catalysts, several important families of acceptors, such as $\alpha, \beta$-unsaturated ketones, could thus far not be utilized. In recent years, bidentate directing groups have emerged as a new powerful tool in $\mathrm{C}-\mathrm{H}$ functionalization. A major progress was achieved by Daugulis, ${ }^{68 c, 77,104}$ Chatani, ${ }^{105}$ and Ackermann ${ }^{78, f, 106}$ et al. Thus, Chatani and coworkers developed the ruthenium-catalyzed $\mathrm{C}-\mathrm{H}$ hydroarylation of aromatic substrates with $\alpha, \beta$-unsaturated ketones via a removable 8-aminoquinoline bidentate directing group. ${ }^{46}$ Herein, we developed ruthenium-catalyzed $\mathrm{C}-\mathrm{H}$ bond hydroarylation and oxidative annulation with $\alpha, \beta$-unsaturated ketones via more atom-economical monodentate directing group.

\subsection{Optimization Studies}

We initiated our studies by testing the feasibility of the ruthenium(II)-catalyzed $\mathrm{C}-\mathrm{H}$ bond hydroarylation of methyl vinyl ketone (41a) with benzamide 2a (Table 5.1). Thus far, the reported ruthenium-catalyzed direct hydroarylation with MVK by Chatani was accomplished with $\mathrm{RuCl}_{2}\left(\mathrm{PPh}_{3}\right)_{3}$ as the catalyst. ${ }^{46}$ However, catalyst delivered no desired product with the assistance of monodentate amide (entries 1-2). Similar observations were made when employing combinations of $\left[\mathrm{RuCl}_{2}(p \text {-cymene) }]_{2}\right.$ and additives (entries 3-5). A significant breakthrough was made using $\mathrm{MesCO}_{2} \mathrm{~K}$ and $\mathrm{MesCO}_{2} \mathrm{H}$ as the cocatalysts (entry 6).

\footnotetext{
104 a) D. Shabashov, O. Daugulis, J. Am. Chem. Soc. 2010, 132, 3965-3972; b) E. T. Nadres, O. Daugulis, J. Am. Chem. Soc. 2012, 134, 7-10; c) L. D. Tran, I. Popov, O. Daugulis, J. Am. Chem. Soc. 2012, 134, 18237-18240.

105 a) Y. Aihara, N. Chatani, J. Am. Chem. Soc. 2013, 135, 5308-5311; b) Y. Aihara, N. Chatani, Chem. Sci. 2013, 4, 664-670; c) Y. Ano, M. Tobisu, N. Chatani, Org. Lett. 2012, 14, 354-357; d) Y. Ano, M. Tobisu, N. Chatani, J. Am. Chem. Soc. 2011, 133, 12984-12986.

${ }^{106}$ H. H. Al Mamari, E. Diers, L. Ackermann, Chem. Eur. J. 2014, 20, 9739-9743.
} 
Stoichiometric $\mathrm{MesCO}_{2} \mathrm{H}$ loading provided the optimal result (entry 7). Notably, omission of either of the two additives, or employing $\left[\mathrm{Ru}\left(\mathrm{MesCO}_{2}\right)_{2}(p\right.$-cymene $\left.)\right]$ as the catalyst, or reducing the catalyst loading resulted in significantly reduced yield of the desired product 120 aa (entries 8-11). Furthermore, it is worth noting that the combination of KOAc and HOAc was found to be suitable as well, albeit with lower efficacy (entry 12).

Table 5.1. Optimization of ruthenium(II)-catalyzed $\mathrm{C}-\mathrm{H}$ bond hydroarylation in benzamide $2 \mathrm{a}^{[\mathrm{a}}{ }^{\text {] }}$<smiles>[N+]NC(=O)c1ccccc1</smiles>

$2 a$

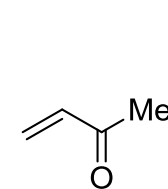

$41 \mathrm{a}$

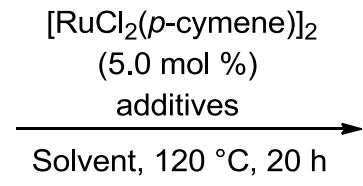

Additive B [equiv]

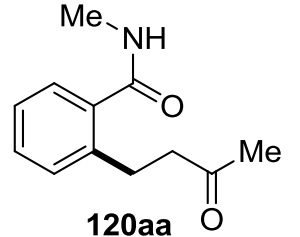

Solvent $\quad$ Yield $[\%]^{[b]}$

\begin{tabular}{|c|c|c|c|c|}
\hline Entry & Additive A [equiv] & Additive $\mathbf{B}$ [equiv] & Solvent & Yield $[\%]^{[b]}$ \\
\hline 1 & $\mathrm{NaOAc}(0.3)$ & -- & $\mathrm{PhMe}$ & $0^{[\mathrm{c}]}$ \\
\hline 2 & $\mathrm{NaOAc}(0.3)$ & -- & $\mathrm{H}_{2} \mathrm{O}$ & $0^{[\mathrm{c}]}$ \\
\hline 3 & $\mathrm{KPF}_{6}(0.2)$ & -- & $\mathrm{H}_{2} \mathrm{O}$ & 0 \\
\hline 4 & $\mathrm{KPF}_{6}(0.2)$ & $\mathrm{NaOAc}(2.0)$ & $\mathrm{H}_{2} \mathrm{O}$ & 0 \\
\hline 5 & $\mathrm{PPh}_{3}(0.15)$ & $\mathrm{NaO}_{2} \mathrm{CH}(0.3)$ & $\mathrm{PhMe}$ & 0 \\
\hline 6 & $\mathrm{MesCO}_{2} \mathrm{~K}(0.3)$ & $\mathrm{MesCO}_{2} \mathrm{H}(0.3)$ & $\mathrm{H}_{2} \mathrm{O}$ & 69 \\
\hline 7 & $\mathrm{MesCO}_{2} \mathrm{~K}(0.3)$ & $\mathrm{MesCO}_{2} \mathrm{H}(1.0)$ & $\mathrm{H}_{2} \mathrm{O}$ & 80 \\
\hline 8 & $\mathrm{MesCO}_{2} \mathrm{~K}(0.3)$ & -- & $\mathrm{H}_{2} \mathrm{O}$ & 51 \\
\hline 9 & -- & $\mathrm{MesCO}_{2} \mathrm{H}(1.0)$ & $\mathrm{H}_{2} \mathrm{O}$ & 29 \\
\hline 10 & -- & -- & $\mathrm{H}_{2} \mathrm{O}$ & $58^{[\mathrm{d}]}$ \\
\hline 11 & $\mathrm{MesCO}_{2} \mathrm{~K}(0.3)$ & $\mathrm{MesCO}_{2} \mathrm{H}(1.0)$ & $\mathrm{H}_{2} \mathrm{O}$ & $46^{[\mathrm{e}]}$ \\
\hline 12 & KOAc (1.0) & HOAc (1.0) & $\mathrm{H}_{2} \mathrm{O}$ & 64 \\
\hline
\end{tabular}

[a]General reaction conditions: $\mathbf{2 a}(0.5 \mathrm{mmol}), \mathbf{4 1 a}(1.0 \mathrm{mmol}),\left[\mathrm{RuCl}_{2}(p-c y m e n e)\right]_{2}(5.0 \mathrm{~mol} \%)$, $\mathrm{Mes}_{2} \mathrm{CO}_{2} \mathrm{~K}$ (0.3 equiv), $\mathrm{MesCO}_{2} \mathrm{H}$ (1.0 equiv), $\mathrm{H}_{2} \mathrm{O}\left(2.0 \mathrm{~mL}\right.$ ), under $\mathrm{N}_{2}, 120 \mathrm{C}, 20 \mathrm{~h}$. [b] Isolated yields. [c] $\mathrm{RuCl}_{2}\left(\mathrm{PPh}_{3}\right)_{3}(10 \mathrm{~mol} \%)$. [d] $\left[\mathrm{Ru}\left(\mathrm{MesCO}_{2}\right)_{2}(p\right.$-cymene $\left.)\right](10 \mathrm{~mol} \%)$. [e] $\left[\mathrm{RuCl}_{2}(p \text {-cymene })\right]_{2}(2.5 \mathrm{~mol} \%)$. 


\subsection{Scope of the Alkylation with $\alpha, \beta$-unsaturated Ketones}

With the optimized ruthenium(II) catalyst in hand, we tested its versatility in the $\mathrm{C}-\mathrm{H}$ bond hydroarylation with weakly-coordinating ${ }^{7 \mathrm{~b}}$ benzamides 2 (Scheme 5.1). Notably, in these chelation-assisted direct hydroarylations, both electron-rich and electron-poor para-substituted benzamides $\mathbf{2 a - 2 f}$ were identified as viable substrates. Furthermore, a variation of the substitution pattern on the nitrogen atom was also investigated, such as benzyl (2g-2i), cyclohexyl (2j) and methoxyethyl (2k), which proved to be suitable and did not significantly alter the catalytic efficacy. More sterically hindered ortho-substituted benzamide 2l was successfully employed as well, albeit furnishing the desired product in a slightly reduced yield. Intramolecular competition experiments with meta-substituted benzamides $\mathbf{2 m}$ and $\mathbf{2 n}$ featured a considerable secondary directing group effect ${ }^{102}$ or ortho-orienting effect, ${ }^{103}$ respectively, thus leading to a site-selective formation of sterically more hindered compounds $120 \mathrm{ma}$ and 120 na as the sole product. In contrast, the conversion of the meta-methyl and meta-trifluoromethyl substituted arenes $\mathbf{2 0 -}-\mathbf{2 q}$ was largely governed by steric interactions to deliver the products 1200a-120qa at the less sterically hindered position. The widely applicable ruthenium(II) catalyst was not limited to aromatic benzamides. Indeed, the reaction of the heteroaromatic indole derivative $2 \mathbf{r}$ also led to the site-selective hydroarylation in modest yie ld. Interestingly, $N$-( $p$-tolyl)acetamide (121a) was proved to be a suitable substrate as well, although a modest yield was obtained under the optimal conditions. However, among a set of varied $\alpha, \beta$-unsaturated ketones, the aromatic ketone $41 \mathrm{~b}$ only delivered the desired product $120 \mathrm{cb}$ in only low yield, presumably due to a polymerization tendency of the ketone. Yet, vinyl alkyl ketones 41c and 41d gave the alkylated products $120 \mathrm{dc}$ and $120 \mathrm{dd}$, respectively, in relatively high yields.<smiles>[R]NC(=O)C1=CC=C[R1]C=C1</smiles>

2

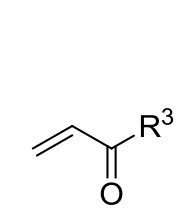

41

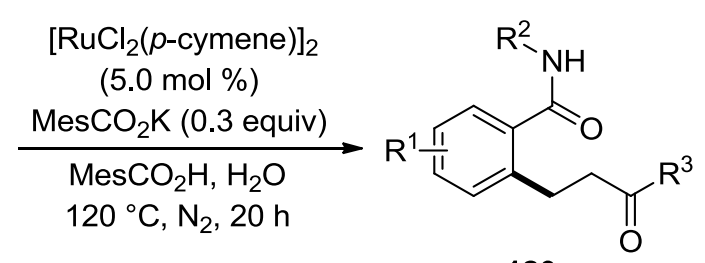

120 


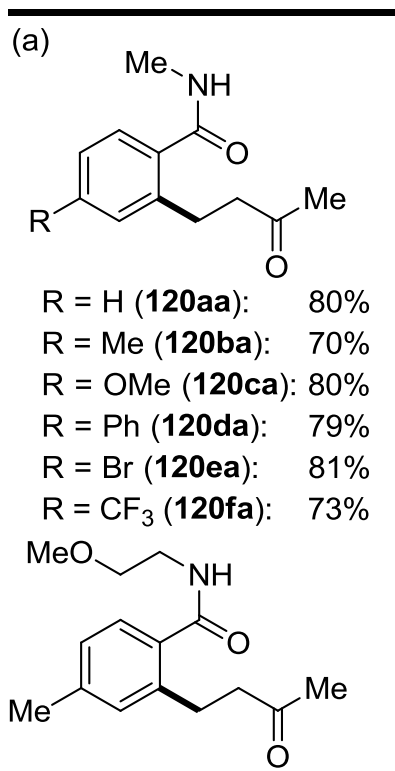

120ka: $58 \%$<smiles>CNC(=O)c1cccc(F)c1CCC(C)=O</smiles>

120na: $77 \%$<smiles>[R]c1ccc(C(=O)NCc2ccccc2)c(CCC(C)=O)c1</smiles>

$\mathrm{R}=\mathrm{OMe}$ (120ga): $68 \%$

$\mathrm{R}=\mathrm{F}$ (120ha): $\quad 73 \%$

$\mathrm{R}=\mathrm{Cl}(120 \mathrm{ia}): \quad 61 \%$<smiles>CNC(=O)c1c(CCC(C)=O)ccc2ccccc12</smiles>

120la: $45 \%$<smiles>[R]c1ccc(CCC(C)=O)c(C(=O)NC)c1</smiles>

$\mathrm{R}=\mathrm{Me}(120 \mathrm{oa}): 62 \%$

$\mathrm{R}=\mathrm{CF}_{3}$ (120pa): $62 \%$<smiles>CC(=O)CCc1cc(C)ccc1C(=O)NC1CCCCC1</smiles>

120ja: $70 \%$<smiles>CNC(=O)c1ccc2c(c1CCC(C)=O)OCO2</smiles>

120ma: $80 \%$<smiles>CC(=O)CCc1ccc(C)cc1C(=O)NCc1ccccc1</smiles>

120qa: $64 \%$<smiles>CNC(=O)c1c(CCC(C)=O)n(C)c2ccccc12</smiles>

120ra: $50 \%$<smiles>CC(=O)CCc1cc(C)ccc1NC(C)=O</smiles>

139aa: $47 \%$

(b)<smiles>CNC(=O)c1ccc(OC)cc1CCC(=O)c1ccccc1</smiles>

120cb: $21 \%$<smiles>CNC(=O)c1ccc(-c2ccccc2)cc1CCC(=O)C1CCCCC1</smiles>

120dc: $45 \%$<smiles>CNC(=O)c1ccc(-c2ccccc2)cc1CCC(=O)OPO</smiles>

120dd: $50 \%$

Scheme 5.1. Ruthenium(II)-catalyzed hydroarylation of $\alpha, \beta$-unsaturated ketones 41 with substituted benzamides 2 .

\subsection{Mechanistic Studies}

\subsubsection{Comparison of the Directing Group Power}

Intermolecular competition experiments between arenes with different directing groups clearly highlighted the amide as directing group is more powerful than the ketone in the 
chelation-assisted $\mathrm{C}-\mathrm{H}$ hydroarylation under the standard reaction conditions (Scheme 5.2).<smiles>CNC(=O)c1ccc(C)cc1</smiles>

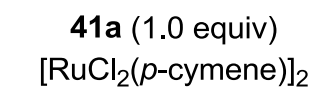
$(5.0 \mathrm{~mol} \%)$<smiles>CC=CCC</smiles>
$\mathrm{MesCO}_{2} \mathrm{~K}(30 \mathrm{~mol} \%)$<smiles>CC(=O)c1ccc(C)cc1</smiles><smiles>CCCCCC(=O)c1ccc(C)cc1CCC(C)=O</smiles>

120ba: $39 \%$

each $0.5 \mathrm{mmol}$

Scheme 5.2. Competition experiments between aromatic amide $2 \mathbf{b}$ and ketone $30 \mathbf{a}$.

\subsubsection{Reaction in the Presence of Isotopically Labeled Solvent}

Obvious evidence of H/D exchange on both the reisolated starting material $\left[D_{n}\right]-2 \mathbf{b}$ and the desired profuct $\left[D_{n}\right]-\mathbf{1 2 0 b a}$ was obtained with the deuterated cosolvent $\mathrm{D}_{2} \mathrm{O}$ (Scheme 5.3), which can be rationalized in terms of a reversible $\mathrm{C}-\mathrm{H}$ bond metalation step in the ruthenium(II)-catalyzed $\mathrm{C}-\mathrm{H}$ hydroarylations.

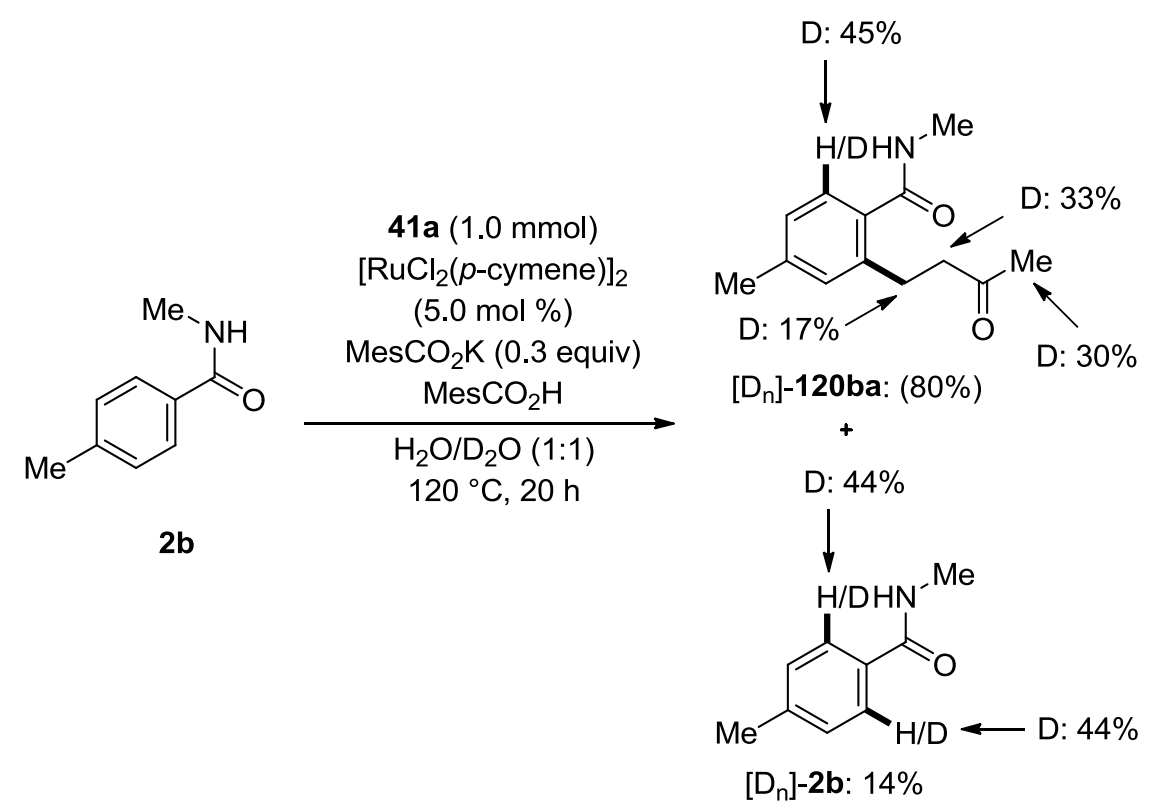

Scheme 5.3. H/D exchange experiments.

Moreover, ruthenium(II)-catalyzed hydroarylations with the product compound and the isotopically labeled substrate $\left[\mathrm{D}_{5}\right]-\mathbf{2}$ a disclosed a significant kinetic isotope effect (KIE) of $k_{\mathrm{H}} / k_{\mathrm{D}} \approx 1.1$ for the intermolecular KIE experiment (Scheme 5.4). This data is in agreement with the $\mathrm{C}-\mathrm{H}$ bond metalation not to be the rate-determining step. 


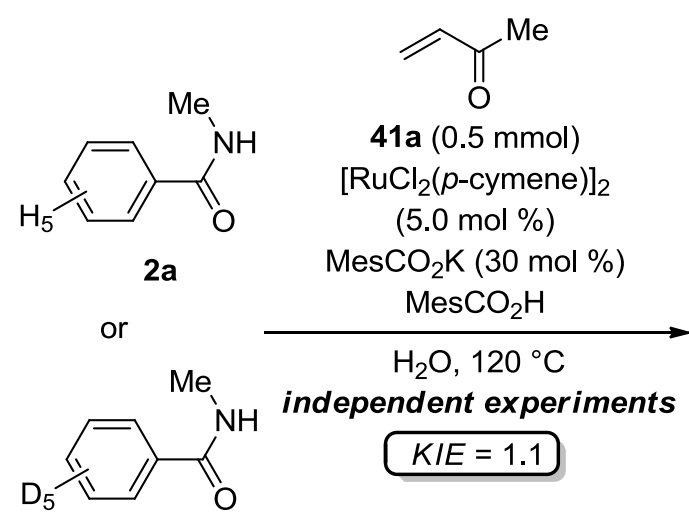

$\left[D_{5}\right]-2 a$<smiles>CNC(=O)c1ccccc1CCC(C)=O</smiles>

120 aa

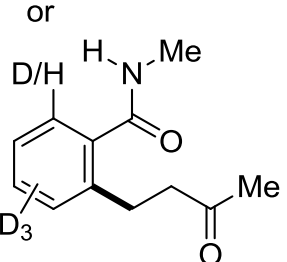

$\left[D_{n}\right]-120 a a$

Scheme 5.4. Kinetic isotope effect (KIE) studies.

\subsection{Scope of the Oxidative Annulation with $\alpha, \beta$-Unsaturated Ketone}

Thereafter, inspired by our previous work on oxidative alkenylations, ${ }^{5,7,100}$ we probed the oxidative annulation of differently decorated acetanilides $\mathbf{1 2 1}$ in the presence of cocatalytic $\mathrm{AgSbF}_{6}$ and stoichimetric $\mathrm{Cu}(\mathrm{OAc})_{2}$ (Scheme 5.5). Importantly, the catalytic system was not limited to the use of electron-rich $N$-phenyleacetanilides $121 \mathrm{a}-121 \mathrm{c}$, but also allowed for the transformation of electron-poor substrates. Valuable electrophilic functional groups, such as fluoro, chloro, bromo, trifluoro and ester substituents, were well tolerated by the ruthenium(II) catalytic system when utilizing substituted acetanilides $\mathbf{1 2 1 d - 1 2 1 h}$. Upon intramolecular competition experiment with substrate 121i bearing a meta-methyl substituent, the cyclization was governed by steric interaction to deliver the product $\mathbf{1 2 2}$ ia in a good yield. However, substrates $121 \mathbf{j}$ and $121 \mathbf{k}$ bearing an ortho-substituent provided the desired products $122 \mathbf{j a}$ and $122 \mathrm{ka}$, respectively, in lower yields. Phenyl vinyl ketone ketone (41b) demonstrated again a lower reactivity.

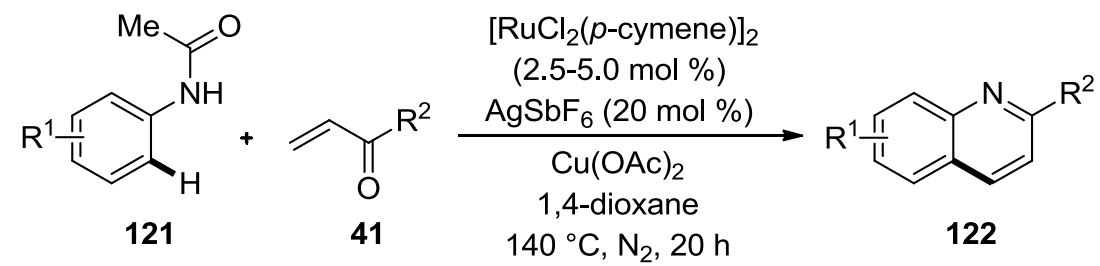




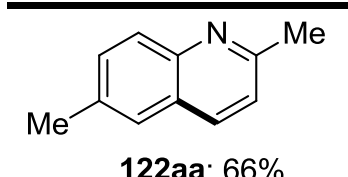

122aa: $66 \%$<smiles>Cc1ccc2cc(F)ccc2n1</smiles>

122da: $43 \%(58 \%)^{[a]}$<smiles>Cc1ccc2cc(C(F)(F)F)ccc2n1</smiles>

122ga: $28 \%$ [a]<smiles>Cc1ccc2cccc(C)c2n1</smiles>

122ja: $34 \%$

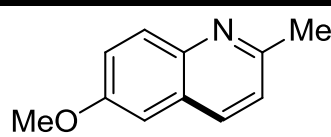

122ba: $69 \%$<smiles>Cc1ccc2cc(Cl)ccc2n1</smiles>

122ea: $52 \%(60 \%)^{[a]}$<smiles>CC(=O)Oc1ccc2nc(C)ccc2c1</smiles>

122ha: $51 \%{ }^{[a]}$<smiles>Cc1ccc2cccc(F)c2n1</smiles>

122ka: $30 \%$ [a]<smiles>Cc1ccc2cc(-c3ccccc3)ccc2n1</smiles>

122ca: $52 \%$<smiles>Cc1ccc2cc(Br)ccc2n1</smiles>

122fa: $51 \%{ }^{[a]}$<smiles>Cc1ccc2ccc(C)nc2c1</smiles>

122ia: $62 \%$<smiles>COc1ccc2nc(-c3ccccc3)ccc2c1</smiles>

Scheme 5.5. Scope of oxidative annulations with substituted $N$-phenylacetanilides 121. [a]

$\left[\mathrm{RuCl}_{2}(p \text {-cymene })\right]_{2}(5.0 \mathrm{~mol} \%), \operatorname{AgSbF}_{6}(20 \mathrm{~mol} \%)$.

\subsection{Proposed Catalytic Cycle}

Based on our previous work, ${ }^{7,107}$ we propose the initial $\mathrm{C}-\mathrm{H}$ ruthenation formed cycloruthenated complex 134b then followed by a migratory insertion with alkene 41 to generate the intermediate $134 \mathbf{c}$. Subsequently, $\beta$-hydrideelimination furnish the product of oxidative alkenylation $\mathbf{1 3 4 d}$, the catalytically active ruthenium(II) complex is regenerated after reductive elimination and reoxidation, while the desired product $\mathbf{1 2 2}$ is obtained through an intramolecular nucleophilic addition of compound 134d, followed by elimination of acetic acid to furnish desired product $\mathbf{1 2 2}$.

107 L. Ackermann, L. Wang, R. Wolfram, A. V. Lygin, Org. Lett. 2012, 14, 728-731. 


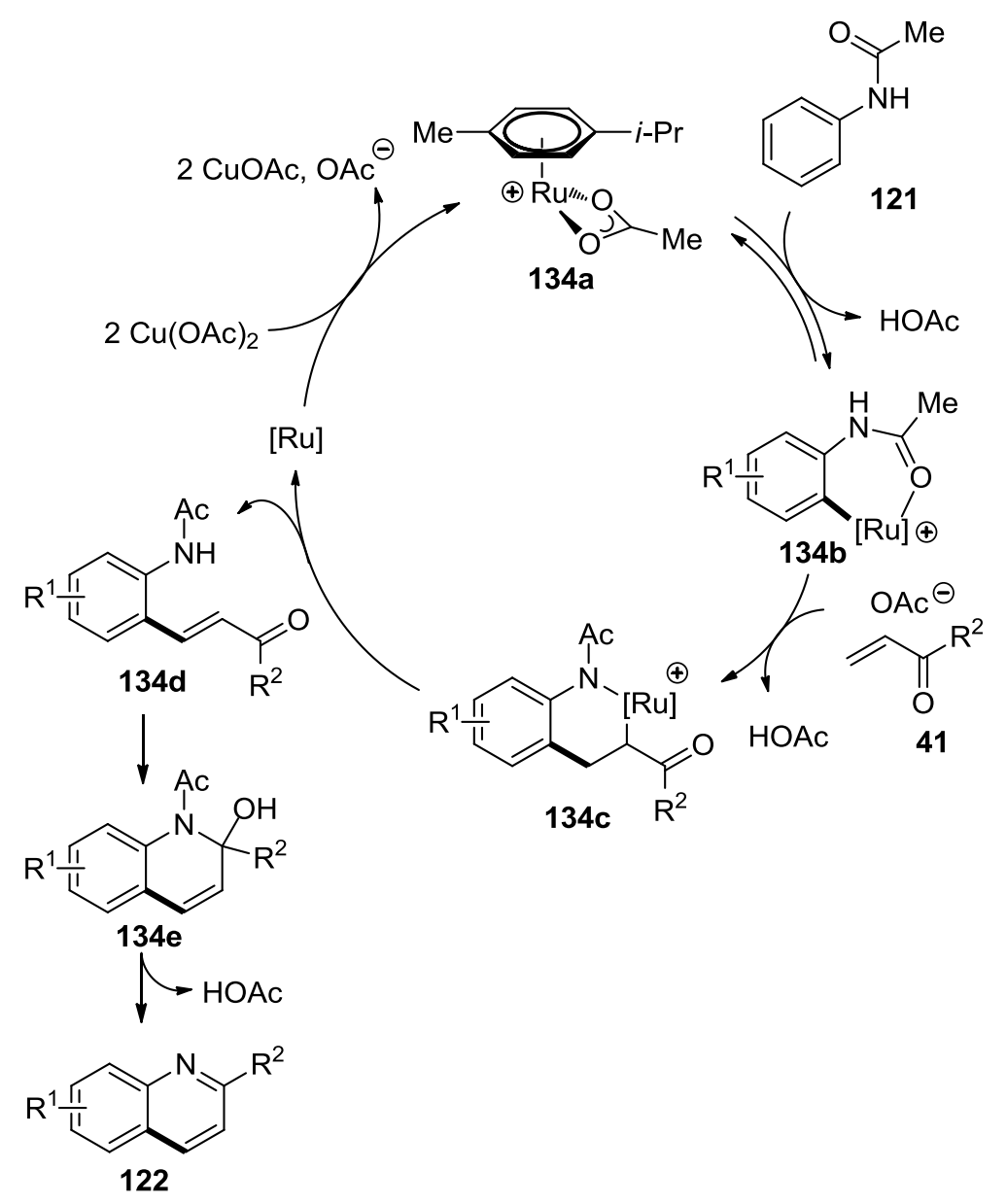

\subsection{Conclusion}

In summary, we have developed the first ruthenium(II)-catalyzed hydroarylation and oxidative annulation of $\alpha, \beta$-unsaturated ketones $\mathbf{4 1}$ with easily accessible monodentate benzamides 2, acetanilides 121 under simple reaction conditions. Furthermore, inexpensive, non-toxic $\mathrm{H}_{2} \mathrm{O}$ was proved to be the suitable reaction medium in the $\mathrm{C}-\mathrm{H}$ bond ortho-alkylation. This methodology highlighted the fact that monodentate directing groups can be used to achieve these challenging transformations. The latter proceeded only with difficulty when traditional methods were used and represent the utilization of $\alpha, \beta$-unsaturated ketones 41 in the ruthenium(II)-catalyzed direct $\mathrm{C}-\mathrm{H}$ bond hydroarylation, which was only realized with bidentate directing groups as of yet. Detailed KIE experiments indicated $\mathrm{C}-\mathrm{H}$ metalation to be not the rate-determining step. Moreover, novel oxidative annulations of $N$-phenylacetanilides 121 with $\alpha, \beta$-unsaturated ketones 41 to deliver decorated quinolines 122 were also viable with the versatile ruthenium(II) catalyst. 


\section{Cobalt-Catalyzed C-H Arylation with Weakly-Coordinating Amides and Tetrazoles: Expedient Route to Angiotensin-I I-Receptor Blocke rs}

Angiotensin-II-Receptor Blockers (ARBs), such as Valsartan, Losartanare or Candesartan, are important antihypertensives. ${ }^{108,5 \mathrm{e}}$ As of yet, the biaryl moieties in ARBs were predominantly synthesized through palladium-catalyzed cross-coupling reactions. ${ }^{109}$ A significant advance to access these building blocks has been accomplished with ruthenium(II) catalysts through direct $\mathrm{C}-\mathrm{H}$ bond arylation reactions. ${ }^{5 e, 110}$ In consideration of the progress in cobalt-catalyzed direct $\mathrm{C}-\mathrm{H}$ bond arylation, ${ }^{67,75}$ we devised cobalt-catalyzed $\mathrm{C}-\mathrm{H}$ bond arylations with weakly-coordinating ${ }^{7 \mathrm{~b}}$ benzamides $\mathbf{2}$ as well as direct arylations of aryl tetrazoles $\mathbf{1 2 3}$. Our strategy for the synthesis of derivatives $\mathbf{1 3 9}$ as ARBs constituents is depicted in scheme 6.1. ${ }^{11}$

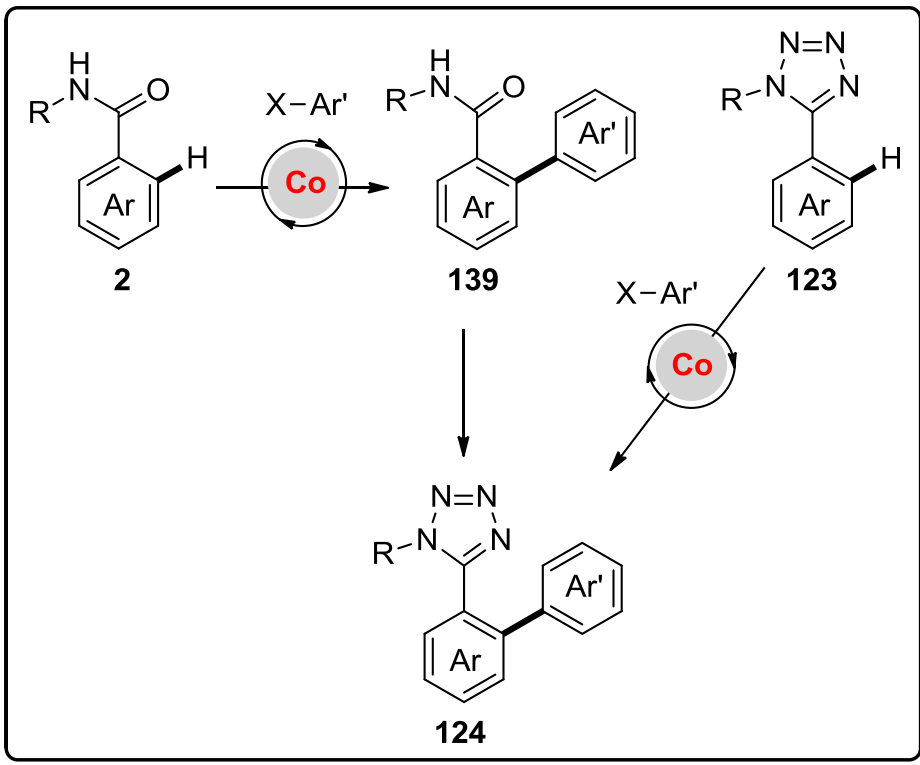

Scheme 6.1. Strategies for the synthesis of derivatives $\mathbf{1 2 4}$ as ARBs building blocks.

\subsection{Optimization}

\subsubsection{Optimization Studies}

We initialed our studies by probing different reaction conditions for the envisioned $\mathrm{C}-\mathrm{H}$ bond

\footnotetext{
108 a) J. H. Kim, J. H. Lee, S. H. Paik, J. H. Kim, Y. H. Chi, Arch. Pharmacal Res. 2012, 35, 1123-1126; b) A. R. De Ca terina, A. R. Ha rer, F. Cuculi, Vasc. Health Risk Manage. 2012, 8, 299-305.

109 a) S. Aalla, G. Gilla, Y. Bojja, R. R. Anumula, P. R. Vummenthala, P. R. Padi, Org. Process Res. Dev. 2012, 16, 682-686; b) G. Wang, B. Sun, C. Peng, Org. Process Res. Dev. 2011, 15, 986-988.

110 a) M. Seki, ACS Catal. 2014, 4, 4047-4050; b) E. Diers, N. Y. P. Kumar, T. Mejuch, I. Marek, L. Ackermann, Tetrahedron 2013, 69, 4445-4453; c) M. Seki, M. Nagaha ma, J. Org. Chem. 2011, 76, 10198-10206.

111 J. L., L. Ackermann, Chem. Eur. J. 2015, 21, 5718-5722.
} 
arylation of weakly-coordinating benzamide $\mathbf{2 a}$ with inexpensive $p$-tolylchloride (88a) (Table 6.1).

Table 6.1. Optimization of cobalt-catalyzed $\mathrm{C}-\mathrm{H}$ bond arylation. ${ }^{[\mathrm{a}]}$<smiles>CNC(=O)c1ccccc1</smiles><smiles>Cc1ccc(Cl)cc1</smiles>

$2 a$ $88 \mathbf{a}$<smiles></smiles>

$\mathrm{R}=$ Mes

$\mathrm{R}=2,6-i \mathrm{Pr}_{2} \mathrm{C}_{6} \mathrm{H}_{4}$

$\mathrm{R}=i \mathrm{Pr}$

$\mathrm{R}=t \mathrm{Bu}$

$\mathrm{R}=\mathrm{Cy}$

(140b)

(140f)

$(140 \mathrm{~g})$<smiles>CNC(=O)c1ccccc1OCCOCCOC(=O)OCC(C)(C)C</smiles>

$60^{\circ} \mathrm{C}, 16 \mathrm{~h}$

\section{9aa}

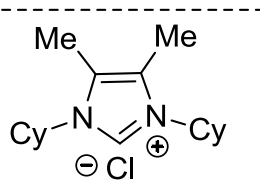

$\begin{array}{ll}R=\text { Mes } & (140 c) \\ R=2,6-i \operatorname{Pr}_{2} \mathrm{C}_{6} \mathrm{H}_{4}(\mathbf{1 4 0 d})\end{array}$<smiles></smiles>

$-\mathrm{N} \vee \frac{\mathrm{N}}{\oplus}-\mathrm{R}$

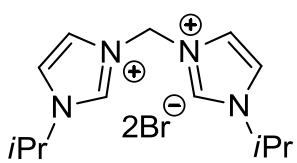

$i \mathrm{Pr}-\mathrm{N}_{\mathrm{Br}}^{=} \underset{\oplus}{\mathrm{N}}-\mathrm{Cy}$

$\Theta$

140j

\begin{tabular}{|c|c|c|c|}
\hline Entry & {$\left[\mathrm{Co}(\mathrm{acac})_{2}\right][\mathrm{mol} \%]$} & Ligand & Yield $^{[\mathrm{b}]}[\%]$ \\
\hline 1 & 10.0 & IMesHCl (140a) & $23^{[\mathrm{c}]}$ \\
\hline 2 & 10.0 & IPrHCl (140b) & $<5^{[\mathrm{c}]}$ \\
\hline 3 & 10.0 & sIMesHCl (140c) & $16^{[\mathrm{c}]}$ \\
\hline 4 & 10.0 & $\mathrm{sIPrHCl}$ (140d) & $0^{[\mathrm{c}]}$ \\
\hline 5 & 10.0 & $\mathrm{PPh}_{3}$ & $13^{[\mathrm{c}]}$ \\
\hline 6 & 10.0 & $140 \mathrm{e}$ & $29^{[\mathrm{c}]}$ \\
\hline 7 & 10.0 & $140 f$ & $<5^{[\mathrm{c}]}$ \\
\hline 8 & 10.0 & $140 \mathrm{~g}$ & $47^{[\mathrm{c}]}$ \\
\hline 9 & 5.0 & $140 \mathrm{~g}$ & 78 \\
\hline 10 & -- & $140 \mathrm{~g}$ & 0 \\
\hline 11 & 5.0 & $140 h$ & 32 \\
\hline 12 & 5.0 & $140 i$ & $<5$ \\
\hline 13 & 5.0 & $140 j$ & 26 \\
\hline
\end{tabular}

[a] General reaction conditions: $2 \mathrm{a}(0.5 \mathrm{mmol}), \mathbf{8 8 a}(0.6 \mathrm{mmol})$, cat. $\mathrm{Co}(\mathrm{acac})_{2}$, ligand $(5 \mathrm{~mol} \%)$,

$\mathrm{CyMgCl}$ (3.0 equiv), DMPU (1.0 mL), $60^{\circ} \mathrm{C}, 16 \mathrm{~h}$. [b] Yield of isolated product. [c] 88a $(0.75$ 
mmol), ligand (20 mol \%).

Thus far, all reported cobalt-catalyzed direct arylations with organic (pseudo)halides were accomplished with one of the two $N$-heterocyclic carbene $(\mathrm{NHC})^{112-113}$ preligands IMesHCl (140a) or IPrHCl (140b). ${ }^{67,75}$ However, preligands 140a and 140b delivered the desired product 139aa in only unsatisfactory low yields, even with a relatively high catalyst loading of $10 \mathrm{~mol} \%$ (entries 1 and 2). A similar observation was made when employing the saturated analogues sIMesHCl (140c), and sIPrHCl (140d) (entries 3 and 4), or the tertiary phosphine $\mathrm{PPh}_{3}$ (entry 5). Cobalt catalysts derived from isopropyl-substituted NHC 140e were previously shown to be effective for direct alkylations, ${ }^{65 \mathrm{c}}$ but proved unsuitable for the $\mathrm{C}-\mathrm{H}$ bond arylation with benzamides 2a (entries 6 and 7), thus highlighting the challenge associated with the use of weakly-coordinating amides. In contrast, among various NHC precursors, $\mathrm{ICyHCl}(\mathbf{1 4 0 g})$ proved to be optimal with an ideal ligand to cobalt ratio of 1:1 (entries 8 and 9). Under these reaction conditions the catalyst loading could also be significantly reduced (entry 9). The more electron-rich pre-NHC 140h, the bidentate derivative $140 \mathbf{i}$ and the unsymmetrically substituted pre-NHC $140 \mathbf{j}$ failed to improve the catalytic efficacy (entries 10-13).

\subsubsection{Effect of the Directing Groups}

Notably, we also tried the cobalt-catalyzed $\mathrm{C}-\mathrm{H}$ bond arylation with differently substituted aryl amides 141a-141f (Scheme 6.2). These test reactions under the optimized reaction conditions verified the crucial importance of the amide directing group. For substrates without free $\mathrm{N}-\mathrm{H}$ moieties or with sterically demanding directing group, no catalytic reaction was observed. The same result was obtained with benzenesulfonamide 141c or with substrate $141 \mathrm{f}$ bearing a bidentate-type directing groups as well.

\footnotetext{
112 M. N. Hopkinson, C. Richter, M. Schedler, F. Glorius, Nature 2014, 510, 485-496.

113 S. Díez-González, N. Marion, S. P. Nolan, Chem. Rev. 2009, 109, 3612-3676.
} 


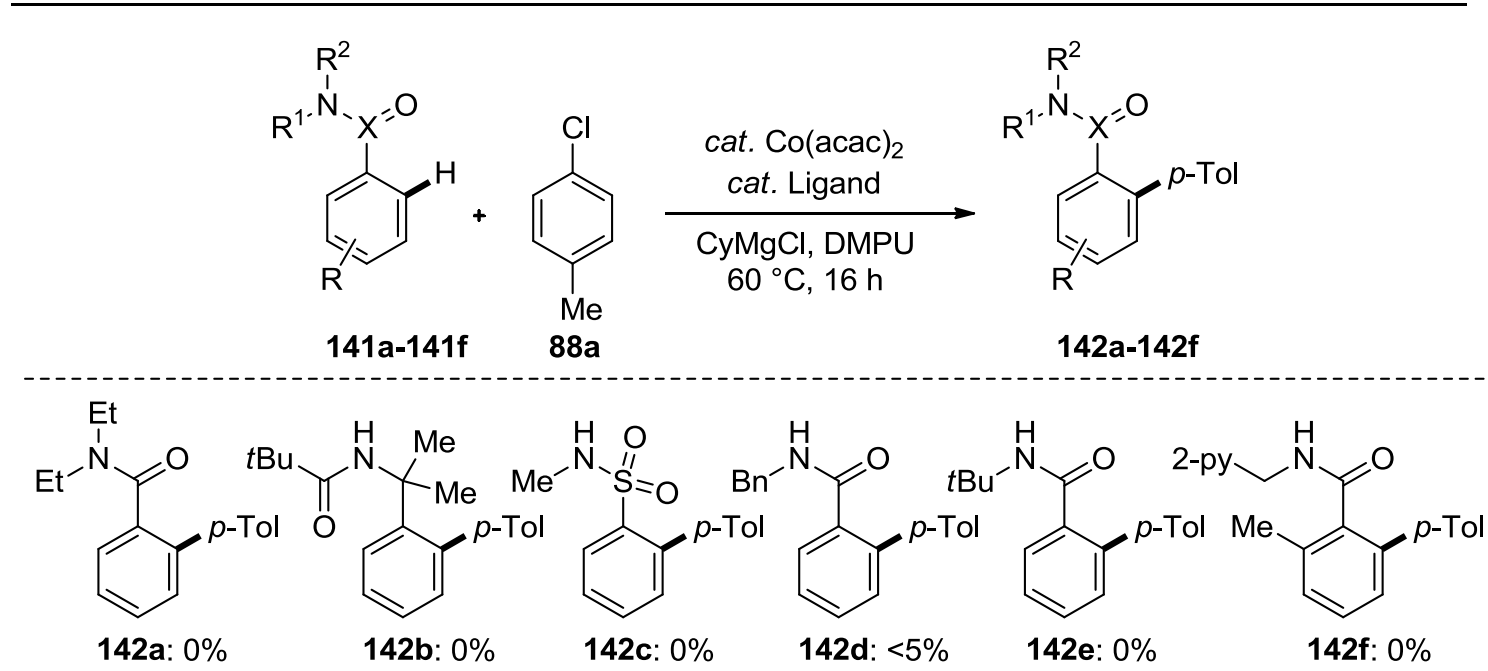

Scheme 6.2. Screening of directing groups for the arylation reactions.

\subsection{Scope and Limitations}

Having identified a highly effective catalyst for the selective arylation of $N$-methylbenzamide (2a) with 4-chlorotoluene (88a), we further extended the reaction scope to include other benzamides with a broad range of aryl chlorides (Scheme 6.3). In order to test the efficiency of the cobalt-catalyzed direct arylation, we subsequently set up a $10 \mathrm{mmol}$ scale reaction of 2a with 88a which provided biaryl product 139aa in 64\% yield when employing $2.0 \mathrm{~mol} \%$ of $\mathrm{Co}(\mathrm{acac})_{2}$ and $\mathrm{ICyHCl}$ in DMPU at $60 \mathcal{C}$ for $16 \mathrm{~h}$. Notably, various aryl chlorides 88a-88g were successfully employed. The TBS-protected (4-chlorophenyl)methanol $\mathbf{8 8 d}$ and 4-chlorophenol $\mathbf{8 8 f}$ were readily converted to the corresponding biaryl products 139ad and 139of in good yields of $57 \%$ and $70 \%$, respectively. The arylation with sterically more demanding ortho substituent $o$-tolylchloride afforded the target product 139ac as well, albe it in a modest yield (55\%). Similarly lower yields were observed for benzamides $2 \mathbf{s}, \mathbf{2 f}$, and $\mathbf{2 p}$ with electron-deficient functional groups, even when employing an increased loading of $\mathrm{Co}(\mathrm{acac})_{2}$ and carbene preligand. Likew ise, we exploited the use of the amide group for the direct arylation of the biologically active indole ${ }^{114}$ derivatives, de livering the desired products 139ua, 139ra and 139rg were isolated in good yields. Upon arylation of meta-substituted benzamide $\mathbf{2} \mathbf{m}$, an influence of the secondary directing group chelation effect ${ }^{102}$ was minor.

\footnotetext{
${ }^{114}$ Modern Heterocyclic Chemistry (Eds.: J. Alvarez-Builla, J. J. Vaquero, J. Barluenga,), Wiley-VCH, Weinheim, 2011.
} 
<smiles>CNC(=O)c1ccccc1</smiles>

2<smiles>Clc1ccccc1</smiles>

88
$\mathrm{Co}(\mathrm{acac})_{2}(5.0 \mathrm{~mol} \%)$ $\mathrm{ICyHCl}(140 \mathrm{~g} 5.0 \mathrm{~mol} \%)$

CyMgCl, DMPU $60^{\circ} \mathrm{C}, 16 \mathrm{~h}$<smiles>CNC(=O)c1ccccc1-c1ccccc1</smiles>

139

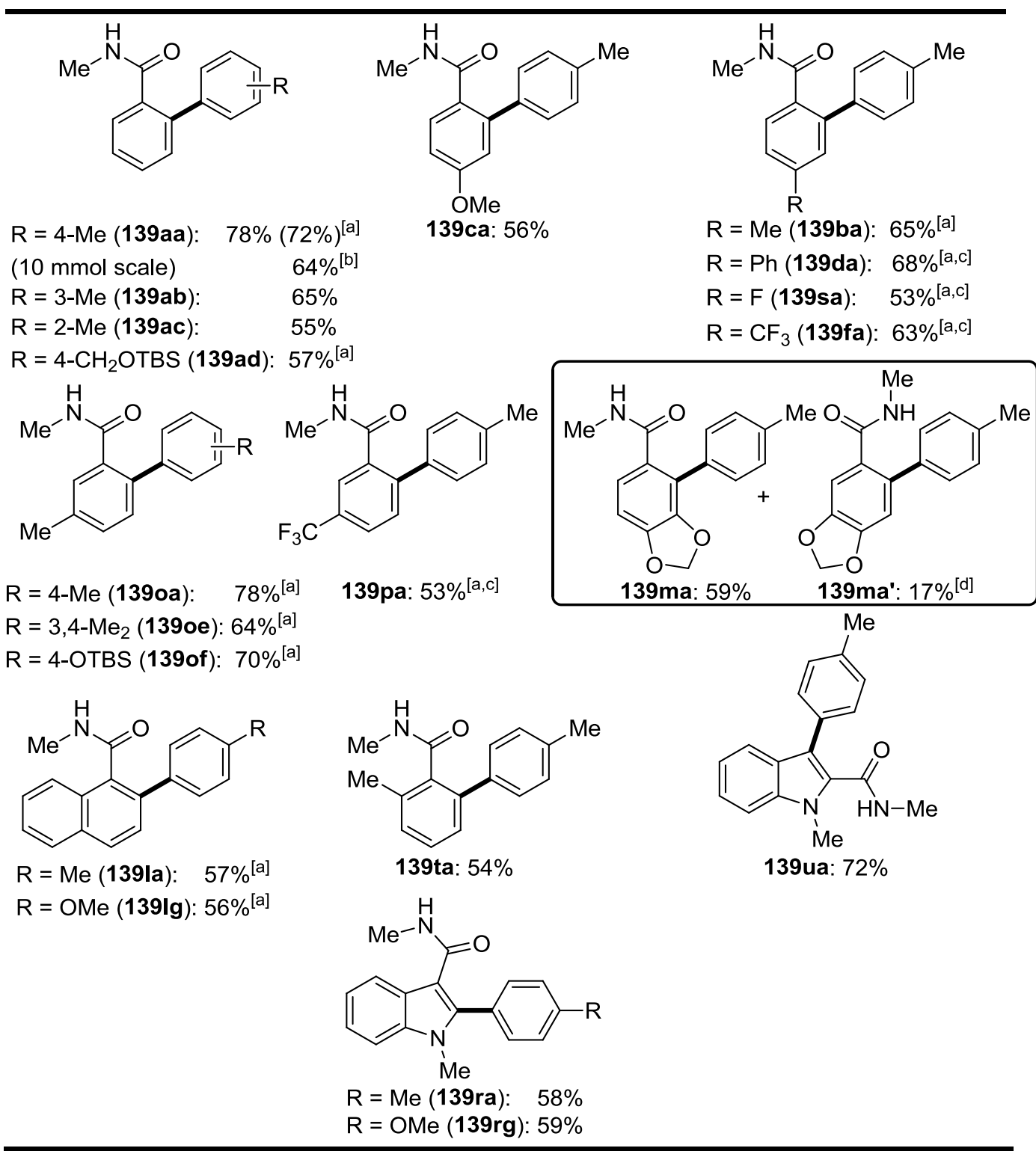

Scheme 6.3. Substrate scope of cobalt-catalyzed C-H bond arylation. [a] 0.5 h. [b] Co(acac $)_{2}(2.0$ $\mathrm{mol} \%), \mathbf{1 4 0 g}(2.0 \mathrm{~mol} \%)$. [c] $\mathrm{Co}(\mathrm{acac})_{2}(10 \mathrm{~mol} \%), \mathbf{1 4 0 g}(10 \mathrm{~mol} \%)$.

Thereafter, the substrate scope was extended to competition experiments of various benzamides 2 (Scheme 6.4). Remarkably, the cobalt-catalyzed $\mathrm{C}-\mathrm{H}$ functionalizations occurred on the benzamides 2 with excellent chemo-selectivity. Both electron-donating and electron-withdrawing substituents on the aryl chloride $\mathbf{8 8}$ were tolerated selectively delivering the mono-arylated biaryls 139va-139xd in good yields. Moreover, chlorobenzenes with 
sterically hindered substituents in the ortho-position also delivered the desired products $\mathbf{1 3 9}$ with high catalytic efficacy.<smiles>[R]c1ccc(NC(=O)c2ccccc2[2H])c([1H])c1</smiles>

2<smiles>Clc1ccccc1</smiles>

88
$\mathrm{Co}(\mathrm{acac})_{2}(5.0 \mathrm{~mol} \%)$ $\mathrm{ICyHCl}(\mathbf{1 4 0 g}, 5.0 \mathrm{~mol} \%)$ $\mathrm{CyMgCl}, \mathrm{DMPU}$ $60^{\circ} \mathrm{C}, 16 \mathrm{~h}$<smiles>[R]c1ccc(NC(=O)c2ccccc2-c2ccc([R])cc2)cc1</smiles>

139

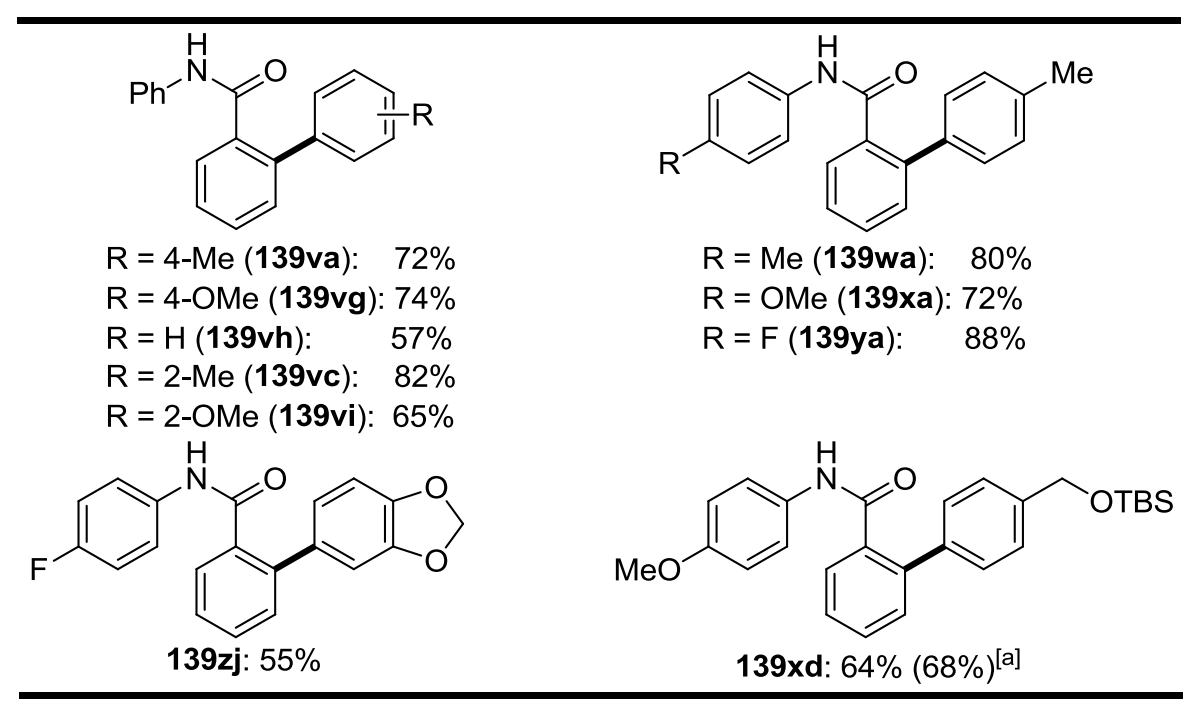

Scheme 6. 4. Cobalt-catalyzed direct $\mathrm{C}-\mathrm{H}$ bond arylation of anilides 2 . [a]: $0.5 \mathrm{~h}$.

\subsection{Mechanistic Studies}

The results of a set of intermolecular competition experiments revealed electron-deficient benzamides $\mathbf{2}$ and electron-deficient aryl chlorides $\mathbf{8 8}$ to be more reactive than their electron-rich counterparts (Scheme 6.5).

Thereafter, we performed studies to delineate the catalyst working mode. Independent experiments with isotopically labeled substrates indicated the $\mathrm{C}-\mathrm{H}$ bond cobaltation not to be kinetically relevant $(\mathrm{KIE} \approx 1.0$ ), and provided evidence for a reversible $\mathrm{D} / \mathrm{H}$ exchange reaction (Scheme 6.6). 


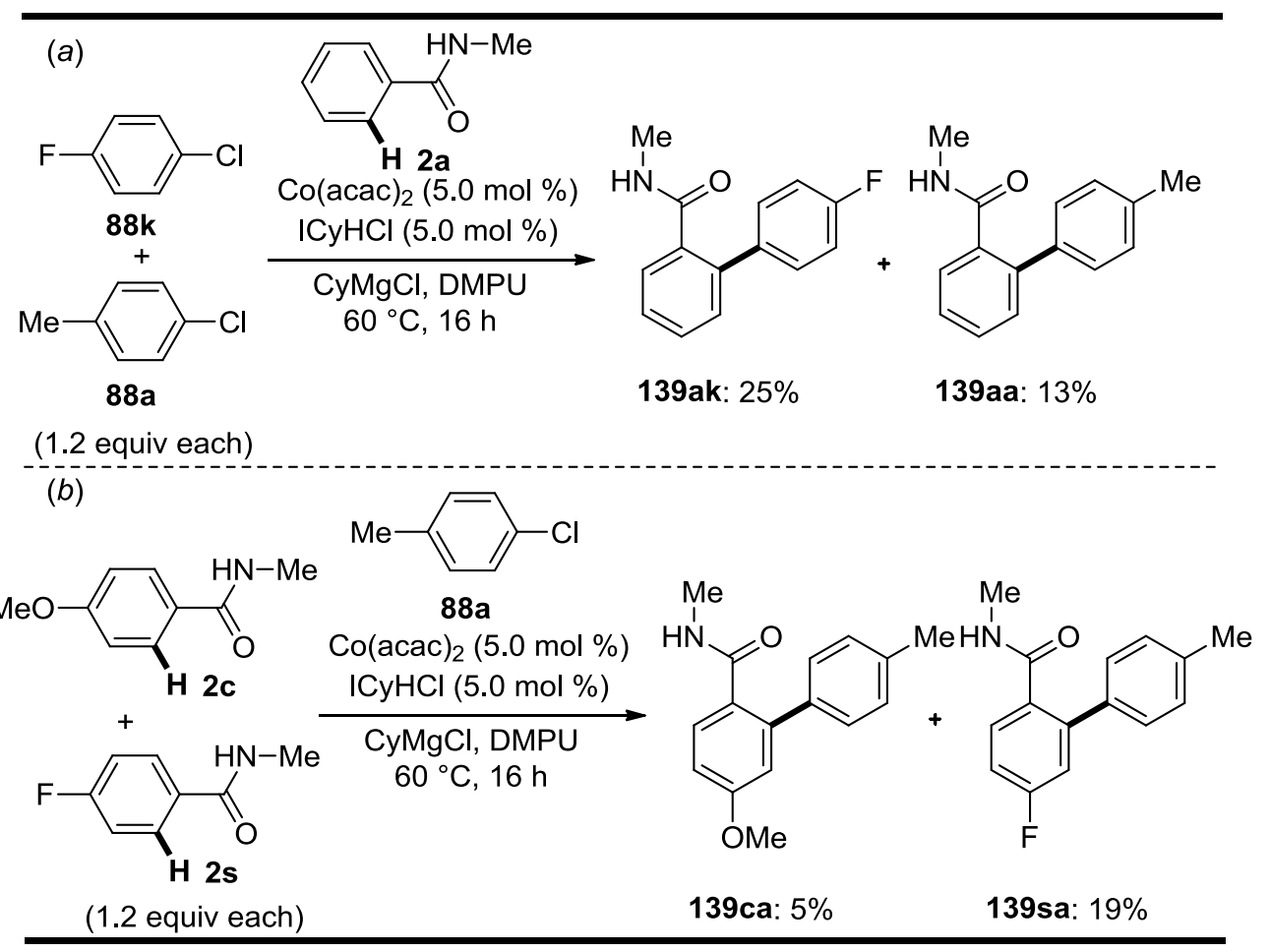

Scheme 6.5. (a) Competition experiments between aryl chlorides $\mathbf{8 8}$, (b) and different aryl amides 2.

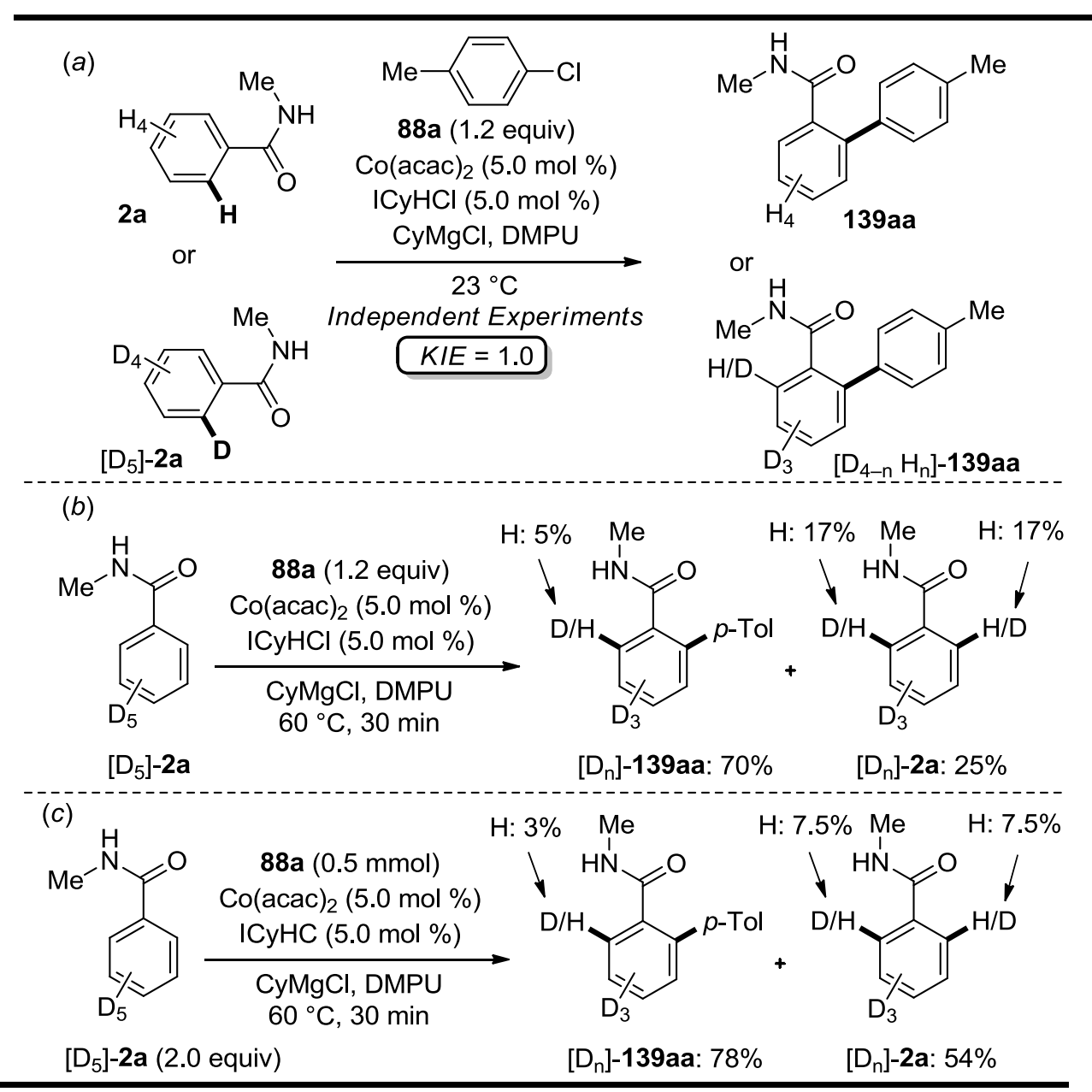


Scheme 6.6. (a) Kinetic isotope effect (KIE) study. (b, c) Cobalt-catalyzed H/D exchange experiments with deuterated $\mathbf{2 a}$.

\subsection{Synthesis of Biaryl Tetrazoles}

As discussed above, benzamides can be easy transformed into phenyl tetrazoles according to the previously published protocols. ${ }^{115}$ Consequently, a simple route to synthesize ARBs building blocks and derivatives 124a-124d was designed via initial cobalt-catalyzed direct $\mathrm{C}-\mathrm{H}$ bond arylation by weak assistance of amides group in substrates 2 (Scheme 6.3) followed by the transformation of the amido substituent into the tetrazole moiety (Scheme 6.7).

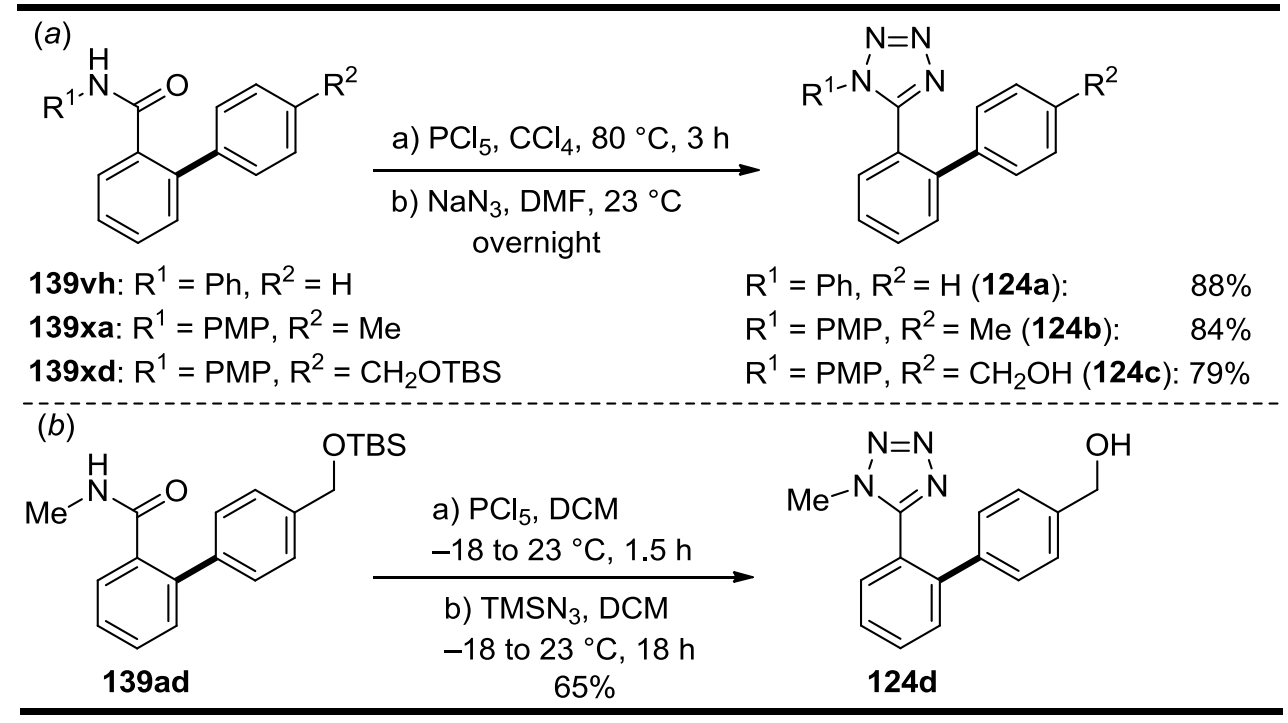

Scheme 6.7. Facile preparation of biaryl tetrazoles 124.

Alternatively, we explored the possibility of devising an even more step-economical approach to biaryl tetrazoles $\mathbf{1 2 4}$ through the unprecedented cobalt-catalyzed $\mathrm{C}-\mathrm{H}$ bond activation by tetrazole assistance in substrates $\mathbf{1 2 3}$. Intriguingly, a low-valent cobalt catalyst derived from preligand 140a proved effective here, thereby chemo-selectively delivering the desired products 124. It is noteworthy that the tetrazole-assisted $\mathrm{C}-\mathrm{H}$ bond arylation proceeded by $\mathrm{C}-\mathrm{H} / \mathrm{C}-\mathrm{O}$ bonds cleavages with challenging aryl carbamates $\mathbf{8 6}$ as the electrophiles (Scheme 6.8), although the products $\mathbf{1 2 4}$ were obtained in rather modest yields.

115 S. N. Rao, T. Ravisankar, J. Latha, K. S. Babu, Pharma Chem. 2012, 4, 1093-1103. 
<smiles>[R]n1nnnc1-c1ccccc1[123I]</smiles>

123

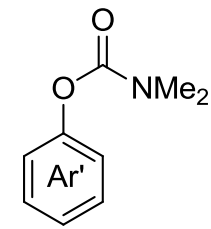

86

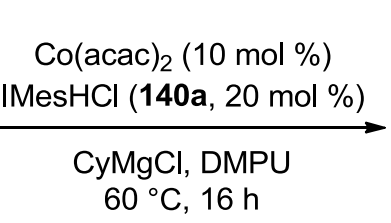

$60^{\circ} \mathrm{C}, 16 \mathrm{~h}$

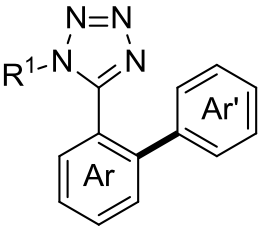

124

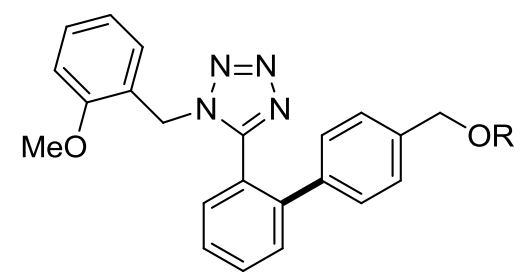

$\mathrm{R}=\mathrm{PMB}(124 \mathrm{e}): 43 \%$

$\mathrm{R}=\mathrm{MOM}(\mathbf{1 2 4 f}): 41 \%$

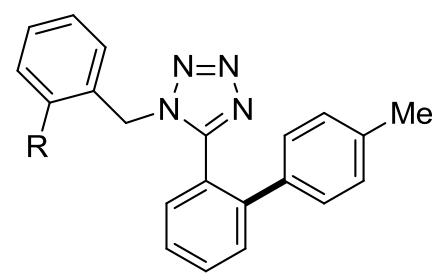

$\mathrm{R}=\mathrm{H}(\mathbf{1 2 4 g}): 51 \%$

Scheme 6.8. Tetrazole-assisted C-H bond arylation with carbamates $\mathbf{8 6}$.

\subsection{Oxidative Annulation}

Finally, we successfully exploited the products of direct arylation 139 for the synthesis of substituted phenanthridin-6(5H)-ones 143a and 143b, which were obtained in 83 and 64\% yield, respectively (Scheme 6.9).<smiles>[R]NC(=O)c1ccccc1-c1ccc(C)cc1</smiles>

139

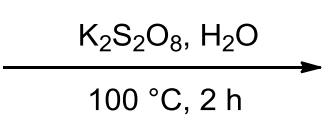

$\mathrm{R}=\mathrm{Me}(143 \mathrm{~b}): 64 \%$

Scheme 6.9. Oxidative annulations of $\mathbf{1 3 9}$ to phenanthridin-6(5H)-ones $\mathbf{1 4 3 .}$

\subsection{Conclusion}

In summary, we have developed novel strategies for cobalt-catalyzed syntheses of biaryl tetrazoles 124 through $\mathrm{C}-\mathrm{H}$ bond functionalization. Thus, NHC-ligated low-valent cobalt catalysts enabled the first direct arylation assisted by weak coordination. The arylated benzamides 139 were formed with high site- and chemo-selectivities as well as ample scope. This reaction provided expedient access to biaryl tetrazoles, which represent key scaffolds of ARB blockbuster drugs. The results of mechanistic studies were in line with a reversible $\mathrm{C}-\mathrm{H}$ bond cobaltation and a rate-determining reductive elimination. The power of the user-friendly 
cobalt catalysis was further illustrated by unprecedented tetrazole-assisted $\mathrm{C}-\mathrm{H}$ bond activations with $3 \mathrm{~d}$ transition metal complexes, thus constituting an alternative approach to ARBs building blocks. 


\section{Cobalt(III)-Catalyzed C-H Bond Cyanation of Arenes and Heteroarenes}

Functionalizations of otherwise inert $\mathrm{C}-\mathrm{H}$ bonds provide an approach for improving the atom- and step-economy in organic synthesis. While most of the achievements were accomplished with expensive second-row transition-metal catalysts, largely focused on ruthenium, rhodium and palladium complexes, further applications of these methods are limited. ${ }^{5,7116}$ Inexpensive cobalt catalysts, for $\mathrm{C}-\mathrm{H}$ bonds activations have been recognized as an increasingly viable tool for organic syntheses, also in the field of $\mathrm{C}-\mathrm{H}$ bond activation including arylations, alkylations, alkenylations, benzylations and hydroarylations. ${ }^{5 \mathrm{j}-5 \mathrm{k}}$ The general cobalt catalytic systems for most of these studies were assisted by phosphine or $N$-heterocyclic carbene (NHC) ligands, a long with a strong base. Grignard reagents were used as base and reductants to generate the reactive low-valent cobalt-catalysts, which devised methods for new $\mathrm{C}-\mathrm{C}$ bonds formation. Although several functional groups can be tolerated, the Grignard reagents could induce some other undesirable byproducts. Therefore, in order to avoid these kinds of disadvantages, the research groups of Kanai, Ackermann, Daugulis and Song developed other mild reaction systems with cobalt catalysts, including high-valent $[\mathrm{Cp} * \mathrm{Co}(\mathrm{III})]$ derivatives, which allowed for the oxidative alkyne annulations, oxidative alkenylations and alkoxylation under mild reaction conditions without Grignard reagents. ${ }^{68,79,82-84,86}$ Based on this progress, we hence became interested in developing unprecedented cobalt(III)-catalyzed $\mathrm{C}-\mathrm{H}$ bond cyanations of arenes and heteroarenes, which are discussed below in this Chapter. ${ }^{89}$

\subsection{Optimization Studies}

Initially we probed various reaction conditions for the desired cobalt-catalyzed $\mathrm{C}-\mathrm{H}$ bond functionalization utilizing 2-phenylpyridine (28a) and $N$-cyano- $N$-phenyl$p$-toluenesulfonamide $(\mathbf{1 2 5}, \mathrm{NCTS})$ as the cyanating reagent. Preliminary experiments identified $\left[\mathrm{Cp}^{*} \mathrm{CoI}_{2}(\mathrm{CO})\right]$ to be the efficient metal catalyst of choice, along with $\mathrm{AgSbF}_{6}$ and $\mathrm{NaOAc}$ as the cocatalytic additives (Table 7.1, entries 1-4, 10). Experiments without this additive or with $\mathrm{AgOAc}$ instead of $\mathrm{AgSbF}_{6}$ inhibited the reaction immediately (entries 6 and

\footnotetext{
${ }^{116}$ L. Ackermann, Top. Curr. Chem. 2010, 292, 211-229, and references cited the rein.
} 
8). Among a representative set of acetate source, KOAc provided optimal yields (entries 1, 5, 7 and 9). A C-H bond cyanation with an excess of substrate $\mathbf{1 2 5}$ formed the monocyanated product 126a as the sole product (entry 11), which unraveled the prominent chemoselectivity of the cobalt(III) catalyst.

Table 7.1 Optimization of cobalt-catalyzed C-H bond cyanation. ${ }^{[a]}$<smiles>Cc1ccccc1-c1ccccn1</smiles>

28a<smiles>N#[Y]N([13F])c1ccccc1</smiles>

125

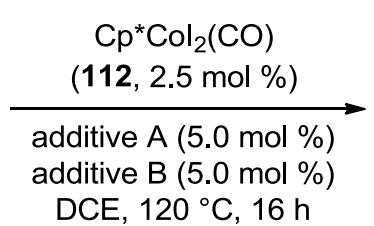

$\mathrm{DCE}, 120^{\circ} \mathrm{C}, 16 \mathrm{~h}$<smiles>N#Cc1ccccc1-c1ccccn1</smiles>

$126 a$

\begin{tabular}{|c|c|c|c|c|}
\hline Entry & {$[\mathrm{Co}]$} & Additive A & Additive B & Yield $(\%)^{[b]}$ \\
\hline 1 & {$\left[C p^{*} \operatorname{CoI}_{2}(C O)\right]$} & $\operatorname{AgSbF}_{6}$ & $\mathrm{NaOAc}$ & 83 \\
\hline 2 & $\mathrm{CoI}_{2}$ & $\mathrm{AgSbF}_{6}$ & $\mathrm{NaOAc}$ & 0 \\
\hline 3 & $\mathrm{Co}(\mathrm{acac})_{2}$ & $\mathrm{AgSbF}_{6}$ & $\mathrm{NaOAc}$ & 0 \\
\hline 4 & {$\left[\mathrm{Cp} * \mathrm{CoCl}_{2}\right]_{2}$} & $\mathrm{AgSbF}_{6}$ & $\mathrm{NaOAc}$ & 30 \\
\hline 5 & {$\left[\mathrm{Cp}^{*} \mathrm{CoI}_{2}(\mathrm{CO})\right]$} & $\mathrm{AgSbF}_{6}$ & $\boldsymbol{K O} A \boldsymbol{c}$ & 90 \\
\hline 6 & {$\left[\mathrm{Cp}^{*} \mathrm{CoI}_{2}(\mathrm{CO})\right]$} & $\mathrm{AgOAc}$ & $\mathrm{NaOAc}$ & 0 \\
\hline 7 & {$\left[\mathrm{Cp}^{*} \mathrm{CoI}_{2}(\mathrm{CO})\right]$} & $\mathrm{AgSbF}_{6}$ & $\mathrm{AgOAc}$ & 66 \\
\hline 8 & {$\left[\mathrm{Cp}^{*} \mathrm{CoI}_{2}(\mathrm{CO})\right]$} & -- & KOAc & 0 \\
\hline 9 & {$\left[\mathrm{Cp}^{*} \mathrm{CoI}_{2}(\mathrm{CO})\right]$} & $\mathrm{AgSbF}_{6}$ & -- & 33 \\
\hline 10 & -- & $\mathrm{AgSbF}_{6}$ & KOAc & 0 \\
\hline 11 & {$\left[\mathrm{Cp} * \mathrm{CoI}_{2}(\mathrm{CO})\right]$} & $\mathrm{AgSbF}_{6}$ & KOAc & $93^{[\mathrm{cc}]}$ \\
\hline 12 & {$\left[\mathrm{Cp}^{*} \mathrm{CoI}_{2}(\mathrm{CO})\right]$} & $\mathrm{AgSbF}_{6}$ & KOAc & $39^{[\mathrm{d}]}$ \\
\hline
\end{tabular}

[a] Reaction conditions: 28a (0.5 mmol), 2 (0.75 mmol), [Cp*CoI $\left.{ }_{2}(\mathrm{CO})\right](\mathbf{1 1 2}, 2.5 \mathrm{~mol} \%)$, additive A(5.0 mol \%), additive B (5.0 mol \%), DCE (2.0 mL), $120 \mathrm{C}, 16 \mathrm{~h}$. [b] Yield of isolated product. [c] $125(1.5 \mathrm{mmol})$. [d] $4.0 \mathrm{~h}$.

\subsection{Scope and Limitations}

\subsubsection{Substrate Scope of Cobalt-Catalyzed C-H Bond Cyanation}

With the optimized catalytic system in hand, the scope of the cyanation was evaluated (Scheme 7.1). 
<smiles>[X]c1ccccc1-c1ccccn1</smiles>

28<smiles>N#CN(c1ccccc1)c1ccccc1</smiles>

125

$$
\begin{gathered}
\mathrm{Cp}^{\star} \mathrm{Col}_{2}(\mathrm{CO})(2.5 \mathrm{~mol} \%) \\
\mathrm{AgSbF}_{6}(5.0 \mathrm{~mol} \%) \\
\underset{\mathrm{KOAc}(5.0 \mathrm{~mol} \%)}{\longrightarrow}
\end{gathered}
$$<smiles>[R]c1ccc(C#N)c(-c2ccccn2)c1</smiles><smiles>COc1ccc(-c2ccccn2)c(N)c1</smiles>

$R=H(126 a): 90 \%$ 126d: $98 \%$

$\mathrm{R}=\mathrm{Me}(126 \mathrm{~b}): 72 \%$

$\mathrm{R}=\mathrm{CF}_{3}(126 \mathrm{c}): 89 \%$<smiles>N#Cc1ccccc1-c1ccc(F)cn1</smiles>

126g: $73 \%$<smiles>CC(=O)c1ccnc(-c2ccccc2C#N)c1</smiles>

126h: $65 \%$<smiles>CC(C)(C)c1ccc(-c2ccccn2)c(C#N)c1</smiles>

126k: $67 \%$<smiles>COc1ccc(-c2cc(C)ccn2)c(C#N)c1</smiles><smiles>CC(=O)c1ccc(-c2ccccn2)c(C#N)c1</smiles>

126e: $97 \%$<smiles>N#Cc1c(-c2ccccn2)ccc(F)c1F</smiles>
126i: $72 \%$<smiles>[R]c1ccc(-c2ncccn2)c(C#N)c1</smiles>

126I: $79 \%$
$R=F(126 m): \quad 60 \%$ $R=$ OMe (126n): $90 \%$<smiles>N#Cc1ccccc1-c1ncccn1</smiles>

126

Scheme 7.1. Substrate scope of cobalt-catalyzed C-H bond cyanation.

The chelation-assisted $\mathrm{C}-\mathrm{H}$ bond functionalization of meta-disubstituted arenes $\mathbf{2 8 a}-\mathbf{2 8 c}$ proceeded with excellent site-selectivity at the less sterically hindered position. Significantly, the cobalt(III) catalyst displayed a remarkable chemo-selectivity, and a number of valuable electrophilic groups such as esters $(\mathbf{1 2 6 d})$ or ketones (126e and $\mathbf{1 2 6 h})$ were well tolerated. Cyanation of the substrate $\mathbf{2 8 i}$ with a fluorine substituent featured a considerable secondary directing group effect, ${ }^{102}$ thereby leading to the site-selectively afforded the more sterically hindered compound $\mathbf{1 2 6} \mathbf{i}$ as the sole product in good yield. ${ }^{103}$ However, the 3,4,5-trifluorosubstituted substrate $\mathbf{2 8} \mathbf{j}$ delivered the corresponding product $\mathbf{1 2 6} \mathbf{j}$ with an inferior result. Subsequently, a variation of the substitution pattern on the Lewis-basic 
pyridine moiety proved to be viable, but did not significantly alter the catalytic efficacy. We were delighted to observe that synthetically useful substrates substituted with heterocyclic moieties, such as pyridyl (py), pyrimidinyl (pym), and pyrazolyl, could be utilized as the selectivity-ensuring entities. In contrast, other directing groups, such as oximes or esters, have been thus far not been suitable substrates.

\subsubsection{Scope of the $\mathbf{C}-\mathbf{H}$ Bond Cyanation with Indoles}

Thereafter, we examined the use of the removable pyrimidine directing group for the cyanation of biologically active indole ${ }^{117}$ derivatives $7^{118}$ (Scheme 7.2).

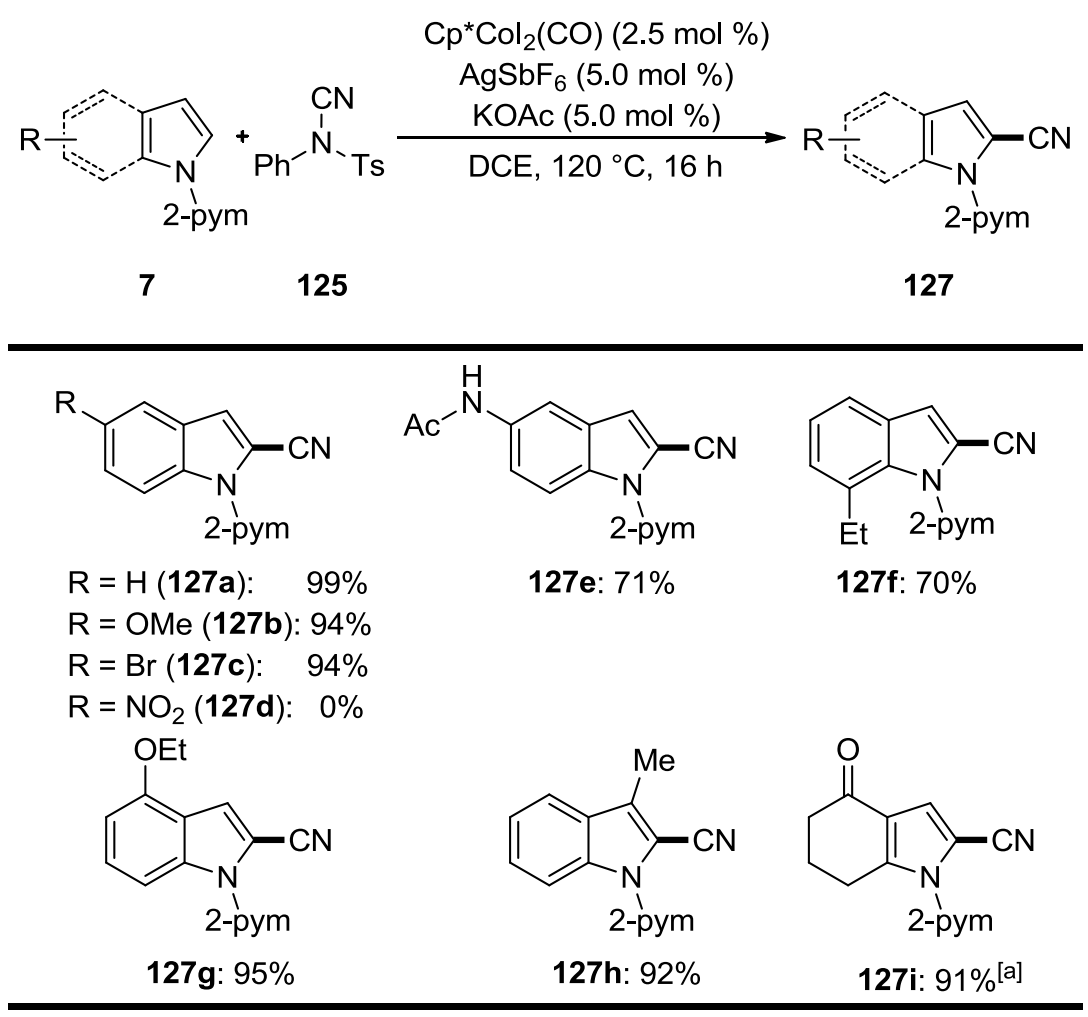

Scheme 7.2. Cobalt-catalyzed $\mathrm{C}-\mathrm{H}$ bond cyanation of indoles 7. [a] $\left[\mathrm{Cp}^{*} \mathrm{CoI}_{2}(\mathrm{CO})\right](\mathbf{1 1 2}, 5.0$

$$
\mathrm{mol} \%), \operatorname{AgSbF}_{6}(10 \mathrm{~mol} \%), \mathrm{KOAc}(10 \mathrm{~mol} \%) \text {. }
$$

Applying this method, the parent compound $\mathbf{1 2 7}$ a was synthesized in virtually quantitative yield. Remarkably, 5-substituted indoles bearing various functional groups, such as methoxy (7b), bromo (7c) or amido (7e), were efficiently cyanated, which allowed the synthesis of serotonin derivatives in a step-economical fashion. Unfortunately, the reaction with 5-nitro

\footnotetext{
${ }^{117}$ Modern Heterocyclic Chemistry (Eds.: J. Alvarez-Builla, J. J. Vaquero, J. Barluenga), Wiley-VCH, Weinheim, 2011.

${ }^{118}$ L. Ackermann, A. V. Lygin, Org. Lett. 2011, 13, 3332-3335.
} 
substituted indole $\mathbf{7 d}$ was not successful. Generally, substitution on the carbocyclic moiety of the substrates 7 was well tolerated by the $\mathrm{C}-\mathrm{H}$ bond functionalization catalyst. Interestingly, the sterically more congested 3-methyl indole derivative $\mathbf{7 h}$ delivered the desired cyanated product $\mathbf{1 2 7} \mathbf{h}$ in excellent isolated yield as well. Even the sensitive ketone functionality on the tetrahydroindolone $\mathbf{7 i}$ was cyanated with remarkably high efficacy.

\subsubsection{Scope of the C-H Bond Cyanation with Heteroarenes}

The versatile cobalt(III) catalyst was not limited to cyanations on the indole heterocycle. Indeed, the direct $\mathrm{C}-\mathrm{H}$ bond cyanation of pyrroles (144, Scheme 7.3a) and thiophenes (146, Scheme 7.3b) occurred with excellent levels of efficacy and positional selectivity as well.

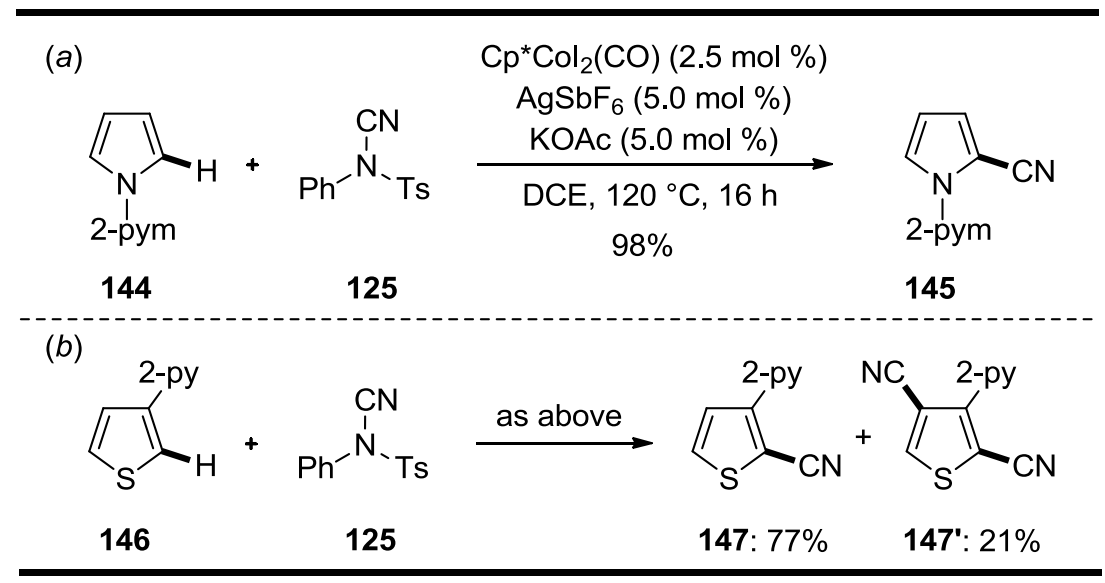

Scheme 7.3. Cobalt-catalyzed C-H bond cyanation of the heteroarenes 144 and 146.

\subsection{Mechanistic Studies}

\subsubsection{Inte rmolecular Competition Experiments}

Intrigued by the outstanding activity of the cobalt catalyst through for cobalt mechanistically unusual carboxylate assistance, we sought to unravel the mode of action. To this end, intermolecular competition experiments between the differently substituted arenes $\mathbf{2 8} \mathbf{b}$ and 28c highlighted a slight preference in the $\mathrm{C}-\mathrm{H}$ bond cyanation for the more electron-rich substrate (Scheme 7.4). 


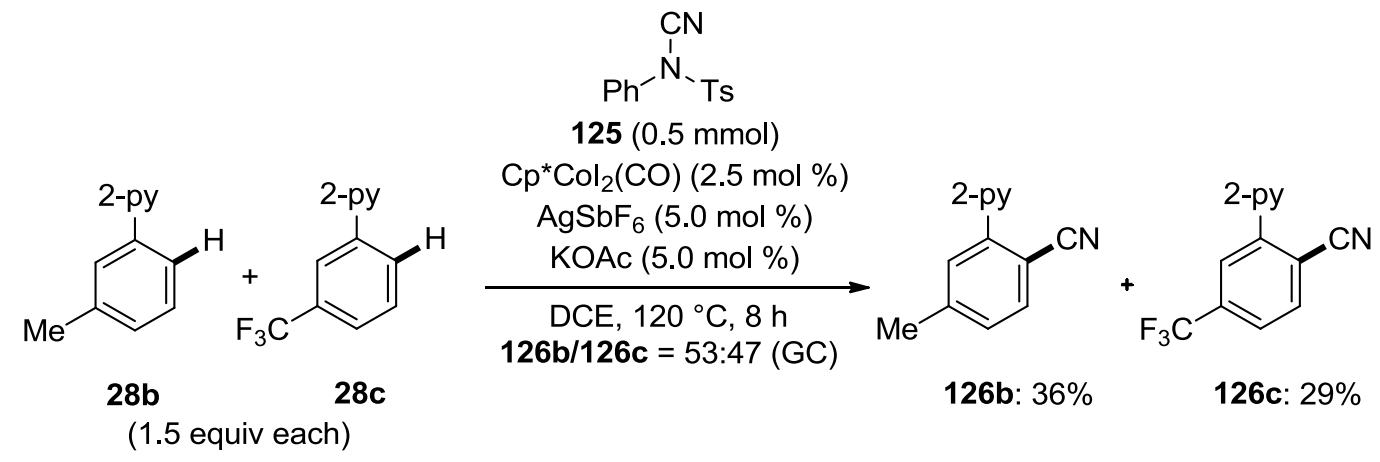

Scheme 7.4. Competition experiment between differently substituted arenes $\mathbf{2 8 b}$ and $\mathbf{2 8 c}$.

\subsubsection{Reactions with Isotopically Labelled Reagents}

The use of a deuterated cosolvent clearly indicated a significant H/D exchange solely occurring in the ortho position of arene 28a (Scheme 7.5a).

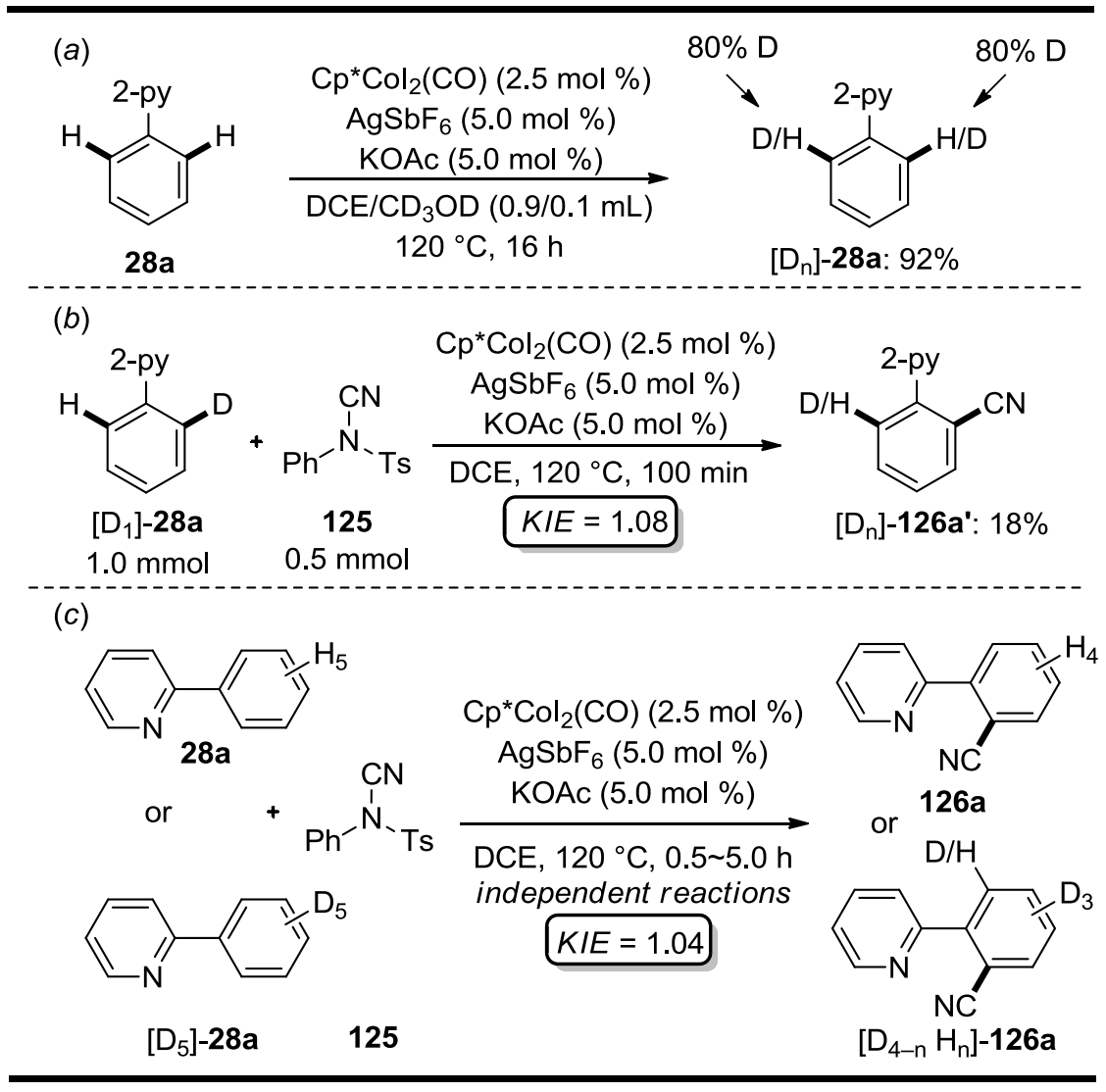

Scheme 7.5. Cobalt-catalyzed H/D exchange and determination of KIE values with arene 28a.

Accordingly, cobalt-catalyzed $\mathrm{C}-\mathrm{H}$ bond cyanations with isotopically labeled substrates led to a minor kinetic isotope effect (KIE) values of $k_{\mathrm{H}} / k_{\mathrm{D}} \approx 1.0$ and $k_{\mathrm{H}} / k_{\mathrm{D}} \approx 1.1$ for the inter- and intramolecular KIE-determination experiments, respectively (Scheme 7.5b and Scheme 7.5c). These data were in agreement with the $\mathrm{C}-\mathrm{H}$ bond metalation step not be ing rate-determining. 
Moreover, a Hammett plot correlation ${ }^{119}$ (See Chapter 10) indicated a change in the rate-determining reaction step depending on the substitution pattern of the arene.

\subsubsection{Proposed Cataly tic Cycle}

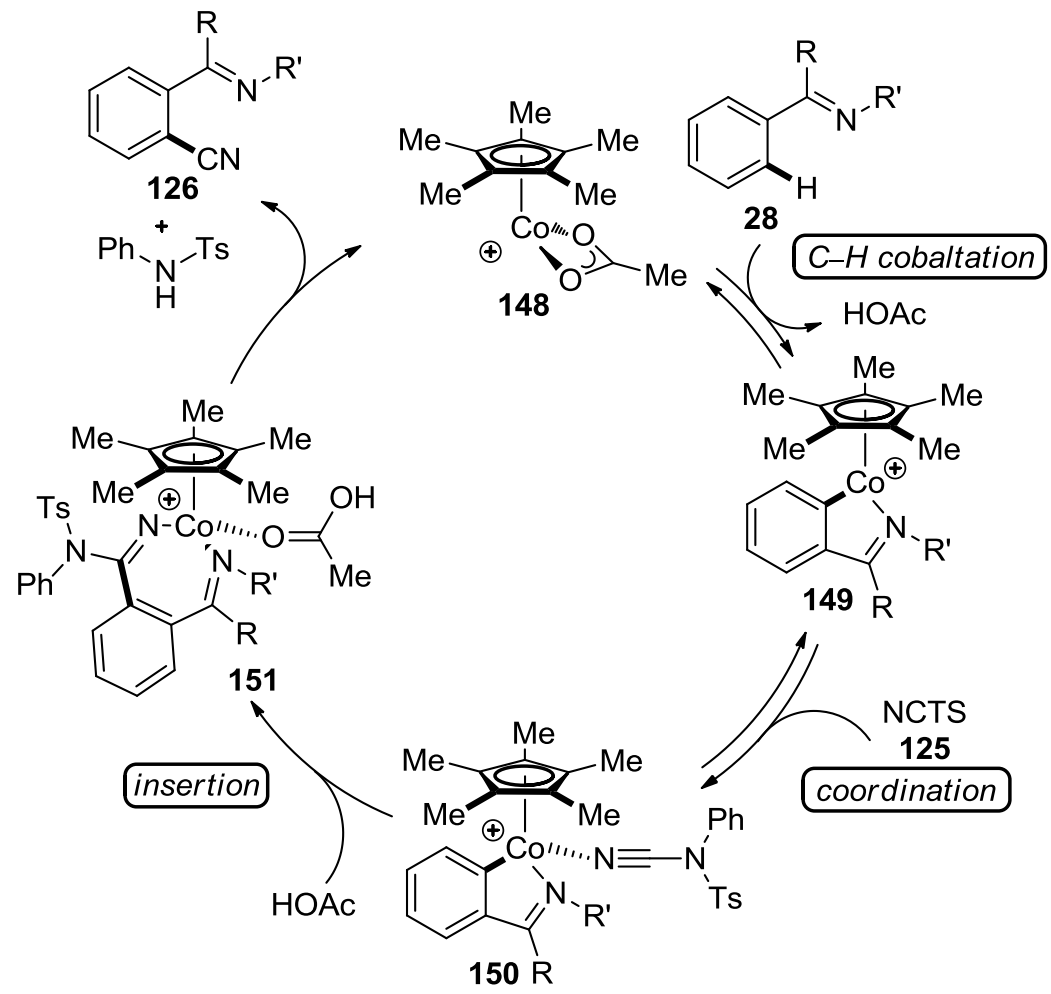

Scheme 7.6. Proposed catalytic cycle.

Based on our mechanistic studies, we proposed the catalytic cycle initiated by a reversible carboxylate-assisted $\mathrm{C}-\mathrm{H}$ bond metalation, thus yielding the cyclometalated complex $\mathbf{1 4 9}$ (Scheme 7.6). Subsequent coordination and insertion of 125 furnished the key intermediates 150 and 151, respectively. Finally, $\beta$-elimination provides the desired product 126, while proto-demetalation regenerates the catalytically active cobalt(III) carboxylate catalyst 148 .

\subsection{Application}

To illustrate the unique potential of the cobalt(III)-catalyzed cyanation protocol, we removed the directing group on the indole 127a (Scheme 7.7a), ${ }^{118}$ and devised a reaction sequence which resulted in the formal direct carboxylation of unactivated $\mathrm{C}-\mathrm{H}$ bonds. ${ }^{120}$ Thus, a

\footnotetext{
119 a) L. P. Hammett, J. Am. Chem. Soc, 1937, 59, 96-103; b) Y. Aihara, N. Chatani, Chem. Sci. 2011, 4, 664-670. 120 a) B. Yu, L.-N. He, Chem SusChem 2015, 8, 52-62; b) M. T. Johnson, O. F. Wendt, J. Organomet. Chem. 2014, 751, 213-220; c) L. Ackermann, Angew. Chem. Int. Ed. 2011, 50, 3842-3844; d) K. Huang, C.-L. Sunwa, Z.-J. Shi, Chem. Soc. Rev. 2011, 40, 2435-2452.
} 
one-pot procedure initiated by the cobalt-catalyzed $\mathrm{C}-\mathrm{H}$ bond cyanation, along $\mathrm{w}$ ith a facile base-mediated saponification, de livered the desired carboxylic acid 152 in high yie ld (Scheme $7.7 b)$.

(a)<smiles>[Y6][R6]n1c(C#N)cc2ccccc21</smiles>

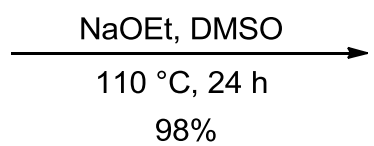<smiles>N#Cc1cc2ccccc2[nH]1</smiles>

$127 a$

127j

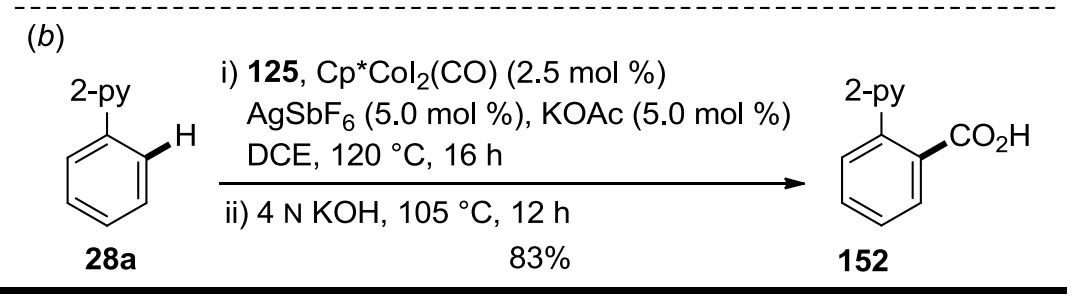

Scheme 7.7. (a) Removal of directing group. (b) Cobalt-catalyzed one-pot synthesis of the carboxylic acid $\mathbf{1 5 2}$.

Finally, we exploited the cobalt-catalyzed $\mathrm{C}-\mathrm{H}$ bond cyanation for the synthesis of the functionalized indoles 153-155 (Scheme 7.8). ${ }^{118,121}$ Particularly, the high-yielding preparation of the 2-tetrazolyl derivative $\mathbf{1 5 3}$ should prove instrumental for the design of novel bioactive drugs.

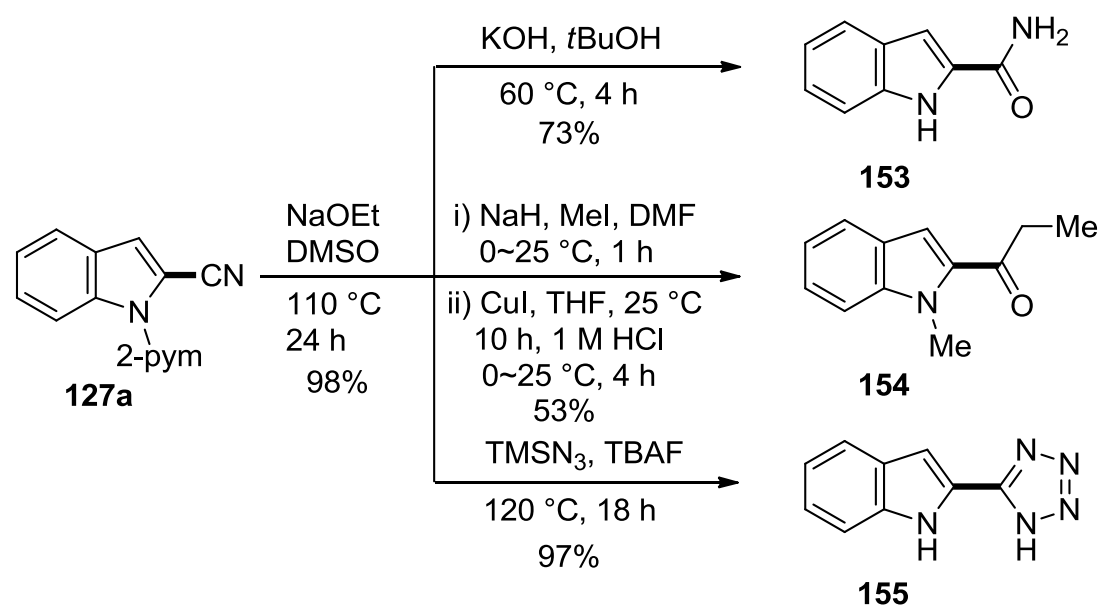

Scheme 7.8. Cobalt-catalyzed C-H bond activation towards substituted indoles.

\subsection{Conclusion}

In summary, we have reported on the first cobalt-catalyzed cyanation of unactivated $\mathrm{C}-\mathrm{H}$

\footnotetext{
121 a) M. Chaitanya, D. Yadagi ri, P. Anbarasan, Org. Lett. 2013, 15, 4960-4963; b) S. M. Kim, J. H. Park, Y. K. Kang, Y. K. Chung, Angew. Chem. Int. Ed. 2009, 48, 4543-4545; c) D. Amantini, R. Beleggia, F. Fringuelli, F. Pi zzo, L. Vacca ro, J. Org. Chem. 2004, 69, 2896-2898.
} 
bonds in arenes 28 and heteroarenes 7, 144 and 146. Thus, carboxylate assistance led to an enhanced activity of cationic cobalt(III) catalyst towards the direct cyanation of arenes and heteroarenes with ample scope. The optimized catalytic system tolerated various functional groups and proved applicable with removable directing groups. Mechanistic studies revealed an effecient cobalt-catalyzed site-selective ortho deuteration of heteroarylarenes by reversible $\mathrm{C}-\mathrm{H}$ bond activation. 


\section{Cobalt(III)-Catalyzed Aryl- and Alkenyl-C-H Bond Aminocarbonylation with Isocyanates and Acyl Azides}

In recent years, transition-metal-catalyzed direct insertion of isocyanates into $\mathrm{C}-\mathrm{H}$ bonds is in high demand, because they efficiently provide synthetically valuable amide moieties. ${ }^{122}$ Theoretically, acyl azides can be employed as precursors for isocyanates via the "Curtius rearrangement" at elevated temperature. ${ }^{123}$ To the best of our knowledge, acyl azides were only found to work as amino sources in the iridium- and ruthenium-catalyzed amidation. ${ }^{124-125}$ Another sole example of the use of acyl azides was developed applying rhodium catalysts. ${ }^{125}$ However, further application of acyl azides was strongly limited by the difficulty in controlling the ir dual reactivity, leading to a mixture of products with $\mathrm{C}-\mathrm{C}$ and $\mathrm{C}-\mathrm{N}$ bond formation. Described herein is the first cobalt(III)-catalyzed $\mathrm{C}-\mathrm{H}$ bond aminocarbonylation with isocyanates and acyl azides. ${ }^{126}$

\subsection{Optimization}

We commenced our studies by exploring the reaction conditions for the cobalt(III)-catalyzed $\mathrm{C}-\mathrm{H}$ bond aminocarbonylations of 1-phenylpyrazole (128a) with phenyl isocyanate (129a) (Table 8.1). With a combination of $\mathrm{AgNTf}_{2}$ or $\mathrm{AgPF}_{6}$ and $\mathrm{AgOAc}$, the desired product 130aa was obtained in low yields (entries 1-2). Among a set of representative carboxylate salts, AgOPiv provided the optimal results (entries 3-9). The nature of the silver salts appeared to be crucial, and both $\mathrm{AgSbF}_{6}$ and $\mathrm{AgNTf}_{2}$ combined with $\mathrm{AgOP}$ iv gave the best yields (entries 10-13). Moreover, omission of either of the catalyst's components or replacement of the $\left[\mathrm{Cp}^{*} \mathrm{CoI}_{2}(\mathrm{CO})\right]^{85-86}$ by other cobalt sources failed to deliver the desired product (entries 14-19), whereas reducing the amount of isocyanate 129a or changing the reaction media resulted in significantly reduced yields or inhibited the reaction (entries 20-22).

Table 8.1. Optimization of cobalt(III)-catalyzed C-H bond aminocarbonylation. ${ }^{[a]}$

\footnotetext{
122 a) S. D. Sarkar, L. Ackemann, Chem. Eur. J. 2014, 20, 13932-13936; b) B. Zhou, W. Hou, Y. Yang, Y. L, Chem. Eur. J. 2013, 19, 4701-4706; c) K. D. Hesp, R. G. Bergman, J. Ellman, J. Am. Chem. Soc. 2013, 135, 11430-11433; d) K. Murali rajan, K. Parthasa rathy, C. H. Cheng, Org. Lett. 2012, 14, 4262-4265; e) Y. Kuninobu, Y. Tokunaga, A. Kawata, K. Takai, J. Am. Chem. Soc. 2006, 128, 202-209.

123 T. Curtius, Ber. Dtsch. Chem. Ges. 1890, 23, 3023-3033.

124 J. Ryu, J. Kwak, K. Shin, D. Lee, S. Chang, J. Am. Chem. Soc. 2013, 135, 12861-12868.

125 K. Shin, J. Ryu, S. Chang, Org. Lett. 2014, 16, 2022-2025.

126 J. L. L. Ackermann, Angew. Chem. Int. Ed. 2015, 54, (DOI: 10.1002/anie.201501926).
} 

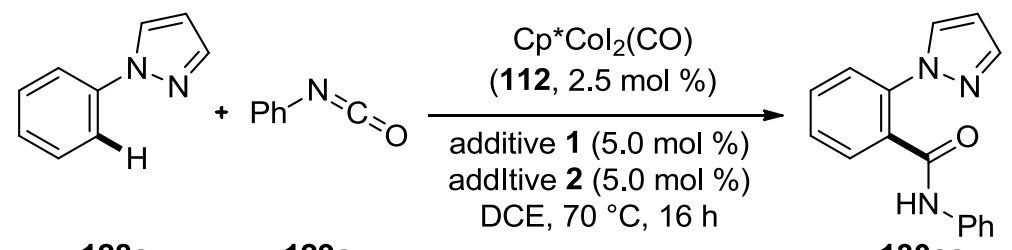

$128 a$

$129 a$ DCE, $70^{\circ} \mathrm{C}, 16 \mathrm{~h}$

130aa

\begin{tabular}{|c|c|c|c|c|}
\hline Entry & [Co] & Addi tive 1 & Additive 2 & Yield $(\%)^{[b]}$ \\
\hline 1 & {$\left[\mathrm{Cp}^{*} \mathrm{CoI}_{2}(\mathrm{CO})\right]$} & $\mathrm{AgNTf}_{2}$ & AgOAc & 35 \\
\hline 2 & {$\left[\mathrm{Cp}^{*} \mathrm{CoI}_{2}(\mathrm{CO})\right]$} & $\mathrm{AgPF}_{6}$ & $\mathrm{AgOAc}$ & 23 \\
\hline 3 & {$\left[\mathrm{Cp}^{*} \mathrm{CoI}_{2}(\mathrm{CO})\right]$} & $\mathrm{AgPF}_{6}$ & $\mathrm{AgCO}_{2} \mathrm{Ad}$ & 25 \\
\hline 4 & {$\left[\mathrm{Cp}^{*} \mathrm{CoI}_{2}(\mathrm{CO})\right]$} & $\mathrm{AgPF}_{6}$ & AgOPiv & 42 \\
\hline 5 & {$\left[\mathrm{Cp}^{*} \mathrm{CoI}_{2}(\mathrm{CO})\right]$} & $\mathrm{AgPF}_{6}$ & KOAc & 24 \\
\hline 6 & {$\left[\mathrm{Cp}^{*} \mathrm{CoI}_{2}(\mathrm{CO})\right]$} & $\mathrm{KPF}_{6}$ & $\mathrm{AgOAc}$ & 0 \\
\hline 7 & {$\left[\mathrm{Cp}^{*} \mathrm{CoI}_{2}(\mathrm{CO})\right]$} & $\mathrm{AgPF}_{6}$ & $\mathrm{CsOAc}$ & 0 \\
\hline 8 & {$\left[\mathrm{Cp}^{*} \mathrm{CoI}_{2}(\mathrm{CO})\right]$} & $\mathrm{AgPF}_{6}$ & KOPiv & 0 \\
\hline 9 & {$\left[\mathrm{Cp}^{*} \mathrm{CoI}_{2}(\mathrm{CO})\right]$} & $\mathrm{AgPF}_{6}$ & $\mathrm{NaOPiv}$ & 0 \\
\hline 10 & {$\left[\mathrm{Cp}^{*} \mathrm{CoI}_{2}(\mathrm{CO})\right]$} & $\mathrm{AgSbF}_{6}$ & AgOPiv & 46 \\
\hline 11 & {$\left[\mathrm{Cp}^{*} \mathrm{CoI}_{2}(\mathrm{CO})\right]$} & $\mathrm{AgNTf}_{2}$ & AgOPiv & 57 \\
\hline 12 & {$\left[\mathrm{Cp}^{*} \mathrm{CoI}_{2}(\mathrm{CO})\right]$} & $\mathrm{AgPF}_{6}$ & AgOPiv & $64^{[\mathrm{c}]}$ \\
\hline 13 & {$\left[C p * \mathrm{CoI}_{2}(\mathrm{CO})\right]$} & $\operatorname{AgSbF}_{6}$ & AgOPiv & $67^{[c]}$ \\
\hline 14 & {$\left[\mathrm{Cp}^{*} \mathrm{CoI}_{2}(\mathrm{CO})\right]$} & $\mathrm{AgPF}_{6}$ & -- & 0 \\
\hline 15 & {$\left[\mathrm{Cp}^{*} \mathrm{CoI}_{2}(\mathrm{CO})\right]$} & -- & AgOPiv & 0 \\
\hline 16 & -- & $\mathrm{AgPF}_{6}$ & AgOPiv & 0 \\
\hline 17 & $\mathrm{CoI}_{2}$ & $\mathrm{AgSbF}_{6}$ & AgOPiv & 0 \\
\hline 18 & $\mathrm{Co}(\mathrm{OAc})_{2}$ & $\mathrm{AgSbF}_{6}$ & AgOPiv & 0 \\
\hline 19 & $\mathrm{Co}(\mathrm{acac})_{2}$ & $\mathrm{AgSbF}_{6}$ & AgOPiv & 0 \\
\hline 20 & {$\left[\mathrm{Cp}^{*} \mathrm{CoI}_{2}(\mathrm{CO})\right]$} & $\mathrm{AgSbF}_{6}$ & AgOPiv & $53^{[\mathrm{c}, \mathrm{d}]}$ \\
\hline 21 & {$\left[\mathrm{Cp}^{*} \mathrm{CoI}_{2}(\mathrm{CO})\right]$} & $\mathrm{AgPF}_{6}$ & AgOPiv & $0^{[\mathrm{e}]}$ \\
\hline 22 & {$\left[\mathrm{Cp}^{*} \mathrm{CoI}_{2}(\mathrm{CO})\right]$} & $\mathrm{AgPF}_{6}$ & AgOPiv & $0^{[\mathrm{f}]}$ \\
\hline
\end{tabular}

[a] General reaction conditions: 128a $(0.5 \mathrm{mmol}), \mathbf{1 2 9 a}(1.0 \mathrm{mmol}), \mathrm{Cp} * \mathrm{CoI}_{2}(\mathrm{CO})(\mathbf{1 1 2}, 2.5$ $\mathrm{mol} \%), \mathrm{AgSbF}_{6}(5.0 \mathrm{~mol} \%), \mathrm{KOAc}(5.0 \mathrm{~mol} \%), \mathrm{DCE}(2.0 \mathrm{~mL}), 70^{\circ} \mathrm{C}, 16 \mathrm{~h}$. [b] Isolated yields. 
[c] $\left[\mathrm{Cp} * \mathrm{CoI}_{2}(\mathrm{CO})\right](5.0 \mathrm{~mol} \%)$, additives $(10 \mathrm{~mol} \%)$. [d] 129a $(0.75 \mathrm{mmol})$. [e] NMP $(2.0 \mathrm{~mL})$.

[f] PhMe $(2.0 \mathrm{~mL})$.

\subsection{Scope and Limitations}

\subsubsection{Scope of Aminocarbonylation with Substrates 128}

With the optimized cobalt(III) catalytic system in hand, we explored its substrate scope with various $N$-heteroarenes (Scheme 8.1).

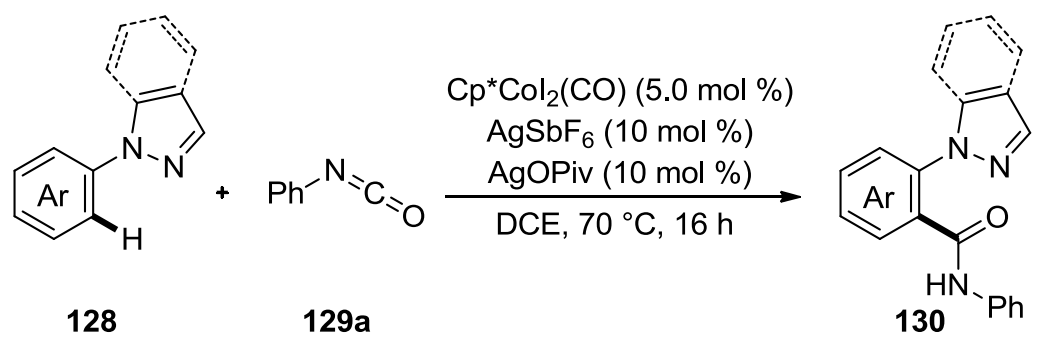

(a) arenes

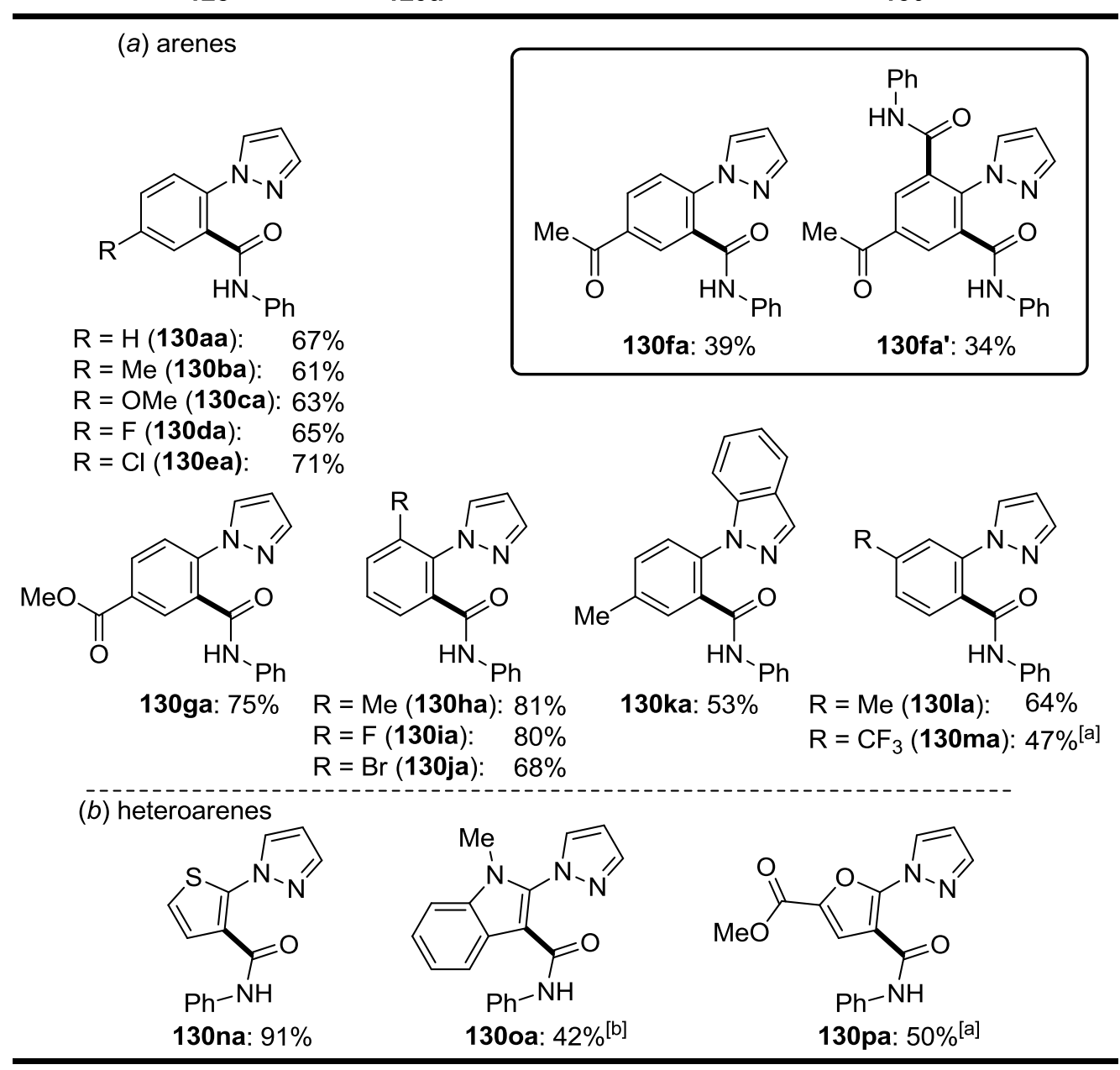

Scheme 8.1. Scope of cobalt-catalyzed aminocarbonylation of $\mathrm{C}-\mathrm{H}$ bonds. [a] $\left[\mathrm{Cp}^{*} \mathrm{CoI}_{2}(\mathrm{CO})\right](10$ mol \%), $\operatorname{AgSbF}_{6}(20 \mathrm{~mol} \%), \operatorname{AgOPiv}(20 \mathrm{~mol} \%)$. [b] [Cp*CoI $\left.(\mathrm{CO})\right](10 \mathrm{~mol} \%), \operatorname{AgNTf}_{2}(20$ mol \%), AgOPiv (20 mol \%). 
Notably, the chelation-assisted $\mathrm{C}-\mathrm{H}$ bond aminocarbonylation proved to be broadly applicable. Both electron-rich as well as electron-deficient arylpyrazoles were converted into nitriles with moderate to high isolated yields 130aa-130ea. Importantly, the cobalt(III) catalyst displayed an excellent chemoselectivity, wherein a number of va luable electrophilic groups, such as ketones or esters, were well tolerated. Interestingly, a certain amount of disubstituted products like 130fa' were also isolated. Furthermore, a set of more sterically hindered substrates bearing an ortho-methyl, fluoro or bromo group generated the desired products in good yields $\mathbf{1 3 0 h a - 1 3 0 j a . ~ W e ~ w e r e ~ d e l i g h t e d ~ t o ~ o b s e r v e ~ t h a t ~ s y n t h e t i c a l l y ~ u s e f u l ~}$ indazoles $\mathbf{2 8 k}$ could be utilized as the selectivity ensuring entity. In intramolecular competition experiments with meta-substituted arenes $\mathbf{2 8 I}$ and $\mathbf{2 8 m}$, the site-selectivities were largely governed by steric interactions. The widely applicable cobalt catalyst was not limited to transformations of only aromatic pyrazoles. Indeed, heteroaromatic thiophene, indole and furan derivatives $\mathbf{1 2 8} \mathbf{n}-\mathbf{1 2 8} \mathbf{p}$ also led to site-selective $\mathrm{C}-\mathrm{H}$ bond aminocarbonylations.

\subsubsection{Scope of Aminoca rbonylation with Decorated Is ocyanates 129}

Next, we were pleased to observe that isocyanates $\mathbf{1 2 9 b}-\mathbf{1 2 9 f}$ bearing various functional groups were well tolerated by the cobalt catalyst (Scheme 8.2).
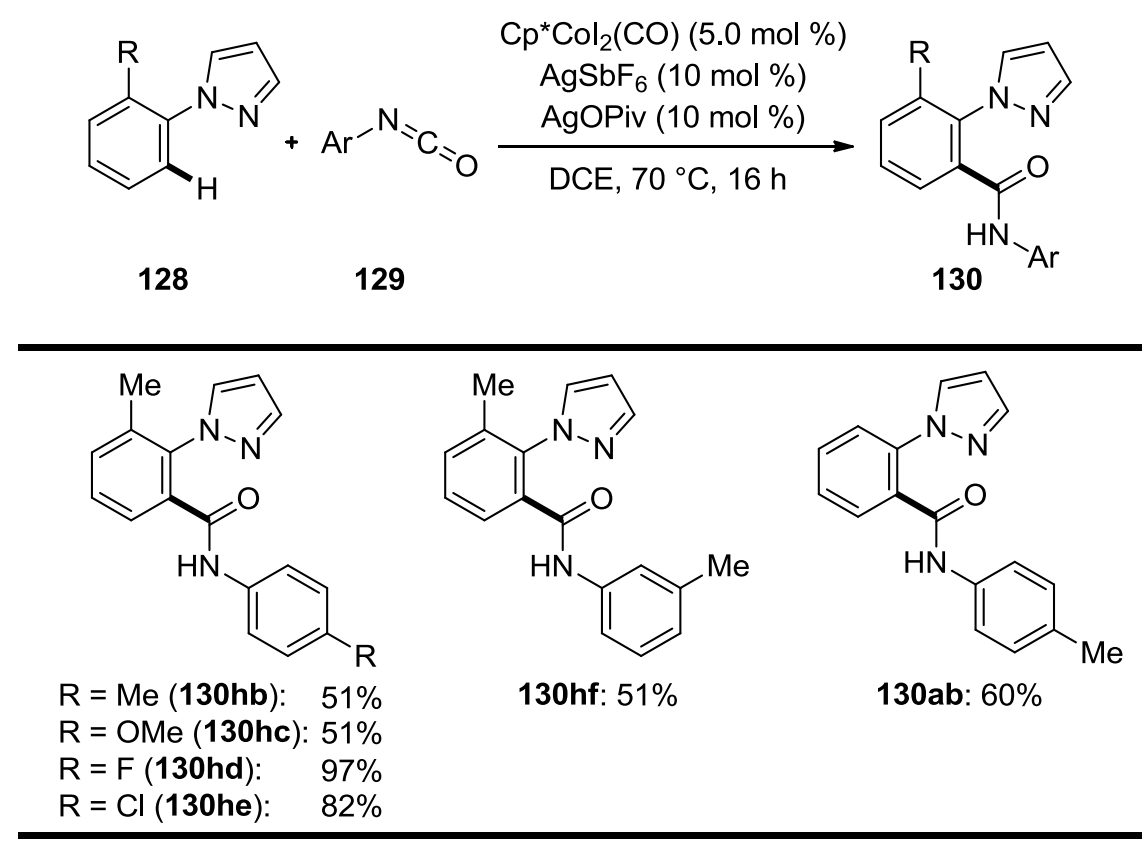

Scheme 8.2. Cobalt-catalyzed aminocarbonylation of C-H bonds.

Acceptable yields were obtained with electron-rich substituted isocyanates $129 \mathbf{b}$ and $129 c$, 
whereas electron-deficient reagents $\mathbf{1 2 9} \mathbf{d}$ were found to be most reactive, and the desired product $\mathbf{1 3 0 h d}$ was produced in virtually quantitative yield. Likewise a chloro substituent in isocyanate 129e was also found to be tolerated by the catalytic system, which can be useful for further functionalizations by cross-coupling chemistry.

\subsubsection{Scope of Aminocarbonylation with Acyl Azides 131}

As discussed above, acyl azides $\mathbf{1 3 1}$ can either be employed as aminocarbonylation reagents or precursors for isocyanates 129. Yet, the synthetic approach to acyl azides is much simpler than those to isocyanates. Thus, we were delighted to explore that a wide substrate scope was observed to be competent when employing differently decorated acyl azides 131 (Scheme 8.3). Good yields were obtained with para-substituted electron-rich acyl azides 131 b, 131c and $131 \mathrm{~g}$ as well as with azides 130ah and 130ai bearing electron-withdrawing substituents. Finally, meta- and ortho-substituted acyl azides $131 \mathbf{f}$ and $\mathbf{1 3 1} \mathbf{j}$ were tested, delivering the desired products 130af and 130aj, respectively, in high isolated yields.
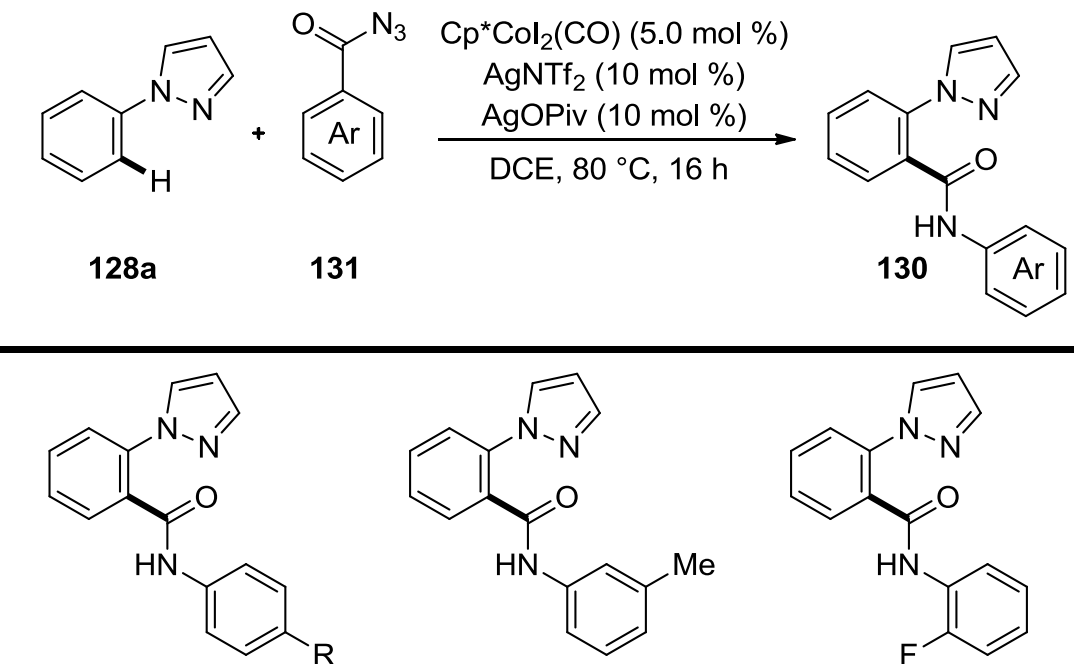

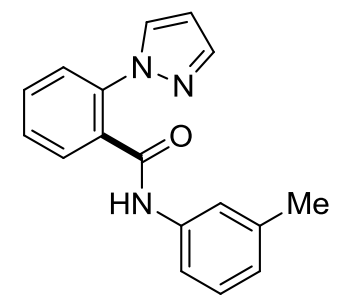

130af: $63 \%$<smiles>O=C(Nc1ccccc1F)c1ccccc1-n1cccn1</smiles>

130aj: $78 \%$

$\mathrm{R}=\mathrm{Me}(130 \mathrm{ab}): \quad 52 \%$

$\mathrm{R}=\mathrm{OMe}$ (130ac): $60 \%$

$\mathrm{R}=\mathrm{Ph}(130 \mathrm{ag}): \quad 57 \%$

$\mathrm{R}=\mathrm{Br}(130 \mathrm{ah}): \quad 63 \%$

$\mathrm{R}=\mathrm{CF}_{3}$ (130ai): $66 \%$

Scheme 8.3. Cobalt-catalyzed C-H bond aminocarbonylation with acyl azides $\mathbf{1 3 1}$.

\subsubsection{Scope of Aminocarbonylation with Vinyl Pyrazole 132}

Interestingly, cobalt(III)-catalyzed aminocarbonylation was not only applicable on (hetero)arenes, but also successfully on olefins via alkenyl C-H bond activation (Scheme 8.4). 
Both (E)-1-styryl-1H-pyrazole (132a) and 1-(1-phenylvinyl)-1H-pyrazole (132b) as well as un- or para-substituted aryl isocyanates showed good reactivity in direct aminocarbonylations under remarkable mild reaction conditions, thereby furnishing the thermodynamically less stable Z-olefins as the sole products $\mathbf{1 3 3}$.
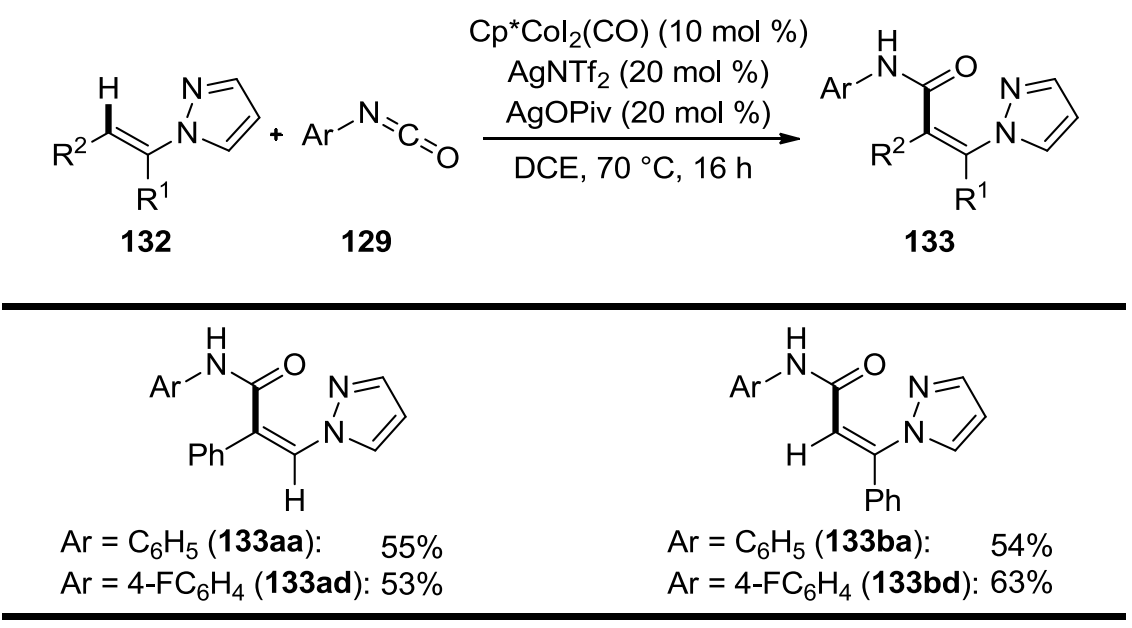

Scheme 8. 4. Cobalt-catalyzed C-H bond aminocarbonylation with vinyl pyrazoles 133.

\subsection{Mechanistic Studies}

\subsubsection{H/D Exchange Experiments and Kinetic Isotope Expe riments}

Intrigued by the outstanding catalytic activity of this cobalt catalyst through for cobalt mechanistically unusual carboxylate assistance, we performed studies to delineate its working mode. To this end, an experiment with deuterated substrate $\left[\mathrm{D}_{5}\right]-\mathbf{1 2 8}$ a clearly demonstrated a significant $H / D$ exchange solely occurring in the ortho position of $\left[D_{n}\right]-128 a$ and $\left[D_{n}\right]-130 a a$ (Scheme 8.5a).

Independent experiments with isotopically labeled substrates indicated the $\mathrm{C}-\mathrm{H}$ bond cobaltation can be characterized with a kinetic isotope effect (KIE) value of $k_{\mathrm{H}} / k_{\mathrm{D}}=1.4$ (Scheme 8.5b). A similar KIE value was also obtained in the intramolecular KIE-determination experiment (Scheme 8.5c). 


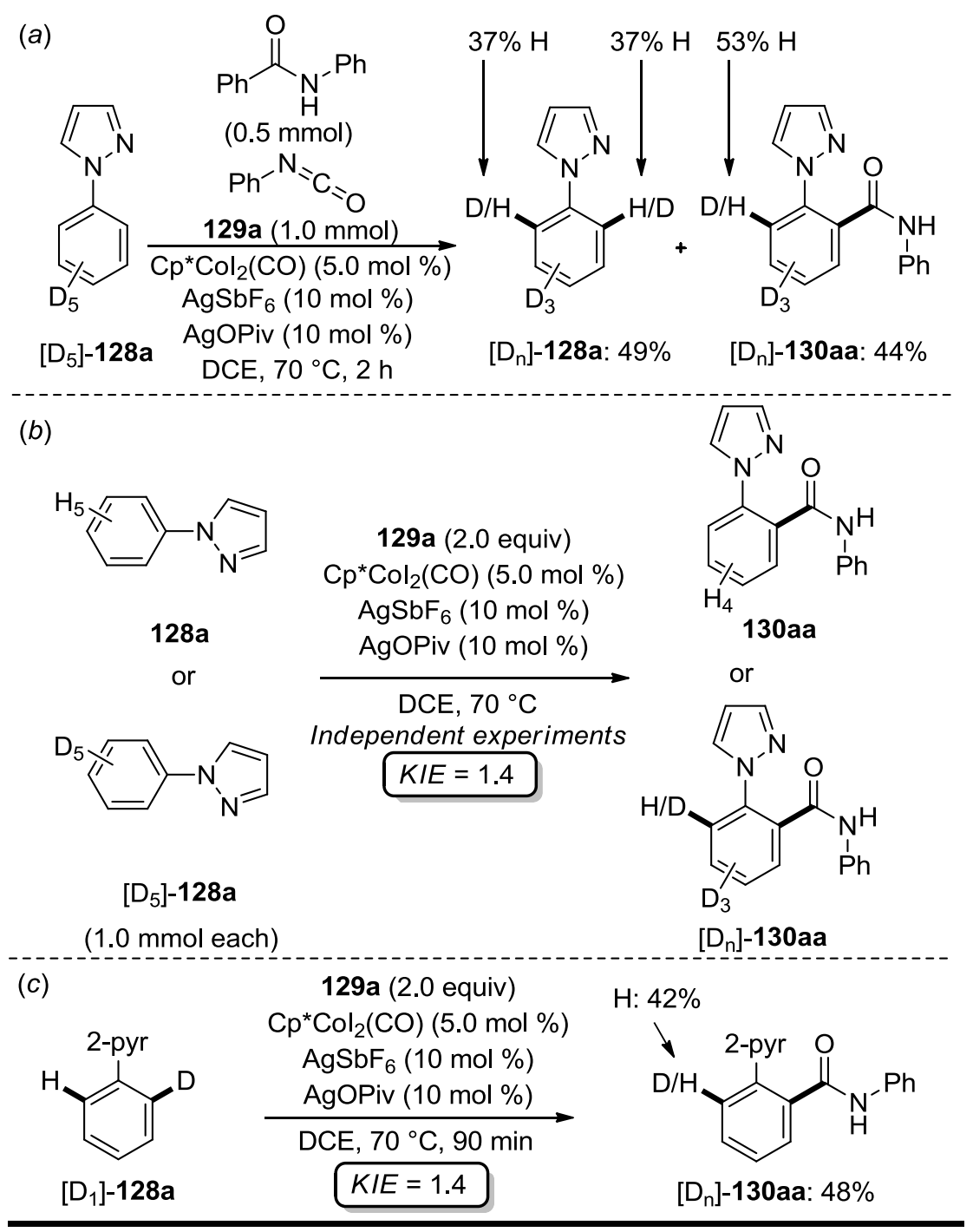

Scheme 8.5. Mechanistic studies. (a) H/D exchange reaction. (b) Intermolecular competition experiment: Determination of kinetic isotope effect (KIE). (c) Intramolecular competition experiment: Determination of kinetic isotope effect (KIE).

\subsubsection{Competition Experiments}

A set of intermolecular competition experiments between the differently substituted arenes 128 revealed a distinct preference in the $\mathrm{C}-\mathrm{H}$ bond aminocarbonylation for the more electron-rich substrate 128b (Scheme 8.6a). On the contrary, the results from the competition experiments between differently substituted isocyanates $\mathbf{1 2 9}$ showed the latter as an electron-defic ient one to be more reactive than its electron-rich counterparts (Scheme 8.6b). 

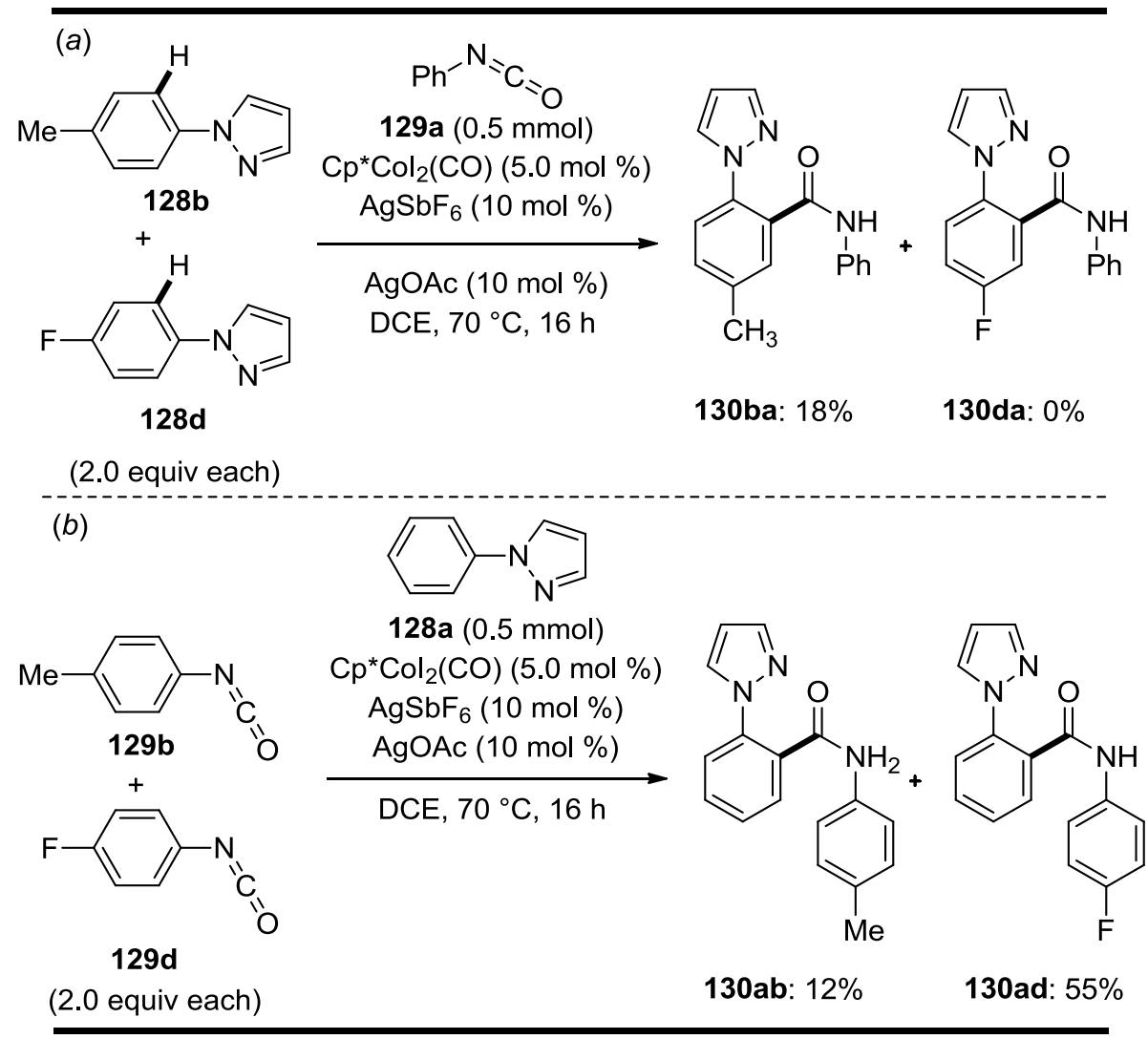

Scheme 8.6. (a) Competition experiment with different arenes 128. (b) Competition experiment with different isocyanates $\mathbf{1 2 9}$.

\subsubsection{Proposed Catalytic Cycle}

Based on our mechanistic studies, we proposed the catalytic cycle to be initiated by a reversible $\mathrm{C}-\mathrm{H}$ bond metalation, thus yie lding the cyclometalated complex 157 (Scheme 8.7). Subsequent coordination and insertion of isocyanate $\mathbf{1 2 9}$ furnish the key intermediates $\mathbf{1 5 8}$ and 159, respectively. Finally, proto-demetalation regenerates the catalytically active cobalt(III) carboxylate catalyst $\mathbf{1 5 6}$ and provides the desired product $\mathbf{1 3 0}$. 


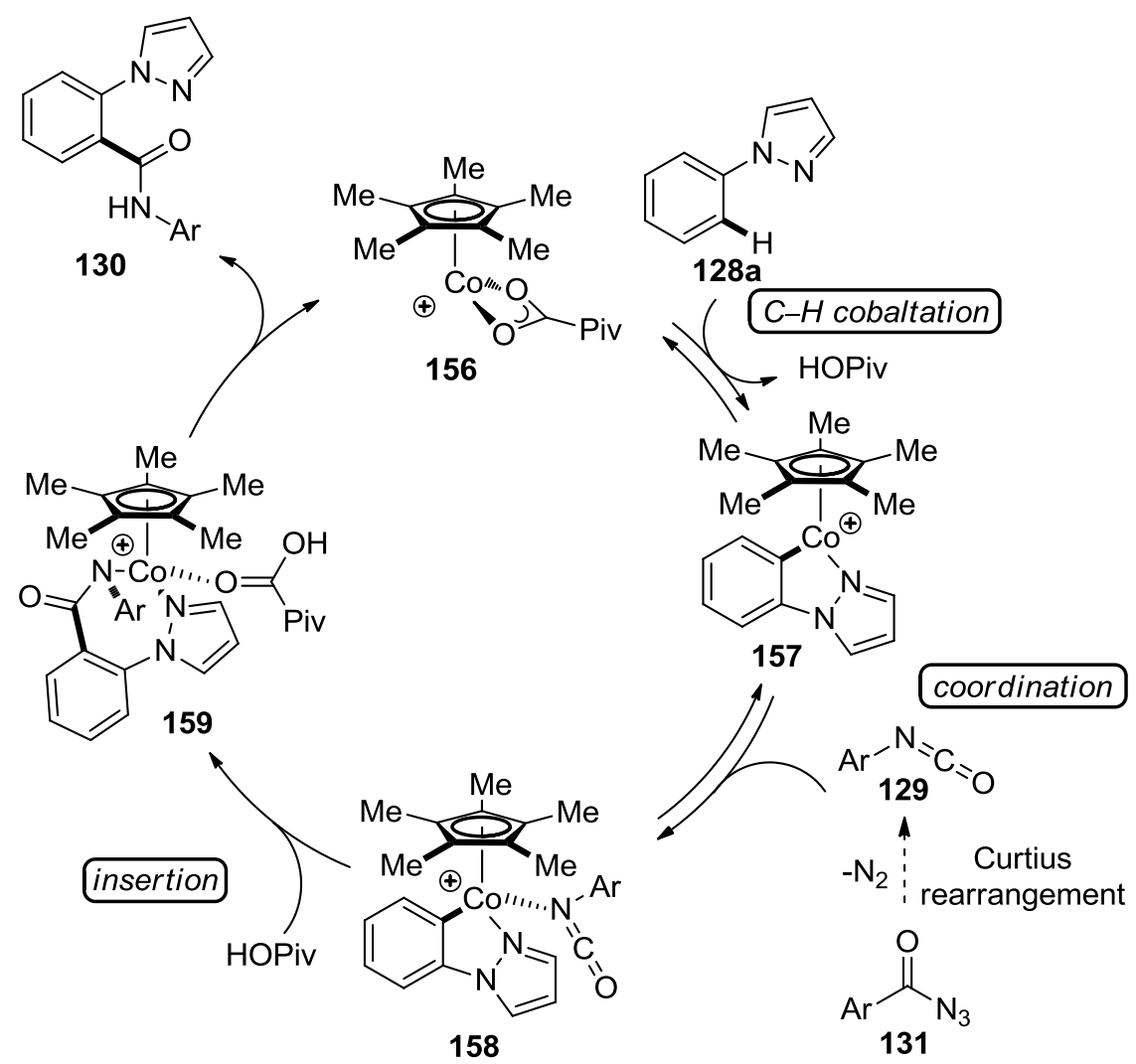

Schem e 8.7. Proposed catalytic cycle.

\subsection{Applications}

To demonstrate the synthetic versatility of the products synthesized by this method, several derivatization reactions were performed (Scheme 8.8).<smiles>O=c1c2ccccc2n2nccc2n1-c1ccccc1</smiles>

$$
\text { 160: } 86 \%
$$

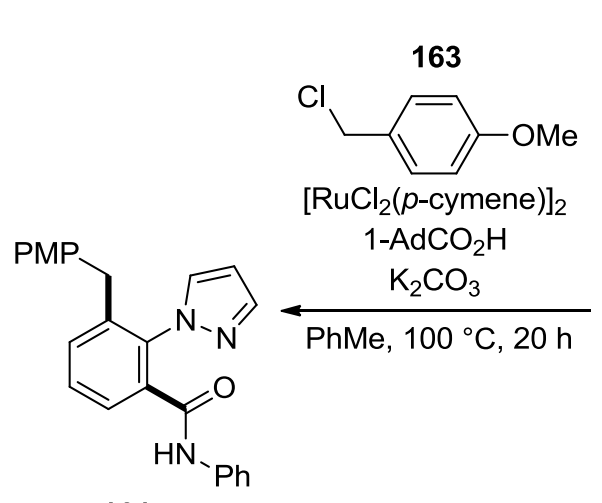

161: $47 \%$

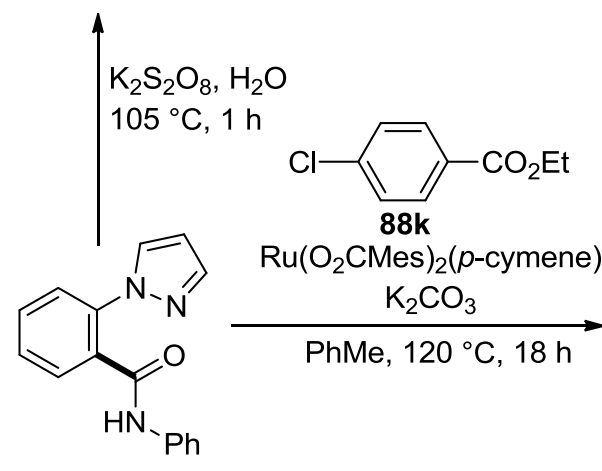

130 aa<smiles>CCOC(=O)c1ccc(-c2cccc(C(=O)Nc3ccccc3)c2-n2cccn2)cc1</smiles>

162: $75 \%$

Scheme 8.8. Derivatization of products $\mathbf{1 3 0}$ obtained by cobalt-catalyzed C-H bond aminocarbonylation. 
Oxidative annulation of 130 aa afforded the heterocyclic product 160 . Direct $\mathrm{C}-\mathrm{H}$ bond benzylation ${ }^{127}$ and arylation ${ }^{128}$ using ruthenium(II) catalysts delivered the corresponding products 161 and 162 in modest to good yie lds, respectively.

\subsection{Conclusion}

In summary, we have established the first cobalt-catalyzed aminocarbonylation of unactivated $\mathrm{C}-\mathrm{H}$ bonds with isocyanates 129 and acyl azides 131. Thus, carboxylate assistance led to a highly active cationic cobalt(III) catalyst for the direct aminocarbonylation of arenes $\mathbf{1 2 8}$ and alkenes $\mathbf{1 3 2}$ with ample scope. The optimized catalytic system tolerated various functional groups and proved applicable to olefins via alkenyl $\mathrm{C}-\mathrm{H}$ bond activation. Mechanistic studies revealed an effective site-selective cobalt catalyst for the site-selective ortho deuteration of heteroarylarenes by reversible $\mathrm{C}-\mathrm{H}$ bond activation.

127 L. Ackermann, P. Novák, Org. Lett. 2009, 11, 4966-4969.

128 L. Ackermann, R. Vicente, H. K. Potukuchi, V. Pi rova no, Org. Lett. 2010, 12, 5032-5035. 


\section{$9 \quad$ Summary and Outlook}

To improve the atom- and step-economy of organic syntheses, major efforts have been made on transformations of inert carbon-hydrogen $(\mathrm{C}-\mathrm{H})$ bonds into carbon-carbon $(\mathrm{C}-\mathrm{C})$ or carbon-heteroatom (C-N, C-O, C-Hal) bonds. Significant advances in organometallic chemistry have set the stage for the development of increasingly viable metal catalysts for $\mathrm{C}-\mathrm{H}$ bond activation reactions and their applications in the preparation of pharmaceutical, agrochemical and functional materials. Thus, the work presented within this thesis focused on the development of versatile ruthenium- and cobalt-catalyzed direct $\mathrm{C}-\mathrm{H}$ bond functionalizations.

In the first project, an effective protocol for the oxidative annulation of ketimines $\mathbf{7 3}$ with internal alkynes 1 was established, and the catalytic system consisting of $\left[\operatorname{RuCl}_{2}(p \text {-cymene })\right]_{2}$, $\mathrm{AgSbF}_{6}$ and $\mathrm{Cu}(\mathrm{OAc})_{2} \cdot \mathrm{H}_{2} \mathrm{O}$ tolerated well various functional groups (Scheme 9.1).

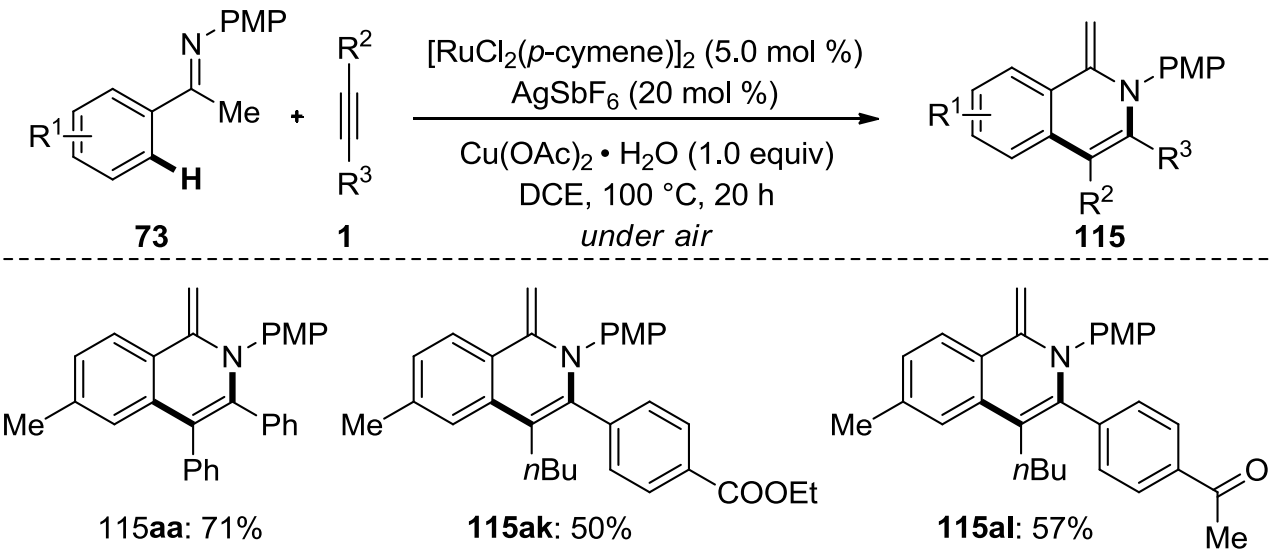

Scheme 9.1. Oxidative $\mathrm{C}-\mathrm{H}$ bond functionalization with decorated ketimines and alkynes.

In order to access the corresponding reduced 1,2-dihydroisoquinolines $\mathbf{1 1 6}$, we subsequently devised a two-step reaction sequence consisting of an initial ruthenium(II)-catalyzed $\mathrm{C}-\mathrm{H}$ bond activation followed by palladium-catalyzed hydrogenation (Scheme 9.2). 


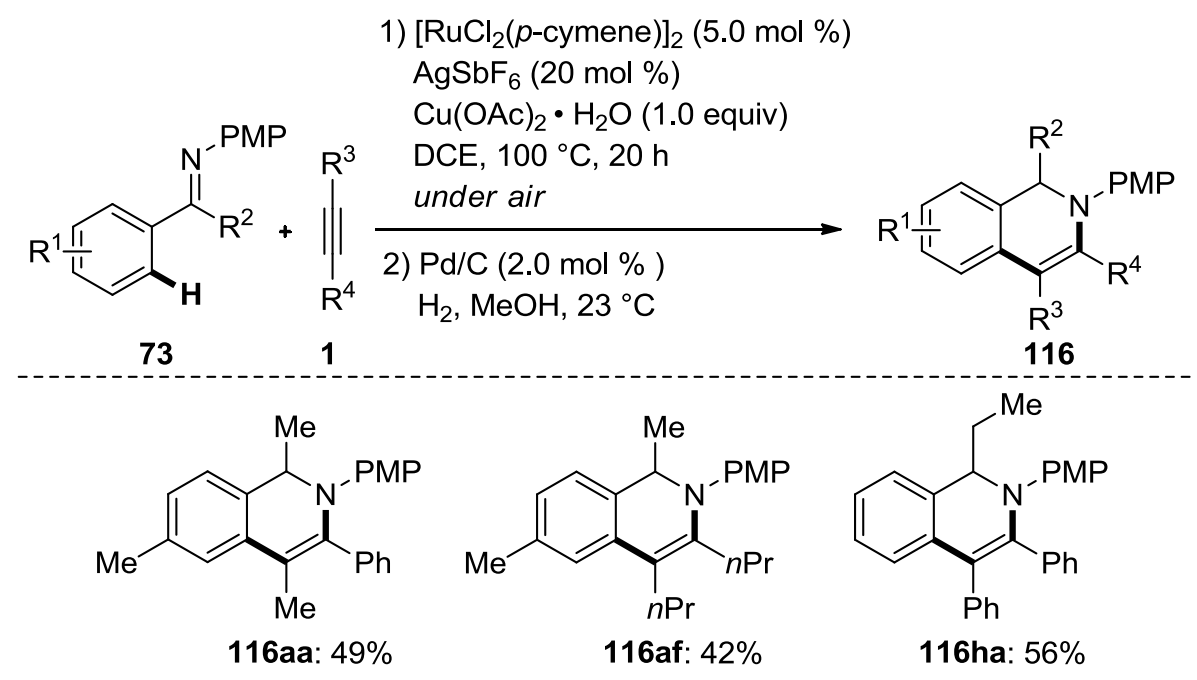

Scheme 9.2. Multicatalytic synthesis of 1,2-dihydroisoquinolines 116.

Based on the above findings, we managed to expand the scope of aromatic substrates to particularly challenging (hetero)aryl amidines 117. A method for ruthenium(II)-catalyzed oxidative $\mathrm{C}-\mathrm{H}$ bond annulations was developed by employing amidines and internal alkynes 1. The reaction provided expedient access to differently decorated aminoisoquinolines $\mathbf{1 1 8}$ with ample scope. In addition, intramolecular competition experiments with substrates bearing meta-methyl or meta-trifluoromethyl substituents proceeded with excellent levels of site selectivity, producing aminoisoquinolines substituted at the less sterically hindered position (Scheme 9.3)

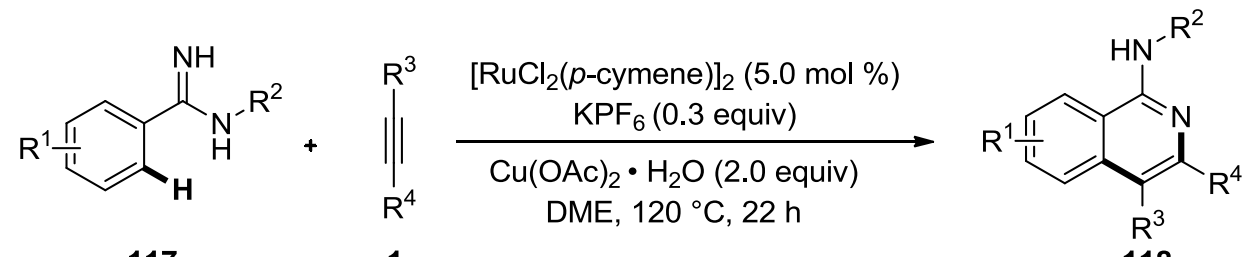<smiles>[R]c1ccc2c(-c3ccccc3)c(-c3ccccc3)nc(NC(C)(C)C)c2c1</smiles>

$R=\operatorname{Me~(118ia):~} 62 \%$ $\mathrm{R}=\mathrm{CF}_{3}(118 \mathrm{ja}): 71 \%$

118pg: $42 \%$<smiles>CC(C)c1c(C(C)(C)C)nc(NC(C)(C)C)c2ccsc12</smiles>

118qg: $57 \%$<smiles>Cc1ccc2c(c1)C(c1ccccc1)=C(c1ccccc1)N1CCN=C21</smiles>

118ra: $62 \%$
118

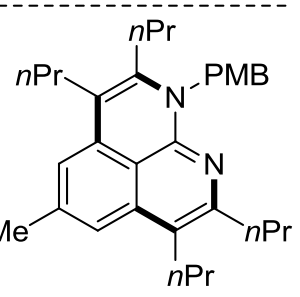

118rg: $64 \%$

Scheme 9.3. Substrates scope for ruthenium(II)-catalyzed oxidative C-H bond annulations. Inspired by the successful synthesis of aminois oquinolines 118, we subsequently became interesting in developing novel oxidative twofold $\mathrm{C}-\mathrm{H}$ bond functionalizations with alkenes 
20. The reaction proceeded not only with excellent chemo-, site-, and regio-selectivities, but also with perfect diastereoselectivity (Scheme 9.4).

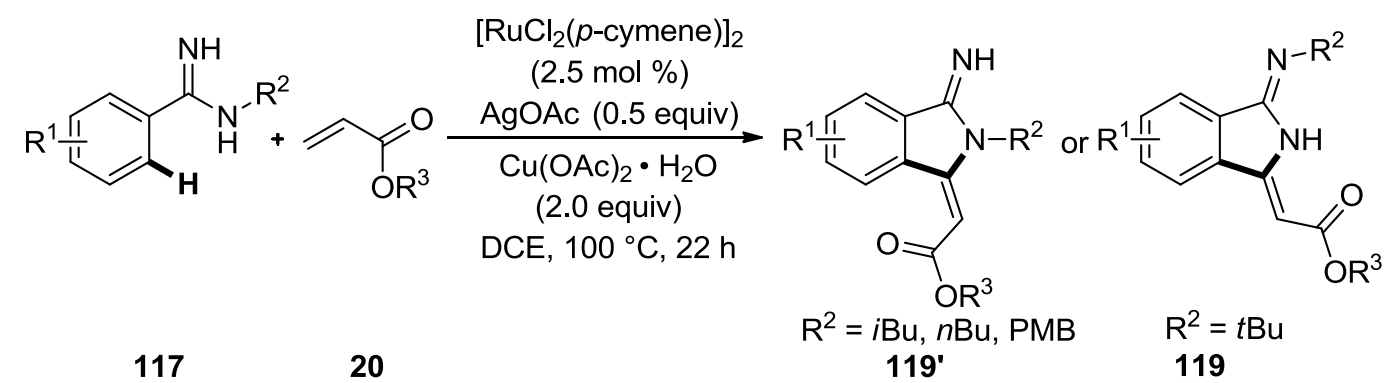<smiles>[R]N1C(=CC(=O)OCC)c2cc(C)ccc2C1=NC</smiles>

$\mathrm{R}=i \mathrm{Bu}\left(\mathbf{1 1 9}^{\prime} \mathrm{ma}\right): \mathbf{7 7 \%}$

$\mathrm{R}=n \mathrm{Bu}$ (119'na): $45 \%$

$\mathrm{R}=\mathrm{PMB}$ (119'ra): $74 \%$<smiles>CCOC(=O)/C=C1\N/C(=N\C(C)(C)C)c2cc(C)ccc21</smiles>

119ba: $73 \%$<smiles>CC#CN=C1N/C(=C\C(=O)OCC)c2ccc3c(c21)OCO3</smiles>

119ka: $68 \%$

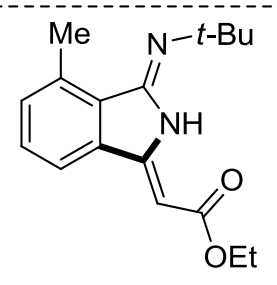

119la: $64 \%$

Scheme 9.4. Ruthenium(II)-catalyzed oxidative C-H bond alkenylation.

We subsequently established ruthenium(II)-catalyzed direct hydroarylation of $\alpha, \beta$-unsaturated ketones 41 with aromatic amides 2 through monodentate coordination (Scheme 9.5). Meanwhile, oxidative annulations of $\alpha, \beta$-unsaturated ketones 41 with acetanilides 121 furnished decorated quinolines 122 (Scheme 9.6).

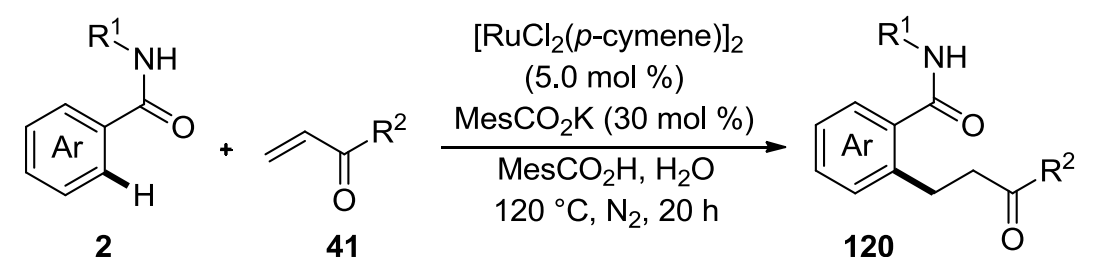<smiles>COCCNC(=O)c1ccc(C)cc1CCC(C)=O</smiles>

120ka: $58 \%$<smiles></smiles>

120ma: $80 \%$<smiles>CNC(=O)c1c(CCC(C)=O)n(C)c2ccccc12</smiles>

120ra: $50 \%$<smiles>CNC(=O)c1ccc(-c2ccccc2)cc1CCC(=O)C1CCCCC1</smiles>

120dc: $45 \%$

Scheme 9.5. Direct C-H bond hydroarylation of $\alpha, \beta$-unsaturated ketones 41 with decorated amides $\mathbf{2}$. 

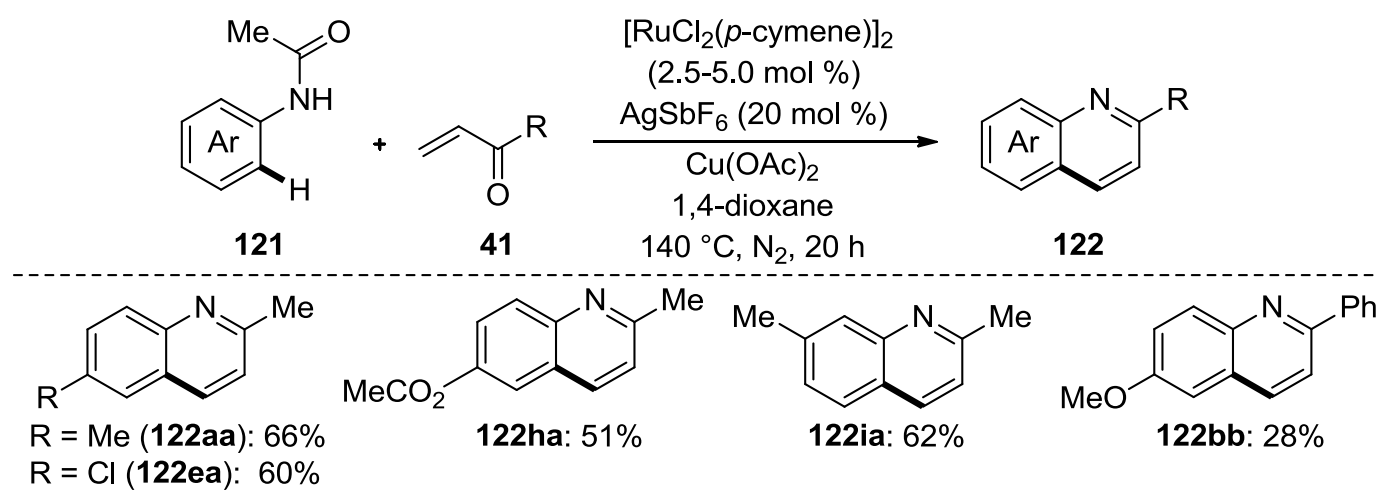

Scheme 9.6. Oxidative annulations of $\alpha, \beta$-unsaturated ketones 41 with acetanilides 121 .

Thereafter, we developed cobalt-catalyzed direct $\mathrm{C}-\mathrm{H}$ bond arylation with weakly-coordinating benzamides. Various aryl chlorides $\mathbf{8 8}$ as well as un- or para-substituted benzamides 2 were identified as viable substrates for direct arylations under remarkably mild reaction conditions. Moreover, the unique synthetic utility of this reaction was illustrated by the facile transformation of the ortho-arylated benzamides $\mathbf{1 3 9}$ to the desired biaryl tetrazoles 124 (Scheme 9.7).

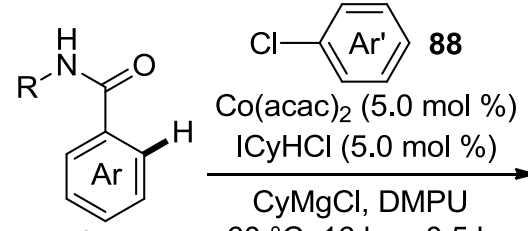

2<smiles>[X]NC(=O)c1ccccc1-c1ccc([R])cc1</smiles>

$\mathrm{R}=4-\mathrm{Me}$ (139aa):

(10 mmol scale)

$\mathrm{R}=4-\mathrm{CH}_{2}$ OTBS (139ad): $57 \%$

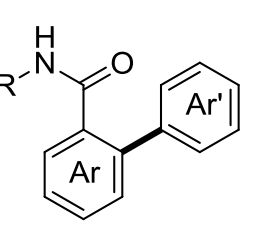

139<smiles>[X]c1ccc(-c2ccccc2C(=O)Nc2ccc(OC)cc2)cc1</smiles>

$72 \%$

$\mathrm{R}=4-\mathrm{Me}$ (139pa):

$\mathrm{R}=4-\mathrm{CH}_{2} \mathrm{OTBS}(139 \mathrm{pd}): 68 \%$

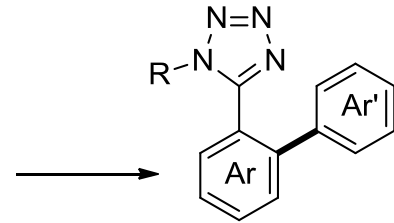

124<smiles>[R]c1ccc(-c2ccccc2-c2nnnn2[Z1])cc1</smiles>

Scheme 9.7. Scope of cobalt-catalyzed $\mathrm{C}-\mathrm{H}$ bond arylation and facile transformation of arylated

benzamides 139 to biaryl tetrazoles 124 .

Very recently, inexpensive high-valent $[\mathrm{Cp} * \mathrm{Co}(\mathrm{III})]$ derivatives have been identified as efficient catalysts for the site-selective functionalization of unactivated $\mathrm{C}-\mathrm{H}$ bonds. We developed first cobalt(III)-catalyzed $\mathrm{C}-\mathrm{H}$ bond cyanation of arenes and heteroarenes. The chelation-assisted $\mathrm{C}-\mathrm{H}$ bond cyanation proceeded with excellent site- and chemo-selectivity, and a wide range of functional groups, such as halogens, ketones, esters and amides, were tolerated under the optimized reaction conditions (Scheme 9.8). 


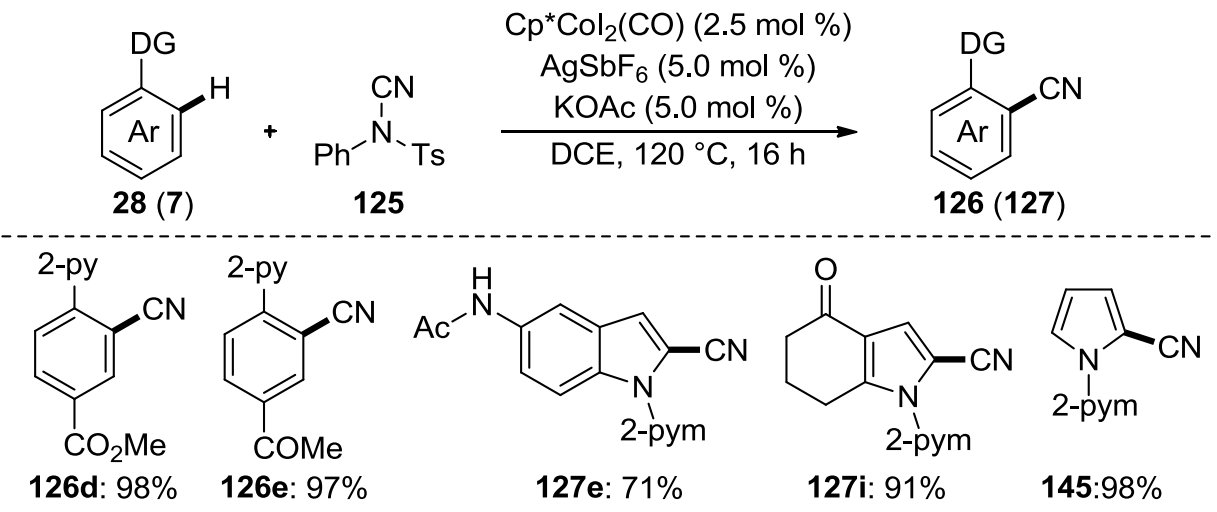

Scheme 9.8. Cobalt-catalyzed C-H bond cyanation.

Finally, described herein is the first cobalt(III)-catalyzed $\mathrm{C}-\mathrm{H}$ bond aminocarbonylation with isocyanates $\mathbf{1 2 9}$ or acyl azides $\mathbf{1 3 1}$ as an electrophilic partner (Scheme 9.9). Notably, a broad scope of isocyanates $\mathbf{1 2 9}$ or acyl azides $\mathbf{1 3 1}$ makes this strategy especially attractive.<smiles>[R]/C(=C/[NH2+])n1ncc2ccccc21</smiles><smiles>NC(=O)OC=CC=[W]</smiles>

131

$128 / 132$
$\mathrm{Cp}^{*} \mathrm{Col}_{2}(\mathrm{CO})$

$(5.0 \mathrm{mmol} \%)$

$\mathrm{AgSbF}_{6}(10 \mathrm{mmol} \%)$

$\underset{\text { DCE, } 70{ }^{\circ} \mathrm{C}, 16 \mathrm{~h}}{\stackrel{\text { AgOPiv }}{\stackrel{\mathrm{mmol}}{\mathrm{C}})}}$<smiles>[R]C(N[Ga])=C([R])n1ncc2ccccc21</smiles>

$130 / 133$<smiles>Cc1ccc(-n2ncc3cc(C)ccc32)c(C(=O)Nc2ccccc2)c1</smiles><smiles>O=C(Nc1ccccc1)c1ccsc1-n1cccn1</smiles><smiles></smiles>

130he: $82 \%$<smiles>O=C(Nc1ccccc1F)c1ccccc1-n1cccn1</smiles>

130aj: $78 \%$<smiles>O=C(/C=C(\c1ccccc1)n1cccn1)Nc1ccc(F)cc1</smiles>

133bd: $63 \%$

Scheme 9.9. Cobalt-catalyzed aminocarbonylation of $\mathrm{C}-\mathrm{H}$ bonds

In summary, major efforts in this thesis were focused on step- and atom-economical synthetic strategies based on transition-metal-catalyzed direct $\mathrm{C}-\mathrm{H}$ bond functionalizations. In this context, we have developed ruthenium- and cobalt-catalyzed chelation-assisted direct $\mathrm{C}-\mathrm{H}$ bond annulations, hydroarylations, arylations, cyanations and aminocarbonylations. Considering the rapid expansion of transition-metal-catalyzed $\mathrm{C}-\mathrm{H}$ bond functionalizations, the researches towards further applications of the cobalt(III) is actively undertaken in the Ackermann group, more exciting progress is expected. 


\section{Experimental Section}

\subsection{General Remarks}

Unless otherwise noted, all reactions were performed under $\mathrm{N}_{2}$ or Ar atmosphere using pre-dried glassware and standard Schlenk techniques.

\section{Solvents}

All solvents for reactions involving moisture-sensitive reagents were dried, distilled and stored under an inert atmosphere ( $\mathrm{Ar}$ or $\mathrm{N}_{2}$ ) according to the follow ing standard procedures. 1,2-Dimethoxyether (DME) and tert-amylalcohol $(t-\mathrm{AmOH})$ were used as supplied by Merck or stirred over sodium chips for $5 \mathrm{~h}$ at $120^{\circ} \mathrm{C}$ and then distilled at ambient pressure.

Water $\left(\mathrm{H}_{2} \mathrm{O}\right)$ was degassed before its use applying repeated freeze-pump-thaw degassing procedure.

\section{1,2-Dichloroethane (DCE) and 1,3-dime thyl-3,4,5,6-tetrahydro-2(1H)-pyrimidinone} (DMPU) were dried over $\mathrm{CaH}_{2}$ for $8 \mathrm{~h}$, degassed and distilled under reduced pressure.

Dichloromethane (DCM), $N, N$-dimethylformamide (DMF) and tetrahydrofuran (THF) were purified using a solvent purification system (SPS) from MBRAUN. $\mathrm{N}$-Methyl-2-pyrrolidone (NMP) was dried over $\mathrm{CaH}_{2}$ for $4 \mathrm{~h}$ at $150{ }^{\circ} \mathrm{C}$ and subsequently distilled under reduced pressure.

Methanol (MeOH) was distilled from magnes ium methanolate.

Tolue ne was pre-dried over KH followed by distillation from sodium benzophenone ketyl. 1,4-Dioxane was dried over sodium benzophenone ketyl and distilled afterwards.

\section{Vacuum}

The following pressures were measured on the used vacuum pump and were not corrected: membrane pump vacuum (MPV): 0.5 mbar, oil pump vacuum (OPV): 0.1 mbar.

\section{Melting Points (M. p.)}

Melting points were measured using a Stuart ${ }^{\circledR}$ Melting Point Apparatus SMP3 from BARLOWORLD SCIENTIFIC. Reported values are uncorrected.

\section{Chromatography}

Analytical thin layer chromatography (TLC) was performed on $0.25 \mathrm{~mm}$ silica gel $60 \mathrm{~F}$-plates 
(Merck) with $254 \mathrm{~nm}$ fluorescent indicator from MERCK. Plates were visualized under UV-light or developed by treatment with a $\mathrm{KMnO}_{4}$ solution followed by carefully heating. Chromatographic purification of products was accomplished by flash column chromatography on MERCK silica gel, grade $60(0.040-0.063 \mathrm{~mm}$ and $0.063-0.200 \mathrm{~mm})$.

\section{Gas Chromatography (GC)}

The conversion of the reactions was monitored by coupled gas chromatography/mass spectrometry using G1760C GCDplus with mass detector HP 5971, 5890 Series II with mass detector HP 5972 from HEWLETT-PACKARD and 7890A GC-System with mass detector 5975C (Triplex-Axis-Detector) from AGILENT TECHNOLOGIES equipped with HP-5MS columns $(30 \mathrm{~m} \times 0.25 \mathrm{~mm}, \varnothing 0.25 \mathrm{~m})$.

\section{High Performance Liquid Chromatography (HPLC)}

Preparative and analytical separations were performed on an HPLC-System from KNAUER (Smartline Pump 100, Dynamic Mixing Chamber, Injection- and Control-Valve, Smartline UV Detector 2500). Separation normal phase column $(250 \times 10 \mathrm{~mm})$ from MACHEREY-NAGEL (MN) was used. Organic solvents of HPLC grade were employed. All samples were filtered through Polytetrafluoroethylene Filter from ROTH (Ø $25 \mathrm{~mm}, 0.2 \mu \mathrm{m})$ or VWR (Ø $13 \mathrm{~mm}, 0.2 \mu \mathrm{m})$ prior to separation.

\section{Nucle ar Magnetic Resonance Spe ctros copy (NMR)}

Nuclear magnetic resonance (NMR) spectroscopy was performed at 300, 400, 500 or 600 $\mathrm{MHz}\left({ }^{1} \mathrm{H}\right.$ NMR), 75, 100 or $125 \mathrm{MHz}\left({ }^{13} \mathrm{C} \mathrm{NMR}, \mathrm{APT}\right)$ and $282 \mathrm{MHz}\left({ }^{19} \mathrm{~F} \mathrm{NMR}\right)$ on BRUKER $A M$ 250, VARIAN Unity-300 and Inova 500 instruments. Chemical shifts are reported as $\delta$-values in ppm relative to the residual proton peak of the deuterated solvent or its carbon atom, respectively, or the standard trimethylsilyl (TMS) peak. For characterization of the observed resonance multiplicities the following abbreviations were applied: $s$ (singlet), $d$ (doublet), $t$ (triplet), $q$ (quartet), $m$ (multiplet), $d d$ (doublet of doublet), $d t$ (doublet of triplet), or analogue representations. The coupling constants $J$ are reported in $\mathrm{Hertz}(\mathrm{Hz})$.

\section{Infrared Spectroscopy (IR)}

Infrared spectra were recorded on a BRUKER Alpha-P ATR-spectrometer. Liquid probes have been measured as films between the plates of $\mathrm{NaCl}$ and solid probes neat applying Attenuated Total Reflection (ATR) technique which enables the samples to be examined 
directly. Analysis of the spectral data has been done by using the OPUS 3.1 software from BRUKER, respectively OPUS 6. Absorption ( $\tilde{v})$ is given in wave numbers $\left(\mathrm{cm}^{-1}\right)$. Spectra were recorded in the range of 4000 to $400 \mathrm{~cm}^{-1}$.

\section{Mass Spectrometry (MS)}

MS (EI) and HR-MS (EI) were measured on a Time-of-Flight mass spectrometer AccuTOF from JOEL. ESI-mass spectra were recorded on an Ion-Trap mass spectrometer $L C Q$ from FINNIGAN or on a Time-of-Flight mass spectrometer microTOF from BRUKER. ESI-HR-MS spectra were recorded on a BRUKER APEX IV or a BRUKER DALTONIC \{7T, Fourier Transform Ion Cyclotron Resonance (FTICR) \} mass spectrometer. The ratios of mass to charge $(\mathrm{m} / \mathrm{z})$ are indicated, intensities relative to the base peak $(\mathrm{I}=100)$ are written in parentheses.

\section{Reagents}

Chemicals obtained from commercial sources with purity above $95 \%$ were used without further purification.

\subsection{Synthes is of the Starting Materials}

The follow ing starting materials were synthesized according to previously described methods: ketimines $\mathbf{7 3} \mathbf{a}-\mathbf{7 3} \mathbf{i} ;{ }^{129}$ alkynes $\mathbf{1 b}-\mathbf{1 d}, \mathbf{1 d}-\mathbf{1 g}, \mathbf{1} \mathbf{h}-\mathbf{1 k}, \mathbf{1} \mathbf{i}$; amidines $\mathbf{1 1 7} \mathbf{a}-\mathbf{1 1 7} \mathbf{r} ;{ }^{130}$ aryl amides 2a-2z, $\left[\mathrm{D}_{5}\right]-\mathbf{- 2 a} ;{ }^{131}$ acetanilides $121 \mathbf{a}-121 \mathbf{k} ;{ }^{132} \alpha, \beta$-unsaturated ketones $41 \mathbf{b}-\mathbf{4 1} \mathbf{c} ;{ }^{133}$ aryl tetrazoles 123a-123b; $;{ }^{110 \mathrm{c}}$ aryl chlorides $\mathbf{8 8 d}, \mathbf{8 8 f} ;{ }^{134}$ carbamates $\mathbf{8 6} ;{ }^{135}$ phenylpyridines 28a-28e, 28g-281, 146, phenyl pyrimidines 28m-28n; ${ }^{136}$ (pyrimidin-2-yl)- $1 H$-indoles $7 \mathbf{a}-\mathbf{7 h}$, 144; ${ }^{137} N$-cyano- $N$-phenyl- $p$-toluenesulfonamide $(\mathbf{1 2 5}, \mathrm{NCTS}) ;{ }^{138}$ 2-[D]-phenylpyridine $\left[\mathrm{D}_{1}\right]-\mathbf{2 8 a} ;{ }^{139} \mathrm{Cp}^{*} \mathrm{Co}^{\mathrm{III}} \mathrm{I}_{2}(\mathrm{CO}) \quad(\mathbf{1 1 2}) ;^{85,86}$ aryl pyrazoles $\mathbf{1 2 8 b}-\mathbf{1 2 8 i}, \mathbf{1 2 8 k}-\mathbf{1 2 8 n}, \mathbf{1 2 8} \mathbf{p}$,

\footnotetext{
129 G. Ghattas, D. Chen, F. Pan, J. Klanke mayer, Dalton Trans. 2012, 41, 9026-9028.

130 a) X. H. Wei, M. Zhao, Z. Y. Du, X. W. L, Org. Lett. 2011, 13, 4636-4639; b) Y. Wang, H. G. Wang, J. L. Peng, Q. Zhu, Org. Lett. 2011, 13, 4604-4607.

131 Q. Tang, D. Xia, X. Jin, Q. Zhang, X.-Q. Sun, C. Wang, J. Am. Chem. Soc. 2013, 135, 4628-4631.

132 S. Ueda, H. Nagasawa, J. Org. Chem. 2009, 74, 4272-4277.

133 A. Buga rin, K. D. Jones, B. T. Connell, Chem. Commun. 2010, 46, 1715-1717.

134 H. M. L. Davies, S. J. Hedley, B. R. Bohall, J. Org. Chem. 2005, 70, 10737-10742.

135 X. Sun, Y. Sun, C. Zhang, Y. Rao, Chem. Commun. 2014, 50, 1262-1264.

136 V. P. W. Böhm, T. Weskamp, C. W. K. Gstöttma yr, W. A. Herrmann, Angew. Chem. Int. Ed. 2000, 39, 1602-1604.

137 L. Ackermann, A. V. Lygin, Org. Lett. 2011, 13, 3332-3335.

138 P. Anbarasan, H. Neumann, M. Beller, Chem. Eur. J. 2011, 17, 4217-4222.

139 C. Xu, Q. Shen, Org. Lett. 2014, 16, 2046-2049.
} 
$\left[D_{5}\right]-128 a ;{ }^{140} \quad$ 128j; ${ }^{141} \quad \mathbf{1 2 8 0} ;{ }^{142} \quad$ 132a; ${ }^{143} \quad$ 132b; ${ }^{144}$ 2-[D]-1-phenyl- $1 H$-pyrazole $\left(\left[D_{1}\right]-\mathbf{1 2 8 a}\right){ }^{141,139}$ isocyanates 129b-129c, 129e-129f and acyl azides $131 .{ }^{145}$

\subsection{General Procedures}

General procedure A for the ruthenium(II)-catalyzed oxidative alkyne annulation with ketimines

A suspension of ketimine $73(0.50 \mathrm{mmol})$, alkyne 1 (1.00 mmol), $\left[\mathrm{RuCl}_{2}(p \text {-cymene })\right]_{2}(15.3$ $\mathrm{mg}, 5.0 \mathrm{~mol} \%), \operatorname{AgSbF}_{6}(34.4 \mathrm{mg}, 20.0 \mathrm{~mol} \%)$, and $\mathrm{Cu}(\mathrm{OAc})_{2} \cdot \mathrm{H}_{2} \mathrm{O}(99.5 \mathrm{mg}, 0.50 \mathrm{mmol})$ in DCE $(2.0 \mathrm{~mL})$ was stirred at $100 \mathcal{C}$ for $20 \mathrm{~h}$ under an atmosphere of air. After cooling down to ambient temperature, the reaction mixture was extracted with EtOAc $(3 \times 20 \mathrm{~mL})$, washed with brine $(20 \mathrm{~mL})$ and dried over $\mathrm{Na}_{2} \mathrm{SO}_{4}$. The solvent was evaporated under reduced pressure, and the residue was purified by column chromatography ( $n$-hexane/EtOAc/Et ${ }_{3} \mathrm{~N}$ ) to afford product 115.

\section{Ge ne ral procedure B for Multicatalytic Synthes is of Dihydroisoquinolines}

A suspension of ketimine 73 (0.50 mmol), alkyne 1 (1.00 mmol), $\left[\operatorname{RuCl}_{2}(p \text {-cymene })\right]_{2}(15.3$ mg, $5.0 \mathrm{~mol} \%), \operatorname{AgSbF}_{6}(34.4 \mathrm{mg}, 20.0 \mathrm{~mol} \%)$, and $\mathrm{Cu}(\mathrm{OAc})_{2} \cdot \mathrm{H}_{2} \mathrm{O}(99.5 \mathrm{mg}, 0.50 \mathrm{mmol})$ in DCE $(2.0 \mathrm{~mL})$ was stirred at $100 \mathrm{C}$ for $20 \mathrm{~h}$ under an atmosphere of air. After cooling to ambient temperature, the reaction mixture was filtrated, and $\mathrm{Pd} / \mathrm{C}(10 \% \mathrm{w} / \mathrm{w})$ was added. The reaction mixture was stirred at ambient temperature for $24 \mathrm{~h}$ under an atmosphere of $\mathrm{H}_{2}$. The reaction mixture was extracted with EtOAc $(3 \times 20 \mathrm{~mL})$, washed with brine $(20 \mathrm{~mL})$ and dried over $\mathrm{Na}_{2} \mathrm{SO}_{4}$. The solvent was evaporated under reduced pressure, and the residue was purified by column chromatography ( $n$-hexane/EtOAc) to afford product 116.

General procedure $C$ for the ruthenium(II)-catalyzed oxidative alkyne annulation with amidines

A suspension of amidine $117(0.50 \mathrm{mmol})$, alkyne 1 (1.00 mmol), [RuCl$l_{2}(p$-cymene $\left.)\right]_{2}(15.3$ $\mathrm{mg}, 5.0 \mathrm{~mol} \%), \mathrm{KPF}_{6}(27.6 \mathrm{mg}, 30 \mathrm{~mol} \%)$ and $\mathrm{Cu}(\mathrm{OAc})_{2} \cdot \mathrm{H}_{2} \mathrm{O}(199 \mathrm{mg}, 1.00 \mathrm{mmol})$ in DME $(2.0 \mathrm{~mL})$ was stirred at $120{ }^{\circ} \mathrm{C}$ for $22 \mathrm{~h}$ under an atmosphere of $\mathrm{N}_{2}$. At ambient temperature,

\footnotetext{
140 A. Correa, C. Bolm, Adv. Synth. Catal. 2007, 349, 2673-2676.

141 F. Diness, D. P. Fai rlie, Angew. Chem. Int. Ed. 2012, 51, 8102-8106.

142 W.-B. Wu, J.-M. Huang, Org. Lett. 2012, 14, 5832-5835.

143 J. Ma o, Q. Hua, J. Guo, D. Shi, S. Ji, Synlett 2008, 13, 2011-2016.

144 Q. Liao, Y. Wang, L. Zhang, C. X, J. Org. Chem. 2009, 74, 6371-6373.

145 K. Shi, J. Ryu, S. Chang, Org. Lett. 2014, 16, 2022-2025.
} 
$\mathrm{H}_{2} \mathrm{O}(20 \mathrm{~mL})$ was added, and the reaction mixture was extracted with EtOAc $(3 \times 20 \mathrm{~mL})$. The combined organic layers were washed with brine $(20 \mathrm{~mL})$ and dried over $\mathrm{Na}_{2} \mathrm{SO}_{4}$. The solvent was evaporated in vacuo, and the residue was purified by column chromatography on silica gel ( $n$-hexane/EtOAc) to yield products 118.

Ge ne ral procedure D for the ruthe nium(II)-catalyzed oxidative alkenylation of amidines A suspension of amidine $117(0.50 \mathrm{mmol})$, alkene $20(0.75 \mathrm{mmol}),\left[\mathrm{RuCl}_{2}(p \text {-cymene })\right]_{2}$ (7.7-15.3 mg, 2.5-5.0 mol \%), AgOAc (41.5 mg, $50 \mathrm{~mol} \%)$ and $\mathrm{Cu}(\mathrm{OAc})_{2} \cdot \mathrm{H}_{2} \mathrm{O}(199 \mathrm{mg}$, $1.00 \mathrm{mmol})$ in DCE $(2.0 \mathrm{~mL})$ was stirred at $100-120{ }^{\circ} \mathrm{C}$ for $22 \mathrm{~h}$ under an atmosphere of $\mathrm{N}_{2}$. At ambient temperature, the reaction mixture was extracted with EtOAc $(3 \times 20 \mathrm{~mL})$, washed with brine $(20 \mathrm{~mL})$ and dried over $\mathrm{Na}_{2} \mathrm{SO}_{4}$. The solvent was evaporated in vacuo, and the residue was purified by column chromatography on silica gel ( $n$-hexane/EtOAc) to yield products 119.

General procedure $\mathrm{E}$ for the ruthenium(II)-catalyzed $\mathrm{C}-\mathrm{H}$ hydroarylation with aromatic amides

A suspension of aryl amides 2 or acetanilides $121(0.50 \mathrm{mmol}), \alpha, \beta$-unsaturated ketones 41 (1.00 mmol), $\left[\mathrm{RuCl}_{2}(p \text {-cymene) }]_{2}\right.$ (15.3 mg, $\left.5.0 \mathrm{~mol} \%\right), \mathrm{MesCO}_{2} \mathrm{~K}(30.3 \mathrm{mg}, 30 \mathrm{~mol} \%)$ and $\mathrm{MesCO}_{2} \mathrm{H}\left(82 \mathrm{mg}, 1.0\right.$ equiv) in degassed $\mathrm{H}_{2} \mathrm{O}(2.0 \mathrm{~mL})$ was stirred at $120{ }^{\circ} \mathrm{C}$ for $20 \mathrm{~h}$ under an atmosphere of $\mathrm{N}_{2}$. At ambient temperature, aq. Sat. $\mathrm{NaCl}$ solution $(15 \mathrm{~mL})$ was added, the reaction mixture was extracted with EtOAc $(3 \times 20 \mathrm{~mL})$ and the combined organic phases were dried over $\mathrm{Na}_{2} \mathrm{SO}_{4}$. Evaporation of the solvents in vacuo and purification of the residue by column chromatography on silica gel ( $n$-hexane/EtOAc) yielded products $\mathbf{1 2 0}$.

General procedure $F$ for the ruthenium(II)-catalyzed oxidative annulation by ace tanilides

A suspension of respective acetanilide $121(0.50 \mathrm{mmol}), \alpha, \beta$-unsaturated ketone 41 (1.00 mmol), $\left[\mathrm{RuCl}_{2}(p \text {-cymene) }]_{2}(7.7 \mathrm{mg}, 2.5 \mathrm{~mol} \%), \mathrm{AgSbF}_{6}(17.2 \mathrm{mg}, 20 \mathrm{~mol} \%)\right.$ and $\mathrm{Cu}(\mathrm{OAc})_{2}$ (190.0 mg, 2.1 equiv) in 1,4-dioxane $(2,0 \mathrm{~mL})$ was stirred at $140{ }^{\circ} \mathrm{C}$ for $20 \mathrm{~h}$ under an atmosphere of $\mathrm{N}_{2}$. At ambient temperature, $\mathrm{H}_{2} \mathrm{O}(15 \mathrm{~mL})$ was added. The reaction mixture was extracted with EtOAc $(3 \times 20 \mathrm{~mL})$. The combined organic phases were washed with brine $(20 \mathrm{~mL})$ and dried over $\mathrm{Na}_{2} \mathrm{SO}_{4}$. Evaporation of the solvents in vacuo and purification of the residue by column chromatography on silica gel ( $n$-hexane/EtOAc) yielded products 
122.

Gene ral procedure $\mathbf{G}$ for the cobalt-catalyze $\mathrm{d} \mathbf{C}-\mathrm{H}$ bond arylation by aromatic amides A suspension of $\mathrm{Co}(\mathrm{acac})_{2}(6.5 \mathrm{mg}, 5.0 \mathrm{~mol} \%), \mathrm{ICyHCl}(6.8 \mathrm{mg}, 5.0 \mathrm{~mol} \%)$, aryl amide 2 $(0.50 \mathrm{mmol})$ and DMPU $(1.0 \mathrm{~mL})$ was stirred for $5 \mathrm{~min}$ at $0{ }^{\circ} \mathrm{C}$. To this mixture, a 2-Me-THF solution of $\mathrm{CyMgCl}$ (1.0 M solution in 2-Me-THF, $1.5 \mathrm{~mL}, 3.0$ equiv) was added dropwise at the same temperature, followed by addition of aryl chloride $\mathbf{8 8}$ (1.2 equiv). Then the mixture was stirred at $60{ }^{\circ} \mathrm{C}$ for $16 \mathrm{~h}$ (or for $30 \mathrm{~min}$ in the indicated cases) under an atmosphere of argon. At ambient temperature, aq. sat. $\mathrm{NH}_{4} \mathrm{Cl}$ solution $(2.0 \mathrm{~mL})$ and $\mathrm{H}_{2} \mathrm{O}(15 \mathrm{~mL})$ were added. The reaction mixture was extracted with $\operatorname{MTBE}(3 \times 20 \mathrm{~mL})$, the combined organic phases were washed with brine $(20 \mathrm{~mL})$ and dried over $\mathrm{Na}_{2} \mathrm{SO}_{4}$. Evaporation of the solvents in vacuo and purification of the residue by column chromatography on silica gel (n-hexane/EtOAc) yielded products 139.

\section{Ge ne ral procedure $\mathrm{H}$ for the cobalt-catalyze $\mathrm{C}-\mathrm{C}$ bond arylation in aryl tetrazoles}

A suspension of $\mathrm{Co}(\mathrm{acac})_{2}(12.9 \mathrm{mg}, 10.0 \mathrm{~mol} \%)$, IMesHCl (34.0 mg, $\left.20.0 \mathrm{~mol} \%\right)$, aryl tetrazole 123 ( $0.50 \mathrm{mmol})$, aryl carbamate 86 (1.2 equiv) and DMPU (1.0 mL) was stirred for $5 \mathrm{~min}$ at $0{ }^{\circ} \mathrm{C}$. To this mixture, a 2-Me-THF solution of $\mathrm{CyMgCl}(1.0 \mathrm{M}$ solution in 2-Me-THF, $0.8 \mathrm{~mL}, 1.6$ equiv) was added dropwise at the same temperature. Then the mixture was stirred at $60{ }^{\circ} \mathrm{C}$ for $16 \mathrm{~h}$ under an atmosphere of argon. At ambient temperature, aq. Sat. $\mathrm{NH}_{4} \mathrm{Cl}$ solution $(2.0 \mathrm{~mL})$ and $\mathrm{H}_{2} \mathrm{O}(15 \mathrm{~mL})$ were added. The reaction mixture was extracted with MTBE $(3 \times 20 \mathrm{~mL})$, the combined organic phases were washed with brine $(20$ $\mathrm{mL}$ ) and dried over $\mathrm{Na}_{2} \mathrm{SO}_{4}$. Evaporation of the solvents in vacuo and purification of the residue by column chromatography on silica gel ( $n$-hexane/EtOAc) yielded products $\mathbf{1 2 4}$.

General procedure I for the cobalt-catalyzed $\mathrm{C}-\mathrm{H}$ bond cyanation by (he tero)are nes A suspension of 28 or $7(0.50 \mathrm{mmol})$, NCTS (125) $(204 \mathrm{mg}, 0.75 \mathrm{mmol}),\left[\mathrm{Cp}^{*} \mathrm{CoI}_{2}(\mathrm{CO})\right]$ $(112,6.0 \mathrm{mg}, 2.5 \mathrm{~mol} \%), \mathrm{AgSbF}_{6}(8.6 \mathrm{mg}, 5.0 \mathrm{~mol} \%)$ and $\mathrm{KOAc}(2.5 \mathrm{mg}, 5.0 \mathrm{~mol} \%)$ in DCE $(2.0 \mathrm{~mL})$ was stirred at $120{ }^{\circ} \mathrm{C}$ for $16 \mathrm{~h}$ under an atmosphere of argon. At ambient temperature, the solvent was evaporated in vacuo, and the residue was purified by column chromatography on silica gel ( $n$-hexane/EtOAc/ $\mathrm{CH}_{2} \mathrm{Cl}_{2} / \mathrm{Et}_{3} \mathrm{~N}$ ) to yield products $\mathbf{1 2 6}$ or 127.

Ge ne ral procedure $\mathbf{J}$ for the cobalt-catalyze $\mathbf{C}-\mathbf{H}$ bond carbonylation by isocyanates

A suspension of $128(0.50 \mathrm{mmol})$, aryl isocyanate $129(1.00 \mathrm{mmol}),\left[\mathrm{Cp} * \mathrm{CoI}_{2}(\mathrm{CO})\right](\mathbf{1 1 2}$, 
$12.0 \mathrm{mg}, 5.0 \mathrm{~mol} \%), \mathrm{AgSbF}_{6}(17.2 \mathrm{mg}, 10.0 \mathrm{~mol} \%$ ) and AgOPiv (10.4 mg, $10.0 \mathrm{~mol} \%)$ in DCE $(2.0 \mathrm{~mL})$ was stirred at $70{ }^{\circ} \mathrm{C}$ for $16 \mathrm{~h}$ under an atmosphere of argon. At ambient temperature, the solvent was evaporated in vacuo, and the residue was purified by column chromatography on silica gel ( $n$-hexane/ $\mathrm{CH}_{2} \mathrm{Cl}_{2} / \mathrm{EtOAc} / \mathrm{Et}_{3} \mathrm{~N}$ ) to yield products $\mathbf{1 3 0}$.

Ge ne ral procedure $\mathrm{K}$ for the cobalt-catalyze $\mathrm{d}-\mathrm{C}$ bond carbonylation by acyl azides A suspension of 128 (0.50 mmol), acyl azide 131 (1.0 mmol), $\mathrm{Cp}^{*} \mathrm{CoI}_{2}(\mathrm{CO})(\mathbf{1 1 2}, 12.0 \mathrm{mg}$, $5.0 \mathrm{~mol} \%), \operatorname{AgNTf}_{2}(19.9 \mathrm{mg}, 10.0 \mathrm{~mol} \%)$ and AgOPiv $(10.4 \mathrm{mg}, 10.0 \mathrm{~mol} \%)$ in DCE $(2.0$ $\mathrm{mL}$ ) was stirred at $80{ }^{\circ} \mathrm{C}$ for $16 \mathrm{~h}$ under an atmosphere of argon. At ambient temperature, the solvent was evaporated in vacuo, and the residue was purified by column chromatography on silica gel ( $n$-hexane/ $\left.\mathrm{CH}_{2} \mathrm{Cl}_{2} / \mathrm{EtOAc} / \mathrm{Et}_{3} \mathrm{~N}\right)$ to yield products $\mathbf{1 3 0}$.

\subsection{Analytical Data}

\subsubsection{Analytical Data for the Product of R uthenium(II)-Catalyzed}

Oxidative Alkyne Annulation with Ketimines

\section{2-(4-Methoxyphenyl)-6-me thyl-1-methyle ne-3,4-diphe nyl-1,2-dihydroisoquinoline} (115aa)<smiles>C=C1c2ccc(C)cc2C(c2ccccc2)=C(c2ccccc2)N1c1ccc(OC)cc1</smiles>

The general procedure $\mathbf{A}$ was followed using 73a $(119.5 \mathrm{mg}, 0.50 \mathrm{mmol})$ and diphenylacetylene (1a) $(178.0 \mathrm{mg}, 1.00 \mathrm{mmol})$. Purification by column chromatography ( $n$-hexane/EtOAc/Et 3 N: 2:1:0.05) yielded 115aa (147 mg, 71\%) as a yellow solid. M. p.: $190-192^{\circ} \mathrm{C}$.

${ }^{1}$ H-NMR $\left(300 \mathrm{MHz}, \mathrm{DMSO}-d_{6}\right): \delta=7.66(\mathrm{~d}, J=8.2 \mathrm{~Hz}, 1 \mathrm{H}), 7.14(\mathrm{~m}, 2 \mathrm{H}), 7.01-7.04(\mathrm{~m}$, $6 \mathrm{H}), 6.84-6.94(\mathrm{~m}, 5 \mathrm{H}), 6.76(\mathrm{~d}, J=8.8 \mathrm{~Hz}, 2 \mathrm{H}), 6.41(\mathrm{~s}, 1 \mathrm{H}), 4.46(\mathrm{~s}, 1 \mathrm{H}), 3.63(\mathrm{~s}, 3 \mathrm{H}), 3.07$ (s, 1H), $2.13(\mathrm{~s}, 3 \mathrm{H})$.

${ }^{13}$ C-NMR (75 MHz, DMSO- $\left.d_{6}\right): \delta=157.5\left(\mathrm{C}_{\mathrm{q}}\right), 147.2\left(\mathrm{C}_{\mathrm{q}}\right), 142.0\left(\mathrm{C}_{\mathrm{q}}\right), 138.0\left(\mathrm{C}_{\mathrm{q}}\right), 137.4\left(\mathrm{C}_{\mathrm{q}}\right)$, $135.9\left(\mathrm{C}_{\mathrm{q}}\right), 134.8\left(\mathrm{C}_{\mathrm{q}}\right), 133.6\left(\mathrm{C}_{\mathrm{q}}\right), 131.8(\mathrm{CH}), 131.6(\mathrm{CH}), 130.7(\mathrm{CH}), 127.7(\mathrm{CH}), 127.1$ $(\mathrm{CH}), 126.7(\mathrm{CH}), 126.5(\mathrm{CH}), 125.9(\mathrm{CH}), 124.7\left(\mathrm{C}_{\mathrm{q}}\right), 124.0(\mathrm{CH}), 123.8(\mathrm{CH}), 114.3(\mathrm{CH})$, $112.1\left(\mathrm{C}_{\mathrm{q}}\right), 79.2\left(\mathrm{CH}_{2}\right), 55.0\left(\mathrm{CH}_{3}\right), 20.9\left(\mathrm{CH}_{3}\right)$. 
IR (ATR): 3024, 1654, 1507, 1245, 1028, $697 \mathrm{~cm}^{-1}$.

MS (EI) $m / z$ (relative intensity) 415 (55) $\left[\mathrm{M}^{+}\right], 414$ (100), 400 (45), 383 (15), 370 (10), 294 (10).

HR-MS (ESI) $m / z$ calcd for $\mathrm{C}_{30} \mathrm{H}_{25} \mathrm{NO}\left[\mathrm{M}+\mathrm{H}^{+}\right]$416.2009, found 416.2014.

2-(4-Methoxyphenyl)-1-me thyle ne-3,4-diphe nyl-1,2-dihydroisoquinoline (115ba)<smiles>C=C1c2ccccc2C(c2ccccc2)=C(c2ccccc2)N1c1ccc(OC)cc1</smiles>

The general procedure $\mathbf{A}$ was followed using $\mathbf{7 3 b}(112.5 \mathrm{mg}, 0.50 \mathrm{mmol})$ and diphenylacetylene (1a) (178 mg, $1.00 \mathrm{mmol})$. Purification by column chromatography ( $n$-hexane/EtOAc/Et $3 \mathrm{~N}:$ 2:1:0.02) yielded $115 \mathrm{ba}(122 \mathrm{mg}, 61 \%$ ) as an off white solid. M. p.: $189-190^{\circ} \mathrm{C}$.

${ }^{1}$ H-NMR (300 MHz, DMSO-d $\left.d_{6}\right): \delta=7.76(\mathrm{~m}, 1 \mathrm{H}), 7.20-6.85(\mathrm{~m}, 14 \mathrm{H}), 6.77(\mathrm{~d}, J=8.7 \mathrm{~Hz}$, 2H), $6.61(\mathrm{~m}, 1 \mathrm{H}), 4.52(\mathrm{~s}, 1 \mathrm{H}), 3.63(\mathrm{~s}, 3 \mathrm{H}), 3.13(\mathrm{~s}, 1 \mathrm{H})$.

${ }^{13}$ C-NMR (75 MHz, DMSO-d $\left.d_{6}\right): \delta=157.3\left(\mathrm{C}_{\mathrm{q}}\right), 147.0\left(\mathrm{C}_{\mathrm{q}}\right), 141.8\left(\mathrm{C}_{\mathrm{q}}\right), 137.2\left(\mathrm{C}_{\mathrm{q}}\right), 135.5\left(\mathrm{C}_{\mathrm{q}}\right)$, $134.6\left(\mathrm{C}_{\mathrm{q}}\right), 133.4\left(\mathrm{C}_{\mathrm{q}}\right), 131.6(\mathrm{CH}), 131.4(\mathrm{CH}), 130.5(\mathrm{CH}), 128.7(\mathrm{CH}), 127.6(\mathrm{CH}), 127.0$ $\left(\mathrm{C}_{\mathrm{q}}\right), 126.6(\mathrm{CH}), 126.4(\mathrm{CH}), 125.9(\mathrm{CH}), 125.8(\mathrm{CH}), 123.8(\mathrm{CH}), 123.6(\mathrm{CH}), 114.3(\mathrm{CH})$, $112.1\left(\mathrm{C}_{\mathrm{q}}\right), 80.1\left(\mathrm{CH}_{2}\right), 54.9\left(\mathrm{CH}_{3}\right)$.

IR (ATR): 2993, 1621, 1507, 1244, 758, $699 \mathrm{~cm}^{-1}$.

MS (EI) $m / z$ (relative intensity) 401 (50) $\left[\mathrm{M}^{+}\right], 400$ (100), 388 (10), 356 (5), 209 (10).

HR-MS (EI) $m / z$ calcd for $\mathrm{C}_{29} \mathrm{H}_{22} \mathrm{NO}\left[\mathrm{M}-\mathrm{H}^{+}\right]$400.1701, found 400.1699 .

\section{2-(3,5-Dime thylphe nyl)-6-methyl-1-me thyle ne-3,4-diphe nyl-1,2-dihydro is oquinoline} (115da)<smiles>C=C1c2ccc(C)cc2C(c2ccccc2)=C(c2ccccc2)N1c1cc(C)cc(C)c1</smiles> 
The general procedure $\mathbf{A}$ was followed using 73d (118.5 $\mathrm{mg}, 0.50 \mathrm{mmol})$ and diphenylacetylene (1a) (178 mg, $1.00 \mathrm{mmol}$ ). Purification by column chromatography $(n$-hexane/EtOAc/Et $3 \mathrm{~N}: 5: 1: 0.01 \rightarrow 5: 1: 0.02)$ yielded $115 \mathrm{da}(125 \mathrm{mg}, 62 \%)$ as an off white solid. M. p.: $194-196{ }^{\circ} \mathrm{C}$.

${ }^{1}$ H-NMR $\left(300 \mathrm{MHz}, \mathrm{DMSO}-d_{6}\right): \delta=7.65(\mathrm{~d}, J=8.3 \mathrm{~Hz}, 1 \mathrm{H}), 7.17-7.00(\mathrm{~m}, 6 \mathrm{H}), 6.94-6.83$ (m, 5H), 6.72 (br s, 3H), 6.41 (s, 1H), 4,45 (s, 1H), 3.12 (s, 1H), 2.14 (s, 3H), 2.10 (s, 6H). ${ }^{13}$ C-NMR $\left(75 \mathrm{MHz}\right.$, DMSO- $\left.d_{6}\right): \delta=146.6\left(\mathrm{C}_{\mathrm{q}}\right), 141.8\left(\mathrm{C}_{\mathrm{q}}\right), 141.6\left(\mathrm{C}_{\mathrm{q}}\right), 138.0\left(\mathrm{C}_{\mathrm{q}}\right), 137.8\left(\mathrm{C}_{\mathrm{q}}\right)$, $137.4\left(\mathrm{C}_{\mathrm{q}}\right), 135.7\left(\mathrm{C}_{\mathrm{q}}\right), 133.5\left(\mathrm{C}_{\mathrm{q}}\right), 131.7(\mathrm{CH}), 130.6(\mathrm{CH}), 128.1(\mathrm{CH}), 127.9(\mathrm{CH}), 127.6$ $(\mathrm{CH}), 127.0(\mathrm{CH}), 126.4(\mathrm{CH}), 126.3(\mathrm{CH}), 125.8(\mathrm{CH}), 124.7\left(\mathrm{C}_{\mathrm{q}}\right), 123.9(\mathrm{CH}), 123.6(\mathrm{CH})$, $112.1\left(\mathrm{C}_{\mathrm{q}}\right), 79.6\left(\mathrm{CH}_{2}\right), 20.7\left(\mathrm{CH}_{3}\right), 20.3\left(\mathrm{CH}_{3}\right)$.

IR (ATR): 3011, 1738, 1600, 1481, 1302, $698 \mathrm{~cm}^{-1}$.

MS (EI) $m / z$ (relative intensity) 413 (5) [M+], 400 (10), 291 (5).131 (5), 69 (30), 44 (100).

HR-MS (ESI) $m / z$ calcd for $\mathrm{C}_{31} \mathrm{H}_{26} \mathrm{~N}\left[\mathrm{M}-\mathrm{H}^{+}\right]$412.2060, found 412.2069.

\section{2-(4-Methoxyphenyl)-8-me thyl-1-methyle ne-3,4-diphe nyl-1,2-dihydroisoquinoline}

(115ea)<smiles>C=C1c2c(C(=O)c3ccccc3)cccc2C(c2ccccc2)=C(c2ccc(OC)cc2)N1c1ccccc1</smiles>

The general procedure $\mathbf{A}$ was followed using 73e (119.5 $\mathrm{mg}, 0.50 \mathrm{mmol})$ and diphenylacetylene (1a) (178 mg, $1.00 \mathrm{mmol})$. Purification by column chromatography ( $n$-hexane/EtOAc/Et 3 N: 3:1:0.02) yielded 115ea (94 mg, 45\%) as a yellow solid. M. p.: $186-188^{\circ} \mathrm{C}$.

${ }^{1} \mathbf{H}-\mathbf{N M R}\left(300 \mathrm{MHz}, \mathrm{DMSO}-d_{6}\right): \delta=7.08-7.18(\mathrm{~m}, 5 \mathrm{H}), 7.02(\mathrm{~d}, J=8.7 \mathrm{~Hz}, 2 \mathrm{H}), 7.00-6.97$ (m, 2H), 6.91-6.89 (m, 5H), $6.75(\mathrm{~d}, J=8.7 \mathrm{~Hz}, 2 \mathrm{H}), 6.42(\mathrm{dd}, J=7.6,1.5 \mathrm{~Hz}, 1 \mathrm{H}), 4,31$ (s, 1H), $3.92(\mathrm{~s}, 1 \mathrm{H}), 3.65(\mathrm{~s}, 3 \mathrm{H}), 2.52(\mathrm{~s}, 3 \mathrm{H})$.

${ }^{13}$ C-NMR (75 MHz, DMSO-d $\left.d_{6}\right): \delta=157.1\left(\mathrm{C}_{\mathrm{q}}\right), 146.3\left(\mathrm{C}_{\mathrm{q}}\right), 142.1\left(\mathrm{C}_{\mathrm{q}}\right), 137.9\left(\mathrm{C}_{\mathrm{q}}\right), 135.8\left(\mathrm{C}_{\mathrm{q}}\right)$, $135.7\left(\mathrm{C}_{\mathrm{q}}\right), 135.1\left(\mathrm{C}_{\mathrm{q}}\right), 133.8\left(\mathrm{C}_{\mathrm{q}}\right), 131.7(\mathrm{CH}), 131.1(\mathrm{CH}), 130.3(\mathrm{CH}), 129.2(\mathrm{CH}), 127.6$ $(\mathrm{CH}), 127.3(\mathrm{CH}), 126.8\left(\mathrm{C}_{\mathrm{q}}\right), 126.6(\mathrm{CH}), 126.3(\mathrm{CH}), 125.7(\mathrm{CH}), 120.7(\mathrm{CH}), 114.1(\mathrm{CH})$, 
$113.7\left(\mathrm{C}_{\mathrm{q}}\right), 96.1\left(\mathrm{CH}_{2}\right), 54.9\left(\mathrm{CH}_{3}\right), 22.5\left(\mathrm{CH}_{3}\right)$.

IR (ATR): 2959, 1616, 1507, 1239, 1034, $760 \mathrm{~cm}^{-1}$.

MS (EI) $m / z$ (relative intensity) 415 (75) $\left[\mathrm{M}^{+}\right], 400$ (100), 369 (10), 294 (5), 279 (5), 121 (10).

HR-MS (EI) $m / z$ calcd for $\mathrm{C}_{30} \mathrm{H}_{25} \mathrm{NO}\left[\mathrm{M}^{+}\right]$415.1936, found 415.1927.

\section{2-(4-Methoxyphenyl)-7-me thyl-1-methyle ne-3,4-diphe nyl-1,2-dihydroisoquinoline}

(115fa)<smiles>C=C1c2cc(C)ccc2C(c2ccccc2)=C(c2ccccc2)N1c1ccc(OC)cc1</smiles>

The general procedure A was followed using $\mathbf{7 3 f}(119.5 \mathrm{mg}, 0.50 \mathrm{mmol})$ and diphenylacetylene (1a) (178 mg, $1.00 \mathrm{mmol})$. Purification by column chromatography $(n$-hexane/EtOAc/Et $3 \mathrm{~N}: 1: 1: 0.01 \rightarrow 1: 1: 0.03)$ yielded $\mathbf{1 1 5 f a}(148 \mathrm{mg}, 71 \%)$ as an off white solid. M. p.: $148-150{ }^{\circ} \mathrm{C}$.

${ }^{1}$ H-NMR $\left(300 \mathrm{MHz}\right.$, DMSO- $\left.d_{6}\right): \delta=7.60(\mathrm{~s}, 1 \mathrm{H}), 7.12(\mathrm{~d}, J=7.3 \mathrm{~Hz}, 2 \mathrm{H}), 7.07-6.84(\mathrm{~m}$, 11H), 6.77 (d, $J=8.6 \mathrm{~Hz}, 2 \mathrm{H}), 6.53(\mathrm{~d}, J=8.0 \mathrm{~Hz}, 1 \mathrm{H}), 4,50(\mathrm{~s}, 1 \mathrm{H}), 3.64$ (s, 3H), 3.11 (s, $1 \mathrm{H}), 2.31(\mathrm{~s}, 3 \mathrm{H})$.

${ }^{13}$ C-NMR (75 MHz, DMSO- $\left.d_{6}\right): \delta=157.5\left(\mathrm{C}_{\mathrm{q}}\right), 147.1\left(\mathrm{C}_{\mathrm{q}}\right), 141.0\left(\mathrm{C}_{\mathrm{q}}\right), 137.5\left(\mathrm{C}_{\mathrm{q}}\right), 135.9\left(\mathrm{C}_{\mathrm{q}}\right)$, $135.3\left(\mathrm{C}_{\mathrm{q}}\right), 134.9\left(\mathrm{C}_{\mathrm{q}}\right), 131.7(\mathrm{CH}), 131.6(\mathrm{CH}), 131.1\left(\mathrm{C}_{\mathrm{q}}\right), 130.7(\mathrm{CH}), 129.7\left(\mathrm{C}_{\mathrm{q}}\right), 127.7$ $(\mathrm{CH}), 127.0\left(\mathrm{C}_{\mathrm{q}}\right), 126.7(\mathrm{CH}), 126.4(\mathrm{CH}), 125.9(\mathrm{CH}), 124.0(\mathrm{CH}), 123.8(\mathrm{CH}), 114.4(\mathrm{CH})$, $112.1\left(\mathrm{C}_{\mathrm{q}}\right), 79.7\left(\mathrm{CH}_{2}\right), 55.0\left(\mathrm{CH}_{3}\right), 20.8\left(\mathrm{CH}_{3}\right)$.

IR (ATR): 2997, 1621, 1507, 1244, 1030, $698 \mathrm{~cm}^{-1}$.

MS (EI) $m / z$ (relative intens ity) 415 (100) $\left[\mathrm{M}^{+}\right], 414$ (85), 400 (45), 383 (10), 369 (10), 306 (5).

HR-MS (ESI) $m / z$ calcd for $\mathrm{C}_{30} \mathrm{H}_{26} \mathrm{NO}\left[\mathrm{M}+\mathrm{H}^{+}\right]$416.2009, found 416.2014 . 
<smiles>C=C1c2ccc(C)cc2C(c2ccc(OC)cc2)=C(c2ccc(OC)cc2)N1c1ccc(OC)cc1</smiles>

The general procedure $\mathbf{A}$ was followed using 73a (119.5 $\mathrm{mg}, 0.50 \mathrm{mmol})$ and 1,2-bis(4-methoxyphenyl)acetylene (1b) (238 mg, $1.00 \mathrm{mmol}$ ). Purification by column chromatography ( $n$-hexane/EtOAc/Et $\left.{ }_{3} \mathrm{~N}: 2: 1: 0 \rightarrow 2: 1: 0.05\right)$ yielded 115ab (142 $\mathrm{mg}, 60 \%$ ) as a yellow solid. M. p.: $125-126^{\circ} \mathrm{C}$.

${ }^{1}$ H-NMR (300 MHz, DMSO- $\left.d_{6}\right): \delta=7.63(\mathrm{~d}, J=8.5 \mathrm{~Hz}, 1 \mathrm{H}), 7.01(\mathrm{~d}, J=8.9 \mathrm{~Hz}, 3 \mathrm{H}), 6.93$ $(\mathrm{d}, J=8.9 \mathrm{~Hz}, 2 \mathrm{H}), 6.84(\mathrm{~d}, J=8.9 \mathrm{~Hz}, 2 \mathrm{H}), 6.78(\mathrm{~d}, J=8.9 \mathrm{~Hz}, 2 \mathrm{H}), 6.72(\mathrm{~d}, J=8.9 \mathrm{~Hz}, 2 \mathrm{H})$, $6.44(\mathrm{~d}, J=8.9 \mathrm{~Hz}, 2 \mathrm{H}), 6.42(\mathrm{~s}, 1 \mathrm{H}), 4,42(\mathrm{~s}, 1 \mathrm{H}), 3.67$ (s, 3H), 3.66 (s, 3H), 3.52 (s, 3H), $3.01(\mathrm{~s}, 1 \mathrm{H}), 2.14(\mathrm{~s}, 3 \mathrm{H})$.

${ }^{13}$ C-NMR $\left(75 \mathrm{MHz}\right.$, DMSO-d $\left.d_{6}\right): \delta=157.4\left(\mathrm{C}_{\mathrm{q}}\right), 157.1\left(\mathrm{C}_{\mathrm{q}}\right), 157.0\left(\mathrm{C}_{\mathrm{q}}\right), 147.3\left(\mathrm{C}_{\mathrm{q}}\right), 141.9\left(\mathrm{C}_{\mathrm{q}}\right)$, $137.9\left(\mathrm{C}_{\mathrm{q}}\right), 135.1\left(\mathrm{C}_{\mathrm{q}}\right), 134.1\left(\mathrm{C}_{\mathrm{q}}\right), 132.7(\mathrm{CH}), 131.8(\mathrm{CH}), 131.5(\mathrm{CH}), 129.7\left(\mathrm{C}_{\mathrm{q}}\right), 128.5$ $\left(\mathrm{C}_{\mathrm{q}}\right), 126.9(\mathrm{CH}), 124.7\left(\mathrm{C}_{\mathrm{q}}\right), 124.0(\mathrm{CH}), 123.7(\mathrm{CH}), 114.4(\mathrm{CH}), 113.2(\mathrm{CH}), 112.2(\mathrm{CH})$, $111.8\left(\mathrm{C}_{\mathrm{q}}\right), 79.0\left(\mathrm{CH}_{2}\right), 55.0\left(\mathrm{CH}_{3}\right), 54.7\left(\mathrm{CH}_{3}\right), 54.5\left(\mathrm{CH}_{3}\right), 20.9\left(\mathrm{CH}_{3}\right)$.

IR (ATR): 2955, 1737, 1605, 1506, 1235, $807 \mathrm{~cm}^{-1}$.

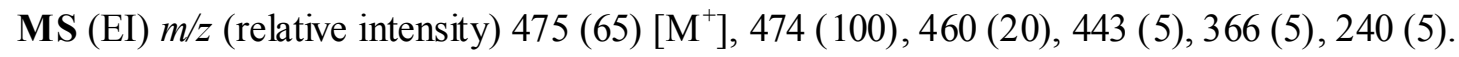

HR-MS (ESI) $m / z$ calcd for $\mathrm{C}_{32} \mathrm{H}_{30} \mathrm{NO}_{3}\left[\mathrm{M}+\mathrm{H}^{+}\right]$476.2220, found 476.2226.

\section{2-(4-Methoxyphenyl)-6-me thyl-1-methyle ne-3,4-di-p-tolyl-1,2-dihydrois oquinoline} (115ac)<smiles>C=C1c2ccc(C)cc2C(c2ccc(C)cc2)=C(c2ccc(C)cc2)N1c1ccc(OC)cc1</smiles>

The general procedure $\mathbf{A}$ was followed using 73a (119.5 $\mathrm{mg}, 0.50 \mathrm{mmol})$ and 1,2-bis(4-methylphenyl)ethylne (1c) (206 mg, $1.00 \mathrm{mmol})$. Purification by column 
chromatography ( $n$-hexane/EtOAc/Et $\left.{ }_{3} \mathrm{~N}: 1: 1: 0 \rightarrow 1: 1: 0.03\right)$ yielded 115ac (134 mg, 61\%) as a yellow solid. M. p.: $163-165^{\circ} \mathrm{C}$.

${ }^{1} \mathbf{H}-\mathbf{N M R}\left(300 \mathrm{MHz}, \mathrm{DMSO}-d_{6}\right): \delta=7.63(\mathrm{~d}, J=8.3 \mathrm{~Hz}, 1 \mathrm{H}), 7.01(\mathrm{~d}, J=8.8 \mathrm{~Hz}, 2 \mathrm{H}), 6.96$ (m, 1H), $6.95(\mathrm{~d}, J=8.1 \mathrm{~Hz}, 2 \mathrm{H}), 6.90(\mathrm{~d}, J=8.1 \mathrm{~Hz}, 2 \mathrm{H}), 6.81$ (d, $J=7.8 \mathrm{~Hz}, 2 \mathrm{H}), 6.76(\mathrm{~d}, J$ $=8.8 \mathrm{~Hz}, 2 \mathrm{H}), 6.68(\mathrm{~d}, J=8.1 \mathrm{~Hz}, 2 \mathrm{H}), 6.41(\mathrm{~s}, 1 \mathrm{H}), 4,43(\mathrm{~s}, 1 \mathrm{H}), 3.63(\mathrm{~s}, 3 \mathrm{H}), 3.03(\mathrm{~s}, 1 \mathrm{H})$, $2.18(\mathrm{~s}, 3 \mathrm{H}), 2.12(\mathrm{~s}, \mathrm{H}), 1.99$ (s, 3H).

${ }^{13}$ C-NMR (75 MHz, DMSO- $\left.d_{6}\right): \delta=157.4\left(\mathrm{C}_{\mathrm{q}}\right), 147.3\left(\mathrm{C}_{\mathrm{q}}\right), 141.9\left(\mathrm{C}_{\mathrm{q}}\right), 137.9\left(\mathrm{C}_{\mathrm{q}}\right), 135.2\left(\mathrm{C}_{\mathrm{q}}\right)$, $135.0\left(\mathrm{C}_{\mathrm{q}}\right), 134.6\left(\mathrm{C}_{\mathrm{q}}\right), 134.5\left(\mathrm{C}_{\mathrm{q}}\right), 133.9\left(\mathrm{C}_{\mathrm{q}}\right), 133.2\left(\mathrm{C}_{\mathrm{q}}\right), 131.6(\mathrm{CH}), 131.5(\mathrm{CH}), 130.5$ $(\mathrm{CH}), 128.4(\mathrm{CH}), 127.4(\mathrm{CH}), 126.9(\mathrm{CH}), 124.7\left(\mathrm{C}_{\mathrm{q}}\right), 124.0(\mathrm{CH}), 123.7(\mathrm{CH}), 114.3(\mathrm{CH})$, $112.0\left(\mathrm{C}_{\mathrm{q}}\right), 79.1\left(\mathrm{CH}_{2}\right), 54.9\left(\mathrm{CH}_{3}\right), 20.9\left(\mathrm{CH}_{3}\right), 20.6\left(\mathrm{CH}_{3}\right), 20.5\left(\mathrm{CH}_{3}\right)$.

IR (ATR): 2956, 1606, 1506, 1239, 1033, $804 \mathrm{~cm}^{-1}$.

MS (EI) $m / z$ (relative intensity) 443 (40) [M+], 430 (100), 411 (5), 386 (5), 342 (15), 237 (60).

HR-MS (EI) $m / z$ calcd for $\mathrm{C}_{32} \mathrm{H}_{28} \mathrm{NO}\left[\mathrm{M}-\mathrm{H}^{+}\right]$442.2171, found 442.2179 .

HR-MS (EI) $m / z$ calcd for $\mathrm{C}_{30} \mathrm{H}_{22} \mathrm{~N}^{35} \mathrm{Cl}_{2} \mathrm{O}\left[\mathrm{M}-\mathrm{H}^{+}\right]$482.1078, found 482.1069 .

\section{3,4-B is (4-fluorophe nyl)-2-(4-me thoxyphe nyl)-6-methyl-1-me thyle ne-1,2-dihydroisoquino} line (115ad)<smiles>C=C1c2ccc(C)cc2C(c2ccc(F)cc2)=C(c2ccc(F)cc2)N1c1ccc(OC)cc1</smiles>

The general procedure $\mathbf{A}$ was followed using 73a $(119.5 \mathrm{mg}, 0.50 \mathrm{mmol})$ and 1,2-bis(4-fluorophenyl)ethyne (1d) $(214 \mathrm{mg}, 1.00 \mathrm{mmol})$ at $120{ }^{\circ} \mathrm{C}$. Purification by column chromatography ( $n$-hexane/EtOAc/Et $3 \mathrm{~N}: 2: 1: 0 \rightarrow 2: 1: 0.05)$ yielded 115ad (122 mg, 54\%) as an off white solid. M. p.: $196-198^{\circ} \mathrm{C}$.

${ }^{1}$ H-NMR $\left(300 \mathrm{MHz}\right.$, DMSO- $\left.d_{6}\right): \delta=7.67(\mathrm{~d}, J=8.1 \mathrm{~Hz}, 1 \mathrm{H}), 7.06-6.97(\mathrm{~m}, 9 \mathrm{H}), 6.79(\mathrm{~d}, J=$ $8.9 \mathrm{~Hz}, 2 \mathrm{H}), 6.73(\mathrm{t}, J=8.9 \mathrm{~Hz}, 2 \mathrm{H}), 6.40(\mathrm{~s}, 1 \mathrm{H}), 4,48(\mathrm{~s}, 1 \mathrm{H}), 3.65(\mathrm{~s}, 3 \mathrm{H}), 3.08(\mathrm{~s}, 1 \mathrm{H})$, $2.15(\mathrm{~s}, 3 \mathrm{H})$.

${ }^{13}$ C-NMR $\left(75 \mathrm{MHz}, \mathrm{DMSO}-d_{6}\right): \delta=160.9\left(\mathrm{C}_{\mathrm{q}}, J_{\mathrm{C}-\mathrm{F}}=243.0 \mathrm{~Hz}\right), 159.8\left(\mathrm{C}_{\mathrm{q}}, J_{\mathrm{C}-\mathrm{F}}=243.0 \mathrm{~Hz}\right)$, 
$157.6\left(\mathrm{C}_{\mathrm{q}}\right), 147.0\left(\mathrm{C}_{\mathrm{q}}\right), 141.3\left(\mathrm{C}_{\mathrm{q}}\right), 138.2\left(\mathrm{C}_{\mathrm{q}}\right), 134.7\left(\mathrm{C}_{\mathrm{q}}\right), 133.6\left(\mathrm{CH}, J_{\mathrm{C}-\mathrm{F}}=8.2 \mathrm{~Hz}\right), 133.5$ $\left(\mathrm{C}_{\mathrm{q}}\right), 132.8\left(\mathrm{CH}, J_{\mathrm{C}-\mathrm{F}}=8.2 \mathrm{~Hz}\right), 132.3\left(\mathrm{C}_{\mathrm{q}}\right), 132.3\left(\mathrm{C}_{\mathrm{q}}\right), 131.5(\mathrm{CH}), 127.3(\mathrm{CH}), 124.7\left(\mathrm{C}_{\mathrm{q}}\right)$, $123.9(\mathrm{CH}), 123.8(\mathrm{CH}), 114.7\left(\mathrm{CH}, J_{\mathrm{C}-\mathrm{F}}=21.1 \mathrm{~Hz}\right), 114.5(\mathrm{CH}), 113.7\left(\mathrm{CH}, J_{\mathrm{C}-\mathrm{F}}=21.7 \mathrm{~Hz}\right)$, $111.3\left(\mathrm{C}_{\mathrm{q}}\right), 79.4\left(\mathrm{CH}_{2}\right), 55.0\left(\mathrm{CH}_{3}\right), 20.8\left(\mathrm{CH}_{3}\right)$.

${ }^{19}$ F-NMR $\left(282 \mathrm{MHz}, \mathrm{DMSO}-d_{6}\right): \delta=-109.9,-111.4$.

IR (ATR): 2971, 1601, 1505, 1213, $828 \mathrm{~cm}^{-1}$.

MS (EI) $m / z$ (relative intensity) 451 (75) $\left[\mathrm{M}^{+}\right], 450$ (100), 436 (45), 419 (15), 406 (5), 330 (5).

HR-MS (EI) $m / z$ calcd for $\mathrm{C}_{30} \mathrm{H}_{22} \mathrm{NOF}_{2}\left[\mathrm{M}-\mathrm{H}^{+}\right] 450.1669$, found 450.1672 .

\section{2-(4-Methoxyphenyl)-6-me thyl-1-methyle ne-3,4-di-n-propyl-1,2-dihydroisoquinoline} (115ae)<smiles>C=C1c2ccc(C)cc2C(C(C)C)=C(C(C)C)N1C(=O)NC</smiles>

The general procedure A was followed using 73a (119.5 mg, $0.50 \mathrm{mmol})$ and oct-4-yne (1e) (110 mg, $1.00 \mathrm{mmol}$ ). Purification by column chromatography ( $n$-hexane/EtOAc/Et ${ }_{3} \mathrm{~N}$ : 3:1:0.01 $\rightarrow 3: 1: 0.05)$ yielded 115ae (77 $\mathrm{mg}, 44 \%$ ) as a yellow oil.

${ }^{1}$ H-NMR (300 MHz, DMSO-d $\left.d_{6}\right): \delta=7.50(\mathrm{~d}, J=8.1 \mathrm{~Hz}, 1 \mathrm{H}), 7.15(\mathrm{~d}, J=8.9 \mathrm{~Hz}, 2 \mathrm{H}), 7.08$ (d, $J=8.9 \mathrm{~Hz}, 2 \mathrm{H}), 7.04(\mathrm{~s}, 1 \mathrm{H}), 6.93(\mathrm{~d}, J=8.1 \mathrm{~Hz}, 1 \mathrm{H}), 4.24$ (s, 1H), 3.81 (s, 3H), 2.81 (s, $1 \mathrm{H}), 2.43-2.38(\mathrm{~m}, 2 \mathrm{H}), 2.31(\mathrm{~s}, 3 \mathrm{H}), 2.06-2.00(\mathrm{~m}, 2 \mathrm{H}), 1.52-1.44(\mathrm{~m}, 2 \mathrm{H}), 1.33-1.25(\mathrm{~m}$, 2H), $1.00(\mathrm{t}, J=7.8 \mathrm{~Hz}, 3 \mathrm{H}), 0.62(\mathrm{t}, J=8.0 \mathrm{~Hz}, 3 \mathrm{H})$.

${ }^{13}$ C-NMR (75 MHz, DMSO- $\left.d_{6}\right): \delta=158.3\left(\mathrm{C}_{\mathrm{q}}\right), 147.4\left(\mathrm{C}_{\mathrm{q}}\right), 140.4\left(\mathrm{C}_{\mathrm{q}}\right), 138.0\left(\mathrm{C}_{\mathrm{q}}\right), 134.7\left(\mathrm{C}_{\mathrm{q}}\right)$, $132.7\left(\mathrm{C}_{\mathrm{q}}\right), 131.0(\mathrm{CH}), 126.1(\mathrm{CH}), 124.8\left(\mathrm{C}_{\mathrm{q}}\right), 132.7(\mathrm{CH}), 121.9(\mathrm{CH}), 115.1(\mathrm{CH}), 106.1$ $\left(\mathrm{C}_{\mathrm{q}}\right), 78.1\left(\mathrm{CH}_{2}\right), 55.2\left(\mathrm{CH}_{3}\right), 31.6\left(\mathrm{CH}_{2}\right), 28.9\left(\mathrm{CH}_{2}\right), 22.8\left(\mathrm{CH}_{2}\right), 21.9\left(\mathrm{CH}_{2}\right), 21.1\left(\mathrm{CH}_{3}\right)$, $14.1\left(\mathrm{CH}_{2}\right), 14.0\left(\mathrm{CH}_{3}\right)$.

IR (ATR): 2957, 1650, 1506, 1241, 1031, $827 \mathrm{~cm}^{-1}$.

MS (EI) $m / z$ (relative intensity) 347 (55) $\left[\mathrm{M}^{+}\right], 334$ (65), 320 (100), 304 (50), 291 (45), 176 (45).

HR-MS (EI) $m / z$ calcd for $\mathrm{C}_{24} \mathrm{H}_{28} \mathrm{NO}\left[\mathrm{M}-\mathrm{H}^{+}\right] 346.2171$, found 346.2167 . 


\section{2-(4-Methoxyphenyl)-4,6-dime thyl-1-methyle ne-3-phe nyl-1,2-dihydroisoquinoline}

(115af)<smiles>C=C1c2ccc(C)cc2C([N+](=O)[O-])=C(c2ccccc2)N1C(=O)c1ccccc1</smiles>

The general procedure $\mathbf{A}$ was followed using 73a (119.5 $\mathrm{mg}, 0.50 \mathrm{mmol})$ and prop-1-yn-1-ylbenzene (1f) (116 mg, $1.00 \mathrm{mmol})$. Purification by column chromatography $(n$-hexane/EtOAc/Et 3 N : 1:2:0 $\rightarrow 1: 2: 0.03)$ yielded 115af $(125 \mathrm{mg}, 69 \%$ ) as an off white solid. M. p.: $121-123^{\circ} \mathrm{C}$.

${ }^{1}$ H-NMR (300 MHz, DMSO- $\left.d_{6}\right): \delta=7.62(\mathrm{~d}, J=8.9 \mathrm{~Hz}, 1 \mathrm{H}), 7.17-7.05(\mathrm{~m}, 7 \mathrm{H}), 6.97(\mathrm{~d}, J=$ $8.9 \mathrm{~Hz}, 2 \mathrm{H}), 6.95$ (d, J = 8.9 Hz, 2H), 4,35 (s, 1H), 3.64 (s, 3H), 2.96 (s, 1H), $2.34(\mathrm{~s}, 3 \mathrm{H})$ ), $1.66(\mathrm{~s}, 3 \mathrm{H})$.

${ }^{13}$ C-NMR $\left(75 \mathrm{MHz}, \mathrm{DMSO}-d_{6}\right): \delta=157.4\left(\mathrm{C}_{\mathrm{q}}\right), 147.1\left(\mathrm{C}_{\mathrm{q}}\right), 141.0\left(\mathrm{C}_{\mathrm{q}}\right), 138.3\left(\mathrm{C}_{\mathrm{q}}\right), 136.6\left(\mathrm{C}_{\mathrm{q}}\right)$, $135.2\left(\mathrm{C}_{\mathrm{q}}\right), 133.3\left(\mathrm{C}_{\mathrm{q}}\right), 131.7(\mathrm{CH}), 130.1(\mathrm{CH}), 127.5(\mathrm{CH}), 127.0(\mathrm{CH}), 126.9(\mathrm{CH}), 125.2$ $\left(\mathrm{C}_{\mathrm{q}}\right), 123.5(\mathrm{CH}), 122.6(\mathrm{CH}), 114.2(\mathrm{CH}), 102.5\left(\mathrm{C}_{\mathrm{q}}\right), 78.1\left(\mathrm{CH}_{2}\right), 55.0\left(\mathrm{CH}_{3}\right), 21.0\left(\mathrm{CH}_{3}\right)$, $14.4\left(\mathrm{CH}_{3}\right)$.

IR (ATR): 2920, 1609, 1506, 1298, 1242, $698 \mathrm{~cm}^{-1}$.

MS (EI) $m / z$ (relative intensity) 353 (45) $\left[\mathrm{M}^{+}\right], 352$ (100), 340 (40), 321 (10), 244 (15), 217 (15).

HR-MS (ESI) $m / z$ calcd for $\mathrm{C}_{25} \mathrm{H}_{22} \mathrm{NO}\left[\mathrm{M}-\mathrm{H}^{+}\right]$352.1701, found 352.1761 .

\section{4-Ethyl-2-(4-methoxyphe nyl)-6-me thyl-1-me thylene-3-phe nyl-1,2-dihydro is oquinoline} (115ag)<smiles>C=C1c2ccc(C)cc2C(CC)=C(c2ccccc2)N1P[N+]([O-])(F)c1ccccc1</smiles>

The general procedure $\mathbf{A}$ was followed using 73a $(119.5 \mathrm{mg}, 0.50 \mathrm{mmol})$ and but-1-yn-1-ylbenzene (1g) (130 mg, $1.00 \mathrm{mmol})$. Purification by column chromatography $(n$-hexane/EtOAc/Et $3 \mathrm{~N}:$ 1:1:0 $\rightarrow 1: 1: 0.03)$ yielded 115ag (116 mg, 63\%) as an off white solid. M. p.: $199-201^{\circ} \mathrm{C}$. 
${ }^{1}$ H-NMR (300 MHz, DMSO- $\left.d_{6}\right): \delta=7.62(\mathrm{~d}, J=8.5 \mathrm{~Hz}, 1 \mathrm{H}), 7.18-7.08(\mathrm{~m}, 6 \mathrm{H}), 7.02(\mathrm{~d}, J=$ $8.5 \mathrm{~Hz}, 1 \mathrm{H}), 6.96(\mathrm{~d}, J=9.0 \mathrm{~Hz}, 2 \mathrm{H}), 6.74(\mathrm{~d}, J=9.0 \mathrm{~Hz}, 2 \mathrm{H}), 4,33(\mathrm{~s}, 1 \mathrm{H}), 3.63(\mathrm{~s}, 3 \mathrm{H}), 2.92$ (s, 1H), 2.34 (s, 3H), 2.08 (q, $J=7.1 \mathrm{~Hz}, 2 \mathrm{H}), 0.92$ (t, $J=7.1 \mathrm{~Hz}, 3 \mathrm{H})$.

${ }^{13}$ C-NMR (75 MHz, DMSO- $\left.d_{6}\right): \delta=157.4\left(\mathrm{C}_{\mathrm{q}}\right), 147.2\left(\mathrm{C}_{\mathrm{q}}\right), 141.0\left(\mathrm{C}_{\mathrm{q}}\right), 138.2\left(\mathrm{C}_{\mathrm{q}}\right), 136.3\left(\mathrm{C}_{\mathrm{q}}\right)$, $135.1\left(\mathrm{C}_{\mathrm{q}}\right), 132.0\left(\mathrm{C}_{\mathrm{q}}\right), 131.7(\mathrm{CH}), 129.9(\mathrm{CH}), 127.5(\mathrm{CH}), 127.1(\mathrm{CH}), 126.8(\mathrm{CH}), 125.5$ $\left(\mathrm{C}_{\mathrm{q}}\right), 123.9(\mathrm{CH}), 122.4(\mathrm{CH}), 114.2(\mathrm{CH}), 108.8\left(\mathrm{C}_{\mathrm{q}}\right), 78.0\left(\mathrm{CH}_{2}\right), 54.9\left(\mathrm{CH}_{3}\right), 21.0\left(\mathrm{CH}_{3}\right)$, $20.6\left(\mathrm{CH}_{2}\right), 14.3\left(\mathrm{CH}_{3}\right)$.

IR (ATR): 2970, 1737, 1584, 1508, 1229, 1027, $736 \mathrm{~cm}^{-1}$.

MS (EI) $m / z$ (relative intensity) 367 (50) $\left[\mathrm{M}^{+}\right], 366$ (100), 352 (25), 336 (5), 320 (5), 244 (5).

HR-MS (ESI) $m / z$ calcd for $\mathrm{C}_{26} \mathrm{H}_{26} \mathrm{NO}\left[\mathrm{M}+\mathrm{H}^{+}\right]$368.2014, found 368.2010.

\section{4-n-Butyl-2,3-bis(4-methoxyphenyl)-6-me thyl-1-me thyle ne-1,2-dihydroisoquinoline} (115ah)<smiles>C=C1C(=[NH+])C(c2ccc(OC)cc2)=C(CCCC)c2cc(C)ccc21</smiles>

The general procedure A was followed using 73a $(119.5 \mathrm{mg}, 0.50 \mathrm{mmol})$ and 1-(hex-1-yn-1-yl)-4-methoxybenzene (1h) (188 mg, $1.00 \mathrm{mmol}$ ). Purification by column chromatography ( $n$-hexane/EtOAc/Et 3 N: 3:1:0.01 $\rightarrow 3: 1: 0.05)$ yielded 115ah (125 mg, 59\%) as an off white solid. M. p.: $133-133^{\circ} \mathrm{C}$.

${ }^{1}$ H-NMR (300 MHz, DMSO- $\left.d_{6}\right): \delta=7.60(\mathrm{~d}, J=8.3 \mathrm{~Hz}, 1 \mathrm{H}), 7.10(\mathrm{~s}, 1 \mathrm{H}), 6.99(\mathrm{~m}, 1 \mathrm{H})$, $6.99(\mathrm{~d}, J=8.8 \mathrm{~Hz}, 2 \mathrm{H}), 6.95(\mathrm{~d}, J=8.8 \mathrm{~Hz}, 2 \mathrm{H}), 6.76(\mathrm{~d}, J=8.8 \mathrm{~Hz}, 2 \mathrm{H}), 6.71(\mathrm{~d}, J=8.8 \mathrm{~Hz}$, 2H), 4,31 (s, 1H), 3.65 (s, 6H), $2.90(\mathrm{~s}, 1 \mathrm{H}), 2.33$ (s, 3H), 2.05 (t, $J=7.3 \mathrm{~Hz}, 2 \mathrm{H}), 1.34$ (m, 2H), $1.16(\mathrm{~m}, 2 \mathrm{H}), 0.71(\mathrm{t}, J=7.3 \mathrm{~Hz}, 3 \mathrm{H})$.

${ }^{13}$ C-NMR (75 MHz, DMSO- $\left.d_{6}\right): \delta=157.6\left(\mathrm{C}_{\mathrm{q}}\right), 157.1\left(\mathrm{C}_{\mathrm{q}}\right), 147.1\left(\mathrm{C}_{\mathrm{q}}\right), 140.9\left(\mathrm{C}_{\mathrm{q}}\right), 138.0\left(\mathrm{C}_{\mathrm{q}}\right)$, $135.3\left(\mathrm{C}_{\mathrm{q}}\right), 132.3\left(\mathrm{C}_{\mathrm{q}}\right), 131.5(\mathrm{CH}), 131.1(\mathrm{CH}), 128.5\left(\mathrm{C}_{\mathrm{q}}\right), 126.6(\mathrm{CH}), 125.3\left(\mathrm{C}_{\mathrm{q}}\right), 123.7$ $(\mathrm{CH}), 122.3(\mathrm{CH}), 114.2(\mathrm{CH}), 112.7(\mathrm{CH}), 108.0\left(\mathrm{C}_{\mathrm{q}}\right), 77.9\left(\mathrm{CH}_{2}\right), 54.9\left(\mathrm{CH}_{3}\right), 54.7\left(\mathrm{CH}_{3}\right)$, 31.6 $\left(\mathrm{CH}_{2}\right), 27.2\left(\mathrm{CH}_{2}\right), 22.1\left(\mathrm{CH}_{2}\right), 21.1\left(\mathrm{CH}_{3}\right), 13.4\left(\mathrm{CH}_{3}\right)$.

IR (ATR): 2928, 1737, 1507, 1240, 1033, $829 \mathrm{~cm}^{-1}$. 
MS (EI) $m / z$ (relative intensity) $425(65)\left[\mathrm{M}^{+}\right], 424$ (100), 412 (25), 384 (75), 274 (5), 240 (10).

HR-MS (EI) $m / z$ calcd for $\mathrm{C}_{29} \mathrm{H}_{31} \mathrm{NO}_{2}\left[\mathrm{M}^{+}\right]$425.2355, found 425.2344.

4-n-B utyl-3-(4-fluorophe nyl)-2-(4-me thoxyphe nyl)-6-methyl-1-methyle ne-1,2-dihydroiso qui-noline (115ai)<smiles>C=C1C(=NP=N)C(c2ccc(F)cc2)=C(CCCC)c2cc(C)ccc21</smiles>

The general procedure A was followed using 73a (119.5 mg, $0.50 \mathrm{mmol})$ and 1-fluoro-4-(hex-1-yn-1-yl) benzene (1i) (176 mg, $1.00 \mathrm{mmol})$ at $120{ }^{\circ} \mathrm{C}$. Purification by column chromatography ( $n$-hexane/EtOAc/ $\left.\mathrm{Et}_{3} \mathrm{~N}: 3: 1: 0 \rightarrow 3: 1: 0.05\right)$ yielded 115ai (148 mg, $72 \%$ ) as an off white solid. M. p.: $96-98^{\circ} \mathrm{C}$.

${ }^{1}$ H-NMR (300 MHz, DMSO- $\left.d_{6}\right): \delta=7.62(\mathrm{~d}, J=8.4 \mathrm{~Hz}, 1 \mathrm{H}), 7.16-7.11(\mathrm{~m}, 3 \mathrm{H}), 7.02-6.96$ (m, 5H), $6.77(\mathrm{~d}, J=8.8 \mathrm{~Hz}, 2 \mathrm{H}), 4,34(\mathrm{~s}, 1 \mathrm{H}), 3.65(\mathrm{~s}, 3 \mathrm{H}), 2.93(\mathrm{~s}, 1 \mathrm{H}), 2.34(\mathrm{~s}, 3 \mathrm{H}), 2.04$ (t, $J=7.2 \mathrm{~Hz}, 2 \mathrm{H}), 1.34(\mathrm{~m}, 2 \mathrm{H}), 1.15(\mathrm{~m}, 2 \mathrm{H}), 0.70(\mathrm{t}, J=7.2 \mathrm{~Hz}, 3 \mathrm{H})$.

${ }^{13}$ C-NMR (75 MHz, DMSO- $\left.d_{6}\right): \delta=160.6\left(\mathrm{C}_{\mathrm{q}} J_{\mathrm{C}-\mathrm{F}}=245 \mathrm{~Hz}\right), 157.3\left(\mathrm{C}_{\mathrm{q}}\right), 147.0\left(\mathrm{C}_{\mathrm{q}}\right), 140.0$ $\left(\mathrm{C}_{\mathrm{q}}\right), 138.1\left(\mathrm{C}_{\mathrm{q}}\right), 135.0\left(\mathrm{C}_{\mathrm{q}}\right), 132.6\left(\mathrm{C}_{\mathrm{q}}, J_{\mathrm{C}-\mathrm{F}}=3.5 \mathrm{~Hz}\right), 132.4\left(\mathrm{C}_{\mathrm{q}}\right), 132.3\left(\mathrm{CH}, J_{\mathrm{C}-\mathrm{F}}=8.0 \mathrm{~Hz}\right)$, $131.5(\mathrm{CH}), 126.8(\mathrm{CH}), 125.3\left(\mathrm{C}_{\mathrm{q}}\right), 123.7(\mathrm{CH}), 122.4(\mathrm{CH}), 114.3\left(\mathrm{CH}, J_{\mathrm{C}-\mathrm{F}}=21.5 \mathrm{~Hz}\right)$, $114.2(\mathrm{CH}), 108.0\left(\mathrm{C}_{\mathrm{q}}\right), 78.1\left(\mathrm{CH}_{2}\right), 55.0\left(\mathrm{CH}_{3}\right), 31.5\left(\mathrm{CH}_{2}\right), 27.1\left(\mathrm{CH}_{2}\right), 22.0\left(\mathrm{CH}_{2}\right), 20.9$ $\left(\mathrm{CH}_{3}\right), 13.4\left(\mathrm{CH}_{3}\right)$.

${ }^{19}$ F-NMR $\left(282 \mathrm{MHz}\right.$, DMSO- $\left.d_{6}\right): \delta=-114.4$.

IR (ATR): 2954, 1599, 1507, 1243, 1033, $825 \mathrm{~cm}^{-1}$.

MS (EI) $m / z$ (relative intensity) 413 (50) $\left[\mathrm{M}^{+}\right], 412$ (100), 398 (20), 372 (40), 354 (10), 262 (10).

HR-MS (ESI) $m / z$ calcd for $\mathrm{C}_{28} \mathrm{H}_{29} \mathrm{NOF}\left[\mathrm{M}+\mathrm{H}^{+}\right]$414.2233, found 414.2228.

Ethyl 4-[4-n-Butyl-2-(4-me thoxyphe nyl)-6-me thyl-1-methylene-1,2-dihydroisoquinolin3-yl]be nzoate (115aj) 
<smiles>C=C1c2ccc(C)cc2C(CCCCC)=C(c2ccc(C(=O)OCC)cc2)N1C(=O)[O-]</smiles>

The general procedure A was followed using 73a (119.5 $\mathrm{mg}, 0.50 \mathrm{mmol}$ ) and ethyl 4-(hex-1-ynyl)benzoate (1j) $(230 \mathrm{mg}, 1.00 \mathrm{mmol})$ at $120{ }^{\circ} \mathrm{C}$. Purification by column chromatography ( $n$-hexane/EtOAc/Et $3 \mathrm{~N}: 3: 1: 0 \rightarrow 3: 1: 0.05$ ) yielded 115aj (116 mg, 50\%) as an off white solid. M. p.: $151-153^{\circ} \mathrm{C}$.

${ }^{1}$ H-NMR (300 MHz, DMSO-d $\left.d_{6}\right): \delta=7.75(\mathrm{~d}, J=8.5 \mathrm{~Hz}, 2 \mathrm{H}), 7.62(\mathrm{~d}, J=8.4 \mathrm{~Hz}, 1 \mathrm{H}), 7.26$ (d, $J=8.4 \mathrm{~Hz}, 2 \mathrm{H}), 7.13(\mathrm{~s}, 1 \mathrm{H}), 7.05(\mathrm{~d}, J=8.5 \mathrm{~Hz}, 1 \mathrm{H}), 6.99(\mathrm{~d}, J=9.0 \mathrm{~Hz}, 2 \mathrm{H}), 6.75(\mathrm{~d}, J$ $=9.0 \mathrm{~Hz}, 2 \mathrm{H}), 4,36(\mathrm{~s}, 1 \mathrm{H}), 4.26(\mathrm{q}, J=7.2 \mathrm{~Hz}, 2 \mathrm{H}), 3.63(\mathrm{~s}, 3 \mathrm{H}), 2.98(\mathrm{~s}, 1 \mathrm{H}), 2.35(\mathrm{~s}, 3 \mathrm{H})$, $2.02(\mathrm{~m}, 2 \mathrm{H}), 1.34(\mathrm{~m}, 2 \mathrm{H}), 1.29(\mathrm{t}, J=7.2 \mathrm{~Hz}, 3 \mathrm{H}), 1.12(\mathrm{~m}, 2 \mathrm{H}), 0.68(\mathrm{t}, J=7.2 \mathrm{~Hz}, 3 \mathrm{H})$.

${ }^{13}$ C-NMR (75 MHz, DMSO- $\left.d_{6}\right): \delta=165.1\left(\mathrm{C}_{\mathrm{q}}\right), 157.5\left(\mathrm{C}_{\mathrm{q}}\right), 147.0\left(\mathrm{C}_{\mathrm{q}}\right), 141.1\left(\mathrm{C}_{\mathrm{q}}\right), 140.0\left(\mathrm{C}_{\mathrm{q}}\right)$, $138.1\left(\mathrm{C}_{\mathrm{q}}\right), 134.8\left(\mathrm{C}_{\mathrm{q}}\right), 132.0\left(\mathrm{C}_{\mathrm{q}}\right), 131.6(\mathrm{CH}), 130.5(\mathrm{CH}), 128.3\left(\mathrm{C}_{\mathrm{q}}\right), 128.1(\mathrm{CH}), 127.0$ $(\mathrm{CH}), 125.5\left(\mathrm{C}_{\mathrm{q}}\right), 123.8(\mathrm{CH}), 122.4(\mathrm{CH}), 114.3(\mathrm{CH}), 107.9\left(\mathrm{C}_{\mathrm{q}}\right), 78.3\left(\mathrm{CH}_{2}\right), 60.5\left(\mathrm{CH}_{2}\right)$, $54.9\left(\mathrm{CH}_{3}\right), 31.4\left(\mathrm{CH}_{2}\right), 26.9\left(\mathrm{CH}_{2}\right), 21.8\left(\mathrm{CH}_{2}\right), 20.9\left(\mathrm{CH}_{3}\right), 13.8\left(\mathrm{CH}_{3}\right), 13.2\left(\mathrm{CH}_{3}\right)$.

IR (ATR): 2928, 1718, 1507, 1272, 1098, $747 \mathrm{~cm}^{-1}$.

MS (EI) $m / z$ (relative intens ity) 467 (60) $\left[\mathrm{M}^{+}\right], 466$ (100), 452 (20), 438 (20), 424 (10), 394 (10).

HR-MS (ESI) $m / z$ calcd for $\mathrm{C}_{31} \mathrm{H}_{34} \mathrm{NO}_{3}\left[\mathrm{M}+\mathrm{H}^{+}\right]$468.2539, found 468.2533.

\section{1-\{4-[4-n-Butyl-2-(4-methoxyphe nyl)-6-methyl-1-me thyle ne-1,2-dihydroisoquinolin-3-} yl]phenyl\}e than-1-one (115ak)<smiles>C=C1c2ccc(C)cc2C(CCCCC)=C(c2ccc(C(C)=O)cc2)N1P(=O)(F)F</smiles>

The general procedure A was followed using 73a (119.5 $\mathrm{mg}, 0.50 \mathrm{mmol})$ and 1-(4-(hex-1-ynyl)phenyl)ethanone (1k) $(200 \mathrm{mg}, 1.00 \mathrm{mmol})$ at $120{ }^{\circ} \mathrm{C}$. Purification by column chromatography ( $n$-hexane/EtOAc/Et 3 N: $3: 1: 0.01 \rightarrow 3: 1: 0.03)$ yielded 115ak (125 mg, 
$57 \%)$ as an off white solid. M. p.: $129-131^{\circ} \mathrm{C}$.

${ }^{1}$ H-NMR (300 MHz, DMSO- $\left.d_{6}\right): \delta=7.75(\mathrm{~d}, J=8.3 \mathrm{~Hz}, 2 \mathrm{H}), 7.56(\mathrm{~s}, 1 \mathrm{H}), 7.27(\mathrm{~d}, J=8.3$ $\mathrm{Hz}, 2 \mathrm{H}), 7.26(\mathrm{~d}, J=8.5 \mathrm{~Hz}, 1 \mathrm{H}), 7.22$ (d, $J=8.5 \mathrm{~Hz}, 1 \mathrm{H}), 7.01$ (d, $J=8.4 \mathrm{~Hz}, 2 \mathrm{H}), 6.76$ (d, $J$ $=8.4 \mathrm{~Hz}, 2 \mathrm{H}), 4,41(\mathrm{~s}, 1 \mathrm{H}), 3.63(\mathrm{~s}, 3 \mathrm{H}), 3.00(\mathrm{~s}, 1 \mathrm{H}), 2.49(\mathrm{~s}, 3 \mathrm{H}), 2.33(\mathrm{~s}, 3 \mathrm{H}), 1.98(\mathrm{~m}, 2 \mathrm{H})$, $1.33(\mathrm{~m}, 2 \mathrm{H}), 1.11(\mathrm{~m}, 2 \mathrm{H}), 0.68(\mathrm{t}, J=7.6 \mathrm{~Hz}, 3 \mathrm{H})$.

${ }^{13}$ C-NMR (75 MHz, DMSO- $\left.d_{6}\right): \delta=197.3\left(\mathrm{C}_{\mathrm{q}}\right), 157.5\left(\mathrm{C}_{\mathrm{q}}\right), 147.1\left(\mathrm{C}_{\mathrm{q}}\right), 141.2\left(\mathrm{C}_{\mathrm{q}}\right), 139.2\left(\mathrm{C}_{\mathrm{q}}\right)$, $135.3\left(\mathrm{C}_{\mathrm{q}}\right), 135.2\left(\mathrm{C}_{\mathrm{q}}\right), 135.0\left(\mathrm{C}_{\mathrm{q}}\right), 131.7(\mathrm{CH}), 130.6(\mathrm{CH}), 130.0(\mathrm{CH}), 129.6\left(\mathrm{C}_{\mathrm{q}}\right), 127.9$ $\left(\mathrm{C}_{\mathrm{q}}\right), 127.3(\mathrm{CH}), 124.1(\mathrm{CH}), 122.5(\mathrm{CH}), 114.4(\mathrm{CH}), 108.0\left(\mathrm{C}_{\mathrm{q}}\right), 79.0\left(\mathrm{CH}_{2}\right), 55.0\left(\mathrm{CH}_{3}\right)$, 31.6 $\left(\mathrm{CH}_{2}\right), 27.2\left(\mathrm{CH}_{2}\right), 26.5\left(\mathrm{CH}_{3}\right), 22.0\left(\mathrm{CH}_{2}\right), 20.8\left(\mathrm{CH}_{2}\right), 13.4\left(\mathrm{CH}_{3}\right)$.

IR (ATR): 2929, 1681, 1507, 1243, 1028, $830 \mathrm{~cm}^{-1}$.

MS (EI) $m / z$ (relative intens ity) 437 (60) [M+1, 436 (85), 424 (100), 408 (10), 396 (20), 354 (90).

HR-MS (EI) $m / z$ calcd for $\mathrm{C}_{30} \mathrm{H}_{30} \mathrm{NO}_{2}\left[\mathrm{M}-\mathrm{H}^{+}\right]$436.2277, found 436.2286.

\section{2-(4-Me thoxyphe nyl)-1,6-dime thyl-3,4-diphenyl-1,2-dihydroisoquinoline (116aa)}<smiles>COc1ccc(N2C(c3ccccc3)=C(c3ccccc3)c3cc(C)ccc3C2C)cc1</smiles>

The general procedure $\mathbf{B}$ was followed using 73a (119.5 $\mathrm{mg}, 0.50 \mathrm{mmol})$ and $1 \mathbf{a}(178 \mathrm{mg}, 1.00$ mmol). Purification by column chromatography ( $n$-hexane/EtOAc: 120:1) yielded 116aa (83 mg, $40 \%$ ) as a white solid. M. P.: $168-170{ }^{\circ} \mathrm{C}$.

${ }^{1} \mathbf{H}-\mathrm{NMR}\left(300 \mathrm{MHz}, \mathrm{CDCl}_{3}\right): \delta=7.32-7.24(\mathrm{~m}, 4 \mathrm{H}), 7.24-7.05(\mathrm{~m}, 4 \mathrm{H}), 6.99-6.91(\mathrm{~m}, 5 \mathrm{H})$, 6.90-6.86 (m, 2H), 6.63-6.57 (m, 2H), 5.02 (q, $J=6.8 \mathrm{~Hz}, 1 \mathrm{H}), 3.65(\mathrm{~d}, J=0.9 \mathrm{~Hz}, 3 \mathrm{H}), 2.26$ (s, $3 \mathrm{H}), 1.67(\mathrm{~d}, J=6.8 \mathrm{~Hz}, 3 \mathrm{H})$.

${ }^{13}$ C-NMR $\left(75 \mathrm{MHz}, \mathrm{CDCl}_{3}\right): \delta=154.6\left(\mathrm{C}_{\mathrm{q}}\right), 140.9\left(\mathrm{C}_{\mathrm{q}}\right), 138.6\left(\mathrm{C}_{\mathrm{q}}\right), 137.8\left(\mathrm{C}_{\mathrm{q}}\right), 137.6\left(\mathrm{C}_{\mathrm{q}}\right)$, $136.2\left(\mathrm{C}_{\mathrm{q}}\right), 132.1\left(\mathrm{C}_{\mathrm{q}}\right), 132.1(\mathrm{CH}), 131.0(\mathrm{CH}), 130.7\left(\mathrm{C}_{\mathrm{q}}\right), 127.8(\mathrm{CH}), 127.0(\mathrm{CH}), 126.9$ $(\mathrm{CH}), 126.6(\mathrm{CH}), 126.0(\mathrm{CH}), 124.8(\mathrm{CH}), 123.8(\mathrm{CH}), 123.6(\mathrm{CH}), 121.7\left(\mathrm{C}_{\mathrm{q}}\right), 113.6(\mathrm{CH})$, $60.2(\mathrm{CH}), 55.3\left(\mathrm{CH}_{3}\right), 22.5\left(\mathrm{CH}_{3}\right), 21.5\left(\mathrm{CH}_{3}\right)$.

IR (ATR): 2980, 1755, 1510, 1243, 1027, $750 \mathrm{~cm}^{-1}$. 


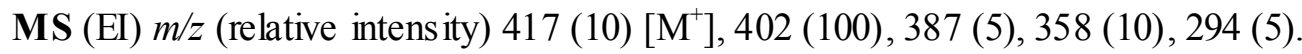

HR-MS (EI) $m / z$ calcd for $\mathrm{C}_{30} \mathrm{H}_{27} \mathrm{NO}\left[\mathrm{M}^{+}\right]$417.2093, found 417.2085.

\section{2-(4-Methoxyphenyl)-1,4,6-trimethyl-3-phe nyl-1,2-dihydroisoquinoline (116af)}<smiles>Cc1ccc2c(=O)n(C(=O)[O-])c(-c3ccccc3)c(C)c2c1</smiles>

The general procedure $\mathbf{B}$ was followed using 73a $(119.5 \mathrm{mg}, 0.50 \mathrm{mmol})$ and prop-1-ynylbenzene (1f) (116 mg, $1.00 \mathrm{mmol}$ ). Purification by column chromatography ( $n$-hexane/EtOAc: 100:1) yielded 116af (87 mg, 49\%) as a white solid. M. p.: $150-152{ }^{\circ} \mathrm{C}$. ${ }^{1} \mathbf{H}-\mathbf{N M R}\left(300 \mathrm{MHz}, \mathrm{CDCl}_{3}\right): \delta=7.42(\mathrm{dd}, J=8.0,1.5 \mathrm{~Hz}, 2 \mathrm{H}), 7.16-7.28(\mathrm{~m}, 4 \mathrm{H}), 7.00(\mathrm{~d}, J$ $=8.0 \mathrm{~Hz}, 1 \mathrm{H}), 6.90(\mathrm{~d}, J=7.6 \mathrm{~Hz}, 1 \mathrm{H}), 6.78(\mathrm{~d}, J=8.7 \mathrm{~Hz}, 2 \mathrm{H}), 6.57(\mathrm{~d}, J=8.7 \mathrm{~Hz}, 2 \mathrm{H})$, $4.90(\mathrm{q}, J=7.0 \mathrm{~Hz}, 1 \mathrm{H}), 3.63(\mathrm{~s}, 3 \mathrm{H}), 2.40(\mathrm{~s}, 3 \mathrm{H}), 2.23(\mathrm{~s}, 3 \mathrm{H}), 1.50$ (d, $J=7.0 \mathrm{~Hz}, 3 \mathrm{H})$.

${ }^{13}$ C-NMR (75 MHz, $\left.\mathrm{CDCl}_{3}\right): \delta=154.3\left(\mathrm{C}_{\mathrm{q}}\right), 141.5\left(\mathrm{C}_{\mathrm{q}}\right), 137.9\left(\mathrm{C}_{\mathrm{q}}\right), 136.2\left(\mathrm{C}_{\mathrm{q}}\right), 136.1\left(\mathrm{C}_{\mathrm{q}}\right)$, $133.7\left(\mathrm{C}_{\mathrm{q}}\right), 132.1\left(\mathrm{C}_{\mathrm{q}}\right), 130.8(\mathrm{CH}), 127.5(\mathrm{CH}), 127.1(\mathrm{CH}), 126.8(\mathrm{CH}), 124.5(\mathrm{CH}), 123.3$ $(\mathrm{CH}), 122.9(\mathrm{CH}), 113.9\left(\mathrm{C}_{\mathrm{q}}\right), 113.6(\mathrm{CH}), 60.1(\mathrm{CH}), 55.3\left(\mathrm{CH}_{3}\right), 22.0\left(\mathrm{CH}_{3}\right), 21.5\left(\mathrm{CH}_{3}\right)$, $15.3\left(\mathrm{CH}_{3}\right)$.

IR (ATR): 2964, 1738, 1505, 1229, 1033, $772 \mathrm{~cm}^{-1}$.

MS (EI) $m / z$ (relative intensity) $355(15)\left[\mathrm{M}^{+}\right], 340$ (100), 325 (5), 296 (10), 282 (5).

HR-MS (EI) $m / z$ calcd for $\mathrm{C}_{25} \mathrm{H}_{25} \mathrm{NO}\left[\mathrm{M}^{+}\right] 355.1936$, found 355.1939 .

\section{2-(4-Methoxyphenyl)-1,6-dime thyl-3,4-di-n-propyl-1,2-dihydroisoquinoline (116ae)}<smiles>Cc1ccc2c(c1)C(C(C)C)=C(C(C)C)N([18OH])C2C</smiles>

The general procedure $\mathbf{B}$ was followed using 73a (119.5 $\mathrm{mg}, 0.50 \mathrm{mmol})$ and oct-4-yne (1e) (110 mg, $1.00 \mathrm{mmol}$ ). Purification by column chromatography ( $n$-hexane/EtOAc: 50:1) yie lded 116ae (75 mg, 42\%) as a green oil.

${ }^{1}$ H-NMR (300 MHz, $\left.\mathrm{CDCl}_{3}\right): \delta=7.08(\mathrm{~s}, 1 \mathrm{H}), 6.91(\mathrm{~d}, J=7.6 \mathrm{~Hz}, 1 \mathrm{H}), 6.87(\mathrm{~d}, J=9.2 \mathrm{~Hz}$, 2H), $6.78(\mathrm{~d}, J=7.6 \mathrm{~Hz}, 1 \mathrm{H}), 6.75(\mathrm{~d}, J=9.2 \mathrm{~Hz}, 2 \mathrm{H}), 4.60(\mathrm{q}, J=6.8 \mathrm{~Hz}, 1 \mathrm{H}), 3.74(\mathrm{~s}, 3 \mathrm{H})$, 
2.70-2.47 (m, 2H), 2.42-2.34 (m, 1H), $2.34(\mathrm{~s}, 3 \mathrm{H}), 2.16-2.06(\mathrm{~m}, 1 \mathrm{H}), 1.41-1.68(\mathrm{~m}, 4 \mathrm{H})$, $1.32(\mathrm{~d}, J=6.8 \mathrm{~Hz}, 3 \mathrm{H}), 1.05(\mathrm{t}, J=7.4 \mathrm{~Hz}, 3 \mathrm{H}), 0.87$ (t, $J=7.4 \mathrm{~Hz}, 3 \mathrm{H})$.

${ }^{13}$ C-NMR $\left(75 \mathrm{MHz}, \mathrm{CDCl}_{3}\right): \delta=155.0\left(\mathrm{C}_{\mathrm{q}}\right), 141.5\left(\mathrm{C}_{\mathrm{q}}\right), 137.6\left(\mathrm{C}_{\mathrm{q}}\right), 135.8\left(\mathrm{C}_{\mathrm{q}}\right), 132.0\left(\mathrm{C}_{\mathrm{q}}\right)$, $131.7\left(\mathrm{C}_{\mathrm{q}}\right), 126.0(\mathrm{CH}), 124.7(\mathrm{CH}), 124.0(\mathrm{CH}), 121.9(\mathrm{CH}), 118.3\left(\mathrm{C}_{\mathrm{q}}\right), 113.9(\mathrm{CH}), 60.3$ $(\mathrm{CH}), 55.4\left(\mathrm{CH}_{3}\right), 31.4\left(\mathrm{CH}_{2}\right), 29.8\left(\mathrm{CH}_{2}\right), 23.4\left(\mathrm{CH}_{2}\right), 22.1\left(\mathrm{CH}_{3}\right), 22.1\left(\mathrm{CH}_{2}\right), 21.6\left(\mathrm{CH}_{3}\right)$, $14.6\left(\mathrm{CH}_{3}\right), 14.2\left(\mathrm{CH}_{3}\right)$.

IR (ATR): 2958, 2869, 1505, 1237, 1035, 827 $\mathrm{cm}^{-1}$.

MS (EI) $m / z$ (relative intensity) 349 (10) $\left[\mathrm{M}^{+}\right], 334$ (100), 320 (10), 304 (5), 290 (5), 276 (5).

HR-MS (EI) $m / z$ calcd for $\mathrm{C}_{24} \mathrm{H}_{31} \mathrm{NO}\left[\mathrm{M}^{+}\right] 349.2406$, found 349.2410.

2-(4-Me thoxyphe nyl)-1,7-dime thyl-3,4-diphenyl-1,2-dihydroisoquinoline (116fa)<smiles>COc1ccc(N2C(c3ccccc3)=C(c3ccccc3)c3ccc(C)cc3C2C)cc1</smiles>

The general procedure $\mathbf{B}$ was followed using $\mathbf{7 3 f}(119.5 \mathrm{mg}, 0.50 \mathrm{mmol})$ and diphenylacetylene (1a) (178 mg, $1.00 \mathrm{mmol}$ ). Purification by column chromatography ( $n$-hexane/EtOAc: 100:1) yielded 116fa (94 mg, 45\%) as an off white solid. M. p.: $160-163^{\circ} \mathrm{C}$.

${ }^{1} \mathbf{H}-\mathbf{N M R}\left(300 \mathrm{MHz}, \mathrm{CDCl}_{3}\right): \delta=7.25$ (d, $\left.J=4.4 \mathrm{~Hz}, 4 \mathrm{H}\right), 7.22-7.09$ (m, 4H), 7.00-6.89 (m, $4 \mathrm{H}), 6.86(\mathrm{~d}, J=8.9 \mathrm{~Hz}, 3 \mathrm{H}), 6.86(\mathrm{~s}, 1 \mathrm{H}), 6.58(\mathrm{~d}, J=8.9 \mathrm{~Hz}, 2 \mathrm{H}), 4.98(\mathrm{q}, J=6.7 \mathrm{~Hz}, 1 \mathrm{H})$, $2.29(\mathrm{~s}, 3 \mathrm{H}), 1.65(\mathrm{~d}, J=6.7 \mathrm{~Hz}, 3 \mathrm{H})$.

${ }^{13}$ C-NMR $\left(75 \mathrm{MHz}, \mathrm{CDCl}_{3}\right): \delta=154.5\left(\mathrm{C}_{\mathrm{q}}\right), 141.0\left(\mathrm{C}_{\mathrm{q}}\right), 138.7\left(\mathrm{C}_{\mathrm{q}}\right), 137.6\left(\mathrm{C}_{\mathrm{q}}\right), 136.8\left(\mathrm{C}_{\mathrm{q}}\right)$, $135.9\left(\mathrm{C}_{\mathrm{q}}\right), 133.6\left(\mathrm{C}_{\mathrm{q}}\right), 131.9(\mathrm{CH}), 131.0(\mathrm{CH}), 129.7\left(\mathrm{C}_{\mathrm{q}}\right), 127.8(\mathrm{CH}), 127.5(\mathrm{CH}), 127.0$ $(\mathrm{CH}), 126.5(\mathrm{CH}), 126.0(\mathrm{CH}), 125.5(\mathrm{CH}), 123.5(\mathrm{CH}), 123.3(\mathrm{CH}), 122.1\left(\mathrm{C}_{\mathrm{q}}\right), 113.7(\mathrm{CH})$, $60.4(\mathrm{CH}), 55.3\left(\mathrm{CH}_{3}\right), 22.4\left(\mathrm{CH}_{3}\right), 21.1\left(\mathrm{CH}_{3}\right)$.

IR (ATR): 2972, 1756, 1509, 1243, 1027, $749 \mathrm{~cm}^{-1}$.

MS (EI) $m / z$ (relative intensity) 417 (10) $\left[\mathrm{M}^{+}\right], 402$ (100), 387 (5), 358 (10), 294 (5).

HR-MS (EI) $m / z$ calcd for $\mathrm{C}_{30} \mathrm{H}_{27} \mathrm{NO}\left[\mathrm{M}^{+}\right]$417.2093, found 417.2088. 


\section{1-Ethyl-2-(4-methoxyphe nyl)-3,4-diphe nyl-1,2-dihydroisoquinoline (116ha)}<smiles>CCC1c2ccccc2C(c2ccccc2)=C(c2ccccc2)N1C(F)(F)F</smiles>

The general procedure $\mathbf{B}$ was followed using $73 \mathbf{h ~}(119.5 \mathrm{mg}, 0.50 \mathrm{mmol})$ and diphenylacetylene (1a) (178 mg, $1.00 \mathrm{mmol}$ ). Purification by column chromatography (n-hexane/EtOAc: 100:1) yielded 116ha (116 mg, 56\%) as an off white solid. M. p.: 100-103 ${ }^{\circ} \mathrm{C}$.

${ }^{1} \mathbf{H}-\mathrm{NMR}\left(300 \mathrm{MHz}, \mathrm{CDCl}_{3}\right): \delta=7.32-7.27$ (m, 5H), 7.22-7.14 (m, 5H), 6.99-6.89 (m, 6H), $6.60(\mathrm{dd}, J=9.0,1.3 \mathrm{~Hz}, 2 \mathrm{H}), 4.68(\mathrm{dd}, J=6.9,6.9 \mathrm{~Hz}, 1 \mathrm{H}), 3.65(\mathrm{~d}, J=1.4 \mathrm{~Hz}, 3 \mathrm{H})$, $2.18-2.08(\mathrm{~m}, 1 \mathrm{H}), 1.92-1.83(\mathrm{~m}, 1 \mathrm{H}), 1.23(\mathrm{t}, J=7.6 \mathrm{~Hz}, 3 \mathrm{H})$.

${ }^{13}$ C-NMR (75 MHz, $\left.\mathrm{CDCl}_{3}\right): \delta=154.7\left(\mathrm{C}_{\mathrm{q}}\right), 141.2\left(\mathrm{C}_{\mathrm{q}}\right), 138.6\left(\mathrm{C}_{\mathrm{q}}\right), 138.5\left(\mathrm{C}_{\mathrm{q}}\right), 137.6\left(\mathrm{C}_{\mathrm{q}}\right)$, $132.4\left(\mathrm{C}_{\mathrm{q}}\right), 131.9(\mathrm{CH}), 131.8\left(\mathrm{C}_{\mathrm{q}}\right), 131.3(\mathrm{CH}), 127.8(\mathrm{CH}), 127.0(\mathrm{CH}), 126.7(\mathrm{CH}), 126.7$ $(\mathrm{CH}), 126.1(\mathrm{CH}), 125.8(\mathrm{CH}), 125.8(\mathrm{CH}), 124.1(\mathrm{CH}), 123.2(\mathrm{CH}), 112.7\left(\mathrm{C}_{\mathrm{q}}\right), 113.7(\mathrm{CH})$, $67.0(\mathrm{CH}), 55.3\left(\mathrm{CH}_{3}\right), 28.6\left(\mathrm{CH}_{2}\right), 11.2\left(\mathrm{CH}_{3}\right)$.

IR (ATR): 2953, 1738, 1505, 1229, 1028, $700 \mathrm{~cm}^{-1}$.

MS (EI) $m / z$ (relative intensity) 417 (65) $\left[\mathrm{M}^{+}\right], 388$ (100), 372 (5), 344 (10), 280 (5), 252 (5).

HR-MS (EI) $m / z$ calcd for $\mathrm{C}_{30} \mathrm{H}_{27} \mathrm{NO}\left[\mathrm{M}^{+}\right]$417.2093, found 417.2081.

\section{5,6,7-Trime thoxy-2-(4-methoxyphe nyl)-1-methyl-3,4-diphe nyl-1,2-dihydroisoquinoline} (116ia)<smiles>COc1ccc(N2C(c3ccccc3)=C(c3ccccc3)c3c(cc(OC)c(OC)c3OC)C2C)cc1</smiles>

The general procedure $\mathbf{B}$ was followed using $73 \mathbf{i}(157.5 \mathrm{mg}, 0.50 \mathrm{mmol})$ and diphenylacetylene (1a) (178 mg, $1.00 \mathrm{mmol})$. Purification by column chromatography (n-hexane/EtOAc: 100:1) yielded 116ia (118 $\mathrm{mg}, 48 \%$ ) as an off white solid. M. p.: $138-140{ }^{\circ} \mathrm{C}$.

${ }^{1} \mathbf{H}-\mathbf{N M R}\left(300 \mathrm{MHz}, \mathrm{CDCl}_{3}\right): \delta=7.38-7.33(\mathrm{~m}, 2 \mathrm{H}), 7.29-7.24(\mathrm{~m}, 2 \mathrm{H}), 7.17-7.07(\mathrm{~m}, 2 \mathrm{H})$, 
7.07-7.00 (m, 1H), 7.00-6.89 (m, 3H), 6.86 (d, $J=9.0 \mathrm{~Hz}, 2 \mathrm{H}), 6.59$ (d, $J=8.9 \mathrm{~Hz}, 2 \mathrm{H}), 6.39$ (s, 1H), 4.91 (q, $J=6.7 \mathrm{~Hz}, 1 \mathrm{H}), 3.81$ (s, 3H), 3.75 (s, 3H), 3.63 (s, 3H), 2.99 (s, 3H). ${ }^{13}$ C-NMR $\left(75 \mathrm{MHz}, \mathrm{CDCl}_{3}\right): \delta=154.3\left(\mathrm{C}_{\mathrm{q}}\right), 152.3\left(\mathrm{C}_{\mathrm{q}}\right), 150.5\left(\mathrm{C}_{\mathrm{q}}\right), 141.8\left(\mathrm{C}_{\mathrm{q}}\right), 140.7\left(\mathrm{C}_{\mathrm{q}}\right)$, $140.5\left(\mathrm{C}_{\mathrm{q}}\right), 137.4\left(\mathrm{C}_{\mathrm{q}}\right), 137.3\left(\mathrm{C}_{\mathrm{q}}\right), 132.4(\mathrm{CH}), 132.1\left(\mathrm{C}_{\mathrm{q}}\right), 131.1(\mathrm{CH}), 126.8(\mathrm{CH}), 126.7$ $(\mathrm{CH}), 126.3(\mathrm{CH}), 125.5(\mathrm{CH}), 122.6(\mathrm{CH}), 121.8\left(\mathrm{C}_{\mathrm{q}}\right), 120.5\left(\mathrm{C}_{\mathrm{q}}\right), 113.7(\mathrm{CH}), 103.9(\mathrm{CH})$, $60.5(\mathrm{CH}), 60.2\left(\mathrm{CH}_{3}\right), 59.7\left(\mathrm{CH}_{3}\right), 55.9\left(\mathrm{CH}_{3}\right), 55.3\left(\mathrm{CH}_{3}\right), 21.9\left(\mathrm{CH}_{3}\right)$.

IR (ATR): 2987, 1742, 1511, 1226, $708 \mathrm{~cm}^{-1}$.

MS (EI) $m / z$ (relative intensity) $493(5)\left[\mathrm{M}^{+}\right], 478$ (100), 463 (5), 448 (10), 417 (5).

HR-MS (EI) $m / z$ calcd for $\mathrm{C}_{32} \mathrm{H}_{31} \mathrm{NO}_{4}\left[\mathrm{M}^{\dagger}\right]$ 493.2253, found 493.2259.

\section{Inte rmolecular Compe tition Experiment Between Alkynes Subs trates 1a and 11:}

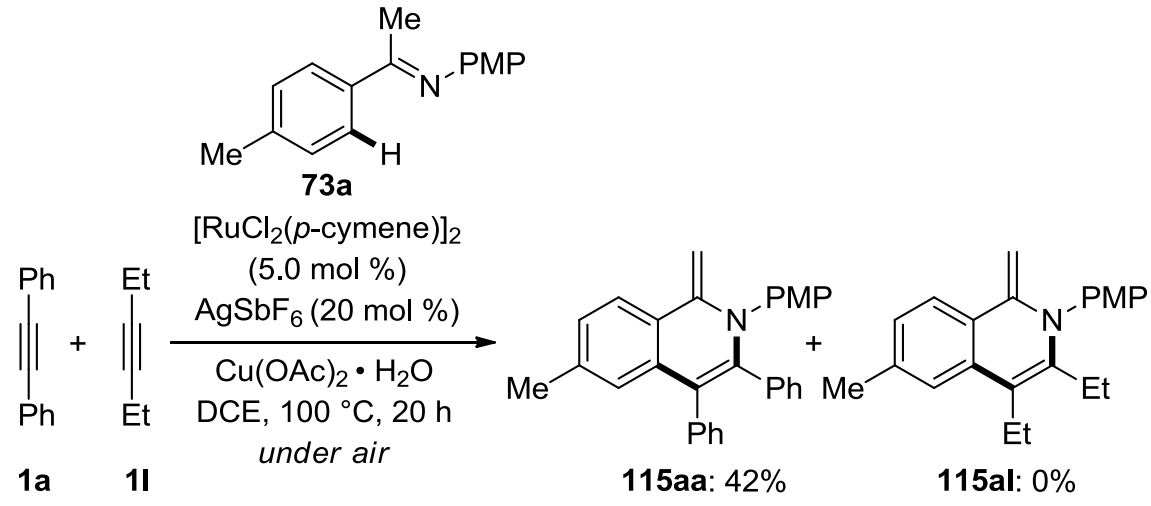

A suspension of (E)-4-methoxy- $N$-[1-(p-tolyl)ethylidene]aniline (73a) $(120 \mathrm{mg}, 0.50 \mathrm{mmol})$, 1,2-diphenylethyne (1a) (178 mg, $1.00 \mathrm{mmol}$ ), hex-3-yne (11) (82.0 mg, $1.00 \mathrm{mmol}$ ), $\left[\mathrm{RuCl}_{2}(p \text {-cymene })\right]_{2}(15.3 \mathrm{mg}, 5.0 \mathrm{~mol} \%), \mathrm{AgSbF}_{6}(34.4 \mathrm{mg}, 20 \mathrm{~mol} \%)$ and $\mathrm{Cu}(\mathrm{OAc})_{2} \cdot \mathrm{H}_{2} \mathrm{O}$ $(100 \mathrm{mg}, 0.50 \mathrm{mmol})$ in DCE $(2.0 \mathrm{~mL})$ was stirred at $100 \mathcal{C}$ for $20 \mathrm{~h}$ under an atmosphere of air. At ambient temperature, $\mathrm{H}_{2} \mathrm{O}(20 \mathrm{~mL})$ was added, and the mixture was extracted with EtOAc $(3 \times 20 \mathrm{~mL})$. The combined organic layers were washed with brine $(20 \mathrm{~mL})$ and dried over $\mathrm{Na}_{2} \mathrm{SO}_{4}$. The solvent was evaporated in vacuo, and the residue was purified by column chromatography on silica gel ( $n$-hexane/EtOAc/Et ${ }_{3} \mathrm{~N}$ : 20:1:0.05) to yield 115aa (78 mg, 42\%) as the sole product.

Ruthenium(II)-Catalyzed H/D Exchange with Substrate $73 \mathrm{a}$ in $\left[\mathrm{D}_{4}\right]-\mathrm{MeOH}$ as the cosolvent in the Presence of Alkyne 1e: 


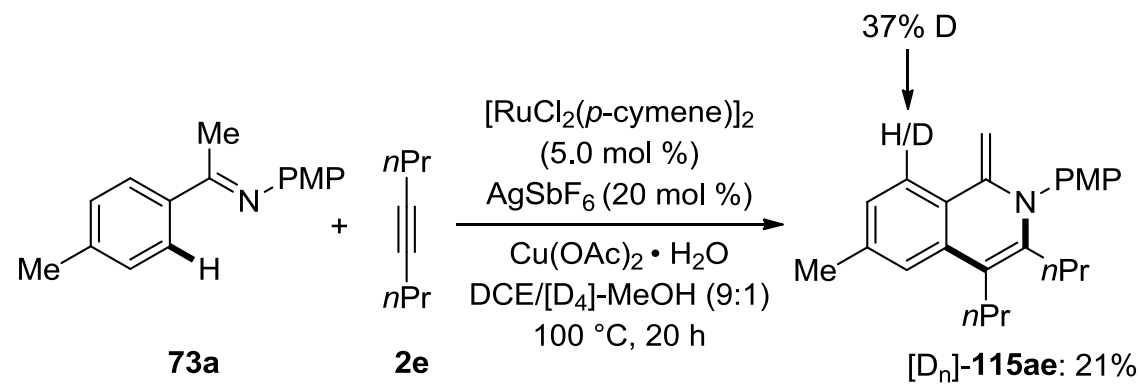

A suspension of (E)-4-methoxy- $N$-[1-( $p$-tolyl)ethylidene]aniline (73a) $(120 \mathrm{mg}, 0.50 \mathrm{mmol})$, oct-4-yne (1e) $(110 \mathrm{mg}, 1.00 \mathrm{mmol}),\left[\mathrm{RuCl}_{2}(p \text {-cymene })\right]_{2}(15.3 \mathrm{mg}, 5.0 \mathrm{~mol} \%), \mathrm{AgSbF}_{6}$ (34.4 mg, $20 \mathrm{~mol} \%$ ) and $\mathrm{Cu}(\mathrm{OAc})_{2} \cdot \mathrm{H}_{2} \mathrm{O}(100 \mathrm{mg}, 0.50 \mathrm{mmol})$ in a solvent mixture of DCE and $\left[\mathrm{D}_{4}\right]-\mathrm{MeOH}(1.8 / 0.2 \mathrm{~mL})$ was stirred at $100 \mathrm{C}$ for $20 \mathrm{~h}$ under an atmosphere of air. At ambient temperature, $\mathrm{H}_{2} \mathrm{O}(20 \mathrm{~mL})$ was added, and the mixture was extracted with EtOAc (3 $\times 20 \mathrm{~mL})$. The combined organic layers were washed with brine $(20 \mathrm{~mL})$ and dried over $\mathrm{Na}_{2} \mathrm{SO}_{4}$. The solvent was evaporated in vacuo, and the residue was purified by column chromatography ( $n$-hexane/EtOAc/Et $\left.{ }_{3} \mathrm{~N}: 1: 2: 0.03\right)$ to yield $\left[\mathrm{D}_{\mathrm{n}}\right]-\mathbf{1 1 5} \mathbf{a e}(32.0 \mathrm{mg}, \mathbf{2 1 \%})$ as an off white solid. The D-incorporation in $\left[\mathrm{D}_{\mathrm{n}}\right]$-115ae was estimated by ${ }^{1} \mathrm{H}-\mathrm{NMR}$ spectroscopy.

\subsubsection{Analytical Data for the Products of the Ruthenium(II)-Catalyzed}

Oxidative C-H Activation with Internal Alkynes and Acrylates

\section{$N$-(tert-B utyl)-3,4-diphe nylis oquinolin-1-amine (118aa)}<smiles>CC(C)(C)Nc1nc(-c2ccccc2)c(-c2ccccc2)c2ccccc12</smiles>

The general procedure $\mathbf{C}$ was followed using $117 \mathbf{a}(88.0 \mathrm{mg}, 0.50 \mathrm{mmol})$ and $\mathbf{1 a}(178 \mathrm{mg}$, 1.00 mmol). Purification by column chromatography ( $n$-hexane/EtOAc: 100:1) yielded 118aa (141 mg, 80\%) as a yellow solid. M. p.: $139-142{ }^{\circ} \mathrm{C}$.

${ }^{1} \mathbf{H}-\mathbf{N M R}\left(300 \mathrm{MHz}, \mathrm{CDCl}_{3}\right): \delta=7.76(\mathrm{~d}, J=7.9 \mathrm{~Hz}, 1 \mathrm{H}), 7.55(\mathrm{~d}, J=7.3 \mathrm{~Hz}, 1 \mathrm{H})$, 7.48-7.41 (m, 4H), 7.35-7.30 (m, 3H), 7.26-7.23 (m, 2H), 7.18-7.16 (m, 3H), $5.23(\mathrm{~s}, 1 \mathrm{H})$, $1.66(\mathrm{~s}, 9 \mathrm{H})$.

${ }^{13}$ C-NMR $\left(75 \mathrm{MHz}, \mathrm{CDCl}_{3}\right): \delta=153.3\left(\mathrm{C}_{\mathrm{q}}\right), 148.0\left(\mathrm{C}_{\mathrm{q}}\right), 141.9\left(\mathrm{C}_{\mathrm{q}}\right), 138.8\left(\mathrm{C}_{\mathrm{q}}\right), 137.4\left(\mathrm{C}_{\mathrm{q}}\right)$, $132.1(\mathrm{CH}), 130.4(\mathrm{CH}), 129.1(\mathrm{CH}), 128.2(\mathrm{CH}), 127.1(\mathrm{CH}), 126.5(\mathrm{CH}), 126.4(\mathrm{CH}), 126.3$ $(\mathrm{CH}), 125.2(\mathrm{CH}), 121.0(\mathrm{CH}), 120.2\left(\mathrm{C}_{\mathrm{q}}\right), 117.0\left(\mathrm{C}_{\mathrm{q}}\right), 51.9\left(\mathrm{C}_{\mathrm{q}}\right), 29.4\left(\mathrm{CH}_{3}\right)$. 
IR (ATR): 3437, 2956, 1515, 1416, 1210, $697 \mathrm{~cm}^{-1}$.

MS (EI) $\mathrm{m} / z$ (relative intensity) $352(30)\left[\mathrm{M}^{+}\right], 337$ (15), 296 (100), 278 (25), 190 (10), 165 (10).

HR-MS (EI) $m / z$ calcd for $\mathrm{C}_{25} \mathrm{H}_{24} \mathrm{~N}_{2}\left[\mathrm{M}^{+}\right] 352.1939$, found 352.1934 .

The spectral data were in accordance with those reported in the literature. ${ }^{146}$

\section{$N$-(tert-B utyl)-3,4-bis (4-me thoxyphe nyl)isoquinolin-1-amine (118ab)}<smiles>COc1ccc(-c2nc(NCC(C)C)c3ccccc3c2-c2ccc(OC)cc2)cc1</smiles>

The general procedure $\mathbf{C}$ was followed using $117 \mathbf{a}(88.0 \mathrm{mg}, 0.50 \mathrm{mmol})$ and $\mathbf{1 b}(238 \mathrm{mg}$, 1.00 mmol). Purification by column chromatography ( $n$-hexane/EtOAc: $100 / 1 \rightarrow 50 / 1$ ) yielded 118ab (131 mg, 64\%) as a white solid. M. p.: $176-179^{\circ} \mathrm{C}$.

${ }^{1}$ H-NMR $\left(300 \mathrm{MHz}, \mathrm{CDCl}_{3}\right): \delta=7.72(\mathrm{~d}, J=8.4 \mathrm{~Hz}, 1 \mathrm{H}), 7.53(\mathrm{~d}, J=7.8 \mathrm{~Hz}, 1 \mathrm{H}), 7.55(\mathrm{~d}, J$ $=6.9,1.4 \mathrm{~Hz}, 1 \mathrm{H}), 7.41-7.35(\mathrm{~m}, 3 \mathrm{H}), 7.12(\mathrm{~d}, J=8.4 \mathrm{~Hz}, 2 \mathrm{H}), 6.89(\mathrm{~d}, J=8.9 \mathrm{~Hz}, 2 \mathrm{H}), 6.71$ $(\mathrm{d}, J=8.4 \mathrm{~Hz}, 2 \mathrm{H}), 5.16(\mathrm{~s}, 1 \mathrm{H}), 3.83(\mathrm{~s}, 3 \mathrm{H}), 3.76(\mathrm{~s}, 3 \mathrm{H}), 1.63(\mathrm{~s}, 9 \mathrm{H})$.

${ }^{13}$ C-NMR $\left(75 \mathrm{MHz}, \mathrm{CDCl}_{3}\right): \delta=158.2\left(\mathrm{C}_{\mathrm{q}}\right), 158.1\left(\mathrm{C}_{\mathrm{q}}\right), 153.1\left(\mathrm{C}_{\mathrm{q}}\right), 147.6\left(\mathrm{C}_{\mathrm{q}}\right), 137.9\left(\mathrm{C}_{\mathrm{q}}\right)$, $134.5\left(\mathrm{C}_{\mathrm{q}}\right), 133.0(\mathrm{CH}), 131.6(\mathrm{CH}), 131.2\left(\mathrm{C}_{\mathrm{q}}\right), 129.0(\mathrm{CH}), 126.2(\mathrm{CH}), 124.9(\mathrm{CH}), 121.0$ $(\mathrm{CH}), 119.2\left(\mathrm{C}_{\mathrm{q}}\right), 116.9\left(\mathrm{C}_{\mathrm{q}}\right), 113.8(\mathrm{CH}), 112.6(\mathrm{CH}), 55.2\left(\mathrm{CH}_{3}\right), 55.1\left(\mathrm{CH}_{3}\right), 51.8\left(\mathrm{C}_{\mathrm{q}}\right), 29.4$ $\left(\mathrm{CH}_{3}\right)$.

IR (ATR): 3439, 2949, 1512, 1417, 1234, $763 \mathrm{~cm}^{-1}$.

MS (EI) $m / z$ (relative intensity) $412(30)\left[\mathrm{M}^{+}\right], 397$ (15), 356 (100), 341 (10), 312 (10).

HR-MS (EI) $m / z$ calcd for $\mathrm{C}_{27} \mathrm{H}_{28} \mathrm{~N}_{2} \mathrm{O}_{2}\left[\mathrm{M}^{+}\right]$412.2151, found 412.2141 .

$N$-(tert-B utyl)-3,4-di-p-tolylisoquinolin-1-amine (118ac)

${ }_{146}$ X. H. Wei, M. Zhao, Z. Y. Du, X. W. Li, Org. Lett. 2011, 13, 4636-4639. 
<smiles>Cc1ccc(-c2nc(NC(C)(C)C)c3ccccc3c2-c2ccc(C)cc2)cc1</smiles>

The general procedure $\mathbf{C}$ was followed using $117 \mathbf{a}(88.0 \mathrm{mg}, 0.50 \mathrm{mmol})$ and $1 \mathbf{c}(206 \mathrm{mg}$, $1.00 \mathrm{mmol})$. Purification by column chromatography ( $n$-hexane/EtOAc: $150 / 1)$ yielded 118ac (171 mg, 90\%) as a white solid. M. p.: $173-174{ }^{\circ} \mathrm{C}$.

${ }^{1}$ H-NMR $\left(300 \mathrm{MHz}, \mathrm{CDCl}_{3}\right): \delta=7.73(\mathrm{~d}, J=8.0 \mathrm{~Hz}, 1 \mathrm{H}), 7.53(\mathrm{dd}, J=8.1,2.1 \mathrm{~Hz}, 1 \mathrm{H})$, 7.46-7.39 (m, 2H), 7.34 (d, $J=8.1 \mathrm{~Hz}, 2 \mathrm{H}), 7.15(\mathrm{~d}, J=8.2 \mathrm{~Hz}, 2 \mathrm{H}), 7.11(\mathrm{~d}, J=8.2 \mathrm{~Hz}, 2 \mathrm{H})$, $6.98(\mathrm{~d}, J=8.1 \mathrm{~Hz}, 2 \mathrm{H}), 5.18(\mathrm{~s}, 1 \mathrm{H}), 2.39$ (s, 3H), 2.31 (s, 3H), 1.64 (s, 9H).

${ }^{13}$ C-NMR (75 MHz, $\left.\mathrm{CDCl}_{3}\right): \delta=153.0\left(\mathrm{C}_{\mathrm{q}}\right), 147.7\left(\mathrm{C}_{\mathrm{q}}\right), 139.0\left(\mathrm{C}_{\mathrm{q}}\right), 137.6\left(\mathrm{C}_{\mathrm{q}}\right), 135.9\left(\mathrm{C}_{\mathrm{q}}\right)$, $135.9\left(\mathrm{C}_{\mathrm{q}}\right), 135.8\left(\mathrm{C}_{\mathrm{q}}\right), 131.6(\mathrm{CH}), 130.0(\mathrm{CH}), 129.0(\mathrm{CH}), 128.9(\mathrm{CH}), 127.7(\mathrm{CH}), 126.2$ $(\mathrm{CH}), 124.7(\mathrm{CH}), 121.0(\mathrm{CH}), 119.8\left(\mathrm{C}_{\mathrm{q}}\right), 116.9\left(\mathrm{C}_{\mathrm{q}}\right), 51.8\left(\mathrm{C}_{\mathrm{q}}\right), 29.5\left(\mathrm{CH}_{3}\right), 21.4\left(\mathrm{CH}_{3}\right), 21.3$ $\left(\mathrm{CH}_{3}\right)$.

IR (ATR): 3452, 2989, 1573, 1512, 1210, $762 \mathrm{~cm}^{-1}$.

MS (EI) $m / z$ (relative intensity) $380(35)\left[\mathrm{M}^{+}\right], 365$ (15), 324 (100), 308 (10), 291 (5). HR-MS (EI) $m / z$ calcd for $\mathrm{C}_{27} \mathrm{H}_{28} \mathrm{~N}_{2}\left[\mathrm{M}^{+}\right] 380.2252$, found 380.2250 .

\section{$N$-(tert-B utyl)-3,4-bis(4-fluorophe nyl)isoquinolin-1-amine (118ad)}<smiles>CC(C)(C)Nc1nc(-c2ccc(F)cc2)c(-c2ccc(F)cc2)c2ccccc12</smiles>

The general procedure $\mathbf{C}$ was followed using $117 \mathbf{a}(88.0 \mathrm{mg}, 0.50 \mathrm{mmol})$ and $\mathbf{1 d}(214 \mathrm{mg}$, $1.00 \mathrm{mmol}$ ) at $140{ }^{\circ} \mathrm{C}$. Purification by column chromatography ( $n$-hexane/EtOAc: $120 / 1$ ) yielded 118ad (131 mg, 68\%) as a yellow solid. M. p.: $195-198^{\circ} \mathrm{C}$.

${ }^{1} \mathbf{H}-\mathbf{N M R}\left(300 \mathrm{MHz}, \mathrm{CDCl}_{3}\right): \delta=7.76(\mathrm{~d}, J=7.9 \mathrm{~Hz}, 1 \mathrm{H}), 7.49-7.40(\mathrm{~m}, 3 \mathrm{H}), 7.36-7.31(\mathrm{~m}$, 
2H), 7.17-7.12 (m, 2H), $7.06(\mathrm{t}, J=8.8 \mathrm{~Hz}, 2 \mathrm{H}), 6.88(\mathrm{t}, J=8.8 \mathrm{~Hz}, 2 \mathrm{H}), 5.24(\mathrm{~s}, 1 \mathrm{H}), 1.64(\mathrm{~s}$, $9 \mathrm{H})$.

${ }^{13}$ C-NMR (75 MHz, CDCl $): \delta=161.9\left(\mathrm{C}_{\mathrm{q}}, J_{\mathrm{C}-\mathrm{F}}=245.5 \mathrm{~Hz}\right), 161.2\left(\mathrm{C}_{\mathrm{q}}, J_{\mathrm{C}-\mathrm{F}}=245.5 \mathrm{~Hz}\right)$, $153.3\left(\mathrm{C}_{\mathrm{q}}\right), 147.2\left(\mathrm{C}_{\mathrm{q}}\right), 137.7\left(\mathrm{C}_{\mathrm{q}}, J_{\mathrm{C}-\mathrm{F}}=3.5 \mathrm{~Hz}\right), 137.3\left(\mathrm{C}_{\mathrm{q}}\right), 134.4\left(\mathrm{C}_{\mathrm{q}}, J_{\mathrm{C}-\mathrm{F}}=3.5 \mathrm{~Hz}\right), 133.4$ $\left(\mathrm{CH}, J_{\mathrm{C}-\mathrm{F}}=7.9 \mathrm{~Hz}\right), 131.9\left(\mathrm{CH}, J_{\mathrm{C}-\mathrm{F}}=7.9 \mathrm{~Hz}\right), 129.3(\mathrm{CH}), 125.9(\mathrm{CH}), 125.3(\mathrm{CH}), 121.1$ $(\mathrm{CH}), 118.8\left(\mathrm{C}_{\mathrm{q}}\right), 117.0\left(\mathrm{C}_{\mathrm{q}}\right), 115.3\left(\mathrm{CH}, J_{\mathrm{C}-\mathrm{F}}=21.4 \mathrm{~Hz}\right), 114.1\left(\mathrm{CH}, J_{\mathrm{C}-\mathrm{F}}=21.4 \mathrm{~Hz}\right), 51.9$ $\left(\mathrm{C}_{\mathrm{q}}\right), 29.4\left(\mathrm{CH}_{3}\right)$.

${ }^{19}$ F-NMR (282 MHz, $\left.\mathrm{CDCl}_{3}\right): \delta=-(115.75-115.83)(\mathrm{m}),-(115.85-116.00)(\mathrm{m})$.

IR (ATR): 3472, 2963, 1506, 1417, 1214, $825 \mathrm{~cm}^{-1}$.

MS (EI) $m / z$ (relative intens ity) $388(30)\left[\mathrm{M}^{+}\right], 373$ (15), 332 (100), 331 (95), 314 (20), 294 (5).

HR-MS (EI) $m / z$ calcd for $\mathrm{C}_{25} \mathrm{H}_{22} \mathrm{~N}_{2} \mathrm{~F}_{2}\left[\mathrm{M}^{+}\right] 388.1751$, found 388.1746 .

\section{$N$-tert-B utyl-3,4-bis(4-chlorophe nyl)isoquinolin-1-amine (118am)}<smiles>CC(C)(C)Nc1nc(-c2ccc(Cl)cc2)c(-c2ccc(Cl)cc2)c2ccccc12</smiles>

The general procedure $\mathbf{C}$ was followed using $\mathbf{1 1 7} \mathbf{a}(88.0 \mathrm{mg}, 0.50 \mathrm{mmol})$ and $\mathbf{1} \mathbf{m}(246 \mathrm{mg}$, $1.00 \mathrm{mmol}$ ) at $140{ }^{\circ} \mathrm{C}$. Purification by column chromatography ( $n$-hexane/EtOAc: 120/1) yie lded 118am (76 mg, 36\%) as an off white solid. M. p.: $198-200^{\circ} \mathrm{C}$.

${ }^{1} \mathbf{H}-\mathbf{N M R}\left(300 \mathrm{MHz}, \mathrm{CDCl}_{3}\right): \delta=7.74(\mathrm{~m}, 1 \mathrm{H}, \mathrm{ArH}), 7.41-7.47(\mathrm{~m}, 3 \mathrm{H}), 7.27-7.33(\mathrm{~m}, 4 \mathrm{H})$, 7.12-7.17 (m, 4H), $5.24(\mathrm{~s}, 1 \mathrm{H}), 1.61(\mathrm{~s}, 9 \mathrm{H})$.

${ }^{13}$ C-NMR (75 MHz, $\left.\mathrm{CDCl}_{3}\right): \delta=153.5\left(\mathrm{C}_{\mathrm{q}}\right), 147.0\left(\mathrm{C}_{\mathrm{q}}\right), 140.1\left(\mathrm{C}_{\mathrm{q}}\right), 137.1\left(\mathrm{C}_{\mathrm{q}}\right), 137.0\left(\mathrm{C}_{\mathrm{q}}\right)$, $133.3(\mathrm{CH}), 132.7\left(\mathrm{C}_{\mathrm{q}}\right), 132.6\left(\mathrm{C}_{\mathrm{q}}\right), 131.6(\mathrm{CH}), 129.5(\mathrm{CH}), 128.6(\mathrm{CH}), 127.5(\mathrm{CH}), 125.9$ $(\mathrm{CH}), 125.6(\mathrm{CH}), 121.2(\mathrm{CH}), 118.9\left(\mathrm{C}_{\mathrm{q}}\right), 117.1\left(\mathrm{C}_{\mathrm{q}}\right), 51.9\left(\mathrm{C}_{\mathrm{q}}\right), 29.3\left(\mathrm{CH}_{3}\right)$.

IR (ATR): 3450, 2952, 1517, 1418, 1088, $763 \mathrm{~cm}^{-1}$.

MS (EI) m/z (relative intensity) 420 (25) $\left[\mathrm{M}^{+}\right], 405$ (15), 364 (100), 328 (30), 311 (15), 292 (10). 
HR-MS (EI) $m / z$ calcd for $\mathrm{C}_{25} \mathrm{H}_{22} \mathrm{~N}_{2} \mathrm{Cl}_{2}\left[\mathrm{M}^{+}\right] 420.1160$, found 420.1149 .

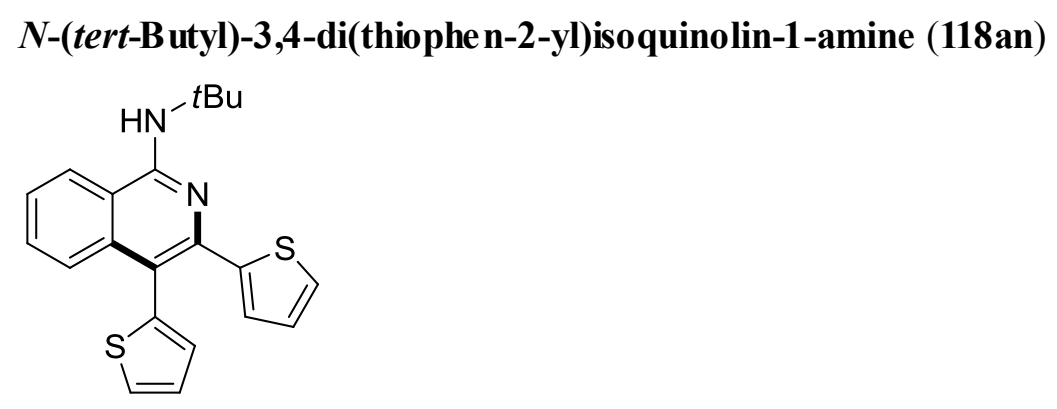

The general procedure $\mathbf{C}$ was followed using $\mathbf{1 1 7 a}(88.0 \mathrm{mg}, 0.50 \mathrm{mmol})$ and $\mathbf{1 n}(190 \mathrm{mg}$, $1.00 \mathrm{mmol})$ at $140{ }^{\circ} \mathrm{C}$. Purification by column chromatography ( $n$-hexane/EtOAc: $\left.120 / 1\right)$ yie lded 118an (142 mg, 75\%) as an off white solid. M. p.: $165-168{ }^{\circ} \mathrm{C}$.

${ }^{1}$ H-NMR $\left(300 \mathrm{MHz}, \mathrm{CDCl}_{3}\right): \delta=7.67(\mathrm{~d}, J=8.2 \mathrm{~Hz}, 1 \mathrm{H}), 7.55(\mathrm{dd}, J=5.2,1.2 \mathrm{~Hz}, 1 \mathrm{H})$, 7.48-7.44 (m, 2H), 7.41-7.36 (m, 1H), 7.27-7.25 (m, 2H), $7.03(\mathrm{dd}, J=3.8,1.2 \mathrm{~Hz}, 1 \mathrm{H})$, 6.89-6.86 (m, 1H), $6.46(\mathrm{dd}, J=3.8,1.2 \mathrm{~Hz}, 1 \mathrm{H}), 5.30(\mathrm{~s}, 1 \mathrm{H}), 1.70(\mathrm{~s}, 9 \mathrm{H})$.

${ }^{13}$ C-NMR $\left(75 \mathrm{MHz}, \mathrm{CDCl}_{3}\right): \delta=153.2\left(\mathrm{C}_{\mathrm{q}}\right), 146.9\left(\mathrm{C}_{\mathrm{q}}\right), 143.0\left(\mathrm{C}_{\mathrm{q}}\right), 139.5\left(\mathrm{C}_{\mathrm{q}}\right), 139.0\left(\mathrm{C}_{\mathrm{q}}\right)$, 129.7 (CH), $128.9(\mathrm{CH}), 127.8(\mathrm{CH}), 127.8(\mathrm{CH}), 127.1(\mathrm{CH}), 126.9(\mathrm{CH}), 126.4(\mathrm{CH}), 126.1$ $(\mathrm{CH}), 125.2(\mathrm{CH}), 120.9(\mathrm{CH}), 117.1\left(\mathrm{C}_{\mathrm{q}}\right), 109.4\left(\mathrm{C}_{\mathrm{q}}\right), 52.0\left(\mathrm{C}_{\mathrm{q}}\right), 29.0\left(\mathrm{CH}_{3}\right)$.

IR (ATR): 3444, 2962, 1513, 1426, 1223, $699 \mathrm{~cm}^{-1}$.

MS (EI) $m / z$ (relative intens ity) 364 (60) $\left[\mathrm{M}^{+}\right], 349$ (20), 308 (100), 290 (10), 275 (40), 263 (10).

HR-MS (EI) $m / z$ calcd for $\mathrm{C}_{21} \mathrm{H}_{20} \mathrm{~N}_{2} \mathrm{~S}_{2}\left[\mathrm{M}^{+}\right] 364.1068$, found 364.1064 .

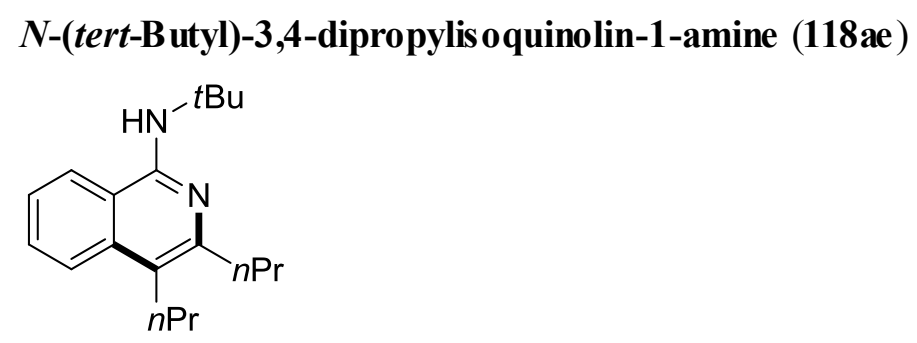

The general procedure $\mathbf{C}$ was followed using $117 \mathbf{a}(88.0 \mathrm{mg}, 0.50 \mathrm{mmol})$ and $1 \mathrm{e}(110 \mathrm{mg}$, 1.00 mmol). Purification by column chromatography ( $n$-hexane/EtOAc: $150 / 1)$ yielded 118ae (112 $\mathrm{mg}, 78 \%)$ as a green oil.

${ }^{1} \mathbf{H}-\mathbf{N M R}\left(300 \mathrm{MHz}, \mathrm{CDCl}_{3}\right) \delta=7.83(\mathrm{~d}, J=8.4 \mathrm{~Hz}, 1 \mathrm{H}), 7.68(\mathrm{~d}, J=8.4 \mathrm{~Hz}, 1 \mathrm{H}), 7.58-7.52$ 
(m, 1H), 7.38-7.32 (m, 1H), 4.95 (s, 1H), 2.87-2.75 (m, 4H), 1.89-1.79 (m, 2H), 1.66-1.57 (m, 2H), $1.60(\mathrm{~s}, 9 \mathrm{H}), 1.07$ (t, $J=7.3 \mathrm{~Hz}, 3 \mathrm{H}), 1.01(\mathrm{t}, J=7.3 \mathrm{~Hz}, 3 \mathrm{H})$.

${ }^{13}$ C-NMR $\left(75 \mathrm{MHz}, \mathrm{CDCl}_{3}\right) \delta=152.3\left(\mathrm{C}_{\mathrm{q}}\right), 150.4\left(\mathrm{C}_{\mathrm{q}}\right), 136.7\left(\mathrm{C}_{\mathrm{q}}\right), 128.7(\mathrm{CH}), 123.8(\mathrm{CH})$, 123.7 (CH), 121.6 (CH), 117.1 $\left(\mathrm{C}_{\mathrm{q}}\right), 116.5\left(\mathrm{C}_{\mathrm{q}}\right), 51.5\left(\mathrm{C}_{\mathrm{q}}\right), 37.1\left(\mathrm{CH}_{2}\right), 29.5\left(\mathrm{CH}_{2}\right), 29.4\left(\mathrm{CH}_{3}\right)$, $24.1\left(\mathrm{CH}_{2}\right), 22.7\left(\mathrm{CH}_{2}\right), 14.6\left(\mathrm{CH}_{3}\right), 14.4\left(\mathrm{CH}_{3}\right)$.

IR (ATR): 3482, 2957, 1518, 1416, 1209, $758 \mathrm{~cm}^{-1}$.

MS (EI) $m / z$ (relative intensity) $284(20)\left[\mathrm{M}^{+}\right], 269$ (15), 255 (10), 228 (25), 213 (10), 199 (100).

HR-MS (EI) $m / z$ calcd for $\mathrm{C}_{19} \mathrm{H}_{28} \mathrm{~N}_{2}\left[\mathrm{M}^{+}\right]$284.2252, found 284.2239 .

\section{$N$-(tert-B utyl)-3,4-die thylisoquinolin-1-amine (118al)}<smiles>CCc1nc(NC(C)(C)C)c2ccccc2c1CC</smiles>

The general procedure $\mathbf{C}$ was followed us ing $\mathbf{1 1 7} \mathbf{a}(88.0 \mathrm{mg}, 0.50 \mathrm{mmol})$ and $\mathbf{1 1}(82 \mathrm{mg}, 1.00$ mmol). Purification by column chromatography ( $n$-hexane/EtOAc: 100/1) yielded 118al (107 $\mathrm{mg}, 84 \%)$ as a green oil.

${ }^{1}$ H-NMR $\left(300 \mathrm{MHz}, \mathrm{CDCl}_{3}\right): \delta=7.83(\mathrm{~d}, J=8.5 \mathrm{~Hz}, 1 \mathrm{H}), 7.66(\mathrm{~d}, J=8.5 \mathrm{~Hz}, 1 \mathrm{H}$ ), 7.53 (ddd, $J=8.5,6.8,1.1 \mathrm{~Hz}, 1 \mathrm{H}), 7.33$ (ddd, $J=8.5,6.8,1.1 \mathrm{~Hz}, 1 \mathrm{H}), 4.94$ (s, 1H), 2.89 (q, $J=$ $7.6 \mathrm{~Hz}, 2 \mathrm{H}), 2.81(\mathrm{q}, J=7.6 \mathrm{~Hz}, 2 \mathrm{H}), 1.58(\mathrm{~s}, 9 \mathrm{H}), 1.33(\mathrm{t}, J=7.6 \mathrm{~Hz}, 3 \mathrm{H}), 1.21$ (t, $J=7.6 \mathrm{~Hz}$, $3 \mathrm{H})$.

${ }^{13}$ C-NMR $\left(75 \mathrm{MHz}, \mathrm{CDCl}_{3}\right): \delta=152.4\left(\mathrm{C}_{\mathrm{q}}\right), 151.2\left(\mathrm{C}_{\mathrm{q}}\right), 136.4\left(\mathrm{C}_{\mathrm{q}}\right), 128.8(\mathrm{CH}), 123.8(\mathrm{CH})$, 123.4 (CH), 121.6 (CH), 117.3 $\left(\mathrm{C}_{\mathrm{q}}\right), 117.1\left(\mathrm{C}_{\mathrm{q}}\right), 51.5\left(\mathrm{C}_{\mathrm{q}}\right), 29.3\left(\mathrm{CH}_{3}\right), 28.0\left(\mathrm{CH}_{2}\right), 20.2\left(\mathrm{CH}_{2}\right)$, $15.2\left(\mathrm{CH}_{3}\right), 14.1\left(\mathrm{CH}_{3}\right)$.

IR (ATR): 3458, 2961, 1518, 1417, 1210, $758 \mathrm{~cm}^{-1}$.

MS (EI) $m / z$ (relative intensity) $256(20)\left[\mathrm{M}^{+}\right], 241$ (20), 228 (10), 200 (45), 185 (100), 171 (60).

HR-MS (EI) $m / z$ calcd for $\mathrm{C}_{17} \mathrm{H}_{24} \mathrm{~N}_{2}\left[\mathrm{M}^{+}\right] 256.1939$, found 256.1946 . 


\section{$N$-(tert-B utyl)-4-n-butyl-3-(4-methoxyphenyl)isoquinolin-1-amine (118ah)}<smiles>CCCCc1c(-c2ccc(OC)cc2)nc(NC(C)(C)C)c2ccccc12</smiles>

The general procedure $\mathbf{C}$ was followed using $\mathbf{1 1 7 a}(88.0 \mathrm{mg}, 0.50 \mathrm{mmol})$ and $\mathbf{1 h}(188 \mathrm{mg}$, 1.00 mmol). Purification by column chromatography ( $n$-hexane/EtOAc: $100 / 1)$ yielded 118ah (83 mg, 46\%) as a white solid. M. p.: $115-117^{\circ} \mathrm{C}$.

${ }^{1}$ H-NMR $\left(300 \mathrm{MHz}, \mathrm{CDCl}_{3}\right): \delta=7.99(\mathrm{~d}, J=8.8 \mathrm{~Hz}, 1 \mathrm{H}), 7.72(\mathrm{~d}, J=8.4 \mathrm{~Hz}, 1 \mathrm{H}), 7.59$ (dd, $J=8.8,7.2 \mathrm{~Hz}, 1 \mathrm{H}), 7.52(\mathrm{~d}, J=8.4,7.2 \mathrm{~Hz}, 2 \mathrm{H}), 7.41(\mathrm{~d}, J=7.8 \mathrm{~Hz}, 1 \mathrm{H}), 6.97(\mathrm{~d}, J=8.6$ $\mathrm{Hz}, 2 \mathrm{H}), 5.01(\mathrm{~s}, 1 \mathrm{H}), 3.87(\mathrm{~s}, 3 \mathrm{H}), 2.93-2.87(\mathrm{~m}, 2 \mathrm{H}), 1.69-1.56(\mathrm{~m}, 2 \mathrm{H}), 1.55(\mathrm{~s}, 9 \mathrm{H})$, $1.42-1.35(\mathrm{~m}, 2 \mathrm{H}), 0.99(\mathrm{t}, J=7.3 \mathrm{~Hz}, 3 \mathrm{H})$.

${ }^{13}$ C-NMR (75 MHz, $\left.\mathrm{CDCl}_{3}\right): \delta=158.5\left(\mathrm{C}_{\mathrm{q}}\right), 152.0\left(\mathrm{C}_{\mathrm{q}}\right), 149.0\left(\mathrm{C}_{\mathrm{q}}\right), 136.9\left(\mathrm{C}_{\mathrm{q}}\right), 135.4\left(\mathrm{C}_{\mathrm{q}}\right)$, $130.4(\mathrm{CH}), 128.7(\mathrm{CH}), 124.6(\mathrm{CH}), 124.4(\mathrm{CH}), 121.4(\mathrm{CH}), 117.8\left(\mathrm{C}_{\mathrm{q}}\right), 117.3\left(\mathrm{C}_{\mathrm{q}}\right), 112.9$ $(\mathrm{CH}), 55.2\left(\mathrm{CH}_{3}\right), 51.4\left(\mathrm{C}_{\mathrm{q}}\right), 33.4\left(\mathrm{CH}_{2}\right), 29.4\left(\mathrm{CH}_{3}\right), 27.8\left(\mathrm{CH}_{2}\right), 22.8\left(\mathrm{CH}_{2}\right), 14.0\left(\mathrm{CH}_{3}\right)$.

IR (ATR): 3448, 2953, 1510, 1419, 1228, $830 \mathrm{~cm}^{-1}$.

MS (EI) $m / z$ (relative intensity) $362(30)\left[\mathrm{M}^{+}\right], 347$ (10), 319 (10), 306 (35), 263 (100), 248 (20).

HR-MS (EI) $m / z$ calcd for $\mathrm{C}_{24} \mathrm{H}_{30} \mathrm{~N}_{2} \mathrm{O}\left[\mathrm{M}^{+}\right] 362.2358$, found 362.2347 .

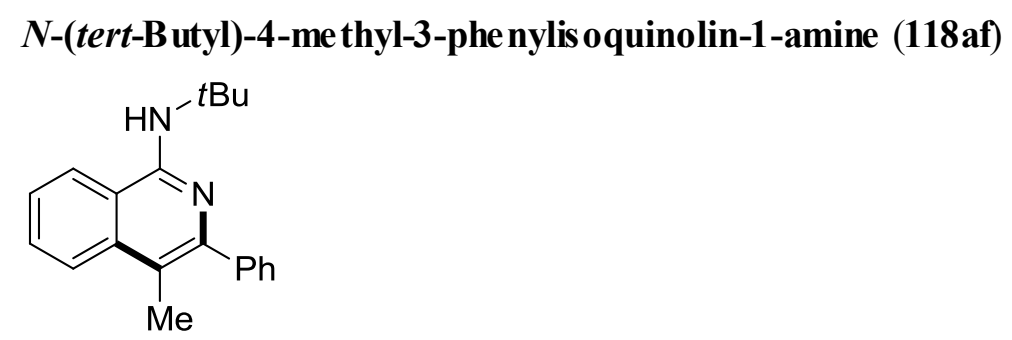

The general procedure $\mathbf{C}$ was followed using $117 \mathbf{a}(88.0 \mathrm{mg}, 0.50 \mathrm{mmol})$ and $\mathbf{1 f}(116 \mathrm{mg}$, 1.00 mmol). Purification by column chromatography ( $n$-hexane/EtOAc: $150 / 1)$ yie lded 118af (105 $\mathrm{mg}, 72 \%)$ as a yellow oil.

${ }^{1} \mathbf{H}-\mathbf{N M R}\left(300 \mathrm{MHz}, \mathrm{CDCl}_{3}\right): \delta=7.93(\mathrm{~d}, J=8.4 \mathrm{~Hz}, 1 \mathrm{H}), 7.75(\mathrm{~d}, J=8.4 \mathrm{~Hz}, 1 \mathrm{H})$, 7.70-7.66 (m, 2H), 7.65-7.62 (m, 1H), 7.49-7.43 (m, 3H), 7.40-7.34 (m, 1H), 5.05 (s, 1H), 
$2.53(\mathrm{~s}, 3 \mathrm{H}), 1.60(\mathrm{~s}, 9 \mathrm{H})$.

${ }^{13}$ C-NMR (75 MHz, $\left.\mathrm{CDCl}_{3}\right): \delta=152.2\left(\mathrm{C}_{\mathrm{q}}\right), 149.0\left(\mathrm{C}_{\mathrm{q}}\right), 142.5\left(\mathrm{C}_{\mathrm{q}}\right), 137.7\left(\mathrm{C}_{\mathrm{q}}\right), 130.1(\mathrm{CH})$, $129.2(\mathrm{CH}), 127.5(\mathrm{CH}), 126.8(\mathrm{CH}), 124.9(\mathrm{CH}), 124.4(\mathrm{CH}), 121.5(\mathrm{CH}), 117.6\left(\mathrm{C}_{\mathrm{q}}\right), 112.2$ $\left(\mathrm{C}_{\mathrm{q}}\right), 51.7\left(\mathrm{C}_{\mathrm{q}}\right), 29.4\left(\mathrm{CH}_{3}\right), 15.2\left(\mathrm{CH}_{3}\right)$.

IR (ATR): 3452, 2960, 1517, 1421, 1211, $699 \mathrm{~cm}^{-1}$.

MS (EI) $m / z$ (relative intensity) $290(25)\left[\mathrm{M}^{+}\right], 275$ (15), 273 (95), 234 (100), 216 (20).

HR-MS (ESI) $m / z$ calcd for $\mathrm{C}_{20} \mathrm{H}_{23} \mathrm{~N}_{2}\left[\mathrm{M}+\mathrm{H}^{+}\right] 291.1861$, found 291.1856 .

\section{$N$-(tert-B utyl)-6-methyl-3,4-diphe nylisoquinolin-1-amine (118ba)}<smiles>Cc1ccc2c(NC(C)C)nc(-c3ccccc3)c(-c3ccccc3)c2c1</smiles>

The general procedure $\mathbf{C}$ was followed using $\mathbf{1 1 7 b}(95.0 \mathrm{mg}, 0.50 \mathrm{mmol})$ and diphenylacetylene (1a) (178 mg, $1.00 \mathrm{mmol})$. Purification by column chromatography ( $n$-hexane/EtOAc: $150 / 1)$ yielded $\mathbf{1 1 8 b a}\left(131 \mathrm{mg}, 72 \%\right.$ ) as a yellow solid. M. p.: $92-95^{\circ} \mathrm{C}$. ${ }^{1}$ H-NMR (300 MHz, $\left.\mathrm{CDCl}_{3}\right): \delta=7.64(\mathrm{~d}, J=8.9 \mathrm{~Hz}, 1 \mathrm{H}), 7.40-7.25(\mathrm{~m}, 7 \mathrm{H}), 7.22-7.12(\mathrm{~m}$, 5H), $5.15(\mathrm{~s}, 1 \mathrm{H}), 2.36(\mathrm{~s}, 3 \mathrm{H}), 1.63(\mathrm{~s}, 9 \mathrm{H})$.

${ }^{13}$ C-NMR (75 MHz, $\left.\mathrm{CDCl}_{3}\right): \delta=153.3\left(\mathrm{C}_{\mathrm{q}}\right), 148.1\left(\mathrm{C}_{\mathrm{q}}\right), 142.1\left(\mathrm{C}_{\mathrm{q}}\right), 139.2\left(\mathrm{C}_{\mathrm{q}}\right), 139.0\left(\mathrm{C}_{\mathrm{q}}\right)$, $137.6\left(\mathrm{C}_{\mathrm{q}}\right), 132.1(\mathrm{CH}), 130.3(\mathrm{CH}), 128.1(\mathrm{CH}), 127.1(\mathrm{CH}), 127.0(\mathrm{CH}), 126.4(\mathrm{CH}), 126.3$ $(\mathrm{CH}), 125.3(\mathrm{CH}), 121.0(\mathrm{CH}), 120.0\left(\mathrm{C}_{\mathrm{q}}\right), 115.2\left(\mathrm{C}_{\mathrm{q}}\right), 51.9\left(\mathrm{C}_{\mathrm{q}}\right), 29.4\left(\mathrm{CH}_{3}\right), 21.9\left(\mathrm{CH}_{3}\right)$.

IR (ATR): 3456, 2957, 1573, 1415, 1206, $697 \mathrm{~cm}^{-1}$.

MS (EI) $m / z$ (relative intensity) $366(40)\left[\mathrm{M}^{+}\right], 351$ (20), 310 (100), 309 (95), 292 (20), 277 (20).

HR-MS (EI) $m / z$ calcd for $\mathrm{C}_{26} \mathrm{H}_{26} \mathrm{~N}_{2}\left[\mathrm{M}^{+}\right]$366.2096, found 366.2086.

$N$-(tert-B utyl)-6-me thoxy-3,4-diphe nylis oquinolin-1-amine (118ca)<smiles>COc1ccc2c(NCC(C)C)nc(-c3ccccc3)c(-c3ccccc3)c2c1</smiles> 
The general procedure $\mathbf{C}$ was followed using $117 \mathrm{c}(103 \mathrm{mg}, 0.50 \mathrm{mmol})$ and diphenylacetylene (1a) (178 mg, $1.00 \mathrm{mmol}$ ). Purification by column chromatography ( $n$-hexane/EtOAc: $100 / 1)$ yielded $118 \mathrm{ca}\left(133 \mathrm{mg}, 70 \%\right.$ ) as a white solid. M. p.: $138-139{ }^{\circ} \mathrm{C}$. ${ }^{1} \mathbf{H}-\mathbf{N M R}\left(300 \mathrm{MHz}, \mathrm{CDCl}_{3}\right): \delta=7.66(\mathrm{~d}, J=9.4 \mathrm{~Hz}, 1 \mathrm{H}), 7.40-7.38(\mathrm{~m}, 1 \mathrm{H}), 7.37(\mathrm{~d}, J=$ $1.5 \mathrm{~Hz}, 1 \mathrm{H}), 7.34-7.25(\mathrm{~m}, 3 \mathrm{H}), 7.22-7.19(\mathrm{~m}, 2 \mathrm{H}), 7.16-7.12(\mathrm{~m}, 3 \mathrm{H}), 7.03(\mathrm{dd}, J=9.0,2.6$ $\mathrm{Hz}, 1 \mathrm{H}), 6.85(\mathrm{~d}, J=2.6 \mathrm{~Hz}, 1 \mathrm{H}), 5.08$ (s, 1H), 3.68 (s, 3H), 1.62 (s, 9H).

${ }^{13}$ C-NMR (75 MHz, $\left.\mathrm{CDCl}_{3}\right): \delta=160.1\left(\mathrm{C}_{\mathrm{q}}\right), 153.3\left(\mathrm{C}_{\mathrm{q}}\right), 148.7\left(\mathrm{C}_{\mathrm{q}}\right), 142.0\left(\mathrm{C}_{\mathrm{q}}\right), 139.4\left(\mathrm{C}_{\mathrm{q}}\right)$, $139.0\left(\mathrm{C}_{\mathrm{q}}\right), 132.0(\mathrm{CH}), 130.4(\mathrm{CH}), 128.2(\mathrm{CH}), 127.0(\mathrm{CH}), 126.5(\mathrm{CH}), 126.4(\mathrm{CH}), 123.0$ $(\mathrm{CH}), 120.0\left(\mathrm{C}_{\mathrm{q}}\right), 116.3(\mathrm{CH}), 112.1\left(\mathrm{C}_{\mathrm{q}}\right), 105.6(\mathrm{CH}), 55.1\left(\mathrm{CH}_{3}\right), 51.8\left(\mathrm{C}_{\mathrm{q}}\right), 29.5\left(\mathrm{CH}_{3}\right)$.

IR (ATR): 3426, 2954, 1610, 1515, 1417, 1213, $703 \mathrm{~cm}^{-1}$.

MS (EI) $m / z$ (relative intensity) $382(25)\left[\mathrm{M}^{+}\right], 367$ (15), 326 (100), 308 (10), 293 (10), 282 (15).

HR-MS (EI) $m / z$ calcd for $\mathrm{C}_{26} \mathrm{H}_{26} \mathrm{~N}_{2} \mathrm{O}\left[\mathrm{M}^{+}\right]$382.2045, found 382.2047.

The spectral data were in accordance with those reported in the literature. ${ }^{146}$

\section{$N$-(tert-B utyl)-6-fluoro-3,4-diphe nylis oquinolin-1-amine (118da)}<smiles>CC(C)CNc1nc(-c2ccccc2)c(-c2ccccc2)c2cc(F)ccc12</smiles>

The general procedure $\mathbf{C}$ was followed using 117d $(97.0 \mathrm{mg}, 0.50 \mathrm{mmol})$ and diphenylacetylene (1a) (178 mg, $1.00 \mathrm{mmol})$. Purification by column chromatography ( $n$-hexane/EtOAc: $120 / 1)$ yielded $118 d a\left(142 \mathrm{mg}, 77 \%\right.$ ) as a yellow solid. M. p.: $175-176^{\circ} \mathrm{C}$. ${ }^{1} \mathbf{H}-\mathbf{N M R}\left(300 \mathrm{MHz}, \mathrm{CDCl}_{3}\right): \delta=7.77-7.72(\mathrm{~m}, 1 \mathrm{H}), 7.40-7.37(\mathrm{~m}, 2 \mathrm{H}), 7.37-7.28(\mathrm{~m}, 3 \mathrm{H})$, 7.20-7.12 (m, 7H), $5.15(\mathrm{~s}, 1 \mathrm{H}), 1.63(\mathrm{~s}, 9 \mathrm{H})$.

${ }^{13}$ C-NMR $\left(75 \mathrm{MHz}, \mathrm{CDCl}_{3}\right): \delta=163.0\left(\mathrm{C}_{\mathrm{q}}, J_{\mathrm{C}-\mathrm{F}}=249.0 \mathrm{~Hz}\right), 153.1\left(\mathrm{C}_{\mathrm{q}}\right), 149.2\left(\mathrm{C}_{\mathrm{q}}\right), 141.5$ $\left(\mathrm{C}_{\mathrm{q}}\right), 139.7\left(\mathrm{C}_{\mathrm{q}}, J_{\mathrm{C}-\mathrm{F}}=9.1 \mathrm{~Hz}\right), 138.3\left(\mathrm{C}_{\mathrm{q}}\right), 131.9(\mathrm{CH}), 130.3(\mathrm{CH}), 128.4(\mathrm{CH}), 127.1(\mathrm{CH})$, $126.8(\mathrm{CH}), 126.7(\mathrm{CH}), 123.9\left(\mathrm{CH}, J_{\mathrm{C}-\mathrm{F}}=8.1 \mathrm{~Hz}\right), 120.0\left(\mathrm{C}_{\mathrm{q}}\right), 114.6\left(\mathrm{CH}, J_{\mathrm{C}-\mathrm{F}}=24.8 \mathrm{~Hz}\right)$, $114.0\left(\mathrm{C}_{\mathrm{q}}\right), 110.2\left(\mathrm{CH}, J_{\mathrm{C}-\mathrm{F}}=22.2 \mathrm{~Hz}\right), 52.0\left(\mathrm{C}_{\mathrm{q}}\right), 29.4\left(\mathrm{CH}_{3}\right)$.

${ }^{19}$ F-NMR $\left(282 \mathrm{MHz}, \mathrm{CDCl}_{3}\right): \delta=-(109.9-110.0),(\mathrm{m})$. 
IR (ATR): 3432, 2970, 1575, 1519, 1208, $696 \mathrm{~cm}^{-1}$.

MS (EI) $m / z$ (relative intensity) 370 (30) $\left[\mathrm{M}^{+}\right], 355$ (15), 314 (95), 313 (100), 296 (20), 208

HR-MS (EI) $m / z$ calcd for $\mathrm{C}_{25} \mathrm{H}_{23} \mathrm{FN}_{2}\left[\mathrm{M}^{+}\right] 370.1845$, found 370.1842 .

\section{$N$-(tert-B utyl)-6-chloro-3,4-diphenylisoquinolin-1-amine (118ea)}<smiles>CC(C)(C)Nc1nc(-c2ccccc2)c(-c2ccccc2)c2cc(Cl)ccc12</smiles>

The general procedure $\mathbf{C}$ was followed using $117 \mathrm{e}(105 \mathrm{mg}, 0.50 \mathrm{mmol})$ and diphenylacetylene (1a) (178 mg, $1.00 \mathrm{mmol})$. Purification by column chromatography ( $n$-hexane/EtOAc: $120 / 1)$ yielded 118 ea (152 mg, 79\%) as a yellow solid. M. p. : $175-176{ }^{\circ} \mathrm{C}$.

${ }^{1}$ H-NMR $\left(300 \mathrm{MHz}, \mathrm{CDCl}_{3}\right): \delta=7.67(\mathrm{~d}, J=8.8 \mathrm{~Hz}, 1 \mathrm{H}), 7.49(\mathrm{~d}, J=2.1 \mathrm{~Hz}, 1 \mathrm{H})$, 7.39-7.29 (m, 6H), 7.20-7.13 (m, 5H), $5.15(\mathrm{~s}, 1 \mathrm{H}), 1.62(\mathrm{~s}, 9 \mathrm{H})$.

${ }^{13}$ C-NMR (75 MHz, CDCl $): \delta=153.1\left(\mathrm{C}_{\mathrm{q}}\right), 149.4\left(\mathrm{C}_{\mathrm{q}}\right), 141.5\left(\mathrm{C}_{\mathrm{q}}\right), 138.8\left(\mathrm{C}_{\mathrm{q}}\right), 138.1\left(\mathrm{C}_{\mathrm{q}}\right)$, $135.6\left(\mathrm{C}_{\mathrm{q}}\right), 131.9(\mathrm{CH}), 130.3(\mathrm{CH}), 128.4(\mathrm{CH}), 127.2(\mathrm{CH}), 126.8(\mathrm{CH}), 126.7(\mathrm{CH}), 125.8$ $(\mathrm{CH}), 125.2(\mathrm{CH}), 122.9(\mathrm{CH}), 119.5\left(\mathrm{C}_{\mathrm{q}}\right), 115.2\left(\mathrm{C}_{\mathrm{q}}\right), 52.1\left(\mathrm{C}_{\mathrm{q}}\right), 29.4\left(\mathrm{CH}_{3}\right)$.

IR (ATR): 3465, 2963, 1571, 1414, 1211, $695 \mathrm{~cm}^{-1}$.

MS (EI) $m / z$ (relative intens ity) $386(35)\left[\mathrm{M}^{+}\right], 371$ (15), 330 (100), 312 (25), 277 (20), 199 (5).

HR-MS (EI) $m / z$ calcd for $\mathrm{C}_{25} \mathrm{H}_{23} \mathrm{CIN}_{2}\left[\mathrm{M}^{+}\right] 386.1550$, found 386.1538 .

The spectral data were in accordance with those reported in the literature. ${ }^{146}$

\section{6-Bromo- $N$-(tert-butyl)-3,4-diphe nylisoquinolin-1-amine (118fa)}<smiles>CC(C)(C)CNc1nc(-c2ccccc2)c(-c2ccccc2)c2cc(Br)ccc12</smiles>

The general procedure $\mathbf{C}$ was followed using $117 \mathbf{f}(127 \mathrm{mg}, 0.50 \mathrm{mmol})$ and diphenylacetylene (1a) (178 mg, $1.00 \mathrm{mmol})$. Purification by column chromatography 
( $n$-hexane/EtOAc: $150 / 1)$ yielded $\mathbf{1 1 8 f a}\left(154 \mathrm{mg}, 72 \%\right.$ ) as a yellow solid. M. p.: $176-178{ }^{\circ} \mathrm{C}$. ${ }^{1} \mathbf{H}-\mathbf{N M R}\left(300 \mathrm{MHz}, \mathrm{CDCl}_{3}\right): \delta=7.69(\mathrm{~d}, J=1.8 \mathrm{~Hz}, 1 \mathrm{H}), 7.62(\mathrm{~d}, J=8.8 \mathrm{~Hz}, 1 \mathrm{H}), 7.50$ (dd, $J=8.8,1.8 \mathrm{~Hz}, 1 \mathrm{H}), 7.40-7.31(\mathrm{~m}, 5 \mathrm{H}), 7.21-7.15$ (m, 5H), 5.17 (s, 1H), 1.64 (s, 9H).

${ }^{13}$ C-NMR $\left(75 \mathrm{MHz}, \mathrm{CDCl}_{3}\right): \delta=153.2\left(\mathrm{C}_{\mathrm{q}}\right), 149.4\left(\mathrm{C}_{\mathrm{q}}\right), 141.5\left(\mathrm{C}_{\mathrm{q}}\right), 139.0\left(\mathrm{C}_{\mathrm{q}}\right), 138.0\left(\mathrm{C}_{\mathrm{q}}\right)$, $131.9(\mathrm{CH}), 130.3(\mathrm{CH}), 128.4(\mathrm{CH}), 128.4(\mathrm{CH}), 128.4(\mathrm{CH}), 127.2(\mathrm{CH}), 126.8(\mathrm{CH}), 126.7$ $(\mathrm{CH}), 124.1\left(\mathrm{C}_{\mathrm{q}}\right), 122.9(\mathrm{CH}), 119.3\left(\mathrm{C}_{\mathrm{q}}\right), 115.5\left(\mathrm{C}_{\mathrm{q}}\right), 52.1\left(\mathrm{C}_{\mathrm{q}}\right), 29.3\left(\mathrm{CH}_{3}\right)$.

IR (ATR): 3443, 2965, 1568, 1414, 1211, $695 \mathrm{~cm}^{-1}$.

MS (EI) $m / z$ (relative intensity) 430, $432(30)\left[\mathrm{M}^{+}\right], 415,417$ (15), 374, 376 (100), 356, 358 (10), 293, $295(10), 277(25)$.

HR-MS (ESI) $m / z$ calcd for $\mathrm{C}_{25} \mathrm{H}_{24} \mathrm{~N}_{2} \mathrm{Br}\left[\mathrm{M}+\mathrm{H}^{+}\right]$431.1123, found 431.1113 .

\section{$N$-(tert-B utyl)-6-iodo-3,4-diphenylis oquinolin-1-amine (118ga)}<smiles>CC(C)(C)Nc1nc(-c2ccccc2)c(-c2ccccc2)c2cc(I)ccc12</smiles>

The general procedure $\mathbf{C}$ was followed using $\mathbf{1 1 7 g}(151 \mathrm{mg}, 0.50 \mathrm{mmol})$ and diphenylacetylene (1a) (178 mg, $1.00 \mathrm{mmol})$. Purification by column chromatography (n-hexane/EtOAc: 120/1) yielded 118ga (105 mg, 44\%) as white solid. M. p.: $179-181{ }^{\circ} \mathrm{C}$. ${ }^{1} \mathbf{H}-\mathbf{N M R}\left(300 \mathrm{MHz}, \mathrm{CDCl}_{3}\right): \delta=7.89(\mathrm{~d}, J=1.8 \mathrm{~Hz}, 1 \mathrm{H}), 7.67(\mathrm{dd}, J=8.8,1.8 \mathrm{~Hz}, 1 \mathrm{H})$, $7.44(\mathrm{dd}, J=8.8 \mathrm{~Hz}, 1 \mathrm{H}), 7.38-7.29(\mathrm{~m}, 5 \mathrm{H}), 7.19-7.13(\mathrm{~m}, 5 \mathrm{H}), 5.15(\mathrm{~s}, 1 \mathrm{H}), 1.62(\mathrm{~s}, 9 \mathrm{H})$.

${ }^{13}$ C-NMR (75 MHz, $\left.\mathrm{CDCl}_{3}\right): \delta=153.3\left(\mathrm{C}_{\mathrm{q}}\right), 149.2\left(\mathrm{C}_{\mathrm{q}}\right), 141.5\left(\mathrm{C}_{\mathrm{q}}\right), 139.2\left(\mathrm{C}_{\mathrm{q}}\right), 138.0\left(\mathrm{C}_{\mathrm{q}}\right)$, $135.0(\mathrm{CH}), 133.8(\mathrm{CH}), 132.0(\mathrm{CH}), 130.3(\mathrm{CH}), 128.4(\mathrm{CH}), 127.1(\mathrm{CH}), 126.8(\mathrm{CH}), 126.7$ $(\mathrm{CH}), 122.7(\mathrm{CH}), 119.0\left(\mathrm{C}_{\mathrm{q}}\right), 115.8\left(\mathrm{C}_{\mathrm{q}}\right), 96.5\left(\mathrm{C}_{\mathrm{q}}\right), 52.0\left(\mathrm{C}_{\mathrm{q}}\right), 29.3\left(\mathrm{CH}_{3}\right)$.

IR (ATR): 3452, 2958, 1566, 1413, 1210, $693 \mathrm{~cm}^{-1}$.

MS (EI) $m / z$ (relative intensity) $478(50)\left[\mathrm{M}^{+}\right], 463$ (15), 422 (100), 421 (90), 404 (5), 277 (20).

HR-MS (EI) $m / z$ calcd for $\mathrm{C}_{25} \mathrm{H}_{23} \mathrm{~N}_{2} \mathrm{I}\left[\mathrm{M}^{+}\right]$478.0906, found 478.0905. 
<smiles>CC(C)(C)Nc1nc(-c2ccccc2)c(-c2ccccc2)c2cc([N+](=O)[O-])ccc12</smiles>

The general procedure $\mathbf{C}$ was followed using $\mathbf{1 1 7 h}(111 \mathrm{mg}, 0.50 \mathrm{mmol})$ and diphenylacetylene (1a) (178 mg, $1.00 \mathrm{mmol})$. Purification by column chromatography ( $n$-hexane/EtOAc: 80/1) yielded 118ha (99 mg, 59\%) as a red solid. M. p.: 200-202 ${ }^{\circ} \mathrm{C}$. ${ }^{1}$ H-NMR $\left(300 \mathrm{MHz}, \mathrm{CDCl}_{3}\right): \delta=8.44(\mathrm{~d}, J=2.3 \mathrm{~Hz}, 1 \mathrm{H}), 8.14(\mathrm{dd}, J=9.0,2.3 \mathrm{~Hz}, 1 \mathrm{H})$, $7.88(\mathrm{~d}, J=9.0 \mathrm{~Hz}, 1 \mathrm{H}), 7.39-7.33(\mathrm{~m}, 5 \mathrm{H}), 7.20-7.16(\mathrm{~m}, 5 \mathrm{H}), 5.32(\mathrm{~s}, 1 \mathrm{H}), 1.64(\mathrm{~s}, 9 \mathrm{H})$.

${ }^{13}$ C-NMR $\left(75 \mathrm{MHz}, \mathrm{CDCl}_{3}\right): \delta=152.9\left(\mathrm{C}_{\mathrm{q}}\right), 148.1\left(\mathrm{C}_{\mathrm{q}}\right), 140.9\left(\mathrm{C}_{\mathrm{q}}\right), 137.6\left(\mathrm{C}_{\mathrm{q}}\right), 137.2\left(\mathrm{C}_{\mathrm{q}}\right)$, $131.8(\mathrm{CH}), 130.3(\mathrm{CH}), 128.7(\mathrm{CH}), 127.4(\mathrm{CH}), 127.3(\mathrm{CH}), 127.1(\mathrm{CH}), 123.3(\mathrm{CH}), 122.9$ $\left(\mathrm{C}_{\mathrm{q}}\right), 122.4(\mathrm{CH}), 121.0\left(\mathrm{C}_{\mathrm{q}}\right), 119.0\left(\mathrm{C}_{\mathrm{q}}\right), 118.5(\mathrm{CH}), 52.4\left(\mathrm{C}_{\mathrm{q}}\right), 29.2\left(\mathrm{CH}_{3}\right)$.

IR (ATR): 3444, 2916, 1530, 1342, 1210, $697 \mathrm{~cm}^{-1}$.

MS (EI) $m / z$ (relative intens ity) 397 (30) $\left[\mathrm{M}^{+}\right], 382$ (10), 341 (100), 311 (20), 294 (30), 277 (20).

HR-MS (EI) $m / z$ calcd for $\mathrm{C}_{25} \mathrm{H}_{23} \mathrm{~N}_{3} \mathrm{O}_{2}\left[\mathrm{M}^{+}\right] 397.1790$, found 397.1793.

\section{$N$-(tert-B utyl)-7-methyl-3,4-diphe nylisoquinolin-1-amine (118ia)}<smiles>Cc1ccc2c(-c3ccccc3)c(-c3ccccc3)nc(NC(C)C)c2c1</smiles>

The general procedure $\mathbf{C}$ was followed using $\mathbf{1 1 7 i}(95.0 \mathrm{mg}, 0.50 \mathrm{mmol})$ and diphenylacetylene (1a) (178 mg, $1.00 \mathrm{mmol})$. Purification by column chromatography ( $n$-hexane/EtOAc: 100/1) yielded 118ia (113 mg, 62\%) as a white solid. M. p.: 215-217 ${ }^{\circ} \mathrm{C}$. ${ }^{1}$ H-NMR $\left(300 \mathrm{MHz}, \mathrm{CDCl}_{3}\right): \delta=7.50(\mathrm{~s}, 1 \mathrm{H}), 7.44(\mathrm{~d}, J=8.6 \mathrm{~Hz}, 1 \mathrm{H}), 7.41-7.38(\mathrm{~m}, 2 \mathrm{H})$, 7.34-7.27 (m, 4H), 7.22-7.19 (m, 2H), 7.16-7.11 (m, 3H), 5.15 (s, 1H), 2.51 (s, 3H), 1.64 (s, 9H).

${ }^{13}$ C-NMR $\left(75 \mathrm{MHz}, \mathrm{CDCl}_{3}\right): \delta=152.9\left(\mathrm{C}_{\mathrm{q}}\right), 147.0\left(\mathrm{C}_{\mathrm{q}}\right), 141.9\left(\mathrm{C}_{\mathrm{q}}\right), 139.0\left(\mathrm{C}_{\mathrm{q}}\right), 135.4\left(\mathrm{C}_{\mathrm{q}}\right)$, $135.0\left(\mathrm{C}_{\mathrm{q}}\right), 132.0(\mathrm{CH}), 131.0(\mathrm{CH}), 130.3(\mathrm{CH}), 128.1(\mathrm{CH}), 127.1(\mathrm{CH}), 126.4(\mathrm{CH}), 126.3$ $(\mathrm{CH}), 126.2(\mathrm{CH}), 120.3(\mathrm{CH}), 120.2\left(\mathrm{C}_{\mathrm{q}}\right), 117.1\left(\mathrm{C}_{\mathrm{q}}\right), 51.8\left(\mathrm{C}_{\mathrm{q}}\right), 29.4\left(\mathrm{CH}_{3}\right), 21.7\left(\mathrm{CH}_{3}\right)$. 
IR (ATR): 3461, 2953, 1570, 1419, 1205, $694 \mathrm{~cm}^{-1}$.

MS (EI) $\mathrm{m} / z$ (relative intensity) $366(30)\left[\mathrm{M}^{+}\right], 351$ (15), 310 (100), 309 (80), 292 (15), 277 (15).

HR-MS (EI) $m / z$ calcd for $\mathrm{C}_{26} \mathrm{H}_{26} \mathrm{~N}_{2}\left[\mathrm{M}^{+}\right]$366.2096, found 366.2078.

The spectral data were in accordance with those reported in the literature. ${ }^{146}$

\section{$N$-(tert-B utyl)-7-(trifluoromethyl)-3,4-diphe nylisoquinolin-1-amine (118ja)}<smiles>CC(C)(C)Nc1nc(-c2ccccc2)c(-c2ccccc2)c2ccc(C(F)(F)F)cc12</smiles>

The general procedure $\mathbf{C}$ was followed using 117j (122 $\mathrm{mg}, 0.50 \mathrm{mmol})$ and diphenylacetylene (1a) (178 mg, $1.00 \mathrm{mmol})$. Purification by column chromatography ( $n$-hexane/EtOAc: $150 / 1)$ yielded $\mathbf{1 1 8 j a ~ ( 1 4 9 ~ m g , ~} 71 \%$ ) as a yellow solid. M. p.: $176-177^{\circ} \mathrm{C}$. ${ }^{1} \mathbf{H}-\mathbf{N M R}\left(300 \mathrm{MHz}, \mathrm{CDCl}_{3}\right): \delta=7.98(\mathrm{~s}, 1 \mathrm{H}), 7.65-7.58(\mathrm{~m}, 2 \mathrm{H}), 7.40-7.37(\mathrm{~m}, 2 \mathrm{H})$, 7.34-7.29 (m, 3H), $7.19(\mathrm{~d}, J=2.1 \mathrm{~Hz}, 1 \mathrm{H}), 7.17(\mathrm{~d}, J=2.0 \mathrm{~Hz}, 2 \mathrm{H}), 7.15(\mathrm{~d}, J=2.8 \mathrm{~Hz}, 2 \mathrm{H})$, $5.24(\mathrm{~s}, 1 \mathrm{H}), 1.64(\mathrm{~s}, 9 \mathrm{H})$.

${ }^{13}$ C-NMR (75 MHz, $\left.\mathrm{CDCl}_{3}\right): \delta=153.4\left(\mathrm{C}_{\mathrm{q}}\right), 150.3\left(\mathrm{C}_{\mathrm{q}}\right), 141.2\left(\mathrm{C}_{\mathrm{q}}\right), 139.3\left(\mathrm{C}_{\mathrm{q}}\right), 138.0\left(\mathrm{C}_{\mathrm{q}}\right)$, $131.8(\mathrm{CH}), 130.3(\mathrm{CH}), 128.3(\mathrm{CH}), 127.2\left(\mathrm{CH}, J_{\mathrm{C}-\mathrm{F}}=6.0 \mathrm{~Hz}\right), 127.2(\mathrm{CH}), 126.8(\mathrm{CH})$, $126.8(\mathrm{CH}), 126.2\left(\mathrm{C}_{\mathrm{q}}, J_{\mathrm{C}-\mathrm{F}}=32.0 \mathrm{~Hz}\right), 124.8\left(\mathrm{CH}, J_{\mathrm{C}-\mathrm{F}}=3.5 \mathrm{~Hz}\right), 124.5\left(\mathrm{C}_{\mathrm{q}}, J_{\mathrm{C}-\mathrm{F}}=271.3 \mathrm{~Hz}\right)$, $119.9\left(\mathrm{C}_{\mathrm{q}}\right), 118.9\left(\mathrm{CH}, J_{\mathrm{C}-\mathrm{F}}=4.5 \mathrm{~Hz}\right), 115.9\left(\mathrm{C}_{\mathrm{q}}\right), 52.3\left(\mathrm{C}_{\mathrm{q}}\right), 29.4\left(\mathrm{CH}_{3}\right)$.

${ }^{19}$ F-NMR $\left(282 \mathrm{MHz}, \mathrm{CDCl}_{3}\right): \delta=-61.96$ (s).

IR (ATR): 3474, 2960, 1524, 1303, 1105, $692 \mathrm{~cm}^{-1}$.

MS (EI) $m / z$ (relative intens ity) 420 (30) $\left[\mathrm{M}^{+}\right], 405$ (15), 364 (100), 363 (95), 346 (15), 277 (10).

HR-MS (EI) $m / z$ calcd for $\mathrm{C}_{26} \mathrm{H}_{23} \mathrm{~N}_{2} \mathrm{~F}_{3}\left[\mathrm{M}-\mathrm{H}^{-}\right]$419.1735, found 419.1733. 
<smiles>CC(C)CNc1nc(CC(C)C)c(C(C)C)c2c3c(ccc12)OCO3</smiles>

The general procedure $\mathbf{C}$ was followed using 117k (110 $\mathrm{mg}, 0.50 \mathrm{mmol})$ and 4-octyne (1e) (110 mg, $1.00 \mathrm{mmol})$. Purification by column chromatography ( $n$-hexane/EtOAc: $100 / 1 \rightarrow 80 / 1)$ yielded 118ke (100 mg, 60\%) and 118ke' (10 mg, 7\%) as a green oil.

${ }^{1}$ H-NMR (300 MHz, CDCl $\left.)_{3}\right): \delta=7.22(\mathrm{~d}, J=8.9 \mathrm{~Hz}, 1 \mathrm{H}), 6.97(\mathrm{~d}, J=8.9 \mathrm{~Hz}, 1 \mathrm{H}), 6.04(\mathrm{~s}$, 2H), $4.79(\mathrm{~s}, 1 \mathrm{H}), 2.90-2.85(\mathrm{~m}, 2 \mathrm{H}), 2.72-2.67(\mathrm{~m}, 2 \mathrm{H}), 1.85-1.73(\mathrm{~m}, 2 \mathrm{H}), 1.58-1.50(\mathrm{~m}$, 2H), 1.54 (s, 9H), 0.99 (t, $J=7.3 \mathrm{~Hz}, 6 \mathrm{H})$.

${ }^{13}$ C-NMR (75 MHz, $\left.\mathrm{CDCl}_{3}\right): \delta=151.9\left(\mathrm{C}_{\mathrm{q}}\right), 151.0\left(\mathrm{C}_{\mathrm{q}}\right), 146.3\left(\mathrm{C}_{\mathrm{q}}\right), 141.4\left(\mathrm{C}_{\mathrm{q}}\right), 124.4\left(\mathrm{C}_{\mathrm{q}}\right)$, $116.2(\mathrm{CH}), 114.6\left(\mathrm{C}_{\mathrm{q}}\right), 114.1\left(\mathrm{C}_{\mathrm{q}}\right), 107.7(\mathrm{CH}), 100.7\left(\mathrm{CH}_{2}\right), 51.6\left(\mathrm{C}_{\mathrm{q}}\right), 36.5\left(\mathrm{CH}_{2}\right), 30.7$ $\left(\mathrm{CH}_{2}\right), 29.4\left(\mathrm{CH}_{3}\right), 25.1\left(\mathrm{CH}_{2}\right), 22.8\left(\mathrm{CH}_{2}\right), 14.4\left(\mathrm{CH}_{3}\right), 14.4\left(\mathrm{CH}_{3}\right)$.

IR (ATR): 3459, 2958, 1525, 1442, 1210, $738 \mathrm{~cm}^{-1}$.

MS (EI) $m / z$ (relative intensity) 328 (55) $\left[\mathrm{M}^{+}\right], 313$ (15), 299 (25), 272 (30), 243 (100), 227 (5).

HR-MS (EI) $m / z$ calcd for $\mathrm{C}_{20} \mathrm{H}_{28} \mathrm{~N}_{2} \mathrm{O}_{2}\left[\mathrm{M}^{+}\right]$328.2151, found 328.2151 .

$N$-(tert-B utyl)-7,8-di-n-propyl-[1,3]dioxolo[4,5-g] is oquinolin-5-amine (118ke ')<smiles>CC(C)CNc1nc(C(C)C)c(C(C)C)c2cc3c(cc12)OCO3</smiles>

${ }^{1} \mathbf{H}-\mathrm{NMR}\left(300 \mathrm{MHz}, \mathrm{CDCl}_{3}\right): \delta=7.11(\mathrm{~s}, 1 \mathrm{H}), 6.97$ (s, 1H), 5.99 (s, 2H), 4.50 (s, 1H), 2.75-2.68 (m, 4H), 1.83-1.76 (m, 2H), 1.60-1.50 (m, 2H), $1.53(\mathrm{~s}, 9 \mathrm{H}), 1.03$ (t, $J=7.2 \mathrm{~Hz}$, $3 \mathrm{H}), 0.99$ (t, $J=7.2 \mathrm{~Hz}, 3 \mathrm{H})$.

${ }^{13}$ C-NMR (75 MHz, $\left.\mathrm{CDCl}_{3}\right): \delta=151.7\left(\mathrm{C}_{\mathrm{q}}\right), 149.6\left(\mathrm{C}_{\mathrm{q}}\right), 149.4\left(\mathrm{C}_{\mathrm{q}}\right), 145.8\left(\mathrm{C}_{\mathrm{q}}\right), 134.4\left(\mathrm{C}_{\mathrm{q}}\right)$, $116.9\left(\mathrm{C}_{\mathrm{q}}\right), 112.6\left(\mathrm{C}_{\mathrm{q}}\right), 101.1\left(\mathrm{CH}_{2}\right), 100.8(\mathrm{CH}), 98.9(\mathrm{CH}), 51.5\left(\mathrm{C}_{\mathrm{q}}\right), 37.0\left(\mathrm{CH}_{2}\right), 30.0\left(\mathrm{CH}_{2}\right)$, $29.5\left(\mathrm{CH}_{3}\right), 23.9\left(\mathrm{CH}_{2}\right), 22.8\left(\mathrm{CH}_{2}\right), 14.6\left(\mathrm{CH}_{3}\right), 14.4\left(\mathrm{CH}_{3}\right)$.

IR (ATR): 3444, 2929, 1634, 1357, 1056, $694 \mathrm{~cm}^{-1}$.

MS (EI) $m / z$ (relative intensity) $328(30)\left[\mathrm{M}^{+}\right], 328$ (40), 313 (25), 299 (10), 272 (45), 243 
(100).

HR-MS (EI) $m / z$ calcd for $\mathrm{C}_{20} \mathrm{H}_{28} \mathrm{~N}_{2} \mathrm{O}_{2}\left[\mathrm{M}^{+}\right] 328.2151$, found 328.2151 .

\section{$N$-(tert-B utyl)-8-methyl-3,4-diphe nylisoquinolin-1-amine (118la)}<smiles>Cc1cccc2c(-c3ccccc3)c(-c3ccccc3)nc(NC(C)(C)C)c12</smiles>

The general procedure $\mathbf{C}$ was followed using $1171(95.0 \mathrm{mg}, 0.50 \mathrm{mmol})$ and diphenylacetylene (1a) (178 mg, $1.00 \mathrm{mmol})$ in $\mathrm{H}_{2} \mathrm{O}$. Purification by column chromatography ( $n$-hexane/EtOAc: 200/1) yielded 118la (83 mg, 45\%) as an off white solid. M. p.: $136-139^{\circ} \mathrm{C}$.

${ }^{1}$ H-NMR $\left(300 \mathrm{MHz}, \mathrm{CDCl}_{3}\right): \delta=7.42-7.38(\mathrm{~m}, 2 \mathrm{H}), 7.36-7.26(\mathrm{~m}, 4 \mathrm{H}), 7.24-7.20(\mathrm{~m}, 2 \mathrm{H})$, 7.19-7.12 (m, 5H), $5.57(\mathrm{~s}, 1 \mathrm{H}), 2.96(\mathrm{~s}, 3 \mathrm{H}), 1.63(\mathrm{~s}, 9 \mathrm{H})$.

${ }^{13}$ C-NMR (75 MHz, $\left.\mathrm{CDCl}_{3}\right): \delta=154.4\left(\mathrm{C}_{\mathrm{q}}\right), 147.5\left(\mathrm{C}_{\mathrm{q}}\right), 141.8\left(\mathrm{C}_{\mathrm{q}}\right), 139.5\left(\mathrm{C}_{\mathrm{q}}\right), 139.4\left(\mathrm{C}_{\mathrm{q}}\right)$, $132.5\left(\mathrm{C}_{\mathrm{q}}\right), 132.2(\mathrm{CH}), 130.3(\mathrm{CH}), 129.1(\mathrm{CH}), 128.3(\mathrm{CH}), 128.2(\mathrm{CH}), 127.1(\mathrm{CH}), 126.4$ $(\mathrm{CH}), 126.4(\mathrm{CH}), 124.6(\mathrm{CH}), 120.2\left(\mathrm{C}_{\mathrm{q}}\right), 117.8\left(\mathrm{C}_{\mathrm{q}}\right), 52.3\left(\mathrm{C}_{\mathrm{q}}\right), 29.4\left(\mathrm{CH}_{3}\right), 25.0\left(\mathrm{CH}_{3}\right)$.

IR (ATR): 3510, 2963, 1503, 1406, 1222, $705 \mathrm{~cm}^{-1}$.

MS (EI) $m / z$ (relative intens ity) $366(20)\left[\mathrm{M}^{+}\right], 351$ (10), 310 (100), 309 (65), 292 (20), 277 (10).

HR-MS (EI) $m / z$ calcd for $\mathrm{C}_{26} \mathrm{H}_{26} \mathrm{~N}_{2}\left[\mathrm{M}^{+}\right]$366.2096, found 366.2093.

\section{$N$-Isobutyl-6-methyl-3,4-diphe nylisoquinolin-1-amine (118ma)}<smiles>Cc1ccc2c(NCC(C)C)nc(-c3ccccc3)c(-c3ccccc3)c2c1</smiles>

The general procedure $\mathbf{C}$ was followed using $117 \mathrm{~m}(95.0 \mathrm{mg}, 0.50 \mathrm{mmol})$ and diphenylacetylene (1a) (178 mg, $1.00 \mathrm{mmol})$. Purification by column chromatography ( $n$-hexane/EtOAc: $100 / 1)$ yielded $118 \mathrm{ma}\left(110 \mathrm{mg}, 60 \%\right.$ ) as a yellow solid. M. p.: $83-86^{\circ} \mathrm{C}$. ${ }^{1}$ H-NMR $\left(300 \mathrm{MHz}, \mathrm{CDCl}_{3}\right): \delta=7.73(\mathrm{~d}, J=8.4 \mathrm{~Hz}, 1 \mathrm{H}), 7.41-7.30(\mathrm{~m}, 7 \mathrm{H}), 7.23-7.20(\mathrm{~m}$, 
2H), 7.16-7.13 (m, 3H), $5.31(\mathrm{t}, J=5.6 \mathrm{~Hz}, 1 \mathrm{H}), 3.56-3.52(\mathrm{~m}, 2 \mathrm{H}), 2.38(\mathrm{~s}, 3 \mathrm{H}), 2.13-2.04$ $(\mathrm{m}, 1 \mathrm{H}), 1.07(\mathrm{~d}, J=6.5 \mathrm{~Hz}, 6 \mathrm{H})$.

${ }^{13}$ C-NMR $\left(75 \mathrm{MHz}, \mathrm{CDCl}_{3}\right): \delta=153.9\left(\mathrm{C}_{\mathrm{q}}\right), 148.3\left(\mathrm{C}_{\mathrm{q}}\right), 141.7\left(\mathrm{C}_{\mathrm{q}}\right), 139.4\left(\mathrm{C}_{\mathrm{q}}\right), 138.8\left(\mathrm{C}_{\mathrm{q}}\right)$, $137.6\left(\mathrm{C}_{\mathrm{q}}\right), 132.0(\mathrm{CH}), 130.3(\mathrm{CH}), 128.1(\mathrm{CH}), 127.2(\mathrm{CH}), 127.2(\mathrm{CH}), 127.1(\mathrm{CH}), 126.4$ $(\mathrm{CH}), 125.3(\mathrm{CH}), 120.8(\mathrm{CH}), 120.2\left(\mathrm{C}_{\mathrm{q}}\right), 114.8\left(\mathrm{C}_{\mathrm{q}}\right), 49.2\left(\mathrm{CH}_{2}\right), 28.6(\mathrm{CH}), 22.0\left(\mathrm{CH}_{3}\right)$, $20.7\left(\mathrm{CH}_{3}\right)$.

IR (ATR): 2957, 1573, 1318, 1055, $697 \mathrm{~cm}^{-1}$.

MS (EI) $m / z$ (relative intensity) $366(15)\left[\mathrm{M}^{+}\right], 351$ (15), 323 (20), 310 (55), 293 (70), 222 (55), 105 (100).

HR-MS (ESI) $m / z$ calcd for $\mathrm{C}_{26} \mathrm{H}_{27} \mathrm{~N}_{2}\left[\mathrm{M}+\mathrm{H}^{+}\right]$367.2174, found 367.2169.

\section{$N$-n-B utyl-6-me thyl-3,4-diphe nylisoquinolin-1-amine (118na)}<smiles>CCCCNc1nc(-c2ccccc2)c(-c2ccccc2)c2cc(C)ccc12</smiles>

The general procedure $\mathbf{C}$ was followed using $117 \mathrm{n}(95.0 \mathrm{mg}, 0.50 \mathrm{mmol})$ and diphenylacetylene (1a) (178 mg, $1.00 \mathrm{mmol})$. Purification by column chromatography ( $n$-hexane/EtOAc: $100 / 1)$ yielded $117 \mathrm{na}$ ( $88 \mathrm{mg}, 48 \%$ ) as a yellow solid. M. p.: $93-96{ }^{\circ} \mathrm{C}$. ${ }^{1} \mathbf{H}-\mathbf{N M R}\left(300 \mathrm{MHz}, \mathrm{CDCl}_{3}\right): \delta=7.69(\mathrm{~d}, J=8.5 \mathrm{~Hz}, 1 \mathrm{H}), 7.41-7.39(\mathrm{~m}, 1 \mathrm{H}), 7.32-7.27(\mathrm{~d}$, $J=1.9 \mathrm{~Hz}, 1 \mathrm{H}), 7.31(\mathrm{~m}, 5 \mathrm{H}), 7.21-7.18(\mathrm{~m}, 2 \mathrm{H}), 7.16-7.11(\mathrm{~m}, 3 \mathrm{H}), 5.20$ (s, 1H), 3.74-3.68 (m, 2H), $2.36(\mathrm{~s}, 3 \mathrm{H}), 1.80-1.70(\mathrm{~m}, 2 \mathrm{H}), 1.57-1.44(\mathrm{~m}, 2 \mathrm{H}), 0.99(\mathrm{t}, J=7.3 \mathrm{~Hz}, 3 \mathrm{H})$. ${ }^{13}$ C-NMR $\left(75 \mathrm{MHz}, \mathrm{CDCl}_{3}\right): \delta=153.9\left(\mathrm{C}_{\mathrm{q}}\right), 148.3\left(\mathrm{C}_{\mathrm{q}}\right), 141.7\left(\mathrm{C}_{\mathrm{q}}\right), 139.4\left(\mathrm{C}_{\mathrm{q}}\right), 138.8\left(\mathrm{C}_{\mathrm{q}}\right)$, $137.5\left(\mathrm{C}_{\mathrm{q}}\right), 132.0(\mathrm{CH}), 130.3(\mathrm{CH}), 128.0(\mathrm{CH}), 127.2(\mathrm{CH}), 127.1(\mathrm{CH}), 126.5(\mathrm{CH}), 126.4$ $(\mathrm{CH}), 125.2(\mathrm{CH}), 120.9(\mathrm{CH}), 120.3\left(\mathrm{C}_{\mathrm{q}}\right), 114.8\left(\mathrm{C}_{\mathrm{q}}\right), 41.4\left(\mathrm{CH}_{2}\right), 32.1\left(\mathrm{CH}_{2}\right), 22.0\left(\mathrm{CH}_{3}\right)$, $20.5\left(\mathrm{CH}_{2}\right), 14.1\left(\mathrm{CH}_{3}\right)$.

IR (ATR): 2958, 1599, 1573, 1448, 1318, $697 \mathrm{~cm}^{-1}$.

MS (EI) $m / z$ (relative intensity) $366(15)\left[\mathrm{M}^{+}\right], 351$ (20), 323 (30), 310 (100), 294 (25). HR-MS (EI) $m / z$ calcd for $\mathrm{C}_{26} \mathrm{H}_{26} \mathrm{~N}_{2}\left[\mathrm{M}^{+}\right]$366.2096, found 366.2091. 


\section{8-Methyl-5,6-diphe nyl-2,3-dihydroimidazo[2,1-a] is oquinoline (1180a)}<smiles>Cc1ccc2c(c1)C(c1ccccc1)=C(c1ccccc1)N1CCN=C21</smiles>

The general procedure $\mathbf{C}$ was followed using $1170(80.0 \mathrm{mg}, 0.50 \mathrm{mmol})$ and diphenylacetylene (1a) (178 mg, $1.00 \mathrm{mmol})$. Purification by column chromatography $(n$-hexane/EtOAc/Et $3 \mathrm{~N}: 3 / 1 / 0 \rightarrow 3 / 1 / 0.1)$ yielded $1180 a(105 \mathrm{mg}, 62 \%)$ as an off white solid. M. p.: $234-237^{\circ} \mathrm{C}$.

${ }^{1}$ H-NMR $\left(300 \mathrm{MHz}, \mathrm{CDCl}_{3}\right): \delta=8.14(\mathrm{~d}, J=8.1 \mathrm{~Hz}, 1 \mathrm{H}), 7.26(\mathrm{~d}, J=1.5 \mathrm{~Hz}, 1 \mathrm{H})$, 7.21-7.14 (m, 8H), 7.07-7.04 (m, 2H), $6.82(\mathrm{~s}, 1 \mathrm{H}), 3.99(\mathrm{t}, J=9.5 \mathrm{~Hz}, 2 \mathrm{H}), 3.74(\mathrm{t}, J=9.5$ $\mathrm{Hz}, 2 \mathrm{H}), 2.28(\mathrm{~s}, 3 \mathrm{H})$.

${ }^{13}$ C-NMR $\left(75 \mathrm{MHz}, \mathrm{CDCl}_{3}\right): \delta=157.2\left(\mathrm{C}_{\mathrm{q}}\right), 141.9\left(\mathrm{C}_{\mathrm{q}}\right), 139.1\left(\mathrm{C}_{\mathrm{q}}\right), 137.0\left(\mathrm{C}_{\mathrm{q}}\right), 136.4\left(\mathrm{C}_{\mathrm{q}}\right)$, $134.8\left(\mathrm{C}_{\mathrm{q}}\right), 131.8(\mathrm{CH}), 129.4(\mathrm{CH}), 128.1(\mathrm{CH}), 128.0(\mathrm{CH}), 127.9(\mathrm{CH}), 127.9(\mathrm{CH}), 126.5$ $(\mathrm{CH}), 126.3(\mathrm{CH}), 125.2(\mathrm{CH}), 119.0\left(\mathrm{C}_{\mathrm{q}}\right), 113.6\left(\mathrm{C}_{\mathrm{q}}\right), 51.6\left(\mathrm{CH}_{2}\right), 50.4\left(\mathrm{CH}_{2}\right), 22.0\left(\mathrm{CH}_{3}\right)$.

IR (ATR): 3045, 2869, 1623, 1419, 1274, $699 \mathrm{~cm}^{-1}$.

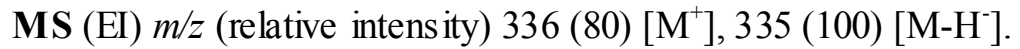

HR-MS (EI) $m / z$ calcd for $\mathrm{C}_{24} \mathrm{H}_{20} \mathrm{~N}_{2}\left[\mathrm{M}^{+}\right] 336.1626$, found 336.1615 .

\section{$N$-(tert-B utyl)-3,4-dipropyl-5H-pyrido[4,3-b]indol-1-amine (118pe)}<smiles>CC(C)Cc1nc(NC(C)C)c2c([nH]c3ccccc32)c1C(C)C(C)C</smiles>

The general procedure $\mathbf{C}$ was followed using $\mathbf{1 1 7 p}$ (108 $\mathrm{mg}, 0.50 \mathrm{mmol}$ ) and 4-octyne (1e) (110 mg, $1.00 \mathrm{mmol}$ ). Purification by column chromatography ( $n$-hexane/EtOAc: 10/1) yielded 118pe (68 mg, $42 \%$ ) as a yellow solid. M. p.: $88-91{ }^{\circ} \mathrm{C}$.

${ }^{1}$ H-NMR $\left(300 \mathrm{MHz}, \mathrm{CDCl}_{3}\right): \delta=7.96($ br s, $1 \mathrm{H}), 7.73(\mathrm{~d}, J=7.7 \mathrm{~Hz}, 1 \mathrm{H}), 7.42(\mathrm{~d}, J=8.0 \mathrm{~Hz}$, 1H), 7.35-7.29 (m, 1H), 7.27-7.21 (m, 1H), $4.67(\mathrm{~s}, 1 \mathrm{H}), 2.79-2.70(\mathrm{~m}, 4 \mathrm{H}), 1.92-1.80(\mathrm{~m}$, 2H), 1.71-1.58 (m, 2H), 1.65 (s, 9H), 1.07-0.98 (m, 6H).

${ }^{13}$ C-NMR $\left(75 \mathrm{MHz}, \mathrm{CDCl}_{3}\right): \delta=153.4\left(\mathrm{C}_{\mathrm{q}}\right), 151.6\left(\mathrm{C}_{\mathrm{q}}\right), 145.2\left(\mathrm{C}_{\mathrm{q}}\right), 138.3(\mathrm{CH}), 123.6(\mathrm{CH})$, 
$122.5\left(\mathrm{C}_{\mathrm{q}}\right), 120.0(\mathrm{CH}), 119.3(\mathrm{CH}), 110.3(\mathrm{CH}), 107.2\left(\mathrm{C}_{\mathrm{q}}\right), 102.5\left(\mathrm{C}_{\mathrm{q}}\right), 51.7\left(\mathrm{C}_{\mathrm{q}}\right), 36.4\left(\mathrm{CH}_{2}\right)$,

$29.9\left(\mathrm{CH}_{3}\right), 29.1\left(\mathrm{CH}_{2}\right), 23.5\left(\mathrm{CH}_{2}\right), 22.8\left(\mathrm{CH}_{2}\right), 14.4\left(\mathrm{CH}_{3}\right), 14.3\left(\mathrm{CH}_{3}\right)$.

IR (ATR): 3448, 2957, 1582, 1439, 1238, $733 \mathrm{~cm}^{-1}$.

MS (EI) $m / z$ (relative intensity) $323(60)\left[\mathrm{M}^{+}\right], 308$ (70), 267 (100), 252 (60), 238 (100), 224 (35), $211(80)$.

HR-MS (EI) $m / z$ calcd for $\mathrm{C}_{21} \mathrm{H}_{29} \mathrm{~N}_{3}\left[\mathrm{M}^{+}\right]$323.2361, found 323.2364.

\section{$N$-(tert-B utyl)-6,7-dipropylthie no[3,2-c]pyridin-4-amine (118qe)}<smiles>CC(C)c1nc(NC(C)(C)C)c2ccsc2c1C(C)C</smiles>

The general procedure $\mathbf{C}$ was followed using $\mathbf{1 1 7 q}$ (91.0 $\mathrm{mg}, 0.50 \mathrm{mmol})$ and 4-octyne (1e) (110 mg, $1.00 \mathrm{mmol}$ ) in $\mathrm{H}_{2} \mathrm{O}$. Purification by column chromatography (n-hexane/EtOAc: $120 / 1 \rightarrow 100 / 1$ ) yie lded 118qe ( $83 \mathrm{mg}, 57 \%$ ) as a yellow oil.

${ }^{1} \mathbf{H}-\mathbf{N M R}\left(300 \mathrm{MHz}, \mathrm{CDCl}_{3}\right): \delta=7.18(\mathrm{~d}, J=5.5 \mathrm{~Hz}, 1 \mathrm{H}), 7.10(\mathrm{~d}, J=5.5 \mathrm{~Hz}, 1 \mathrm{H}), 4.43(\mathrm{~s}$, $1 \mathrm{H}), 2.73-2.67(\mathrm{~m}, 4 \mathrm{H}), 1.85-1.77(\mathrm{~m}, 2 \mathrm{H}), 1.69-1.61(\mathrm{~m}, 2 \mathrm{H}), 1.53(\mathrm{~s}, 9 \mathrm{H}), 1.03-0.96(\mathrm{~m}$, 6H). ${ }^{13} \mathbf{C}-\mathbf{N M R}\left(75 \mathrm{MHz}, \mathrm{CDCl}_{3}\right): \delta=150.8\left(\mathrm{C}_{\mathrm{q}}\right), 150.8\left(\mathrm{C}_{\mathrm{q}}\right), 149.3\left(\mathrm{C}_{\mathrm{q}}\right), 122.8(\mathrm{CH}), 120.8$ $\left(\mathrm{C}_{\mathrm{q}}\right), 119.9(\mathrm{CH}), 117.2\left(\mathrm{C}_{\mathrm{q}}\right), 51.6\left(\mathrm{C}_{\mathrm{q}}\right), 36.1\left(\mathrm{CH}_{2}\right), 33.2\left(\mathrm{CH}_{2}\right), 29.5\left(\mathrm{CH}_{3}\right), 22.9\left(\mathrm{CH}_{2}\right), 22.7$ $\left(\mathrm{CH}_{2}\right), 14.5\left(\mathrm{CH}_{3}\right), 14.3\left(\mathrm{CH}_{3}\right)$.

IR (ATR): 3443, 2957, 1556, 1489, 1419, 1215, $686 \mathrm{~cm}^{-1}$.

MS (EI) $m / z$ (relative intensity) 290 (60) $\left[\mathrm{M}^{+}\right], 275$ (75), 234 (75), 219 (35), 205 (100), 178 (40).

HR-MS (EI) $m / z$ calcd for $\mathrm{C}_{17} \mathrm{H}_{26} \mathrm{~N}_{2} \mathrm{~S}\left[\mathrm{M}^{+}\right] 290.1817$, found 290.1821.

\section{1-(4-Methoxybe nzyl)-5-me thyl-2,3,7,8-tetrapropyl-1 $H$-be nzo [de $][1,8]$ naphthyridine} (118re) 
<smiles>COc1ccc(CN2C(C(C)C)=C(C(C)C)c3cc(C)cc4c(C(C)C)c(C(C)C)nc2c34)cc1</smiles>

The general procedure $\mathbf{C}$ was followed using $\mathbf{1 1 8}$ (127 mg, $0.50 \mathrm{mmol})$ and oct-4-yne (1e) (110 mg, $1.00 \mathrm{mmol}$ ). Purification by column chromatography ( $n$-hexane/EtOAc: 100/1) yielded 118re (150 mg, 64\%) as a yellow solid. M. p.: $107-108^{\circ} \mathrm{C}$.

${ }^{1}$ H-NMR (300 MHz, DMSO- $\left.d_{6}\right): \delta=7.07$ (d, $\left.J=8.6 \mathrm{~Hz}, 2 \mathrm{H}\right), 6.92(\mathrm{~s}, 1 \mathrm{H}), 6.83(\mathrm{~d}, J=8.6$ $\mathrm{Hz}, 2 \mathrm{H}), 6.70$ (s, 1H), 5.44 (br s, 2H), 3.68 (s, 3H), 2.64-2.43 (m, 8H), 2.40 (s, 3H), $1.61-1.45(\mathrm{~m}, 8 \mathrm{H}), 1.02-0.92(\mathrm{~m}, 9 \mathrm{H}), 0.80(\mathrm{t}, J=7.4 \mathrm{~Hz}, 3 \mathrm{H})$.

${ }^{13}$ C-NMR (75 MHz, DMSO-d $\left.d_{6}\right): \delta=157.9\left(\mathrm{C}_{\mathrm{q}}\right), 150.9\left(\mathrm{C}_{\mathrm{q}}\right), 149.5\left(\mathrm{C}_{\mathrm{q}}\right), 141.3\left(\mathrm{C}_{\mathrm{q}}\right), 140.7\left(\mathrm{C}_{\mathrm{q}}\right)$, $138.1\left(\mathrm{C}_{\mathrm{q}}\right), 135.4\left(\mathrm{C}_{\mathrm{q}}\right), 130.7\left(\mathrm{C}_{\mathrm{q}}\right), 127.2(\mathrm{CH}), 117.4\left(\mathrm{C}_{\mathrm{q}}\right), 116.3\left(\mathrm{C}_{\mathrm{q}}\right), 113.9(\mathrm{CH}), 113.7$ $(\mathrm{CH}), 112.5(\mathrm{CH}), 112.1\left(\mathrm{C}_{\mathrm{q}}\right), 54.9\left(\mathrm{CH}_{3}\right), 45.7\left(\mathrm{CH}_{2}\right), 36.0\left(\mathrm{CH}_{2}\right), 30.7\left(\mathrm{CH}_{2}\right), 29.1\left(\mathrm{CH}_{2}\right)$, $28.8\left(\mathrm{CH}_{2}\right), 22.6\left(\mathrm{CH}_{2}\right), 22.4\left(\mathrm{CH}_{3}\right), 21.8\left(\mathrm{CH}_{2}\right), 21.7\left(\mathrm{CH}_{2}\right), 20.9\left(\mathrm{CH}_{2}\right), 14.1\left(\mathrm{CH}_{3}\right), 14.0$ $\left(\mathrm{CH}_{3}\right), 13.9\left(\mathrm{CH}_{3}\right), 13.8\left(\mathrm{CH}_{3}\right)$.

IR (ATR): 2954, 1624, 1513, 1345, 1252, $829 \mathrm{~cm}^{-1}$.

MS (EI) $m / z$ (relative intensity) 470 (40) $\left[\mathrm{M}^{+}\right], 441$ (5), 349 (45), 306 (10), 277 (10), 121 (100).

HR-MS (EI) $m / z$ calcd for $\mathrm{C}_{32} \mathrm{H}_{42} \mathrm{~N}_{2} \mathrm{O}\left[\mathrm{M}^{+}\right]$470.3297, found 470.3293 .

\section{$N$-(4-Methoxybenzyl)-6-methyl-3,4-diphe nylisoquinolin-1-amine (118ra)}<smiles>COc1ccc(CNc2nc(-c3ccccc3)c(-c3ccccc3)c3cc(C)ccc23)cc1</smiles>

The general procedure $\mathbf{C}$ was followed using $\mathbf{1 1 7} \mathbf{r}$ (127 mg, $0.50 \mathrm{mmol})$ and $\mathbf{1 a}(178 \mathrm{mg}, 1.00$ mmol). Purification by column chromatography ( $n$-hexane/EtOAc: 100/1) yielded 118ra (73 mg, 34\%) as a yellow solid. M. p.: $97-98^{\circ} \mathrm{C}$.

${ }^{1} \mathbf{H}-\mathbf{N M R}\left(\mathrm{CDCl}_{3}, 300 \mathrm{MHz}\right): \delta 7.70(\mathrm{~d}, J=8.8 \mathrm{~Hz}, 2 \mathrm{H}), 7.44-7.27(\mathrm{~m}, 9 \mathrm{H}), 7.25-7.13(\mathrm{~m}$, $5 \mathrm{H}), 6.92(\mathrm{dd}, J=8.3,2.4 \mathrm{~Hz}, 2 \mathrm{H}), 5.42(\mathrm{t}, J=5.0 \mathrm{~Hz}, 1 \mathrm{H}), 4.86(\mathrm{~d}, J=5.0 \mathrm{~Hz}, 2 \mathrm{H}), 3.82(\mathrm{~s}$, 
$3 \mathrm{H}), 2.38(\mathrm{~s}, 3 \mathrm{H})$.

${ }^{13}$ C-NMR $\left(\mathrm{CDCl}_{3}, 75 \mathrm{MHz}\right): \delta=158.8\left(\mathrm{C}_{\mathrm{q}}\right), 153.5\left(\mathrm{C}_{\mathrm{q}}\right), 148.2\left(\mathrm{C}_{\mathrm{q}}\right), 141.6\left(\mathrm{C}_{\mathrm{q}}\right), 139.6\left(\mathrm{C}_{\mathrm{q}}\right)$, $138.7\left(\mathrm{C}_{\mathrm{q}}\right), 132.1\left(\mathrm{C}_{\mathrm{q}}\right), 132.0(\mathrm{CH}), 130.3(\mathrm{CH}), 129.5(\mathrm{CH}), 128.1(\mathrm{CH}), 127.3(\mathrm{CH}), 127.1$

$(\mathrm{CH}), 126.5(\mathrm{CH}), 126.4(\mathrm{CH}), 125.3(\mathrm{CH}), 121.0(\mathrm{CH}), 120.9\left(\mathrm{C}_{\mathrm{q}}\right), 114.8\left(\mathrm{C}_{\mathrm{q}}\right), 113.9(\mathrm{CH})$, $55.4\left(\mathrm{CH}_{3}\right), 45.5\left(\mathrm{CH}_{2}\right), 22.0\left(\mathrm{CH}_{3}\right)$.

IR (ATR): 3436, 2910, 1573, 1509, 1245, $699 \mathrm{~cm}^{-1}$.

MS (EI) m/z (relative intensity) 430 (100) $\left[\mathrm{M}^{+}\right], 415$ (20), 309 (15), 294 (30), 136 (35), 121 (70).

HR-MS (EI) $\mathrm{m} / \mathrm{z}$ calcd for $\mathrm{C}_{30} \mathrm{H}_{26} \mathrm{~N}_{2} \mathrm{O}\left[\mathrm{M}^{+}\right]$430.2045, found 430.2051 .

Intermolecular Competition Experiment between Substrates 117c and 117e:
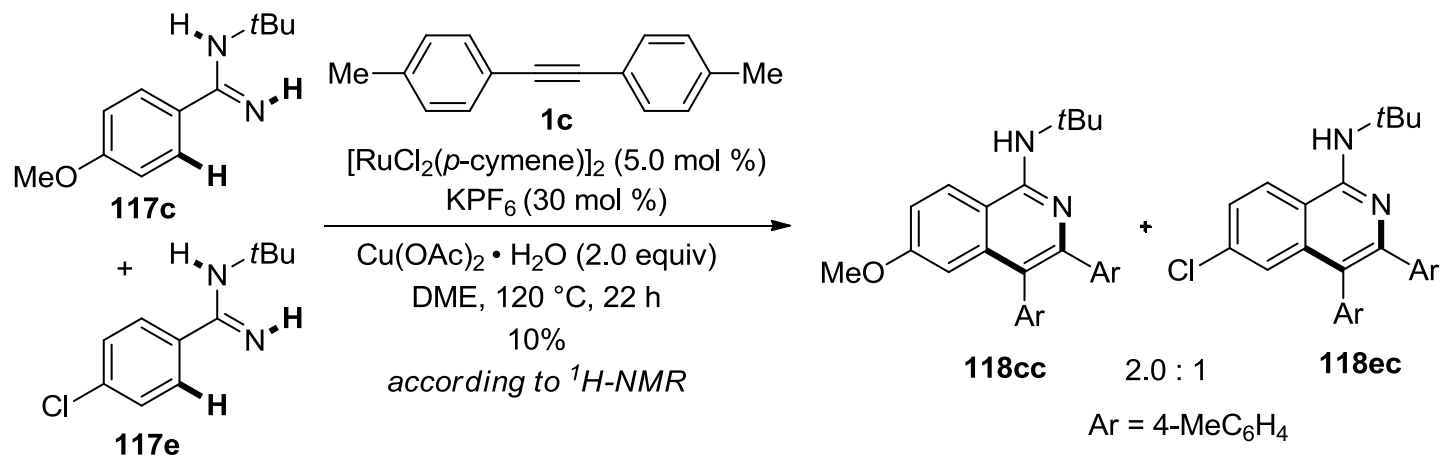

(2.0 equiv each)

A suspension of 1,2-di- $p$-tolylethyne (1c) $\quad(103 \quad \mathrm{mg}, \quad 0.50 \quad \mathrm{mmol})$, $N$-(tert-butyl)-4-methoxybenzimidamide $\quad(\mathbf{1 1 7 c}) \quad(206 \quad \mathrm{mg}, \quad 1.00 \quad \mathrm{mmol})$, $N$-(tert-butyl)-4-chlorobenzimidamide (117e) (210 mg, $1.00 \mathrm{mmol}),\left[\mathrm{RuCl}_{2}(p \text {-cymene) }]_{2}\right.$ (15.3 mg, $5.0 \mathrm{~mol} \%), \mathrm{KPF}_{6}(27.6 \mathrm{mg}, 30 \mathrm{~mol} \%)$ and $\mathrm{Cu}(\mathrm{OAc})_{2} \cdot \mathrm{H}_{2} \mathrm{O}(199 \mathrm{mg}, 1.00 \mathrm{mmol})$ in DME $(2.0 \mathrm{~mL})$ was stirred at $120 \mathcal{C}$ for $22 \mathrm{~h}$ under an atmosphere of $\mathrm{N}_{2}$. At ambient temperature, $\mathrm{H}_{2} \mathrm{O}(20 \mathrm{~mL})$ was added, and the mixture was extracted with EtOAc $(3 \times 20 \mathrm{~mL})$. The combined organic layers were washed with brine $(20 \mathrm{~mL})$ and dried over $\mathrm{Na}_{2} \mathrm{SO}_{4}$. The solvent was evaporated in vacuo, and the residue was purified by column chromatography on silica gel ( $n$-hexane/EtOAc: 100/1) to yield a mixture of $118 c c$ and $118 e c(21 \mathrm{mg}, 10 \%)$ as a solid. The ratio was estimated by ${ }^{1} \mathrm{H}-\mathrm{NMR}$ spectroscopy.

Inte rmolecular Compe tition Expe riment Between Alkynes Substrates 1d and 1e: 


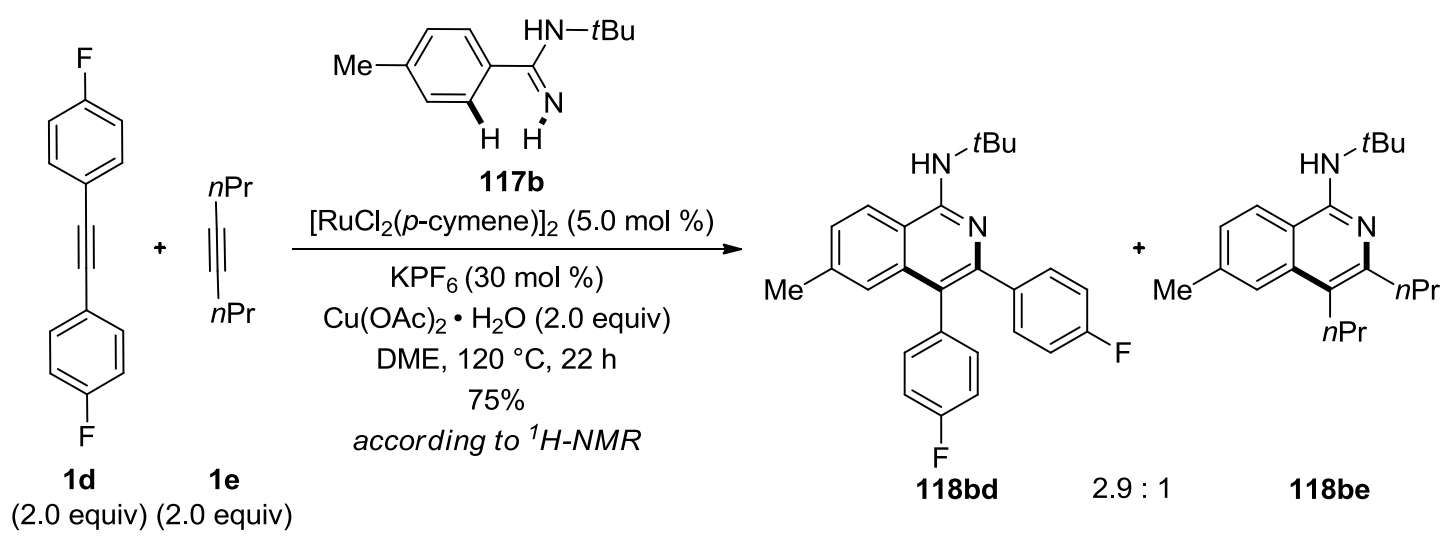

A suspension of $N$-(tert-butyl)-4-methylbenzimidamide (117b) (95.0 mg, $0.50 \mathrm{mmol}$ ), 1,2-bis(4-fluorophenyl)ethyne (1d) (214 mg, $1.00 \mathrm{mmol})$, 4-octyne (1e) (110 mg, $1.00 \mathrm{mmol})$, $\left[\mathrm{RuCl}_{2}(p \text {-cymene })\right]_{2}(15.3 \mathrm{mg}, 5.0 \mathrm{~mol} \%), \mathrm{KPF}_{6}(27.6 \mathrm{mg}, 30 \mathrm{~mol} \%)$ and $\mathrm{Cu}(\mathrm{OAc})_{2} \cdot \mathrm{H}_{2} \mathrm{O}$ (199 mg, $1.00 \mathrm{mmol})$ in DME $(2.0 \mathrm{~mL})$ was stirred at $120 \mathrm{C}$ for $22 \mathrm{~h}$ under an atmosphere of $\mathrm{N}_{2}$. At ambient temperature, $\mathrm{H}_{2} \mathrm{O}(20 \mathrm{~mL})$ was added, and the mixture was extracted with EtOAc $(3 \times 20 \mathrm{~mL})$. The combined organic layers were washed with brine $(20 \mathrm{~mL})$ and dried over $\mathrm{Na}_{2} \mathrm{SO}_{4}$. The solvent was evaporated in vacuo, and the residue was purified by column chromatography on silica gel ( $n$-hexane/EtOAc: $150: 1 \rightarrow 120: 1)$ to yield a mixture products of 118bd and 118be (141 mg, 75\%) as a solid. The ratio was estimated by ${ }^{1} \mathrm{H}-\mathrm{NMR}$ spectroscopy.

\section{Ruthenium(II)-Catalyzed H/D Exchange in Subs trate $117 \mathrm{~b}$ with $\mathrm{D}_{2} \mathrm{O}$ as the Cosolvent in} the Absence of Alkyne:

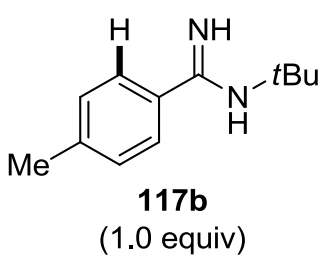

(1.0 equiv)
$\left[\mathrm{RuCl}_{2}(p \text {-cymene })\right]_{2}(5.0 \mathrm{~mol} \%)$ $\mathrm{KPF}_{6}(30 \mathrm{~mol} \%)$

$$
\mathrm{Cu}(\mathrm{OAc})_{2} \cdot \mathrm{H}_{2} \mathrm{O}(2.0 \text { equiv) }
$$

$\mathrm{DME} / \mathrm{D}_{2} \mathrm{O}(9: 1)$ $120^{\circ} \mathrm{C}, \mathrm{N} 2,30 \mathrm{~min}$ $58 \%$<smiles></smiles><smiles>Cc1cc(O)c(C(=N)NC(C)(C)C)c([18OH])c1</smiles>

$\left[D_{n}\right]-117 b$

A suspension of $N$-(tert-butyl)-4-methylbenzimidamide (117b) $(95.0 \mathrm{mg}, 0.50 \mathrm{mmol}$ ), $\left[\mathrm{RuCl}_{2}(p \text {-cymene })\right]_{2}(15.3 \mathrm{mg}, 5.0 \mathrm{~mol} \%), \mathrm{KPF}_{6}(27.6 \mathrm{mg}, 30 \mathrm{~mol} \%)$ and $\mathrm{Cu}(\mathrm{OAc})_{2} \cdot \mathrm{H}_{2} \mathrm{O}$ (199 $\mathrm{mg}, 1.00 \mathrm{mmol})$ in a solvent mixture of DME and $\mathrm{D}_{2} \mathrm{O}(1.8 / 0.2 \mathrm{~mL})$ was stirred at $120 \mathrm{C}$ for $0.5 \mathrm{~h}$ under an atmosphere of $\mathrm{N}_{2}$. At ambient temperature, $\mathrm{H}_{2} \mathrm{O}(20 \mathrm{~mL})$ was added, and the mixture was extracted with EtOAc $(3 \times 20 \mathrm{~mL})$. The combined organic layers were 
washed with brine $(20 \mathrm{~mL})$ and dried over $\mathrm{Na}_{2} \mathrm{SO}_{4}$. The solvent was evaporated in vacuo, and the residue was purified by column chromatography ( $n$-hexane/EtOAc/Et ${ }_{3} \mathrm{~N}: 1 / 1 / 0.02$ ) to yield $\left[\mathrm{D}_{\mathrm{n}}\right]-\mathbf{1 1 7} \mathbf{b}(55.0 \mathrm{mg}, 58 \%)$ as a white solid. The D-incorporation in $\left[\mathrm{D}_{\mathrm{n}}\right]-\mathbf{1 1 7 \mathbf { b }}$ was estimated by ${ }^{1} \mathrm{H}-\mathrm{NMR}$ spectroscopy.

\section{Ruthe nium-Catalyzed $\mathrm{H} / \mathrm{D}$ Exchange in Substrate $117 \mathrm{~b}$ with $\mathrm{D}_{2} \mathrm{O}$ as the Cosolvent in the} Presence of Alkyne:
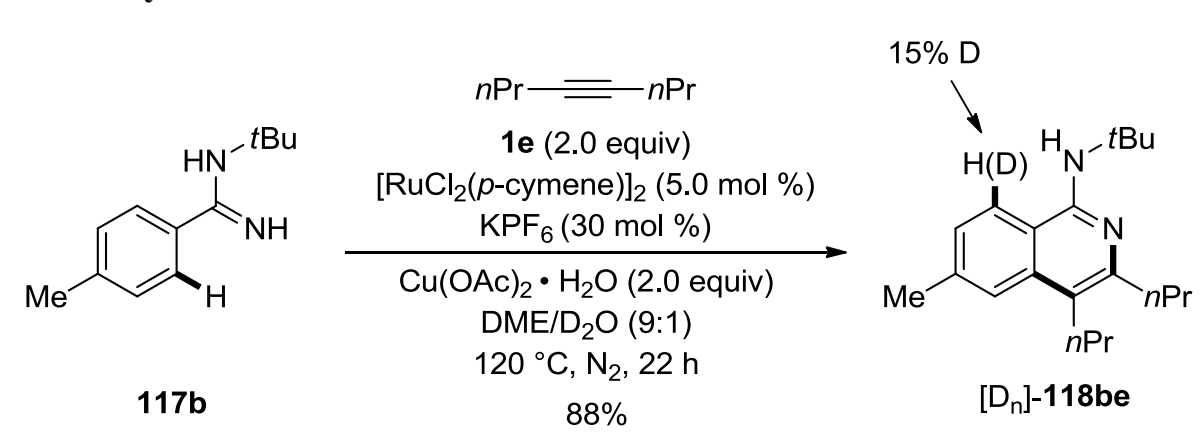

$\left[D_{n}\right]-118 b e$

A suspension of $N$-(tert-butyl)-4-methylbenzimidamide (117b) $(95.0 \mathrm{mg}, 0.50 \mathrm{mmol}$ ), oct-4-yne (1e) (110 mg, $1.00 \mathrm{mmol}),\left[\mathrm{RuCl}_{2}(p \text {-cymene) }]_{2}(15.3 \mathrm{mg}, 5.0 \mathrm{~mol} \%), \mathrm{KPF}_{6}(27.6\right.$ $\mathrm{mg}, 30 \mathrm{~mol} \%)$ and $\mathrm{Cu}(\mathrm{OAc})_{2} \cdot \mathrm{H}_{2} \mathrm{O}(199 \mathrm{mg}, 1.00 \mathrm{mmol})$ in a solvent mixture of DME and $\mathrm{D}_{2} \mathrm{O}(1.8 / 0.2 \mathrm{~mL})$ was stirred at $120 \mathrm{C}$ for $22 \mathrm{~h}$ under an atmosphere of $\mathrm{N}_{2}$. At ambient temperature, $\mathrm{H}_{2} \mathrm{O}(20 \mathrm{~mL})$ was added, and the mixture was extracted with EtOAc $(3 \times 20 \mathrm{~mL})$. The combined organic layers were washed with brine $(20 \mathrm{~mL})$ and dried over $\mathrm{Na}_{2} \mathrm{SO}_{4}$. The solvent was evaporated in vacuo, and the residue was purified by column chromatography on silica gel ( $n$-hexane/EtOAc: $100 / 1)$ to yield $\left[\mathrm{D}_{\mathrm{n}}\right]-\mathbf{1 1 8 b e}(131 \mathrm{mg}, 88 \%)$ as a green oil. The D-incorporation in $\left[D_{n}\right]$-118be was estimated by ${ }^{1} \mathrm{H}-\mathrm{NMR}$ spectroscopy.

\section{Analytical Data for the Products of the Ruthenium(II)-Catalyzed Oxidative C-H Bond Activation with Acrylates}

Ethyl (E)-2-(3-imino-2-isobutyl-6-methylisoindolin-1-ylidene)acetate (119'ma)<smiles>CCCN1C(=N)c2ccc(C)cc2/C1=C\C(=O)OCC</smiles>

The general procedure $\mathbf{D}$ was followed using $\mathbf{1 1 7} \mathbf{m}$ (95.0 mg, $0.50 \mathrm{mmol}), \mathbf{2 0 a}(75.0 \mathrm{mg}, 0.75$ 
mmol) and $\left[\operatorname{RuCl}_{2}(p \text {-cymene })\right]_{2}(7.7 \mathrm{mg}, 2.5 \mathrm{~mol} \%)$ at $100 \mathrm{C}$. Purification by column chromatography ( $n$-hexane/EtOAc: $8 / 1)$ yie lded 119'ma (110 mg, 77\%) as a white solid. M.p.: $68-71^{\circ} \mathrm{C}$.

${ }^{1} \mathbf{H}-\mathbf{N M R}\left(300 \mathrm{MHz}, \mathrm{CDCl}_{3}\right): \delta=8.96(\mathrm{~s}, 1 \mathrm{H}), 7.68($ br s, $1 \mathrm{H}), 7.50(\mathrm{~d}, J=7.9 \mathrm{~Hz}, 1 \mathrm{H}), 7.31$ (d, $J=7.9 \mathrm{~Hz}, 1 \mathrm{H}), 5.42(\mathrm{~s}, 1 \mathrm{H}), 4.21$ (q, $J=7.0 \mathrm{~Hz}, 2 \mathrm{H}), 3.66$ (d, $J=7.7 \mathrm{~Hz}, 2 \mathrm{H}), 2.45$ (s, $3 \mathrm{H}), 2.24-2.15(\mathrm{~m}, 1 \mathrm{H}), 1.31(\mathrm{t}, J=7.0 \mathrm{~Hz}, 3 \mathrm{H}), 0.93(\mathrm{~d}, J=6.8 \mathrm{~Hz}, 6 \mathrm{H})$.

${ }^{13}$ C-NMR (75 MHz, $\left.\mathrm{CDCl}_{3}\right): \delta=166.8\left(\mathrm{C}_{\mathrm{q}}\right), 162.3\left(\mathrm{C}_{\mathrm{q}}\right), 151.4\left(\mathrm{C}_{\mathrm{q}}\right), 142.2\left(\mathrm{C}_{\mathrm{q}}\right), 133.0\left(\mathrm{C}_{\mathrm{q}}\right)$, $131.2(\mathrm{CH}), 128.3(\mathrm{CH}), 127.9\left(\mathrm{C}_{\mathrm{q}}\right), 120.4(\mathrm{CH}), 93.5(\mathrm{CH}), 59.9\left(\mathrm{CH}_{2}\right), 47.2\left(\mathrm{CH}_{2}\right), 26.9$ $(\mathrm{CH}), 22.0\left(\mathrm{CH}_{3}\right), 20.1\left(\mathrm{CH}_{3}\right), 14.4\left(\mathrm{CH}_{3}\right)$.

IR (ATR): 3385, 3228, 2961, 1640, 1157, 1069, $826 \mathrm{~cm}^{-1}$.

MS (EI) $m / z$ (relative intensity) $286(20)\left[\mathrm{M}^{+}\right], 241$ (15), 213 (100), 197 (15), 185 (15), 158 (35), $142(10)$.

HR-MS (EI) $m / z$ calcd for $\mathrm{C}_{17} \mathrm{H}_{22} \mathrm{~N}_{2} \mathrm{O}_{2}\left[\mathrm{M}^{+}\right] 286.1681$, found 286.1678 .

Ethyl (E)-2-(2-n-butyl-6-me thylis oindolin-3-imino-1-ylide ne)acetate (119'na)<smiles>CCCCCN1C(=N)c2ccc(C)cc2/C1=C/C(=O)OCC</smiles>

The general procedure $\mathbf{D}$ was followed us ing $117 \mathbf{n}(95.0 \mathrm{mg}, 0.50 \mathrm{mmol}), \mathbf{2 0 a}(75.0 \mathrm{mg}, 0.75$ mmol) and $\left[\mathrm{RuCl}_{2}(p \text {-cymene })\right]_{2}(7.7 \mathrm{mg}, 2.5 \mathrm{~mol} \%)$ at $100 \mathrm{C}$. Purification by column chromatography ( $n$-hexane/EtOAc: $8 / 1)$ yielded 119 'na (64 mg, 45\%) as a white solid. M. p. : $101-102{ }^{\circ} \mathrm{C}$.

${ }^{1} \mathbf{H}-\mathbf{N M R}\left(300 \mathrm{MHz}, \mathrm{CDCl}_{3}\right): \delta=8.97(\mathrm{~s}, 1 \mathrm{H}), 7.51(\mathrm{~d}, J=7.6 \mathrm{~Hz}, 1 \mathrm{H}), 7.32(\mathrm{~d}, J=7.6 \mathrm{~Hz}$, 1H), 5.43 (s, 1H), 4.23 (q, $J=7.2 \mathrm{~Hz}, 2 \mathrm{H}), 3.81$ (t, $J=7.7 \mathrm{~Hz}, 2 \mathrm{H}), 2.46$ (s, 3H), 1.68-1.57 (m, 2H), 1.45-1.30 (m, 2H), $1.33(\mathrm{t}, J=7.2 \mathrm{~Hz}, 3 \mathrm{H}), 0.94(\mathrm{t}, J=7.3 \mathrm{~Hz}, 3 \mathrm{H})$.

${ }^{13}$ C-NMR (75 MHz, $\left.\mathrm{CDCl}_{3}\right): \delta=166.9\left(\mathrm{C}_{\mathrm{q}}\right), 161.8\left(\mathrm{C}_{\mathrm{q}}\right), 151.0\left(\mathrm{C}_{\mathrm{q}}\right), 142.2\left(\mathrm{C}_{\mathrm{q}}\right), 133.2\left(\mathrm{C}_{\mathrm{q}}\right)$, $131.1(\mathrm{CH}), 128.4(\mathrm{CH}), 128.0\left(\mathrm{C}_{\mathrm{q}}\right), 120.3(\mathrm{CH}), 93.1(\mathrm{CH}), 59.9\left(\mathrm{CH}_{2}\right), 40.0\left(\mathrm{CH}_{2}\right), 29.3$ $\left(\mathrm{CH}_{2}\right), 22.1\left(\mathrm{CH}_{3}\right), 20.2\left(\mathrm{CH}_{2}\right), 14.4\left(\mathrm{CH}_{3}\right), 13.8\left(\mathrm{CH}_{3}\right)$.

IR (ATR): 3235, 2934, 1598, 1153, 1069, $822 \mathrm{~cm}^{-1}$. 
MS (EI) $m / z$ (relative intensity) $286(5)\left[\mathrm{M}^{+}\right], 241$ (10), 213 (100), 185 (15), 158 (20), 142 (10).

HR-MS (EI) $m / z$ calcd for $\mathrm{C}_{17} \mathrm{H}_{22} \mathrm{~N}_{2} \mathrm{O}_{2}\left[\mathrm{M}^{+}\right] 286.1681$, found 286.1684 .

Ethyl (E)-2-\{3-imino-2-(4-methoxybe nzyl)-6-me thylis oindolin-1-ylide ne\}acetate (119'ra)<smiles>CCOC(=O)/C=C1\c2cc(C)ccc2C(=N)N1Cc1ccc(OC)cc1</smiles>

The general procedure $\mathbf{D}$ was followed using $117 \mathbf{r}(127 \mathrm{mg}, 0.50 \mathrm{mmol}), \mathbf{2 0 a}(75.0 \mathrm{mg}, 0.75$ mmol $)\left[\mathrm{RuCl}_{2}(p \text {-cymene })\right]_{2}(7.7 \mathrm{mg}, 2.5 \mathrm{~mol} \%)$ at $100 \mathrm{C}$. Purification by column chromatography ( $n$-hexane/EtOAc: $5 / 1)$ yielded 117 'ra (129 mg, $74 \%$ ) as a white solid. M. p.: $165-166^{\circ} \mathrm{C}$.

${ }^{1} \mathbf{H}-\mathbf{N M R}\left(300 \mathrm{MHz}, \mathrm{CDCl}_{3}\right): \delta=8.99(\mathrm{~s}, 1 \mathrm{H}), 7.58(\mathrm{~d}, J=7.9 \mathrm{~Hz}, 1 \mathrm{H}), 7.37(\mathrm{~d}, J=7.9 \mathrm{~Hz}$, $1 \mathrm{H}), 7.15(\mathrm{~d}, J=8.9 \mathrm{~Hz}, 2 \mathrm{H}), 6.82$ (d, $J=8.9 \mathrm{~Hz}, 2 \mathrm{H}), 5.44(\mathrm{~s}, 1 \mathrm{H}), 5.03(\mathrm{~s}, 2 \mathrm{H}), 4.17$ (q, $J=$ $7.1 \mathrm{~Hz}, 2 \mathrm{H}), 3.75$ (s, 3H), 2.49 (s, 3H), 1.28 (t, $J=7.1 \mathrm{~Hz}, 3 \mathrm{H})$.

${ }^{13}$ C-NMR (75 MHz, $\left.\mathrm{CDCl}_{3}\right): \delta=166.7\left(\mathrm{C}_{\mathrm{q}}\right), 162.1\left(\mathrm{C}_{\mathrm{q}}\right), 158.7\left(\mathrm{C}_{\mathrm{q}}\right), 150.8\left(\mathrm{C}_{\mathrm{q}}\right), 142.5\left(\mathrm{C}_{\mathrm{q}}\right)$, $142.5\left(\mathrm{C}_{\mathrm{q}}\right), 133.3\left(\mathrm{C}_{\mathrm{q}}\right), 131.4(\mathrm{CH}), 128.6(\mathrm{CH}), 128.3\left(\mathrm{C}_{\mathrm{q}}\right), 127.9(\mathrm{CH}), 120.6(\mathrm{CH}), 114.1$ (CH), $94.4(\mathrm{CH}), 59.9\left(\mathrm{CH}_{2}\right), 55.2\left(\mathrm{CH}_{3}\right), 43.2\left(\mathrm{CH}_{2}\right), 22.1\left(\mathrm{CH}_{3}\right), 14.4\left(\mathrm{CH}_{3}\right)$.

IR (ATR): 3261, 2955, 1604, 1115, 1035, $819 \mathrm{~cm}^{-1}$.

MS (EI) $m / z$ (relative intensity) 350 (70) $\left[\mathrm{M}^{+}\right], 335$ (5), 277 (60), 185 (100), 121 (45).

HR-MS (EI) $m / z$ calcd for $\mathrm{C}_{21} \mathrm{H}_{22} \mathrm{~N}_{2} \mathrm{O}_{3}\left[\mathrm{M}^{+}\right] 350.1630$, found 350.1627 .

n-Butyl

(E)-2-\{3-imino-2-(4-me thoxybe nzyl)-6-me thylisoindolin-1-ylidene\}acetate (119'rb) 
<smiles>CCCCCCOC(=O)/C=C1\c2cc(C)ccc2C(=N)N1Cc1ccc(OC)cc1</smiles>

The general procedure $\mathbf{D}$ was followed using $\mathbf{1 1 7}$ r (127 mg, $0.50 \mathrm{mmol}), \mathbf{2 0 b}$ (96.0 mg, 0.75 mmol) $\left[\mathrm{RuCl}_{2}(p \text {-cymene })\right]_{2}(7.7 \mathrm{mg}, 2.5 \mathrm{~mol} \%)$ at $100 \mathrm{C}$. Purification by column chromatography ( $n$-hexane/EtOAc: $5 / 1)$ to afford 119'rb (140 mg, 74\%) as a white solid. M.p.: $111-113{ }^{\circ} \mathrm{C}$.

${ }^{1} \mathbf{H}-\mathbf{N M R}\left(300 \mathrm{MHz}, \mathrm{CDCl}_{3}\right): \delta=8.99(\mathrm{~s}, 1 \mathrm{H}), 7.58(\mathrm{~d}, J=7.9 \mathrm{~Hz}, 1 \mathrm{H}), 7.37$ (d, $J=7.9 \mathrm{~Hz}$, $1 \mathrm{H}), 7.15(\mathrm{~d}, J=8.9 \mathrm{~Hz}, 2 \mathrm{H}), 6.82(\mathrm{~d}, J=8.9 \mathrm{~Hz}, 2 \mathrm{H}), 5.44(\mathrm{~s}, 1 \mathrm{H}), 5.03(\mathrm{~s}, 2 \mathrm{H}), 4.17$ (q, $J=$ $6.8 \mathrm{~Hz}, 2 \mathrm{H}), 3.75$ (s, 3H), 2.49 (s, 3H), 1.65 (m, 2H), 1.39 (m, 2H), 0.94 (t, $J=7.1 \mathrm{~Hz}, 3 \mathrm{H})$. ${ }^{13}$ C-NMR (75 MHz, $\left.\mathrm{CDCl}_{3}\right): \delta=166.8\left(\mathrm{C}_{\mathrm{q}}\right), 162.1\left(\mathrm{C}_{\mathrm{q}}\right), 158.8\left(\mathrm{C}_{\mathrm{q}}\right), 150.8\left(\mathrm{C}_{\mathrm{q}}\right), 142.5\left(\mathrm{C}_{\mathrm{q}}\right)$, $142.0\left(\mathrm{C}_{\mathrm{q}}\right), 133.4\left(\mathrm{C}_{\mathrm{q}}\right), 131.5(\mathrm{CH}), 128.6(\mathrm{CH}), 128.3\left(\mathrm{C}_{\mathrm{q}}\right), 127.9(\mathrm{CH}), 120.6(\mathrm{CH}), 114.1$ $(\mathrm{CH}), 94.5(\mathrm{CH}), 64.0\left(\mathrm{CH}_{2}\right), 55.2\left(\mathrm{CH}_{3}\right), 43.2\left(\mathrm{CH}_{2}\right), 30.8\left(\mathrm{CH}_{2}\right), 22.1\left(\mathrm{CH}_{3}\right), 19.2\left(\mathrm{CH}_{2}\right)$, $13.8\left(\mathrm{CH}_{3}\right)$.

IR (ATR): 3250, 2965, 1596, 1247, 1097, $822 \mathrm{~cm}^{-1}$.

MS (EI) $m / z$ (relative intensity) $378(55)\left[\mathrm{M}^{+}\right], 363$ (5), 277 (45), 185 (100), 121 (65).

HR-MS (EI) $m / z$ calcd for $\mathrm{C}_{23} \mathrm{H}_{26} \mathrm{~N}_{2} \mathrm{O}_{3}\left[\mathrm{M}^{+}\right] 378.1943$, found 378.1942 .

\section{Benzyl}

(E)-2-\{3-imino-2-(4-me thoxybe nzyl)-6-me thylisoindolin-1-ylidene\}acetate (119'rc).<smiles>COc1ccc(CN2C(=N)c3ccc(C)cc3/C2=C\C(=O)OCc2ccccc2)cc1</smiles>

The general procedure D was followed using $117 \mathbf{r}(127 \mathrm{mg}, 0.50 \mathrm{mmol}), \mathbf{2 0 c}(122 \mathrm{mg}, 0.75$ mmol $)\left[\operatorname{RuCl}_{2}(p \text {-cymene })\right]_{2}(7.7 \mathrm{mg}, 2.5 \mathrm{~mol} \%)$ at $100 \mathrm{C}$. Purification by column chromatography ( $n$-hexane/EtOAc: $5 / 1)$ yie lded 119'rc (142 mg, 69\%) as a white solid. M. p.: 
$136-137^{\circ} \mathrm{C}$.

${ }^{1}$ H-NMR (300 MHz, CDCl $): \delta=8.99(\mathrm{~s}, 1 \mathrm{H}), 7.58(\mathrm{~d}, J=7.7 \mathrm{~Hz}, 1 \mathrm{H}), 7.30-7.39(\mathrm{~m}, 6 \mathrm{H})$, $7.13(\mathrm{~d}, J=8.8 \mathrm{~Hz}, 2 \mathrm{H}), 6.81(\mathrm{~d}, J=8.8 \mathrm{~Hz}, 2 \mathrm{H}), 5.50(\mathrm{~s}, 1 \mathrm{H}), 5.17(\mathrm{~s}, 2 \mathrm{H}), 5.03(\mathrm{~s}, 2 \mathrm{H}), 3.75$ (s, 3H), 2.48 (s, 3H).

${ }^{13}$ C-NMR (75 MHz, $\left.\mathrm{CDCl}_{3}\right): \delta=166.5\left(\mathrm{C}_{\mathrm{q}}\right), 162.1\left(\mathrm{C}_{\mathrm{q}}\right), 158.8\left(\mathrm{C}_{\mathrm{q}}\right), 151.3\left(\mathrm{C}_{\mathrm{q}}\right), 142.6\left(\mathrm{C}_{\mathrm{q}}\right)$, $136.4\left(\mathrm{C}_{\mathrm{q}}\right), 133.3\left(\mathrm{C}_{\mathrm{q}}\right), 131.5(\mathrm{CH}), 128.7(\mathrm{CH}), 128.5(\mathrm{CH}), 128.2(\mathrm{CH}), 128.2\left(\mathrm{C}_{\mathrm{q}}\right), 128.2$ $\left(\mathrm{C}_{\mathrm{q}}\right), 128.1(\mathrm{CH}), 127.9(\mathrm{CH}), 120.6(\mathrm{CH}), 114.1(\mathrm{CH}), 93.9(\mathrm{CH}), 65.8\left(\mathrm{CH}_{2}\right), 55.2\left(\mathrm{CH}_{3}\right)$, $43.2\left(\mathrm{CH}_{2}\right), 22.1\left(\mathrm{CH}_{3}\right)$.

IR (ATR): 3255, 2832, 1707, 1637, 1120, $824 \mathrm{~cm}^{-1}$.

MS (EI) $m / z$ (relative intensity) $412(10)\left[\mathrm{M}^{+}\right], 321$ (10), 277 (25), 185 (100), 142 (5), 121 (45).

HR-MS (EI) $m / z$ calcd for $\mathrm{C}_{26} \mathrm{H}_{24} \mathrm{~N}_{2} \mathrm{O}_{3}\left[\mathrm{M}^{+}\right] 412.1787$, found 412.1790 .

\section{Ethyl 2-\{(1Z,3Z)-3-(tert-Butylimino)-6-me thylis oindolin-1-ylide ne $\}$ ace tate (119ba)}

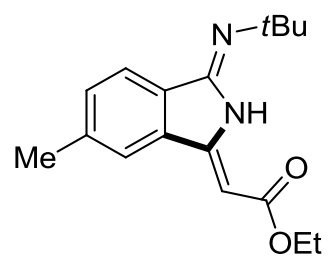

The general procedure $\mathbf{D}$ was followed us ing $\mathbf{1 1 9 b}(95.0 \mathrm{mg}, 0.50 \mathrm{mmol}), \mathbf{2 0 a}(75.0 \mathrm{mg}, 0.75$ mmol) and $\left[\operatorname{RuCl}_{2}(p \text {-cymene })\right]_{2}(7.7 \mathrm{mg}, 2.5 \mathrm{~mol} \%)$ at $100{ }^{\circ} \mathrm{C}$. Purification by column chromatography ( $n$-hexane/EtOAc: $12 / 1)$ yielded 119 ba (105 mg, 73\%) as a white solid. M.p.: $142-144^{\circ} \mathrm{C}$.

${ }^{1} \mathbf{H}-\mathbf{N M R}\left(300 \mathrm{MHz}, \mathrm{CDCl}_{3}\right): \delta=9.88(\mathrm{~s}, 1 \mathrm{H}), 7.74(\mathrm{~d}, J=7.8 \mathrm{~Hz}, 1 \mathrm{H}), 7.39$ (s, 1H), $7.32(\mathrm{~d}$, $J=7.8 \mathrm{~Hz}, 1 \mathrm{H}), 5.45(\mathrm{~s}, 1 \mathrm{H}), 4.25(\mathrm{q}, J=7.2 \mathrm{~Hz}, 2 \mathrm{H}), 2.44(\mathrm{~s}, 3 \mathrm{H}), 1.46(\mathrm{~s}, 9 \mathrm{H}), 1.34(\mathrm{t}, J=$ $7.2 \mathrm{~Hz}, 3 \mathrm{H})$.

${ }^{13}$ C-NMR (75 MHz, $\left.\mathrm{CDCl}_{3}\right): \delta=169.6\left(\mathrm{C}_{\mathrm{q}}\right), 151.9\left(\mathrm{C}_{\mathrm{q}}\right), 148.0\left(\mathrm{C}_{\mathrm{q}}\right), 140.7\left(\mathrm{C}_{\mathrm{q}}\right), 134.5\left(\mathrm{C}_{\mathrm{q}}\right)$, $132.9\left(\mathrm{C}_{\mathrm{q}}\right), 132.1(\mathrm{CH}), 122.4(\mathrm{CH}), 120.9(\mathrm{CH}), 84.0(\mathrm{CH}), 59.9\left(\mathrm{C}_{\mathrm{q}}\right), 54.1\left(\mathrm{CH}_{2}\right), 30.5$ $\left(\mathrm{CH}_{3}\right), 21.7\left(\mathrm{CH}_{3}\right), 14.5\left(\mathrm{CH}_{3}\right)$.

IR (ATR): 3397, 2969, 1637, 1193, 1053, $801 \mathrm{~cm}^{-1}$.

MS (EI) $m / z$ (relative intensity) $286(10)\left[\mathrm{M}^{+}\right], 271$ (100), 225 (90), 184 (30), $158(45), 143$ 
(15).

HR-MS (EI) $m / z$ calcd for $\mathrm{C}_{17} \mathrm{H}_{22} \mathrm{~N}_{2} \mathrm{O}_{2}\left[\mathrm{M}^{+}\right] 286.1681$, found 286.1676 .

n-B utyl 2-\{(1Z,3Z)-3-(tert-B utylimino)-6-me thylis oindolin-1-ylide ne $\}$ ace tate (119bb)<smiles>CCOC(=O)/C=C1\N/C(=N\C(C)C)c2ccc(C)cc21</smiles>

The general procedure $\mathbf{D}$ was followed using $\mathbf{1 1 9 b}(95.0 \mathrm{mg}, 0.50 \mathrm{mmol}), \mathbf{2 0 b}(75.0 \mathrm{mg}, 0.75$ mmol) and $\left[\mathrm{RuCl}_{2}(p \text {-cymene })\right]_{2}(7.7 \mathrm{mg}, 2.5 \mathrm{~mol} \%)$ at $100 \mathrm{C}$. Purification by column chromatography ( $n$-hexane/EtOAc: $15 / 1)$ yielded $119 b b$ (100 mg, 64\%) as a white solid. M.p.: $75-78^{\circ} \mathrm{C}$.

${ }^{1}$ H-NMR (300 MHz, $\left.\mathrm{CDCl}_{3}\right): \delta=9.87(\mathrm{~s}, 1 \mathrm{H}), 7.72(\mathrm{~d}, J=7.9 \mathrm{~Hz}, 1 \mathrm{H}), 7.38(\mathrm{~s}, 1 \mathrm{H}), 7.30$ (d, $J=7.9 \mathrm{~Hz}, 1 \mathrm{H}), 5.45(\mathrm{~s}, 1 \mathrm{H}), 4.17(\mathrm{t}, J=6.7 \mathrm{~Hz}, 2 \mathrm{H}), 2.43(\mathrm{~s}, 3 \mathrm{H}), 1.72-1.62(\mathrm{~m}, 2 \mathrm{H}), 1.45$ (s, 9H), 1.50-1.36 (m, 2H), 0.94 (t, $J=7.3 \mathrm{~Hz}, 3 \mathrm{H})$.

${ }^{13}$ C-NMR (75 MHz, $\left.\mathrm{CDCl}_{3}\right): \delta=169.7\left(\mathrm{C}_{\mathrm{q}}\right), 151.9\left(\mathrm{C}_{\mathrm{q}}\right), 148.0\left(\mathrm{C}_{\mathrm{q}}\right), 140.7\left(\mathrm{C}_{\mathrm{q}}\right), 134.5\left(\mathrm{C}_{\mathrm{q}}\right)$, $132.8\left(\mathrm{C}_{\mathrm{q}}\right), 132.1(\mathrm{CH}), 122.4(\mathrm{CH}), 120.9(\mathrm{CH}), 84.0(\mathrm{CH}), 63.9\left(\mathrm{C}_{\mathrm{q}}\right), 54.0\left(\mathrm{CH}_{2}\right), 30.8$ $\left(\mathrm{CH}_{2}\right), 30.4\left(\mathrm{CH}_{3}\right), 21.7\left(\mathrm{CH}_{3}\right), 19.2\left(\mathrm{CH}_{2}\right), 13.7\left(\mathrm{CH}_{3}\right)$.

IR (ATR): 3396, 2968, 1634, 1189, 1051, $702 \mathrm{~cm}^{-1}$.

MS (EI) $m / z$ (relative intensity) 314 (10) $\left[\mathrm{M}^{+}\right], 299$ (100), 285 (5), 225 (85), 185 (20), 158 (25).

HR-MS (EI) $m / z$ calcd for $\mathrm{C}_{19} \mathrm{H}_{26} \mathrm{~N}_{2} \mathrm{O}_{2}\left[\mathrm{M}^{+}\right]$314.1994, found 314.1996.

Benzyl 2-\{(1Z,3Z)-3-(tert-Butylimino)-6-me thylis oindolin-1-ylide ne $\}$ ace tate (119bc)<smiles>CCCCOC(=O)/C=C1\N/C(=N/CC(C)C)c2ccc(C)cc21</smiles>

The general procedure $\mathbf{D}$ was followed us ing $\mathbf{1 1 7 b}(95.0 \mathrm{mg}, 0.50 \mathrm{mmol}), \mathbf{2 0 c}(75.0 \mathrm{mg}, 0.75$ mmol) and $\left[\mathrm{RuCl}_{2}(p \text {-cymene })\right]_{2}(7.7 \mathrm{mg}, 2.5 \mathrm{~mol} \%)$ at $100 \mathrm{C}$. Purification by column chromatography ( $n$-hexane/EtOAc: $10 / 1)$ yie lded $119 b c(119 \mathrm{mg}, 68 \%)$ as a white solid. M. p.: 
$126-127^{\circ} \mathrm{C}$

${ }^{1} \mathbf{H}-\mathbf{N M R}\left(300 \mathrm{MHz}, \mathrm{CDCl}_{3}\right): \delta=9.82(\mathrm{~s}, 1 \mathrm{H}), 7.74(\mathrm{~d}, J=7.8 \mathrm{~Hz}, 1 \mathrm{H}), 7.42-7.30(\mathrm{~m}, 7 \mathrm{H})$, $5.52(\mathrm{~s}, 1 \mathrm{H}), 5.25(\mathrm{~s}, 2 \mathrm{H}), 2.42(\mathrm{~s}, 3 \mathrm{H}), 1.44(\mathrm{~s}, 9 \mathrm{H})$.

${ }^{13}$ C-NMR (75 MHz, $\left.\mathrm{CDCl}_{3}\right): \delta=169.1\left(\mathrm{C}_{\mathrm{q}}\right), 152.2\left(\mathrm{C}_{\mathrm{q}}\right), 147.9\left(\mathrm{C}_{\mathrm{q}}\right), 140.7\left(\mathrm{C}_{\mathrm{q}}\right), 136.2\left(\mathrm{C}_{\mathrm{q}}\right)$, $134.4\left(\mathrm{C}_{\mathrm{q}}\right), 132.8\left(\mathrm{C}_{\mathrm{q}}\right), 132.2(\mathrm{CH}), 128.6(\mathrm{CH}), 128.2(\mathrm{CH}), 128.1(\mathrm{CH}), 122.4(\mathrm{CH}), 120.9$ $(\mathrm{CH}), 83.6(\mathrm{CH}), 65.8\left(\mathrm{C}_{\mathrm{q}}\right), 54.1\left(\mathrm{CH}_{2}\right), 30.4\left(\mathrm{CH}_{3}\right), 21.7\left(\mathrm{CH}_{3}\right)$.

IR (ATR): 3396, 2968, 1634, 1189, 1050, $754 \mathrm{~cm}^{-1}$.

MS (EI) $m / z$ (relative intensity) $348(10)\left[\mathrm{M}^{+}\right], 333$ (100), 225 (80), 185 (15), 158 (25), 91 (60).

HR-MS (EI) $m / z$ calcd for $\mathrm{C}_{22} \mathrm{H}_{24} \mathrm{~N}_{2} \mathrm{O}_{2}\left[\mathrm{M}^{+}\right] 348.1838$, found 348.1831.

\section{Ethyl 2-\{(1Z,3Z)-3-(tert-Butylimino)isoindolin-1-ylide ne $\}$ acetate (119aa)}

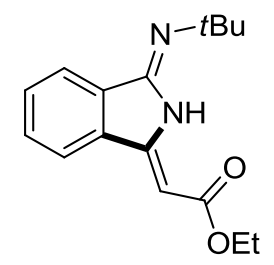

The general procedure $\mathbf{D}$ was followed us ing $117 \mathbf{a}(88.0 \mathrm{mg}, 0.50 \mathrm{mmol}), \mathbf{2 0 a}(75.0 \mathrm{mg}, 0.75$ mmol) and $\left[\mathrm{RuCl}_{2}(p \text {-cymene })\right]_{2}(7.7 \mathrm{mg}, 2.5 \mathrm{~mol} \%)$ at $100 \mathrm{C}$. Purification by column chromatography ( $n$-hexane/EtOAc: $12 / 1)$ yielded 119aa (79 mg, 58\%) as a white solid. M. p.: $68-70{ }^{\circ} \mathrm{C}$.

${ }^{1}$ H-NMR $\left(300 \mathrm{MHz}, \mathrm{CDCl}_{3}\right): \delta=9.92(\mathrm{~s}, 1 \mathrm{H}), 7.85-7.82(\mathrm{~m}, 1 \mathrm{H}), 7.58-7.56(\mathrm{~m}, 1 \mathrm{H})$, 7.51-7.42 (m, 2H), 5.47 (s, 1H), 4.22 (q, $J=7.2 \mathrm{~Hz}, 2 \mathrm{H}), 1.45$ (s, 9H), 1.31 (t, $J=7.2 \mathrm{~Hz}$, $3 \mathrm{H})$.

${ }^{13}$ C-NMR (75 MHz, $\left.\mathrm{CDCl}_{3}\right): \delta=169.4\left(\mathrm{C}_{\mathrm{q}}\right), 151.7\left(\mathrm{C}_{\mathrm{q}}\right), 147.8\left(\mathrm{C}_{\mathrm{q}}\right), 135.3\left(\mathrm{C}_{\mathrm{q}}\right), 134.2\left(\mathrm{C}_{\mathrm{q}}\right)$, $131.0(\mathrm{CH}), 130.2(\mathrm{CH}), 122.6(\mathrm{CH}), 120.5(\mathrm{CH}), 84.2(\mathrm{CH}), 59.9\left(\mathrm{C}_{\mathrm{q}}\right), 54.1\left(\mathrm{CH}_{2}\right), 30.4$ $\left(\mathrm{CH}_{3}\right), 14.4\left(\mathrm{CH}_{3}\right)$.

IR (ATR): 3387, 2971, 1656, 1179, 1058, $808 \mathrm{~cm}^{-1}$. MS (EI) $m / z$ (relative intens ity) $272(15)\left[\mathrm{M}^{+}\right], 257$ (100), 211 (95), 170 (35), 144 (40), 128 (20).

HR-MS (EI) $m / z$ calcd for $\mathrm{C}_{16} \mathrm{H}_{20} \mathrm{~N}_{2} \mathrm{O}_{2}\left[\mathrm{M}^{+}\right] 272.1525$, found 272.1522 . 
Ethyl 2-\{(1Z,3Z)-3-(tert-Butylimino)-6-me thoxyisoindolin-1-ylidene\}acetate (119ca)<smiles>CCOC(=O)/C=C1\N/C(=N\CC(C)C)c2ccc(OC)cc21</smiles>

The general procedure $\mathbf{D}$ was followed using $117 \mathrm{c}(103 \mathrm{mg}, 0.50 \mathrm{mmol}), \mathbf{2 0 a}(75.0 \mathrm{mg}, 0.75$ mmol) and $\left[\mathrm{RuCl}_{2}(p \text {-cymene })\right]_{2}(15.3 \mathrm{mg}, 5.0 \mathrm{~mol} \%)$ at $120 \mathrm{C}$. Purification by column chromatography ( $n$-hexane/EtOAc: 4/1) yielded 119ca (127 mg, 84\%) as a white solid. M. p.: $100-101^{\circ} \mathrm{C}$.

${ }^{1} \mathbf{H}-\mathbf{N M R}\left(300 \mathrm{MHz}, \mathrm{CDCl}_{3}\right): \delta=9.81(\mathrm{~s}, 1 \mathrm{H}), 7.72(\mathrm{~d}, J=9.2 \mathrm{~Hz}, 1 \mathrm{H}), 7.02(\mathrm{~d}, J=2.2 \mathrm{~Hz}$, 1H), $7.00(\mathrm{dd}, J=9.2,2.2 \mathrm{~Hz}, 1 \mathrm{H}), 5.41(\mathrm{~s}, 1 \mathrm{H}), 4.22(\mathrm{q}, J=7.2 \mathrm{~Hz}, 2 \mathrm{H}), 3.82(\mathrm{~s}, 3 \mathrm{H}), 1.43$ (s, 9H), 1.30 (t, $J=7.2 \mathrm{~Hz}, 3 \mathrm{H})$.

${ }^{13}$ C-NMR $\left(75 \mathrm{MHz}, \mathrm{CDCl}_{3}\right): \delta=169.4\left(\mathrm{C}_{\mathrm{q}}\right), 161.8\left(\mathrm{C}_{\mathrm{q}}\right), 151.6\left(\mathrm{C}_{\mathrm{q}}\right), 147.6\left(\mathrm{C}_{\mathrm{q}}\right), 135.9\left(\mathrm{C}_{\mathrm{q}}\right)$, $128.0\left(\mathrm{C}_{\mathrm{q}}\right), 123.8(\mathrm{CH}), 118.0(\mathrm{CH}), 104.6(\mathrm{CH}), 84.0(\mathrm{CH}), 59.9\left(\mathrm{C}_{\mathrm{q}}\right), 55.6\left(\mathrm{CH}_{3}\right), 54.0\left(\mathrm{CH}_{2}\right)$, $30.5\left(\mathrm{CH}_{3}\right), 14.4\left(\mathrm{CH}_{3}\right)$.

IR (ATR): 3418, 2969, 1634, 1490, 1282, 1057, $800 \mathrm{~cm}^{-1}$.

MS (EI) $m / z$ (relative intensity) $302(15)\left[\mathrm{M}^{+}\right], 287$ (100), 241 (90), 200 (30), 174 (40), 158 (20).

HR-MS (EI) $m / z$ calcd for $\mathrm{C}_{17} \mathrm{H}_{22} \mathrm{~N}_{2} \mathrm{O}_{3}\left[\mathrm{M}^{+}\right] 302.1630$, found 302.1636 .

\section{Ethyl 2-\{(1Z,3Z)-3-(tert-Butylimino)-6-fluorois oindolin-1-ylide ne $\}$ ace tate (119da)}<smiles>CCOC(=O)/C=C1\N/C(=N\C(C)C)c2ccc(F)cc21</smiles>

The general procedure $\mathbf{D}$ was followed us ing $\mathbf{1 1 7 d}(97.0 \mathrm{mg}, 0.50 \mathrm{mmol}), \mathbf{2 0 a}(75.0 \mathrm{mg}, 0.75$ $\mathrm{mmol})$ and $\left[\mathrm{RuCl}_{2}(p \text {-cymene })\right]_{2}(15.3 \mathrm{mg}, 5.0 \mathrm{~mol} \%)$ at $120 \mathrm{C}$. Purification by column chromatography (n-hexane/EtOAc: $20 / 1)$ yielded 119da (105 mg, 72\%) as a white solid. M.p.: $87-88^{\circ} \mathrm{C}$.

${ }^{1}$ H-NMR $\left(300 \mathrm{MHz}, \mathrm{CDCl}_{3}\right): \delta=9.90(\mathrm{~s}, 1 \mathrm{H}), 7.81(\mathrm{dd}, J=8.4,5.0 \mathrm{~Hz}, 1 \mathrm{H}), 7.23(\mathrm{dd}, J=$ 
8.4, $2.1 \mathrm{~Hz}, 1 \mathrm{H}), 7.17$ (dd, $J=8.4,2.1 \mathrm{~Hz}, 1 \mathrm{H}), 5.42$ (s, 1H), 4.23 (q, $J=7.2 \mathrm{~Hz}, 2 \mathrm{H}), 1.44$ (s, 9H), $1.32(\mathrm{t}, J=7.2 \mathrm{~Hz}, 3 \mathrm{H})$.

${ }^{13}$ C-NMR $\left(75 \mathrm{MHz}, \mathrm{CDCl}_{3}\right): \delta=169.3\left(\mathrm{C}_{\mathrm{q}}\right), 164.3\left(\mathrm{C}_{\mathrm{q}}, J_{\mathrm{C}-\mathrm{F}}=250.2 \mathrm{~Hz}\right), 150.6\left(\mathrm{C}_{\mathrm{q}}, J_{\mathrm{C}-\mathrm{F}}=4.1\right.$ $\mathrm{Hz}), 146.8\left(\mathrm{C}_{\mathrm{q}}\right), 136.2\left(\mathrm{C}_{\mathrm{q}}, J_{\mathrm{C}-\mathrm{F}}=10.0 \mathrm{~Hz}\right), 131.3\left(\mathrm{C}_{\mathrm{q}}\right), 124.5\left(\mathrm{CH}, J_{\mathrm{C}-\mathrm{F}}=9.4 \mathrm{~Hz}\right), 118.6(\mathrm{CH}$, $\left.J_{\mathrm{C}-\mathrm{F}}=23.6 \mathrm{~Hz}\right), 107.5\left(\mathrm{CH}, J_{\mathrm{C}-\mathrm{F}}=24.6 \mathrm{~Hz}\right), 84.7(\mathrm{CH}), 60.1\left(\mathrm{C}_{\mathrm{q}}\right), 54.2\left(\mathrm{CH}_{2}\right), 30.4\left(\mathrm{CH}_{3}\right)$, $14.2\left(\mathrm{CH}_{3}\right)$.

${ }^{19}$ F-NMR (282 MHz, $\mathrm{CDCl}_{3}$ ): $\delta=-(109.5-109.6)$. (m). IR (ATR): 3391, 2970, 1667, 1210, $1054,799 \mathrm{~cm}^{-1}$.

MS (EI) $m / z$ (relative intensity) $290(5)\left[\mathrm{M}^{+}\right], 275$ (100), 229 (90), 188 (25), 162 (35), 146 (20).

HR-MS (EI) $m / z$ calcd for $\mathrm{C}_{16} \mathrm{H}_{19} \mathrm{~N}_{2} \mathrm{O}_{2} \mathrm{~F}\left[\mathrm{M}^{+}\right] 290.1431$, found 290.1423.

Ethyl 2-\{(1Z,3Z)-6-Bromo-3-(tert-butylimino)is oindolin-1-ylidene $\}$ ace tate (119fa)<smiles>CCOC(=O)/C=C1\N/C(=N\CC(C)C)c2ccc(Br)cc21</smiles>

The general procedure $\mathbf{D}$ was followed using $119 \mathrm{f}$ (127 $\mathrm{mg}, 0.50 \mathrm{mmol})$, ethyl acrylate (20a) (75.0 mg, $0.75 \mathrm{mmol})$ and $\left[\mathrm{RuCl}_{2}(p \text {-cymene })\right]_{2}(15.3 \mathrm{mg}, 5.0 \mathrm{~mol} \%)$ at $120 \mathrm{C}$. Purification by column chromatography ( $n$-hexane/EtOAc: 20/1) yielded 119fa (112 mg, 64\%) as a white solid. M. p.: $115-116^{\circ} \mathrm{C}$.

${ }^{1}$ H-NMR (300 MHz, $\left.\mathrm{CDCl}_{3}\right): \delta=9.92(\mathrm{~s}, 1 \mathrm{H}), 7.75-7.72(\mathrm{~m}, 2 \mathrm{H}), 7.64(\mathrm{~d}, J=8.1 \mathrm{~Hz}, 1 \mathrm{H})$, $5.46(\mathrm{~s}, 1 \mathrm{H}), 4.26(\mathrm{q}, J=6.8 \mathrm{~Hz}, 2 \mathrm{H}), 1.46(\mathrm{~s}, 9 \mathrm{H}), 1.34(\mathrm{t}, J=6.8 \mathrm{~Hz}, 3 \mathrm{H})$.

${ }^{13}$ C-NMR (75 MHz, $\left.\mathrm{CDCl}_{3}\right): \delta=169.3\left(\mathrm{C}_{\mathrm{q}}\right), 150.3\left(\mathrm{C}_{\mathrm{q}}\right), 146.9\left(\mathrm{C}_{\mathrm{q}}\right), 136.1\left(\mathrm{C}_{\mathrm{q}}\right), 134.2(\mathrm{CH})$, $134.0\left(\mathrm{C}_{\mathrm{q}}\right), 124.5(\mathrm{CH}), 124.1\left(\mathrm{C}_{\mathrm{q}}\right), 123.7(\mathrm{CH}), 85.1(\mathrm{CH}), 60.1\left(\mathrm{C}_{\mathrm{q}}\right), 54.3\left(\mathrm{CH}_{2}\right), 30.4\left(\mathrm{CH}_{3}\right)$, $14.4\left(\mathrm{CH}_{3}\right)$.

IR (ATR): 3382, 2970, 1647, 1197, 1046, $799 \mathrm{~cm}^{-1}$.

MS (EI) $m / z$ (relative intensity) 350; $352(5)\left[\mathrm{M}^{+}\right], 335 ; 337$ (100), 289; 291 (70), 222; 224 (20), 207; 209 (15).

HR-MS (ESI) $m / z$ calcd for $\mathrm{C}_{16} \mathrm{H}_{19} \mathrm{~N}_{2} \mathrm{O}_{2}{ }^{79} \mathrm{Br}\left[\mathrm{M}+\mathrm{H}^{+}\right]$351.0708; 353.0688, found 351.0703; 
Ethyl 2-\{(1Z,3Z)-3-(tert-Butylimino)-6-nitroisoindolin-1-ylidene\}acetate (119ha)<smiles>CCOC(=O)/C=C1\N/C(=N\CC(C)C)c2ccc([N+](=O)[O-])cc21</smiles>

The general procedure $\mathbf{D}$ was followed using $117 \mathbf{h}(111 \mathrm{mg}, 0.50 \mathrm{mmol}), \mathbf{2 0 a}(75.0 \mathrm{mg}, 0.75$ mmol) and $\left[\mathrm{RuCl}_{2}(p \text {-cymene })\right]_{2}(15.3 \mathrm{mg}, 5.0 \mathrm{~mol} \%)$ at $120 \mathrm{C}$. Purification by column chromatography ( $n$-Hexane/EtOAc: $20 / 1)$ yielded 119 ha (42 mg, 26\%) as a white solid. M. p.: $190-191^{\circ} \mathrm{C}$.

${ }^{1}$ H-NMR (300 MHz, $\left.\mathrm{CDCl}_{3}\right): \delta=10.09(\mathrm{~s}, 1 \mathrm{H}), 8.44(\mathrm{~d}, J=1.9 \mathrm{~Hz}, 1 \mathrm{H}), 8.35(\mathrm{dd}, J=8.6$, $1.9 \mathrm{~Hz}, 1 \mathrm{H}), 7.99$ (d, $J=8.6 \mathrm{~Hz}, 1 \mathrm{H}), 5.60(\mathrm{~s}, 1 \mathrm{H}), 4.26$ (q, $J=7.2 \mathrm{~Hz}, 2 \mathrm{H}), 1.45$ (s, 9H), 1.33 (t, $J=7.2 \mathrm{~Hz}, 3 \mathrm{H})$.

${ }^{13}$ C-NMR (75 MHz, $\left.\mathrm{CDCl}_{3}\right): \delta=169.1\left(\mathrm{C}_{\mathrm{q}}\right), 149.5\left(\mathrm{C}_{\mathrm{q}}\right), 149.5\left(\mathrm{C}_{\mathrm{q}}\right), 145.8\left(\mathrm{C}_{\mathrm{q}}\right), 140.4\left(\mathrm{C}_{\mathrm{q}}\right)$, $135.2\left(\mathrm{C}_{\mathrm{q}}\right), 125.9(\mathrm{CH}), 123.7(\mathrm{CH}), 116.3(\mathrm{CH}), 86.3(\mathrm{CH}), 60.4\left(\mathrm{C}_{\mathrm{q}}\right), 54.8\left(\mathrm{CH}_{2}\right), 30.2\left(\mathrm{CH}_{3}\right)$, $14.4\left(\mathrm{CH}_{3}\right)$.

IR (ATR): 3339, 2966, 1623, 1259, 1030, $800 \mathrm{~cm}^{-1}$.

MS (EI) $m / z$ (relative intens ity) $317(10)\left[\mathrm{M}^{+}\right], 302$ (100), 272 (30), 256 (75), 226 (20), 189 (20), 57 (25).

HR-MS (ESI) $m / z$ calcd for $\mathrm{C}_{16} \mathrm{H}_{20} \mathrm{~N}_{3} \mathrm{O}_{4}\left[\mathrm{M}+\mathrm{H}^{+}\right] 318.1454$, found 318.1449 .

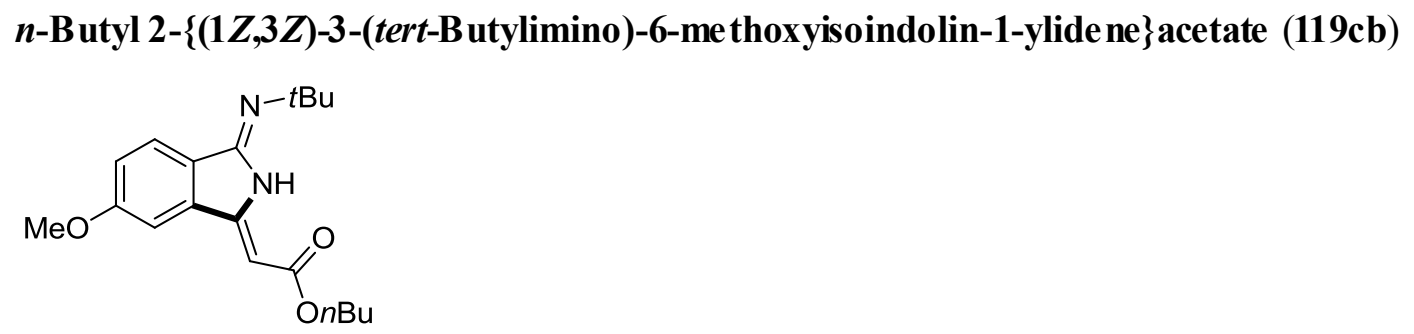

The general procedure $\mathbf{D}$ was followed using $117 \mathbf{c}(103 \mathrm{mg}, 0.50 \mathrm{mmol}), \mathbf{2 0 b}(96.0 \mathrm{mg}, 0.75$ mmol) $\left[\operatorname{RuCl}_{2}(p \text {-cymene })\right]_{2}(7.7 \mathrm{mg}, 2.5 \mathrm{~mol} \%)$ at $100 \mathrm{C}$. Purification by column chromatography ( $n$-hexane/EtOAc: 8/1) yielded 119cb $(117 \mathrm{mg}, 71 \%)$ as a yellow oil. ${ }^{1}$ H-NMR (300 MHz, $\left.\mathrm{CDCl}_{3}\right): \delta=9.83(\mathrm{~s}, 1 \mathrm{H}), 7.74(\mathrm{~d}, J=8.6 \mathrm{~Hz}, 1 \mathrm{H}), 7.06-7.02(\mathrm{~m}, 2 \mathrm{H})$, 
$5.45(\mathrm{~s}, 1 \mathrm{H}), 4.17(\mathrm{t}, J=6.6 \mathrm{~Hz}, 2 \mathrm{H}), 3.85(\mathrm{~s}, 3 \mathrm{H}), 1.72-1.62(\mathrm{~m}, 2 \mathrm{H}), 1.44(\mathrm{~s}, 9 \mathrm{H}), 1.49-1.36$ (m, 2H), $1.31(\mathrm{t}, J=7.4 \mathrm{~Hz}, 3 \mathrm{H})$.

${ }^{13}$ C-NMR $\left(75 \mathrm{MHz}, \mathrm{CDCl}_{3}\right): \delta=169.6\left(\mathrm{C}_{\mathrm{q}}\right), 161.8\left(\mathrm{C}_{\mathrm{q}}\right), 151.7\left(\mathrm{C}_{\mathrm{q}}\right), 147.7\left(\mathrm{C}_{\mathrm{q}}\right), 135.9\left(\mathrm{C}_{\mathrm{q}}\right)$, $128.1\left(\mathrm{C}_{\mathrm{q}}\right), 123.8(\mathrm{CH}), 118.1(\mathrm{CH}), 104.6(\mathrm{CH}), 84.1(\mathrm{CH}), 63.9\left(\mathrm{C}_{\mathrm{q}}\right), 55.7\left(\mathrm{CH}_{3}\right), 54.0\left(\mathrm{CH}_{2}\right)$, $30.9\left(\mathrm{CH}_{2}\right), 30.5\left(\mathrm{CH}_{3}\right), 19.2\left(\mathrm{CH}_{2}\right), 13.8\left(\mathrm{CH}_{3}\right)$.

IR (ATR): 3399, 2962, 1621, 1157, 1053, $800 \mathrm{~cm}^{-1}$.

MS (EI) $m / z$ (relative intensity) 330 (10) $\left[\mathrm{M}^{+}\right], 315$ (100), 274 (5), 241 (75), 200 (25), 174 (30), $158(20)$.

HR-MS (EI) $m / z$ calcd for $\mathrm{C}_{19} \mathrm{H}_{26} \mathrm{~N}_{2} \mathrm{O}_{3}\left[\mathrm{M}^{+}\right] 330.1943$, found 330.1947.

Ethyl 2-\{(1Z,3Z)-3-(tert-Butylimino)-6-chloroisoindolin-1-ylide ne $\}$ acetate (119ea)<smiles>CCOC(=O)/C=C1\N/C(=N\CC(C)C)c2ccc(Cl)cc21</smiles>

The general procedure $\mathbf{D}$ was followed using $117 \mathrm{e}(105 \mathrm{mg}, 0.50 \mathrm{mmol}), \mathbf{2 0 a}(75.0 \mathrm{mg}, 0.75$ mmol $)$ and $\left[\mathrm{RuCl}_{2}(p \text {-cymene })\right]_{2}(15.3 \mathrm{mg}, 5.0 \mathrm{~mol} \%)$ at $120 \mathrm{C}$. Purification by column chromatography ( $n$-hexane/EtOAc: $15 / 1)$ yie lded 119ea (107 mg, 70\%) as a white solid. M. p.: $120-121^{\circ} \mathrm{C}$.

${ }^{1} \mathbf{H}-\mathbf{N M R}\left(300 \mathrm{MHz}, \mathrm{CDCl}_{3}\right): \delta=9.91(\mathrm{~s}, 1 \mathrm{H}), 7.76(\mathrm{~d}, J=8.1 \mathrm{~Hz}, 1 \mathrm{H}), 7.54(\mathrm{~d}, J=1.8 \mathrm{~Hz}$, 1H), 7.45 (dd, $J=8.1,1.8 \mathrm{~Hz}, 1 \mathrm{H}), 5.44(\mathrm{~s}, 1 \mathrm{H}), 4.23$ (q, $J=7.2 \mathrm{~Hz}, 2 \mathrm{H}), 1.44(\mathrm{~s}, 9 \mathrm{H}), 1.32$ (t, $J=7.2 \mathrm{~Hz}, 3 \mathrm{H})$.

${ }^{13}$ C-NMR (75 MHz, $\left.\mathrm{CDCl}_{3}\right): \delta=169.2\left(\mathrm{C}_{\mathrm{q}}\right), 150.4\left(\mathrm{C}_{\mathrm{q}}\right), 146.8\left(\mathrm{C}_{\mathrm{q}}\right), 136.5\left(\mathrm{C}_{\mathrm{q}}\right), 135.8\left(\mathrm{C}_{\mathrm{q}}\right)$, $133.7\left(\mathrm{C}_{\mathrm{q}}\right), 131.2(\mathrm{CH}), 123.9(\mathrm{CH}), 120.7(\mathrm{CH}), 85.1(\mathrm{CH}), 60.1\left(\mathrm{C}_{\mathrm{q}}\right), 54.3\left(\mathrm{CH}_{2}\right), 30.3$ $\left(\mathrm{CH}_{3}\right), 14.4\left(\mathrm{CH}_{3}\right)$.

IR (ATR): 3382, 2967, 1643, 1176, 1049, $800 \mathrm{~cm}^{-1}$.

MS (EI) $m / z$ (relative intensity) $306(5)\left[\mathrm{M}^{+}\right], 291$ (85), 245 (60), 204 (20), 178 (25), 43 (100).

HR-MS (ESI) $m / z$ calcd for $\mathrm{C}_{16} \mathrm{H}_{19} \mathrm{~N}_{2} \mathrm{O}_{2}{ }^{35} \mathrm{Cl}\left[\mathrm{M}^{+}\right]$306.1135, found 306.1096.

n-B utyl 2-\{(1Z,3Z)-3-(tert-B utylimino)-6-chloroisoindolin-1-ylide ne $\}$ acetate (119eb) 
<smiles>CCCCC(=O)/C=C1\N/C(=N\CC(C)C)c2ccc(Cl)cc21</smiles>

The general procedure $\mathbf{D}$ was followed using $117 \mathrm{e}(105 \mathrm{mg}, 0.50 \mathrm{mmol}), \mathbf{2 0 b}(96.0 \mathrm{mg}, 0.75$ mmol) and $\left[\mathrm{RuCl}_{2}(p \text {-cymene })\right]_{2}(15.3 \mathrm{mg}, 5.0 \mathrm{~mol} \%)$ at $120 \mathrm{C}$. Purification by column chromatography ( $n$-hexane/EtOAc: $20 / 1)$ yielded 119eb (123 mg, 74\%) as a white solid. M. p.: $81-82{ }^{\circ} \mathrm{C}$.

${ }^{1} \mathbf{H}-\mathbf{N M R}\left(300 \mathrm{MHz}, \mathrm{CDCl}_{3}\right): \delta=9.91(\mathrm{~s}, 1 \mathrm{H}), 7.77(\mathrm{~d}, J=8.2 \mathrm{~Hz}, 1 \mathrm{H}), 7.54(\mathrm{~d}, J=1.9 \mathrm{~Hz}$, 1H), $7.45(\mathrm{dd}, J=8.2,1.9 \mathrm{~Hz}, 1 \mathrm{H}), 5.45(\mathrm{~s}, 1 \mathrm{H}), 4.17(\mathrm{t}, J=6.6 \mathrm{~Hz}, 2 \mathrm{H}), 1.72-1.62(\mathrm{~m}, 2 \mathrm{H})$, $1.44(\mathrm{~s}, 9 \mathrm{H}), 1.45-1.37(\mathrm{~m}, 2 \mathrm{H}), 0.94(\mathrm{t}, J=7.3 \mathrm{~Hz}, 3 \mathrm{H})$.

${ }^{13}$ C-NMR (75 MHz, CDCl $): \delta=169.4\left(\mathrm{C}_{\mathrm{q}}\right), 150.4\left(\mathrm{C}_{\mathrm{q}}\right), 146.9\left(\mathrm{C}_{\mathrm{q}}\right), 136.5\left(\mathrm{C}_{\mathrm{q}}\right), 135.8\left(\mathrm{C}_{\mathrm{q}}\right)$, $133.7\left(\mathrm{C}_{\mathrm{q}}\right), 131.2(\mathrm{CH}), 124.0(\mathrm{CH}), 120.7(\mathrm{CH}), 85.1(\mathrm{CH}), 64.1\left(\mathrm{C}_{\mathrm{q}}\right), 54.3\left(\mathrm{CH}_{2}\right), 30.8$ $\left(\mathrm{CH}_{2}\right), 30.3\left(\mathrm{CH}_{3}\right), 19.2\left(\mathrm{CH}_{2}\right), 13.7\left(\mathrm{CH}_{3}\right)$.

IR (ATR): 3367, 2958, 1651, 1172, 1061, $828 \mathrm{~cm}^{-1}$.

MS (EI) $m / z$ (relative intensity) $334(5)\left[\mathrm{M}^{+}\right], 319$ (100), 245 (75), 204 (20), 178 (20), 163 (15).

HR-MS (EI) $m / z$ calcd for $\mathrm{C}_{18} \mathrm{H}_{23} \mathrm{~N}_{2} \mathrm{O}_{2}{ }^{35} \mathrm{Cl}\left[\mathrm{M}^{+}\right] 334.1448$, found 334.1445 .

\section{Benzyl 2-\{(1Z,3Z)-3-(tert-Butylimino)-6-chloroisoindolin-1-ylide ne $\}$ acetate (119ec)}<smiles>CC(C)(C)N=C1N/C(=C\C(=O)OCc2ccccc2)c2cc(Cl)ccc21</smiles>

The general procedure D was followed using 117 e (105 mg, $0.50 \mathrm{mmol}), \mathbf{2 0 c}(122 \mathrm{mg}, 0.75$ mmol) and $\left[\mathrm{RuCl}_{2}(p \text {-cymene })\right]_{2}(15.3 \mathrm{mg}, 5.0 \mathrm{~mol} \%)$ at $120 \mathrm{C}$. Purification by column chromatography ( $n$-hexane/EtOAc: $15 / 1)$ yie lded 119ec (130 mg, 71\%) as a white solid. M. p.: $114-116^{\circ} \mathrm{C}$.

${ }^{1} \mathbf{H}-\mathbf{N M R}\left(300 \mathrm{MHz}, \mathrm{CDCl}_{3}\right): \delta=9.85(\mathrm{~s}, 1 \mathrm{H}), 7.77(\mathrm{~d}, J=8.4 \mathrm{~Hz}, 1 \mathrm{H}), 7.55(\mathrm{~d}, J=1.8 \mathrm{~Hz}$, 1H), 7.47 (dd, $J=8.4,1.8 \mathrm{~Hz}, 1 \mathrm{H}), 7.41-7.33(\mathrm{~m}, 5 \mathrm{H}), 5.51$ (s, 1H), 5.23 (s, 2H), 1.42 (s, 9H). ${ }^{13}$ C-NMR $\left(75 \mathrm{MHz}, \mathrm{CDCl}_{3}\right): \delta=168.9\left(\mathrm{C}_{\mathrm{q}}\right), 150.8\left(\mathrm{C}_{\mathrm{q}}\right), 146.7\left(\mathrm{C}_{\mathrm{q}}\right), 136.5\left(\mathrm{C}_{\mathrm{q}}\right), 136.0\left(\mathrm{C}_{\mathrm{q}}\right)$, 
$135.7\left(\mathrm{C}_{\mathrm{q}}\right), 133.7\left(\mathrm{C}_{\mathrm{q}}\right), 131.3(\mathrm{CH}), 128.6(\mathrm{CH}), 128.3(\mathrm{CH}), 128.2(\mathrm{CH}), 123.9(\mathrm{CH}), 120.8$ $(\mathrm{CH}), 84.7(\mathrm{CH}), 66.0\left(\mathrm{C}_{\mathrm{q}}\right), 54.3\left(\mathrm{CH}_{2}\right), 30.8\left(\mathrm{CH}_{3}\right)$.

IR (ATR): 3385, 2970, 1638, 1161, 1048, $602 \mathrm{~cm}^{-1}$.

MS (EI) $m / z$ (relative intensity) $368(10)\left[\mathrm{M}^{+}\right], 353$ (100), 309 (5), 245 (80), 205 (20), 178 (20).

HR-MS (EI) $m / z$ calcd for $\mathrm{C}_{21} \mathrm{H}_{21} \mathrm{~N}_{2} \mathrm{O}_{2} \mathrm{Cl}\left[\mathrm{M}^{+}\right]$368.1292, found 368.1297.

\section{Ethyl 2-\{(1Z,3Z)-3-(tert-Butylimino)-4-me thylis oindolin-1-ylide ne $\}$ acetate (119la)}

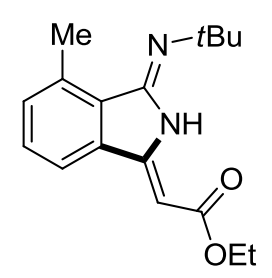

The general procedure $\mathbf{D}$ was followed using 1171 (95.0 mg, $0.50 \mathrm{mmol}), \mathbf{2 0 a}(75.0 \mathrm{mg}, 0.75$ mmol $)\left[\mathrm{RuCl}_{2}(p \text {-cymene })\right]_{2}(7.7 \mathrm{mg}, 2.5 \mathrm{~mol} \%)$ at $100 \mathrm{C}$. Purification by column chromatography ( $n$-hexane/EtOAc: 20/1) yielded $119 l a(91 \mathrm{mg}, 64 \%$ ) as a white solid. M. p.: $91-92{ }^{\circ} \mathrm{C}$.

${ }^{1}$ H-NMR $\left(300 \mathrm{MHz}, \mathrm{CDCl}_{3}\right): \delta=9.85(\mathrm{~s}, 1 \mathrm{H}), 7.42(\mathrm{dd}, J=9.0,3.0 \mathrm{~Hz}, 1 \mathrm{H}), 7.31(\mathrm{dd}, J=$ 9.0, $9.0 \mathrm{~Hz}, 1 \mathrm{H}), 7.25$ (dd, $J=9.0,3.0 \mathrm{~Hz}, 1 \mathrm{H}), 5.42$ (s, 1H), 4.23 (q, $J=6.0 \mathrm{~Hz}, 2 \mathrm{H}), 2.69$ (s, $3 \mathrm{H}), 1.44(\mathrm{~s}, 9 \mathrm{H}), 1.32(\mathrm{t}, J=6.0 \mathrm{~Hz}, 3 \mathrm{H})$.

${ }^{13}$ C-NMR (75 MHz, $\left.\mathrm{CDCl}_{3}\right): \delta=169.7\left(\mathrm{C}_{\mathrm{q}}\right), 152.1\left(\mathrm{C}_{\mathrm{q}}\right), 148.3\left(\mathrm{C}_{\mathrm{q}}\right), 137.3\left(\mathrm{C}_{\mathrm{q}}\right), 134.8\left(\mathrm{C}_{\mathrm{q}}\right)$, 133.6 (CH), $131.8\left(\mathrm{C}_{\mathrm{q}}\right), 129.3(\mathrm{CH}), 118.1(\mathrm{CH}), 82.7(\mathrm{CH}), 59.8\left(\mathrm{C}_{\mathrm{q}}\right), 54.4\left(\mathrm{CH}_{2}\right), 30.3\left(\mathrm{CH}_{3}\right)$, $18.4\left(\mathrm{CH}_{3}\right), 14.5\left(\mathrm{CH}_{3}\right)$.

IR (ATR): 3400, 2965, 1618, 1178, 1054, $780 \mathrm{~cm}^{-1}$.

MS (EI) $m / z$ (relative intensity) $286(55)\left[\mathrm{M}^{+}\right], 271$ (50), 225 (100), 184 (90), $156(30), 142$ (15).

HR-MS (EI) $m / z$ calcd for $\mathrm{C}_{17} \mathrm{H}_{22} \mathrm{~N}_{2} \mathrm{O}_{2}\left[\mathrm{M}^{+}\right] 286.1681$, found 286.1685 . 
<smiles>CCOC(=O)/C=C1\N/C(=N\CC(C)C)c2cc(C)ccc21</smiles>

The general procedure D was followed using $117 \mathbf{i}(95.0 \mathrm{mg}, 0.50 \mathrm{mmol}), \mathbf{2 0 a}(75.0 \mathrm{mg}, 0.75$ mmol $)\left[\mathrm{RuCl}_{2}(p \text {-cymene })\right]_{2}(7.7 \mathrm{mg}, 2.5 \mathrm{~mol} \%)$ at $100 \mathrm{C}$. Purification by column chromatography ( $n$-hexane/EtOAc: $5 / 1)$ yielded 119ia (114 mg, 80\%) as a white solid. M. p.: 79- $81{ }^{\circ} \mathrm{C}$.

${ }^{1} \mathbf{H}-\mathbf{N M R}\left(300 \mathrm{MHz}, \mathrm{CDCl}_{3}\right): \delta=9.88(\mathrm{br} \mathrm{s}, 1 \mathrm{H}), 7.65(\mathrm{~s}, 1 \mathrm{H}), 7.45(\mathrm{~d}, J=8.0 \mathrm{~Hz}, 1 \mathrm{H}), 7.35$ $(\mathrm{d}, J=8.0 \mathrm{~Hz}, 1 \mathrm{H}), 5.42(\mathrm{~s}, 1 \mathrm{H}), 4.22(\mathrm{q}, J=7.1 \mathrm{~Hz}, 2 \mathrm{H}), 2.41(\mathrm{~s}, 3 \mathrm{H}), 1.45(\mathrm{~s}, 9 \mathrm{H}), 1.31(\mathrm{t}, J$ $=7.1 \mathrm{~Hz}, 3 \mathrm{H})$.

${ }^{13}$ C-NMR $\left(75 \mathrm{MHz}, \mathrm{CDCl}_{3}\right): \delta=169.6\left(\mathrm{C}_{\mathrm{q}}\right), 151.9\left(\mathrm{C}_{\mathrm{q}}\right), 148.1\left(\mathrm{C}_{\mathrm{q}}\right), 141.7\left(\mathrm{C}_{\mathrm{q}}\right), 135.5\left(\mathrm{C}_{\mathrm{q}}\right)$, $131.7\left(\mathrm{C}_{\mathrm{q}}\right), 131.3(\mathrm{CH}), 122.8(\mathrm{CH}), 120.4(\mathrm{CH}), 83.8(\mathrm{CH}), 59.9\left(\mathrm{CH}_{2}\right), 54.0\left(\mathrm{C}_{\mathrm{q}}\right), 30.4$ $\left(\mathrm{CH}_{3}\right), 21.6\left(\mathrm{CH}_{3}\right), 14.4\left(\mathrm{CH}_{3}\right)$.

IR (ATR): 3406, 2962, 1214, 1161, 1030, $796 \mathrm{~cm}^{-1}$.

MS (EI) $m / z$ (relative intensity) $286(10)\left[\mathrm{M}^{+}\right], 271$ (100), 225 (90), 185 (25), 158 (30).

HR-MS (EI) $m / z$ calcd for $\mathrm{C}_{17} \mathrm{H}_{22} \mathrm{~N}_{2} \mathrm{O}_{2}\left[\mathrm{M}^{+}\right] 286.1681$, found 286.1681 .

Ethyl 2-\{(1Z,3Z)-3-(tert-Butylimino)-5-(trifluoromethyl)isoindolin-1-ylidene $\}$ acetate (119ja)<smiles>CCOC(=O)/C=C1\N/C(=N\CC(C)C)c2cc(C(F)(F)F)ccc21</smiles>

The general procedure D was followed using $\mathbf{1 1 7} \mathbf{j}$ (122 mg, $0.50 \mathrm{mmol}), \mathbf{2 0 a}(75.0 \mathrm{mg}, 0.75$ mmol $)$ and $\left[\mathrm{RuCl}_{2}(p \text {-cymene })\right]_{2}(15.3 \mathrm{mg}, 5.0 \mathrm{~mol} \%)$ at $120 \mathrm{C}$. Purification by column chromatography ( $n$-hexane/EtOAc: $10 / 1)$ yielded 119ja (85 mg, 50\%) as a white solid. M. p.: 139- $140{ }^{\circ} \mathrm{C}$.

${ }^{1} \mathbf{H}-\mathbf{N M R}\left(300 \mathrm{MHz}, \mathrm{CDCl}_{3}\right): \delta=10.0(\mathrm{br} \mathrm{s}, 1 \mathrm{H}), 8.13(\mathrm{~s}, 1 \mathrm{H}), 7.74(\mathrm{dd}, J=8.0,1.0 \mathrm{~Hz}, 1 \mathrm{H})$, $7.69(\mathrm{~d}, J=8.0 \mathrm{~Hz}, 1 \mathrm{H}), 5.55(\mathrm{~s}, 1 \mathrm{H}), 4.25$ (q, $J=7.2 \mathrm{~Hz}, 2 \mathrm{H}), 1.46(\mathrm{~s}, 9 \mathrm{H}), 1.33$ (t, $J=7.2$ $\mathrm{Hz}, 3 \mathrm{H})$. 
${ }^{13}$ C-NMR (75 MHz, $\left.\mathrm{CDCl}_{3}\right): \delta=169.2\left(\mathrm{C}_{\mathrm{q}}\right), 150.3\left(\mathrm{C}_{\mathrm{q}}\right), 146.5\left(\mathrm{C}_{\mathrm{q}}\right), 137.2\left(\mathrm{C}_{\mathrm{q}}\right), 136.0\left(\mathrm{C}_{\mathrm{q}}\right)$, $133.1\left(\mathrm{C}_{\mathrm{q}}, J_{\mathrm{C}-\mathrm{F}}=32.8 \mathrm{~Hz}\right), 127.2\left(\mathrm{CH}, J_{\mathrm{C}-\mathrm{F}}=7.4 \mathrm{~Hz}\right), 123.8\left(\mathrm{C}_{\mathrm{q}}, J_{\mathrm{C}-\mathrm{F}}=273.0 \mathrm{~Hz}\right), 121.1(\mathrm{CH})$, $120.1\left(\mathrm{CH}, J_{\mathrm{C}-\mathrm{F}}=7.6 \mathrm{~Hz}\right), 85.8(\mathrm{CH}), 60.3\left(\mathrm{CH}_{2}\right), 54.5\left(\mathrm{C}_{\mathrm{q}}\right), 30.3\left(\mathrm{CH}_{3}\right), 14.4\left(\mathrm{CH}_{3}\right)$.

IR (ATR): 3278, 2942, 1725, 1642, 1184, 1050, $778 \mathrm{~cm}^{-1}$.

MS (EI) $m / z$ (relative intensity) 340 (10) $\left[\mathrm{M}^{+}\right], 325$ (80), 279 (85), 238 (15), 212 (15), 43 (100).

HR-MS (ESI) $m / z$ calcd for $\mathrm{C}_{17} \mathrm{H}_{20} \mathrm{~F}_{3} \mathrm{~N}_{2} \mathrm{O}_{2}\left[\mathrm{M}+\mathrm{H}^{+}\right] 341.1477$ found 341.1471.

Ethyl 2-\{(1Z,3Z)-3-(tert-Butylimino)-7-fluorois oindolin-1-ylide ne $\}$ ace tate (119sa)<smiles>CCOC(=O)/C=C1\N/C(=N\CC(C)C)c2cccc(F)c21</smiles>

The general procedure $\mathbf{D}$ was followed using $\mathbf{1 1 7} \mathbf{s}(97.0 \mathrm{mg}, 0.50 \mathrm{mmol}), \mathbf{2 0 a}(75.0 \mathrm{mg}, 0.75$ mmol $)$ and $\left[\mathrm{RuCl}_{2}(p \text {-cymene })\right]_{2}(15.3 \mathrm{mg}, 5.0 \mathrm{~mol} \%)$ at $120 \mathrm{C}$. Purification by column chromatography ( $n$-hexane/EtOAc: $15 / 1)$ yie lded 119sa (71 mg, 49\%) and 119sa' (7 mg, 5\%) as a white solids. 119sa: M. p.: $101-102{ }^{\circ} \mathrm{C}$.

${ }^{1} \mathbf{H}\left\{{ }^{19} \mathbf{F}\right\}$-NMR $\left(300 \mathrm{MHz}, \mathrm{CDCl}_{3}\right): \delta=10.10(\mathrm{~s}, 1 \mathrm{H}), 7.74(\mathrm{~d}, J=7.6 \mathrm{~Hz}, 1 \mathrm{H}), 7.53(\mathrm{dd}, J=$ 8.2, 7.6 Hz, 1H), $7.23(\mathrm{~d}, J=8.2 \mathrm{~Hz}, 1 \mathrm{H}), 5.80(\mathrm{~s}, 1 \mathrm{H}), 4.32$ (q, $J=7.2 \mathrm{~Hz}, 2 \mathrm{H}), 1.53(\mathrm{~s}, 9 \mathrm{H})$, $1.41(\mathrm{t}, J=7.2 \mathrm{~Hz}, 3 \mathrm{H})$.

${ }^{13}$ C-NMR $\left(75 \mathrm{MHz}, \mathrm{CDCl}_{3}\right): \delta=169.6\left(\mathrm{C}_{\mathrm{q}}\right), 158.3\left(\mathrm{C}_{\mathrm{q}}, J_{\mathrm{C}-\mathrm{F}}=256.7 \mathrm{~Hz}\right), 148.6\left(\mathrm{C}_{\mathrm{q}}, J_{\mathrm{C}-\mathrm{F}}=3.6\right.$ $\mathrm{Hz}), 147.1\left(\mathrm{C}_{\mathrm{q}}, J_{\mathrm{C}-\mathrm{F}}=2.7 \mathrm{~Hz}\right), 138.2\left(\mathrm{C}_{\mathrm{q}}, J_{\mathrm{C}-\mathrm{F}}=3.3 \mathrm{~Hz}\right), 132.4\left(\mathrm{CH}, J_{\mathrm{C}-\mathrm{F}}=7.5 \mathrm{~Hz}\right), 121.3\left(\mathrm{C}_{\mathrm{q}}\right.$, $\left.J_{\mathrm{C}-\mathrm{F}}=14.1 \mathrm{~Hz}\right), 118.6\left(\mathrm{CH}, J_{\mathrm{C}-\mathrm{F}}=3.6 \mathrm{~Hz}\right), 117.4\left(\mathrm{CH}, J_{\mathrm{C}-\mathrm{F}}=19.8 \mathrm{~Hz}\right), 89.1\left(\mathrm{CH}, J_{\mathrm{C}-\mathrm{F}}=7.3\right.$ $\mathrm{Hz}), 60.1\left(\mathrm{C}_{\mathrm{q}}\right), 54.2\left(\mathrm{CH}_{2}\right), 30.2\left(\mathrm{CH}_{3}\right), 14.4\left(\mathrm{CH}_{3}\right)$.

${ }^{19}$ F-NMR $\left(282 \mathrm{MHz}, \mathrm{CDCl}_{3}\right): \delta=-(116.9-117.0)$. (m).

IR (ATR): 3388, 2970, 1634, 1210, 1073, $812 \mathrm{~cm}^{-1}$.

MS (EI) $m / z$ (relative intensity) 290 (10) $\left[\mathrm{M}^{+}\right], 275$ (90), 229 (100), 188 (30), 162 (30), 147 (15).

HR-MS (EI) $m / z$ calcd for $\mathrm{C}_{16} \mathrm{H}_{19} \mathrm{~N}_{2} \mathrm{O}_{2} \mathrm{~F}\left[\mathrm{M}^{+}\right] 290.1431$, found 290.1428 . 


\section{Ethyl 2-\{(1Z,3Z)-3-(tert-Butylimino)-5-fluorois oindolin-1-ylide ne $\}$ ace tate (119sa')}

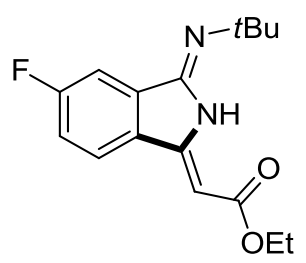

M. p.: $96-97^{\circ} \mathrm{C}$.

${ }^{1} \mathbf{H}-\mathrm{NMR}\left(300 \mathrm{MHz}, \mathrm{CDCl}_{3}\right): \delta=9.99(\mathrm{~s}, 1 \mathrm{H}), 7.58(\mathrm{dd}, J=8.4,4.6 \mathrm{~Hz}, 2 \mathrm{H}), 7.19(\mathrm{td}, J=8.6$, $2.4 \mathrm{~Hz}, 1 \mathrm{H}), 5.45(\mathrm{~s}, 1 \mathrm{H}), 4.27$ (q, $J=7.1 \mathrm{~Hz}, 2 \mathrm{H}), 1.48(\mathrm{~s}, 9 \mathrm{H}), 1.34$ (t, $J=7.1 \mathrm{~Hz}, 3 \mathrm{H})$. ${ }^{13}$ C-NMR (75 MHz, $\left.\mathrm{CDCl}_{3}\right): \delta=169.4\left(\mathrm{C}_{\mathrm{q}}\right), 165.3\left(\mathrm{C}_{\mathrm{q}}, J_{\mathrm{C}-\mathrm{F}}=251.7 \mathrm{~Hz}\right), 150.9\left(\mathrm{C}_{\mathrm{q}}\right), 146.7$ $\left(\mathrm{C}_{\mathrm{q}}\right), 138.0\left(\mathrm{C}_{\mathrm{q}}\right), 129.0\left(\mathrm{C}_{\mathrm{q}}\right), 122.4\left(\mathrm{CH}, J_{\mathrm{C}-\mathrm{F}}=9.5 \mathrm{~Hz}\right), 118.2\left(\mathrm{CH}, J_{\mathrm{C}-\mathrm{F}}=28.2 \mathrm{~Hz}\right), 109.5(\mathrm{CH}$, $\left.J_{\mathrm{C}-\mathrm{F}}=23.0 \mathrm{~Hz}\right), 84.0(\mathrm{CH}), 60.1\left(\mathrm{C}_{\mathrm{q}}\right), 54.3\left(\mathrm{CH}_{2}\right), 30.3\left(\mathrm{CH}_{3}\right), 14.4\left(\mathrm{CH}_{3}\right)$.

IR (ATR): 3382, 2967, 1628, 1207, 1053, $798 \mathrm{~cm}^{-1}$.

MS (EI) $m / z$ (relative intensity) 290 (10) $\left[\mathrm{M}^{+}\right], 275$ (100), 229 (95), 188 (30), 162 (30), 147 (15).

HR-MS (ESI) $m / z$ calcd for $\mathrm{C}_{16} \mathrm{H}_{19} \mathrm{~N}_{2} \mathrm{O}_{2} \mathrm{~F}\left[\mathrm{M}^{+}\right] 290.1431$, found 290.1391.

Ethyl $(Z)-2-\{(Z)-6-(t e r t-B$ utylimino)-6,7-dihydro-8H-[1,3]dioxolo [4,5-e]isoindol-8-ylide ne $\}$ ace tate $(119 \mathrm{ka})$<smiles>CCOC(=O)/C=C1\N/C(=N\C(C)C)c2ccc3c(c21)OCO3</smiles>

The general procedure $\mathbf{D}$ was followed using $117 \mathbf{k}(110 \mathrm{mg}, 0.50 \mathrm{mmol}), \mathbf{2 0 a}(75.0 \mathrm{mg}, 0.75$ mmol) and $\left[\mathrm{RuCl}_{2}(p \text {-cymene })\right]_{2}(15.3 \mathrm{mg}, 5.0 \mathrm{~mol} \%)$ at $100 \mathrm{C}$. Purification by column chromatography ( $n$-hexane/EtOAc: $5 / 1)$ yie lded 119ka (108 mg, 68\%) as a white solid. M. p.: $181-182{ }^{\circ} \mathrm{C}$.

${ }^{1}$ H-NMR (300 MHz, CDCl $): \delta=9.79(\mathrm{~s}, 1 \mathrm{H}), 7.37(\mathrm{~d}, J=8.0 \mathrm{~Hz}, 1 \mathrm{H}), 6.92(\mathrm{~d}, J=8.0 \mathrm{~Hz}$, $1 \mathrm{H}), 6.11(\mathrm{~s}, 2 \mathrm{H}), 5.52(\mathrm{~s}, 1 \mathrm{H}), 4.24(\mathrm{q}, J=7.2 \mathrm{~Hz}, 2 \mathrm{H}), 1.43(\mathrm{~s}, 9 \mathrm{H}), 1.31(\mathrm{t}, J=7.2 \mathrm{~Hz}, 3 \mathrm{H})$. ${ }^{13}$ C-NMR (75 MHz, $\left.\mathrm{CDCl}_{3}\right): \delta=169.5\left(\mathrm{C}_{\mathrm{q}}\right), 150.1\left(\mathrm{C}_{\mathrm{q}}\right), 148.9\left(\mathrm{C}_{\mathrm{q}}\right), 147.4\left(\mathrm{C}_{\mathrm{q}}\right), 142.4\left(\mathrm{C}_{\mathrm{q}}\right)$, $129.6\left(\mathrm{C}_{\mathrm{q}}\right), 116.3(\mathrm{CH}), 116.1\left(\mathrm{C}_{\mathrm{q}}\right), 110.5(\mathrm{CH}), 102.7\left(\mathrm{C} \mathrm{H}_{2}\right), 87.6(\mathrm{CH}), 59.9\left(\mathrm{C}_{\mathrm{q}}\right), 54.0$ $\left(\mathrm{CH}_{2}\right), 30.4\left(\mathrm{CH}_{3}\right), 14.4\left(\mathrm{CH}_{3}\right)$. 
IR (ATR): 3400, 2981, 1620, 1178, 1036, $800 \mathrm{~cm}^{-1}$.

MS (EI) $m / z$ (relative intensity) $316(15)\left[\mathrm{M}^{+}\right], 301$ (95), 255 (100), 214 (35), 188 (45), 173 (10).

HR-MS (EI) $m / z$ calcd for $\mathrm{C}_{17} \mathrm{H}_{20} \mathrm{~N}_{2} \mathrm{O}_{4}\left[\mathrm{M}^{+}\right] 316.1423$, found 316.1424 .

Ethyl 2-\{3-(te rt-Butylamino)-6-methoxy-1 $H$-is oindol-1-yl $\}$ ace tate (135ca)<smiles>CCOC(=O)CC1N=C(NC(C)(C)C)c2ccc(OC)cc21</smiles>

A suspension of $N$-(tert-butyl)-4-methoxybenzimidamide (117c) $(15.30 \mathrm{mg}, 0.075 \mathrm{mmol}), \mathbf{2 0 a}$ (7.500 mg, $0.075 \mathrm{mmol}),\left[\mathrm{RuCl}_{2}(p \text {-cymene })\right]_{2}(23.0 \mathrm{mg}, 50.0 \mathrm{~mol} \%)$ and $\mathrm{AgOAc}(12.50 \mathrm{mg}$, $0.075 \mathrm{mmol})$ in DCE $(1.0 \mathrm{~mL})$ was stirred at $60^{\circ} \mathrm{C}$ for $22 \mathrm{~h}$ under an atmosphere of $\mathrm{N}_{2}$. At ambient temperature, the reaction mixture was extracted with EtOAc $(3 \times 10 \mathrm{~mL})$, washed with brine $(20 \mathrm{~mL})$ and dried over $\mathrm{Na}_{2} \mathrm{SO}_{4}$. The solvent was evaporated under reduced pressure, and the residue was purified by column chromatography on silica gel ( $n$-hexane/EtOAc/Et 3 N : 5/1/0.05) to afford $135 \mathrm{ca}(10 \mathrm{mg}, 44 \%)$ as a yellow oil.

${ }^{1} \mathbf{H}-\mathbf{N M R}\left(300 \mathrm{MHz}, \mathrm{CDCl}_{3}\right): \delta=7.17(\mathrm{~d}, J=8.9 \mathrm{~Hz}, 1 \mathrm{H}), 7.02(\mathrm{~d}, J=2.0 \mathrm{~Hz}, 1 \mathrm{H}), 6.86(\mathrm{dd}$, $J=8.9,2.0 \mathrm{~Hz}, 1 \mathrm{H}), 5.06$ (dd, $J=8.5,6.4 \mathrm{~Hz}, 1 \mathrm{H}), 4.41$ (br s, 1H), 4.19-4.11 (m, 2H), 3.82 (s, 3H), 2.99 (dd, $J=15.6,6.2 \mathrm{~Hz}, 1 \mathrm{H}), 2.48$ (dd, $J=15.6,8.6 \mathrm{~Hz}, 1 \mathrm{H}), 1.48$ (s, 9H), 1.24 (t, $J$ $=7.2 \mathrm{~Hz}, 1 \mathrm{H})$.

${ }^{13}$ C-NMR (75 MHz, $\left.\mathrm{CDCl}_{3}\right): \delta=171.9\left(\mathrm{C}_{\mathrm{q}}\right), 160.5\left(\mathrm{C}_{\mathrm{q}}\right), 160.1\left(\mathrm{C}_{\mathrm{q}}\right), 154.2\left(\mathrm{C}_{\mathrm{q}}\right), 128.8\left(\mathrm{C}_{\mathrm{q}}\right)$, $118.8(\mathrm{CH}), 113.4(\mathrm{CH}), 107.7(\mathrm{CH}), 66.7(\mathrm{CH}), 60.3\left(\mathrm{C}_{\mathrm{q}}\right), 55.5\left(\mathrm{CH}_{3}\right), 51.4\left(\mathrm{CH}_{2}\right), 39.7$ $\left(\mathrm{CH}_{2}\right), 29.0\left(\mathrm{CH}_{3}\right), 14.2\left(\mathrm{CH}_{3}\right)$.

IR (film): 3402, 2965, 1725, 1605, 1480, 1242, $1023 \mathrm{~cm}^{-1}$.

MS (ESI) $m / z$ (relative intensity) 305 (90) $\left[\mathrm{M}+\mathrm{H}^{+}\right], 291$ (100), 249 (5), 235 (10), 161 (5).

HR-MS (ESI) $m / z$ calcd for $\mathrm{C}_{17} \mathrm{H}_{25} \mathrm{~N}_{2} \mathrm{O}_{3}\left[\mathrm{M}+\mathrm{H}^{+}\right] 305.1865$, found 305.1862 .

Inte rmolecular Compe tition Experiment between Substrates 117b and 117d: 


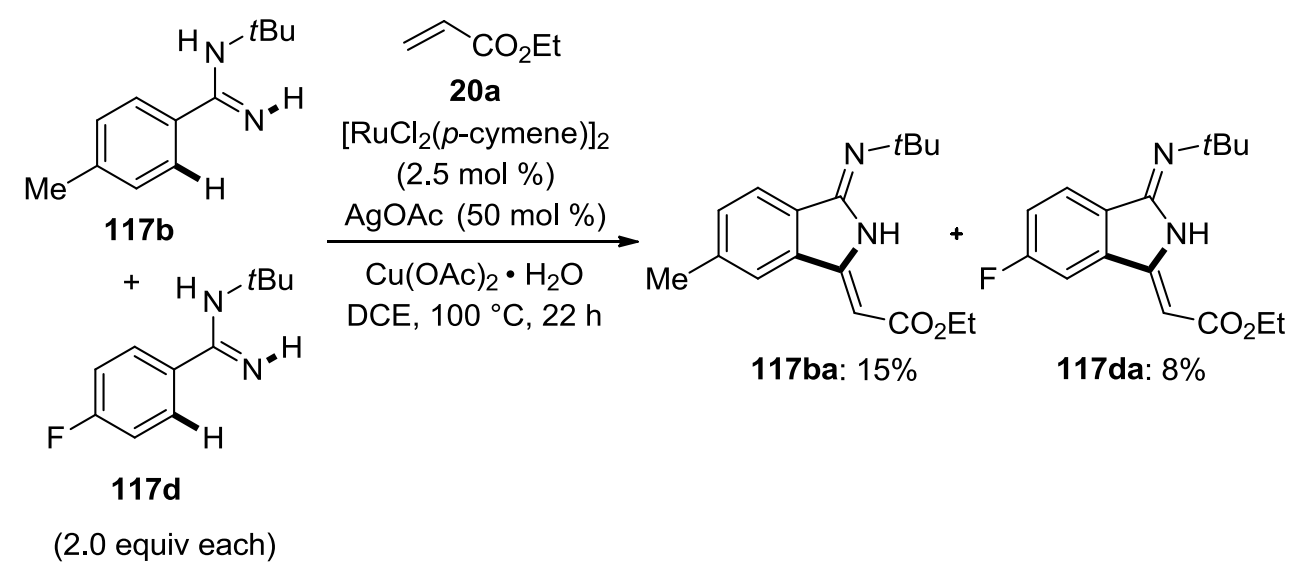

A suspension of $N$-(tert-butyl)-4-methylbenzimidamide (117b) $(190 \mathrm{mg}, 1.00 \mathrm{mmol})$, $N$-(tert-butyl)-4-fluorobenzimidamide (117d) (194 mg, $1.00 \mathrm{mmol}), \mathbf{2 0 a}(50.0 \mathrm{mg}, 0.50$ mmol), $\left[\operatorname{RuCl}_{2}(p \text {-cymene })\right]_{2}(7.7 \mathrm{mg}, 2.5 \mathrm{~mol} \%), \operatorname{AgOAc}(41.5 \mathrm{mg}, 50 \mathrm{~mol} \%)$ and $\mathrm{Cu}(\mathrm{OAc})_{2} \cdot \mathrm{H}_{2} \mathrm{O}(199 \mathrm{mg}, 1.00 \mathrm{mmol})$ in DCE $(2.0 \mathrm{~mL})$ was stirred at $100{ }^{\circ} \mathrm{C}$ for $22 \mathrm{~h}$ under an atmosphere of $\mathrm{N}_{2}$. At ambient temperature, the reaction mixture was extracted with EtOAc $(3 \times 20 \mathrm{~mL})$, washed with brine $(20 \mathrm{~mL})$ and dried over $\mathrm{Na}_{2} \mathrm{SO}_{4}$. The solvent was evaporated under reduced pressure and the residue was purified by column chromatography on silica gel ( $n$-hexane/EtOAc: $15 / 1 \rightarrow 10 / 1)$ to yield $119 b a(21.0 \mathrm{mg}, 15 \%)$ and $119 \mathrm{da}(11.0 \mathrm{mg}, 8 \%)$ as colorless solids.

\section{Ruthenium(II)-Catalyzed H/D Exchange with Substrate 117c in $\mathrm{CD}_{3} \mathrm{OD}$ as the}

Cosolvent: A suspension of $N$-(tert-butyl)-4-methoxybenzimidamide (117c) (103 $\mathrm{mg}, 0.50$ mmol), ethyl acrylate (20a) (100 mg, $0.75 \mathrm{mmol}),\left[\mathrm{RuCl}_{2}(p \text {-cymene) }]_{2}(7.7 \mathrm{mg}, 2.5 \mathrm{~mol} \%)\right.$, $\mathrm{AgOAc}(41.5 \mathrm{mg}, 50 \mathrm{~mol} \%)$ and $\mathrm{Cu}(\mathrm{OAc})_{2} \cdot \mathrm{H}_{2} \mathrm{O}(199 \mathrm{mg}, 1.00 \mathrm{mmol})$ was stirred at $100{ }^{\circ} \mathrm{C}$ for $22 \mathrm{~h}$ in a solvent mixture of $\mathrm{DCE}$ and $\mathrm{CD}_{3} \mathrm{OD}(1.8 / 0.2 \mathrm{~mL})$ as the solvent under an atmosphere of $\mathrm{N}_{2}$. At ambient temperature, the reaction mixture was extracted with EtOAc (3 $\times 20 \mathrm{~mL}$ ), washed with brine $(20 \mathrm{~mL})$ and dried over $\mathrm{Na}_{2} \mathrm{SO}_{4}$. The solvent was evaporated under reduced pressure, and the residue was purified by column chromatography on silica gel $(n$-hexane/EtOAc/Et $3 \mathrm{~N}: 5 / 1 / 0 \rightarrow 1 / 1 / 0.02)$ yielded $\left[\mathrm{D}_{\mathrm{n}}\right]-119 \mathrm{ca}(71.0 \mathrm{mg}, 47 \%)$ as a green oil, $\left[\mathrm{D}_{\mathrm{n}}\right]-\mathbf{1 3 5} \mathrm{ca}(26.0 \mathrm{mg}, 17 \%)$ as a green oil and reisolated starting material $\left[\mathrm{D}_{\mathrm{n}}\right]-\mathbf{- 1 1 7 c}(11.0 \mathrm{mg}$, $11 \%)$ as a colorless solid. The D-incorporation in $\left[D_{n}\right]-119 \mathbf{c a},\left[D_{n}\right]-135 \mathbf{c a}$ and $\left[D_{n}\right]-117 \mathbf{c}$ was estimated by ${ }^{1} \mathrm{H}-\mathrm{NMR}$ spectroscopy. 


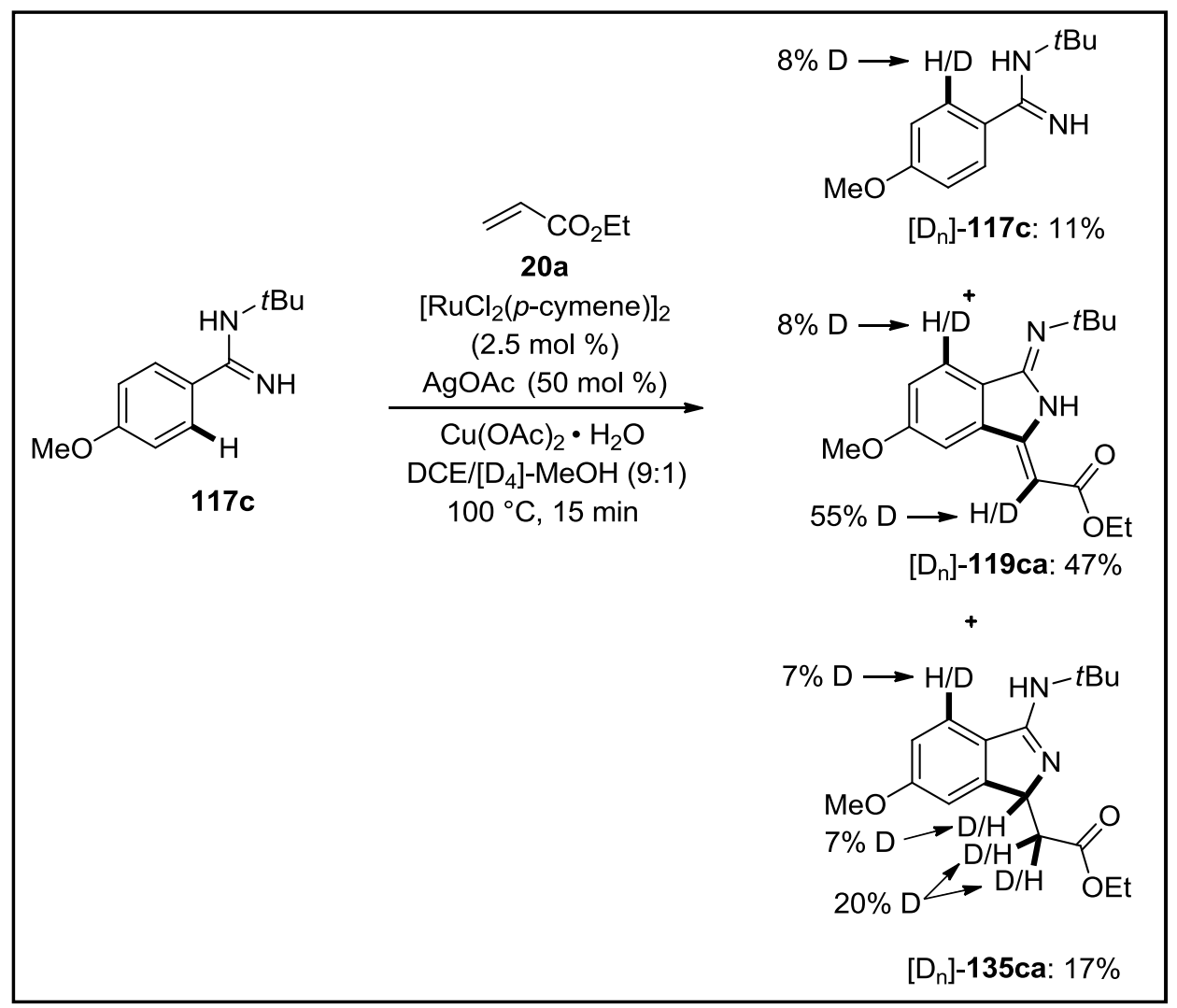

\subsubsection{Analytical Data for the Products of Ruthenium(II)-Catalyzed C-H}

\section{Bond Hydroarylation and Oxidative Annulation with}

$\alpha, \beta-U n s a t u r a t e d$ Ketones via Monodentate Coordination

\section{$N$-Me thyl-2-(3-oxobutyl)be nzamide (120aa)}<smiles>CC(=O)CCc1ccccc1C(=O)N[Na]</smiles>

The general procedure $\mathbf{E}$ was followed using $2 \mathbf{a}(68 \mathrm{mg}, 0.5 \mathrm{mmol})$ and methyl vinyl ketone (41a) (70 mg, $1.0 \mathrm{mmol})$ for $20 \mathrm{~h}$. Purification by column chromatography ( $n$-hexane/EtOAc $1: 1 \rightarrow 1: 2)$ yielded 120aa ( $82 \mathrm{mg}, 80 \%)$ as a colorless solid. M. p.: $=117-119^{\circ} \mathrm{C}$.

${ }^{1}$ H-NMR (300 MHz, $\left.\mathrm{CDCl}_{3}\right): \delta=7.36-7.27$ (m, 2H), 7.21-7.13 (m, 2H), 6.36 (br s, 1H), 2.96 (d, $J=4.9 \mathrm{~Hz}, 3 \mathrm{H}), 2.93$ (t, $J=6.0 \mathrm{~Hz}, 2 \mathrm{H}), 2.87$ (t, $J=6.0 \mathrm{~Hz}, 2 \mathrm{H}), 2.10$ (s, 3H).

${ }^{13}$ C-NMR (125MHz, $\left.\mathrm{CDCl}_{3}\right): \delta=208.6\left(\mathrm{C}_{\mathrm{q}}\right), 170.6\left(\mathrm{C}_{\mathrm{q}}\right), 138.9\left(\mathrm{C}_{\mathrm{q}}\right), 136.7\left(\mathrm{C}_{\mathrm{q}}\right), 129.9(\mathrm{CH})$, 129.7 (CH), $127.2(\mathrm{CH}), 126.2(\mathrm{CH}), 45.2\left(\mathrm{CH}_{2}\right), 30.0\left(\mathrm{CH}_{3}\right), 27.2\left(\mathrm{CH}_{2}\right), 26.6\left(\mathrm{CH}_{3}\right)$.

IR (ATR): 3290, 1707, 1631, 1368, 1166, $661 \mathrm{~cm}^{-1}$. 
MS (EI) m/z (relative intensity) 205 (10) $\left[\mathrm{M}^{+}\right], 175$ (10), 162 (100), 144 (10), 131 (45), 103 (5).

HR-MS (ESI) m/z calcd for $\mathrm{C}_{12} \mathrm{H}_{16} \mathrm{NO}_{2}\left[\mathrm{M}+\mathrm{H}^{+}\right]$206.1181, found 206.1177.

N,4-Dime thyl-2-(3-oxobutyl)be nzamide (120ba)<smiles>CC(=O)CCc1cc(C)ccc1C(=O)N[Na]</smiles>

The general procedure $\mathbf{E}$ was followed using $\mathbf{2 b}(75 \mathrm{mg}, 0.5 \mathrm{mmol})$ and methyl vinyl ketone (41a) (70 mg, $1.0 \mathrm{mmol})$ for $20 \mathrm{~h}$. Purification by column chromatography ( $n$-hexane/EtOAc 2:1 $\rightarrow 1: 1)$ yielded $\mathbf{1 2 0 b a}(77 \mathrm{mg}, 70 \%)$ as a colorless solid. M. p.: $=108-109^{\circ} \mathrm{C}$.

${ }^{1}$ H-NMR (300 MHz, $\left.\mathrm{CDCl}_{3}\right): \delta=7.24$ (dd, $\left.J=7.1,1.4 \mathrm{~Hz}, 1 \mathrm{H}\right), 7.00(\mathrm{~s}, 1 \mathrm{H}), 6.99$ (d, $J=7.1$ Hz, 1H), 6.26 (br s, 1H), 2.96 (d, $J=4.9 \mathrm{~Hz}, 3 \mathrm{H}), 2.90$ (t, $J=6.0 \mathrm{~Hz}, 2 \mathrm{H}), 2.89$ (t, $J=6.0 \mathrm{~Hz}$, $2 \mathrm{H}), 2.30(\mathrm{~s}, 3 \mathrm{H}), 2.11(\mathrm{~s}, 3 \mathrm{H})$.

${ }^{13}$ C-NMR (125 MHz, $\left.\mathrm{CDCl}_{3}\right): \delta=208.5\left(\mathrm{C}_{\mathrm{q}}\right), 170.5\left(\mathrm{C}_{\mathrm{q}}\right), 139.9\left(\mathrm{C}_{\mathrm{q}}\right), 139.0\left(\mathrm{C}_{\mathrm{q}}\right), 133.6\left(\mathrm{C}_{\mathrm{q}}\right)$, $130.5(\mathrm{CH}), 127.1(\mathrm{CH}), 126.8(\mathrm{CH}), 45.4\left(\mathrm{CH}_{2}\right), 30.0\left(\mathrm{CH}_{3}\right), 27.3\left(\mathrm{CH}_{2}\right), 26.7\left(\mathrm{CH}_{3}\right), 21.3$ $\left(\mathrm{CH}_{3}\right)$.

IR (ATR): 3287, 1710, 1632, 1542, 1164, $693 \mathrm{~cm}^{-1}$.

MS (EI) m/z (relative intensity) 219 (10) $\left[\mathrm{M}^{+}\right], 189$ (5), 176 (100), 161 (10), 145 (45), 115 (15).

HR-MS (EI) m/z calcd for $\mathrm{C}_{13} \mathrm{H}_{17} \mathrm{NO}_{2}\left[\mathrm{M}^{+}\right]$219.1259, found 219.1256 .

\section{4-Methoxy- $N$-me thyl-2-(3-oxobutyl)benzamide (120ca)}<smiles>CNC(=O)c1ccc(OC)cc1CCC(C)=O</smiles>

The general procedure $\mathbf{E}$ was followed using $2 \mathbf{c}(83 \mathrm{mg}, 0.5 \mathrm{mmol})$ and methyl vinyl ketone (41a) (70 mg, $1.0 \mathrm{mmol})$ for $20 \mathrm{~h}$. Purification by column chromatography ( $n$-hexane/EtOAc $1: 1 \rightarrow 1: 2)$ yielded 120ca (94 mg, 80\%) as a colorless solid. M. p.: $=107-109^{\circ} \mathrm{C}$. 
${ }^{1} \mathbf{H}-\mathbf{N M R}\left(300 \mathrm{MHz}, \mathrm{CDCl}_{3}\right): \delta=7.29(\mathrm{dd}, J=8.1,0.8 \mathrm{~Hz}, 1 \mathrm{H}), 6.70(\mathrm{~s}, 1 \mathrm{H}), 6.66(\mathrm{~d}, J=8.1$ Hz, 1H), 6.34 (br s, 1H), 3.76 (s, 3H), 2.93 (d, $J=5.1 \mathrm{~Hz}, 3 \mathrm{H}), 2.93$ (t, $J=6.4 \mathrm{~Hz}, 2 \mathrm{H}), 2.86$ (t, $J=6.4 \mathrm{~Hz}, 2 \mathrm{H}), 2.10(\mathrm{~s}, 3 \mathrm{H})$.

${ }^{13}$ C-NMR (125 MHz, $\left.\mathrm{CDCl}_{3}\right): \delta=208.6\left(\mathrm{C}_{\mathrm{q}}\right), 170.3\left(\mathrm{C}_{\mathrm{q}}\right), 160.6\left(\mathrm{C}_{\mathrm{q}}\right), 141.4\left(\mathrm{C}_{\mathrm{q}}\right), 128.9\left(\mathrm{C}_{\mathrm{q}}\right)$, $128.9(\mathrm{CH}), 115.4(\mathrm{CH}), 111.2(\mathrm{CH}), 55.2\left(\mathrm{CH}_{3}\right), 45.2\left(\mathrm{CH}_{2}\right), 29.9\left(\mathrm{CH}_{3}\right), 27.5\left(\mathrm{CH}_{2}\right), 26.6$ $\left(\mathrm{CH}_{3}\right)$.

IR (ATR): 3288, 1705, 1543, 1247, 1157, $696 \mathrm{~cm}^{-1}$.

MS (EI) m/z (relative intensity) 235 (15) $\left[\mathrm{M}^{+}\right], 205$ (10), 192 (100), 177 (5), 161 (60), 135 (10).

HR-MS (EI) m/z calcd for $\mathrm{C}_{13} \mathrm{H}_{17} \mathrm{NO}_{3}\left[\mathrm{M}^{+}\right]$235.1208, found 235.1206.

$N$-Me thyl-3-(3-oxobutyl)-[1,1'-biphe nyl]-4-carboxamide (120da)<smiles>CNC(=O)c1ccc(-c2ccccc2)cc1CCC(C)=O</smiles>

The general procedure $\mathbf{E}$ was followed using $\mathbf{2 d}(106 \mathrm{mg}, 0.5 \mathrm{mmol})$ and methyl vinyl ketone (41a) (70 mg, $1.0 \mathrm{mmol})$ for $20 \mathrm{~h}$. Purification by column chromatography ( $n$-hexane/EtOAc $1: 1 \rightarrow 1: 2)$ yielded $120 \mathrm{da}(111 \mathrm{mg}, 79 \%)$ as a colorless solid. M. p.: $=153-155^{\circ} \mathrm{C}$.

${ }^{1}$ H-NMR (300 MHz, $\left.\mathrm{CDCl}_{3}\right): \delta=7.60-7.48(\mathrm{~m}, 2 \mathrm{H}), 7.48-7.38(\mathrm{~m}, 5 \mathrm{H}), 7.38-7.28(\mathrm{~m}, 1 \mathrm{H})$, 6.42 (br s, 1H), 3.10-3.00 (m, 2H), 2.98 (d, $J=4.9 \mathrm{~Hz}, 3 \mathrm{H}), 2.96-2.83$ (m, 2H), 2.12 (s, 3H). ${ }^{13}$ C-NMR (75 MHz, $\left.\mathrm{CDCl}_{3}\right): \delta=208.5\left(\mathrm{C}_{\mathrm{q}}\right), 170.4\left(\mathrm{C}_{\mathrm{q}}\right), 142.8\left(\mathrm{C}_{\mathrm{q}}\right), 140.1\left(\mathrm{C}_{\mathrm{q}}\right), 139.6\left(\mathrm{C}_{\mathrm{q}}\right)$, $135.3\left(\mathrm{C}_{\mathrm{q}}\right), 128.8(\mathrm{CH}), 128.6(\mathrm{CH}), 127.7(\mathrm{CH}), 127.7(\mathrm{CH}), 127.1(\mathrm{CH}), 124.9(\mathrm{CH}), 45.3$ $\left(\mathrm{CH}_{2}\right), 29.9\left(\mathrm{CH}_{3}\right), 27.4\left(\mathrm{CH}_{2}\right), 26.6\left(\mathrm{CH}_{3}\right)$.

IR (ATR): 3287, 1709, 1630, 1543, 1160, $697 \mathrm{~cm}^{-1}$.

MS (EI) m/z (relative intensity) 281 (15) [M+ $], 250$ (5), 238 (100), 207 (40), 178 (20), 165 (15).

HR-MS (EI) m/z calcd for $\mathrm{C}_{18} \mathrm{H}_{19} \mathrm{NO}_{2}\left[\mathrm{M}^{+}\right]$281.1416, found 281.1417.

\section{4-Bromo- $N$-methyl-2-(3-oxobutyl)be nzamide (120ea)}


<smiles>CC(=O)CCc1cc(Br)ccc1C(=O)NO</smiles>

The general procedure $\mathbf{E}$ was followed using $2 \mathbf{e}(107 \mathrm{mg}, 0.5 \mathrm{mmol})$ and methyl vinyl ketone (41a) (70 mg, $1.0 \mathrm{mmol}$ ) for $20 \mathrm{~h}$. Purification by column chromatography ( $n$-hexane/EtOAc $2: 1 \rightarrow 1: 1)$ yielded 120 ea $(113 \mathrm{mg}, 81 \%)$ as a colorless solid. M. p.: $=132-134{ }^{\circ} \mathrm{C}$.

${ }^{1}$ H-NMR $\left(300 \mathrm{MHz}, \mathrm{CDCl}_{3}\right): \delta=7.40-7.29(\mathrm{~m}, 2 \mathrm{H}), 7.27-7.21(\mathrm{~m}, 1 \mathrm{H}), 6.52$ (br s, 1H), 2.99-2.92 (m, 4H), $2.92(\mathrm{~d}, J=1.6 \mathrm{~Hz}, 3 \mathrm{H}), 2.15(\mathrm{~s}, 3 \mathrm{H})$.

${ }^{13}$ C-NMR (125 MHz, $\left.\mathrm{CDCl}_{3}\right): \delta=208.0\left(\mathrm{C}_{\mathrm{q}}\right), 169.5\left(\mathrm{C}_{\mathrm{q}}\right), 141.1\left(\mathrm{C}_{\mathrm{q}}\right), 135.6\left(\mathrm{C}_{\mathrm{q}}\right), 132.4(\mathrm{CH})$, $129.3(\mathrm{CH}), 128.8(\mathrm{CH}), 124.0\left(\mathrm{C}_{\mathrm{q}}\right), 44.8\left(\mathrm{CH}_{2}\right), 30.0\left(\mathrm{CH}_{3}\right), 26.8\left(\mathrm{CH}_{2}\right), 26.8\left(\mathrm{CH}_{3}\right)$.

IR (ATR): 3287, 1705, 1639, 1543, 1167, $686 \mathrm{~cm}^{-1}$.

MS (EI) m/z (relative intensity) 283 (5) [ $\left.\mathrm{M}^{+}\right], 240$ (100), 225 (10), 211 (35), 183 (10), 102 (20).

HR-MS (ESI) $\mathrm{m} / \mathrm{z}$ calcd for $\mathrm{C}_{12} \mathrm{H}_{14} \mathrm{NO}_{2}{ }^{79} \mathrm{Br}[\mathrm{M}+]$ 283.0208, found 283.0210.

\section{$N$-Methyl-2-(3-oxobutyl)-4-(trifluorome thyl)be nzamide (120fa)}<smiles>CNC(=O)c1ccc(C(F)(F)F)cc1CCC(C)=O</smiles>

The general procedure $\mathbf{E}$ was followed using $\mathbf{2 f}$ (102 $\mathrm{mg}, 0.5 \mathrm{mmol}$ ) and methyl vinyl ketone (41a) (70 mg, $1.0 \mathrm{mmol})$ for $20 \mathrm{~h}$. Purification by column chromatography ( $n$-hexane/EtOAc $1: 1 \rightarrow 1: 2)$ yielded $\mathbf{1 2 0 f a}\left(99 \mathrm{mg}, 73 \%\right.$ ) as a colorless solid. M. p.: $=117-119{ }^{\circ} \mathrm{C}$.

${ }^{1} \mathbf{H}-\mathbf{N M R}\left(300 \mathrm{MHz}, \mathrm{CDCl}_{3}\right): \delta=7.45$ (s, 3H), 6.58 (br s, 1H), $2.99(\mathrm{~d}, J=4.9 \mathrm{~Hz}, 3 \mathrm{H}), 2.96$ (t, $J=5.0 \mathrm{~Hz}, 2 \mathrm{H}), 2.94(\mathrm{t}, J=5.0 \mathrm{~Hz}, 2 \mathrm{H}), 2.13(\mathrm{~s}, 3 \mathrm{H})$.

${ }^{13}$ C-NMR $\left.\left(100 \mathrm{MHz}, \mathrm{CDCl}_{3}\right)\right): \delta=207.9\left(\mathrm{C}_{\mathrm{q}}\right), 169.2\left(\mathrm{C}_{\mathrm{q}}\right), 140.1\left(\mathrm{C}_{\mathrm{q}}\right), 139.6\left(\mathrm{C}_{\mathrm{q}}\right), 131.8\left(\mathrm{C}_{\mathrm{q}}\right.$, $\left.J_{\mathrm{C}-\mathrm{F}}=32.4 \mathrm{~Hz}\right), 127.8(\mathrm{CH}), 126.2\left(\mathrm{CH}, J_{\mathrm{C}-\mathrm{F}}=3.7 \mathrm{~Hz}\right), 124.0\left(\mathrm{C}_{\mathrm{q}}, J_{\mathrm{C}-\mathrm{F}}=271.5 \mathrm{~Hz}\right), 123.1(\mathrm{CH}$, $J=3.7 \mathrm{~Hz}), 44.68\left(\mathrm{CH}_{2}\right), 30.02\left(\mathrm{CH}_{3}\right), 26.8\left(\mathrm{CH}_{2}\right), 26.8\left(\mathrm{CH}_{3}\right)$.

${ }^{19}$ F-NMR $\left(\mathrm{CDCl}_{3}, 282 \mathrm{MHz}\right): \delta-62.9(\mathrm{~s})$.

IR (ATR): 3294, 1713, 1550, 1333, 1114, $696 \mathrm{~cm}^{-1}$. 
MS (EI) m/z (relative intensity) 273 (5) [M+ $], 254$ (5), 230 (60), 199 (20), 151 (10), 43 (100).

HR-MS (EI) $\mathrm{m} / \mathrm{z}$ calcd for $\mathrm{C}_{13} \mathrm{H}_{14} \mathrm{~F}_{3} \mathrm{NO}_{2}\left[\mathrm{M}^{+}\right]$273.0977, found 273.0973.

\section{$N$-Benzyl-4-me thoxy-2-(3-0xobutyl)be nzamide (120ga)}<smiles>COc1ccc(C(=O)NCc2ccccc2)c(CCC(C)=O)c1</smiles>

The general procedure $\mathbf{E}$ was followed using $\mathbf{2 g}(121 \mathrm{mg}, 0.5 \mathrm{mmol})$ and methyl vinyl ketone (41a) (70 mg, $1.0 \mathrm{mmol})$ for $20 \mathrm{~h}$. Purification by column chromatography ( $n$-hexane/EtOAc 2:1) yielded 120ga (106 mg, 68\%) as a colorless solid. M. p.: $=131-133{ }^{\circ} \mathrm{C}$.

${ }^{1}$ H-NMR (300 MHz, $\left.\mathrm{CDCl}_{3}\right): \delta=7.37-7.31(\mathrm{~m}, 5 \mathrm{H}), 7.32-7.25(\mathrm{~m}, 1 \mathrm{H}), 6.74(\mathrm{~d}, J=2.5 \mathrm{~Hz}$, 1H), 6.70 (dd, $J=8.4,2.6 \mathrm{~Hz}, 1 \mathrm{H}), 6.44$ (br s, 1H), 4.59 (d, $J=5.8 \mathrm{~Hz}, 2 \mathrm{H}), 3.78$ (s, 3H), 2.99 (t, $J=7.1 \mathrm{~Hz}, 2 \mathrm{H}), 2.86(\mathrm{t}, J=7.1 \mathrm{~Hz}, 2 \mathrm{H}), 2.09$ (s, 3H).

${ }^{13}$ C-NMR (125 MHz, $\left.\mathrm{CDCl}_{3}\right): \delta=208.3\left(\mathrm{C}_{\mathrm{q}}\right), 169.4\left(\mathrm{C}_{\mathrm{q}}\right), 160.8\left(\mathrm{C}_{\mathrm{q}}\right), 141.9\left(\mathrm{C}_{\mathrm{q}}\right), 138.3\left(\mathrm{C}_{\mathrm{q}}\right)$, $128.8(\mathrm{CH}), 128.8(\mathrm{CH}), 128.6\left(\mathrm{C}_{\mathrm{q}}\right), 127.8(\mathrm{CH}), 127.5(\mathrm{CH}), 115.7(\mathrm{CH}), 111.3(\mathrm{CH}), 55.3$ $\left(\mathrm{CH}_{3}\right), 45.3\left(\mathrm{CH}_{2}\right), 44.0\left(\mathrm{CH}_{2}\right), 29.9\left(\mathrm{CH}_{3}\right), 27.7\left(\mathrm{CH}_{2}\right)$.

IR (ATR): 3280, 1706, 1628, 1268, 1025, $694 \mathrm{~cm}^{-1}$.

MS (EI) m/z (relative intensity) $311(15)\left[\mathrm{M}^{+}\right], 268$ (45), 205 (20), 161 (30), 106 (25), 91 (100).

HR-MS (ESI) m/z calcd for $\mathrm{C}_{19} \mathrm{H}_{22} \mathrm{NO}_{3}\left[\mathrm{M}+\mathrm{H}^{+}\right]$312.1600, found 312.1600.

The spectral data were in accordance with those reported in the literature. ${ }^{147}$

\section{$N$-Benzyl-4-fluoro-2-(3-oxobutyl)be nzamide (120ha)}<smiles>CC(=O)CCc1cc(F)ccc1C(=O)NCc1ccccc1</smiles>

The general procedure $\mathbf{E}$ was followed using $\mathbf{2 h}(115 \mathrm{mg}, 0.5 \mathrm{mmol})$ and methyl vinyl ketone (41a) $(70 \mathrm{mg}, 1.0 \mathrm{mmol})$ for $20 \mathrm{~h}$. Purification by column chromatography ( $n$-hexane/EtOAc $5: 1 \rightarrow 2: 1)$ yielded 120 ha $(109 \mathrm{mg}, 73 \%)$ as a colorless solid. M. p.: $=112-114{ }^{\circ} \mathrm{C}$.

${ }^{147}$ R. Manoharan, M. Jeganmohan, Chem. Commun. 2015, 51, 2929-2932. 
${ }^{1} \mathbf{H}-\mathbf{N M R}\left(300 \mathrm{MHz}, \mathrm{CDCl}_{3}\right): \delta=7.40-7.25(\mathrm{~m}, 6 \mathrm{H}), 6.93-6.81(\mathrm{~m}, 2 \mathrm{H}), 6.74(\mathrm{t}, J=5.8 \mathrm{~Hz}$, $1 \mathrm{H}), 4.57(\mathrm{~d}, J=5.8 \mathrm{~Hz}, 2 \mathrm{H}), 2.94(\mathrm{t}, J=6.6 \mathrm{~Hz}, 2 \mathrm{H}), 2.83(\mathrm{t}, J=6.6 \mathrm{~Hz}, 2 \mathrm{H}), 2.07(\mathrm{~s}, 3 \mathrm{H})$.

${ }^{13}$ C-NMR $\left(75 \mathrm{MHz}, \mathrm{CDCl}_{3}\right): \delta=208.0\left(\mathrm{C}_{\mathrm{q}}\right), 168.9\left(\mathrm{C}_{\mathrm{q}}\right), 163.4\left(\mathrm{C}_{\mathrm{q}}, J_{\mathrm{C}-\mathrm{F}}=249.7 \mathrm{~Hz}\right), 142.3\left(\mathrm{C}_{\mathrm{q}}\right.$, $\left.J_{\mathrm{C}-\mathrm{F}}=7.8 \mathrm{~Hz}\right), 138.1\left(\mathrm{C}_{\mathrm{q}}\right), 132.5\left(\mathrm{C}_{\mathrm{q}}, J_{\mathrm{C}-\mathrm{F}}=3.1 \mathrm{~Hz}\right), 129.3\left(\mathrm{CH}, J_{\mathrm{C}-\mathrm{F}}=8.8 \mathrm{~Hz}\right), 128.8(\mathrm{CH})$, $127.8(\mathrm{CH}), 127.6(\mathrm{CH}), 116.6\left(\mathrm{CH}, J_{\mathrm{C}-\mathrm{F}}=21.4 \mathrm{~Hz}\right), 113.2\left(\mathrm{CH}, J_{\mathrm{C}-\mathrm{F}}=21.6 \mathrm{~Hz}\right), 44.7\left(\mathrm{CH}_{2}\right)$, $44.0\left(\mathrm{CH}_{2}\right), 29.9\left(\mathrm{CH}_{3}\right), 27.1\left(\mathrm{CH}_{2}, J_{\mathrm{C}-\mathrm{F}}=1.6 \mathrm{~Hz}\right)$.

${ }^{19}$ F-NMR $\left(282 \mathrm{MHz}, \mathrm{CDCl}_{3}\right): \delta=-110.4$ (ddd, $\left.J=9.8,8.1,5.8 \mathrm{~Hz}\right)$.

IR (ATR): 3281, 1709, 1635, 1234, 1168, $694 \mathrm{~cm}^{-1}$.

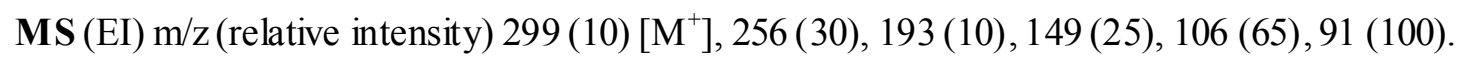

HR-MS (EI) $\mathrm{m} / \mathrm{z}$ calcd for $\mathrm{C}_{18} \mathrm{H}_{18} \mathrm{FNO}_{2}\left[\mathrm{M}^{\dagger}\right] 299.1322$, found 299.1323.

\section{$N$-Benzyl-4-chloro-2-(3-0xobutyl)be nzamide (120ia)}<smiles>CC(=O)CCc1cc(Cl)ccc1C(=O)NCc1ccccc1</smiles>

The general procedure $\mathbf{E}$ was followed using $2 \mathbf{i}$ (123 $\mathrm{mg}, 0.5 \mathrm{mmol}$ ) and methyl vinyl ketone (41a) (70 mg, $1.0 \mathrm{mmol}$ ) for $20 \mathrm{~h}$. Purification by column chromatography ( $n$-hexane/EtOAc 2:1) yielded 120ia (97 mg, 62\%) as a colorless solid. M. p.: $=125-126{ }^{\circ} \mathrm{C}$.

${ }^{1}$ H-NMR (300 MHz, $\left.\mathrm{CDCl}_{3}\right): \delta=7.37-7.25(\mathrm{~m}, 6 \mathrm{H}), 7.20-7.18(\mathrm{~m}, 1 \mathrm{H}), 7.16(\mathrm{dd}, J=8.1,2.1$ $\mathrm{Hz}, 1 \mathrm{H}), 6.67(\mathrm{~d}, J=6.4 \mathrm{~Hz}, 1 \mathrm{H}), 4.59(\mathrm{~d}, J=5.8 \mathrm{~Hz}, 2 \mathrm{H}), 2.93(\mathrm{t}, J=6.6 \mathrm{~Hz}, 2 \mathrm{H}), 2.85(\mathrm{t}, J=$ $6.6 \mathrm{~Hz}, 2 \mathrm{H}), 2.09$ (s, 3H).

${ }^{13}$ C-NMR $\left(75 \mathrm{MHz}, \mathrm{CDCl}_{3}\right): \delta=207.7\left(\mathrm{C}_{\mathrm{q}}\right), 168.6\left(\mathrm{C}_{\mathrm{q}}\right), 141.2\left(\mathrm{C}_{\mathrm{q}}\right), 137.9\left(\mathrm{C}_{\mathrm{q}}\right), 135.8\left(\mathrm{C}_{\mathrm{q}}\right)$, $134.7\left(\mathrm{C}_{\mathrm{q}}\right), 129.8(\mathrm{CH}), 128.7(\mathrm{CH}), 128.6(\mathrm{CH}), 127.8(\mathrm{CH}), 127.6(\mathrm{CH}), 126.4(\mathrm{CH}), 44.8$ $\left(\mathrm{CH}_{2}\right), 44.1\left(\mathrm{CH}_{2}\right), 30.0\left(\mathrm{CH}_{3}\right), 27.0\left(\mathrm{CH}_{2}\right)$.

IR (ATR): 3279, 1708, 1637, 1541, 1163, $692 \mathrm{~cm}^{-1}$.

MS (EI) m/z (relative intens ity) 315 (10) [M+ $], 272$ (30), 209 (5), 165 (15), 106 (75), 91 (100).

HR-MS (EI) $\mathrm{m} / \mathrm{z}$ calcd for $\mathrm{C}_{18} \mathrm{H}_{18} \mathrm{~N}^{35} \mathrm{ClO}_{2}\left[\mathrm{M}^{+}\right] 315.1026$, found 315.1030 . 
<smiles>CC(=O)CCc1cc(C)ccc1C(=O)NC1CCCC1</smiles>

The general procedure $\mathbf{E}$ was followed using $\mathbf{2} \mathbf{j}$ (109 $\mathrm{mg}, 0.5 \mathrm{mmol}$ ) and methyl vinyl ketone (41a) (70 mg, $1.0 \mathrm{mmol})$ for $20 \mathrm{~h}$. Purification by column chromatography ( $n$-hexane/EtOAc 3:1) yielded $120 \mathrm{ja}$ (100 mg, 70\%) as a colorless solid. M. p.: $=143-145^{\circ} \mathrm{C}$.

${ }^{1} \mathbf{H}-\mathbf{N M R}\left(300 \mathrm{MHz}, \mathrm{CDCl}_{3}\right): \delta=7.30-7.20(\mathrm{~m}, 1 \mathrm{H}), 7.02(\mathrm{~s}, 1 \mathrm{H}), 7.01(\mathrm{~d}, J=6.8 \mathrm{~Hz}, 1 \mathrm{H})$, $6.04(\mathrm{~d}, J=8.2 \mathrm{~Hz}, 1 \mathrm{H}), 4.13-3.82(\mathrm{~m}, 1 \mathrm{H}), 2.96(\mathrm{t}, J=6.2 \mathrm{~Hz}, 2 \mathrm{H}), 2.87(\mathrm{t}, J=6.2 \mathrm{~Hz}, 2 \mathrm{H})$, $2.32(\mathrm{~s}, 3 \mathrm{H}), 2.12(\mathrm{~s}, 3 \mathrm{H}), 2.13-2.02(\mathrm{~m}, 2 \mathrm{H}), 1.88-1.69(\mathrm{~m}, 3 \mathrm{H}), 1.54-1.32(\mathrm{~m}, 2 \mathrm{H}), 1.31-1.09$ $(\mathrm{m}, 3 \mathrm{H})$.

${ }^{13}$ C-NMR (125 MHz, $\left.\mathrm{CDCl}_{3}\right): \delta=208.2\left(\mathrm{C}_{\mathrm{q}}\right), 169.0\left(\mathrm{C}_{\mathrm{q}}\right), 139.8\left(\mathrm{C}_{\mathrm{q}}\right), 138.9\left(\mathrm{C}_{\mathrm{q}}\right), 134.1\left(\mathrm{C}_{\mathrm{q}}\right)$, $130.5(\mathrm{CH}), 127.0(\mathrm{CH}), 126.8(\mathrm{CH}), 48.6(\mathrm{CH}), 45.4\left(\mathrm{CH}_{2}\right), 33.2\left(\mathrm{CH}_{2}\right), 30.0\left(\mathrm{CH}_{3}\right), 27.4$ $\left(\mathrm{CH}_{2}\right), 25.6\left(\mathrm{CH}_{2}\right), 25.0\left(\mathrm{CH}_{2}\right), 21.3\left(\mathrm{CH}_{3}\right)$.

IR (ATR): 3278, 2923, 1712, 1631, 1537, $699 \mathrm{~cm}^{-1}$.

MS (EI) m/z (relative intensity) $287(35)\left[\mathrm{M}^{+}\right], 244$ (100), 189 (70), 162 (70), 145 (60).

HR-MS (EI) m/z calcd for $\mathrm{C}_{18} \mathrm{H}_{25} \mathrm{NO}_{2}\left[\mathrm{M}^{+}\right]$287.1885, found 287.1879.

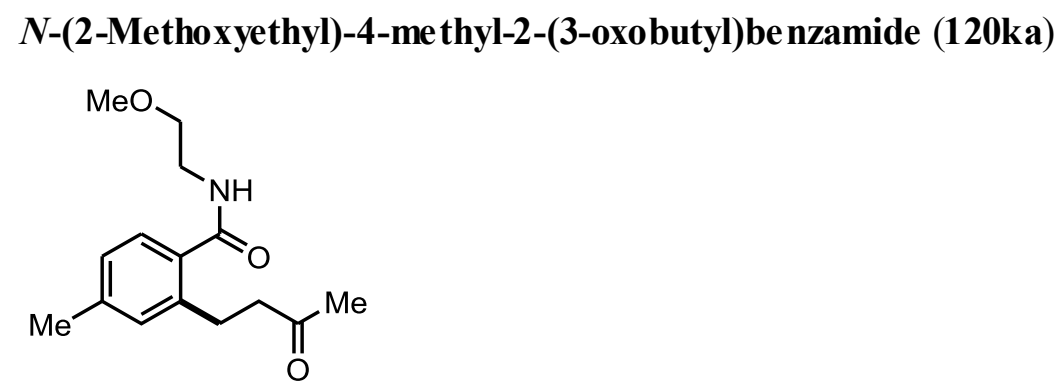

The general procedure $\mathbf{E}$ was followed using $\mathbf{2 k}$ (97 mg, $0.5 \mathrm{mmol})$ and methyl vinyl ketone (41a) (70 mg, $1.0 \mathrm{mmol})$ for $20 \mathrm{~h}$. Purification by column chromatography ( $n$-hexane/EtOAc $1: 1 \rightarrow 1: 2)$ yie lded $120 \mathrm{ka}$ ( $76 \mathrm{mg}, 58 \%$ ) as a yellow oil.

${ }^{1} \mathbf{H}-\mathbf{N M R}\left(300 \mathrm{MHz}, \mathrm{CDCl}_{3}\right): \delta=7.25(\mathrm{~d}, J=7.7 \mathrm{~Hz}, 1 \mathrm{H}), 7.01(\mathrm{~s}, 1 \mathrm{H}), 7.00(\mathrm{~d}, J=7.7 \mathrm{~Hz}, 1 \mathrm{H})$, 6.40 (br s, 1H), $3.60(\mathrm{dt}, J=7.8,1.7 \mathrm{~Hz}, 2 \mathrm{H}), 3.53(\mathrm{dt}, J=7.8,1.7 \mathrm{~Hz}, 2 \mathrm{H}), 3.35$ (s, 3H), 2.95 $(\mathrm{dt}, J=6.0,1.3 \mathrm{~Hz}, 2 \mathrm{H}), 2.83(\mathrm{dt}, J=6.0,1.3 \mathrm{~Hz}, 2 \mathrm{H}), 2.31(\mathrm{~s}, 3 \mathrm{H}), 2.11(\mathrm{~s}, 3 \mathrm{H})$. 
${ }^{13}$ C-NMR (125 MHz, $\left.\mathrm{CDCl}_{3}\right): \delta=208.1\left(\mathrm{C}_{\mathrm{q}}\right), 169.8\left(\mathrm{C}_{\mathrm{q}}\right), 140.1\left(\mathrm{C}_{\mathrm{q}}\right), 139.3\left(\mathrm{C}_{\mathrm{q}}\right), 133.4\left(\mathrm{C}_{\mathrm{q}}\right)$, $130.8(\mathrm{CH}), 127.1(\mathrm{CH}), 126.8(\mathrm{CH}), 71.2\left(\mathrm{CH}_{2}\right), 58.8\left(\mathrm{CH}_{3}\right), 45.6\left(\mathrm{CH}_{2}\right), 39.6\left(\mathrm{CH}_{2}\right), 30.0$ $\left(\mathrm{CH}_{3}\right), 27.6\left(\mathrm{CH}_{2}\right), 21.3\left(\mathrm{CH}_{3}\right)$.

IR (ATR): 3313, 2928, 1640, 1530, 1302, $1121 \mathrm{~cm}^{-1}$.

MS (EI) m/z (relative intensity) 263 (5) [M] 248 (10), 231 (10), 220 (30), 189 (75), 145 (100).

HR-MS (EI) m/z calcd for $\mathrm{C}_{15} \mathrm{H}_{21} \mathrm{NO}_{3}\left[\mathrm{M}^{+}\right]$263.1521, found 263.1524 .

\section{N-Methyl-2-(3-oxobutyl)-1-naphthamide (120la)}<smiles>CNC(=O)c1c(CCC(C)=O)ccc2ccccc12</smiles>

The general procedure $\mathbf{E}$ was followed using 21 (93 $\mathrm{mg}, 0.5 \mathrm{mmol})$ and methyl vinyl ketone (41a) (70 mg, $1.0 \mathrm{mmol})$ for $20 \mathrm{~h}$. Purification by column chromatography ( $n$-hexane/EtOAc 2:1) yielded $1201 \mathrm{la}(57 \mathrm{mg}, 45 \%)$ as a colorless solid. M. p.: $=153-155^{\circ} \mathrm{C}$.

${ }^{1} \mathbf{H}-\mathbf{N M R}\left(500 \mathrm{MHz}, \mathrm{CDCl}_{3}\right): \delta=7.91-7.62(\mathrm{~m}, 3 \mathrm{H}), 7.54-7.35(\mathrm{~m}, 2 \mathrm{H}), 7.25(\mathrm{~d}, J=8.3 \mathrm{~Hz}$, 1H), 6.46 (br s, 1H), 3.08 (d, $J=5.4 \mathrm{~Hz}, 3 \mathrm{H}), 3.01-2.78$ (m, 4H), 2.09 (s, 3H).

${ }^{13}$ C-NMR $\left(125 \mathrm{MHz}, \mathrm{CDCl}_{3}\right): \delta=208.0\left(\mathrm{C}_{\mathrm{q}}\right), 170.1\left(\mathrm{C}_{\mathrm{q}}\right), 134.6\left(\mathrm{C}_{\mathrm{q}}\right), 134.1\left(\mathrm{C}_{\mathrm{q}}\right), 131.8\left(\mathrm{C}_{\mathrm{q}}\right)$, $130.2\left(\mathrm{C}_{\mathrm{q}}\right), 129.1(\mathrm{CH}), 127.8(\mathrm{CH}), 126.8(\mathrm{CH}), 126.3(\mathrm{CH}), 125.6(\mathrm{CH}), 124.8(\mathrm{CH}), 44.7$ $\left(\mathrm{CH}_{2}\right), 30.1\left(\mathrm{CH}_{3}\right), 27.4\left(\mathrm{CH}_{2}\right), 26.6\left(\mathrm{CH}_{3}\right)$.

IR (ATR): 3261, 1704, 1627, 1260, 745, $448 \mathrm{~cm}^{-1}$.

MS (EI) m/z (relative intensity) 255 (20) $\left[\mathrm{M}^{+}\right], 224$ (10), 212 (100), 197 (20), 181 (50), 155 (25).

HR-MS (EI) m/z calcd for $\mathrm{C}_{16} \mathrm{H}_{17} \mathrm{NO}_{2}\left[\mathrm{M}^{+}\right]$255.1259, found 255.1261 .

$N$-Me thyl-4-(3-oxobutyl)be nzo $[d][1,3]$ dioxole-5-carboxamide (120ma)<smiles>CNC(=O)c1ccc2c(c1CCC(C)=O)OCO2</smiles> 
The general procedure $\mathbf{E}$ was followed using $\mathbf{2} \mathbf{m}(90 \mathrm{mg}, 0.5 \mathrm{mmol})$ and methyl vinyl ketone (41a) (70 mg, $1.0 \mathrm{mmol})$ for $20 \mathrm{~h}$. Purification by column chromatography ( $n$-hexane/EtOAc $1: 1 \rightarrow 1: 2)$ yielded $120 \mathrm{ma}(100 \mathrm{mg}, 80 \%)$ as a colorless solid. M. p.: $=139-141{ }^{\circ} \mathrm{C}$.

${ }^{1}$ H-NMR (300 MHz, $\left.\mathrm{CDCl}_{3}\right): \delta=6.93(\mathrm{~d}, J=8.0 \mathrm{~Hz}, 1 \mathrm{H}), 6.64(\mathrm{~d}, J=8.0 \mathrm{~Hz}, 1 \mathrm{H}), 6.47$ (br s, 1H), 5.95 (s, 2H), 2.94 (d, J = 4.9 Hz, 3H), 2.94-2.88 (m, 4H), $2.15(\mathrm{~s}, 3 \mathrm{H})$.

${ }^{13}$ C-NMR (125 MHz, $\left.\mathrm{CDCl}_{3}\right): \delta=208.7\left(\mathrm{C}_{\mathrm{q}}\right), 169.5\left(\mathrm{C}_{\mathrm{q}}\right), 148.1\left(\mathrm{C}_{\mathrm{q}}\right), 146.2\left(\mathrm{C}_{\mathrm{q}}\right), 131.0\left(\mathrm{C}_{\mathrm{q}}\right)$, $121.5(\mathrm{CH}), 121.1\left(\mathrm{C}_{\mathrm{q}}\right), 106.2(\mathrm{CH}), 101.1\left(\mathrm{CH}_{2}\right), 43.0\left(\mathrm{CH}_{2}\right), 29.9\left(\mathrm{CH}_{3}\right), 26.7\left(\mathrm{CH}_{3}\right), 21.7$ $\left(\mathrm{CH}_{2}\right)$.

IR (ATR): 3301, 1706, 1543, 1251, 1040, $705 \mathrm{~cm}^{-1}$.

MS (EI) m/z (relative intensity) 249 (25) [M+ $], 218$ (15), 206 (100), 188 (5), 175 (65), 149 (15).

HR-MS (EI) m/z calcd for $\mathrm{C}_{13} \mathrm{H}_{15} \mathrm{NO}_{4}\left[\mathrm{M}^{+}\right]$249.1001, found 249.1006.

\section{3-Fluoro- $N$-methyl-2-(3-oxobutyl)be nzamide (120na)}<smiles>CNC(=O)c1cccc(F)c1CCC(C)=O</smiles>

The general procedure $\mathbf{E}$ was followed using $2 \mathbf{n}(77 \mathrm{mg}, 0.5 \mathrm{mmol})$ and methyl vinyl ketone (41a) (70 mg, $1.0 \mathrm{mmol})$ for $20 \mathrm{~h}$. Purification by column chromatography ( $n$-hexane/EtOAc 1:1) yielded 120na (86 mg, 70\%) as a colorless solid. M. p.: $=123-125^{\circ} \mathrm{C}$.

${ }^{1}$ H-NMR (300 MHz, CDCl $): \delta=7.21-7.11(\mathrm{~m}, 2 \mathrm{H}), 7.09-6.93(\mathrm{~m}, 1 \mathrm{H}), 6.55$ (br s, 1H), 2.96 (d, $J=4.9 \mathrm{~Hz}, 3 \mathrm{H}), 2.94$ (t, $J=5.4 \mathrm{~Hz}, 2 \mathrm{H}), 2.89$ (t, $J=5.4 \mathrm{~Hz}, 2 \mathrm{H}), 2.12$ (s, 3H).

${ }^{13}$ C-NMR (75 MHz, $\left.\mathrm{CDCl}_{3}\right): \delta=208.7\left(\mathrm{C}_{\mathrm{q}}\right), 169.3\left(\mathrm{C}_{\mathrm{q}}, J=3.2 \mathrm{~Hz}\right), 161.4\left(\mathrm{C}_{\mathrm{q}}, J=246.1 \mathrm{~Hz}\right)$, $139.1\left(\mathrm{C}_{\mathrm{q}}, J=4.3 \mathrm{~Hz}\right), 127.8(\mathrm{CH}, J=8.9 \mathrm{~Hz}), 126.1\left(\mathrm{C}_{\mathrm{q}}, J=16.7 \mathrm{~Hz}\right), 122.9(\mathrm{CH}, J=3.4 \mathrm{~Hz})$, $116.7(\mathrm{CH}, J=23.0 \mathrm{~Hz}), 43.5\left(\mathrm{CH}_{2}, J=2.4 \mathrm{~Hz}\right), 29.8\left(\mathrm{CH}_{3}\right), 26.7\left(\mathrm{CH}_{3}\right), 21.1\left(\mathrm{CH}_{2}, J=2.9 \mathrm{~Hz}\right)$. ${ }^{19}$ F-NMR (282 MHz, $\left.\mathrm{CDCl}_{3}\right): \delta=-116.1$ (dd, $\left.J=10.2,4.4 \mathrm{~Hz}\right)$.

IR (ATR): 3291, 1703, 1547, 1319, 1163, $710 \mathrm{~cm}^{-1}$.

MS (EI) m/z (relative intensity) $223(10)\left[\mathrm{M}^{+}\right], 180$ (100), 165 (15), 149 (60), 121 (15), 101 (15).

HR-MS (EI) m/z calcd for $\mathrm{C}_{12} \mathrm{H}_{14} \mathrm{NO}_{2} \mathrm{~F}\left[\mathrm{M}^{+}\right]$223.1009, found 223.1006 . 


\section{N,5-Dime thyl-2-(3-oxobutyl)be nzamide (1200a)}<smiles>CC(=O)CCc1ccc(C)cc1C(=O)N[Na]</smiles>

The general procedure $\mathbf{E}$ was followed using 20 (75 $\mathrm{mg}, 0.5 \mathrm{mmol})$ and methyl vinyl ketone (41a) (70 mg, $1.0 \mathrm{mmol})$ for $20 \mathrm{~h}$. Purification by column chromatography ( $n$-hexane/EtOAc $2: 1 \rightarrow 1: 1)$ yielded $1200 a(68 \mathrm{mg}, 62 \%)$ as a colorless solid. M. p.: $=112-114{ }^{\circ} \mathrm{C}$.

${ }^{1}$ H-NMR $\left(300 \mathrm{MHz}, \mathrm{CDCl}_{3}\right): \delta=7.15(\mathrm{~d}, J=1.8 \mathrm{~Hz}, 1 \mathrm{H}), 7.10(\mathrm{dd}, J=7.9,1.8 \mathrm{~Hz}, 1 \mathrm{H}), 7.07$ (d, $J=7.9 \mathrm{~Hz}, 1 \mathrm{H}), 6.41$ (br s, 1H), 2.98 (d, $J=4.9 \mathrm{~Hz}, 3 \mathrm{H}), 2.89$ (t, $J=5.0 \mathrm{~Hz}, 2 \mathrm{H}), 2.87$ (t, $J$ $=5.0 \mathrm{~Hz}, 2 \mathrm{H}), 2.30(\mathrm{~s}, 3 \mathrm{H}), 2.12(\mathrm{~s}, 3 \mathrm{H})$.

${ }^{13}$ C-NMR (125 MHz, $\left.\mathrm{CDCl}_{3}\right): \delta=208.6\left(\mathrm{C}_{\mathrm{q}}\right), 170.6\left(\mathrm{C}_{\mathrm{q}}\right), 136.4\left(\mathrm{C}_{\mathrm{q}}\right), 135.8\left(\mathrm{C}_{\mathrm{q}}\right), 135.6\left(\mathrm{C}_{\mathrm{q}}\right)$, 130.6 (CH), $129.5(\mathrm{CH}), 127.8(\mathrm{CH}), 45.3\left(\mathrm{CH}_{2}\right), 30.1\left(\mathrm{CH}_{3}\right), 26.7\left(\mathrm{CH}_{2}\right), 26.7\left(\mathrm{CH}_{3}\right), 20.9$ $\left(\mathrm{CH}_{3}\right)$.

IR (ATR): 3285, 1709, 1541, 1319, 1160, $697 \mathrm{~cm}^{-1}$.

MS (EI) m/z (relative intensity) $219(15)\left[\mathrm{M}^{+}\right], 189$ (5), 176 (100), 161 (10), 145 (60), 117 (15).

HR-MS (EI) m/z calcd for $\mathrm{C}_{13} \mathrm{H}_{17} \mathrm{NO}_{2}\left[\mathrm{M}^{+}\right] 219.1259$, found 219.1252 .

\section{$N$-Me thyl-2-(3-oxobutyl)-5-(trifluorome thyl)be nzamide (120pa)}<smiles>CNC(=O)c1cc(C(F)(F)F)ccc1CCC(C)=O</smiles>

The general procedure $\mathbf{E}$ was followed using $\mathbf{2 p}(102 \mathrm{mg}, 0.5 \mathrm{mmol})$ and methyl vinyl ketone (41a) (70 mg, $1.0 \mathrm{mmol})$ for $20 \mathrm{~h}$. Purification by column chromatography ( $n$-hexane/EtOAc $1: 1 \rightarrow 1: 2)$ yielded 120pa ( $85 \mathrm{mg}, 62 \%)$ as a colorless solid. M. p.: $=96-98^{\circ} \mathrm{C}$.

${ }^{1} \mathbf{H}-\mathrm{NMR}\left(300 \mathrm{MHz}, \mathrm{CDCl}_{3}\right.$ ): $\delta=7.60$ (dt, $\left.J=1.6,0.7 \mathrm{~Hz}, 1 \mathrm{H}\right), 7.56-7.49$ (m, 1H), 7.32 (dt, $J=8.3,0.8 \mathrm{~Hz}, 1 \mathrm{H}), 6.61($ br s, $1 \mathrm{H}), 2.98$ (d, $J=5.2 \mathrm{~Hz}, 3 \mathrm{H}), 2.97$ (t, $J=5.8 \mathrm{~Hz}, 2 \mathrm{H}), 2.89$ (t, $J=5.8 \mathrm{~Hz}, 2 \mathrm{H}), 2.11(\mathrm{~s}, 3 \mathrm{H})$.

${ }^{13}$ C-NMR (125 MHz, $\left.\mathrm{CDCl}_{3}\right): \delta=207.9\left(\mathrm{C}_{\mathrm{q}}\right), 169.0\left(\mathrm{C}_{\mathrm{q}}\right), 142.9\left(\mathrm{C}_{\mathrm{q}}, J_{\mathrm{C}-\mathrm{F}}=1.7 \mathrm{~Hz}\right), 137.2$ 
$\left(\mathrm{C}_{\mathrm{q}}\right), 130.1(\mathrm{CH}), 128.6\left(\mathrm{C}_{\mathrm{q}}, J_{\mathrm{C}-\mathrm{F}}=32.8 \mathrm{~Hz}\right), 126.4\left(\mathrm{CH}, J_{\mathrm{C}-\mathrm{F}}=3.7 \mathrm{~Hz}\right), 124.3\left(\mathrm{CH}, J_{\mathrm{C}-\mathrm{F}}=3.8\right.$

$\mathrm{Hz}), 123.2\left(\mathrm{C}_{\mathrm{q}}, J_{\mathrm{C}-\mathrm{F}}=271.5 \mathrm{~Hz}\right), 44.7\left(\mathrm{CH}_{2}\right), 30.0\left(\mathrm{CH}_{3}\right), 26.9\left(\mathrm{CH}_{2}\right), 26.8\left(\mathrm{CH}_{3}\right)$.

${ }^{19}$ F-NMR $\left(282 \mathrm{MHz}, \mathrm{CDCl}_{3}\right): \delta=-62.6(\mathrm{~s})$.

IR (ATR): 3286, 1705, 1552, 1311, 1115, $641 \mathrm{~cm}^{-1}$.

MS (EI) m/z (relative intensity) 273 (5) [M+], 243 (5), 230 (100), 215 (10), 199 (35), 189 (10).

HR-MS (EI) $\mathrm{m} / \mathrm{z}$ calcd for $\mathrm{C}_{13} \mathrm{H}_{14} \mathrm{NO}_{2} \mathrm{~F}_{3}\left[\mathrm{M}^{+}\right]$273.0977, found 273.0979.

\section{$N$-Benzyl-5-me thyl-2-(3-oxobutyl)benzamide (120qa)}<smiles>CC(=O)CCc1ccc(C)cc1C(=O)NCc1ccccc1</smiles>

The general procedure $\mathbf{E}$ was followed using $\mathbf{2 q}(113 \mathrm{mg}, 0.5 \mathrm{mmol})$ and methyl vinyl ketone (41a) (70 mg, $1.0 \mathrm{mmol})$ for $20 \mathrm{~h}$. Purification by column chromatography ( $n$-hexane/EtOAc 2:1) yielded 120qa (94 mg, 64\%) as a colorless solid. M. p.: $=130-132{ }^{\circ} \mathrm{C}$.

${ }^{1} \mathbf{H}-\mathbf{N M R}\left(300 \mathrm{MHz}, \mathrm{CDCl}_{3}\right): \delta=7.43-7.25(\mathrm{~m}, 5 \mathrm{H}), 7.17(\mathrm{~d}, J=0.9 \mathrm{~Hz}, 1 \mathrm{H}), 7.14-7.06(\mathrm{~m}$, 2H), $6.52($ br s, 1H), $4.60(\mathrm{~d}, J=5.8 \mathrm{~Hz}, 2 \mathrm{H}), 3.01(\mathrm{dt}, J=6.8,1.5 \mathrm{~Hz}, 2 \mathrm{H}),, 2.82(\mathrm{dt}, J=6.8,1.5$ $\mathrm{Hz}, 2 \mathrm{H}), 2.29$ (s, 3H), 2.07 (s, 3H).

${ }^{13}$ C-NMR (75 MHz, $\left.\mathrm{CDCl}_{3}\right): \delta=208.5\left(\mathrm{C}_{\mathrm{q}}\right), 169.9\left(\mathrm{C}_{\mathrm{q}}\right), 138.2\left(\mathrm{C}_{\mathrm{q}}\right), 136.2\left(\mathrm{C}_{\mathrm{q}}\right), 136.0\left(\mathrm{C}_{\mathrm{q}}\right)$, $136.0\left(\mathrm{C}_{\mathrm{q}}\right), 130.8(\mathrm{CH}), 129.9(\mathrm{CH}), 128.8(\mathrm{CH}), 127.9(\mathrm{CH}), 127.7(\mathrm{CH}), 127.6(\mathrm{CH}), 45.4$ $\left(\mathrm{CH}_{2}\right), 44.0\left(\mathrm{CH}_{2}\right), 29.9\left(\mathrm{CH}_{3}\right), 26.9\left(\mathrm{CH}_{2}\right), 20.8\left(\mathrm{CH}_{3}\right)$.

IR (ATR): 3242, 1707, 1634, 1311, 821, $702 \mathrm{~cm}^{-1}$.

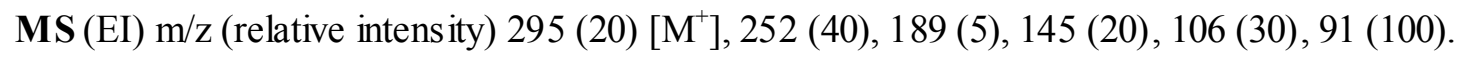

HR-MS (EI) m/z calcd for $\mathrm{C}_{19} \mathrm{H}_{21} \mathrm{NO}_{2}\left[\mathrm{M}^{+}\right]$295.1572, found 295.1580 .

\section{$N, 1-D i m e$ thyl-2-(3-oxobutyl)-1H-indole-3-carboxamide (120ra)}<smiles>CC(=O)CCc1c(C(=O)NN)c2ccccc2n1C</smiles> 
The general procedure $\mathbf{E}$ was followed using $2 \mathbf{r}(94 \mathrm{mg}, 0.5 \mathrm{mmol})$ and methyl vinyl ketone (41a) (70 mg, $1.0 \mathrm{mmol})$ for $20 \mathrm{~h}$. Purification by column chromatography ( $n$-hexane/EtOAc $2: 1 \rightarrow 1: 1)$ yielded 120ra (65 mg, $50 \%)$ as a colorless solid. M. p.: $=151-153{ }^{\circ} \mathrm{C}$.

${ }^{1} \mathbf{H}-\mathbf{N M R}\left(300 \mathrm{MHz}, \mathrm{CDCl}_{3}\right): \delta=7.67(\mathrm{~m}, 1 \mathrm{H}), 7.35-7.29(\mathrm{~m}, 1 \mathrm{H}), 7.26-7.15(\mathrm{~m}, 2 \mathrm{H}), 6.08$ (br s, 1H), $3.74(\mathrm{~s}, 3 \mathrm{H}), 3.35$ (t, $J=7.5 \mathrm{~Hz}, 2 \mathrm{H}), 3.04$ (d, $J=4.9 \mathrm{~Hz}, 3 \mathrm{H}), 2.93$ (t, $J=7.5 \mathrm{~Hz}, 2 \mathrm{H})$, $2.15(\mathrm{~s}, 3 \mathrm{H})$.

${ }^{13}$ C-NMR (75 MHz, $\left.\mathrm{CDCl}_{3}\right): \delta=207.8\left(\mathrm{C}_{\mathrm{q}}\right), 166.6\left(\mathrm{C}_{\mathrm{q}}\right), 144.9\left(\mathrm{C}_{\mathrm{q}}\right), 136.6\left(\mathrm{C}_{\mathrm{q}}\right), 124.8\left(\mathrm{C}_{\mathrm{q}}\right)$, 121.8 (CH), $121.2(\mathrm{CH}), 118.4(\mathrm{CH}), 110.0(\mathrm{CH}), 107.6\left(\mathrm{C}_{\mathrm{q}}\right), 43.3\left(\mathrm{CH}_{2}\right), 29.9\left(\mathrm{CH}_{3}\right), 29.6$ $\left(\mathrm{CH}_{3}\right), 26.3\left(\mathrm{CH}_{3}\right), 19.8\left(\mathrm{CH}_{2}\right)$.

IR (ATR): 3293, 1711, 1619, 1539, 1167, $734 \mathrm{~cm}^{-1}$.

MS (EI) m/z (relative intensity) 258 (55) $\left[\mathrm{M}^{+}\right], 227$ (15), 215 (80), 200 (10), 184 (100), 172 (15), $158(85)$.

HR-MS (EI) $\mathrm{m} / \mathrm{z}$ calcd for $\mathrm{C}_{15} \mathrm{H}_{18} \mathrm{~N}_{2} \mathrm{O}_{2}\left[\mathrm{M}^{+}\right]$258.1368, found 258.1363.

\section{4-Methoxy- $N$-me thyl-2-(3-oxo-3-phenylpropyl)be nzamide (120cb)}<smiles>CNC(=O)c1ccc(OC)cc1CCC(=O)c1ccccc1</smiles>

The general procedure $\mathbf{E}$ was followed using $2 \mathbf{c}(83 \mathrm{mg}, 0.5 \mathrm{mmol})$ and $\mathbf{4 1} \mathbf{b}$ (132 $\mathrm{mg}, 1.0 \mathrm{mmol})$ for $20 \mathrm{~h}$. Purification by column chromatography ( $n$-hexane/EtOAc $1: 1 \rightarrow 1: 2$ ) yielded $120 \mathrm{cb}$ (31 mg, 21\%) as a colorless solid. M. p.:=130-132 C .

${ }^{1} \mathbf{H}-\mathbf{N M R}\left(300 \mathrm{MHz}, \mathrm{CDCl}_{3}\right): \delta=7.97-7.88(\mathrm{~m}, 2 \mathrm{H}), 7.51$ (ddt, $\left.J=8.3,6.6,1.4 \mathrm{~Hz}, 1 \mathrm{H}\right)$, 7.45-7.36 (m, 2H), $7.31(\mathrm{~d}, J=8.5 \mathrm{~Hz}, 1 \mathrm{H}), 6.77(\mathrm{~d}, J=2.6 \mathrm{~Hz}, 1 \mathrm{H}), 6.69$ (dd, $J=8.5,2.6 \mathrm{~Hz}$, 1H), 6.38 (br s, 1H), 3.75 (s, 3H), $3.42(\mathrm{t}, J=7.3 \mathrm{~Hz}, 2 \mathrm{H}), 3.12$ (t, $J=7.3 \mathrm{~Hz}, 2 \mathrm{H}), 2.94$ (d, $J=$ $4.9 \mathrm{~Hz}, 3 \mathrm{H})$.

${ }^{13}$ C-NMR $\left(75 \mathrm{MHz}, \mathrm{CDCl}_{3}\right): \delta=199.8\left(\mathrm{C}_{\mathrm{q}}\right), 170.4\left(\mathrm{C}_{\mathrm{q}}\right), 160.6\left(\mathrm{C}_{\mathrm{q}}\right), 141.6\left(\mathrm{C}_{\mathrm{q}}\right), 136.8\left(\mathrm{C}_{\mathrm{q}}\right)$, $133.0(\mathrm{CH}), 129.1\left(\mathrm{C}_{\mathrm{q}}\right), 128.9(\mathrm{CH}), 128.5(\mathrm{CH}), 128.1(\mathrm{CH}), 115.5(\mathrm{CH}), 111.3(\mathrm{CH}), 55.2$ $\left(\mathrm{CH}_{3}\right), 40.5\left(\mathrm{CH}_{2}\right), 28.1\left(\mathrm{CH}_{2}\right), 26.7\left(\mathrm{CH}_{3}\right)$.

IR (ATR): 3277, 1681, 1545, 1277, 1039, $690 \mathrm{~cm}^{-1}$. 
MS (EI) m/z (relative intensity) 297 (15) $\left[\mathrm{M}^{+}\right], 266$ (10), 223 (10), 192 (100), 177 (30), 161 (35).

HR-MS (ESI) m/z calcd for $\mathrm{C}_{18} \mathrm{H}_{20} \mathrm{NO}_{3}\left[\mathrm{M}+\mathrm{H}^{+}\right]$298.1443, found 298.1437.

\section{3-(3-Cyclohexyl-3-oxopropyl)- $N$-methyl-[1,1'-biphe nyl]-4-carboxamide (120dc)}<smiles>CNC(=O)c1ccc(-c2ccccc2)cc1CCC(=O)C1CCCC1</smiles>

The general procedure $\mathbf{E}$ was followed using $\mathbf{2 d}$ (106 mg, $0.5 \mathrm{mmol})$ and $41 \mathrm{c}$ (138 $\mathrm{mg}, 1.0$ mmol) for $20 \mathrm{~h}$. Purification by column chromatography ( $n$-hexane/EtOAc $1: 1 \rightarrow 1: 2$ ) yie lded $\operatorname{120dc}(79 \mathrm{mg}, 45 \%)$ as a colorless solid. M. p.: $=155-157^{\circ} \mathrm{C}$.

${ }^{1} \mathbf{H}-\mathbf{N M R}\left(300 \mathrm{MHz}, \mathrm{CDCl}_{3}\right): \delta=7.55(\mathrm{~d}, J=7.4 \mathrm{~Hz}, 2 \mathrm{H}), 7.50-7.30(\mathrm{~m}, 6 \mathrm{H}), 6.56(\mathrm{br} \mathrm{s}, J=4.9$ $\mathrm{Hz}, 1 \mathrm{H}), 3.00$ (d, $J=4.9 \mathrm{~Hz}, 3 \mathrm{H}), 2.99$ (t, $J=5.7 \mathrm{~Hz}, 2 \mathrm{H}), 2.94$ (t, $J=5.7 \mathrm{~Hz}, 2 \mathrm{H}), 2.34-2.25$ (m, 1H), $1.82-1.68(\mathrm{~m}, 4 \mathrm{H}), 1.34-1.10(\mathrm{~m}, 6 \mathrm{H})$.

${ }^{13}$ C-NMR (125 MHz, $\left.\mathrm{CDCl}_{3}\right): \delta=213.7\left(\mathrm{C}_{\mathrm{q}}\right), 170.3\left(\mathrm{C}_{\mathrm{q}}\right), 142.6\left(\mathrm{C}_{\mathrm{q}}\right), 140.2\left(\mathrm{C}_{\mathrm{q}}\right), 139.6\left(\mathrm{C}_{\mathrm{q}}\right)$, $135.4\left(\mathrm{C}_{\mathrm{q}}\right), 128.7(\mathrm{CH}), 128.3(\mathrm{CH}), 127.8(\mathrm{CH}), 127.6(\mathrm{CH}), 127.0(\mathrm{CH}), 124.8(\mathrm{CH}), 50.9$ (CH), $42.2\left(\mathrm{CH}_{2}\right), 28.5\left(\mathrm{CH}_{2}\right), 27.3\left(\mathrm{CH}_{2}\right), 26.8\left(\mathrm{CH}_{3}\right), 25.9\left(\mathrm{CH}_{2}\right), 25.7\left(\mathrm{CH}_{2}\right)$.

IR (ATR): 3289, 2927, 1699, 1630, 1538, 1312, $697 \mathrm{~cm}^{-1}$.

MS (EI) m/z (relative intensity) 349 (20) [M+ $], 318$ (5), 238 (100), 209 (40), 178 (15), 165 (15).

HR-MS (EI) m/z calcd for $\mathrm{C}_{23} \mathrm{H}_{27} \mathrm{NO}_{2}\left[\mathrm{M}^{+}\right]$349.2042, found 249.2039.

\section{$N$-Methyl-3-(3-0x ooctyl)-[1,1'-biphe nyl]-4-carbox amide (120dd)}<smiles>CNC(=O)c1ccc(-c2ccccc2)cc1CCC(=O)POCCO</smiles>

The general procedure $\mathbf{E}$ was followed using 2d (106 mg, $0.5 \mathrm{mmol}$ ) and $\mathbf{4 1 d}$ (126 mg, 1.0 mmol) for $20 \mathrm{~h}$. Purification by column chromatography ( $n$-hexane/EtOAc 1:1) yielded $\mathbf{1 2 0 d d}$ (84 mg, 50\%) as a colorless solid. M. p.: $=128-130{ }^{\circ} \mathrm{C}$. 
${ }^{1} \mathbf{H}-\mathbf{N M R}\left(\mathrm{CDCl}_{3}, 500 \mathrm{MHz}\right): \delta=7.57-7.50(\mathrm{~m}, 2 \mathrm{H}), 7.46-7.38(\mathrm{~m}, 5 \mathrm{H}), 7.37-7.32(\mathrm{~m}, 1 \mathrm{H})$, 6.46 (br s, $J=4.9 \mathrm{~Hz}, 1 \mathrm{H}), 3.03$ (t, $J=7.2 \mathrm{~Hz}, 2 \mathrm{H}), 3.00$ (d, $J=4.9 \mathrm{~Hz}, 3 \mathrm{H}), 2.90$ (t, $J=7.2 \mathrm{~Hz}$, 2H), 2.36 (t, $J=7.5 \mathrm{~Hz}, 2 \mathrm{H}), 1.52$ (p, $J=7.5 \mathrm{~Hz}, 2 \mathrm{H}), 1.31-1.13(\mathrm{~m}, 4 \mathrm{H}), 0.83$ (t, $J=7.1 \mathrm{~Hz}$, $3 \mathrm{H})$.

${ }^{13}$ C-NMR $\left(\mathrm{CDCl}_{3}, 125 \mathrm{MHz}\right): \delta=211.1\left(\mathrm{C}_{\mathrm{q}}\right), 170.4\left(\mathrm{C}_{\mathrm{q}}\right), 142.8\left(\mathrm{C}_{\mathrm{q}}\right), 140.2\left(\mathrm{C}_{\mathrm{q}}\right), 139.6\left(\mathrm{C}_{\mathrm{q}}\right)$, $135.5\left(\mathrm{C}_{\mathrm{q}}\right), 128.8(\mathrm{CH}), 128.5(\mathrm{CH}), 127.9(\mathrm{CH}), 127.7(\mathrm{CH}), 127.1(\mathrm{CH}), 124.9(\mathrm{CH}), 44.2$ $\left(\mathrm{CH}_{2}\right), 43.0\left(\mathrm{CH}_{2}\right), 31.4\left(\mathrm{CH}_{2}\right), 27.3\left(\mathrm{CH}_{2}\right), 26.7\left(\mathrm{CH}_{3}\right), 23.5\left(\mathrm{CH}_{2}\right), 22.4\left(\mathrm{CH}_{2}\right), 13.9\left(\mathrm{CH}_{3}\right)$.

IR (ATR): 3287, 1710, 1632, 1542, 1164, $693 \mathrm{~cm}^{-1}$.

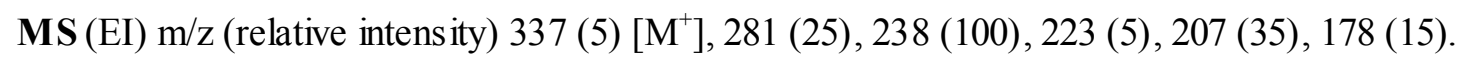

HR-MS (EI) m/z calcd for $\mathrm{C}_{22} \mathrm{H}_{27} \mathrm{NO}_{2}\left[\mathrm{M}^{+}\right]$337.2042, found 337.2048.

\section{$N$-(4-Methyl-2-(3-oxobutyl)phenyl)ace tamide (139aa)}<smiles>CC(=O)CCc1cc(C)ccc1NC(C)=O</smiles>

The general procedure $\mathbf{E}$ was followed using 121a (75 mg, $0.5 \mathrm{mmol})$, methyl vinyl ketone (41a) (70 mg, $1.0 \mathrm{mmol})$ and $\mathrm{KO}_{2} \mathrm{CMes}(51 \mathrm{mg}, 50 \mathrm{~mol} \%$ ) for $20 \mathrm{~h}$. Purification by column chromatography ( $n$-hexane/EtOAc $2: 1 \rightarrow 1: 1)$ yie lded 139aa (51 mg, 47\%) as a colorless solid. M. p.: $=124-126^{\circ} \mathrm{C}$.

${ }^{1}$ H-NMR $\left(300 \mathrm{MHz}, \mathrm{CDCl}_{3}\right): \delta=8.84(\mathrm{br} \mathrm{s}, 1 \mathrm{H}), 7.62(\mathrm{~d}, J=8.2 \mathrm{~Hz}, 1 \mathrm{H}), 6.99(\mathrm{dd}, J=8.0,2.0$ $\mathrm{Hz}, 1 \mathrm{H}), 6.89(\mathrm{~d}, J=2.1 \mathrm{~Hz}, 1 \mathrm{H}), 2.87$ (dt, $J=6.0,1.5 \mathrm{~Hz}, 2 \mathrm{H}), 2.75(\mathrm{dt}, J=6.0,1.5 \mathrm{~Hz}, 2 \mathrm{H})$, $2.26(\mathrm{~s}, 3 \mathrm{H}), 2.24(\mathrm{~s}, 3 \mathrm{H}), 2.12(\mathrm{~s}, 3 \mathrm{H})$.

${ }^{13}$ C-NMR $\left(\mathrm{CDCl}_{3}, 75 \mathrm{MHz}\right): \delta=210.3\left(\mathrm{C}_{\mathrm{q}}\right), 168.8\left(\mathrm{C}_{\mathrm{q}}\right), 134.7\left(\mathrm{C}_{\mathrm{q}}\right), 133.0\left(\mathrm{C}_{\mathrm{q}}\right), 132.6\left(\mathrm{C}_{\mathrm{q}}\right)$, $130.3(\mathrm{CH}), 127.7(\mathrm{CH}), 124.4(\mathrm{CH}), 45.3\left(\mathrm{CH}_{2}\right), 29.9\left(\mathrm{CH}_{3}\right), 24.2\left(\mathrm{CH}_{3}\right), 23.8\left(\mathrm{CH}_{2}\right), 20.8$ $\left(\mathrm{CH}_{3}\right)$.

IR (ATR): 3282, 1709, 1641, 1522, 1287, $811 \mathrm{~cm}^{-1}$.

MS (EI) m/z (relative intensity) 219 (60) $\left[\mathrm{M}^{+}\right], 176$ (75), 162 (40), 134 (100), 120 (85), 107 (10).

HR-MS (EI) m/z calcd for $\mathrm{C}_{13} \mathrm{H}_{17} \mathrm{NO}_{2}\left[\mathrm{M}^{+}\right]$219.1259, found 219.1259. 
Intermolecular Competition Experiments Between Arenes with Different Directing Groups :

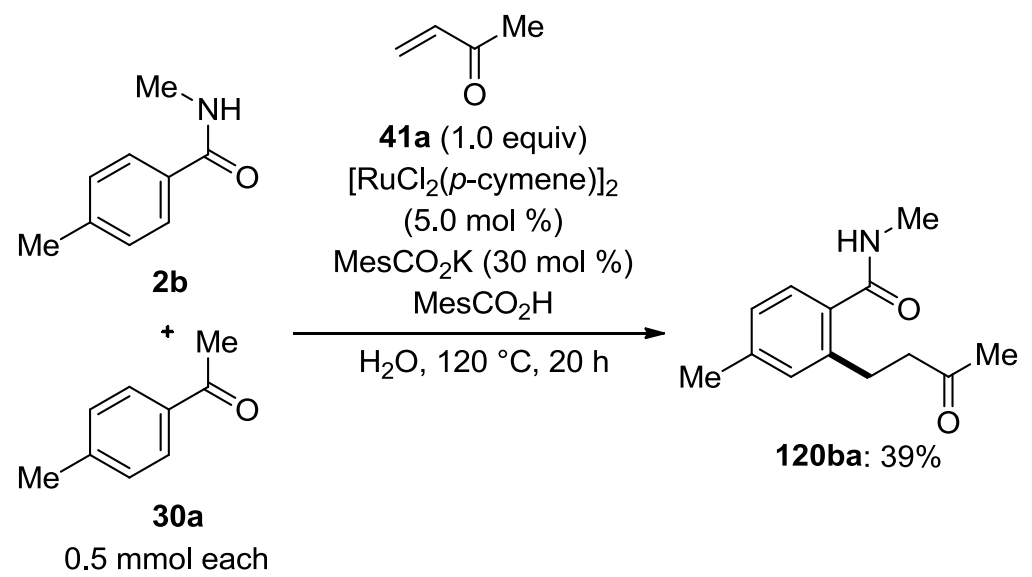

A suspension of MVK (41a) (35 mg, $0.5 \mathrm{mmol}$ ), N,4-dimethylbenzamide (2b) (75 mg, 0.5 mmol), 1-(p-tolyl)ethanone (30a) (67 mg, $0.5 \mathrm{mmol}),\left[\mathrm{RuCl}_{2}(p \text {-cymene) }]_{2}(15.3 \mathrm{mg}, 5.0\right.$ $\mathrm{mol} \%$ ), $\mathrm{MesCO}_{2} \mathrm{~K}\left(30.3 \mathrm{mg}, 30.0 \mathrm{~mol} \%\right.$ ) and $\mathrm{MesCO}_{2} \mathrm{H}\left(82 \mathrm{mg}, 1.0\right.$ equiv) in $\mathrm{H}_{2} \mathrm{O}(2.0 \mathrm{~mL})$ was stirred at $120^{\circ} \mathrm{C}$ for $20 \mathrm{~h}$ under an atmosphere of Ar. At ambient temperature, aq. sat. $\mathrm{NaCl}(15 \mathrm{~mL})$ was added. The reaction mixture was extracted with EtOAc $(3 \times 20 \mathrm{~mL})$. The combined organic phases were dried over $\mathrm{Na}_{2} \mathrm{SO}_{4}$. Evaporation of the solvents in vacuo and purification of the residue by column chromatography on silica gel ( $n$-hexane/EtOAc 1:1) yie lded products $\mathbf{1 2 0 b a}(43 \mathrm{mg}, 39 \%$ ) as the sole product.

\section{H/D Exchange Experiments:}

Ruthe nium(II)-Catalyze d H/D Exchange with Substrate III-1b in $\mathrm{D}_{2} \mathrm{O}$ as the cosolvent: 


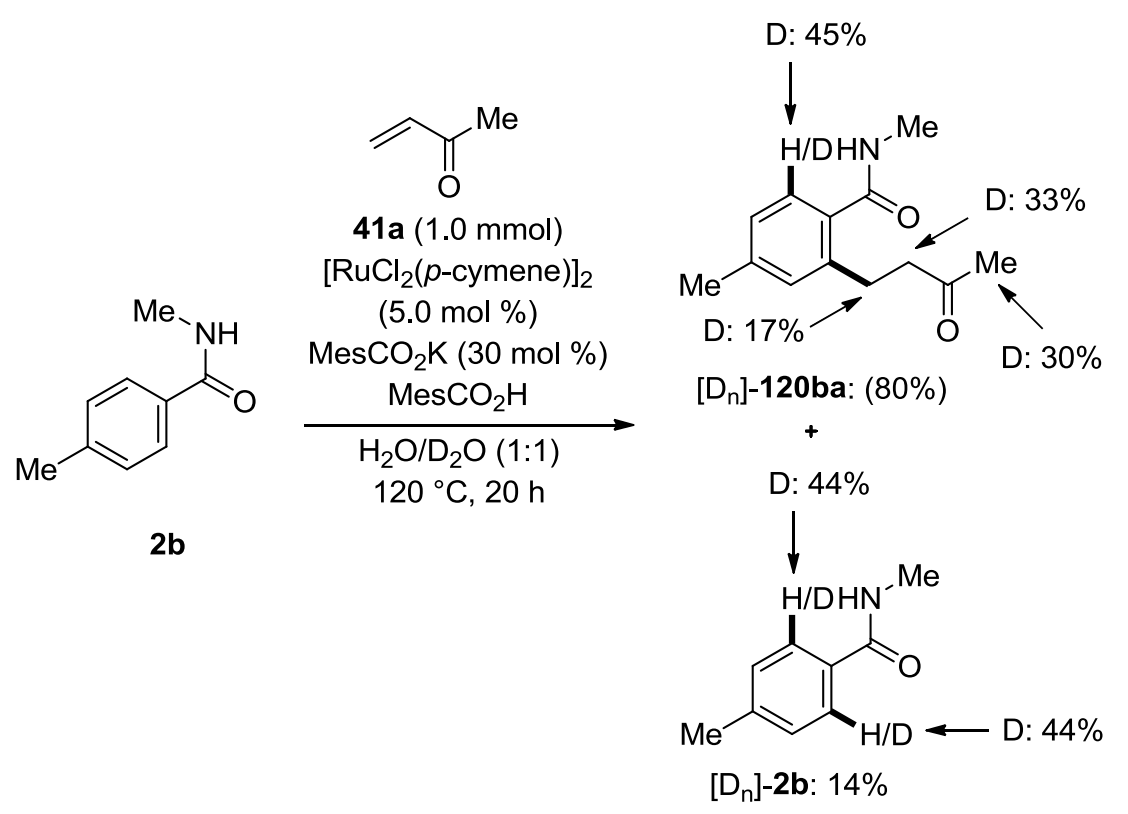

A suspension of MVK (41a) (70 mg, $1.0 \mathrm{mmol}), N$,4-dimethylbenzamide (2b) (75 mg, 0.5 mmol), $\left[\mathrm{RuCl}_{2}(p \text {-cymene })\right]_{2}(15.3 \mathrm{mg}, 5.0 \mathrm{~mol} \%), \mathrm{MesCO}_{2} \mathrm{~K}(30.3 \mathrm{mg}, 30.0 \mathrm{~mol} \%)$ and $\mathrm{MesCO}_{2} \mathrm{H}$ (82 mg, 1.0 equiv) in a solvent mixture of $\mathrm{H}_{2} \mathrm{O}$ and $\mathrm{D}_{2} \mathrm{O}(1.0 / 1.0 \mathrm{~mL})$ was stirred at $120{ }^{\circ} \mathrm{C}$ for $20 \mathrm{~h}$ under an atmosphere of Ar. At ambient temperature, aq. sat. $\mathrm{NaCl}(15 \mathrm{~mL})$ was added. The reaction mixture was extracted with EtOAc $(3 \times 20 \mathrm{~mL})$. The combined organic phases were dried over $\mathrm{Na}_{2} \mathrm{SO}_{4}$. Evaporation of the solvents in vacuo and purification of the residue by column chromatography on silica gel ( $n$-hexane/EtOAc $1: 1 \rightarrow 1: 2)$ yielded product $\left[\mathrm{D}_{\mathrm{n}}\right]-\mathbf{- 1 2 0 b a}(88 \mathrm{mg}, 80 \%)$ as a colorless solid, and re isolated starting material $\left[\mathrm{D}_{\mathrm{n}}\right]-\mathbf{2 b}$ (11 mg, 14\%) as a colorless solid. The D-incorporation in $\left[D_{n}\right]-120 b a$ and $\left[D_{n}\right]-2 b$ was estimated by ${ }^{1} \mathrm{H}-\mathrm{NMR}$ spectroscopy.

\section{Kine tic Isotope Effect:}

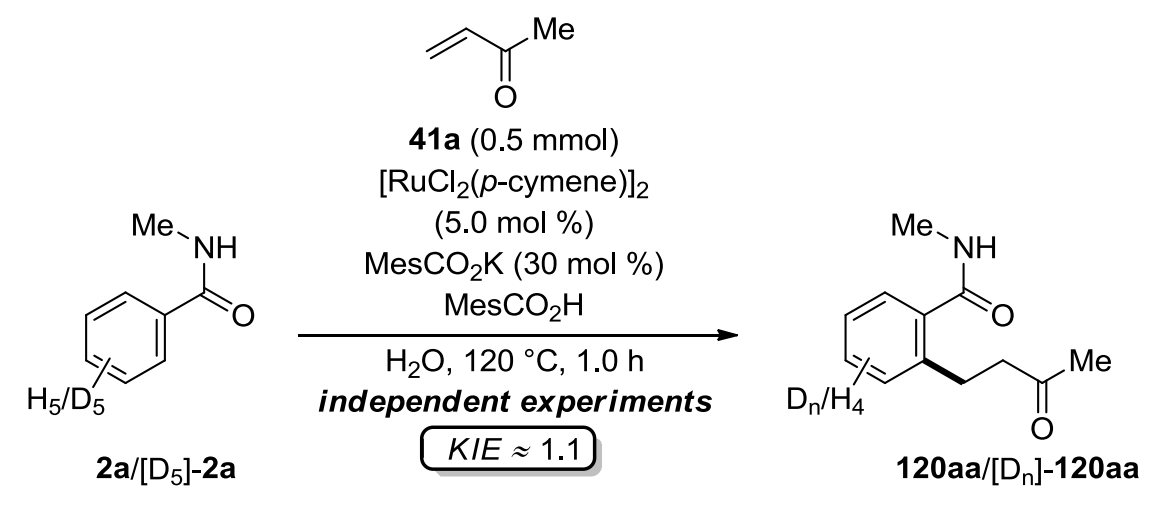


Two independent reactions with $\mathbf{2 a}$ or deuterated substrate $\left[\mathrm{D}_{5}\right]-\mathbf{2 a}$ under the standard conditions were performed: Suspensions of MVK (41a) (70 mg, $1.0 \mathrm{mmol})$, substrates $2 \mathbf{a}(68$ $\mathrm{mg}, 0.5 \mathrm{mmol})$ or $\left[\mathrm{D}_{5}\right]-\mathbf{2 a}(70 \mathrm{mg}, 0.5 \mathrm{mmol}),\left[\mathrm{RuCl}_{2}(p \text {-cymene })\right]_{2}(15.3 \mathrm{mg}, 5.0 \mathrm{~mol} \%)$, $\mathrm{MesCO}_{2} \mathrm{~K}(30.3 \mathrm{mg}, 30.0 \mathrm{~mol} \%)$ and $\mathrm{MesCO}_{2} \mathrm{H}(82 \mathrm{mg}, 0.5 \mathrm{mmol})$ in $\mathrm{H}_{2} \mathrm{O}(2.0 \mathrm{~mL})$ were stirred at $110^{\circ} \mathrm{C}$ for $0.5 \mathrm{~h}, 1.5 \mathrm{~h}, 2.0 \mathrm{~h}, 2.5 \mathrm{~h}, 3.5 \mathrm{~h}, 4.5 \mathrm{~h}, 5.0 \mathrm{~h}$ under an atmosphere of argon, respectively. The consumption of substrate $\mathbf{2 a}$ or $\left[\mathrm{D}_{5}\right]-\mathbf{2} \mathbf{a}$ and the appearance of the products $120 \mathrm{aa}$ or $\left[D_{n}\right]-120$ aa were monitored by GC analysis. These experiments indicated that the $\mathrm{C}-\mathrm{H}$ bond activation is not the turnover-limiting step of the ruthenium(II)-catalyzed $\mathrm{C}-\mathrm{H}$ alkylation reaction.

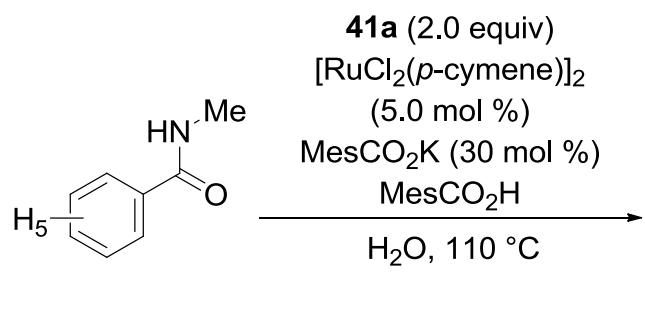

$2 a$<smiles>CNC(=O)c1cc[14cH]cc1CCC(C)=O</smiles>

120aa

\begin{tabular}{|c|c|c|c|c|c|c|c|}
\hline $\mathbf{t} /(\mathbf{h})$ & $\mathbf{0 . 5}$ & $\mathbf{1 . 5}$ & $\mathbf{2 . 0}$ & $\mathbf{2 . 5}$ & $\mathbf{3 . 5}$ & $\mathbf{4 . 5}$ & $\mathbf{5 . 0}$ \\
\hline 120aa & 0.23 & 0.39 & 0.57 & 0.45 & 0.66 & 0.73 & 0.76 \\
\hline 2a & 0.77 & 0.61 & 0.43 & 0.55 & 0.34 & 0.27 & 0.24 \\
\hline
\end{tabular}

41a (2.0 equiv)

$\left[\mathrm{RuCl}_{2}(p \text {-cymene })\right]_{2}$

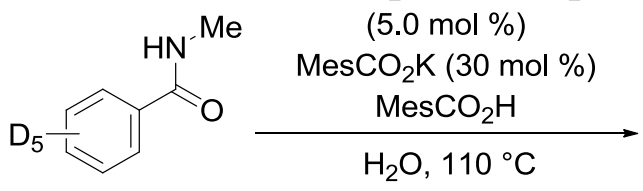

$\left[D_{5}\right]-2 a$<smiles>CNC(=O)c1ccc(Br)cc1CCC(C)=O</smiles>

$\left[D_{n}\right]-120 a a$

\begin{tabular}{|c|c|c|c|c|c|c|c|}
\hline $\mathbf{t} /(\mathbf{h})$ & $\mathbf{0 . 5}$ & $\mathbf{1 . 5}$ & $\mathbf{2 . 0}$ & $\mathbf{2 . 5}$ & $\mathbf{3 . 5}$ & $\mathbf{4 . 5}$ & $\mathbf{5 . 0}$ \\
\hline$\left[\mathrm{D}_{\mathrm{n}}\right]-\mathbf{- 1 2 0 a a}$ & 0.04 & 0.17 & 0.22 & 0.24 & 0.31 & 0.42 & 0.60 \\
\hline$\left[\mathrm{D}_{5}\right]-\mathbf{- 2 a}$ & 0.96 & 0.83 & 0.78 & 0.76 & 0.69 & 0.58 & 0.40 \\
\hline
\end{tabular}
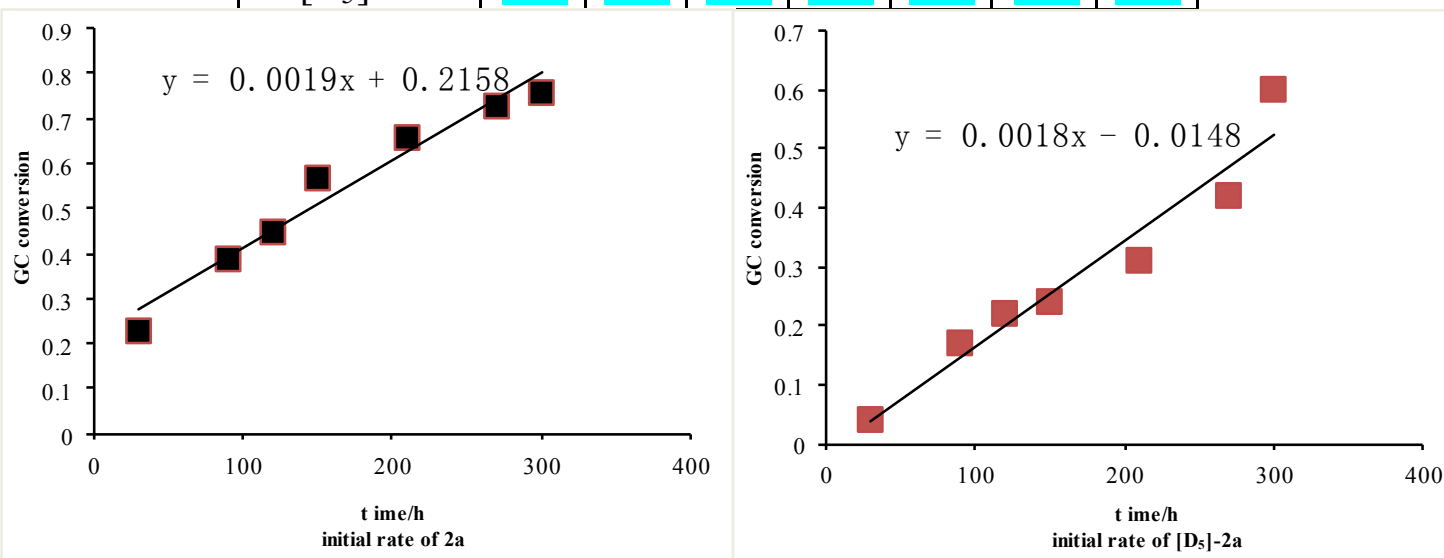


\section{,6-Dime thylquinoline (122aa)}<smiles>Cc1ccc2nc(C)ccc2c1</smiles>

The general procedure $\mathbf{F}$ was followed using 121 a $(75 \mathrm{mg}, 0.5 \mathrm{mmol})$ and methyl vinyl ketone (41a) (70 $\mathrm{mg}, 1.0 \mathrm{mmol})$ for $20 \mathrm{~h}$. Purification by column chromatography ( $n$-hexane/EtOAc 6:1) yielded $122 \mathrm{aa}(52 \mathrm{mg}, 66 \%)$ as a yellow oil.

${ }^{1} \mathbf{H}-\mathbf{N M R}\left(300 \mathrm{MHz}, \mathrm{CDCl}_{3}\right): \delta=7.91(\mathrm{~d}, J=8.7 \mathrm{~Hz}, 1 \mathrm{H}), 7.89(\mathrm{~d}, J=9.0 \mathrm{~Hz}, 1 \mathrm{H}), 7.49(\mathrm{~s}$, 1H), 7.48 (dd, $J=7.6,1.8 \mathrm{~Hz}, 1 \mathrm{H}), 7.20$ (d, $J=8.3 \mathrm{~Hz}, 1 \mathrm{H}), 2.69$ (s, 3H), 2.48 (s, 3H).

${ }^{13}$ C-NMR $\left(125 \mathrm{MHz}, \mathrm{CDCl}_{3}\right): \delta=157.9\left(\mathrm{C}_{\mathrm{q}}\right), 146.4\left(\mathrm{C}_{\mathrm{q}}\right), 135.5(\mathrm{CH}), 135.3\left(\mathrm{C}_{\mathrm{q}}\right), 131.6(\mathrm{CH})$, $128.2(\mathrm{CH}), 126.4\left(\mathrm{C}_{\mathrm{q}}\right), 126.3(\mathrm{CH}), 121.9(\mathrm{CH}), 25.2\left(\mathrm{CH}_{3}\right), 21.4\left(\mathrm{CH}_{3}\right)$.

IR (film): 2917, 1601, 1495, 1119, 825, $592 \mathrm{~cm}^{-1}$.

MS (EI) m/z (relative intens ity) 157 (100) $\left[\mathrm{M}^{+}\right], 142$ (20), 128 (10), 115 (20), 89 (10), 77 (10).

HR-MS (EI) $\mathrm{m} / \mathrm{z}$ calcd for $\mathrm{C}_{11} \mathrm{H}_{11} \mathrm{~N}\left[\mathrm{M}^{+}\right]$157.0891, found 157.0884 .

The spectral data were in accordance with those reported in the literature. ${ }^{148}$

\section{6-Methoxy-2-methylquinoline (122ba)}<smiles>COc1ccc2nc(C)ccc2c1</smiles>

The general procedure $\mathbf{F}$ was followed using $121 \mathbf{b}(83 \mathrm{mg}, 0.5 \mathrm{mmol})$ and methyl vinyl ketone (41a) (70 mg, $1.0 \mathrm{mmol})$ for $20 \mathrm{~h}$. Purification by column chromatography ( $n$-hexane/EtOAc 3:1) yielded III-6ba (60 mg, 69\%) as a yellow oil.

${ }^{1} \mathbf{H}-\mathbf{N M R}\left(300 \mathrm{MHz}, \mathrm{CDCl}_{3}\right): \delta=7.94-7.90(\mathrm{~m}, 1 \mathrm{H}), 7.89-7.85(\mathrm{~m}, 1 \mathrm{H}), 7.30(\mathrm{dd}, J=9.2,2.8$ Hz, 1H), 7.20 (d, $J=8.4 \mathrm{~Hz}, 1 \mathrm{H}), 7.01(\mathrm{~d}, J=2.8 \mathrm{~Hz}, 1 \mathrm{H}), 3.88(\mathrm{~s}, 3 \mathrm{H}), 2.67$ (s, 3H).

${ }^{13}$ C-NMR $\left(75 \mathrm{MHz}, \mathrm{CDCl}_{3}\right): \delta=157.1\left(\mathrm{C}_{\mathrm{q}}\right), 156.3\left(\mathrm{C}_{\mathrm{q}}\right), 143.9\left(\mathrm{C}_{\mathrm{q}}\right), 135.0(\mathrm{CH}), 130.0(\mathrm{CH})$, $127.3\left(\mathrm{C}_{\mathrm{q}}\right), 122.2(\mathrm{CH}), 121.8(\mathrm{CH}), 105.2(\mathrm{CH}), 55.4\left(\mathrm{CH}_{3}\right), 25.0\left(\mathrm{CH}_{3}\right)$.

IR (ATR): 2937, 1602, 1498, 1229, 1029, $830 \mathrm{~cm}^{-1}$.

\footnotetext{
148 a). Y. Matsubara, S. Hirakawa, Y. Yamaguchi, Z.-i. Yoshida, Angew. Chem. Int. Ed., 2011, 50, 7670-7673; b) V. Sridha ran, C. Avendaño, J. C. Menéndez, Tetrahedron, 2007, 63, 673-681.
} 
MS (EI) m/z (relative intens ity) 173 (100) $\left[\mathrm{M}^{+}\right], 158$ (50), 143 (5), 130 (80), 115 (5), 103 (20).

HR-MS (ESI) m/z calcd for $\mathrm{C}_{11} \mathrm{H}_{12} \mathrm{NO}\left[\mathrm{M}+\mathrm{H}^{+}\right]$174.0919, found 174.0921.

The spectral data were in accordance with those reported in the literature. ${ }^{148}$

\section{2-Methyl-6-phe nylquinoline (122ca)}<smiles>Cc1ccc2cc(-c3ccccc3)ccc2n1</smiles>

The general procedure $\mathbf{F}$ was followed using 121c (106 $\mathrm{mg}, 0.5 \mathrm{mmol})$ and methyl vinyl ketone (41a) (70 mg, $1.0 \mathrm{mmol})$ for $20 \mathrm{~h}$. Purification by column chromatography ( $n$-hexane/EtOAc 5:1) yielded $122 \mathrm{ca}(57 \mathrm{mg}, 52 \%$ ) as an off white solid. M. p. : $=92-93 \mathrm{C}$. ${ }^{1} \mathbf{H}-\mathbf{N M R}\left(300 \mathrm{MHz}, \mathrm{CDCl}_{3}\right): \delta=8.09(\mathrm{dd}, J=8.9,2.1 \mathrm{~Hz}, 2 \mathrm{H}), 7.99-7.91$ (m, 2H), 7.76-7.65 (m, 2H), $7.50(\mathrm{~d}, J=7.5 \mathrm{~Hz}, 1 \mathrm{H}), 7.48(\mathrm{~d}, J=6.6 \mathrm{~Hz}, 1 \mathrm{H}), 7.43-7.35(\mathrm{~m}, 1 \mathrm{H}), 7.31(\mathrm{~d}, J=8.4$ $\mathrm{Hz}, 1 \mathrm{H}), 2.77$ (s, 3H).

${ }^{13}$ C-NMR (75 MHz, $\left.\mathrm{CDCl}_{3}\right): \delta=159.0\left(\mathrm{C}_{\mathrm{q}}\right), 147.3\left(\mathrm{C}_{\mathrm{q}}\right), 140.5\left(\mathrm{C}_{\mathrm{q}}\right), 138.4\left(\mathrm{C}_{\mathrm{q}}\right), 136.3(\mathrm{CH})$, $129.1(\mathrm{CH}), 129.0(\mathrm{CH}), 128.9(\mathrm{CH}), 127.5(\mathrm{CH}), 127.4(\mathrm{CH}), 126.6\left(\mathrm{C}_{\mathrm{q}}\right), 125.2(\mathrm{CH}), 122.4$ (CH), $25.4\left(\mathrm{CH}_{3}\right)$.

IR (ATR): 2998, 1595, 1488, 1314, 892, $764 \mathrm{~cm}^{-1}$.

MS (EI) m/z (relative intensity) 219 (100) [M+], 204 (5), 191 (5), 176 (5), 152 (5).

HR-MS (EI) $\mathrm{m} / \mathrm{z}$ calcd for $\mathrm{C}_{16} \mathrm{H}_{13} \mathrm{~N}\left[\mathrm{M}^{+}\right] 219.1048$, found 219.1049 .

The spectral data were in accordance with those reported in the literature. ${ }^{149}$

\section{6-Fluoro-2-me thylquinoline (122da)}<smiles>Cc1ccc2cc(F)ccc2n1</smiles>

The general procedure $\mathbf{E}$ was followed using $121 \mathrm{~d}$ (77 $\mathrm{mg}, 0.5 \mathrm{mmol})$, methyl vinyl ketone (41 a) (70 mg, $1.0 \mathrm{mmol})$ and $\left[\mathrm{RuCl}_{2}(p \text {-cymene) }]_{2}(15.3 \mathrm{mg}, 5.0 \mathrm{~mol} \%)\right.$ for $20 \mathrm{~h}$. Purification

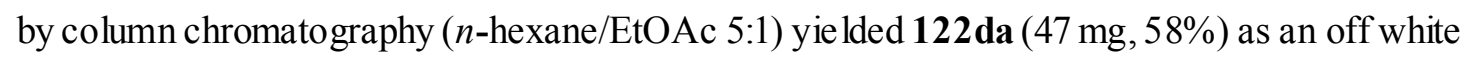
solid. M. p.: $=51-53 \mathbb{C}$.

${ }^{149}$ F. Mongin, L. Mojovic, B. Guillamet, F. Trécourt, G. Quéguiner, J. Org. Chem., 2002, 67, 8991-8994. 
${ }^{1} \mathbf{H}-\mathbf{N M R}\left(300 \mathrm{MHz}, \mathrm{CDCl}_{3}\right): \delta=8.05-7.91(\mathrm{~m}, 2 \mathrm{H}), 7.42(\mathrm{ddd}, J=9.1,8.4,2.8 \mathrm{~Hz}, 1 \mathrm{H}), 7.36$ (dd, $J=8.9,2.8 \mathrm{~Hz}, 1 \mathrm{H}), 7.27(\mathrm{dd}, J=8.4,0.8 \mathrm{~Hz}, 1 \mathrm{H}), 2.71$ (s, 3H).

${ }^{13}$ C-NMR $\left(75 \mathrm{MHz}, \mathrm{CDCl}_{3}\right): \delta=159.9\left(\mathrm{C}_{\mathrm{q}}, J_{\mathrm{C}-\mathrm{F}}=250.1 \mathrm{~Hz}\right), 158.3\left(\mathrm{C}_{\mathrm{q}}\right), 144.9\left(\mathrm{C}_{\mathrm{q}}\right), 135.5(\mathrm{CH}$, $\left.J_{\mathrm{C}-\mathrm{F}}=5.1 \mathrm{~Hz}\right), 131.0\left(\mathrm{CH}, J_{\mathrm{C}-\mathrm{F}}=9.0 \mathrm{~Hz}\right), 126.9\left(\mathrm{C}_{\mathrm{q}}, J_{\mathrm{C}-\mathrm{F}}=10.1 \mathrm{~Hz}\right), 122.7(\mathrm{CH}), 119.4\left(\mathrm{CH}, J_{\mathrm{C}-\mathrm{F}}\right.$ $=25.6 \mathrm{~Hz}), 110.5\left(\mathrm{CH}, J_{\mathrm{C}-\mathrm{F}}=21.8 \mathrm{~Hz}\right), 25.2\left(\mathrm{CH}_{3}\right)$.

${ }^{19}$ F-NMR $\left(\mathrm{CDCl}_{3}, 282 \mathrm{MHz}\right): \delta-115.0(\mathrm{td}, J=8.6,5.3 \mathrm{~Hz})$.

IR (ATR): 3058, 1652, 1439, 1329, 749, $509 \mathrm{~cm}^{-1}$.

MS (EI) m/z (relative intensity) $161(100)\left[\mathrm{M}^{+}\right], 146$ (5), 133 (10).

HR-MS (EI) m/z calcd for $\mathrm{C}_{10} \mathrm{H}_{8} \mathrm{NF}\left[\mathrm{M}^{+}\right]$161.0641, found 161.0638.

The spectral data were in accordance with those reported in the literature. ${ }^{148 a, 150}$

\section{6-Chloro-2-me thylquinoline (122ea)}<smiles>Cc1ccc2cc(Cl)ccc2n1</smiles>

The general procedure $\mathbf{F}$ was followed using 121e ( $85 \mathrm{mg}, 0.5 \mathrm{mmol})$, methyl vinyl ketone (41 a) (70 mg, $1.0 \mathrm{mmol})$ and $\left[\mathrm{RuCl}_{2}(p \text {-cymene })\right]_{2}(15.3 \mathrm{mg}, 5.0 \mathrm{~mol} \%)$ for $20 \mathrm{~h}$. Purification by column chromatography ( $n$-hexane/EtOAc 5:1) yielded $122 \mathrm{e}$ (53 $\mathrm{mg}, 60 \%)$ as an off white solid. M. p.: $=95-97 \mathbb{C}$.

${ }^{1} \mathbf{H}-\mathbf{N M R}\left(300 \mathrm{MHz}, \mathrm{CDCl}_{3}\right): \delta=7.9(\mathrm{~d}, J=8.6 \mathrm{~Hz}, 2 \mathrm{H}), 7.7(\mathrm{~d}, J=2.4 \mathrm{~Hz}, 1 \mathrm{H}), 7.57(\mathrm{dd}, J=$ 9.0, $2.3 \mathrm{~Hz}, 1 \mathrm{H}), 7.26(\mathrm{~d}, J=8.4 \mathrm{~Hz}, 1 \mathrm{H}), 2.70(\mathrm{~s}, 3 \mathrm{H})$.

${ }^{13}$ C-NMR $\left(75 \mathrm{MHz}, \mathrm{CDCl}_{3}\right): \delta=159.3\left(\mathrm{C}_{\mathrm{q}}\right), 146.2\left(\mathrm{C}_{\mathrm{q}}\right), 135.2(\mathrm{CH}), 131.2\left(\mathrm{C}_{\mathrm{q}}\right), 130.2(\mathrm{CH})$, 130.2 (CH), $127.0\left(\mathrm{C}_{\mathrm{q}}\right), 126.1(\mathrm{CH}), 122.8(\mathrm{CH}), 25.3\left(\mathrm{CH}_{3}\right)$.

IR (ATR): 3050, 1597, 1468, 1067, 830, $641 \mathrm{~cm}^{-1}$.

MS (EI) m/z (relative intensity) 177 (100) [M+], $162(10), 142$ (15), $133(15), 115$ (15), 105 (5).

HR-MS (EI) $\mathrm{m} / \mathrm{z}$ calcd for $\mathrm{C}_{10} \mathrm{H}_{8} \mathrm{NCl}\left[\mathrm{M}^{+}\right]$177.0345, found 177.0341.

The spectral data were in accordance with those reported in the literature. ${ }^{148 a, 150}$

\section{6-Bromo-2-methylquinoline (122fa)}

${ }^{150}$ C. Ra mesh, V. Kavala, C.-W. Kuo, C.-F. Yao, Tetrahedron Lett., 2010, 51, 5234-5237. 
<smiles>Cc1ccc2cc(Br)ccc2n1</smiles>

The general procedure $\mathbf{F}$ was followed using $121 \mathrm{f}(107 \mathrm{mg}, 0.5 \mathrm{mmol})$, methyl vinyl ketone (41a) (70 mg, $1.0 \mathrm{mmol})$ and $\left[\mathrm{RuCl}_{2}(p \text {-cymene })\right]_{2}(15.3 \mathrm{mg}, 5.0 \mathrm{~mol} \%)$ for $20 \mathrm{~h}$. Purification by column chromatography ( $n$-hexane/EtOAc 5:1) yielded $122 \mathrm{fa}(56 \mathrm{mg}, 51 \%$ ) as an off white solid. M. p.: $=98-100 \mathbb{C}$.

${ }^{1} \mathbf{H}-\mathbf{N M R}\left(300 \mathrm{MHz}, \mathrm{CDCl}_{3}\right): \delta=7.95-7.90(\mathrm{~m}, 1 \mathrm{H}), 7.89(\mathrm{~d}, J=2.2 \mathrm{~Hz}, 1 \mathrm{H}), 7.87-7.82(\mathrm{~m}$, 1H), $7.71(\mathrm{dd}, J=9.0,2.2 \mathrm{~Hz}, 1 \mathrm{H}), 7.27(\mathrm{~d}, J=8.5 \mathrm{~Hz}, 1 \mathrm{H}), 2.70$ (s, 3H).

${ }^{13}$ C-NMR $\left(75 \mathrm{MHz}, \mathrm{CDCl}_{3}\right): \delta=159.5\left(\mathrm{C}_{\mathrm{q}}\right), 146.4\left(\mathrm{C}_{\mathrm{q}}\right), 135.1(\mathrm{CH}), 132.8(\mathrm{CH}), 130.4(\mathrm{CH})$, $129.5(\mathrm{CH}), 127.6\left(\mathrm{C}_{\mathrm{q}}\right), 122.8(\mathrm{CH}), 119.3\left(\mathrm{C}_{\mathrm{q}}\right), 25.4\left(\mathrm{CH}_{3}\right)$.

IR (ATR): 3048, 1594, 1488, 1300, 1071, $828 \mathrm{~cm}^{-1}$.

MS (EI) m/z (relative intensity) 221 (100) $\left[\mathrm{M}^{+}\right], 205$ (5), 142 (20), 115 (30).

HR-MS (EI) $\mathrm{m} / \mathrm{z}$ calcd for $\mathrm{C}_{10} \mathrm{H}_{8} \mathrm{~N}^{79} \mathrm{Br}\left[\mathrm{M}^{+}\right] 220.9840$, found 220.9838 .

The spectral data were in accordance with those reported in the literature. ${ }^{148 a, 150}$

\section{2-Methyl-6-(trifluoromethyl)quinoline (122ga)}<smiles>Cc1ccc2cc(C(F)(F)F)ccc2n1</smiles>

The general procedure $\mathbf{F}$ was followed using $121 \mathrm{~g}$ (101 $\mathrm{mg}, 0.5 \mathrm{mmol})$, methyl vinyl ketone (41a) (70 mg, $1.0 \mathrm{mmol})$ and $\left[\mathrm{RuCl}_{2}(p \text {-cymene })\right]_{2}(15.3 \mathrm{mg}, 5.0 \mathrm{~mol} \%)$ for $20 \mathrm{~h}$. Purification by column chromatography ( $n$-hexane/EtOAc 5:1) yielded 122ga (30 mg, 28\%) as a yellow oil.

${ }^{1}$ H-NMR (300 MHz, $\left.\mathrm{CDCl}_{3}\right): \delta=8.21-8.04(\mathrm{~m}, 3 \mathrm{H}), 7.83(\mathrm{dd}, J=8.8,2.1 \mathrm{~Hz}, 1 \mathrm{H}), 7.37(\mathrm{~d}, J$ $=8.4 \mathrm{~Hz}, 1 \mathrm{H}), 2.77(\mathrm{~s}, 3 \mathrm{H})$.

${ }^{13}$ C-NMR (125 MHz, $\left.\mathrm{CDCl}_{3}\right): \delta=161.5\left(\mathrm{C}_{\mathrm{q}}\right), 148.9\left(\mathrm{C}_{\mathrm{q}}\right), 136.8(\mathrm{CH}), 129.8(\mathrm{CH}), 127.6\left(\mathrm{C}_{\mathrm{q}}\right.$, $\left.J_{\mathrm{C}-\mathrm{F}}=32.5 \mathrm{~Hz}\right), 124.1\left(\mathrm{C}_{\mathrm{q}}, J_{\mathrm{C}-\mathrm{F}}=273.3 \mathrm{~Hz}\right), 125.5\left(\mathrm{CH}, J_{\mathrm{C}-\mathrm{F}}=4.4 \mathrm{~Hz}\right), 125.4\left(\mathrm{C}_{\mathrm{q}}\right), 125.1(\mathrm{CH}$, $\left.J_{\mathrm{C}-\mathrm{F}}=3.2 \mathrm{~Hz}\right), 123.2(\mathrm{CH}), 25.5\left(\mathrm{CH}_{3}\right)$.

${ }^{19}$ F-NMR (282 MHz, $\left.\mathrm{CDCl}_{3}\right): \delta=-62.2(\mathrm{~s})$.

IR (ATR): 1605, 1484, 1291, 1109, 903, $705 \mathrm{~cm}^{-1}$.

MS (EI) m/z (relative intensity) $211(100)\left[\mathrm{M}^{+}\right], 193$ (5), 168 (5). 
HR-MS (ESI) m/z calcd for $\mathrm{C}_{11} \mathrm{H}_{9} \mathrm{NF}_{3}\left[\mathrm{M}+\mathrm{H}^{+}\right] 212.0687$, found 212.0687.

The spectral data were in accordance with those reported in the literature. ${ }^{151}$

\section{2-Methylquinolin-6-yl ace tate (122 ha)}<smiles>CC(=O)Oc1ccc2nc(C)ccc2c1</smiles>

The general procedure $\mathbf{F}$ was followed using $\mathbf{1 2 1 h}$ (97 mg, $0.5 \mathrm{mmol})$, methyl vinyl ketone (41a) (70 mg, $1.0 \mathrm{mmol})$ and $\left[\mathrm{RuCl}_{2}(p \text {-cymene })\right]_{2}(15.3 \mathrm{mg}, 5.0 \mathrm{~mol} \%)$ for $20 \mathrm{~h}$. Purification by column chromatography ( $n$-hexane/EtOAc 3:1) yie lded $\mathbf{1 2 2} \mathbf{h a}(51 \mathrm{mg}, 51 \%)$ as a yellow oil. ${ }^{1} \mathbf{H}-\mathbf{N M R}\left(300 \mathrm{MHz}, \mathrm{CDCl}_{3}\right): \delta=7.99(\mathrm{~d},=8.8 \mathrm{~Hz}, 1 \mathrm{H}), 7.96(\mathrm{~d},=8.5 \mathrm{~Hz}, 1 \mathrm{H}), 7.48(\mathrm{~d}, J=2.5$ $\mathrm{Hz}, 1 \mathrm{H}), 7.38(\mathrm{dd}, J=9.0,2.6 \mathrm{~Hz}, 1 \mathrm{H}), 7.28-7.22(\mathrm{~m}, 1 \mathrm{H}), 2.71(\mathrm{~s}, 3 \mathrm{H}), 2.32(\mathrm{~s}, 3 \mathrm{H})$.

${ }^{13}$ C-NMR $\left(75 \mathrm{MHz}, \mathrm{CDCl}_{3}\right): \delta=169.4\left(\mathrm{C}_{\mathrm{q}}\right), 158.8\left(\mathrm{C}_{\mathrm{q}}\right), 147.8\left(\mathrm{C}_{\mathrm{q}}\right), 145.8\left(\mathrm{C}_{\mathrm{q}}\right), 135.8(\mathrm{CH})$, $130.1(\mathrm{CH}), 126.6\left(\mathrm{C}_{\mathrm{q}}\right), 124.4(\mathrm{CH}), 122.5(\mathrm{CH}), 118.1(\mathrm{CH}), 25.2\left(\mathrm{CH}_{3}\right), 21.1\left(\mathrm{CH}_{3}\right)$.

IR (ATR): 1713, 1598, 1504, 1429, 1234, $832 \mathrm{~cm}^{-1}$.

MS (EI) m/z (relative intensity) $201(5)\left[\mathrm{M}^{+}\right], 159$ (100), 130 (10), 103 (5).

HR-MS (EI) m/z calcd for $\mathrm{C}_{12} \mathrm{H}_{11} \mathrm{NO}_{2}\left[\mathrm{M}^{+}\right]$201.0790, found 201.0789.

The spectral data were in accordance with those reported in the literature. ${ }^{152}$

\section{2,7-Dimethylquinoline (122ia)}<smiles>Cc1ccc2ccc(C)nc2c1</smiles>

The general procedure $\mathbf{F}$ was followed using $121 \mathbf{i}(75 \mathrm{mg}, 0.5 \mathrm{mmol})$ and methyl vinyl ketone (41a) (70 mg, $1.0 \mathrm{mmol})$ for $20 \mathrm{~h}$. Purification by column chromatography ( $n$-hexane/EtOAc 5:1) yielded 122ia (49 $\mathrm{mg}, 62 \%)$ as a yellow oil.

${ }^{1}$ H-NMR $\left(300 \mathrm{MHz}, \mathrm{CDCl}_{3}\right): \delta=7.96(\mathrm{~d}, J=8.4 \mathrm{~Hz}, 1 \mathrm{H}), 7.78(\mathrm{~d}, J=0.8 \mathrm{~Hz}, 1 \mathrm{H}), 7.63(\mathrm{~d}, J$ $=8.3 \mathrm{~Hz}, 1 \mathrm{H}), 7.29(\mathrm{dd}, J=8.3,1.7 \mathrm{~Hz}, 1 \mathrm{H}), 7.19(\mathrm{~d}, J=8.4 \mathrm{~Hz}, 1 \mathrm{H}), 2.70(\mathrm{~s}, 3 \mathrm{H}), 2.52(\mathrm{~s}$, $3 \mathrm{H})$.

${ }^{13}$ C-NMR $\left(75 \mathrm{MHz}, \mathrm{CDCl}_{3}\right): \delta=158.9\left(\mathrm{C}_{\mathrm{q}}\right), 148.1\left(\mathrm{C}_{\mathrm{q}}\right), 139.6\left(\mathrm{C}_{\mathrm{q}}\right), 135.8(\mathrm{CH}), 127.8(\mathrm{CH})$,

\footnotetext{
${ }^{151}$ K. K. H. Chandrasheka rappa, K. M. Mahadevan, K. B. Ma nja ppa, Tetrahedron Letters 2013, 54, 1368-1370.

152 K. Jyothish, R. R. Avirah, D. Ramaiah, Org. Lett. 2006, 8, 111-114.
} 
127.7 (CH), $127.1(\mathrm{CH}), 124.5\left(\mathrm{C}_{\mathrm{q}}\right), 121.1(\mathrm{CH}), 25.3\left(\mathrm{CH}_{3}\right), 21.9\left(\mathrm{CH}_{3}\right)$.

IR (ATR): 2916, 1601, 1505, 1305, 835, $776 \mathrm{~cm}^{-1}$.

MS (EI) m/z (relative intensity) 157 (100) $\left[\mathrm{M}^{+}\right], 142$ (20), 128 (5), 115 (15), 89 (5).

HR-MS (EI) m/z calcd for $\mathrm{C}_{11} \mathrm{H}_{11} \mathrm{~N}\left[\mathrm{M}^{+}\right]$157.0891, found 157.0889.

The spectral data were in accordance with those reported in the literature. ${ }^{148 a}$

\section{2,8-Dimethylquinoline (122 ja)}<smiles>Cc1ccc2cccc(C)c2n1</smiles>

The general procedure $\mathbf{E}$ was followed using $\mathbf{1 2 1} \mathbf{j}(75 \mathrm{mg}, 0.5 \mathrm{mmol}$ ) and methyl vinyl ketone (41a) (70 mg, $1.0 \mathrm{mmol})$ for $20 \mathrm{~h}$. Purification by column chromatography ( $n$-hexane/EtOAc 5:1) yielded $122 \mathbf{j a}(27 \mathrm{mg}, 34 \%)$ as a yellow oil.

${ }^{1} \mathbf{H}-\mathbf{N M R}\left(300 \mathrm{MHz}, \mathrm{CDCl}_{3}\right): \delta=7.98(\mathrm{~d}, J=8.4 \mathrm{~Hz}, 1 \mathrm{H}), 7.59(\mathrm{~d}, J=8.4,1 \mathrm{H}), 7.51(\mathrm{ddd}, J$ $=7.0,1.6,0.9 \mathrm{~Hz}, 1 \mathrm{H}), 7.34(\mathrm{dd}, J=8.0,7.0 \mathrm{~Hz}, 1 \mathrm{H}), 7.25(\mathrm{~d}, J=8.4 \mathrm{~Hz}, 1 \mathrm{H}), 2.80(\mathrm{~s}, 3 \mathrm{H})$, $2.75(\mathrm{~s}, 3 \mathrm{H})$.

${ }^{13}$ C-NMR (75 MHz, $\left.\mathrm{CDCl}_{3}\right): \delta=157.8\left(\mathrm{C}_{\mathrm{q}}\right), 146.9\left(\mathrm{C}_{\mathrm{q}}\right), 136.5\left(\mathrm{C}_{\mathrm{q}}\right), 136.2(\mathrm{CH}), 129.4(\mathrm{CH})$, $126.3\left(\mathrm{C}_{\mathrm{q}}\right), 125.4(\mathrm{CH}), 125.2(\mathrm{CH}), 121.6(\mathrm{CH}), 25.6\left(\mathrm{CH}_{3}\right), 17.9\left(\mathrm{CH}_{3}\right)$.

IR (ATR): 2919, 1604, 1500, 1424, 829, $758 \mathrm{~cm}^{-1}$.

MS (EI) m/z (relative intensity) 157 (100) $\left[\mathrm{M}^{+}\right], 142$ (20), 128 (5), 115 (15), 89 (5).

HR-MS (EI) m/z calcd for $\mathrm{C}_{11} \mathrm{H}_{11} \mathrm{~N}\left[\mathrm{M}^{+}\right]$157.0891, found 157.0883.

The spectral data were in accordance with those reported in the literature. ${ }^{149}$

\section{8-Fluoro-2-me thylquinoline (122ka)}<smiles>Cc1ccc2cccc(F)c2n1</smiles>

The general procedure $\mathbf{F}$ was followed using $121 \mathrm{k}$ (77 $\mathrm{mg}, 0.5 \mathrm{mmol})$, methyl vinyl ketone (41 a) (70 mg, $1.0 \mathrm{mmol})$ and $\left[\mathrm{RuCl}_{2}(p \text {-cymene })\right]_{2}(15.3 \mathrm{mg}, 5.0 \mathrm{~mol} \%)$ for $20 \mathrm{~h}$. Purification by column chromatography ( $n$-hexane/EtOAc 5:1) yie lded 122ka (24 mg, 30\%) as a yellow oil. 
${ }^{1} \mathbf{H}-\mathbf{N M R}\left(300 \mathrm{MHz}, \mathrm{CDCl}_{3}\right): \delta=8.03(\mathrm{dd}, J=8.5,1.6 \mathrm{~Hz}, 1 \mathrm{H}), 7.58-7.48(\mathrm{~m}, 1 \mathrm{H}), 7.44-7.34$ (m, 2H), $7.32(\mathrm{~d}, J=8.6 \mathrm{~Hz}, 1 \mathrm{H}), 2.77(\mathrm{~s}, 3 \mathrm{H})$.

${ }^{13}$ C-NMR (75 MHz, $\left.\mathrm{CDCl}_{3}\right): \delta=159.5\left(\mathrm{C}_{\mathrm{q}}, J_{\mathrm{C}-\mathrm{F}}=1.6 \mathrm{~Hz}\right), 157.6\left(\mathrm{C}_{\mathrm{q}}, J_{\mathrm{C}-\mathrm{F}}=255.3 \mathrm{~Hz}\right), 138.0$ $\left(\mathrm{C}_{\mathrm{q}}, J_{\mathrm{C}-\mathrm{F}}=11.2 \mathrm{~Hz}\right), 135.8\left(\mathrm{CH}, J_{\mathrm{C}-\mathrm{F}}=3.2 \mathrm{~Hz}\right), 128.1\left(\mathrm{C}_{\mathrm{q}}, J_{\mathrm{C}-\mathrm{F}}=2.7 \mathrm{~Hz}\right), 125.3\left(\mathrm{CH}, J_{\mathrm{C}-\mathrm{F}}=8.0\right.$ $\mathrm{Hz}), 123.1\left(\mathrm{CH}, J_{\mathrm{C}-\mathrm{F}}=4.8 \mathrm{~Hz}\right), 123.0(\mathrm{CH}), 113.5\left(\mathrm{CH}, J_{\mathrm{C}-\mathrm{F}}=19.2 \mathrm{~Hz}\right), 25.5\left(\mathrm{CH}_{3}\right)$.

${ }^{19}$ F-NMR $\left(282 \mathrm{MHz}, \mathrm{CDCl}_{3}\right): \delta=-126.4(\mathrm{ddd}, J=9.9,5.3,1.6 \mathrm{~Hz})$.

IR (ATR): 3057, 1606, 1503, 1234, 1081, $831 \mathrm{~cm}^{-1}$.

MS (EI) m/z (relative intensity) $161(100)\left[\mathrm{M}^{+}\right], 146(10), 132$ (5).

HR-MS (EI) m/z calcd for $\mathrm{C}_{10} \mathrm{H}_{8} \mathrm{NF}\left[\mathrm{M}^{+}\right]$161.0641, found 161.0645.

The spectral data were in accordance with those reported in the literature. ${ }^{151}$

\section{6-Methoxy-2-phenylquinoline (122bb)}<smiles>COc1ccc2nc(-c3ccccc3)ccc2c1</smiles>

The general procedure $\mathbf{F}$ was followed using $\mathbf{1 2 1} \mathbf{b}(83 \mathrm{mg}, 0.5 \mathrm{mmol}), \mathbf{4 1} \mathbf{b}(132 \mathrm{mg}, 1.0 \mathrm{mmol})$ and $\left[\mathrm{RuCl}_{2}(p \text {-cymene })\right]_{2}(15.3 \mathrm{mg}, 5.0 \mathrm{~mol} \%)$ for $20 \mathrm{~h}$. Purification by column chromatography ( $n$-hexane/EtOAc 8:1) yie lded 122 bb (33 mg, 28\%) as a colorless solid. M. p. : $=129-131^{\circ} \mathrm{C}$.

${ }^{1} \mathbf{H}-\mathbf{N M R}\left(300 \mathrm{MHz}, \mathrm{CDCl}_{3}\right): \delta=8.15-8.00(\mathrm{~m}, 4 \mathrm{H}), 7.81(\mathrm{~d}, J=8.6 \mathrm{~Hz}, 1 \mathrm{H}), 7.62-7.46(\mathrm{~m}$, 2H), 7.46-7.41 (m, 1H), 7.37 (dd, $J=9.2,2.8 \mathrm{~Hz}, 1 \mathrm{H}), 7.07$ (d, $J=2.8 \mathrm{~Hz}, 1 \mathrm{H}), 3.93$ (s, 3H). ${ }^{13}$ C-NMR $\left(75 \mathrm{MHz}, \mathrm{CDCl}_{3}\right): \delta=157.6\left(\mathrm{C}_{\mathrm{q}}\right), 155.0\left(\mathrm{C}_{\mathrm{q}}\right), 144.3\left(\mathrm{C}_{\mathrm{q}}\right), 139.8\left(\mathrm{C}_{\mathrm{q}}\right), 135.5(\mathrm{CH})$, $131.2(\mathrm{CH}), 128.9(\mathrm{CH}), 128.8(\mathrm{CH}), 128.1\left(\mathrm{C}_{\mathrm{q}}\right), 127.2(\mathrm{CH}), 122.3(\mathrm{CH}), 119.2(\mathrm{CH}), 105.0$ (CH), $55.5\left(\mathrm{CH}_{3}\right)$.

IR (ATR): 1596, 1490, 1227, 1020, 831, $699 \mathrm{~cm}^{-1}$.

MS (EI) m/z (relative intensity) 235 (100) $\left[\mathrm{M}^{+}\right], 220$ (35), 192 (60), 165 (5).

HR-MS (EI) m/z calcd for $\mathrm{C}_{16} \mathrm{H}_{13} \mathrm{NO}\left[\mathrm{M}^{+}\right]$235.0997, found 235.1000.

The spectral data were in accordance with those reported in the literature. ${ }^{153}$

153 K. A. Reynolds, D. J. Young, W. A. Loughlin, Synthesis 2010, 3645-3648. 


\subsubsection{Analytically Data for the Products of Cobalt-Catalyzed Direct} Arylation of Aromatic Amides

$N, 4$ '-Dime thylbiphe nyl-2-carboxamide (139aa)<smiles>CNC(=O)c1ccccc1-c1ccc(C)cc1</smiles>

The general procedure $\mathbf{G}$ was followed using $\mathbf{2 a}(68 \mathrm{mg}, 0.5 \mathrm{mmol})$ and 4-chlorotoluene (88a) (76 mg, $0.6 \mathrm{mmol}$ ) for $16 \mathrm{~h}$ (or $0.5 \mathrm{~h}$ ). Purification by column chromatography ( $n$-hexane/EtOAc 10:1 $\rightarrow 2: 1)$ yielded 139aa (88 mg, 78\%) and 139aa' (14 mg, 9\%) as color less solids, (or $81 \mathrm{mg}, 72 \%$ yield of 139 aa as the sole product). 139aa: M. p.: $=108-110{ }^{\circ} \mathrm{C}$.

${ }^{1}$ H-NMR $\left(300 \mathrm{MHz}, \mathrm{CDCl}_{3}\right): \delta=7.67$ (ddd, $\left.J=7.5,1.6,0.6 \mathrm{~Hz}, 1 \mathrm{H}\right), 7.49-7.40(\mathrm{~m}, 1 \mathrm{H})$, $7.40-7.35(\mathrm{~m}, 1 \mathrm{H}), 7.33(\mathrm{ddd}, J=7.2,1.6,0.6 \mathrm{~Hz}, 1 \mathrm{H}), 7.28(\mathrm{~d}, J=8.1 \mathrm{~Hz}, 2 \mathrm{H}), 7.20(\mathrm{~d}, J=8.1$ $\mathrm{Hz}, 2 \mathrm{H}), 5.20(\mathrm{~s}, 1 \mathrm{H}), 2.67(\mathrm{~d}, J=5.0 \mathrm{~Hz}, 3 \mathrm{H}), 2.37$ (s, 3H).

${ }^{13}$ C-NMR (75 MHz, $\left.\mathrm{CDCl}_{3}\right): \delta=170.3\left(\mathrm{C}_{\mathrm{q}}\right), 139.3\left(\mathrm{C}_{\mathrm{q}}\right), 137.5\left(\mathrm{C}_{\mathrm{q}}\right), 137.1\left(\mathrm{C}_{\mathrm{q}}\right), 135.5\left(\mathrm{C}_{\mathrm{q}}\right)$, $130.1(\mathrm{CH}), 130.1(\mathrm{CH}), 129.3(\mathrm{CH}), 128.8(\mathrm{CH}), 128.5(\mathrm{CH}), 127.3(\mathrm{CH}), 26.7\left(\mathrm{CH}_{3}\right), 21.2$ $\left(\mathrm{CH}_{3}\right)$.

IR (ATR): 3252, 2921, 1625, 1564, 1020, $801 \mathrm{~cm}^{-1}$.

MS (EI) m/z (relative intensity) 225 (40) $\left[\mathrm{M}^{+}\right], 208$ (15), 195 (100), 165 (50), 152 (45), 115 (5).

HR-MS (EI) $\mathrm{m} / \mathrm{z}$ calcd for $\mathrm{C}_{15} \mathrm{H}_{15} \mathrm{NO}\left[\mathrm{M}^{+}\right] 225.1154$, found 225.1160 .

\section{$N, 4,4^{\prime \prime}$-Trimethyl-[1,1':3',1'--te rphe nyl]-2'-carboxamide (139aa')}<smiles>CNC(=O)c1c(-c2ccc(C)cc2)cccc1-c1ccc(C)cc1</smiles>

M. p.: $=223-225^{\circ} \mathrm{C}$.

${ }^{1}$ H-NMR $\left(400 \mathrm{MHz}, \mathrm{CDCl}_{3}\right): \delta=7.43(\mathrm{dd}, J=8.3,7.0 \mathrm{~Hz}, 1 \mathrm{H}), 7.34(\mathrm{~d}, J=8.1 \mathrm{~Hz}, 4 \mathrm{H}), 7.31$ $(\mathrm{dd}, J=7.7,0.6 \mathrm{~Hz}, 2 \mathrm{H}), 7.20-7.15(\mathrm{~m}, 4 \mathrm{H}), 5.21(\mathrm{~s}, 1 \mathrm{H}), 2.49(\mathrm{~d}, J=5.0 \mathrm{~Hz}, 3 \mathrm{H}), 2.36(\mathrm{~s}, 6 \mathrm{H})$. ${ }^{13}$ C-NMR (125 MHz, $\left.\mathrm{CDCl}_{3}\right): \delta=169.9\left(\mathrm{C}_{\mathrm{q}}\right), 140.0\left(\mathrm{C}_{\mathrm{q}}\right), 137.5\left(\mathrm{C}_{\mathrm{q}}\right), 137.0\left(\mathrm{C}_{\mathrm{q}}\right), 135.6\left(\mathrm{C}_{\mathrm{q}}\right)$, $128.9(\mathrm{CH}), 128.9(\mathrm{CH}), 128.9(\mathrm{CH}), 128.3(\mathrm{CH}), 26.6\left(\mathrm{CH}_{3}\right), 21.3\left(\mathrm{CH}_{3}\right)$. 
IR (ATR): 3253, 2921, 1625, 1564, 1020, $801 \mathrm{~cm}^{-1}$.

MS (EI) m/z (relative intensity) 315 (40) [M $\left.\mathrm{M}^{+}\right], 298$ (5), 285 (100), 267 (10), 242 (20), 226 (10).

HR-MS (EI) m/z calcd for $\mathrm{C}_{22} \mathrm{H}_{21} \mathrm{NO}\left[\mathrm{M}^{+}\right]$315.1623, found 315.1615 .

\section{$N, 3$ '-Dime thylbiphe nyl-2-carboxamide (139ab)}<smiles>CNC(=O)c1ccccc1-c1cccc(C)c1</smiles>

The general procedure $\mathbf{G}$ was followed using $\mathbf{2 a}(68 \mathrm{mg}, 0.5 \mathrm{mmol})$ and 3-chlorotoluene $(\mathbf{8 8 b})$ (76 mg, $0.6 \mathrm{mmol}$ ) for $16 \mathrm{~h}$. Purification by column chromatography ( $n$-hexane/EtOAc $10: 1 \rightarrow 2: 1)$ yielded 139 ab $(73 \mathrm{mg}, 65 \%)$ as a colorless solid. M. p.: $=144-146{ }^{\circ} \mathrm{C}$.

${ }^{1} \mathbf{H}-\mathbf{N M R}\left(300 \mathrm{MHz}, \mathrm{CDCl}_{3}\right): \delta=7.67(\mathrm{dd}, J=7.4,1.5 \mathrm{~Hz}, 1 \mathrm{H}), 7.46-7.31(\mathrm{~m}, 3 \mathrm{H}), 7.27(\mathrm{~d}, J=$ $7.4 \mathrm{~Hz}, 1 \mathrm{H}), 7.20-7.14(\mathrm{~m}, 3 \mathrm{H}), 5.20(\mathrm{~s}, 1 \mathrm{H}), 2.66$ (d, $J=5.1 \mathrm{~Hz}, 3 \mathrm{H}), 2.37$ (s, 3H).

${ }^{13}$ C-NMR (125 MHz, $\left.\mathrm{CDCl}_{3}\right): \delta=170.1\left(\mathrm{C}_{\mathrm{q}}\right), 140.0\left(\mathrm{C}_{\mathrm{q}}\right), 139.3\left(\mathrm{C}_{\mathrm{q}}\right), 138.2\left(\mathrm{C}_{\mathrm{q}}\right), 135.5\left(\mathrm{C}_{\mathrm{q}}\right)$, $130.0(\mathrm{CH}), 129.9(\mathrm{CH}), 129.2(\mathrm{CH}), 128.8(\mathrm{CH}), 128.4(\mathrm{CH}), 128.4(\mathrm{CH}), 127.4(\mathrm{CH}), 125.6$ (CH), $26.7\left(\mathrm{CH}_{3}\right), 21.5\left(\mathrm{CH}_{3}\right)$.

IR (ATR): 3284, 1632, 1313, 757, 700, $449 \mathrm{~cm}^{-1}$.

MS (EI) m/z (relative intensity) 225 (35) $\left[\mathrm{M}^{+}\right], 208$ (10), 195 (100), 165 (50), 152 (45), 115 (5).

HR-MS (EI) m/z calcd for $\mathrm{C}_{15} \mathrm{H}_{15} \mathrm{NO}\left[\mathrm{M}^{+}\right]$225.1154, found 225.1157.

\section{$N, 2$ '-Dime thylbiphe nyl-2-carboxamide (139ac)}<smiles>CNC(=O)c1ccccc1-c1ccccc1C</smiles>

The general procedure $\mathbf{G}$ was followed using $\mathbf{2 a}(68 \mathrm{mg}, 0.5 \mathrm{mmol})$ and 2-chlorotoluene $(\mathbf{8 8 c})$ (76 mg, $0.6 \mathrm{mmol}$ ) for $16 \mathrm{~h}$. Purification by column chromatography ( $n$-hexane/EtOAc $10: 1 \rightarrow 2: 1)$ yielded 139 ac $(62 \mathrm{mg}, 55 \%)$ as a colorless solid. M. p.: $=95-96{ }^{\circ} \mathrm{C}$.

${ }^{1} \mathbf{H}-\mathbf{N M R}\left(300 \mathrm{MHz}, \mathrm{CDCl}_{3}\right): \delta=7.92-7.88(\mathrm{~m}, 1 \mathrm{H}), 7.52-7.37(\mathrm{~m}, 2 \mathrm{H}), 7.32-7.22(\mathrm{~m}, 3 \mathrm{H})$, $7.20-7.12(\mathrm{~m}, 2 \mathrm{H}), 5.22(\mathrm{~s}, 1 \mathrm{H}), 2.60(\mathrm{~d}, J=4.9 \mathrm{~Hz}, 3 \mathrm{H}), 2.09$ (s, 3H). 
${ }^{13}$ C-NMR (125 MHz, $\left.\mathrm{CDCl}_{3}\right): \delta=168.7\left(\mathrm{C}_{\mathrm{q}}\right), 140.1\left(\mathrm{C}_{\mathrm{q}}\right), 139.0\left(\mathrm{C}_{\mathrm{q}}\right), 136.0\left(\mathrm{C}_{\mathrm{q}}\right), 134.7\left(\mathrm{C}_{\mathrm{q}}\right)$, $130.3(\mathrm{CH}), 130.2(\mathrm{CH}), 130.2(\mathrm{CH}), 129.2(\mathrm{CH}), 129.0(\mathrm{CH}), 128.1(\mathrm{CH}), 127.6(\mathrm{CH}), 126.1$ (CH), $26.7\left(\mathrm{CH}_{3}\right), 20.1\left(\mathrm{CH}_{3}\right)$.

IR (ATR): 3318, 1629, 1548, 1310, 755, $467 \mathrm{~cm}^{-1}$.

MS (EI) m/z (relative intensity) 225 (15) $\left[\mathrm{M}^{+}\right], 210$ (20), 195 (100), 165 (50), 152 (25), 139 (5).

HR-MS (EI) m/z calcd for $\mathrm{C}_{15} \mathrm{H}_{15} \mathrm{NO}\left[\mathrm{M}^{+}\right] 225.1154$, found 225.1152 .

\section{$4^{\prime}$-\{[(tert-B utyldime thyls ilyl)oxy]methyl $\}-N$-methyl-[1,1'-biphe nyl]-2-carboxamide}

(139ad)<smiles>CNC(=O)c1ccccc1-c1ccc(CO[Sb](C)(=O)=O)cc1</smiles>

The general procedure $\mathbf{G}$ was followed using 2a (68 $\mathrm{mg}, 0.5 \mathrm{mmol}$ ) and tert-butyl\{(4-chlorobenzyl)oxy\} dimethylsilane $\quad(\mathbf{8 8 d})(154 \mathrm{mg}, 0.6 \mathrm{mmol})$ for $0.5 \mathrm{~h}$. Purification by column chromatography ( $n$-hexane/EtOAc 10:1 $\rightarrow 2: 1)$ yielded 139ad $(102 \mathrm{mg}$, 57\%) as a colorless solid. M. p.: $=91-93^{\circ} \mathrm{C}$.

${ }^{1} \mathbf{H}-\mathbf{N M R}\left(300 \mathrm{MHz}, \mathrm{CDCl}_{3}\right): \delta=7.68(\mathrm{ddd}, J=7.6,1.5,0.5 \mathrm{~Hz}, 1 \mathrm{H}), 7.44(\mathrm{td}, J=7.5,1.5 \mathrm{~Hz}$, 1H), 7.38 (dd, $J=7.5,1.5 \mathrm{~Hz}, 1 \mathrm{H}), 7.36$ (s, 4H), $7.34(\mathrm{ddd}, J=7.6,1.5,0.5 \mathrm{~Hz}, 1 \mathrm{H}), 5.16(\mathrm{~d}, J$ $=5.0 \mathrm{~Hz}, 1 \mathrm{H}), 4.77(\mathrm{~s}, 2 \mathrm{H}), 2.65(\mathrm{~d}, J=5.0 \mathrm{~Hz}, 3 \mathrm{H}), 0.93(\mathrm{~s}, 9 \mathrm{H}), 0.09(\mathrm{~s}, 6 \mathrm{H})$.

${ }^{13}$ C-NMR (125 MHz, $\left.\mathrm{CDCl}_{3}\right): \delta=170.3\left(\mathrm{C}_{\mathrm{q}}\right), 141.1\left(\mathrm{C}_{\mathrm{q}}\right), 139.2\left(\mathrm{C}_{\mathrm{q}}\right), 138.7\left(\mathrm{C}_{\mathrm{q}}\right), 135.6\left(\mathrm{C}_{\mathrm{q}}\right)$, $130.1(\mathrm{CH}), 130.1(\mathrm{CH}), 128.9(\mathrm{CH}), 128.5(\mathrm{CH}), 127.5(\mathrm{CH}), 126.2(\mathrm{CH}), 64.7\left(\mathrm{CH}_{2}\right), 26.7$ $\left(\mathrm{CH}_{3}\right), 25.9\left(\mathrm{CH}_{3}\right), 18.4\left(\mathrm{C}_{\mathrm{q}}\right),-5.2\left(\mathrm{CH}_{3}\right)$.

IR (ATR): 3304, 2929, 1630, 1252, 834, $759 \mathrm{~cm}^{-1}$.

MS (EI) m/z (relative intens ity) 355 (5) [M+ $], 340$ (5), 298 (100), 224 (35), 195 (10), 165 (20).

HR-MS (ESI) $\mathrm{m} / \mathrm{z}$ calcd for $\mathrm{C}_{21} \mathrm{H}_{30} \mathrm{NO}_{2} \mathrm{Si}\left[\mathrm{M}+\mathrm{H}^{+}\right]$356.2046, found 356.2040. 
<smiles>CNC(=O)c1ccc(OC)cc1-c1ccc(C)cc1</smiles>

The general procedure $\mathbf{G}$ was followed using $\mathbf{2 c}(83 \mathrm{mg}, 0.5 \mathrm{mmol})$ and 4-chlorotoluene (88a) (76 mg, $0.6 \mathrm{mmol}$ ) for $16 \mathrm{~h}$. Purification by column chromatography ( $n$-hexane/EtOAc $10: 1 \rightarrow 3: 2)$ yielded $139 \mathrm{ca}(72 \mathrm{mg}, 56 \%)$ as a colorless solid. M. p.: $=128-129^{\circ} \mathrm{C}$.

${ }^{1} \mathbf{H}-\mathbf{N M R}\left(300 \mathrm{MHz}, \mathrm{CDCl}_{3}\right): \delta=7.68(\mathrm{~d}, J=8.6 \mathrm{~Hz}, 1 \mathrm{H}), 7.27(\mathrm{~d}, J=8.0 \mathrm{~Hz}, 2 \mathrm{H}), 7.20(\mathrm{~d}, J=$ $8.0 \mathrm{~Hz}, 2 \mathrm{H}), 6.88(\mathrm{dd}, J=8.6,2.6 \mathrm{~Hz}, 1 \mathrm{H}), 6.80(\mathrm{~d}, J=2.6 \mathrm{~Hz}, 1 \mathrm{H}), 5.15(\mathrm{~s}, 1 \mathrm{H}), 3.82(\mathrm{~s}, 3 \mathrm{H})$, $2.64(\mathrm{~d}, J=4.9 \mathrm{~Hz}, 3 \mathrm{H}), 2.37$ (s, 3H).

${ }^{13}$ C-NMR (125 MHz, $\left.\mathrm{CDCl}_{3}\right): \delta=169.6\left(\mathrm{C}_{\mathrm{q}}\right), 160.5\left(\mathrm{C}_{\mathrm{q}}\right), 141.1\left(\mathrm{C}_{\mathrm{q}}\right), 137.6\left(\mathrm{C}_{\mathrm{q}}\right), 137.2\left(\mathrm{C}_{\mathrm{q}}\right)$, $130.8(\mathrm{CH}), 129.2(\mathrm{CH}), 128.4(\mathrm{CH}), 127.8\left(\mathrm{C}_{\mathrm{q}}\right), 115.3(\mathrm{CH}), 112.7(\mathrm{CH}), 55.4\left(\mathrm{CH}_{3}\right), 26.7$ $\left(\mathrm{CH}_{3}\right), 21.2\left(\mathrm{CH}_{3}\right)$.

IR (ATR): 3304, 1633, 1536, 1287, 1179, $824 \mathrm{~cm}^{-1}$.

MS (EI) m/z (relative intens ity) 255 (35) [M+ $], 238$ (5), 225 (100), 210 (5), 182 (15), 165 (10).

HR-MS (EI) m/z calcd for $\mathrm{C}_{16} \mathrm{H}_{17} \mathrm{NO}_{2}\left[\mathrm{M}^{+}\right]$255.1259, found 255.1263 .

\section{N,4',5-Trimethylbiphe nyl-2-carboxamide (139ba)}<smiles>CNC(=O)c1ccc(C)cc1-c1ccc(C)cc1</smiles>

The general procedure $\mathbf{G}$ was followed using $\mathbf{2 b}(75 \mathrm{mg}, 0.5 \mathrm{mmol})$ and 4-chlorotoluene (88a) (76 mg, $0.6 \mathrm{mmol}$ ) for $0.5 \mathrm{~h}$. Purification by column chromatography ( $n$-hexane/EtOAc $10: 1 \rightarrow 2: 1)$ yielded $\mathbf{1 3 9 b a}\left(78 \mathrm{mg}, 65 \%\right.$ ) as a colorless solid. M. p.: $=137-139{ }^{\circ} \mathrm{C}$.

${ }^{1} \mathbf{H}-\mathbf{N M R}\left(300 \mathrm{MHz}, \mathrm{CDCl}_{3}\right): \delta=7.59(\mathrm{~d}, J=7.8 \mathrm{~Hz}, 1 \mathrm{H}), 7.27(\mathrm{~d}, J=8.1 \mathrm{~Hz}, 2 \mathrm{H}), 7.21-7.10$ (m, 4H), $5.16(\mathrm{~d}, J=5.0 \mathrm{~Hz}, 1 \mathrm{H}), 2.66(\mathrm{~d}, J=4.9 \mathrm{~Hz}, 3 \mathrm{H}), 2.38(\mathrm{~s}, 3 \mathrm{H}), 2.37$ (s, 3H).

${ }^{13}$ C-NMR $\left(75 \mathrm{MHz}, \mathrm{CDCl}_{3}\right): \delta=170.3\left(\mathrm{C}_{\mathrm{q}}\right), 140.2\left(\mathrm{C}_{\mathrm{q}}\right), 139.3\left(\mathrm{C}_{\mathrm{q}}\right), 137.4\left(\mathrm{C}_{\mathrm{q}}\right), 137.3\left(\mathrm{C}_{\mathrm{q}}\right)$, $132.6\left(\mathrm{C}_{\mathrm{q}}\right), 130.8(\mathrm{CH}), 129.2(\mathrm{CH}), 129.0(\mathrm{CH}), 128.5(\mathrm{CH}), 128.1(\mathrm{CH}), 26.7\left(\mathrm{CH}_{3}\right), 21.3$ $\left(\mathrm{CH}_{3}\right), 21.2\left(\mathrm{CH}_{3}\right)$. 
IR (ATR): 3243, 1627, 1563, 1322, 841, $519 \mathrm{~cm}^{-1}$.

MS (EI) m/z (relative intensity) 239 (40) $\left[\mathrm{M}^{+}\right], 222$ (10), 209 (100), 181 (15), 165 (45), 152 (5).

HR-MS (EI) m/z calcd for $\mathrm{C}_{16} \mathrm{H}_{17} \mathrm{NO}\left[\mathrm{M}^{+}\right] 239.1310$, found 239.1302 .

\section{$N, 4^{\prime \prime}$-Dime thyl-[1,1':3',1"-terphe nyl]-4'-carboxamide (139da)}<smiles>CNC(=O)c1ccc(-c2ccccc2)cc1-c1ccc(C)cc1</smiles>

The general procedure $\mathbf{G}$ was followed using $\mathbf{2 d}$ (106 $\mathrm{mg}, 0.5 \mathrm{mmol})$, 4-chlorotoluene (88a) (76 mg, $0.6 \mathrm{mmol}), \mathrm{Co}(\mathrm{acac})_{2}(12.9 \mathrm{mg}, 10 \mathrm{~mol} \%)$ and $\mathrm{ICyHCl}(13.5 \mathrm{mg}, 10 \mathrm{~mol} \%)$ for $0.5 \mathrm{~h}$. Purification by column chromatography ( $n$-hexane/EtOAc 10:1 $\rightarrow 3: 2)$ yielded 139da (102 mg, $68 \%$ ) as a colorless solid. M. p.: $=170-172{ }^{\circ} \mathrm{C}$.

${ }^{1}$ H-NMR $\left(500 \mathrm{MHz}, \mathrm{CDCl}_{3}\right): \delta=7.76(\mathrm{~d}, J=8.0 \mathrm{~Hz}, 1 \mathrm{H}), 7.64-7.57(\mathrm{~m}, 3 \mathrm{H}), 7.55(\mathrm{~d}, J=1.8$ $\mathrm{Hz}, 1 \mathrm{H}), 7.43$ (ddd, $J=7.7,6.7,1.2 \mathrm{~Hz}, 2 \mathrm{H}), 7.39-7.35$ (m, 1H), 7.34 (d, $J=8.0 \mathrm{~Hz}, 2 \mathrm{H})$, 7.26-7.21 (m, 2H), 5.28 (d, $J=4.9 \mathrm{~Hz}, 1 \mathrm{H}), 2.70(\mathrm{~d}, J=4.9 \mathrm{~Hz}, 3 \mathrm{H}), 2.39$ (s, 3H).

${ }^{13}$ C-NMR $\left(125 \mathrm{MHz}, \mathrm{CDCl}_{3}\right): \delta=169.9\left(\mathrm{C}_{\mathrm{q}}\right), 142.8\left(\mathrm{C}_{\mathrm{q}}\right), 140.0\left(\mathrm{C}_{\mathrm{q}}\right), 139.7\left(\mathrm{C}_{\mathrm{q}}\right), 137.6\left(\mathrm{C}_{\mathrm{q}}\right)$, $137.1\left(\mathrm{C}_{\mathrm{q}}\right), 134.1\left(\mathrm{C}_{\mathrm{q}}\right), 129.4(\mathrm{CH}), 129.3(\mathrm{CH}), 128.8(\mathrm{CH}), 128.8(\mathrm{CH}), 128.5(\mathrm{CH}), 127.8$ $(\mathrm{CH}), 127.1(\mathrm{CH}), 125.9(\mathrm{CH}), 26.7\left(\mathrm{CH}_{3}\right), 21.3\left(\mathrm{CH}_{3}\right)$.

IR (ATR): 3301, 1639, 1530, 1304, 757, $719 \mathrm{~cm}^{-1}$.

MS (EI) m/z (relative intensity) 301 (40) $\left[\mathrm{M}^{+}\right], 284$ (10), 271 (100), 241 (10), 228 (30), 165 (10).

HR-MS (EI) m/z calcd for $\mathrm{C}_{21} \mathrm{H}_{19} \mathrm{NO}\left[\mathrm{M}^{+}\right]$301.1467, found 301.1460.

\section{5-Fluoro- $N, 4$ '-dime thylbiphe nyl-2-carboxamide (139sa)}<smiles>CNC(=O)c1ccc(F)cc1-c1ccc(C)cc1</smiles> 
The general procedure $\mathbf{G}$ was followed using $\mathbf{2 s}(77 \mathrm{mg}, 0.5 \mathrm{mmol})$, 4-chlorotoluene (88a) (76 mg, $0.6 \mathrm{mmol}), \mathrm{Co}(\mathrm{acac})_{2}(12.9 \mathrm{mg}, 10 \mathrm{~mol} \%)$ and $\mathrm{ICyHCl}(13.5 \mathrm{mg}, 10 \mathrm{~mol} \%)$ for $0.5 \mathrm{~h}$. Purification by column chromatography ( $n$-hexane/EtOAc $3: 1 \rightarrow 1: 1)$ yielded 139sa $(64 \mathrm{mg}$, $53 \%$ ) as a colorless solid. M. p.: $=144-145^{\circ} \mathrm{C}$.

${ }^{1} \mathbf{H}-\mathbf{N M R}\left(300 \mathrm{MHz}, \mathrm{CDCl}_{3}\right): \delta=7.70(\mathrm{dd}, J=8.4,6.0 \mathrm{~Hz}, 1 \mathrm{H}), 7.29(\mathrm{~d}, J=8.1 \mathrm{~Hz}, 2 \mathrm{H}), 7.23$ (d, $J=8.1 \mathrm{~Hz}, 2 \mathrm{H}), 7.11-7.02(\mathrm{~m}, 2 \mathrm{H}), 5.17(\mathrm{~s}, 1 \mathrm{H}), 2.68(\mathrm{~d}, J=4.8 \mathrm{~Hz}, 3 \mathrm{H}), 2.40(\mathrm{~s}, 3 \mathrm{H})$. ${ }^{13}$ C-NMR $\left(75 \mathrm{MHz}, \mathrm{CDCl}_{3}\right): \delta=169.2\left(\mathrm{C}_{\mathrm{q}}\right), 163.1\left(\mathrm{C}_{\mathrm{q}}, J_{\mathrm{C}-\mathrm{F}}=250.0 \mathrm{~Hz}\right), 141.8\left(\mathrm{C}_{\mathrm{q}}, J_{\mathrm{C}-\mathrm{F}}=8.3\right.$ $\mathrm{Hz}), 138.1\left(\mathrm{C}_{\mathrm{q}}\right), 136.0\left(\mathrm{C}_{\mathrm{q}}, J_{\mathrm{C}-\mathrm{F}}=1.8 \mathrm{~Hz}\right), 131.5\left(\mathrm{C}_{\mathrm{q}}, J_{\mathrm{C}-\mathrm{F}}=3.2 \mathrm{~Hz}\right), 131.2\left(\mathrm{CH}, J_{\mathrm{C}-\mathrm{F}}=9.0 \mathrm{~Hz}\right)$, $129.4(\mathrm{CH}), 128.3(\mathrm{CH}), 116.7\left(\mathrm{CH}, J_{\mathrm{C}-\mathrm{F}}=22.1 \mathrm{~Hz}\right), 114.3\left(\mathrm{CH}, J_{\mathrm{C}-\mathrm{F}}=22.1 \mathrm{~Hz}\right), 26.8\left(\mathrm{CH}_{3}\right)$, $21.3\left(\mathrm{CH}_{3}\right)$.

${ }^{19}$ F-NMR $\left(\mathrm{CDCl}_{3}, 376 \mathrm{MHz}\right): \delta-110.4--110.5(\mathrm{~m})$.

IR (ATR): 3243, 1630, 1557, 1176, 835, $607 \mathrm{~cm}^{-1}$.

MS (EI) m/z (relative intens ity) 243 (5) $\left[\mathrm{M}^{+}\right], 213$ (20), 183 (5), 165 (5), 58 (25), 43 (100).

HR-MS (EI) m/z calcd for $\mathrm{C}_{15} \mathrm{H}_{14} \mathrm{NFO}\left[\mathrm{M}^{+}\right]$243.1059, found 243.1065 .

\section{N,4'-Dime thyl-5-(trifluorome thyl)biphe nyl-2-carboxamide (139fa)}<smiles>CNC(=O)c1ccc(C(F)(F)F)cc1-c1ccc(C)cc1</smiles>

The general procedure $\mathbf{G}$ was followed us ing $\mathbf{2 f}$ (102 $\mathrm{mg}, 0.5 \mathrm{mmol})$, 4-chlorotoluene (88a) (76 mg, $0.6 \mathrm{mmol}), \mathrm{Co}(\mathrm{acac})_{2}(12.9 \mathrm{mg}, 10 \mathrm{~mol} \%)$ and $\mathrm{ICyHCl}(13.5 \mathrm{mg}, 10 \mathrm{~mol} \%)$ for $0.5 \mathrm{~h}$. Purification by column chromatography ( $n$-hexane/EtOAc 6:1 $\rightarrow 2: 1)$ yielded $139 \mathrm{fa}(93 \mathrm{mg}$, $63 \%$ ) as a colorless solid. M. p.: $=157-159^{\circ} \mathrm{C}$.

${ }^{1}$ H-NMR $\left(300 \mathrm{MHz}, \mathrm{CDCl}_{3}\right): \delta=7.74(\mathrm{~d}, J=8.6 \mathrm{~Hz}, 1 \mathrm{H}), 7.61(\mathrm{~d}, J=8.6 \mathrm{~Hz}, 1 \mathrm{H}), 7.60(\mathrm{~s}, 1 \mathrm{H})$, $7.29(\mathrm{~d}, J=8.1 \mathrm{~Hz}, 2 \mathrm{H}), 7.23(\mathrm{~d}, J=8.1 \mathrm{~Hz}, 2 \mathrm{H}), 5.31(\mathrm{~s}, 1 \mathrm{H}), 2.69$ (d, $J=4.9 \mathrm{~Hz}, 3 \mathrm{H}), 2.38$ (s, $3 \mathrm{H})$.

${ }^{13}$ C-NMR (125 MHz, $\left.\mathrm{CDCl}_{3}\right): \delta=168.9\left(\mathrm{C}_{\mathrm{q}}\right), 139.9\left(\mathrm{C}_{\mathrm{q}}\right), 138.6\left(\mathrm{C}_{\mathrm{q}}\right), 138.3\left(\mathrm{C}_{\mathrm{q}}\right), 135.6\left(\mathrm{C}_{\mathrm{q}}\right)$, $131.9\left(\mathrm{C}_{\mathrm{q}}, J_{\mathrm{C}-\mathrm{F}}=32.8 \mathrm{~Hz}\right), 129.5(\mathrm{CH}), 129.3(\mathrm{CH}), 128.3(\mathrm{CH}), 126.9\left(\mathrm{CH}, J_{\mathrm{C}-\mathrm{F}}=7.5 \mathrm{~Hz}\right)$, $124.0\left(\mathrm{CH}, J_{\mathrm{C}-\mathrm{F}}=7.5 \mathrm{~Hz}\right), 123.5\left(\mathrm{C}_{\mathrm{q}}, J_{\mathrm{C}-\mathrm{F}}=271.9 \mathrm{~Hz}\right), 26.8\left(\mathrm{CH}_{3}\right), 21.3\left(\mathrm{CH}_{3}\right)$. 
${ }^{19}$ F-NMR (376 MHz, $\left.\mathrm{CDCl}_{3}\right): \delta=-62.9(\mathrm{~s})$.

IR (ATR): 3280, 1643, 1335, 1117, 814, $545 \mathrm{~cm}^{-1}$.

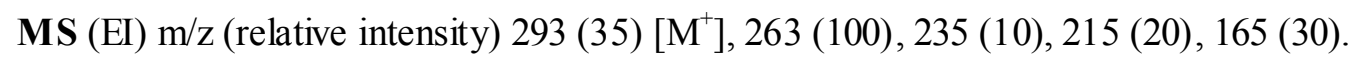

HR-MS (EI) m/z calcd for $\mathrm{C}_{16} \mathrm{H}_{14} \mathrm{NOF}_{3}\left[\mathrm{M}^{+}\right] 293.1027$, found 293.1034.

\section{$N, 4,4^{\prime}$-Trimethylbiphe nyl-2-carboxamide (1390a)}<smiles>CNC(=O)c1cc(C)ccc1-c1ccc(C)cc1</smiles>

The general procedure $\mathbf{G}$ was followed using $20(75 \mathrm{mg}, 0.5 \mathrm{mmol})$ and 4-chlorotoluene (88a) (76 mg, $0.6 \mathrm{mmol}$ ) for $0.5 \mathrm{~h}$. Purification by column chromatography ( $n$-hexane/EtOAc 2:1) yie lded 1390a ( $93 \mathrm{mg}, 78 \%$ ) as a colorless solid. M. p.: $=144-146^{\circ} \mathrm{C}$.

${ }^{1} \mathbf{H}-\mathbf{N M R}\left(300 \mathrm{MHz}, \mathrm{CDCl}_{3}\right): \delta=7.48(\mathrm{~s}, 1 \mathrm{H}), 7.27(\mathrm{~d}, J=8.2 \mathrm{~Hz}, 2 \mathrm{H}), 7.22(\mathrm{~d}, J=8.2 \mathrm{~Hz}, 2 \mathrm{H})$, $7.19(\mathrm{~d}, J=8.2 \mathrm{~Hz}, 2 \mathrm{H}), 5.18(\mathrm{~s}, 1 \mathrm{H}), 2.66$ (d, $J=4.9 \mathrm{~Hz}, 3 \mathrm{H}), 2.37$ (s, 3H), 2.36 (s, 3H). ${ }^{13}$ C-NMR (75 MHz, $\left.\mathrm{CDCl}_{3}\right): \delta=170.5\left(\mathrm{C}_{\mathrm{q}}\right), 137.3\left(\mathrm{C}_{\mathrm{q}}\right), 137.2\left(\mathrm{C}_{\mathrm{q}}\right), 137.1\left(\mathrm{C}_{\mathrm{q}}\right), 136.4\left(\mathrm{C}_{\mathrm{q}}\right)$, $135.3\left(\mathrm{C}_{\mathrm{q}}\right), 130.8(\mathrm{CH}), 130.1(\mathrm{CH}), 129.4(\mathrm{CH}), 129.2(\mathrm{CH}), 128.5(\mathrm{CH}), 26.6\left(\mathrm{CH}_{3}\right), 21.1$ $\left(\mathrm{CH}_{3}\right), 20.9\left(\mathrm{CH}_{3}\right)$.

IR (ATR): 3320, 1633, 1533, 1309, 810, $531 \mathrm{~cm}^{-1}$.

MS (EI) m/z (relative intensity) 239 (45) $\left[\mathrm{M}^{+}\right], 222$ (10), 209 (100), 181 (15), 165 (50), 152 (5).

HR-MS (EI) m/z calcd for $\mathrm{C}_{16} \mathrm{H}_{17} \mathrm{NO}\left[\mathrm{M}^{+}\right] 239.1310$, found 239.1308 .

\section{$N, 3$ ',4,4'-Tetrame thylbiphe nyl-2-carboxamide (139oe)}<smiles>CNC(=O)c1cc(C)ccc1-c1ccc(C)c(C)c1</smiles>

The general procedure $\mathbf{G}$ was followed using $20 \quad(75 \mathrm{mg}, 0.5 \mathrm{mmol})$ and 4-chloro-1,2-dimethylbenzene $(\mathbf{8 8 e})(84 \mathrm{mg}, 0.6 \mathrm{mmol})$ for $0.5 \mathrm{~h}$. Purification by column chromatography ( $n$-hexane/EtOAc 3:1) yielded 139oe ( $82 \mathrm{mg}, 64 \%$ ) as a colorless solid. M. p.: $=123-125^{\circ} \mathrm{C}$. 
${ }^{1} \mathbf{H}-\mathbf{N M R}\left(300 \mathrm{MHz}, \mathrm{CDCl}_{3}\right): \delta=7.77-7.71(\mathrm{~m}, 1 \mathrm{H}), 7.29-7.26(\mathrm{~m}, 1 \mathrm{H}), 7.14(\mathrm{~d}, J=7.8 \mathrm{~Hz}$, 1H), 7.07 (dd, $J=7.5,1.4 \mathrm{~Hz}, 1 \mathrm{H}), 7.05(\mathrm{~d}, J=7.7 \mathrm{~Hz}, 1 \mathrm{H}), 6.98(\mathrm{~s}, 1 \mathrm{H}), 5.24$ (s, 1H), 2.60 (d, $J=4.9 \mathrm{~Hz}, 3 \mathrm{H}), 2.40(\mathrm{~s}, 3 \mathrm{H}), 2.32(\mathrm{~s}, 3 \mathrm{H}), 2.03(\mathrm{~s}, 3 \mathrm{H})$.

${ }^{13}$ C-NMR (125 MHz, $\left.\mathrm{CDCl}_{3}\right): \delta=168.9\left(\mathrm{C}_{\mathrm{q}}\right), 139.9\left(\mathrm{C}_{\mathrm{q}}\right), 137.3\left(\mathrm{C}_{\mathrm{q}}\right), 136.2\left(\mathrm{C}_{\mathrm{q}}\right), 135.6\left(\mathrm{C}_{\mathrm{q}}\right)$, $134.2\left(\mathrm{C}_{\mathrm{q}}\right), 133.0\left(\mathrm{C}_{\mathrm{q}}\right), 131.0(\mathrm{CH}), 130.2(\mathrm{CH}), 130.2(\mathrm{CH}), 129.8(\mathrm{CH}), 129.8(\mathrm{CH}), 128.7$ (CH), $26.8\left(\mathrm{CH}_{3}\right), 21.1\left(\mathrm{CH}_{3}\right), 21.0\left(\mathrm{CH}_{3}\right), 19.5\left(\mathrm{CH}_{3}\right)$.

IR (ATR): 3281, 1634, 1551, 1319, 823, $468 \mathrm{~cm}^{-1}$.

MS (EI) m/z (relative intensity) 253 (40) $\left[\mathrm{M}^{+}\right], 238$ (70), 222 (100), 208 (15), 195 (50), 179 (55).

HR-MS (EI) m/z calcd for $\mathrm{C}_{17} \mathrm{H}_{19} \mathrm{NO}\left[\mathrm{M}^{+}\right]$253.1467, found 253.1473 .

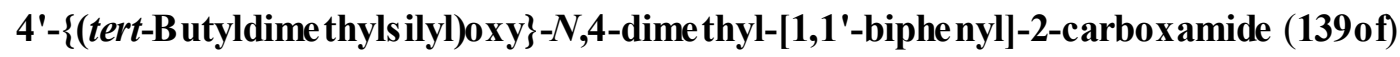<smiles>CNC(=O)c1cc(C)ccc1-c1ccc(OC(C)(C)C)cc1</smiles>

The general procedure $\mathbf{G}$ was followed using 20 (75 $\mathrm{mg}, 0.5 \mathrm{mmol})$ and tert-butyl(4-chlorophenoxy)dimethylsilane (88f) (145 $\mathrm{mg}, 0.6 \mathrm{mmol})$ for $0.5 \mathrm{~h}$. Purification by column chromatography ( $n$-hexane/EtOAc 2:1) yielded 139of (125 mg, 70\%) as a colorless solid. M. p.: $=70-72{ }^{\circ} \mathrm{C}$.

${ }^{1} \mathbf{H}-\mathbf{N M R}\left(300 \mathrm{MHz}, \mathrm{CDCl}_{3}\right): \delta=7.49(\mathrm{~d}, J=1.5 \mathrm{~Hz}, 1 \mathrm{H}), 7.23-7.21(\mathrm{~m}, 4 \mathrm{H}), 6.85(\mathrm{dd}, J=8.6$, $0.6 \mathrm{~Hz}, 2 \mathrm{H}), 5.10$ (d, $J=5.0 \mathrm{~Hz}, 1 \mathrm{H}), 2.65(\mathrm{~d}, J=5.0 \mathrm{~Hz}, 3 \mathrm{H}), 2.37$ (d, $J=0.7 \mathrm{~Hz}, 3 \mathrm{H}), 0.98$ (s, $9 \mathrm{H}), 0.20(\mathrm{~s}, 6 \mathrm{H})$.

${ }^{13}$ C-NMR (125 MHz, $\left.\mathrm{CDCl}_{3}\right): \delta=170.5\left(\mathrm{C}_{\mathrm{q}}\right), 155.4\left(\mathrm{C}_{\mathrm{q}}\right), 137.1\left(\mathrm{C}_{\mathrm{q}}\right), 136.1\left(\mathrm{C}_{\mathrm{q}}\right), 135.3\left(\mathrm{C}_{\mathrm{q}}\right)$, $133.1\left(\mathrm{C}_{\mathrm{q}}\right), 130.8(\mathrm{CH}), 129.9(\mathrm{CH}), 129.8(\mathrm{CH}), 129.5(\mathrm{CH}), 120.2(\mathrm{CH}), 26.6\left(\mathrm{CH}_{3}\right), 25.7$ $\left(\mathrm{CH}_{3}\right), 20.9\left(\mathrm{CH}_{3}\right), 18.3\left(\mathrm{C}_{\mathrm{q}}\right),-4.4\left(\mathrm{CH}_{3}\right)$.

IR (ATR): 3251, 2930, 1621, 1254, 911, $777 \mathrm{~cm}^{-1}$.

MS (EI) m/z (relative intens ity) 355 (45) [M+ $], 298$ (35), 267 (100), 239 (5), 211 (5), 165 (10).

HR-MS (EI) m/z calcd for $\mathrm{C}_{21} \mathrm{H}_{29} \mathrm{NO}_{2} \mathrm{Si}\left[\mathrm{M}^{+}\right] 355.1968$, found 355.1960 . 


\section{N,4'-Dime thyl-4-(trifluorome thyl)biphe nyl-2-carboxamide (139pa)}<smiles>CNC(=O)c1cc(C(F)(F)F)ccc1-c1ccc(C)cc1</smiles>

The general procedure $\mathbf{G}$ was followed us ing $\mathbf{2 p}$ (102 $\mathrm{mg}, 0.5 \mathrm{mmol})$ 4-chlorotoluene (88a) (76 $\mathrm{mg}, 0.6 \mathrm{mmol}), \mathrm{Co}(\mathrm{acac})_{2}(12.9 \mathrm{mg}, 10 \mathrm{~mol} \%)$ and $\mathrm{ICyHCl}(13.5 \mathrm{mg}, 10 \mathrm{~mol} \%)$ for $0.5 \mathrm{~h}$. Purification by column chromatography ( $n$-hexane/EtOAc 3:1) yielded 139pa (78 mg, 53\%) as a colorless solid. M. p.: $=161-163{ }^{\circ} \mathrm{C}$.

${ }^{1} \mathbf{H}-\mathbf{N M R}\left(300 \mathrm{MHz}, \mathrm{CDCl}_{3}\right): \delta=7.96(\mathrm{~s}, 1 \mathrm{H}), 7.70(\mathrm{~d}, J=8.4 \mathrm{~Hz}, 1 \mathrm{H}), 7.48(\mathrm{~d}, J=8.4 \mathrm{~Hz}$, 1H), $7.31(\mathrm{~d}, J=7.9 \mathrm{~Hz}, 2 \mathrm{H}), 7.26(\mathrm{~d}, J=7.9 \mathrm{~Hz}, 2 \mathrm{H}), 5.26(\mathrm{~s}, 1 \mathrm{H}), 2.72(\mathrm{~d}, J=4.8 \mathrm{~Hz}, 3 \mathrm{H})$, $2.41(\mathrm{~s}, 3 \mathrm{H})$.

${ }^{13}$ C-NMR (125 MHz, $\left.\mathrm{CDCl}_{3}\right): \delta=168.7\left(\mathrm{C}_{\mathrm{q}}\right), 142.7\left(\mathrm{C}_{\mathrm{q}}\right), 138.4\left(\mathrm{C}_{\mathrm{q}}\right), 136.0\left(\mathrm{C}_{\mathrm{q}}\right), 135.7\left(\mathrm{C}_{\mathrm{q}}\right)$, $130.6(\mathrm{CH}), 129.6\left(\mathrm{C}_{\mathrm{q}}, J_{\mathrm{C}-\mathrm{F}}=32.6 \mathrm{~Hz}\right), 129.5(\mathrm{CH}), 128.3(\mathrm{CH}), 126.6\left(\mathrm{CH}, J_{\mathrm{C}-\mathrm{F}}=7.2 \mathrm{~Hz}\right)$, $126.0\left(\mathrm{CH}, J_{\mathrm{C}-\mathrm{F}}=7.2 \mathrm{~Hz}\right), 123.7\left(\mathrm{C}_{\mathrm{q}}, J_{\mathrm{C}-\mathrm{F}}=272.4 \mathrm{~Hz}\right), 26.8\left(\mathrm{CH}_{3}\right), 21.3\left(\mathrm{CH}_{3}\right)$.

${ }^{19}$ F-NMR $\left(282 \mathrm{MHz}, \mathrm{CDCl}_{3}\right): \delta=-62.7$ (s).

IR (ATR): 3241, 1633, 1364, 1263, 1125, $825 \mathrm{~cm}^{-1}$.

MS (EI) m/z (relative intensity) 293 (30) [M+ $], 263$ (100), 249 (5), 235 (10), 215 (20), 165 (35).

HR-MS (EI) m/z calcd for $\mathrm{C}_{16} \mathrm{H}_{14} \mathrm{NOF}_{3}\left[\mathrm{M}^{+}\right] 293.1027$, found 293.1027.

\section{$N$-Me thyl-4-p-tolylbe nzo $[d][1,3]$ dioxole-5-carboxamide (139ma)}<smiles>CNC(=O)c1ccc2c(c1-c1ccc(C)cc1)OCO2</smiles>

general procedure $\mathbf{G}$ was followed using $\mathbf{2} \mathbf{m}(90 \mathrm{mg}, 0.5 \mathrm{mmol})$ and 4-chlorotoluene (88a) (76 $\mathrm{mg}, 0.6 \mathrm{mmol}$ ) for $16 \mathrm{~h}$. Purification by column chromatography ( $n$-hexane/EtOAc $10: 1 \rightarrow 2: 1$ ) yie lded 139ma (93 mg, 59\%) and 139ma' (23 mg, 17\%) as colorless solids. IV-5ia: M. p.: = $166-168^{\circ} \mathrm{C}$. 
${ }^{1} \mathbf{H}-\mathbf{N M R}\left(300 \mathrm{MHz}, \mathrm{CDCl}_{3}\right): \delta=7.30(\mathrm{~d}, J=8.1 \mathrm{~Hz}, 2 \mathrm{H}), 7.28(\mathrm{~d}, J=7.9 \mathrm{~Hz}, 1 \mathrm{H}), 7.21(\mathrm{~d}, J=$ $8.1 \mathrm{~Hz}, 2 \mathrm{H}), 6.79(\mathrm{~d}, J=7.9 \mathrm{~Hz}, 1 \mathrm{H}), 5.97(\mathrm{~s}, 2 \mathrm{H}), 5.25(\mathrm{~s}, 1 \mathrm{H}), 2.63(\mathrm{~d}, J=4.9 \mathrm{~Hz}, 3 \mathrm{H}), 2.36(\mathrm{~s}$, $3 \mathrm{H})$.

${ }^{13}$ C-NMR $\left(125 \mathrm{MHz}, \mathrm{CDCl}_{3}\right): \delta=168.9\left(\mathrm{C}_{\mathrm{q}}\right), 148.6\left(\mathrm{C}_{\mathrm{q}}\right), 145.2\left(\mathrm{C}_{\mathrm{q}}\right), 138.0\left(\mathrm{C}_{\mathrm{q}}\right), 130.5\left(\mathrm{C}_{\mathrm{q}}\right)$, $129.5\left(\mathrm{C}_{\mathrm{q}}\right), 129.2(\mathrm{CH}), 129.0(\mathrm{CH}), 123.5(\mathrm{CH}), 121.4\left(\mathrm{C}_{\mathrm{q}}\right), 107.3(\mathrm{CH}), 101.3\left(\mathrm{CH}_{2}\right), 26.7$ $\left(\mathrm{CH}_{3}\right), 21.3\left(\mathrm{CH}_{3}\right)$.

IR (ATR): 3238, 1637, 1443, 1243, 830, $526 \mathrm{~cm}^{-1}$.

MS (EI) m/z (relative intensity) 269 (55) $\left[\mathrm{M}^{+}\right], 252$ (10), 239 (100), 209 (20), 181 (40), 153 (35).

HR-MS (EI) m/z calcd for $\mathrm{C}_{16} \mathrm{H}_{15} \mathrm{NO}_{3}\left[\mathrm{M}^{+}\right]$269.1052, found 269.1050.

\section{$N$-Me thyl-6-p-tolylbe nzo $[d][1,3]$ dioxole-5-carboxamide (139ma')}<smiles>CNC(=O)c1cc2c(cc1-c1ccc(C)cc1)OCO2</smiles>

M. p.: $=190-192^{\circ} \mathrm{C}$.

${ }^{1} \mathbf{H}-\mathbf{N M R}\left(500 \mathrm{MHz}, \mathrm{CDCl}_{3}\right): \delta=7.22(\mathrm{~d}, J=8.2 \mathrm{~Hz}, 2 \mathrm{H}), 7.18(\mathrm{~d}, J=8.2 \mathrm{~Hz}, 3 \mathrm{H}), 7.18(\mathrm{~s}, 1 \mathrm{H})$, $6.00(\mathrm{~s}, 2 \mathrm{H}), 5.07(\mathrm{~d}, J=4.9 \mathrm{~Hz}, 1 \mathrm{H}), 2.61(\mathrm{~d}, J=4.9 \mathrm{~Hz}, 3 \mathrm{H}), 2.36(\mathrm{~s}, 3 \mathrm{H})$.

${ }^{13}$ C-NMR $\left(125 \mathrm{MHz}, \mathrm{CDCl}_{3}\right): \delta=169.4\left(\mathrm{C}_{\mathrm{q}}\right), 148.9\left(\mathrm{C}_{\mathrm{q}}\right), 146.9\left(\mathrm{C}_{\mathrm{q}}\right), 137.4\left(\mathrm{C}_{\mathrm{q}}\right), 137.0\left(\mathrm{C}_{\mathrm{q}}\right)$, $134.3\left(\mathrm{C}_{\mathrm{q}}\right), 129.2(\mathrm{CH}), 129.2\left(\mathrm{C}_{\mathrm{q}}\right), 128.5(\mathrm{CH}), 109.9(\mathrm{CH}), 109.1(\mathrm{CH}), 101.6\left(\mathrm{CH}_{2}\right), 26.8$ $\left(\mathrm{CH}_{3}\right), 21.2\left(\mathrm{CH}_{3}\right)$.

IR (ATR): 3341, 1635, 1480, 1232, 820, $577 \mathrm{~cm}^{-1}$.

MS (EI) m/z (relative intensity) 269 (100) $\left[\mathrm{M}^{+}\right], 252$ (25), 239 (100), 224 (10), 209 (55), 181 (60).

HR-MS (ESI) m/z calcd for $\mathrm{C}_{16} \mathrm{H}_{16} \mathrm{NO}_{3}\left[\mathrm{M}+\mathrm{H}^{+}\right] 270.1130$, found 270.1127 . 
<smiles>CNC(=O)c1c(-c2ccc(C)cc2)ccc2ccccc12</smiles>

The general procedure $\mathbf{G}$ was followed using $\mathbf{2 l}$ (93 $\mathrm{mg}, 0.5 \mathrm{mmol})$ and 4-chlorotoluene (88a) (76 mg, $0.6 \mathrm{mmol}$ ) for $0.5 \mathrm{~h}$. Purification by column chromatography ( $n$-hexane/EtOAc 4:1) yie lded 139la (78 mg, 57\%) as a colorless solid. M. p.: $=196-198{ }^{\circ} \mathrm{C}$.

${ }^{1}$ H-NMR (400 MHz, $\mathrm{CDCl}_{3}$ ): $\delta=8.08-7.97(\mathrm{~m}, 1 \mathrm{H}), 7.90-7.87(\mathrm{~d}, J=8.8 \mathrm{~Hz}, 1 \mathrm{H}), 7.86-7.82$ (m, 1H), 7.57-7.46 (m, 3H), 7.46-7.43 (m, 2H), 7.25-7.21 (m, 2H), $5.40(\mathrm{~d}, J=5.0 \mathrm{~Hz}, 1 \mathrm{H})$, $2.77(\mathrm{~d}, J=5.0 \mathrm{~Hz}, 3 \mathrm{H}), 2.39(\mathrm{~s}, 3 \mathrm{H})$.

${ }^{13}$ C-NMR (100 MHz, $\left.\mathrm{CDCl}_{3}\right): \delta=170.3\left(\mathrm{C}_{\mathrm{q}}\right), 137.4\left(\mathrm{C}_{\mathrm{q}}\right), 137.4\left(\mathrm{C}_{\mathrm{q}}\right), 136.3\left(\mathrm{C}_{\mathrm{q}}\right), 133.3\left(\mathrm{C}_{\mathrm{q}}\right)$, $132.4\left(\mathrm{C}_{\mathrm{q}}\right), 130.4\left(\mathrm{C}_{\mathrm{q}}\right), 129.4(\mathrm{CH}), 129.2(\mathrm{CH}), 128.6(\mathrm{CH}), 127.9(\mathrm{CH}), 127.5(\mathrm{CH}), 127.2$ $(\mathrm{CH}), 126.2(\mathrm{CH}), 125.6(\mathrm{CH}), 26.8\left(\mathrm{CH}_{3}\right), 21.2\left(\mathrm{CH}_{3}\right)$.

IR (ATR): 3280, 1632, 1538, 1292, 811, $548 \mathrm{~cm}^{-1}$.

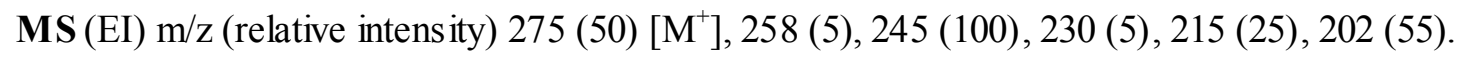

HR-MS (EI) m/z calcd for $\mathrm{C}_{19} \mathrm{H}_{17} \mathrm{NO}\left[\mathrm{M}^{+}\right] 275.1310$, found 275.1305 .

\section{2-(4-Methoxyphenyl)- $N$-methyl-1-naphthamide (139lg)}<smiles>CONC(=O)c1c(-c2ccc(OC)cc2)ccc2ccccc12</smiles>

The general procedure $\mathbf{G}$ was followed using $\mathbf{2 l}$ (93 $\mathrm{mg}, 0.5 \mathrm{mmol}$ ) and 1-chloro-4-methoxybenzene $\mathbf{( 8 8 g}$ ) $(85 \mathrm{mg}, 0.6 \mathrm{mmol}$ ) for $0.5 \mathrm{~h}$. Purification by column chromatography ( $n$-hexane/EtOAc 2:1) yie lded 139lg (81 mg, 56\%) as a color less solid. M. p.: $=190-191^{\circ} \mathrm{C}$.

${ }^{1}$ H-NMR $\left(300 \mathrm{MHz}, \mathrm{CDCl}_{3}\right): \delta=8.04(\mathrm{~d}, J=8.5 \mathrm{~Hz}, 1 \mathrm{H}), 7.87(\mathrm{~d}, J=8.5 \mathrm{~Hz}, 1 \mathrm{H}), 7.80$ (dd, $J$ $=8.5,1.6 \mathrm{~Hz}, 1 \mathrm{H}), 7.61-7.37(\mathrm{~m}, 5 \mathrm{H}), 6.95(\mathrm{~d}, J=8.5 \mathrm{~Hz}, 2 \mathrm{H}), 5.45(\mathrm{~d}, J=5.1 \mathrm{~Hz}, 1 \mathrm{H}), 3.83(\mathrm{~s}$, $3 \mathrm{H}), 2.77(\mathrm{~d}, J=5.1 \mathrm{~Hz}, 3 \mathrm{H})$. 
${ }^{13}$ C-NMR (125 MHz, $\left.\mathrm{CDCl}_{3}\right): \delta=170.3\left(\mathrm{C}_{\mathrm{q}}\right), 159.1\left(\mathrm{C}_{\mathrm{q}}\right), 135.8\left(\mathrm{C}_{\mathrm{q}}\right), 133.0\left(\mathrm{C}_{\mathrm{q}}\right), 132.6\left(\mathrm{C}_{\mathrm{q}}\right)$, $132.1\left(\mathrm{C}_{\mathrm{q}}\right), 130.4\left(\mathrm{C}_{\mathrm{q}}\right), 129.8(\mathrm{CH}), 129.3(\mathrm{CH}), 127.8(\mathrm{CH}), 127.4(\mathrm{CH}), 127.1(\mathrm{CH}), 126.1$ $(\mathrm{CH}), 125.4(\mathrm{CH}), 113.9(\mathrm{CH}), 55.3\left(\mathrm{CH}_{3}\right), 26.8\left(\mathrm{CH}_{3}\right)$.

IR (ATR): 3288, 1633, 1541, 1243, 820, $555 \mathrm{~cm}^{-1}$.

MS (EI) m/z (relative intensity) 291 (60) [M+ $], 274$ (5), 261 (100), 218 (15), 202 (10), 189 (35).

HR-MS (EI) m/z calcd for $\mathrm{C}_{19} \mathrm{H}_{17} \mathrm{NO}_{2}\left[\mathrm{M}^{+}\right]$291.1259, found 291.1264 .

\section{$N, 3,4$ 'Trime thylbiphe nyl-2-carboxamide (139ta)}<smiles>CNC(=O)c1c(C)cccc1-c1ccc(C)cc1</smiles>

The general procedure $\mathbf{G}$ was followed using $\mathbf{2 t}$ (75 $\mathrm{mg}, 0.5 \mathrm{mmol}$ ) and 4-chlorotoluene (88a) (76 mg, $0.6 \mathrm{mmol}$ ) for $16 \mathrm{~h}$. Purification by column chromatography ( $n$-hexane/EtOAc 2:1) yie lded 139ta (64 mg, 54\%) as a colorless solid. M. p.: $=185-186{ }^{\circ} \mathrm{C}$.

${ }^{1} \mathbf{H}-\mathbf{N M R}\left(300 \mathrm{MHz}, \mathrm{CDCl}_{3}\right): \delta=7.31(\mathrm{~d}, J=8.1 \mathrm{~Hz}, 2 \mathrm{H}), 7.27(\mathrm{~d}, J=7.6 \mathrm{~Hz}, 1 \mathrm{H})$, 7.20-7.14 (m, 4H), 5.23 (d, $J=6.0 \mathrm{~Hz}, 1 \mathrm{H}), 2.64$ (d, $J=4.9 \mathrm{~Hz}, 3 \mathrm{H}), 2.39$ (s, 3H), 2.35 (s, $3 \mathrm{H})$.

${ }^{13}$ C-NMR (125 MHz, $\left.\mathrm{CDCl}_{3}\right): \delta=170.6\left(\mathrm{C}_{\mathrm{q}}\right), 138.9\left(\mathrm{C}_{\mathrm{q}}\right), 137.4\left(\mathrm{C}_{\mathrm{q}}\right), 137.0\left(\mathrm{C}_{\mathrm{q}}\right), 136.2\left(\mathrm{C}_{\mathrm{q}}\right)$, $135.6\left(\mathrm{C}_{\mathrm{q}}\right), 129.0(\mathrm{CH}), 129.0(\mathrm{CH}), 128.8(\mathrm{CH}), 128.2(\mathrm{CH}), 127.1(\mathrm{CH}), 26.5\left(\mathrm{CH}_{3}\right), 21.2$ $\left(\mathrm{CH}_{3}\right), 19.6\left(\mathrm{CH}_{3}\right)$.

IR (ATR): 3228, 1625, 1543, 786, 710, $543 \mathrm{~cm}^{-1}$.

MS (EI) m/z (relative intensity) 239 (65) $\left[\mathrm{M}^{+}\right], 224$ (10), 209 (100), 195 (5), 181 (20), 165 (65).

HR-MS (ESI) m/z calcd for $\mathrm{C}_{16} \mathrm{H}_{18} \mathrm{NO}\left[\mathrm{M}+\mathrm{H}^{+}\right] 240.1388$, found 240.1380 . 


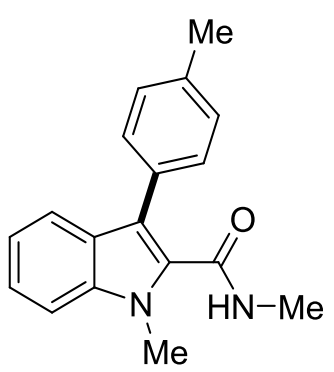

The general procedure $\mathbf{G}$ was followed using $\mathbf{2 u}(94 \mathrm{mg}, 0.5 \mathrm{mmol})$ and 4-chlorotoluene (88a) (76 mg, $0.6 \mathrm{mmol}$ ) for $16 \mathrm{~h}$. Purification by column chromatography ( $n$-hexane/EtOAc 2:1) yie lded 139ua (100 mg, 72\%) as a colorless solid. M. p.: $=167-169^{\circ} \mathrm{C}$.

${ }^{1}$ H-NMR (300 MHz, $\left.\mathrm{CDCl}_{3}\right): \delta=7.56(\mathrm{~d}, J=8.1 \mathrm{~Hz}, 1 \mathrm{H}), 7.41-7.38(\mathrm{~m}, 1 \mathrm{H}), 7.36(\mathrm{~d}, J=8.0$ $\mathrm{Hz}, 2 \mathrm{H}), 7.31(\mathrm{dd}, J=8.1,1.2 \mathrm{~Hz}, 1 \mathrm{H}), 7.27$ (d, $J=8.0 \mathrm{~Hz}, 2 \mathrm{H}), 7.12(\mathrm{dd}, J=8.1,1.2 \mathrm{~Hz}, 1 \mathrm{H})$, $5.64(\mathrm{~s}, 1 \mathrm{H}), 4.02(\mathrm{~s}, 3 \mathrm{H}), 2.77(\mathrm{~d}, J=5.1 \mathrm{~Hz}, 3 \mathrm{H}), 2.42(\mathrm{~s}, 3 \mathrm{H})$.

${ }^{13}$ C-NMR $\left(125 \mathrm{MHz}, \mathrm{CDCl}_{3}\right): \delta=163.2\left(\mathrm{C}_{\mathrm{q}}\right), 137.7\left(\mathrm{C}_{\mathrm{q}}\right), 136.9\left(\mathrm{C}_{\mathrm{q}}\right), 130.7\left(\mathrm{C}_{\mathrm{q}}\right), 129.9(\mathrm{CH})$, $129.5(\mathrm{CH}), 129.1\left(\mathrm{C}_{\mathrm{q}}\right), 126.2\left(\mathrm{C}_{\mathrm{q}}\right), 124.1(\mathrm{CH}), 120.6(\mathrm{CH}), 120.4(\mathrm{CH}), 118.2\left(\mathrm{C}_{\mathrm{q}}\right), 109.9$ $(\mathrm{CH}), 31.6\left(\mathrm{CH}_{3}\right), 26.4\left(\mathrm{CH}_{3}\right), 21.3\left(\mathrm{CH}_{3}\right)$.

IR (ATR): 3242, 1639, 1567, 1365, 751, $517 \mathrm{~cm}^{-1}$.

MS (EI) m/z (relative intensity) 278 (100) $\left[\mathrm{M}^{+}\right], 262$ (5), 248 (30), 233 (15), 221 (15), 204 (20).

HR-MS (EI) m/z calcd for $\mathrm{C}_{18} \mathrm{H}_{18} \mathrm{~N}_{2} \mathrm{O}\left[\mathrm{M}^{+}\right]$278.1419, found 278.1414 .

\section{$N, 1-D i m e$ thyl-2-p-tolyl-1H-indole-3-carboxamide (139ra)}<smiles>CNC(=O)c1c(-c2ccc(C)cc2)n(C)c2ccccc12</smiles>

The general procedure $\mathbf{G}$ was followed using $2 \mathbf{r}$ (94 $\mathrm{mg}, 0.5 \mathrm{mmol})$ and 4-chlorotoluene (88a) (76 mg, $0.6 \mathrm{mmol}$ ) for $16 \mathrm{~h}$. Purification by column chromatography ( $n$-hexane/EtOAc $2: 1 \rightarrow 1: 1)$ yielded 139ra (80 mg, $58 \%$ ) as a colorless solid. M. p.: $=170-171{ }^{\circ} \mathrm{C}$.

${ }^{1} \mathbf{H}-\mathbf{N M R}\left(300 \mathrm{MHz}, \mathrm{CDCl}_{3}\right): \delta=8.36(\mathrm{~d}, J=7.3 \mathrm{~Hz}, 1 \mathrm{H}), 7.35(\mathrm{~d}, J=7.9 \mathrm{~Hz}, 2 \mathrm{H}), 7.32(\mathrm{~d}, J=$ $7.9 \mathrm{~Hz}, 2 \mathrm{H}), 7.31$ (m, 1H), $7.29(\mathrm{dd}, J=6.8,1.5 \mathrm{~Hz}, 1 \mathrm{H}), 7.26(\mathrm{~m}, 1 \mathrm{H}), 5.24(\mathrm{~s}, 1 \mathrm{H}), 3.49$ (s, $3 \mathrm{H}), 2.73(\mathrm{~d}, J=4.7 \mathrm{~Hz}, 3 \mathrm{H}), 2.46(\mathrm{~s}, 3 \mathrm{H})$. 
${ }^{13}$ C-NMR $\left(125 \mathrm{MHz}, \mathrm{CDCl}_{3}\right): \delta=166.0\left(\mathrm{C}_{\mathrm{q}}\right), 140.9\left(\mathrm{C}_{\mathrm{q}}\right), 139.8\left(\mathrm{C}_{\mathrm{q}}\right), 136.6\left(\mathrm{C}_{\mathrm{q}}\right), 130.4(\mathrm{CH})$, $129.8(\mathrm{CH}), 127.9\left(\mathrm{C}_{\mathrm{q}}\right), 127.0\left(\mathrm{C}_{\mathrm{q}}\right), 122.6(\mathrm{CH}), 122.0(\mathrm{CH}), 121.5(\mathrm{CH}), 109.3(\mathrm{CH}), 108.9$ $\left(\mathrm{C}_{\mathrm{q}}\right), 30.6\left(\mathrm{CH}_{3}\right), 26.0\left(\mathrm{CH}_{3}\right), 21.4\left(\mathrm{CH}_{3}\right)$.

IR (ATR): 3338, 1623, 1524, 1222, 750, $511 \mathrm{~cm}^{-1}$.

MS (EI) m/z (relative intensity) 278 (30) [M+], 248 (100), 233 (5), 218 (5), 204 (10), 190 (5).

HR-MS (EI) m/z calcd for $\mathrm{C}_{18} \mathrm{H}_{18} \mathrm{~N}_{2} \mathrm{O}\left[\mathrm{M}^{+}\right]$278.1419, found 278.1422.

\section{2-(4-Methoxyphenyl)-N,1-dime thyl- $1 H$-indole-3-carboxamide (139rg)}<smiles>CNC(=O)c1c(-c2ccc(OC)cc2)n(C)c2ccccc12</smiles>

The general procedure $\mathbf{G}$ was followed using $\mathbf{2 r} \quad(94 \mathrm{mg}, 0.5 \mathrm{mmol}$ ) and 1-chloro-4-methoxybenzene $(\mathbf{8 8 g})(85 \mathrm{mg}, 0.6 \mathrm{mmol})$ for $16 \mathrm{~h}$. Purification by column chromatography ( $n$-hexane/EtOAc 3:2) yielded 139rg (87 mg, 59\%) as a colorless solid. M. p.: $=146-147^{\circ} \mathrm{C}$.

${ }^{1} \mathbf{H}-\mathbf{N M R}\left(300 \mathrm{MHz}, \mathrm{CDCl}_{3}\right): \delta=8.41-8.30(\mathrm{~m}, 1 \mathrm{H}), 7.36(\mathrm{~d}, J=8.7 \mathrm{~Hz}, 2 \mathrm{H}), 7.33-7.19(\mathrm{~m}$, $3 \mathrm{H}), 7.06(\mathrm{~d}, J=8.7 \mathrm{~Hz}, 2 \mathrm{H}), 5.28(\mathrm{~s}, 1 \mathrm{H}), 3.89$ (s, 3H), 3.49 (s, 3H), $2.74(\mathrm{~d}, J=4.8 \mathrm{~Hz}, 3 \mathrm{H})$. ${ }^{13}$ C-NMR $\left(125 \mathrm{MHz}, \mathrm{CDCl}_{3}\right): \delta=165.9\left(\mathrm{C}_{\mathrm{q}}\right), 160.4\left(\mathrm{C}_{\mathrm{q}}\right), 140.6\left(\mathrm{C}_{\mathrm{q}}\right), 136.5\left(\mathrm{C}_{\mathrm{q}}\right), 131.8(\mathrm{CH})$, $126.9\left(\mathrm{C}_{\mathrm{q}}\right), 122.8\left(\mathrm{C}_{\mathrm{q}}\right), 122.6(\mathrm{CH}), 121.9(\mathrm{CH}), 121.5(\mathrm{CH}), 114.5(\mathrm{CH}), 109.2(\mathrm{CH}), 108.8$ $\left(\mathrm{C}_{\mathrm{q}}\right), 55.4\left(\mathrm{CH}_{3}\right), 30.6\left(\mathrm{CH}_{3}\right), 26.1\left(\mathrm{CH}_{3}\right)$.

IR (ATR): 3338, 1619, 1542, 1247, 752, $531 \mathrm{~cm}^{-1}$.

MS (EI) m/z (relative intensity) 294 (45) [M+ $], 264$ (100), 249 (5), 234 (5), 221 (5), 192 (10).

HR-MS (ESI) $\mathrm{m} / \mathrm{z}$ calcd for $\mathrm{C}_{18} \mathrm{H}_{19} \mathrm{~N}_{2} \mathrm{O}_{2}\left[\mathrm{M}+\mathrm{H}^{+}\right]$295.1447, found 295.1445.

4'-Methyl- $N$-phe nylbiphe nyl-2-carboxamide (139va)<smiles>Cc1ccc(-c2ccccc2C(=O)Nc2ccccc2)cc1</smiles> 
The general procedure $\mathbf{G}$ was followed using $\mathbf{2 v}(99 \mathrm{mg}, 0.5 \mathrm{mmol}$ ) and 4-chlorotoluene (88a) (76 mg, $0.6 \mathrm{mmol}$ ) for $16 \mathrm{~h}$. Purification by column chromatography ( $n$-hexane/EtOAc 10:1) yielded 139va (103 mg, 72\%) as a colorless solid. M. p.: $=154-155^{\circ} \mathrm{C}$.

${ }^{1}$ H-NMR (300 MHz, $\left.\mathrm{CDCl}_{3}\right): \delta=7.86(\mathrm{dd}, J=7.5,1.5 \mathrm{~Hz}, 1 \mathrm{H}), 7.64-7.37$ (m, 3H), 7.35 (d, $J$ $=8.1 \mathrm{~Hz}, 2 \mathrm{H}), 7.24-7.16(\mathrm{~m}, 4 \mathrm{H}), 7.11(\mathrm{dd}, J=8.1,1.5 \mathrm{~Hz}, 2 \mathrm{H}), 7.08-6.98(\mathrm{~m}, 1 \mathrm{H}), 6.92(\mathrm{~s}$, $1 \mathrm{H}), 2.37$ (s, 3H).

${ }^{13}$ C-NMR $\left(125 \mathrm{MHz}, \mathrm{CDCl}_{3}\right): \delta=167.0\left(\mathrm{C}_{\mathrm{q}}\right), 139.5\left(\mathrm{C}_{\mathrm{q}}\right), 137.9\left(\mathrm{C}_{\mathrm{q}}\right), 137.5\left(\mathrm{C}_{\mathrm{q}}\right), 136.9\left(\mathrm{C}_{\mathrm{q}}\right)$, $135.1\left(\mathrm{C}_{\mathrm{q}}\right), 130.6(\mathrm{CH}), 130.3(\mathrm{CH}), 129.6(\mathrm{CH}), 129.5(\mathrm{CH}), 128.7(\mathrm{CH}), 128.6(\mathrm{CH}), 127.6$ (CH), $124.2(\mathrm{CH}), 119.8(\mathrm{CH}), 21.2\left(\mathrm{CH}_{3}\right)$.

IR (ATR): 3058, 1652, 1439, 1329, 749, $509 \mathrm{~cm}^{-1}$.

MS (EI) m/z (relative intensity) 287 (35) $\left[\mathrm{M}^{+}\right], 195$ (100), 165 (40), 152 (40).

HR-MS (EI) m/z calcd for $\mathrm{C}_{20} \mathrm{H}_{17} \mathrm{NO}\left[\mathrm{M}^{+}\right]$287.1310, found 287.1304.

The spectral data were in accordance with those reported in the literature. ${ }^{154}$

\section{4'-Methoxy- $N$-phe nylbiphe nyl-2-carboxamide (139vg)}<smiles>COc1ccc(-c2ccccc2C(=O)Nc2ccccc2)cc1</smiles>

The general procedure $\mathbf{G}$ was followed using $\mathbf{2 v}(99 \mathrm{mg}, 0.5 \mathrm{mmol}$ ) and 1-chloro-4-methoxybenzene (88g) (85 $\mathrm{mg}, 0.6 \mathrm{mmol}$ ) for $16 \mathrm{~h}$. Purification by column chromatography ( $n$-hexane/EtOAc 8:1) yielded 139vg (112 mg, 74\%) as a colorless solid. M.p.: $=146-148^{\circ} \mathrm{C}$.

${ }^{1} \mathbf{H}-\mathbf{N M R}\left(300 \mathrm{MHz}, \mathrm{CDCl}_{3}\right): \delta=7.84(\mathrm{dd}, J=7.5,1.4 \mathrm{~Hz}, 1 \mathrm{H}), 7.53-7.37(\mathrm{~m}, 5 \mathrm{H})$, 7.25-7.13 (m, 4H), 7.07-7.01 (m, 1H), 6.97-6.91 (d, $J=8.8 \mathrm{~Hz}, 3 \mathrm{H}), 3.81(\mathrm{~s}, 3 \mathrm{H})$.

${ }^{13}$ C-NMR (75 MHz, $\left.\mathrm{CDCl}_{3}\right): \delta=167.3\left(\mathrm{C}_{\mathrm{q}}\right), 159.5\left(\mathrm{C}_{\mathrm{q}}\right), 139.1\left(\mathrm{C}_{\mathrm{q}}\right), 137.6\left(\mathrm{C}_{\mathrm{q}}\right), 135.1\left(\mathrm{C}_{\mathrm{q}}\right)$, $132.1\left(\mathrm{C}_{\mathrm{q}}\right), 130.6(\mathrm{CH}), 130.4(\mathrm{CH}), 130.0(\mathrm{CH}), 129.4(\mathrm{CH}), 128.8(\mathrm{CH}), 127.4(\mathrm{CH}), 124.3$ (CH), $119.9(\mathrm{CH}), 114.4(\mathrm{CH}), 55.4\left(\mathrm{CH}_{3}\right)$.

IR (ATR): 3238, 1653, 1434, 1238, 755, $526 \mathrm{~cm}^{-1}$.

${ }^{154}$ S. E. Havlik, J. M. Simmons, V. J. Winton, J. B. Johnson, J. Org. Chem. 2011, 76, 3588-3593. 
MS (EI) m/z (relative intensity) 303 (20) [M+], 211 (100), 196 (5), 183 (5), 168 (20), 152 (5).

HR-MS (EI) m/z calcd for $\mathrm{C}_{20} \mathrm{H}_{17} \mathrm{NO}_{2}\left[\mathrm{M}^{+}\right]$303.1259, found 303.1260.

The spectral data were in accordance with those reported in the literature. ${ }^{154}$

\section{$N$-Phe nyl-[1,1'-biphenyl]-2-carboxamide (139vh)}<smiles>O=C(Nc1ccccc1)c1ccccc1-c1ccccc1</smiles>

The general procedure $\mathbf{G}$ was followed using $\mathbf{2 v}$ (99 $\mathrm{mg}, 0.5 \mathrm{mmol})$ and chlorobenzene (88h) (67 mg, $0.6 \mathrm{mmol}$ ) for $16 \mathrm{~h}$. Purification by column chromatography ( $n$-hexane/EtOAc 10:1) and recrystallization ( $n$-hexane/EtOAc) yielded 139vh (78 mg, 57\%) as a colorless solid. M. p. : $=111-113^{\circ} \mathrm{C}$.

${ }^{1} \mathbf{H}-\mathbf{N M R}\left(300 \mathrm{MHz}, \mathrm{CDCl}_{3}\right): \delta=7.88(\mathrm{dd}, J=7.2,1.6 \mathrm{~Hz}, 1 \mathrm{H}), 7.60-7.37(\mathrm{~m}, 8 \mathrm{H}), 7.22(\mathrm{~d}, J=$ $8.5 \mathrm{~Hz}, 1 \mathrm{H}), 7.19$ (d, $J=7.2 \mathrm{~Hz}, 1 \mathrm{H}), 7.13-6.95$ (m, 3H), 6.88 (s, 1H).

${ }^{13}$ C-NMR (125 MHz, $\left.\mathrm{CDCl}_{3}\right): \delta=166.9\left(\mathrm{C}_{\mathrm{q}}\right), 139.8\left(\mathrm{C}_{\mathrm{q}}\right), 139.4\left(\mathrm{C}_{\mathrm{q}}\right), 137.4\left(\mathrm{C}_{\mathrm{q}}\right), 135.2\left(\mathrm{C}_{\mathrm{q}}\right)$, 130.6 (CH), $130.2(\mathrm{CH}), 129.5(\mathrm{CH}), 128.9(\mathrm{CH}), 128.7(\mathrm{CH}), 128.7(\mathrm{CH}), 128.0(\mathrm{CH}), 127.8$ (CH), $124.3(\mathrm{CH}), 119.8(\mathrm{CH})$.

IR (ATR): 3331, 1664, 1516, 1433, 741, $688 \mathrm{~cm}^{-1}$.

MS (EI) m/z (relative intensity) 273 (50) $\left[\mathrm{M}^{+}\right], 181$ (100), 152 (60), 127 (5).

HR-MS (EI) m/z calcd for $\mathrm{C}_{19} \mathrm{H}_{15} \mathrm{NO}\left[\mathrm{M}^{+}\right]$273.1154, found 273.1153 .

The spectral data were in accordance with those reported in the literature. ${ }^{154}$

2'-Methyl- $N$-phe nylbiphe nyl-2-carboxamide $(139 \mathrm{vc})$<smiles>Cc1ccccc1-c1ccccc1C(=O)Nc1ccccc1</smiles>

The general procedure $\mathbf{G}$ was followed using $\mathbf{2 v}(99 \mathrm{mg}, 0.5 \mathrm{mmol})$ and 2-chlorotoluene (88c) (76 mg, $0.6 \mathrm{mmol}$ ) for $16 \mathrm{~h}$. Purification by column chromatography ( $n$-hexane/EtOAc 10:1) yie lded 139vc (118 $\mathrm{mg}, 82 \%)$ as a colorless solid. M. p.: $=134-136{ }^{\circ} \mathrm{C}$. 
${ }^{1} \mathbf{H}-\mathbf{N M R}\left(300 \mathrm{MHz}, \mathrm{CDCl}_{3}\right): \delta=8.12(\mathrm{dd}, J=7.1,2.0 \mathrm{~Hz}, 1 \mathrm{H}), 7.57-7.48$ (m, 2H), 7.41-7.30 (m, 4H), $7.26(\mathrm{dd}, J=7.3,2.1 \mathrm{~Hz}, 1 \mathrm{H}), 7.20(\mathrm{~d}, J=7.3 \mathrm{~Hz}, 1 \mathrm{H}), 7.17(\mathrm{~d}, J=7.1 \mathrm{~Hz}, 1 \mathrm{H}), 7.10$ (s, 1H), $7.01(\mathrm{~d}, J=6.9 \mathrm{~Hz}, 2 \mathrm{H}), 7.00-6.98(\mathrm{~m}, 1 \mathrm{H}), 2.11$ (s, 3H).

${ }^{13}$ C-NMR (125 MHz, $\left.\mathrm{CDCl}_{3}\right): \delta=165.5\left(\mathrm{C}_{\mathrm{q}}\right), 139.9\left(\mathrm{C}_{\mathrm{q}}\right), 138.9\left(\mathrm{C}_{\mathrm{q}}\right), 137.5\left(\mathrm{C}_{\mathrm{q}}\right), 136.5\left(\mathrm{C}_{\mathrm{q}}\right)$, $134.2\left(\mathrm{C}_{\mathrm{q}}\right), 130.9(\mathrm{CH}), 130.8(\mathrm{CH}), 130.4(\mathrm{CH}), 130.1(\mathrm{CH}), 129.2(\mathrm{CH}), 128.7(\mathrm{CH}), 128.6$ $(\mathrm{CH}), 128.0(\mathrm{CH}), 126.6(\mathrm{CH}), 124.1(\mathrm{CH}), 119.6(\mathrm{CH}), 20.1\left(\mathrm{CH}_{3}\right)$.

IR (ATR): 3063, 1647, 1546, 1329, 752, $512 \mathrm{~cm}^{-1}$.

MS (EI) m/z (relative intensity) 287 (5) $\left[\mathrm{M}^{+}\right], 195$ (100), 165 (20), 152 (15), 93 (25).

HR-MS (EI) m/z calcd for $\mathrm{C}_{20} \mathrm{H}_{17} \mathrm{NO}\left[\mathrm{M}^{+}\right]$287.1310, found 287.1308 .

2'-Methoxy- $N$-phe nylbiphe nyl-2-carboxamide (139vi)<smiles>COc1ccccc1-c1ccccc1C(=O)Nc1ccccc1</smiles>

The general procedure $\mathbf{G}$ was followed using $\mathbf{2 v}(99 \mathrm{mg}, 0.5 \mathrm{mmol}$ ) and 1-chloro-2-methoxybenzene $(\mathbf{8 8 i})(85 \mathrm{mg}, 0.6 \mathrm{mmol})$ for $16 \mathrm{~h}$. Purification by column chromatography ( $n$-hexane/EtOAc 10:1) yielded 139vi (99 mg, 65\%) as a colorless solid. M. p.: $=139-141^{\circ} \mathrm{C}$.

${ }^{1} \mathbf{H}-\mathbf{N M R}\left(300 \mathrm{MHz}, \mathrm{CDCl}_{3}\right): \delta=7.90(\mathrm{dd}, J=7.3,1.8 \mathrm{~Hz}, 1 \mathrm{H}), 7.59-7.42(\mathrm{~m}, 2 \mathrm{H}), 7.41-7.27$ (m, 4H), 7.24-7.12 (m, 4H), $7.08(\mathrm{td}, J=7.5,1.1 \mathrm{~Hz}, 1 \mathrm{H}), 7.05-6.98(\mathrm{~m}, 1 \mathrm{H}), 6.90(\mathrm{dd}, J=8.2$, $1.0 \mathrm{~Hz}, 1 \mathrm{H}), 3.65(\mathrm{~s}, 3 \mathrm{H})$.

${ }^{13}$ C-NMR (125 MHz, $\left.\mathrm{CDCl}_{3}\right): \delta=166.7\left(\mathrm{C}_{\mathrm{q}}\right), 156.2\left(\mathrm{C}_{\mathrm{q}}\right), 137.8\left(\mathrm{C}_{\mathrm{q}}\right), 137.8\left(\mathrm{C}_{\mathrm{q}}\right), 135.9\left(\mathrm{C}_{\mathrm{q}}\right)$, $135.7\left(\mathrm{C}_{\mathrm{q}}\right), 130.9(\mathrm{CH}), 130.6(\mathrm{CH}), 130.6(\mathrm{CH}), 129.8(\mathrm{CH}), 128.9(\mathrm{CH}), 128.7(\mathrm{CH}), 127.8$ $(\mathrm{CH}), 123.9(\mathrm{CH}), 121.2(\mathrm{CH}), 119.4(\mathrm{CH}), 111.0(\mathrm{CH}), 55.6\left(\mathrm{CH}_{3}\right)$.

IR (ATR): 3237, 1653, 1434, 1238, 756, $515 \mathrm{~cm}^{-1}$.

MS (EI) m/z (relative intensity) 303 (5) [M+] 272 (10), 211 (100), 196 (20), 168 (10), 139 (10).

HR-MS (EI) m/z calcd for $\mathrm{C}_{20} \mathrm{H}_{17} \mathrm{NO}_{2}\left[\mathrm{M}^{+}\right]$303.1259, found 303.1255.

The spectral data were in accordance with those reported in the literature. ${ }^{154}$ 


\section{4'-Methyl- $N$-p-tolylbiphe nyl-2-carboxamide (139wa)}<smiles>Cc1ccc(NC(=O)c2ccccc2-c2ccc(C)cc2)cc1</smiles>

The general procedure $\mathbf{G}$ was followed using $\mathbf{2 w}$ (105 $\mathrm{mg}, 0.5 \mathrm{mmol})$ and 4-chlorotoluene (88a) (76 mg, $0.6 \mathrm{mmol}$ ) for $16 \mathrm{~h}$. Purification by column chromatography ( $n$-hexane/EtOAc 10:1) yielded 139wa (120 mg, 80\%) as a colorless solid. M. p.: $=134-136{ }^{\circ} \mathrm{C}$.

${ }^{1}$ H-NMR $\left(300 \mathrm{MHz}, \mathrm{CDCl}_{3}\right): \delta=7.87(\mathrm{dd}, J=7.5,1.5 \mathrm{~Hz}, 1 \mathrm{H}), 7.55-7.45(\mathrm{~m}, 2 \mathrm{H})$, $7.42-7.39(\mathrm{~m}, 1 \mathrm{H}), 7.37(\mathrm{~d}, J=8.1 \mathrm{~Hz}, 2 \mathrm{H}), 7.24(\mathrm{~d}, J=8.1 \mathrm{~Hz}, 2 \mathrm{H}), 7.03(\mathrm{~s}, 4 \mathrm{H}), 6.91$ (s, 1H), 2.39 (s, 3H), 2.28 (s, 3H).

${ }^{13}$ C-NMR $\left(125 \mathrm{MHz}, \mathrm{CDCl}_{3}\right): \delta=167.0\left(\mathrm{C}_{\mathrm{q}}\right), 139.4\left(\mathrm{C}_{\mathrm{q}}\right), 137.8\left(\mathrm{C}_{\mathrm{q}}\right), 136.9\left(\mathrm{C}_{\mathrm{q}}\right), 135.2\left(\mathrm{C}_{\mathrm{q}}\right)$, $134.9\left(\mathrm{C}_{\mathrm{q}}\right), 133.9\left(\mathrm{C}_{\mathrm{q}}\right), 130.4(\mathrm{CH}), 130.2(\mathrm{CH}), 129.5(\mathrm{CH}), 129.4(\mathrm{CH}), 129.2(\mathrm{CH}), 128.6$ $(\mathrm{CH}), 127.5(\mathrm{CH}), 119.9(\mathrm{CH}), 21.2\left(\mathrm{CH}_{3}\right), 20.9\left(\mathrm{CH}_{3}\right)$.

IR (ATR): 3044, 1649, 1510, 1327, 755, $510 \mathrm{~cm}^{-1}$.

MS (EI) m/z (relative intensity) $301(15)\left[\mathrm{M}^{+}\right], 285$ (5), 195 (100), 165 (20), 152 (20).

HR-MS (EI) m/z calcd for $\mathrm{C}_{21} \mathrm{H}_{19} \mathrm{NO}\left[\mathrm{M}^{+}\right]$301.1467, found 301.1478 .

The spectral data were in accordance with those reported in the literature. ${ }^{155}$

\section{$N$-(4-Methoxyphe nyl)-4'-me thyl-[1,1'-biphe nyl]-2-carboxamide (139xa)}<smiles>COc1ccc(NC(=O)c2ccccc2-c2ccc(C)cc2)cc1</smiles>

The general procedure $\mathbf{G}$ was followed using $\mathbf{2} \mathbf{x}(114 \mathrm{mg}, 0.5 \mathrm{mmol})$ and 4-chlorotoluene (88a) (76 mg, $0.6 \mathrm{mmol}$ ) for $16 \mathrm{~h}$. Purification by column chromatography ( $n$-hexane/EtOAc 10:1) yielded 139xa (115 mg, 72\%) as a colorless solid. M. p.: = $152-154{ }^{\circ} \mathrm{C}$.

${ }^{1} \mathbf{H}-\mathrm{NMR}\left(400 \mathrm{MHz}, \mathrm{CDCl}_{3}\right): \delta=7.84(\mathrm{ddd}, J=7.6,1.6,0.5 \mathrm{~Hz}, 1 \mathrm{H}), 7.50(\mathrm{td}, J=7.5,1.5 \mathrm{~Hz}$, 1H), $7.43(\mathrm{td}, J=7.5,1.5 \mathrm{~Hz}, 1 \mathrm{H}), 7.38$ (ddd, $J=7.6,1.5,0.5 \mathrm{~Hz}, 1 \mathrm{H}), 7.35(\mathrm{~d}, J=8.1 \mathrm{~Hz}, 2 \mathrm{H})$,

155 U. A. Shah, N. K. Wagh,.H. S. Deokar, S. S. Kadam, V. M. Kulkarni, Int. J. Pharm. Bio Sci. 2010, 1, 501-511. 
$7.22(\mathrm{~d}, J=8.1 \mathrm{~Hz}, 2 \mathrm{H}), 7.02(\mathrm{~d}, J=9.0 \mathrm{~Hz}, 2 \mathrm{H}), 6.81(\mathrm{~s}, 1 \mathrm{H}), 6.75(\mathrm{~d}, J=9.0 \mathrm{~Hz}, 2 \mathrm{H}), 3.74(\mathrm{~s}$, $3 \mathrm{H}), 2.38$ (s, 3H).

${ }^{13}$ C-NMR $\left(125 \mathrm{MHz}, \mathrm{CDCl}_{3}\right): \delta=167.0\left(\mathrm{C}_{\mathrm{q}}\right), 156.3\left(\mathrm{C}_{\mathrm{q}}\right), 139.4\left(\mathrm{C}_{\mathrm{q}}\right), 137.8\left(\mathrm{C}_{\mathrm{q}}\right), 137.0\left(\mathrm{C}_{\mathrm{q}}\right)$, $135.2\left(\mathrm{C}_{\mathrm{q}}\right), 130.7\left(\mathrm{C}_{\mathrm{q}}\right), 130.4(\mathrm{CH}), 130.2(\mathrm{CH}), 129.5(\mathrm{CH}), 129.4(\mathrm{CH}), 128.6(\mathrm{CH}), 127.5$ $(\mathrm{CH}), 121.7(\mathrm{CH}), 113.9(\mathrm{CH}), 55.5\left(\mathrm{CH}_{3}\right), 21.5\left(\mathrm{CH}_{3}\right)$.

IR (ATR): 3250, 1650, 1506, 1239, 836, $515 \mathrm{~cm}^{-1}$.

MS (EI) m/z (relative intensity) 317 (40) $\left[\mathrm{M}^{+}\right], 195$ (100), 165 (30), 152 (35), 122 (5).

HR-MS (EI) $\mathrm{m} / \mathrm{z}$ calcd for $\mathrm{C}_{21} \mathrm{H}_{19} \mathrm{NO}_{2}\left[\mathrm{M}^{+}\right]$317.1416, found 317.1408.

The spectral data were in accordance with those reported in the literature. ${ }^{155}$

$N$-(4-Fluorophe nyl)-4'-me thylbiphe nyl-2-carbox amide (139ya)<smiles>Cc1ccc(-c2ccccc2C(=O)Nc2ccc(F)cc2)cc1</smiles>

The general procedure $\mathbf{G}$ was followed us ing $\mathbf{2 y}$ (108 $\mathrm{mg}, 0.5 \mathrm{mmol})$ and 4-chlorotoluene (88a) (76 mg, $0.6 \mathrm{mmol}$ ) for $16 \mathrm{~h}$. Purification by column chromatography ( $n$-hexane/EtOAc 8:1) yie lded 139ya (134 mg, 88\%) as a colorless solid. M. p.: $=160-162{ }^{\circ} \mathrm{C}$.

${ }^{1} \mathbf{H}-\mathbf{N M R}\left(300 \mathrm{MHz}, \mathrm{CDCl}_{3}\right): \delta=7.84(\mathrm{dd}, J=7.5,1.6 \mathrm{~Hz}, 1 \mathrm{H}), 7.54-7.38(\mathrm{~m}, 3 \mathrm{H}), 7.34(\mathrm{~d}, J=$ $8.1 \mathrm{~Hz}, 2 \mathrm{H}), 7.23(\mathrm{~d}, J=8.1 \mathrm{~Hz}, 2 \mathrm{H}), 7.07-7.03(\mathrm{~m}, 2 \mathrm{H}), 6.93-6.87(\mathrm{~m}, 3 \mathrm{H}), 2.39(\mathrm{~s}, 3 \mathrm{H}), 2.38$ $(\mathrm{s}, 3 \mathrm{H})$.

${ }^{13}$ C-NMR (125 MHz, $\left.\mathrm{CDCl}_{3}\right): \delta=167.1\left(\mathrm{C}_{\mathrm{q}}\right), 159.3\left(\mathrm{C}_{\mathrm{q}}, J_{\mathrm{C}-\mathrm{F}}=243.0 \mathrm{~Hz}\right), 139.4\left(\mathrm{C}_{\mathrm{q}}\right), 137.9$ $\left(\mathrm{C}_{\mathrm{q}}\right), 136.9\left(\mathrm{C}_{\mathrm{q}}\right), 134.9\left(\mathrm{C}_{\mathrm{q}}\right), 133.5\left(\mathrm{C}_{\mathrm{q}}, J_{\mathrm{C}-\mathrm{F}}=2.7 \mathrm{~Hz}\right), 130.6(\mathrm{CH}), 130.3(\mathrm{CH}), 129.6(\mathrm{CH})$, $129.5(\mathrm{CH}), 128.6(\mathrm{CH}), 127.6(\mathrm{CH}), 121.6\left(\mathrm{CH}, J_{\mathrm{C}-\mathrm{F}}=7.9 \mathrm{~Hz}\right), 115.4\left(\mathrm{CH}, J_{\mathrm{C}-\mathrm{F}}=22.5 \mathrm{~Hz}\right)$, $21.2\left(\mathrm{CH}_{3}\right)$.

${ }^{19}$ F-NMR $\left(\mathrm{CDCl}_{3}, 282 \mathrm{MHz}\right): \delta-117.9--118.0(\mathrm{~m})$.

IR (ATR): 3026, 1650, 1505, 1212, 755, $514 \mathrm{~cm}^{-1}$.

MS (EI) m/z (relative intens ity) 305 (20) [M+ $], 267$ (5), 239 (5), 195 (100), 165 (40), 152 (40).

HR-MS (EI) m/z calcd for $\mathrm{C}_{20} \mathrm{H}_{16} \mathrm{NFO}\left[\mathrm{M}^{+}\right]$305.1216, found 305.1209.

The spectral data were in accordance with those reported in the literature. ${ }^{155}$ 
2-(Benzo $[d][1,3]$ dioxol-5-yl)- $N$-(4-fluorophenyl)be nzamide (139zj)<smiles>O=C(Nc1ccc(F)cc1)c1ccccc1-c1ccc2c(c1)OCO2</smiles>

The general procedure $\mathbf{G}$ was followed using $\mathbf{2 z}(108 \mathrm{mg}, 0.5 \mathrm{mmol})$ and 5-chlorobenzo $[d][1,3]$ dioxole $(\mathbf{8 8 j})(94 \mathrm{mg}, 0.6 \mathrm{mmol})$ for $16 \mathrm{~h}$. Purification by column chromatography ( $n$-hexane/EtOAc 8:1) yie lded 139zj (92 mg, 55\%) as a colorless solid. M. p.: $=123-125^{\circ} \mathrm{C}$.

${ }^{1} \mathbf{H}-\mathbf{N M R}\left(300 \mathrm{MHz}, \mathrm{CDCl}_{3}\right): \delta=7.80(\mathrm{dd}, J=7.5,1.5 \mathrm{~Hz}, 1 \mathrm{H}), 7.63-7.40(\mathrm{~m}, 2 \mathrm{H}), 7.38$ (dd, $J$ $=7.5,1.5 \mathrm{~Hz}, 1 \mathrm{H}), 7.18(\mathrm{~m}, 2 \mathrm{H}), 7.03(\mathrm{br} \mathrm{s}, 1 \mathrm{H}), 7.00-6.81(\mathrm{~m}, 5 \mathrm{H}), 5.98(\mathrm{~s}, 2 \mathrm{H})$.

${ }^{13}$ C-NMR (125 MHz, $\left.\mathrm{CDCl}_{3}\right): \delta=167.2\left(\mathrm{C}_{\mathrm{q}}\right), 159.3\left(\mathrm{C}_{\mathrm{q}}, J_{\mathrm{C}-\mathrm{F}}=245.4 \mathrm{~Hz}\right), 148.0\left(\mathrm{C}_{\mathrm{q}}\right), 147.5$ $\left(\mathrm{C}_{\mathrm{q}}\right), 139.0\left(\mathrm{C}_{\mathrm{q}}\right), 135.0\left(\mathrm{C}_{\mathrm{q}}\right), 133.7\left(\mathrm{C}_{\mathrm{q}}\right), 133.5\left(\mathrm{C}_{\mathrm{q}}, J_{\mathrm{C}-\mathrm{F}}=2.8 \mathrm{~Hz}\right), 130.6(\mathrm{CH}), 130.2(\mathrm{CH})$, 129.2 (CH), $127.6(\mathrm{CH}), 122.2(\mathrm{CH}), 121.7\left(\mathrm{CH}, J_{\mathrm{C}-\mathrm{F}}=7.9 \mathrm{~Hz}\right), 115.5\left(\mathrm{CH}, J_{\mathrm{C}-\mathrm{F}}=22.6 \mathrm{~Hz}\right)$, 109.2 (CH), $108.6(\mathrm{CH}), 101.3\left(\mathrm{CH}_{2}\right)$.

${ }^{19}$ F-NMR $\left(376 \mathrm{MHz}, \mathrm{CDCl}_{3}\right): \delta=-117.7--117.8(\mathrm{~m})$.

IR (ATR): 3235, 1646, 1505, 1223, 761, $517 \mathrm{~cm}^{-1}$.

MS (EI) m/z (relative intensity) 335 (40) $\left[\mathrm{M}^{+}\right], 278$ (40), 248 (15), 225 (40), 195 (100), 167 (40).

HR-MS (EI) m/z calcd for $\mathrm{C}_{20} \mathrm{H}_{14} \mathrm{NO}_{3}\left[\mathrm{M}^{+}\right]$335.0958, found 335.0962.

\section{4 '-\{[(tert-B utyldime thyls ilyl)oxy]methyl $\}-N$-(4-me thoxyphe nyl)-[1,1'-biphe nyl]-2-carbox} amide (139xd)<smiles>COc1ccc(NC(=O)c2ccccc2-c2ccc(CO[Sb](C)(C)C)cc2)cc1</smiles>

The general procedure $\mathbf{G}$ was followed using $\mathbf{2 x}(114 \mathrm{mg}, 0.5 \mathrm{mmol})$ and tert-butyl\{(4-chlorobenzyl)oxy\} dimethylsilane (88d) (154 mg, $0.6 \mathrm{mmol})$ for $16 \mathrm{~h}$ (or $0.5 \mathrm{~h})$. 
Purification by column chromatography ( $n$-hexane/EtOAc 5:1) yielded 139xd (143 mg, 64\%; or $152 \mathrm{mg}, 68 \%$ ) as a colorless solid. M. p.: $=97-99^{\circ} \mathrm{C}$.

${ }^{1} \mathbf{H}-\mathrm{NMR}\left(500 \mathrm{MHz}, \mathrm{CDCl}_{3}\right): \delta=7.84(\mathrm{ddd}, J=7.6,1.5,0.5 \mathrm{~Hz}, 1 \mathrm{H}), 7.50(\mathrm{td}, J=7.5,1.5 \mathrm{~Hz}$, 1H), 7.46-7.41 (m, 3H), 7.41-7.36 (m, 3H), 7.00 (d, $J=8.9 \mathrm{~Hz}, 2 \mathrm{H}), 6.80(\mathrm{~s}, 1 \mathrm{H}), 6.74(\mathrm{~d}, J=$ $9.0 \mathrm{~Hz}, 2 \mathrm{H}), 4.76(\mathrm{t}, J=0.8 \mathrm{~Hz}, 2 \mathrm{H}), 3.73$ (s, 3H), 0.93 (s, 9H), 0.09 (s, 6H).

${ }^{13}$ C-NMR (125 MHz, $\left.\mathrm{CDCl}_{3}\right): \delta=167.1\left(\mathrm{C}_{\mathrm{q}}\right), 156.5\left(\mathrm{C}_{\mathrm{q}}\right), 141.4\left(\mathrm{C}_{\mathrm{q}}\right), 139.3\left(\mathrm{C}_{\mathrm{q}}\right), 138.5\left(\mathrm{C}_{\mathrm{q}}\right)$, $135.4\left(\mathrm{C}_{\mathrm{q}}\right), 130.6\left(\mathrm{C}_{\mathrm{q}}\right), 130.5(\mathrm{CH}), 130.3(\mathrm{CH}), 129.5(\mathrm{CH}), 128.7(\mathrm{CH}), 127.7(\mathrm{CH}), 126.5$ $(\mathrm{CH}), 121.8(\mathrm{CH}), 114.0(\mathrm{CH}), 64.6\left(\mathrm{CH}_{2}\right), 55.4\left(\mathrm{CH}_{3}\right), 25.9\left(\mathrm{CH}_{3}\right), 18.4\left(\mathrm{C}_{\mathrm{q}}\right),-5.3\left(\mathrm{CH}_{3}\right)$.

IR (ATR): 3312, 2928, 1646, 1509, 1243, 1087, $823 \mathrm{~cm}^{-1}$.

MS (EI) m/z (relative intensity) 447 (20) [M $\left.{ }^{+}\right], 390$ (100), 375 (5), 316 (15), 195 (10), 165 (30).

HR-MS (EI) m/z calcd for $\mathrm{C}_{27} \mathrm{H}_{33} \mathrm{NO}_{3} \mathrm{Si}\left[\mathrm{M}^{+}\right]$447.2230, found 447.2223.

a) Inte rmolecular Compe tition Experiment between Substrates 88k and 88a:
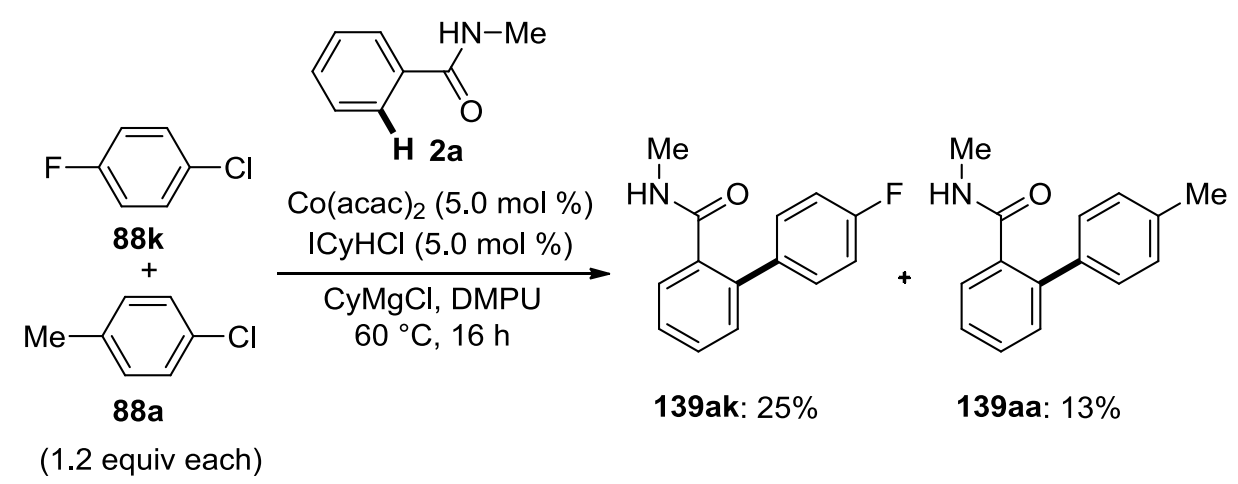

A suspension of $N$-methylbenzamide (2a) (68 mg, $0.5 \mathrm{mmol})$, 4-chlorotoluene (88a) (76 mg, $0.6 \mathrm{mmol})$, 1-chloro-4-fluorobenzene $(\mathbf{8 8 k})(78 \mathrm{mg}, 0.6 \mathrm{mmol}), \mathrm{Co}(\mathrm{acac})_{2}(6.5 \mathrm{mg}, 5.0$ $\mathrm{mol} \%)$, and $\mathrm{ICyHCl}(\mathbf{1 4 0 g})(6.8 \mathrm{mg}, 5.0 \mathrm{~mol} \%)$ in DMPU $(1.0 \mathrm{~mL})$ was stirred for $5 \mathrm{~min}$ at $0{ }^{\circ} \mathrm{C}$. To this mixture, a $1.0 \mathrm{M}$ solution of $\mathrm{CyMgCl}$ in $\mathrm{THF}(1.5 \mathrm{~mL}, 3.0$ equiv) was added dropwise at the same temperature. The reaction mixture was stirred at $60^{\circ} \mathrm{C}$ for $16 \mathrm{~h}$ under an atmosphere of Ar. At ambient temperature, aq. sat. $\mathrm{NH}_{4} \mathrm{Cl}$ solution $(2.0 \mathrm{~mL})$ and $\mathrm{H}_{2} \mathrm{O}(15 \mathrm{~mL})$ were added, and the resulting mixture was extracted with MTBE $(3 \times 20 \mathrm{~mL})$. The combined organic phases were washed with brine $(20 \mathrm{~mL})$ and dried over $\mathrm{Na}_{2} \mathrm{SO}_{4}$. Evaporation of the solvents in vacuo and purification of the residue by column chromatography on silica gel ( $n$-hexane/EtOAc $3: 2 \rightarrow 2: 1)$ yielded 139ak (29 mg, 25\%) and 139aa (15 mg, 13\%) as color less solids. 


\section{4'-Fluoro- $N$-methyl-[1,1'-biphe nyl]-2-carboxamide (139ak)}<smiles>CNC(=O)c1ccccc1-c1ccc(F)cc1</smiles>

M. p.: $=131-133^{\circ} \mathrm{C}$.

${ }^{1} \mathbf{H}-\mathbf{N M R}\left(300 \mathrm{MHz}, \mathrm{CDCl}_{3}\right): \delta=7.61(\mathrm{ddd}, J=7.4,1.6,0.5 \mathrm{~Hz}, 1 \mathrm{H}), 7.45(\mathrm{td}, J=7.4,1.6 \mathrm{~Hz}$, 1H), 7.41-7.33 (m, 3H), 7.33-7.29 (m, 1H), $7.08(\mathrm{t}, J=8.7 \mathrm{~Hz}, 2 \mathrm{H}), 5.26(\mathrm{~s}, 1 \mathrm{H}), 2.69(\mathrm{~d}, J=$ $4.9 \mathrm{~Hz}, 3 \mathrm{H})$.

${ }^{13}$ C-NMR $\left(125 \mathrm{MHz}, \mathrm{CDCl}_{3}\right): \delta=170.0\left(\mathrm{C}_{\mathrm{q}}\right), 162.3\left(\mathrm{C}_{\mathrm{q}}, J_{\mathrm{C}-\mathrm{F}}=246.9 \mathrm{~Hz}\right), 138.2\left(\mathrm{C}_{\mathrm{q}}\right), 136.0\left(\mathrm{C}_{\mathrm{q}}\right.$, $\left.J_{\mathrm{C}-\mathrm{F}}=3.4 \mathrm{~Hz}\right), 135.8\left(\mathrm{C}_{\mathrm{q}}\right), 130.1\left(\mathrm{CH}, J_{\mathrm{C}-\mathrm{F}}=8.1 \mathrm{~Hz}\right), 130.0(\mathrm{CH}), 130.0(\mathrm{CH}), 128.5(\mathrm{CH})$, 127.6 $(\mathrm{CH}), 115.5\left(\mathrm{CH}, J_{\mathrm{C}-\mathrm{F}}=21.4 \mathrm{~Hz}\right), 26.7\left(\mathrm{CH}_{3}\right)$.

${ }^{19}$ F-NMR (282 MHz, $\left.\mathrm{CDCl}_{3}\right): \delta=-114.49--114.62(\mathrm{~m})$.

IR (ATR): 3320, 1633, 1533, 1309, 810, $531 \mathrm{~cm}^{-1}$.

MS (EI) m/z (relative intensity) 229 (30) $\left[\mathrm{M}^{+}\right], 212$ (5), 199 (100), 170 (55), 151 (10).

HR-MS (EI) m/z calcd for $\mathrm{C}_{14} \mathrm{H}_{12} \mathrm{NFO}\left[\mathrm{M}^{+}\right]$229.0903, found 229.0906.

b) Intermolecular Competition Expe riment be tween Substrates $2 \mathrm{c}$ and $2 \mathrm{~s}$ :

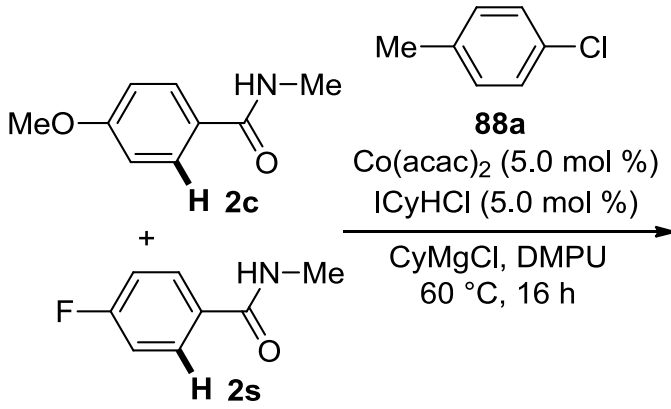

(1.2 equiv each)<smiles>CNC(=O)c1ccc(OC)cc1-c1ccc(C)cc1</smiles>

139ca: $5 \%$

139sa: $19 \%$

A suspension of 4-methoxy- $N$-methylbenzamide (2c) $\quad(99 \quad \mathrm{mg}, 0.6 \mathrm{mmol})$, 4-fluoro- $N$-methylbenzamide (2s) (92 mg, $0.6 \mathrm{mmol}$ ), 4-chlorotoluene (88a) (63 mg, 0.5 $\mathrm{mmol}), \mathrm{Co}(\mathrm{acac})_{2}(6.5 \mathrm{mg}, 5.0 \mathrm{~mol} \%)$, and $\mathrm{ICyHCl}(\mathbf{1 4 0 g})(6.8 \mathrm{mg}, 5.0 \mathrm{~mol} \%)$ in DMPU $(1.0 \mathrm{~mL})$ was stirred for $5 \mathrm{~min}$ at $0{ }^{\circ} \mathrm{C}$. To this mixture, a $1.0 \mathrm{M}$ solution of $\mathrm{CyMgCl}$ in THF ( $2.2 \mathrm{~mL}, 4.4$ equiv) was added dropwise at the same temperature. Then the reaction mixture 
was stirred at $60{ }^{\circ} \mathrm{C}$ for $16 \mathrm{~h}$ under an atmosphere of Ar. At ambient temperature, aq. sat. $\mathrm{NH}_{4} \mathrm{Cl}$ solution $(2.0 \mathrm{~mL})$ and $\mathrm{H}_{2} \mathrm{O}(15 \mathrm{~mL})$ were added and the reaction mixture was extracted with MTBE $(3 \times 20 \mathrm{~mL})$. The combined organic phases were washed with brine $(20$ $\mathrm{mL}$ ) and dried over $\mathrm{Na}_{2} \mathrm{SO}_{4}$. Evaporation of the solvents in vacuo and purification of the residue by column chromatography on silica gel ( $n$-hexane/EtOAc $2: 1 \rightarrow 1: 1)$ yielded 139ca (6 $\mathrm{mg}, 5 \%)$ and 139sa (23 mg, 19\%) as colorless solids.

\section{Cobalt-Catalyzed H/D Exchange in Substrate $\left[D_{5}\right]-2 a$ under the Standard Reaction}

\section{Conditions:}<smiles>CNC(=O)c1ccccc1</smiles>

$\left[D_{5}\right]-2 a$

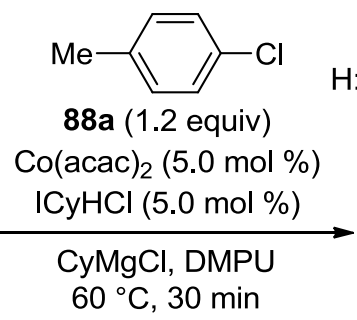

$60^{\circ} \mathrm{C}, 30 \mathrm{~min}$

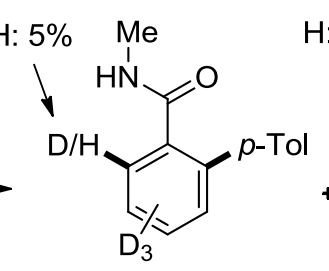

$\left[D_{n}\right]-139 a a: 70 \%$
$\mathrm{H}: 17 \%$ Me $\quad \mathrm{H}: 17 \%$

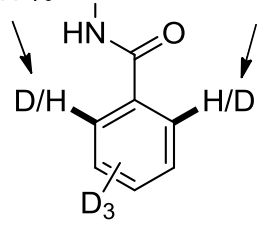

$\left[D_{n}\right]-2 a: 25 \%$

A suspension of $\mathrm{Co}(\mathrm{acac})_{2}(6.5 \mathrm{mg}, 5.0 \mathrm{~mol} \%), \operatorname{ICyHCl}(\mathbf{1 4 0 g})(6.8 \mathrm{mg}, 5.0 \mathrm{~mol} \%),\left[\mathrm{D}_{5}\right]-\mathbf{2 a}$

(70 mg, $0.5 \mathrm{mmol})$ and DMPU $(1.0 \mathrm{~mL})$ was stirred for $5 \mathrm{~min}$ at $0{ }^{\circ} \mathrm{C}$. To this mixture, a 1.0 $\mathrm{M}$ solution of $\mathrm{CyMgCl}$ in $\mathrm{THF}(1.5 \mathrm{~mL}, 3.0$ equiv) was added dropwise at the same temperature followed by 4-chlorotoluene $\mathbf{8 8 a}$ ( $76 \mathrm{mg}, 1.2$ equiv). Then the reaction mixture was stirred at $60{ }^{\circ} \mathrm{C}$ for 30 min under an atmosphere of Ar. At ambient temperature, aq. sat. $\mathrm{NH}_{4} \mathrm{Cl}$ solution $(2.0 \mathrm{~mL})$ and $\mathrm{H}_{2} \mathrm{O}(15 \mathrm{~mL})$ were added. The reaction mixture was extracted with MTBE $(3 \times 20 \mathrm{~mL})$. The combined organic phases were washed with brine $(20 \mathrm{~mL})$ and dried over $\mathrm{Na}_{2} \mathrm{SO}_{4}$. Evaporation of the solvents in vacuo and purification of the residue by column chromatography on silica gel ( $n$-hexane/EtOAc $2: 1)$ yielded product $\left[\mathrm{D}_{n}\right]$-139aa $(81$ $\mathrm{mg}, 70 \%)$ and starting material $\left[\mathrm{D}_{\mathrm{n}}\right]-\mathbf{- 2 a}(18 \mathrm{mg}, 25 \%)$ as colorless solids. The D-incorporation in $\left[D_{n}\right]-139 a a$ and $\left[D_{n}\right]-2 a$ was estimated by ${ }^{1} H-N M R$ spectroscopy. 


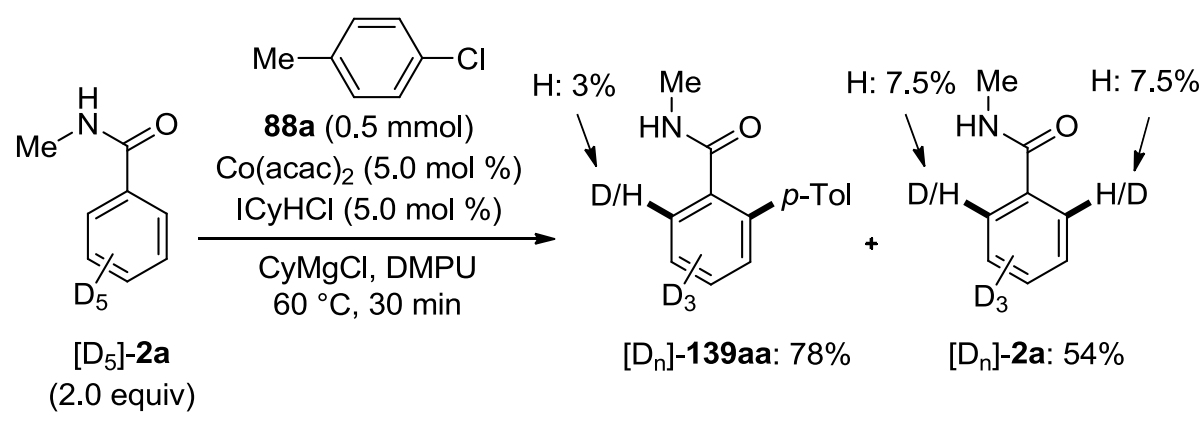

Under the same reaction conditions, from $\left[\mathrm{D}_{5}\right]-\mathbf{2 a}(140 \mathrm{mg}, 1.0 \mathrm{mmol})$ and 4-chlorotoluene 88a (63 mg, $0.5 \mathrm{mmol})$ trated with $1.0 \mathrm{M}$ solution of $\mathrm{CyMgCl}$ in THF ( $2.0 \mathrm{~mL}, 4.0$ equiv) in the presence of $\mathrm{Co}(\mathrm{acac})_{2}(6.5 \mathrm{mg}, 5.0 \mathrm{~mol} \%)$ and $\mathrm{ICyHCl}(\mathbf{1 4 0 g})(6.8 \mathrm{mg}, 5.0 \mathrm{~mol} \%)$ in DMPU $(1.0 \mathrm{~mL})$, product $\left[\mathrm{D}_{\mathrm{n}}\right]-\mathbf{1 3 9} \mathbf{a a}(89 \mathrm{mg}, 78 \%)$ and starting material $\left[\mathrm{D}_{\mathrm{n}}\right]-\mathbf{2 a}(76 \mathrm{mg}$, $54 \%$ ) were obtained as colorless solids after the same workup and column chromatography on silica gel ( $n$-hexane/EtOAc 2:1). The degree of deuterium incorporation in $\left[\mathrm{D}_{\mathrm{n}}\right]-\mathbf{1 3 9} \mathbf{a a}$ and $\left[D_{n}\right]$-2a were estimated by ${ }^{1} H-N M R$ spectroscopy.

KIE De termination Experiments with $2 \mathrm{a}$ and $\left[\mathrm{D}_{5}\right]-2 \mathrm{a}$ as Substrates:

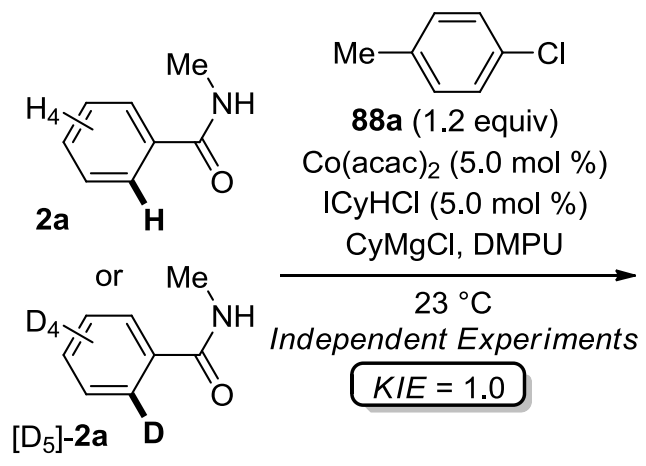<smiles>CNC(=O)c1ccccc1-c1ccc(C)cc1</smiles>

Two parallel reactions with substrate $\mathbf{2 a}$ and deuterated substrate $\left[\mathrm{D}_{5}\right]-\mathbf{2} \mathbf{a}$ were performed under the standard conditions G: In each case, a suspension of 4-chlorotoluene (88a) (151 mg, $1.2 \mathrm{mmol})$, substrates $2 \mathrm{a}(135 \mathrm{mg}, 1.0 \mathrm{mmol})$ or $\left[\mathrm{D}_{5}\right]-\mathbf{2 a}(140 \mathrm{mg}, 1.0 \mathrm{mmol})$, respectively, $\mathrm{Co}(\mathrm{acac})_{2} \quad(12.9 \mathrm{mg}, \quad 5.0 \mathrm{~mol} \%)$, ICyHCl (140g) $\quad(13.5 \mathrm{mg}, \quad 5.0 \mathrm{~mol} \%)$ and 1,3,5-trimethoxybenzene $(168 \mathrm{mg}, 1.0 \mathrm{mmol})$ in DMPU $(2.0 \mathrm{~mL})$ was stirred for $5 \mathrm{~min}$ at $0{ }^{\circ} \mathrm{C}$. To each mixture was added a $1.0 \mathrm{M}$ solution of $\mathrm{CyMgCl}$ in $\mathrm{THF}$ ( $3.0 \mathrm{~mL}, 3.0$ equiv) dropwise at the same temperature, and then the reaction mixtures were stirred at $23{ }^{\circ} \mathrm{C}$ for 2.0 h. For both reactions, an aliquot $(0.2 \mathrm{~mL})$ was removed by syringe every $10 \mathrm{~min}$. The 
consumption of substrate $\mathbf{2 a}$ or $\left[\mathrm{D}_{5}\right] \mathbf{- 2} \mathbf{a}$ and the appearance of the product $\mathbf{1 3 9} \mathbf{a a}$ or $\left[\mathrm{D}_{\mathrm{n}}\right]-\mathbf{1 3 9}$ aa were monitored by $\mathrm{GC}$ analysis. These experiments indicated that the $\mathrm{C}-\mathrm{H}$ bond activation was not the rate-limiting step of the cobalt-catalyzed $\mathrm{C}-\mathrm{H}$ bond arylation reaction.
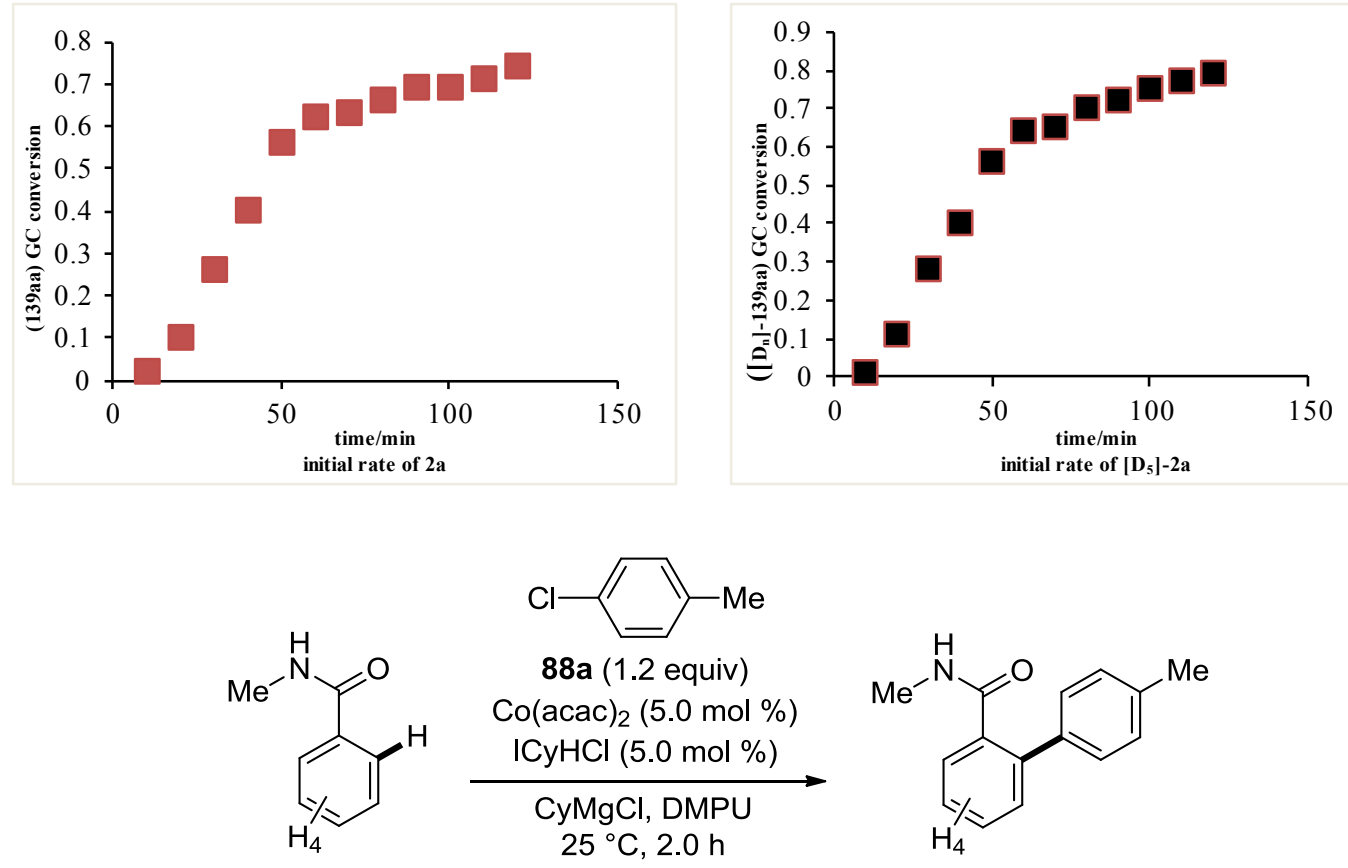

$2 a$

139aa

\begin{tabular}{|c|c|c|c|c|c|c|c|c|c|c|c|c|}
\hline Time/(min) & 10 & 20 & 30 & 40 & 50 & 60 & 70 & 80 & 90 & 100 & 110 & 120 \\
\hline 139aa & $\mathbf{0 . 0 2}$ & $\mathbf{0 . 1}$ & $\mathbf{0 . 2 6}$ & $\mathbf{0 . 4}$ & $\mathbf{0 . 5 6}$ & $\mathbf{0 . 6 2}$ & $\mathbf{0 . 6 3}$ & $\mathbf{0 . 6 6}$ & $\mathbf{0 . 6 9}$ & $\mathbf{0 . 6 9}$ & $\mathbf{0 . 7 1}$ & $\mathbf{0 . 7 4}$ \\
\hline 2a & $\mathbf{0 . 9 8}$ & $\mathbf{0 . 9}$ & $\mathbf{0 . 7 4}$ & $\mathbf{0 . 6}$ & $\mathbf{0 . 4 4}$ & $\mathbf{0 . 3 8}$ & $\mathbf{0 . 3 7}$ & $\mathbf{0 . 3 4}$ & $\mathbf{0 . 3 1}$ & $\mathbf{0 . 3 1}$ & $\mathbf{0 . 2 9}$ & $\mathbf{0 . 2 6}$ \\
\hline
\end{tabular}<smiles>[2H]NC(=O)c1ccccc1[2H]</smiles><smiles>Cc1ccc(C(C(=O)O)(C(=O)O)C(C)(C)C(=O)O)cc1</smiles><smiles>CNC(=O)c1ccccc1-c1ccc(C)cc1</smiles>

$\left[D_{5}\right]-2 a$

$\left[\mathrm{D}_{4-n} \mathrm{H}_{\mathrm{n}}\right]-139 \mathrm{aa}$

\begin{tabular}{|c|c|c|c|c|c|c|c|c|c|c|c|c|}
\hline Time/(min) & 10 & 20 & 30 & 40 & 50 & 60 & 70 & 80 & 90 & 100 & 110 & 120 \\
\hline$\left[\mathrm{D}_{\mathrm{n}}\right]-\mathbf{1 3 9 a a}$ & $\mathbf{0 . 0 1}$ & $\mathbf{0 . 1 1}$ & $\mathbf{0 . 2 8}$ & $\mathbf{0 . 4}$ & $\mathbf{0 . 5 6}$ & $\mathbf{0 . 6 4}$ & $\mathbf{0 . 6 5}$ & $\mathbf{0 . 7}$ & $\mathbf{0 . 7 2}$ & $\mathbf{0 . 7 5}$ & $\mathbf{0 . 7 7}$ & $\mathbf{0 . 7 9}$ \\
\hline$\left[\mathrm{D}_{5}\right]-\mathbf{2 a}$ & $\mathbf{0 . 9 9}$ & $\mathbf{0 . 8 9}$ & $\mathbf{0 . 7 2}$ & $\mathbf{0 . 6}$ & $\mathbf{0 . 4 4}$ & $\mathbf{0 . 3 6}$ & $\mathbf{0 . 3 5}$ & $\mathbf{0 . 3}$ & $\mathbf{0 . 2 8}$ & $\mathbf{0 . 2 5}$ & $\mathbf{0 . 2 3}$ & $\mathbf{0 . 2 1}$ \\
\hline
\end{tabular}

\section{Synthes is of Biaryl Tetrazoles}

\section{5-([1,1'-B iphe nyl]-2-yl)-1-phe nyl-1 $H$-tetrazole (124a)}<smiles>c1ccc(-c2ccccc2-c2nnnn2-c2ccccc2)cc1</smiles> 
To a stirred solution of compound $139 \mathrm{vh}(205 \mathrm{mg}, 0.75 \mathrm{mmol})$ in $\mathrm{CCl}_{4}(10 \mathrm{~mL})$ was added $\mathrm{PCl}_{5}(312 \mathrm{mg}, 1.5 \mathrm{mmol})$ under an atmosphere of Ar. The reaction mixture was stirred at $80{ }^{\circ} \mathrm{C}$ for $3 \mathrm{~h}$. After completion of the reaction, the solvent was removed under reduced pressure. To the residue was added DMF $(5.0 \mathrm{~mL})$ at $0{ }^{\circ} \mathrm{C}$ under an atmosphere of Ar, and the mixture was stirred for $10 \mathrm{~min}$ to get a clear solution. The obtained iminoyl chloride solution was added dropwise to the stirred suspension of $\mathrm{NaN}_{3}(153 \mathrm{mg}, 2.35 \mathrm{mmol})$ in DMF $(5.0 \mathrm{~mL})$ at $0{ }^{\circ} \mathrm{C}$, then the mixture was stirred at $23{ }^{\circ} \mathrm{C}$ overnight. After completion of the reaction as was indicated by TLC monitoring, the reaction mixture was cooled to $0{ }^{\circ} \mathrm{C}$, diluted with $\mathrm{H}_{2} \mathrm{O}$ $(15.0 \mathrm{~mL})^{115}$ and extracted with EtOAc $(3 \times 20 \mathrm{~mL})$ and the combined organic phases were washed with brine $(20 \mathrm{~mL})$ and dried over $\mathrm{Na}_{2} \mathrm{SO}_{4}$. Evaporation of the solvents in vacuo and purification of the residue by column chromatography on silica gel ( $n$-hexane/EtOAc 5:1) yielded product 124a (197 mg, 88\%) as a colorless solid. M. p.: $=124-126^{\circ} \mathrm{C}$.

${ }^{1} \mathbf{H}-\mathbf{N M R}\left(300 \mathrm{MHz}, \mathrm{CDCl}_{3}\right): \delta=7.79(\mathrm{ddd}, J=7.4,1.6,0.5 \mathrm{~Hz}, 1 \mathrm{H}), 7.63-7.51(\mathrm{~m}, 2 \mathrm{H})$, 7.31 (ddd, $J=7.6,1.5,0.5 \mathrm{~Hz}, 1 \mathrm{H}), 7.27-7.21(\mathrm{~m}, 1 \mathrm{H}), 7.16-7.06(\mathrm{~m}, 3 \mathrm{H}), 7.03-6.95$ (m, $2 \mathrm{H}), 6.66-6.51(\mathrm{~m}, 4 \mathrm{H})$.

${ }^{13}$ C-NMR (125 MHz, $\left.\mathrm{CDCl}_{3}\right): \delta=153.9\left(\mathrm{C}_{\mathrm{q}}\right), 141.7\left(\mathrm{C}_{\mathrm{q}}\right), 138.6\left(\mathrm{C}_{\mathrm{q}}\right), 133.5\left(\mathrm{C}_{\mathrm{q}}\right), 131.5(\mathrm{CH})$, $131.2(\mathrm{CH}), 130.3(\mathrm{CH}), 128.9(\mathrm{CH}), 128.9(\mathrm{CH}), 128.3(\mathrm{CH}), 128.1(\mathrm{CH}), 127.9(\mathrm{CH}), 127.1$ (CH), $123.2(\mathrm{CH}), 122.7\left(\mathrm{C}_{\mathrm{q}}\right)$.

IR (ATR): 1503, 1454, 1103, 760, 684, $550 \mathrm{~cm}^{-1}$.

MS (EI) m/z (relative intensity) 298 (15) $\left[\mathrm{M}^{+}\right], 297$ (55), 269 (100), 241 (5), 178 (5), 152 (15).

HR-MS (ESI) m/z calcd for $\mathrm{C}_{19} \mathrm{H}_{15} \mathrm{~N}_{4}\left[\mathrm{M}+\mathrm{H}^{+}\right]$299.1297, found 299.1294.

\section{1-(4-Me thoxyphenyl)-5-(4'-me thyl-[1,1'-biphe nyl]-2-yl)-1H-tetrazole (124b)}<smiles>COc1ccc(-n2nnnc2-c2ccccc2-c2ccc(C)cc2)cc1</smiles>

From the compound 139xa (222 mg, $0.70 \mathrm{mmol})$ in $\mathrm{CCl}_{4}(10 \mathrm{~mL})$ was added $\mathrm{PCl}_{5}(292 \mathrm{mg}$, $1.40 \mathrm{mmol})$ and $\mathrm{NaN}_{3}(137 \mathrm{mg}, 2.10 \mathrm{mmol})$, product 124b (202 $\left.\mathrm{mg}, 84 \%\right)$ was obtained as a 
colorless solid after column chromatography on silica gel ( $n$-hexane/EtOAc 4:1) applying the same protocol as indicated above. M. p.: $=143-145^{\circ} \mathrm{C}$.

${ }^{1}$ H-NMR $\left(300 \mathrm{MHz}, \mathrm{CDCl}_{3}\right): \delta=7.74(\mathrm{dd}, J=7.5,1.6 \mathrm{~Hz}, 1 \mathrm{H}), 7.56(\mathrm{td}, J=7.5,1.6 \mathrm{~Hz}, 1 \mathrm{H})$, $7.49(\mathrm{td}, J=7.5,1.6 \mathrm{~Hz}, 1 \mathrm{H}), 7.30(\mathrm{dd}, J=7.5,1.6 \mathrm{~Hz}, 1 \mathrm{H}), 6.85(\mathrm{~d}, J=8.0 \mathrm{~Hz}, 2 \mathrm{H}), 6.59$ (d, $J$ $=9.0 \mathrm{~Hz}, 2 \mathrm{H}), 6.55-6.44(\mathrm{~m}, 4 \mathrm{H}), 3.75(\mathrm{~s}, 3 \mathrm{H}), 2.26(\mathrm{~s}, 3 \mathrm{H})$.

${ }^{13}$ C-NMR (125 MHz, $\left.\mathrm{CDCl}_{3}\right): \delta=159.8\left(\mathrm{C}_{\mathrm{q}}\right), 154.0\left(\mathrm{C}_{\mathrm{q}}\right), 141.8\left(\mathrm{C}_{\mathrm{q}}\right), 137.0\left(\mathrm{C}_{\mathrm{q}}\right), 135.9\left(\mathrm{C}_{\mathrm{q}}\right)$, $131.3(\mathrm{CH}), 131.1(\mathrm{CH}), 130.1(\mathrm{CH}), 128.9(\mathrm{CH}), 128.0(\mathrm{CH}), 127.6(\mathrm{CH}), 126.6\left(\mathrm{C}_{\mathrm{q}}\right), 124.7$ $(\mathrm{CH}), 122.8\left(\mathrm{C}_{\mathrm{q}}\right), 113.9(\mathrm{CH}), 55.6\left(\mathrm{CH}_{3}\right), 21.1\left(\mathrm{CH}_{3}\right)$.

IR (ATR): 2919, 1515, 1252, 1103, 831, $756 \mathrm{~cm}^{-1}$.

MS (EI) m/z (relative intensity) 342 (10) $\left[\mathrm{M}^{+}\right], 313$ (95), 299 (100), 284 (10), 271 (10), 192 (45).

HR-MS (EI) m/z calcd for $\mathrm{C}_{21} \mathrm{H}_{18} \mathrm{~N}_{4} \mathrm{O}\left[\mathrm{M}^{+}\right]$342.1481, found 342.1484.

\section{\{2'-[1-(4-Me thoxyphe nyl)-1 $H$-te trazol-5-yl]-[1,1'-biphe nyl]-4-yl $\}$ me thanol (124c)}<smiles>COc1ccc(-n2nnnc2-c2ccccc2-c2ccc(CO)cc2)cc1</smiles>

From the compound 139xd (224 mg, $0.50 \mathrm{mmol}), \mathrm{PCl}_{5}(312 \mathrm{mg}, 1.50 \mathrm{mmol})$ and $\mathrm{NaN}_{3}(102$ mg, $1.60 \mathrm{mmol})$, product $124 \mathrm{c}(142 \mathrm{mg}, 79 \%)$ was obtained as a colorless oil after column chromatography on silica gel ( $n$-hexane/EtOAc 4:1) applying the same protocol as indicated above.

${ }^{1} \mathbf{H}-\mathbf{N M R}\left(500 \mathrm{MHz}, \mathrm{CDCl}_{3}\right): \delta=7.77(\mathrm{dd}, J=7.6,1.5 \mathrm{~Hz}, 1 \mathrm{H}), 7.59(\operatorname{tdd}, J=7.6,1.5,0.4 \mathrm{~Hz}$, 1H), 7.54 (tdd, $J=7.6,1.4,0.4 \mathrm{~Hz}, 1 \mathrm{H}), 7.31$ (dd, $J=7.6,1.5 \mathrm{~Hz}, 1 \mathrm{H}), 7.01$ (d, $J=8.2 \mathrm{~Hz}, 2 \mathrm{H})$, $6.63(\mathrm{~d}, J=8.2 \mathrm{~Hz}, 2 \mathrm{H}), 6.61(\mathrm{~d}, J=9.0 \mathrm{~Hz}, 2 \mathrm{H}), 6.51(\mathrm{~d}, J=9.0 \mathrm{~Hz}, 2 \mathrm{H}), 4.23$ (s, 2H), 3.75 (s, $3 \mathrm{H})$.

${ }^{13}$ C-NMR $\left(125 \mathrm{MHz}, \mathrm{CDCl}_{3}\right): \delta=160.0\left(\mathrm{C}_{\mathrm{q}}\right), 153.8\left(\mathrm{C}_{\mathrm{q}}\right), 141.1\left(\mathrm{C}_{\mathrm{q}}\right), 139.0\left(\mathrm{C}_{\mathrm{q}}\right), 134.6\left(\mathrm{C}_{\mathrm{q}}\right)$, $131.5(\mathrm{CH}), 131.4(\mathrm{CH}), 130.3(\mathrm{CH}), 128.6(\mathrm{CH}), 128.3(\mathrm{CH}), 128.2(\mathrm{CH}), 126.6\left(\mathrm{C}_{\mathrm{q}}\right), 124.6$ (CH), $122.9\left(\mathrm{C}_{\mathrm{q}}\right), 114.2(\mathrm{CH}), 55.6\left(\mathrm{CH}_{3}\right), 54.3\left(\mathrm{CH}_{2}\right)$.

IR (ATR): 2099, 1512, 1250, 1025, 831, $694 \mathrm{~cm}^{-1}$. 
MS (EI) m/z (relative intensity) 358 (20) $\left[\mathrm{M}^{+}\right], 357$ (100), 329 (20), 311 (10), 300 (10), 270 (10).

HR-MS (ESI) m/z calcd for $\mathrm{C}_{21} \mathrm{H}_{19} \mathrm{~N}_{4} \mathrm{O}\left[\mathrm{M}+\mathrm{H}^{+}\right]$359.1508, found 359.1511 .

\section{\{2'-(1-Methyl-1 H-te trazol-5-yl)-[1,1'-biphenyl]-4-yl\} me thanol (124d)}<smiles>Cn1nnnc1-c1ccccc1-c1ccc(CO)cc1</smiles>

To a solution of compound 139ad (178 $\mathrm{mg}, 0.50 \mathrm{mmol})$ in $\mathrm{CH}_{2} \mathrm{Cl}_{2}(10 \mathrm{~mL})$ was added phosphorous pentachloride $(125 \mathrm{mg}, 0.60 \mathrm{mmol})$ at -18 to $-20{ }^{\circ} \mathrm{C}$ under an atmosphere of $\mathrm{Ar}$, then the reaction mixture was warmed to $23{ }^{\circ} \mathrm{C}$ for $1 \mathrm{~h}$. The solvent was removed under reduced pressure (bath temperature under $\left.23^{\circ} \mathrm{C}\right)$. The residue was dissolved in DCM $(10 \mathrm{~mL})$, and $\mathrm{TMSN}_{3}(98.0 \mathrm{mg}, 0.85 \mathrm{mmol})$ was added dropwise to this solution at -18 to $-20^{\circ} \mathrm{C}$ under stirring. The reaction mixture was stirred at $23{ }^{\circ} \mathrm{C}$ for $18 \mathrm{~h}$, the reaction was quenched by careful addition of aq. sat. $\mathrm{NaHCO}_{3}$ solution $(15 \mathrm{~mL}) .{ }^{110 \mathrm{c}}$ After extraction with DCM $(3 \times 20$ $\mathrm{mL})$, the combined organic phases were washed with brine $(20 \mathrm{~mL})$ and dried over $\mathrm{Na}_{2} \mathrm{SO}_{4}$. The solvent was evaporated in vacuo, and the residue was purified by column chromatography on silica gel ( $n$-hexane/EtOAc 1:1) to yield products $\mathbf{1 2 4 d}(87.0 \mathrm{mg}, 65 \%)$ as a colorless solid. M. p.: $=143-145^{\circ} \mathrm{C}$.

${ }^{1}$ H-NMR $\left(500 \mathrm{MHz}, \mathrm{CDCl}_{3}\right): \delta=7.63$ (ddd, $\left.J=7.6,1.5,0.5 \mathrm{~Hz}, 1 \mathrm{H}\right), 7.58-7.53(\mathrm{~m}, 2 \mathrm{H})$, 7.49 (td, $J=7.5,1.4 \mathrm{~Hz}, 1 \mathrm{H}), 7.25$ (d, $J=8.5 \mathrm{~Hz}, 2 \mathrm{H}), 7.05$ (d, $J=8.5 \mathrm{~Hz}, 2 \mathrm{H}), 4.65$ (s, 2H), $3.21(\mathrm{~s}, 3 \mathrm{H}), 2.53(\mathrm{~s}, 1 \mathrm{H})$.

${ }^{13}$ C-NMR (125 MHz, $\left.\mathrm{CDCl}_{3}\right): \delta=155.1\left(\mathrm{C}_{\mathrm{q}}\right), 141.5\left(\mathrm{C}_{\mathrm{q}}\right), 141.1\left(\mathrm{C}_{\mathrm{q}}\right), 137.9\left(\mathrm{C}_{\mathrm{q}}\right), 131.7(\mathrm{CH})$, $131.3(\mathrm{CH}), 130.3(\mathrm{CH}), 128.5(\mathrm{CH}), 127.9(\mathrm{CH}), 127.3(\mathrm{CH}), 122.2\left(\mathrm{C}_{\mathrm{q}}\right), 64.3\left(\mathrm{CH}_{2}\right), 33.5$ $\left(\mathrm{CH}_{3}\right)$.

IR (ATR): 3390, 1474, 1206, 1006, 780, $526 \mathrm{~cm}^{-1}$.

MS (EI) m/z (relative intensity) 266 (20) $\left[\mathrm{M}^{+}\right], 265$ (100), 237 (45), 219 (10), 208 (20), 178 (20).

HR-MS (ESI) m/z calcd for $\mathrm{C}_{15} \mathrm{H}_{15} \mathrm{~N}_{4} \mathrm{O}\left[\mathrm{M}+\mathrm{H}^{+}\right]$267.1246, found 267.1238. 
1-(2-Methoxybe nzyl)-5-\{4'-[(4-methoxybe nzyloxy)me thyl]-[1,1'-biphe nyl]-2-yl\}-1 H-te tra zole (124e)<smiles>COc1ccc(COCc2ccc(-c3ccccc3-c3nnnn3Cc3ccccc3OC)cc2)cc1</smiles>

The general procedure $\mathbf{H}$ was followed using 123a (133 $\mathrm{mg}, 0.5 \mathrm{mmol})$ and 4-\{(4-methoxybenzyloxy)methyl\}phenyl dimethylcarbamate (86a) (189 mg, 0.6 mmol). Purification by column chromatography ( $n$-hexane/EtOAc 5:1) yie lded 124e (107 mg, 43\%) as a colorless oil.

${ }^{1} \mathbf{H}-\mathbf{N M R}\left(500 \mathrm{MHz}, \mathrm{CDCl}_{3}\right): \delta=7.61(\mathrm{ddd}, J=7.8,6.4,2.3 \mathrm{~Hz}, 1 \mathrm{H}), 7.54(\mathrm{ddd}, J=7.8,1.3$, $0.7 \mathrm{~Hz}, 1 \mathrm{H}), 7.46-7.39(\mathrm{~m}, 2 \mathrm{H}), 7.27$ (ddd, $J=8.6,8.2,0.6 \mathrm{~Hz}, 4 \mathrm{H}), 7.17$ (ddd, $J=8.3,7.3,1.9$ Hz, 1H), 7.12-7.08 (m, 2H), 6.88 (d, $J=8.7 \mathrm{~Hz}, 2 \mathrm{H}), 6.77$ (ddd, $J=7.6,1.9,0.4 \mathrm{~Hz}, 1 \mathrm{H}), 6.73$ $(\mathrm{td}, J=7.4,1.1 \mathrm{~Hz}, 1 \mathrm{H}), 6.68-6.63(\mathrm{~m}, 1 \mathrm{H}), 4.71(\mathrm{~s}, 2 \mathrm{H}), 4.49(\mathrm{~s}, 2 \mathrm{H}), 4.49(\mathrm{~s}, 2 \mathrm{H}), 3.80(\mathrm{~s}, 3 \mathrm{H})$, $3.48(\mathrm{~s}, 3 \mathrm{H})$.

${ }^{13}$ C-NMR $\left(125 \mathrm{MHz}, \mathrm{CDCl}_{3}\right): \delta=159.2\left(\mathrm{C}_{\mathrm{q}}\right), 156.7\left(\mathrm{C}_{\mathrm{q}}\right), 154.6\left(\mathrm{C}_{\mathrm{q}}\right), 141.5\left(\mathrm{C}_{\mathrm{q}}\right), 138.2\left(\mathrm{C}_{\mathrm{q}}\right)$, $138.2\left(\mathrm{C}_{\mathrm{q}}\right), 138.2\left(\mathrm{C}_{\mathrm{q}}\right), 131.1(\mathrm{CH}), 131.1(\mathrm{CH}), 130.1(\mathrm{CH}), 130.0(\mathrm{CH}), 129.9(\mathrm{CH}), 129.3$ $(\mathrm{CH}), 128.6(\mathrm{CH}), 128.0(\mathrm{CH}), 127.4(\mathrm{CH}), 123.1\left(\mathrm{C}_{\mathrm{q}}\right), 121.5\left(\mathrm{C}_{\mathrm{q}}\right), 120.4(\mathrm{CH}), 113.8(\mathrm{CH})$, $110.2(\mathrm{CH}), 72.1\left(\mathrm{CH}_{2}\right), 71.2\left(\mathrm{CH}_{2}\right), 55.3\left(\mathrm{CH}_{3}\right), 55.1\left(\mathrm{CH}_{3}\right), 46.1\left(\mathrm{CH}_{2}\right)$.

IR (ATR): 2962, 1720, 1257, 1083, 1022, $795 \mathrm{~cm}^{-1}$.

MS (EI) m/z (relative intensity) $492(5)\left[\mathrm{M}^{+}\right], 356$ (30), 250 (10), 235 (10), 207 (20), 121 (100).

HR-MS (ESI) m/z calcd for $\mathrm{C}_{30} \mathrm{H}_{29} \mathrm{~N}_{4} \mathrm{O}_{3}\left[\mathrm{M}+\mathrm{H}^{+}\right]$493.2240, found 493.2225.

1-(2-Methoxybe nzyl)-5-\{4'-[(me thoxyme thoxy)methyl]-[1,1'-biphe nyl]-2-yl\}-1H-tetrazole (124f)<smiles>COCOCc1ccc(-c2ccccc2-c2nnnn2Cc2ccccc2OC)cc1</smiles> 
The general procedure $\mathbf{H}$ was followed using $\mathbf{1 2 3 b}(133 \mathrm{mg}, 0.5 \mathrm{mmol})$ and 4-\{(methoxymethoxy)methyl\}phenyl dimethylcarbamate (84b) (143 mg, $0.6 \mathrm{mmol})$. Purification by column chromatography ( $n$-hexane/EtOAc 4:1) yielded $\mathbf{1 2 4 f}(85 \mathrm{mg}, 41 \%$ ) as a colorless solid. M. p.: $=98-100^{\circ} \mathrm{C}$.

${ }^{1} \mathbf{H}-\mathbf{N M R}\left(500 \mathrm{MHz}, \mathrm{CDCl}_{3}\right): \delta=7.61(\mathrm{ddd}, J=7.8,6.7,2.0 \mathrm{~Hz}, 1 \mathrm{H}), 7.54(\mathrm{ddd}, J=7.8,1.3$, $0.6 \mathrm{~Hz}, 1 \mathrm{H}), 7.47-7.38(\mathrm{~m}, 2 \mathrm{H}), 7.30-7.25(\mathrm{~m}, 2 \mathrm{H}), 7.17(\mathrm{ddd}, J=8.4,7.4,1.9 \mathrm{~Hz}, 1 \mathrm{H}), 7.10(\mathrm{~d}$, $J=8.4 \mathrm{~Hz}, 2 \mathrm{H}), 6.78(\mathrm{ddd}, J=7.5,1.9,0.5 \mathrm{~Hz}, 1 \mathrm{H}), 6.74(\mathrm{td}, J=7.4,1.0 \mathrm{~Hz}, 1 \mathrm{H}), 6.69-6.64(\mathrm{~m}$, 1H), $4.72(\mathrm{~s}, 2 \mathrm{H}), 4.70(\mathrm{~s}, 2 \mathrm{H}), 4.56(\mathrm{~s}, 2 \mathrm{H}), 3.49$ (s, 3H), 3.40 (s, 3H).

${ }^{13}$ C-NMR (125 MHz, $\left.\mathrm{CDCl}_{3}\right): \delta=156.6\left(\mathrm{C}_{\mathrm{q}}\right), 154.6\left(\mathrm{C}_{\mathrm{q}}\right), 141.5\left(\mathrm{C}_{\mathrm{q}}\right), 138.3\left(\mathrm{C}_{\mathrm{q}}\right), 137.7\left(\mathrm{C}_{\mathrm{q}}\right)$, $131.1(\mathrm{CH}), 131.1(\mathrm{CH}), 130.1(\mathrm{CH}), 130.0(\mathrm{CH}), 129.9(\mathrm{CH}), 128.6(\mathrm{CH}), 128.1(\mathrm{CH}), 127.5$ $(\mathrm{CH}), 123.1\left(\mathrm{C}_{\mathrm{q}}\right), 121.5\left(\mathrm{C}_{\mathrm{q}}\right), 120.4(\mathrm{CH}), 110.2(\mathrm{CH}), 95.9\left(\mathrm{CH}_{2}\right), 68.7\left(\mathrm{CH}_{2}\right), 55.5\left(\mathrm{CH}_{3}\right), 55.1$ $\left(\mathrm{CH}_{3}\right), 46.1\left(\mathrm{CH}_{2}\right)$.

IR (ATR): 2950, 1728, 1496, 1249, 1040, $769 \mathrm{~cm}^{-1}$.

MS (EI) m/z (relative intensity) $416(20)\left[\mathrm{M}^{+}\right], 415$ (30), 355 (10), 298 (5), 205 (30), 121 (100).

HR-MS (ESI) m/z calcd for $\mathrm{C}_{24} \mathrm{H}_{25} \mathrm{~N}_{4} \mathrm{O}_{3}\left[\mathrm{M}+\mathrm{H}^{+}\right]$417.1927, found 417.1917.

\section{1-Benzyl-5-(4'-me thyl-[1,1'-biphe nyl]-2-yl)-1 $H$-tetrazole (124g)}<smiles>Cc1ccc(-c2ccccc2-c2nnnn2Cc2ccccc2)cc1</smiles>

The general procedure $\mathbf{H}$ was followed using $123 \mathrm{c}(118 \mathrm{mg}, 0.5 \mathrm{mmol})$ and $p$-tolyl dimethylcarbamate $\mathbf{( 8 4 c )}(107 \mathrm{mg}, 0.6 \mathrm{mmol})$. Purification by column chromatography ( $n$-hexane/EtOAc 5:1) and recrystallization ( $n$-hexane) yielded $\mathbf{1 2 4 g}(83 \mathrm{mg}, 51 \%)$ as a colorless solid. M. p.: $=109-111^{\circ} \mathrm{C}$.

${ }^{1} \mathbf{H}-\mathbf{N M R}\left(300 \mathrm{MHz}, \mathrm{CDCl}_{3}\right): \delta=7.66-7.47(\mathrm{~m}, 2 \mathrm{H}), 7.44-7.27(\mathrm{~m}, 2 \mathrm{H}), 7.22-7.10(\mathrm{~m}, 3 \mathrm{H})$, $7.08(\mathrm{~d}, J=8.2 \mathrm{~Hz}, 2 \mathrm{H}), 7.01(\mathrm{~d}, J=8.2 \mathrm{~Hz}, 2 \mathrm{H}), 6.74(\mathrm{~d}, J=7.8 \mathrm{~Hz}, 2 \mathrm{H}), 4.75$ (s, 2H), 2.32 (s, $3 \mathrm{H})$. 
${ }^{13}$ C-NMR (125 MHz, $\left.\mathrm{CDCl}_{3}\right): \delta=154.6\left(\mathrm{C}_{\mathrm{q}}\right), 141.5\left(\mathrm{C}_{\mathrm{q}}\right), 137.9\left(\mathrm{C}_{\mathrm{q}}\right), 135.8\left(\mathrm{C}_{\mathrm{q}}\right), 133.1\left(\mathrm{C}_{\mathrm{q}}\right)$, 131.4 (CH), $131.2(\mathrm{CH}), 130.1(\mathrm{CH}), 129.6(\mathrm{CH}), 128.6(\mathrm{CH}), 128.4(\mathrm{CH}), 128.4(\mathrm{CH}), 127.7$ $(\mathrm{CH}), 127.5(\mathrm{CH}), 122.6\left(\mathrm{C}_{\mathrm{q}}\right), 50.8\left(\mathrm{CH}_{2}\right), 21.2\left(\mathrm{CH}_{3}\right)$.

IR (ATR): 1435, 1240, 1095, 839, 721, $533 \mathrm{~cm}^{-1}$.

MS (EI) m/z (relative intensity) 326 (55) [M+ $]$, 325 (100), 297 (45), 192 (30), 178 (35), 91 (85).

HR-MS (ESI) m/z calcd for $\mathrm{C}_{21} \mathrm{H}_{19} \mathrm{~N}_{4}\left[\mathrm{M}+\mathrm{H}^{+}\right]$327.1610, found 327.1609.

The spectral data were in accordance with those reported in the literature. ${ }^{109 b}$

\section{3,5-Dimethylphe nanthridin-6(5H)-one (143a)}<smiles>Cc1ccc2c3ccccc3c(=O)n(C)c2c1</smiles>

A suspension of $N, 4^{\prime}$-dimethyl-[1,1'-biphenyl]-2-carboxamide (139aa) (113 mg, $0.5 \mathrm{mmol}$ ) and $\mathrm{K}_{2} \mathrm{~S}_{2} \mathrm{O}_{8}(270 \mathrm{mg}, 1.0 \mathrm{mmol})$ in $\mathrm{H}_{2} \mathrm{O}(5.0 \mathrm{~mL})$ was stirred at $105^{\circ} \mathrm{C}$ for $2.0 \mathrm{~h}$. After completion of the reaction, at ambient temperature the reaction mixture was extracted with MTBE $(3 \times 20$ $\mathrm{mL}$ ) at ambient temperature. The combined organic layers were dried over $\mathrm{Na}_{2} \mathrm{SO}_{4}$ and filtered. The solvent was concentrated in vacuo and purification by column chromatography ( $n$-Hexane/EtOAc 4:1) yielded 143a (93 mg, 83\%) as a colorless solid. M. p.: $=123-125^{\circ} \mathrm{C}$. ${ }^{1}$ H-NMR (300 MHz, CDCl $): \delta=8.53(\mathrm{dd}, J=8.1,0.7 \mathrm{~Hz}, 1 \mathrm{H}), 8.22(\mathrm{~d}, J=8.2 \mathrm{~Hz}, 1 \mathrm{H}), 8.14$ $(\mathrm{dd}, J=8.3,1.1 \mathrm{~Hz}, 1 \mathrm{H}), 7.73(\mathrm{ddt}, J=8.4,7.1,1.4 \mathrm{~Hz}, 1 \mathrm{H}), 7.55(\mathrm{ddt}, J=7.3,5.8,1.3 \mathrm{~Hz}, 1 \mathrm{H})$, 7.29-7.17 (m, 1H), 7.13 (ddd, $J=8.1,1.6,0.8 \mathrm{~Hz}, 1 \mathrm{H}), 3.80$ (d, $J=1.2 \mathrm{~Hz}, 3 \mathrm{H}), 2.51$ (s, 3H). ${ }^{13}$ C-NMR (125 MHz, $\left.\mathrm{CDCl}_{3}\right): \delta=161.6\left(\mathrm{C}_{\mathrm{q}}\right), 139.8\left(\mathrm{C}_{\mathrm{q}}\right), 137.9\left(\mathrm{C}_{\mathrm{q}}\right), 133.6\left(\mathrm{C}_{\mathrm{q}}\right), 132.2(\mathrm{CH})$, $128.8(\mathrm{CH}), 127.3(\mathrm{CH}), 125.1\left(\mathrm{C}_{\mathrm{q}}\right), 123.5(\mathrm{CH}), 123.0(\mathrm{CH}), 121.3(\mathrm{CH}), 116.8\left(\mathrm{C}_{\mathrm{q}}\right), 115.3$ (CH), $30.0\left(\mathrm{CH}_{3}\right), 22.0\left(\mathrm{CH}_{3}\right)$.

IR (ATR): 1644, 1606, 1310, 1098, $769 \mathrm{~cm}^{-1}$.

MS (EI) m/z (relative intensity) 223 (100) [M+], 208 (10), 192 (20), 178 (5), 165 (15), 152 (10).

HR-MS (EI) m/z calcd for $\mathrm{C}_{15} \mathrm{H}_{13} \mathrm{NO}\left[\mathrm{M}^{+}\right]$223.0997, found 223.0994.

The spectral data were in accordance with those reported in the literature. ${ }^{156}$

156 S. A. Glover, A. Goosen, J. Chem. Soc., Perkin Trans. 1 1978, 653-657. 


\section{5-Methylphenanthridin-6(5H)-one (143b)}<smiles>Cn1c(=O)c2ccccc2c2ccccc21</smiles>

Under the same conditions as above, from $N$-methyl-[1,1'-biphenyl]-2-carboxamide (139ah) (106.0 mg, $0.50 \mathrm{mmol})$ and $\mathrm{K}_{2} \mathrm{~S}_{2} \mathrm{O}_{8}(270.0 \mathrm{mg}, 1.00 \mathrm{mmol})$, compound $143 \mathrm{~b}(6.0 \mathrm{mg}, 64 \%)$ was obtained as a colorless solid after column chromatography ( $n$-hexane/EtOAc 4:1). M. p.:= $116-118^{\circ} \mathrm{C}$.

${ }^{1} \mathbf{H}-\mathbf{N M R}\left(300 \mathrm{MHz}, \mathrm{CDCl}_{3}\right): \delta=8.54(\mathrm{dd}, J=8.1,1.3 \mathrm{~Hz}, 1 \mathrm{H}), 8.25(\mathrm{~m}, 2 \mathrm{H}), 7.74(\mathrm{~m}, 1 \mathrm{H})$, $7.55(\mathrm{~m}, 2 \mathrm{H}), 7.39(\mathrm{dd}, J=8.6,1.1 \mathrm{~Hz}, 2 \mathrm{H}), 7.30(\mathrm{~m}, 1 \mathrm{H}), 3.80(\mathrm{~s}, 3 \mathrm{H})$.

${ }^{13}$ C-NMR $\left(125 \mathrm{MHz}, \mathrm{CDCl}_{3}\right): \delta=161.4\left(\mathrm{C}_{\mathrm{q}}\right), 137.9\left(\mathrm{C}_{\mathrm{q}}\right), 133.4\left(\mathrm{C}_{\mathrm{q}}\right), 132.2(\mathrm{CH}), 129.4(\mathrm{CH})$, $128.7(\mathrm{CH}), 127.8(\mathrm{CH}), 125.5\left(\mathrm{C}_{\mathrm{q}}\right), 123.1(\mathrm{CH}), 122.3(\mathrm{CH}), 121.5(\mathrm{CH}), 119.1\left(\mathrm{C}_{\mathrm{q}}\right), 114.9$ (CH), $30.0\left(\mathrm{CH}_{3}\right)$.

IR (ATR): 2918, 1639, 1599, 1300, 1088, $723 \mathrm{~cm}^{-1}$.

MS (EI) m/z (relative intensity) 209 (100) [M+], 178 (40), 166 (5), 152 (25), 139 (10).

HR-MS (EI) m/z calcd for $\mathrm{C}_{14} \mathrm{H}_{11} \mathrm{NO}\left[\mathrm{M}^{+}\right]$209.0841, found 209.0843 .

The spectral data were in accordance with those reported in the literature. ${ }^{157}$

\subsubsection{Analytical Data for the Products of Cobalt(III)-Catalyzed C-H Bond Cyanation of (Hetero)Arenes}

2-(Pyridin-2-yl)be nzonitrile (126a)<smiles>N#Cc1ccccc1-c1ccccn1</smiles>

The general procedure I was followed using 28 a (78 mg, $0.5 \mathrm{mmol}$ ) and NCTS (204 mg, 0.75 mmol). Purification by column chromatography ( $n$-hexane/EtOAc 6:1) yielded 126a (81 mg, $90 \%$ ) as a slight yellow solid. M. p.: $=63-65^{\circ} \mathrm{C}$.

${ }^{1}$ H-NMR $\left(300 \mathrm{MHz}, \mathrm{CDCl}_{3}\right): \delta=8.84-8.71(\mathrm{~m}, 1 \mathrm{H}), 7.91-7.73(\mathrm{~m}, 4 \mathrm{H}), 7.68(\mathrm{dd}, J=7.5$,

${ }^{157}$ H. I wasaki, T. Eguchi, N. Tsutsui, H. Ohno, T, Tanaka, J. Org. Chem. 2008, 73, 7145-7152. 
$1.4 \mathrm{~Hz}, 1 \mathrm{H}), 7.49(\mathrm{td}, J=7.6,1.3 \mathrm{~Hz}, 1 \mathrm{H}), 7.34$ (ddd, $J=7.2,4.8,1.5 \mathrm{~Hz}, 1 \mathrm{H})$.

${ }^{13}$ C-NMR (125 MHz, $\left.\mathrm{CDCl}_{3}\right): \delta=155.1\left(\mathrm{C}_{\mathrm{q}}\right), 149.8(\mathrm{CH}), 143.4\left(\mathrm{C}_{\mathrm{q}}\right), 136.7(\mathrm{CH}), 134.0$ $(\mathrm{CH}), 132.7(\mathrm{CH}), 129.9(\mathrm{CH}), 128.6(\mathrm{CH}), 123.2(\mathrm{CH}), 123.1(\mathrm{CH}), 118.6\left(\mathrm{C}_{\mathrm{q}}\right), 111.0\left(\mathrm{C}_{\mathrm{q}}\right)$.

IR (ATR): 3350, 2224, 1560, 1464, 758, $509 \mathrm{~cm}^{-1}$.

MS (EI) m/z (relative intensity) 180 (100) $\left[\mathrm{M}^{+}\right], 154$ (5), 140 (5), 126 (5), 102 (5), 75 (5).

HR-MS (EI) $\mathrm{m} / \mathrm{z}$ calcd for $\mathrm{C}_{12} \mathrm{H}_{8} \mathrm{~N}_{2}\left[\mathrm{M}^{+}\right]$180.0687, found 180.0684 .

The spectral data were in accordance with those reported in the literature. ${ }^{158}$

\section{4-Methyl-2-(pyridin-2-yl)be nzonitrile (126b)}<smiles>Cc1ccc(C#N)c(-c2ccccn2)c1</smiles>

The general procedure I was followed using $\mathbf{2 8 b}$ ( $85 \mathrm{mg}, 0.5 \mathrm{mmol}$ ) and NCTS (204 mg, 0.75 mmol). Purification by column chromatography ( $n$-hexane $\left./ \mathrm{CH}_{2} \mathrm{Cl}_{2} 1: 1\right)$ yielded $\mathbf{1 2 6} \mathbf{b}(70 \mathrm{mg}$, $72 \%)$ as a colorless solid. M. p.: $=62-63{ }^{\circ} \mathrm{C}$.

${ }^{1} \mathbf{H}-\mathbf{N M R}\left(300 \mathrm{MHz}, \mathrm{CDCl}_{3}\right): \delta=8.76(\mathrm{dd}, J=5.0,1.4 \mathrm{~Hz}, 1 \mathrm{H}), 8.00-7.74(\mathrm{~m}, 2 \mathrm{H}), 7.68(\mathrm{~d}, J$ $=8.0 \mathrm{~Hz}, 1 \mathrm{H}), 7.66(\mathrm{~s}, 1 \mathrm{H}), 7.44-7.27(\mathrm{~m}, 2 \mathrm{H}), 2.48(\mathrm{~s}, 3 \mathrm{H})$.

${ }^{13}$ C-NMR (125 MHz, $\left.\mathrm{CDCl}_{3}\right): \delta=155.2\left(\mathrm{C}_{\mathrm{q}}\right), 149.8(\mathrm{CH}), 143.7\left(\mathrm{C}_{\mathrm{q}}\right), 143.2\left(\mathrm{C}_{\mathrm{q}}\right), 136.6(\mathrm{CH})$, $133.9(\mathrm{CH}), 130.6(\mathrm{CH}), 129.4(\mathrm{CH}), 123.2(\mathrm{CH}), 123.1(\mathrm{CH}), 118.9\left(\mathrm{C}_{\mathrm{q}}\right), 107.9\left(\mathrm{C}_{\mathrm{q}}\right), 21.9$ $\left(\mathrm{CH}_{3}\right)$.

IR (ATR): 3002, 2218, 1587, 1462, 791, $586 \mathrm{~cm}^{-1}$.

MS (EI) m/z (relative intensity) 194 (100) $\left[\mathrm{M}^{+}\right], 179$ (10), 167 (30), 152 (5), 140 (10), 114 (5).

HR-MS (EI) m/z calcd for $\mathrm{C}_{13} \mathrm{H}_{10} \mathrm{~N}_{2}\left[\mathrm{M}^{+}\right]$194.0844, found 194.0849.

The spectral data were in accordance with those reported in the literature. ${ }^{159}$

2-(Pyridin-2-yl)-4-(trifluorome thyl)be nzonitrile (126c)

158 X. Chen, X.-S. Hao, C. E. Good hue, J.-Q. Yu, J. Am. Chem. Soc. 2006, 128, 6790-6791.
159 J. Kim, S. Chang, J. Am. Chem. Soc. 2010, 132, 10272-10274. 
<smiles>N#Cc1ccc(C(F)(F)F)cc1-c1ccccn1</smiles>

The general procedure I was followed using $\mathbf{2 8 c}$ (112 mg, $0.5 \mathrm{mmol})$ and NCTS (204 mg, 0.75 mmol). Purification by column chromatography (n-hexane $/ \mathrm{CH}_{2} \mathrm{Cl}_{2} / \mathrm{EtOAc}$ $1: 1: 0 \rightarrow 1: 1: 0.1)$ yielded $126 \mathrm{c}(110 \mathrm{mg}, 89 \%)$ as a colorless oil.

${ }^{1} \mathbf{H}-\mathbf{N M R}\left(300 \mathrm{MHz}, \mathrm{CDCl}_{3}\right): \delta=8.79(\mathrm{dt}, J=4.8,1.4 \mathrm{~Hz}, 1 \mathrm{H}), 8.14(\mathrm{~d}, J=1.8 \mathrm{~Hz}, 1 \mathrm{H}), 7.92$ $(\mathrm{d}, J=8.1 \mathrm{~Hz}, 1 \mathrm{H}), 7.90-7.80(\mathrm{~m}, 2 \mathrm{H}), 7.75(\mathrm{dd}, J=8.1,1.8 \mathrm{~Hz}, 1 \mathrm{H}), 7.40$ (ddd, $J=6.8,4.8$, $1.8 \mathrm{~Hz}, 1 \mathrm{H})$.

${ }^{13}$ C-NMR (125 MHz, $\left.\mathrm{CDCl}_{3}\right): \delta=153.6\left(\mathrm{C}_{\mathrm{q}}\right), 150.1(\mathrm{CH}), 144.2\left(\mathrm{C}_{\mathrm{q}}\right), 137.0(\mathrm{CH}), 134.6$ $(\mathrm{CH}), 134.6\left(\mathrm{C}_{\mathrm{q}}, J_{\mathrm{C}-\mathrm{F}}=33.0 \mathrm{~Hz}\right), 127.0\left(\mathrm{CH}, J_{\mathrm{C}-\mathrm{F}}=3.8 \mathrm{~Hz}\right), 125.3\left(\mathrm{CH}, J_{\mathrm{C}-\mathrm{F}}=3.8 \mathrm{~Hz}\right), 123.9$ $(\mathrm{CH}), 123.2(\mathrm{CH}), 123.0\left(\mathrm{C}_{\mathrm{q}}, J_{\mathrm{C}-\mathrm{F}}=253.6 \mathrm{~Hz}\right), 117.4\left(\mathrm{C}_{\mathrm{q}}\right), 114.3\left(\mathrm{C}_{\mathrm{q}}, J_{\mathrm{C}-\mathrm{F}}=1.5 \mathrm{~Hz}\right)$.

${ }^{19}$ F-NMR $\left(282 \mathrm{MHz}, \mathrm{CDCl}_{3}\right): \delta=-63.43(\mathrm{~s})$.

IR (ATR): 3056, 2232, 1568, 1335, 1128, $792 \mathrm{~cm}^{-1}$.

MS (EI) m/z (relative intensity) 248 (100) $\left[\mathrm{M}^{+}\right], 226$ (25), 209 (10), 179 (25), 152 (10).

HR-MS (EI) m/z calcd for $\mathrm{C}_{13} \mathrm{H}_{7} \mathrm{~N}_{2} \mathrm{~F}_{3}\left[\mathrm{M}^{+}\right]$248.0561, found 248.0551.

The spectral data were in accordance with those reported in the literature. ${ }^{160}$

Methyl 3-cyano-4-(pyridin-2-yl)benzoate (126d)<smiles>CC(=O)c1ccc(-c2ccccn2)c(C#N)c1</smiles>

The general procedure I was followed using $\mathbf{2 8 d}(107 \mathrm{mg}, 0.5 \mathrm{mmol})$ and NCTS (204 mg, 0.75 mmol). Purification by column chromatography ( $n$-hexane/EtOAc 3:1) yielded 126d (117 mg, 98\%) as a colorless solid. M. p.: $=106-108^{\circ} \mathrm{C}$.

${ }^{1} \mathbf{H}-\mathbf{N M R}\left(300 \mathrm{MHz}, \mathrm{CDCl}_{3}\right): \delta=8.78(\mathrm{ddd}, J=4.8,1.8,1.1 \mathrm{~Hz}, 1 \mathrm{H}), 8.45(\mathrm{dd}, J=1.8,0.5$

${ }^{160}$ X. Jia, D. Yang, W. Wang, F. Luo, J. Cheng, J. Org. Chem. 2009, 74, 9470-9474. 
$\mathrm{Hz}, 1 \mathrm{H}), 8.30(\mathrm{dd}, J=8.2,1.8 \mathrm{~Hz}, 1 \mathrm{H}), 7.93(\mathrm{dd}, J=8.2,0.5 \mathrm{~Hz}, 1 \mathrm{H}), 7.89-7.79(\mathrm{~m}, 2 \mathrm{H})$, 7.38 (ddd, $J=6.7,4.8,1.8 \mathrm{~Hz}, 1 \mathrm{H}), 3.96(\mathrm{~s}, 3 \mathrm{H})$.

${ }^{13}$ C-NMR $\left(125 \mathrm{MHz}, \mathrm{CDCl}_{3}\right): \delta=164.8\left(\mathrm{C}_{\mathrm{q}}\right), 154.1\left(\mathrm{C}_{\mathrm{q}}\right), 150.0(\mathrm{CH}), 146.8\left(\mathrm{C}_{\mathrm{q}}\right), 136.9(\mathrm{CH})$, $135.3(\mathrm{CH}), 133.5(\mathrm{CH}), 130.6\left(\mathrm{C}_{\mathrm{q}}\right), 130.2(\mathrm{CH}), 123.8(\mathrm{CH}), 123.3(\mathrm{CH}), 117.8\left(\mathrm{C}_{\mathrm{q}}\right), 111.4$ $\left(\mathrm{C}_{\mathrm{q}}\right), 52.7\left(\mathrm{CH}_{3}\right)$.

IR (ATR): 3085, 2230, 1725, 1448, 1294, $756 \mathrm{~cm}^{-1}$.

MS (EI) m/z (relative intensity) $238(85)\left[\mathrm{M}^{+}\right], 207$ (100), 179 (70), 152 (40), 125 (15), 89 (15).

HR-MS (EI) $\mathrm{m} / \mathrm{z}$ calcd for $\mathrm{C}_{14} \mathrm{H}_{10} \mathrm{~N}_{2} \mathrm{O}_{2}\left[\mathrm{M}^{+}\right]$238.0742, found 238.0734.

The spectral data were in accordance with those reported in the literature. ${ }^{159}$

\section{5-Ace tyl-2-(pyridin-2-yl)benzonitrile (126e)}<smiles>CC(=O)c1ccc(-c2ccccn2)c(C#N)c1</smiles>

The general procedure I was followed using 28e (98 mg, $0.5 \mathrm{mmol})$ and NCTS (204 mg, 0.75 mmol). Purification by column chromatography ( $n$-hexane/EtOAc 3:1) yielded 126e (108 mg, 97\%) as a colorless solid. M. p.: $=107-108^{\circ} \mathrm{C}$.

${ }^{1} \mathbf{H}-\mathbf{N M R}\left(300 \mathrm{MHz}, \mathrm{CDCl}_{3}\right): \delta=8.79(\mathrm{ddd}, J=4.8,1.8,1.1 \mathrm{~Hz}, 1 \mathrm{H}), 8.35(\mathrm{dd}, J=1.8,0.6$ $\mathrm{Hz}, 1 \mathrm{H}), 8.22$ (dd, $J=8.2,1.8 \mathrm{~Hz}, 1 \mathrm{H}), 7.96(\mathrm{dd}, J=8.2,0.6 \mathrm{~Hz}, 1 \mathrm{H}), 7.90-7.78(\mathrm{~m}, 2 \mathrm{H})$, 7.39 (ddd, $J=6.7,4.8,1.8 \mathrm{~Hz}, 1 \mathrm{H}), 2.66(\mathrm{~s}, 3 \mathrm{H})$.

${ }^{13}$ C-NMR (125 MHz, $\left.\mathrm{CDCl}_{3}\right): \delta=195.4\left(\mathrm{C}_{\mathrm{q}}\right), 154.0\left(\mathrm{C}_{\mathrm{q}}\right), 150.0(\mathrm{CH}), 146.8\left(\mathrm{C}_{\mathrm{q}}\right), 136.9(\mathrm{CH})$, $136.8\left(\mathrm{C}_{\mathrm{q}}\right), 134.1(\mathrm{CH}), 132.0(\mathrm{CH}), 130.4(\mathrm{CH}), 123.9(\mathrm{CH}), 123.3(\mathrm{CH}), 117.9\left(\mathrm{C}_{\mathrm{q}}\right), 111.6$ $\left(\mathrm{C}_{\mathrm{q}}\right), 26.7\left(\mathrm{CH}_{3}\right)$.

IR (ATR): 3077, 2227, 1685, 1467, 1260, $786 \mathrm{~cm}^{-1}$.

MS (EI) m/z (relative intensity) $222(70)\left[\mathrm{M}^{+}\right], 207$ (100), 179 (70), 152 (35), 125 (15), 78 (15).

HR-MS (EI) m/z calcd for $\mathrm{C}_{14} \mathrm{H}_{10} \mathrm{~N}_{2} \mathrm{O}\left[\mathrm{M}^{+}\right]$222.0793, found 222.0790. 
The spectral data were in accordance with those reported in the literature. ${ }^{91}$

\section{Benzo[ $h]$ quinoline-10-carbonitrile (126f)}<smiles>N#Cc1cccc2ccc3cccnc3c12</smiles>

The general procedure I was followed using $\mathbf{2 8 f}$ ( $90 \mathrm{mg}, 0.5 \mathrm{mmol}$ ) and NCTS (204 mg, 0.75 mmol). Purification by column chromatography ( $n$-hexane/ $\mathrm{CH}_{2} \mathrm{Cl}_{2} / \mathrm{EtOAc} / \mathrm{Et}_{3} \mathrm{~N}$ 8:4:1:0.01) yielded $126 f(84 \mathrm{mg}, 82 \%)$ as a colorless solid. M. p.: $=138-140{ }^{\circ} \mathrm{C}$.

${ }^{1} \mathbf{H}-\mathbf{N M R}\left(300 \mathrm{MHz}, \mathrm{CDCl}_{3}\right): \delta=9.13(\mathrm{dd}, J=4.4,1.8 \mathrm{~Hz}, 1 \mathrm{H}), 8.21(\mathrm{dd}, J=8.1,1.8 \mathrm{~Hz}$, 1H), $8.13(\mathrm{ddd}, J=11.8,7.7,1.4 \mathrm{~Hz}, 2 \mathrm{H}), 7.83-7.79(\mathrm{~m}, 2 \mathrm{H}), 7.74(\mathrm{~m}, 1 \mathrm{H}), 7.62(\mathrm{dd}, J=8.1$, $4.4 \mathrm{~Hz}, 1 \mathrm{H})$.

${ }^{13}$ C-NMR (75 MHz, $\left.\mathrm{CDCl}_{3}\right): \delta=148.4(\mathrm{CH}), 144.4\left(\mathrm{C}_{\mathrm{q}}\right), 136.2(\mathrm{CH}), 135.7(\mathrm{CH}), 134.0\left(\mathrm{C}_{\mathrm{q}}\right)$, $132.7(\mathrm{CH}), 130.7\left(\mathrm{C}_{\mathrm{q}}\right), 127.3(\mathrm{CH}), 127.2(\mathrm{CH}), 127.1(\mathrm{CH}), 126.9\left(\mathrm{C}_{\mathrm{q}}\right), 123.0(\mathrm{CH}), 120.8$ $\left(\mathrm{C}_{\mathrm{q}}\right), 108.9\left(\mathrm{C}_{\mathrm{q}}\right)$.

IR (ATR): 2207, 1618, 1422, 829, 714, $664 \mathrm{~cm}^{-1}$.

MS (EI) m/z (relative intensity) 204 (100) [M+], 177 (20), 150 (10), 124 (5), 102 (5).

HR-MS (EI) m/z calcd for $\mathrm{C}_{14} \mathrm{H}_{8} \mathrm{~N}_{2}\left[\mathrm{M}^{+}\right]$204.0687, found 204.0685.

The spectral data were in accordance with those reported in the literature. ${ }^{159}$

\section{2-(5-Fluoropyridin-2-yl)be nzonitrile (126g)}<smiles>N#Cc1ccccc1-c1ccc(F)cn1</smiles>

The general procedure I was followed using $\mathbf{2 8 g}$ ( $87 \mathrm{mg}, 0.5 \mathrm{mmol}$ ) and NCTS (204 mg, 0.75 mmol). Purification by column chromatography ( $n$-hexane $/ \mathrm{CH}_{2} \mathrm{Cl}_{2} / \mathrm{EtOAc}$ 1:1:0.05) yie lded $126 \mathrm{~g}(72 \mathrm{mg}, 73 \%)$ as a colorless solid. M. p.: $=102-105^{\circ} \mathrm{C}$.

${ }^{1} \mathbf{H}-\mathbf{N M R}\left(300 \mathrm{MHz}, \mathrm{CDCl}_{3}\right): \delta=8.61(\mathrm{~d}, J=2.9 \mathrm{~Hz}, 1 \mathrm{H}), 7.78(\mathrm{~m}, 3 \mathrm{H}), 7.67(\mathrm{td}, J=7.7,1.4$ 
$\mathrm{Hz}, 1 \mathrm{H}), 7.58-7.42(\mathrm{~m}, 2 \mathrm{H})$.

${ }^{13}$ C-NMR $\left(125 \mathrm{MHz}, \mathrm{CDCl}_{3}\right): \delta=159.1\left(\mathrm{C}_{\mathrm{q}}, J_{\mathrm{C}-\mathrm{F}}=258.5 \mathrm{~Hz}\right), 151.3\left(\mathrm{C}_{\mathrm{q}}, J_{\mathrm{C}-\mathrm{F}}=4.3 \mathrm{~Hz}\right)$, $142.3\left(\mathrm{C}_{\mathrm{q}}\right), 138.3\left(\mathrm{CH}, J_{\mathrm{C}-\mathrm{F}}=24.0 \mathrm{~Hz}\right), 134.0(\mathrm{CH}), 132.8(\mathrm{CH}), 129.8(\mathrm{CH}), 128.7(\mathrm{CH})$, $124.1\left(\mathrm{CH}, J_{\mathrm{C}-\mathrm{F}}=4.7 \mathrm{~Hz}\right), 123.5\left(\mathrm{CH}, J_{\mathrm{C}-\mathrm{F}}=20.8 \mathrm{~Hz}\right), 118.5\left(\mathrm{C}_{\mathrm{q}}\right), 110.9\left(\mathrm{C}_{\mathrm{q}}\right)$.

${ }^{19}$ F-NMR $\left(282 \mathrm{MHz}, \mathrm{CDCl}_{3}\right): \delta=-126.8(\mathrm{dd}, J=8.0,4.3 \mathrm{~Hz})$.

IR (ATR): 3043, 2220, 1584, 1446, 1223, $750 \mathrm{~cm}^{-1}$.

MS (EI) m/z (relative intens ity) 198 (100) $\left[\mathrm{M}^{+}\right], 179$ (5), 171 (10), 151 (5), 128 (5), 99 (5).

HR-MS (EI) m/z calcd for $\mathrm{C}_{12} \mathrm{H}_{7} \mathrm{~N}_{2} \mathrm{~F}\left[\mathrm{M}^{+}\right]$198.0593, found 198.0585.

\section{2-(4-Ace tylpyridin-2-yl)be nzonitrile (126h)}<smiles>CC(=O)c1ccnc(-c2ccccc2C#N)c1</smiles>

The general procedure I was followed using $\mathbf{2 8 h}(99 \mathrm{mg}, 0.5 \mathrm{mmol})$ and NCTS (204 mg, 0.75 mmol). Purification by column chromatography ( $n$-Hexane $/ \mathrm{CH}_{2} \mathrm{Cl}_{2} / \mathrm{EtOAc}$ 1:1:0.05) yie lded 126h (72 mg, 65\%) as a colorless solid. M. p.: $=124-125^{\circ} \mathrm{C}$.

${ }^{1} \mathbf{H}-\mathbf{N M R}\left(400 \mathrm{MHz}, \mathrm{CDCl}_{3}\right): \delta=9.29(\mathrm{dd}, J=2.3,0.9 \mathrm{~Hz}, 1 \mathrm{H}), 8.36(\mathrm{dd}, J=8.2,2.2 \mathrm{~Hz}$, 1H), 7.91-7.85 (m, 2H), $7.82(\mathrm{ddd}, J=7.5,1.4,0.6 \mathrm{~Hz}, 1 \mathrm{H}), 7.71$ (dd, $J=7.5,1.4 \mathrm{~Hz}, 1 \mathrm{H})$, $7.55(\mathrm{td}, J=7.5,1.4 \mathrm{~Hz}, 1 \mathrm{H}), 2.67(\mathrm{~s}, 3 \mathrm{H})$.

${ }^{13}$ C-NMR $\left(125 \mathrm{MHz}, \mathrm{CDCl}_{3}\right): \delta=196.0\left(\mathrm{C}_{\mathrm{q}}\right), 158.6\left(\mathrm{C}_{\mathrm{q}}\right), 149.9(\mathrm{CH}), 142.1\left(\mathrm{C}_{\mathrm{q}}\right), 136.4(\mathrm{CH})$, $134.3(\mathrm{CH}), 132.8(\mathrm{CH}), 131.3\left(\mathrm{C}_{\mathrm{q}}\right), 130.0(\mathrm{CH}), 129.5(\mathrm{CH}), 122.9(\mathrm{CH}), 118.3\left(\mathrm{C}_{\mathrm{q}}\right), 111.2$ $\left(\mathrm{C}_{\mathrm{q}}\right), 26.9\left(\mathrm{CH}_{3}\right)$.

IR (ATR): 2224, 1676, 1582, 1370, 1269, $761 \mathrm{~cm}^{-1}$.

MS (EI) m/z (relative intensity) 222 (70) $\left[\mathrm{M}^{+}\right], 207$ (100), 179 (50), 152 (65), 125 (15), 102 (10).

HR-MS (EI) m/z calcd for $\mathrm{C}_{14} \mathrm{H}_{10} \mathrm{~N}_{2} \mathrm{O}\left[\mathrm{M}^{+}\right]$222.0793, found 222.0800 .

\section{2,3-Difluoro-6-(pyridin-2-yl)be nzonitrile (126i)}


<smiles>N#Cc1c(-c2ccccn2)ccc(F)c1F</smiles>

The general procedure I was followed using $\mathbf{2 8 i}$ (96 mg, $0.5 \mathrm{mmol}$ ) and NCTS (204 mg, 0.75 mmol). Purification by column chromatography ( $n$-Hexane $/ \mathrm{CH}_{2} \mathrm{Cl}_{2} / \mathrm{EtOAc}$ 2:1:0.1) yie lded 126i (78 mg, $72 \%)$ as a slight yellow solid. M. p.: $=67-68^{\circ} \mathrm{C}$.

${ }^{1} \mathbf{H}-\mathbf{N M R}\left(300 \mathrm{MHz}, \mathrm{CDCl}_{3}\right): \delta=8.82-8.77(\mathrm{~m}, 1 \mathrm{H}), 7.85(\mathrm{td}, J=7.8,1.7 \mathrm{~Hz}, 1 \mathrm{H}), 7.61-7.56$ (m, 2H), $7.41(\mathrm{ddd}, J=7.8,4.9,1.2 \mathrm{~Hz}, 1 \mathrm{H}), 7.31(\mathrm{td}, J=8.9,7.4 \mathrm{~Hz}, 1 \mathrm{H})$.

${ }^{13}$ C-NMR (125 MHz, $\left.\mathrm{CDCl}_{3}\right): \delta=153.4\left(\mathrm{C}_{\mathrm{q}}, J_{\mathrm{C}-\mathrm{F}}=259.0,13.4 \mathrm{~Hz}\right), 150.1(\mathrm{CH}), 149.3\left(\mathrm{C}_{\mathrm{q}}\right.$, $\left.J_{\mathrm{C}-\mathrm{F}}=2.9 \mathrm{~Hz}\right), 148.5\left(\mathrm{C}_{\mathrm{q}}, J_{\mathrm{C}-\mathrm{F}}=259.0,13.4 \mathrm{~Hz}\right), 136.6(\mathrm{CH}), 134.1\left(\mathrm{C}_{\mathrm{q}}, J_{\mathrm{C}-\mathrm{F}}=13.4 \mathrm{~Hz}\right), 130.5$ $\left(\mathrm{CH}, J_{\mathrm{C}-\mathrm{F}}=7.8,4.7 \mathrm{~Hz}\right), 125.2\left(\mathrm{CH}, J_{\mathrm{C}-\mathrm{F}}=3.7 \mathrm{~Hz}\right), 124.2(\mathrm{CH}), 117.9\left(\mathrm{CH}, J_{\mathrm{C}-\mathrm{F}}=18.5 \mathrm{~Hz}\right)$, $116.5\left(\mathrm{C}_{\mathrm{q}}\right), 109.5\left(\mathrm{C}_{\mathrm{q}}, J_{\mathrm{C}-\mathrm{F}}=3.7 \mathrm{~Hz}\right)$.

${ }^{19}$ F-NMR (282 MHz, $\left.\mathrm{CDCl}_{3}\right): \delta=-126.9(\mathrm{~m}),-138.33(\mathrm{~m})$.

IR (ATR): 3089, 2235, 1570, 1426, 1275, $745 \mathrm{~cm}^{-1}$.

MS (EI) m/z (relative intensity) $216(100)\left[\mathrm{M}^{+}\right], 197$ (15), 189 (15), 163 (10), $136(5), 88$ (5).

HR-MS (EI) m/z calcd for $\mathrm{C}_{12} \mathrm{H}_{6} \mathrm{~N}_{2} \mathrm{~F}_{2}\left[\mathrm{M}^{+}\right] 216.0499$, found 216.0492 .

\section{2,3,4-trifluoro-6-(pyridin-2-yl)be nzonitrile (126j)}<smiles>N#Cc1c(-c2ccccn2)cc(F)c(F)c1F</smiles>

The general procedure I was followed using $\mathbf{2 8 j}$ (105 mg, $0.5 \mathrm{mmol}$ ) and NCTS (204 mg, 0.75 mmol). Purification by column chromatography ( $n$-Hexane $/ \mathrm{CH}_{2} \mathrm{Cl}_{2} / \mathrm{EtOAc}$ 2:1:0.1) yie lded 126j (47 mg, 40\%) as a slight yellow solid. M. p.: $=61-63{ }^{\circ} \mathrm{C}$.

${ }^{1} \mathbf{H}-\mathbf{N M R}\left(300 \mathrm{MHz}, \mathrm{CDCl}_{3}\right): \delta=8.75(\mathrm{ddd}, J=4.8,1.7,1.0 \mathrm{~Hz}, 1 \mathrm{H}), 7.92-7.82(\mathrm{~m}, 1 \mathrm{H}), 7.78$ (dt, $J=8.0,1.1 \mathrm{~Hz}, 1 \mathrm{H}), 7.56(\mathrm{ddd}, J=10.4,6.9,2.1 \mathrm{~Hz}, 1 \mathrm{H}), 7.40(\mathrm{ddd}, J=7.4,4.8,1.3 \mathrm{~Hz}$, 1H). 
${ }^{13}$ C-NMR $\left(125 \mathrm{MHz}, \mathrm{CDCl}_{3}\right): \delta=153.6\left(\mathrm{C}_{\mathrm{q}}\right.$, ddd, $\left.J_{\mathrm{C}-\mathrm{F}}=259.7,16.5,3.2 \mathrm{~Hz}\right), 153.6\left(\mathrm{C}_{\mathrm{q}}\right.$, ddd, $\left.J_{\mathrm{C}-\mathrm{F}}=260.1,15.8,2.2 \mathrm{~Hz}\right), 152.1\left(\mathrm{C}_{\mathrm{q}}\right), 150.1(\mathrm{CH}), 140.0\left(\mathrm{C}_{\mathrm{q}}, \mathrm{dd}, J_{\mathrm{C}-\mathrm{F}}=8.1,4.3 \mathrm{~Hz}\right), 139.7\left(\mathrm{C}_{\mathrm{q}}\right.$, $\left.\mathrm{ddd}, J_{\mathrm{C}-\mathrm{F}}=257.6,15.9,2.1 \mathrm{~Hz}\right), 137.1(\mathrm{CH}), 124.2(\mathrm{CH}), 122.9(\mathrm{CH}), 114.2\left(\mathrm{CH}, \mathrm{dd}, J_{\mathrm{C}-\mathrm{F}}=19.8\right.$, $3.3 \mathrm{~Hz}), 111.8\left(\mathrm{C}_{\mathrm{q}}, \mathrm{t}, J_{\mathrm{C}-\mathrm{F}}=2.7 \mathrm{~Hz}\right), 98.0\left(\mathrm{C}_{\mathrm{q}}, \mathrm{dd}, J_{\mathrm{C}-\mathrm{F}}=13.3,3.7 \mathrm{~Hz}\right)$.

${ }^{19}$ F-NMR (282 MHz, $\mathrm{CDCl}_{3}$ ): $\delta=-122.84--123.12(\mathrm{~m}),-124.79$ (ddd, $\left.J=20.0,11.8,2.2 \mathrm{~Hz}\right)$, $-156.60(\mathrm{ddd}, J=21.3,19.9,6.9 \mathrm{~Hz})$.

IR (ATR): 3068, 2235, 1520, 1397, 1072, $789 \mathrm{~cm}^{-1}$.

MS (EI) m/z (relative intensity) 234 (100) $\left[\mathrm{M}^{+}\right], 215$ (25), 207 (20), 181 (15), 157 (5).

HR-MS (EI) $\mathrm{m} / \mathrm{z}$ calcd for $\mathrm{C}_{12} \mathrm{H}_{5} \mathrm{~N}_{2} \mathrm{~F}_{3}\left[\mathrm{M}^{+}\right]$234.0405, found 2344.0408.

\section{5-(tert-Butyl)-2-(pyridin-2-yl)benzonitrile (126k)}<smiles>CC(C)(C)c1ccc(-c2ccccn2)c(C#N)c1</smiles>

The general procedure I was followed using $\mathbf{2 8 k}$ (106 mg, $0.5 \mathrm{mmol}$ ) and NCTS (204 mg, 0.75 mmol). Purification by column chromatography (n-hexane/ $\mathrm{CH}_{2} \mathrm{Cl}_{2} / \mathrm{EtOAc}$ $1: 1: 0 \rightarrow 1: 1: 0.1)$ yie lded $126 \mathbf{k}(79 \mathrm{mg}, 67 \%)$ as a colorless solid. M. p.: $=68-71{ }^{\circ} \mathrm{C}$.

${ }^{1}$ H-NMR $\left(400 \mathrm{MHz}, \mathrm{CDCl}_{3}\right): \delta=8.74(\mathrm{ddd}, J=4.8,1.8,1.0 \mathrm{~Hz}, 1 \mathrm{H}), 7.83-7.73(\mathrm{~m}, 4 \mathrm{H})$, $7.68(\mathrm{dd}, J=8.3,2.1 \mathrm{~Hz}, 1 \mathrm{H}), 7.31(\mathrm{ddd}, J=7.3,4.8,1.4 \mathrm{~Hz}, 1 \mathrm{H}), 1.34(\mathrm{~s}, 9 \mathrm{H})$.

${ }^{13}$ C-NMR $\left(100 \mathrm{MHz}, \mathrm{CDCl}_{3}\right): \delta=155.2\left(\mathrm{C}_{\mathrm{q}}\right), 152.3\left(\mathrm{C}_{\mathrm{q}}\right), 149.9(\mathrm{CH}), 140.6\left(\mathrm{C}_{\mathrm{q}}\right), 136.7(\mathrm{CH})$, $131.1(\mathrm{CH}), 130.2(\mathrm{CH}), 129.7(\mathrm{CH}), 123.0(\mathrm{CH}), 123.0(\mathrm{CH}), 119.2\left(\mathrm{C}_{\mathrm{q}}\right), 110.6\left(\mathrm{C}_{\mathrm{q}}\right), 34.8$ $\left(\mathrm{C}_{\mathrm{q}}\right), 31.0\left(\mathrm{CH}_{3}\right)$.

IR (ATR): 2964, 2223, 1602, 1461, 897, $789 \mathrm{~cm}^{-1}$.

MS (EI) m/z (relative intensity) $236(30)\left[\mathrm{M}^{+}\right], 221$ (100), 205 (10), 193 (25), 166 (5), 152 (5).

HR-MS (EI) m/z calcd for $\mathrm{C}_{16} \mathrm{H}_{16} \mathrm{~N}_{2}\left[\mathrm{M}^{+}\right]$236.1313, found 236.1314.

The spectral data were in accordance with those reported in the literature. ${ }^{161}$

${ }^{161}$ X. Kou, M. Zhao, X. Qiao, Y. Zhu, X. Tong, Z. Shen, Chem. Eur. J. 2013, 19, 16880-16886. 


\section{5-Methoxy-2-(4-methylpyridin-2-yl)benzonitrile (126I)}<smiles>COc1ccc(-c2cc(C)ccn2)c(C#N)c1</smiles>

The general procedure I was followed using 281 (100 mg, $0.5 \mathrm{mmol})$ and NCTS (204 mg, 0.75 mmol). Purification by column chromatography ( $n$-hexane/EtOAc 5:1) yie lded 1261 (88 mg, 79\%) as a colorless solid. M. p.: $=109-111^{\circ} \mathrm{C}$.

${ }^{1} \mathbf{H}-\mathbf{N M R}\left(400 \mathrm{MHz}, \mathrm{CDCl}_{3}\right): \delta=8.55(\mathrm{dd}, J=5.1,1.0 \mathrm{~Hz}, 1 \mathrm{H}), 7.72(\mathrm{dd}, J=8.7,1.0 \mathrm{~Hz}$, 1H), 7.51 (dt, $J=1.7,0.8 \mathrm{~Hz}, 1 \mathrm{H}), 7.22(\mathrm{~d}, J=2.7 \mathrm{~Hz}, 1 \mathrm{H}), 7.16(\mathrm{dd}, J=8.7,2.7 \mathrm{~Hz}, 1 \mathrm{H})$, 7.09 (ddd, $J=5.1,1.5,0.8 \mathrm{~Hz}, 1 \mathrm{H}), 3.84$ (s, 3H), 2.40 (s, 3H).

${ }^{13}$ C-NMR $\left(125 \mathrm{MHz}, \mathrm{CDCl}_{3}\right): \delta=159.3\left(\mathrm{C}_{\mathrm{q}}\right), 154.8\left(\mathrm{C}_{\mathrm{q}}\right), 149.5(\mathrm{CH}), 147.8\left(\mathrm{C}_{\mathrm{q}}\right), 136.2\left(\mathrm{C}_{\mathrm{q}}\right)$, $131.2(\mathrm{CH}), 123.7(\mathrm{CH}), 123.7(\mathrm{CH}), 119.3(\mathrm{CH}), 118.6\left(\mathrm{C}_{\mathrm{q}}\right), 118.3(\mathrm{CH}), 111.7\left(\mathrm{C}_{\mathrm{q}}\right), 55.7$ $\left(\mathrm{CH}_{3}\right), 21.1\left(\mathrm{CH}_{3}\right)$.

IR (ATR): 2925, 2225, 1608, 1466, 1276, $820 \mathrm{~cm}^{-1}$.

MS (EI) m/z (relative intensity) 224 (100) $\left[\mathrm{M}^{+}\right], 209$ (25), 194 (10), 181 (60), 166 (20), 154 (25).

HR-MS (EI) m/z calcd for $\mathrm{C}_{14} \mathrm{H}_{12} \mathrm{~N}_{2} \mathrm{O}\left[\mathrm{M}^{+}\right] 224.0950$, found 224.0952.

\section{5-Fluoro-2-(pyrimidin-2-yl)benzonitrile (126m)}<smiles>N#Cc1cc(F)ccc1-c1ncccn1</smiles>

The general procedure I was followed using $\mathbf{2 8 m}(87 \mathrm{mg}, 0.5 \mathrm{mmol})$ and NCTS (204 mg, 0.75 mmol). Purification by column chromatography ( $n$-hexane/EtOAc 5:1) yielded $\mathbf{1 2 6}$ (60 mg, $60 \%$ ) as a colorless solid. M. p.: $=170-171^{\circ} \mathrm{C}$.

${ }^{1} \mathbf{H}-\mathbf{N M R}\left(300 \mathrm{MHz}, \mathrm{CDCl}_{3}\right): \delta=8.90(\mathrm{~d}, J=4.9 \mathrm{~Hz}, 2 \mathrm{H}), 8.42(\mathrm{dd}, J=8.9,5.6 \mathrm{~Hz}, 1 \mathrm{H})$, 
$7.54(\mathrm{dd}, J=8.1,2.7 \mathrm{~Hz}, 1 \mathrm{H}), 7.46-7.36(\mathrm{~m}, 1 \mathrm{H}), 7.32(\mathrm{t}, J=4.9 \mathrm{~Hz}, 1 \mathrm{H})$.

${ }^{13}$ C-NMR $\left(125 \mathrm{MHz}, \mathrm{CDCl}_{3}\right): \delta=162.9\left(\mathrm{C}_{\mathrm{q}}, J_{\mathrm{C}-\mathrm{F}}=253.4 \mathrm{~Hz}\right), 161.8\left(\mathrm{C}_{\mathrm{q}}\right), 157.2\left(\mathrm{C}_{\mathrm{q}}\right), 136.5$ $\left(\mathrm{CH}, J_{\mathrm{C}-\mathrm{F}}=3.7 \mathrm{~Hz}\right), 132.7\left(\mathrm{CH}, J_{\mathrm{C}-\mathrm{F}}=8.7 \mathrm{~Hz}\right), 121.8\left(\mathrm{CH}, J_{\mathrm{C}-\mathrm{F}}=24.8 \mathrm{~Hz}\right), 120.0\left(\mathrm{CH}, J_{\mathrm{C}-\mathrm{F}}=\right.$ $10.5 \mathrm{~Hz}), 120.0(\mathrm{CH}), 117.6\left(\mathrm{C}_{\mathrm{q}}, J_{\mathrm{C}-\mathrm{F}}=2.7 \mathrm{~Hz}\right), 113.5\left(\mathrm{C}_{\mathrm{q}}, J_{\mathrm{C}-\mathrm{F}}=9.4 \mathrm{~Hz}\right)$.

${ }^{19}$ F-NMR $\left(282 \mathrm{MHz}, \mathrm{CDCl}_{3}\right): \delta=-108.8(\mathrm{td}, J=7.9,5.5 \mathrm{~Hz})$.

IR (ATR): 3062, 2231, 1560, 1415, 1227, $810 \mathrm{~cm}^{-1}$.

MS (EI) m/z (relative intensity) 119 (100) $\left[\mathrm{M}^{+}\right], 172$ (15), 146 (90), 119 (25), 100 (10), 75 (10).

HR-MS (EI) m/z calcd for $\mathrm{C}_{11} \mathrm{H}_{6} \mathrm{~N}_{3} \mathrm{~F}\left[\mathrm{M}^{+}\right]$199.0546, found 199.0538 .

The spectral data were in accordance with those reported in the literature. ${ }^{162}$

\section{5-Methoxy-2-(pyrimidin-2-yl)be nzonitrile (126n)}<smiles>COc1ccc(-c2ncccn2)c(C#N)c1</smiles>

The general procedure I was followed using $\mathbf{2 8 n}$ (93 mg, $0.5 \mathrm{mmol}$ ) and NCTS (204 mg, 0.75 mmol). Purification by column chromatography ( $n$-hexane/EtOAc $6: 1 \rightarrow 4: 1)$ yielded 126n (95 mg, 90\%) as a colorless solid. M. p.: $=127-129^{\circ} \mathrm{C}$.

${ }^{1} \mathbf{H}-\mathbf{N M R}\left(500 \mathrm{MHz}, \mathrm{CDCl}_{3}\right): \delta=8.84(\mathrm{~d}, J=4.8 \mathrm{~Hz}, 2 \mathrm{H}), 8.32(\mathrm{~d}, J=8.9 \mathrm{~Hz}, 1 \mathrm{H}), 7.30(\mathrm{~d}, J$ $=2.7 \mathrm{~Hz}, 1 \mathrm{H}), 7.25-7.22(\mathrm{~m}, 1 \mathrm{H}), 7.19(\mathrm{dd}, J=8.9,2.7 \mathrm{~Hz}, 1 \mathrm{H}), 3.88(\mathrm{~s}, 3 \mathrm{H})$.

${ }^{13}$ C-NMR $\left(125 \mathrm{MHz}, \mathrm{CDCl}_{3}\right): \delta=162.4\left(\mathrm{C}_{\mathrm{q}}\right), 160.6\left(\mathrm{C}_{\mathrm{q}}\right), 157.1(\mathrm{CH}), 132.6\left(\mathrm{C}_{\mathrm{q}}\right), 131.9(\mathrm{CH})$, $119.7(\mathrm{CH}), 119.4(\mathrm{CH}), 118.8\left(\mathrm{C}_{\mathrm{q}}\right), 118.7(\mathrm{CH}), 112.9\left(\mathrm{C}_{\mathrm{q}}\right), 55.8\left(\mathrm{CH}_{3}\right)$.

IR (ATR): 3082, 2222, 1552, 1404, 1288, $803 \mathrm{~cm}^{-1}$.

MS (EI) m/z (relative intensity) $211(100)\left[\mathrm{M}^{+}\right], 196(15), 168$ (10), 158 (40), $128(10), 115$ (10).

HR-MS (EI) m/z calcd for $\mathrm{C}_{12} \mathrm{H}_{9} \mathrm{~N}_{3} \mathrm{O}\left[\mathrm{M}^{+}\right]$211.0746, found 211.0752 .

The spectral data were in accordance with those reported in the literature. ${ }^{162}$

$\overline{162}$ X. Hong, H. Wang, G. Qian, Q. Tan, B. Xu, J. Org. Chem. 2014, 79, 3228-3237. 


\section{2-(1 H-Pyrazol-1-yl)be nzonitrile (126o)}<smiles>N#Cc1ccccc1-n1cccn1</smiles>

The general procedure I was followed using 128a (72 $\mathrm{mg}, 0.5 \mathrm{mmol}$ ) and NCTS (204 mg, 0.75 mmol). Purification by column chromatography ( $n$-hexane $/ \mathrm{CH}_{2} \mathrm{Cl}_{2} / \mathrm{EtOAc} / \mathrm{Et}_{3} \mathrm{~N}$ 20:7:1:0.01) yielded $\mathbf{1 2 6 0}$ (83 $\mathrm{mg}, 98 \%)$ as a colorless oil.

${ }^{1} \mathbf{H}-\mathbf{N M R}\left(300 \mathrm{MHz}, \mathrm{CDCl}_{3}\right): \delta=8.13(\mathrm{~d}, J=2.6 \mathrm{~Hz}, 1 \mathrm{H}), 7.77(\mathrm{~m}, 3 \mathrm{H}), 7.68(\operatorname{td}, J=7.6,1.8$ $\mathrm{Hz}, 1 \mathrm{H}), 7.40$ (td, $J=7.6,1.2 \mathrm{~Hz}, 1 \mathrm{H}), 6.53$ (dd, $J=2.6,1.8 \mathrm{~Hz}, 1 \mathrm{H})$.

${ }^{13}$ C-NMR (75 MHz, $\left.\mathrm{CDCl}_{3}\right): \delta=142.2(\mathrm{CH}), 142.0\left(\mathrm{C}_{\mathrm{q}}\right), 134.4(\mathrm{CH}), 134.0(\mathrm{CH}), 129.5$ $(\mathrm{CH}), 127.2(\mathrm{CH}), 124.3(\mathrm{CH}), 117.0\left(\mathrm{C}_{\mathrm{q}}\right), 108.5(\mathrm{CH}), 105.3\left(\mathrm{C}_{\mathrm{q}}\right)$.

IR (ATR): 3124, 2161, 1700, 1392, 933, $749 \mathrm{~cm}^{-1}$.

MS (EI) m/z (relative intensity) 169 (100) $\left[\mathrm{M}^{+}\right], 142$ (55), 129 (15), 115 (25), 102 (30), 75 (20).

HR-MS (EI) m/z calcd for $\mathrm{C}_{10} \mathrm{H}_{7} \mathrm{~N}_{3}\left[\mathrm{M}^{+}\right]$169.0640, found 169.0644.

The spectral data were in accordance with those reported in the literature. ${ }^{160}$

\section{1-(Pyrimidin-2-yl)-1H-indole-2-carbonitrile (7a)}<smiles>N#Cc1cc2ccccc2n1-c1ncccn1</smiles>

The general procedure I was followed using 7a (98 mg, $0.5 \mathrm{mmol})$ and NCTS (204 mg, $0.75 \mathrm{mmol}$ ). Purification by column chromatography ( $n$-hexane $/ \mathrm{CH}_{2} \mathrm{Cl}_{2}$ 3:2) yielded $\mathbf{1 2 7}$ a (109 mg, 99\%) as a colorless solid. M. p.: $=125-127^{\circ} \mathrm{C}$.

${ }^{1}$ H-NMR $\left(300 \mathrm{MHz}, \mathrm{CDCl}_{3}\right): \delta=8.82(\mathrm{~d}, J=4.8 \mathrm{~Hz}, 2 \mathrm{H}), 8.68(\mathrm{dq}, J=8.6,0.9 \mathrm{~Hz}, 1 \mathrm{H})$, 7.67 (ddd, $J=8.0,1.3,0.8 \mathrm{~Hz}, 1 \mathrm{H}), 7.49$ (ddd, $J=8.5,7.1,1.3 \mathrm{~Hz}, 1 \mathrm{H}), 7.46$ (d, $J=0.8 \mathrm{~Hz}$, 1H), 7.31 (ddd, $J=8.0,7.1,1.0 \mathrm{~Hz}, 1 \mathrm{H}), 7.22(\mathrm{t}, J=4.8 \mathrm{~Hz}, 1 \mathrm{H})$.

${ }^{13}$ C-NMR $\left(125 \mathrm{MHz}, \mathrm{CDCl}_{3}\right): \delta=158.2(\mathrm{CH}), 156.4\left(\mathrm{C}_{\mathrm{q}}\right), 136.5\left(\mathrm{C}_{\mathrm{q}}\right), 127.7\left(\mathrm{C}_{\mathrm{q}}\right), 127.4(\mathrm{CH})$, 
123.4 (CH), $121.9(\mathrm{CH}), 120.9(\mathrm{CH}), 117.9(\mathrm{CH}), 116.1(\mathrm{CH}), 114.1\left(\mathrm{C}_{\mathrm{q}}\right), 108.9\left(\mathrm{C}_{\mathrm{q}}\right)$.

IR (ATR): 3100, 2225, 1565, 1423, 1333, $738 \mathrm{~cm}^{-1}$.

MS (EI) m/z (relative intensity) 220 (100) $\left[\mathrm{M}^{+}\right], 194$ (15), 169 (25), 141 (15), 114 (25), 79 (30).

HR-MS (ESI) $\mathrm{m} / \mathrm{z}$ calcd for $\mathrm{C}_{13} \mathrm{H}_{9} \mathrm{~N}_{4}\left[\mathrm{M}+\mathrm{H}^{+}\right]$221.0827, found 221.0825.

The spectral data were in accordance with those reported in the literature. ${ }^{91}$

\section{5-Methoxy-1-(pyrimidin-2-yl)-1H-indole-2-carbonitrile (127b)}<smiles>COc1ccc2c(c1)cc(C#N)n2-c1ncccn1</smiles>

The general procedure I was followed using $7 \mathbf{b}$ (113 mg, $0.5 \mathrm{mmol})$ and NCTS (204 mg, 0.75 mmol). Purification by column chromatography ( $n$-hexane $/ \mathrm{CH}_{2} \mathrm{Cl}_{2} / \mathrm{EtOAc}$ 2:1:0.01) yie lded 127b (118 mg, 94\%) as a colorless solid. M. p.: $=185-187^{\circ} \mathrm{C}$.

${ }^{1} \mathbf{H}-\mathbf{N M R}\left(500 \mathrm{MHz}, \mathrm{CDCl}_{3}\right): \delta=8.79(\mathrm{~d}, J=4.8 \mathrm{~Hz}, 2 \mathrm{H}), 8.59(\mathrm{dt}, J=9.3,0.7 \mathrm{~Hz}, 1 \mathrm{H}), 7.36$ $(\mathrm{d}, J=0.8 \mathrm{~Hz}, 1 \mathrm{H}), 7.19(\mathrm{t}, J=4.8 \mathrm{~Hz}, 1 \mathrm{H}), 7.11(\mathrm{dd}, J=9.3,2.6 \mathrm{~Hz}, 1 \mathrm{H}), 7.07-7.00(\mathrm{~m}, 1 \mathrm{H})$, $3.86(\mathrm{~s}, 3 \mathrm{H})$.

${ }^{13}$ C-NMR $\left(125 \mathrm{MHz}, \mathrm{CDCl}_{3}\right): \delta=158.1(\mathrm{CH}), 156.4\left(\mathrm{C}_{\mathrm{q}}\right), 156.2\left(\mathrm{C}_{\mathrm{q}}\right), 131.5\left(\mathrm{C}_{\mathrm{q}}\right), 128.4\left(\mathrm{C}_{\mathrm{q}}\right)$, $120.4(\mathrm{CH}), 117.8(\mathrm{CH}), 117.7(\mathrm{CH}), 117.2(\mathrm{CH}), 114.2\left(\mathrm{C}_{\mathrm{q}}\right), 109.0\left(\mathrm{C}_{\mathrm{q}}\right), 102.5(\mathrm{CH}), 55.7$ $\left(\mathrm{CH}_{3}\right)$.

IR (ATR): 2985, 2219, 1568, 1437, 1211, $805 \mathrm{~cm}^{-1}$.

MS (EI) m/z (relative intensity) 250 (100) $\left[\mathrm{M}^{+}\right], 235$ (80), 207 (75), 180 (15), 154 (5), 79 (40).

HR-MS (EI) $\mathrm{m} / \mathrm{z}$ calcd for $\mathrm{C}_{14} \mathrm{H}_{10} \mathrm{~N}_{4} \mathrm{O}\left[\mathrm{M}^{+}\right]$250.0855, found 250.0850 .

The spectral data were in accordance with those reported in the literature. ${ }^{161}$

\section{5-Bromo-1-(pyrimidin-2-yl)-1 $H$-indole-2-carbonitrile (127c)}<smiles>N#Cc1cc2cc(Br)ccc2n1-c1ncccn1</smiles> 
The general procedure I was followed using 7c (137 mg, $0.5 \mathrm{mmol})$ and NCTS (204 mg, 0.75 mmol). Purification by column chromatography ( $n$-hexane/ $\mathrm{CH}_{2} \mathrm{Cl}_{2} / \mathrm{EtOAc} / \mathrm{Et}_{3} \mathrm{~N}$ 8:4:1:0.01) yielded 127c (140 mg, 94\%) as a colorless solid. M. p.: $=234-236{ }^{\circ} \mathrm{C}$.

${ }^{1}$ H-NMR (300 MHz, $\left.\mathrm{CDCl}_{3}\right): \delta=8.80(\mathrm{~d}, J=4.8 \mathrm{~Hz}, 2 \mathrm{H}), 8.56(\mathrm{dt}, J=9.1,0.7 \mathrm{~Hz}, 1 \mathrm{H}), 7.78$ $(\mathrm{dd}, J=2.0,0.7 \mathrm{~Hz}, 1 \mathrm{H}), 7.53(\mathrm{dd}, J=9.1,2.0 \mathrm{~Hz}, 1 \mathrm{H}), 7.35(\mathrm{~d}, J=0.7 \mathrm{~Hz}, 1 \mathrm{H}), 7.22(\mathrm{t}, J=$ $4.8 \mathrm{~Hz}, 1 \mathrm{H})$.

${ }^{13}$ C-NMR $\left(125 \mathrm{MHz}, \mathrm{CDCl}_{3}\right): \delta=158.4(\mathrm{CH}), 156.3\left(\mathrm{C}_{\mathrm{q}}\right), 135.1\left(\mathrm{C}_{\mathrm{q}}\right), 130.4(\mathrm{CH}), 129.3\left(\mathrm{C}_{\mathrm{q}}\right)$, $124.4(\mathrm{CH}), 119.7(\mathrm{CH}), 118.3(\mathrm{CH}), 117.8(\mathrm{CH}), 116.8\left(\mathrm{C}_{\mathrm{q}}\right), 113.6\left(\mathrm{C}_{\mathrm{q}}\right), 110.0\left(\mathrm{C}_{\mathrm{q}}\right)$.

IR (ATR): 3140, 2216, 1576, 1441, 1253, $801 \mathrm{~cm}^{-1}$.

MS (EI) m/z (relative intensity) 298 (100) $\left[\mathrm{M}^{+}\right], 273$ (5), 247 (10), 219 (30), 166 (10), 140 (30).

HR-MS (EI) $\mathrm{m} / \mathrm{z}$ calcd for $\mathrm{C}_{13} \mathrm{H}_{7} \mathrm{~N}_{4}{ }^{79} \mathrm{Br}\left[\mathrm{M}^{+}\right]$297.9854, found 297.9860 .

The spectral data were in accordance with those reported in the literature. ${ }^{161}$

\section{$N$-\{2-Cyano-1-(pyrimidin-2-yl)-1 $H$-indol-5-yl\}acetamide (127e)}<smiles>CC(=O)Nc1ccc2c(c1)cc(C#N)n2-c1ncccn1</smiles>

The general procedure I was followed using 7e (126 mg, $0.5 \mathrm{mmol})$ and NCTS (204 mg, 0.75 mmol). Purification by column chromatography ( $n$-hexane/EtOAc 1:1) yielded 127 e $(98 \mathrm{mg}$, $71 \%$ ) as an off white solid. M. p.: $=284-286{ }^{\circ} \mathrm{C}$.

${ }^{1}$ H-NMR (300 MHz, DMSO- $\left.d_{6}\right): \delta=10.04(\mathrm{~s}, 1 \mathrm{H}), 8.95(\mathrm{dd}, J=4.9,0.7 \mathrm{~Hz}, 2 \mathrm{H}), 8.64-8.40$ $(\mathrm{m}, 1 \mathrm{H}), 8.20(\mathrm{~d}, J=2.2 \mathrm{~Hz}, 1 \mathrm{H}), 7.81(\mathrm{~s}, 1 \mathrm{H}), 7.55(\mathrm{dd}, J=9.2,2.1 \mathrm{~Hz}, 1 \mathrm{H}), 7.49(\mathrm{td}, J=4.9$, $0.7 \mathrm{~Hz}, 1 \mathrm{H}), 2.08$ (s, 3H).

${ }^{13}$ C-NMR (125 MHz, DMSO- $\left.d_{6}\right): \delta=168.0\left(\mathrm{C}_{\mathrm{q}}\right), 158.7(\mathrm{CH}), 155.3\left(\mathrm{C}_{\mathrm{q}}\right), 135.0\left(\mathrm{C}_{\mathrm{q}}\right), 132.0$ $\left(\mathrm{C}_{\mathrm{q}}\right), 127.4\left(\mathrm{C}_{\mathrm{q}}\right), 120.9(\mathrm{CH}), 120.1(\mathrm{CH}), 118.6(\mathrm{CH}), 115.7(\mathrm{CH}), 113.6\left(\mathrm{C}_{\mathrm{q}}\right), 110.9(\mathrm{CH})$, $108.1\left(\mathrm{C}_{\mathrm{q}}\right), 23.9\left(\mathrm{CH}_{3}\right)$.

IR (ATR): 3216, 2215, 1659, 1424, 1260, $809 \mathrm{~cm}^{-1}$. MS (EI) m/z (relative intensity) 277 (45) $\left[\mathrm{M}^{+}\right], 235$ (100), 207 (10), 183 (5), 156 (10), 129 (5). 
HR-MS (EI) $\mathrm{m} / \mathrm{z}$ calcd for $\mathrm{C}_{15} \mathrm{H}_{11} \mathrm{~N}_{5} \mathrm{O}\left[\mathrm{M}^{+}\right]$277.0964, found 277.0973.

\section{7-Ethyl-1-(pyrimidin-2-yl)-1 $H$-indole-2-carbonitrile (127f)}<smiles>CCc1cccc2cc(C#N)n(-c3ncccn3)c12</smiles>

The general procedure I was followed using $7 \mathbf{f}(112 \mathrm{mg}, 0.5 \mathrm{mmol})$ and NCTS (204 mg, 0.75 mmol). Purification by column chromatography ( $n$-hexane $\left./ \mathrm{CH}_{2} \mathrm{Cl}_{2} 2: 1\right)$ yielded $\mathbf{1 2 7} \mathbf{f}(87 \mathrm{mg}$, $70 \%$ ) as a slight yellow oil.

${ }^{1} \mathbf{H}-\mathbf{N M R}\left(500 \mathrm{MHz}, \mathrm{CDCl}_{3}\right): \delta=8.89(\mathrm{~d}, J=4.8 \mathrm{~Hz}, 2 \mathrm{H}), 7.54(\mathrm{~d}, J=1.4 \mathrm{~Hz}, 1 \mathrm{H}), 7.41$ (s, $1 \mathrm{H}), 7.39(\mathrm{t}, J=4.9 \mathrm{~Hz}, 1 \mathrm{H}), 7.30-7.27(\mathrm{~m}, 1 \mathrm{H}), 7.26-7.22(\mathrm{t}, J=7.5 \mathrm{~Hz}, 1 \mathrm{H}), 2.53$ (q, $J=$ $7.5 \mathrm{~Hz}, 2 \mathrm{H}), 0.95(\mathrm{t}, J=7.5 \mathrm{~Hz}, 3 \mathrm{H})$.

${ }^{13}$ C-NMR $\left(125 \mathrm{MHz}, \mathrm{CDCl}_{3}\right): \delta=158.6(\mathrm{CH}), 156.8\left(\mathrm{C}_{\mathrm{q}}\right), 135.7\left(\mathrm{C}_{\mathrm{q}}\right), 129.6\left(\mathrm{C}_{\mathrm{q}}\right), 128.0\left(\mathrm{C}_{\mathrm{q}}\right)$, $127.5(\mathrm{CH}), 123.2(\mathrm{CH}), 120.1(\mathrm{CH}), 120.0(\mathrm{CH}), 118.5(\mathrm{CH}), 113.4\left(\mathrm{C}_{\mathrm{q}}\right), 111.2\left(\mathrm{C}_{\mathrm{q}}\right), 26.3$ $\left(\mathrm{CH}_{2}\right), 13.6\left(\mathrm{CH}_{3}\right)$.

IR (ATR): 2969, 2223, 1561, 1411, 1267, $743 \mathrm{~cm}^{-1}$.

MS (EI) m/z (relative intensity) 248 (100) $\left[\mathrm{M}^{+}\right], 233$ (100), 220 (10), 206 (5), 179 (15), 155 (25).

HR-MS (ESI) $\mathrm{m} / \mathrm{z}$ calcd for $\mathrm{C}_{15} \mathrm{H}_{13} \mathrm{~N}_{4}\left[\mathrm{M}+\mathrm{H}^{+}\right]$249.1140, found 249.1135.

\section{4-Ethoxy-1-(pyrimidin-2-yl)-1 H-indole-2-carbonitrile (127g)}<smiles>CCOc1cccc2c1cc(C#N)n2-c1ncccn1</smiles>

The general procedure I was followed using $7 \mathbf{g}$ (119 mg, $0.5 \mathrm{mmol})$ and NCTS (204 mg, 0.75 mmol). Purification by column chromatography ( $n$-hexane $/ \mathrm{CH}_{2} \mathrm{Cl}_{2}$ 1:1) yielded $\mathbf{1 2 7 g}$ (125 mg, 95\%) as a colorless solid. M. p.: $=170-172{ }^{\circ} \mathrm{C}$.

${ }^{1} \mathbf{H}-\mathbf{N M R}\left(500 \mathrm{MHz}, \mathrm{CDCl}_{3}\right): \delta=8.80(\mathrm{~d}, J=4.8 \mathrm{~Hz}, 2 \mathrm{H}), 8.24-8.09(\mathrm{~m}, 1 \mathrm{H}), 7.59(\mathrm{t}, J=0.6$ 
$\mathrm{Hz}, 1 \mathrm{H}), 7.37$ (dd, $J=8.6,7.9 \mathrm{~Hz}, 1 \mathrm{H}), 7.20$ (t, $J=4.8 \mathrm{~Hz}, 1 \mathrm{H}), 6.65$ (d, $J=7.9 \mathrm{~Hz}, 1 \mathrm{H}), 4.18$ (d, $J=7.0 \mathrm{~Hz}, 2 \mathrm{H}), 1.49(\mathrm{t}, J=7.0 \mathrm{~Hz}, 3 \mathrm{H})$.

${ }^{13}$ C-NMR (125 MHz, $\left.\mathrm{CDCl}_{3}\right): \delta=158.1(\mathrm{CH}), 156.5\left(\mathrm{C}_{\mathrm{q}}\right), 152.7\left(\mathrm{C}_{\mathrm{q}}\right), 137.7\left(\mathrm{C}_{\mathrm{q}}\right), 128.6(\mathrm{CH})$, $119.1\left(\mathrm{C}_{\mathrm{q}}\right), 118.6(\mathrm{CH}), 117.8(\mathrm{CH}), 114.3\left(\mathrm{C}_{\mathrm{q}}\right), 108.5(\mathrm{CH}), 107.2\left(\mathrm{C}_{\mathrm{q}}\right), 103.6(\mathrm{CH}), 63.8$ $\left(\mathrm{CH}_{2}\right), 14.8\left(\mathrm{CH}_{3}\right)$.

IR (ATR): 2977, 2221, 1567, 1431, 1348, $769 \mathrm{~cm}^{-1}$.

MS (EI) m/z (relative intensity) 264 (60) $\left[\mathrm{M}^{+}\right], 236$ (100), 210 (10), 207 (35), 180 (10), 79 (30).

HR-MS (EI) m/z calcd for $\mathrm{C}_{15} \mathrm{H}_{12} \mathrm{~N}_{4} \mathrm{O}\left[\mathrm{M}^{+}\right]$264.1011, found 264.1011 .

3-Methyl-1-(pyrimidin-2-yl)-1 H-indole-2-carbonitrile (127h)<smiles>Cc1c(C#N)n(-c2ncccn2)c2ccccc12</smiles>

The general procedure I was followed using $7 \mathbf{h}$ (105 mg, $0.5 \mathrm{mmol})$ and NCTS (204 mg, 0.75 mmol). Purification by column chromatography ( $n$-hexane/ $\mathrm{CH}_{2} \mathrm{Cl}_{2} / \mathrm{EtOAc} / \mathrm{Et}_{3} \mathrm{~N}$ 8:4:1:0.01) yie lded $\mathbf{1 2 7 h}$ (108 $\mathrm{mg}, 92 \%)$ as a colorless solid. M. p.: $=167-169^{\circ} \mathrm{C}$.

${ }^{1} \mathbf{H}-\mathbf{N M R}\left(300 \mathrm{MHz}, \mathrm{CDCl}_{3}\right): \delta=8.78(\mathrm{~d}, J=4.8 \mathrm{~Hz}, 2 \mathrm{H}), 8.69(\mathrm{dt}, J=8.5,1.0 \mathrm{~Hz}, 1 \mathrm{H}), 7.63$ (ddd, $J=8.0,1.3,0.7 \mathrm{~Hz}, 1 \mathrm{H}), 7.49(\mathrm{ddd}, J=8.5,7.2,1.3 \mathrm{~Hz}, 1 \mathrm{H}), 7.32(\mathrm{ddd}, J=8.0,7.2,1.0$ $\mathrm{Hz}, 1 \mathrm{H}), 7.16(\mathrm{t}, J=4.8 \mathrm{~Hz}, 1 \mathrm{H}), 2.57(\mathrm{~s}, 3 \mathrm{H})$.

${ }^{13}$ C-NMR (125 MHz, $\left.\mathrm{CDCl}_{3}\right): \delta=158.1(\mathrm{CH}), 156.5\left(\mathrm{C}_{\mathrm{q}}\right), 136.4\left(\mathrm{C}_{\mathrm{q}}\right), 131.5\left(\mathrm{C}_{\mathrm{q}}\right), 128.6\left(\mathrm{C}_{\mathrm{q}}\right)$, $127.6(\mathrm{CH}), 123.0(\mathrm{CH}), 120.1(\mathrm{CH}), 117.3(\mathrm{CH}), 116.2(\mathrm{CH}), 113.9\left(\mathrm{C}_{\mathrm{q}}\right), 107.2\left(\mathrm{C}_{\mathrm{q}}\right), 10.0$ $\left(\mathrm{CH}_{3}\right)$.

IR (ATR): 3049, 2215, 1567, 1424, 812, $731 \mathrm{~cm}^{-1}$.

MS (EI) m/z (relative intensity) 234 (100) $\left[\mathrm{M}^{+}\right], 219$ (5), 206 (10), 180 (10), 155 (40), 128 (15).

HR-MS (ESI) m/z calcd for $\mathrm{C}_{14} \mathrm{H}_{11} \mathrm{~N}_{4}\left[\mathrm{M}+\mathrm{H}^{+}\right]$235.0984, found 235.0978 . 
The spectral data were in accordance with those reported in the literature. ${ }^{163}$

\section{4-Oxo-1-(pyrimidin-2-yl)-4,5,6,7-tetrahydro- $1 H$-indole-2-carbonitrile (127i)}<smiles>N#Cc1cc2c(n1-c1ncccn1)CCCC2=O</smiles>

The general procedure I was followed using 7i (107 mg, $0.5 \mathrm{mmol})$, NCTS (204 mg, 0.75 $\mathrm{mmol}), \mathrm{Cp}^{*} \mathrm{CoI}_{2}(\mathrm{CO})(\mathbf{1 1 2}, 12.0 \mathrm{mg}, 5.0 \mathrm{~mol} \%), \mathrm{AgSbF}_{6}(17.2 \mathrm{mg}, 10.0 \mathrm{~mol} \%)$ and $\mathrm{KOAc}$ (5.0 mg, $10 \mathrm{~mol} \%$ ). Purification by column chromatography ( $n$-hexane/EtOAc 1:1) yie lded 127i (108 mg, 91\%) as a yellow solid. M. p.: $=238-241^{\circ} \mathrm{C}$.

${ }^{1} \mathbf{H}-\mathbf{N M R}\left(500 \mathrm{MHz}, \mathrm{CDCl}_{3}\right): \delta=8.83(\mathrm{~d}, J=4.8 \mathrm{~Hz}, 1 \mathrm{H}), 7.38(\mathrm{~s}, 1 \mathrm{H}), 7.36(\mathrm{t}, J=4.8 \mathrm{~Hz}, 1 \mathrm{H})$, $3.25(\mathrm{t}, J=6.2 \mathrm{~Hz}, 2 \mathrm{H}), 2.60-2.52(\mathrm{~m}, 2 \mathrm{H}), 2.24-2.12(\mathrm{~m}, 2 \mathrm{H})$.

${ }^{13}$ C-NMR $\left(125 \mathrm{MHz}, \mathrm{CDCl}_{3}\right): \delta=193.3\left(\mathrm{C}_{\mathrm{q}}\right), 158.6(\mathrm{CH}), 155.2\left(\mathrm{C}_{\mathrm{q}}\right), 147.9\left(\mathrm{C}_{\mathrm{q}}\right), 122.8\left(\mathrm{C}_{\mathrm{q}}\right)$, $120.9(\mathrm{CH}), 119.9(\mathrm{CH}), 113.1\left(\mathrm{C}_{\mathrm{q}}\right), 105.5\left(\mathrm{C}_{\mathrm{q}}\right), 37.7\left(\mathrm{CH}_{2}\right), 25.4\left(\mathrm{CH}_{2}\right), 23.3\left(\mathrm{CH}_{2}\right)$.

IR (ATR): 2963, 2217, 1677, 1577, 1410, $825 \mathrm{~cm}^{-1}$.

MS (EI) m/z (relative intens ity) 238 (80) [M+ $], 223$ (5), 210 (15), 196 (10), 182 (100), 155 (5).

HR-MS (EI) m/z calcd for $\mathrm{C}_{13} \mathrm{H}_{10} \mathrm{~N}_{4} \mathrm{O}\left[\mathrm{M}^{+}\right]$238.0855, found 238.0853 .

\section{1-(Pyrimidin-2-yl)-1 H-pyrrole-2-carbonitrile (145)}<smiles>N#Cc1cccn1-c1ncccn1</smiles>

The general procedure I was followed using 144 (73 mg, $0.5 \mathrm{mmol})$ and NCTS (204 mg, 0.75 mmol). Purification by column chromatography ( $n$-hexane/EtOAc 7:1) yielded $145(83 \mathrm{mg}$, 98\%) as a colorless solid. M. p.: $=120-122^{\circ} \mathrm{C}$.

${ }^{1}$ H-NMR $\left(500 \mathrm{MHz}, \mathrm{CDCl}_{3}\right): \delta=8.71(\mathrm{~d}, J=4.8 \mathrm{~Hz}, 2 \mathrm{H}), 7.96(\mathrm{dd}, J=3.2,1.7 \mathrm{~Hz}, 1 \mathrm{H})$, $7.20(\mathrm{t}, J=4.8 \mathrm{~Hz}, 1 \mathrm{H}), 7.05(\mathrm{dd}, J=3.7,1.7 \mathrm{~Hz}, 1 \mathrm{H}), 6.35(\mathrm{dd}, J=3.7,3.2 \mathrm{~Hz}, 1 \mathrm{H})$.

${ }^{163}$ S. Xu, X. Huang, X. Hong, B. Xu, Org. Lett. 2012, 14, 4614-4617. 
${ }^{13}$ C-NMR $\left(125 \mathrm{MHz}, \mathrm{CDCl}_{3}\right): \delta=158.4(\mathrm{CH}), 155.0\left(\mathrm{C}_{\mathrm{q}}\right), 126.0(\mathrm{CH}), 124.9(\mathrm{CH}), 118.8$ $(\mathrm{CH}), 114.0\left(\mathrm{C}_{\mathrm{q}}\right), 111.7(\mathrm{CH}), 102.8\left(\mathrm{C}_{\mathrm{q}}\right)$.

IR (ATR): 3165, 2219, 1564, 1429, 1177, $742 \mathrm{~cm}^{-1}$.

MS (EI) m/z (relative intensity) 170 (100) $\left[\mathrm{M}^{+}\right], 144$ (10), 118 (10), 91 (5), 79 (40), 52 (20).

HR-MS (EI) $\mathrm{m} / \mathrm{z}$ calcd for $\mathrm{C}_{9} \mathrm{H}_{6} \mathrm{~N}_{4}\left[\mathrm{M}^{+}\right]$170.0592, found 170.0589 .

The spectral data were in accordance with those reported in the literature. ${ }^{163}$

\section{3-(Pyridin-2-yl)thiophe ne-2-carbonitrile (147)}<smiles>N#Cc1sccc1-c1ccccn1</smiles>

The general procedure I was followed using 146 ( $81 \mathrm{mg}, 0.5 \mathrm{mmol})$ and NCTS (204 mg, 0.75 mmol). Purification by column chromatography ( $n$-hexane $/ \mathrm{CH}_{2} \mathrm{Cl}_{2} / \mathrm{EtOAc} / \mathrm{Et}_{3} \mathrm{~N}$ 2:1:0:0 $\rightarrow 8: 4: 1: 0.01)$ yie lded 147 (72 $\mathrm{mg}, 77 \%)$ and 147' (22 mg, 21\%) as slight yellow solids. 147: M. p.: $=43-45^{\circ} \mathrm{C}$.

${ }^{1}$ H-NMR $\left(300 \mathrm{MHz}, \mathrm{CDCl}_{3}\right): \delta=8.70(\mathrm{ddd}, J=4.8,1.8,0.9 \mathrm{~Hz}, 1 \mathrm{H}), 8.02-7.88(\mathrm{~m}, 1 \mathrm{H})$, 7.79 (ddd, $J=8.0,7.5,1.8 \mathrm{~Hz}, 1 \mathrm{H}), 7.66(\mathrm{~d}, J=5.2 \mathrm{~Hz}, 1 \mathrm{H}), 7.59$ (d, $J=5.2 \mathrm{~Hz}, 1 \mathrm{H}), 7.30$ (ddd, $J=7.5,4.8,0.9 \mathrm{~Hz}, 1 \mathrm{H}$ ).

${ }^{13}$ C-NMR $\left(125 \mathrm{MHz}, \mathrm{CDCl}_{3}\right): \delta=150.8\left(\mathrm{C}_{\mathrm{q}}\right), 149.9(\mathrm{CH}), 149.8\left(\mathrm{C}_{\mathrm{q}}\right), 136.9(\mathrm{CH}), 131.6$ $(\mathrm{CH}), 128.2(\mathrm{CH}), 123.5(\mathrm{CH}), 121.8(\mathrm{CH}), 114.6\left(\mathrm{C}_{\mathrm{q}}\right), 106.2\left(\mathrm{C}_{\mathrm{q}}\right)$.

IR (ATR): 3101, 2210, 1582, 1430, 894, $735 \mathrm{~cm}^{-1}$.

MS (EI) m/z (relative intensity) $186(100)\left[\mathrm{M}^{+}\right], 159(30), 142$ (20), 129 (5), 114 (10), 78 (10).

HR-MS (ESI) m/z calcd for $\mathrm{C}_{10} \mathrm{H}_{7} \mathrm{~N}_{2} \mathrm{~S}\left[\mathrm{M}+\mathrm{H}^{+}\right]$187.0330, found 187.0326.

\section{3-(Pyridin-2-yl)thiophe ne-2,4-dicarbonitrile (147')}<smiles>N#Cc1csc(C#N)c1-c1ccccn1</smiles>

M. p.: $=153-155^{\circ} \mathrm{C}$. 
${ }^{1} \mathbf{H}-\mathbf{N M R}\left(300 \mathrm{MHz}, \mathrm{CDCl}_{3}\right): \delta=8.85-8.78(\mathrm{~m}, 1 \mathrm{H}), 8.21(\mathrm{~s}, 1 \mathrm{H}), 7.95-7.84(\mathrm{~m}, 2 \mathrm{H}), 7.43$ (ddd, $J=6.4,4.8,2.4 \mathrm{~Hz}, 1 \mathrm{H})$.

${ }^{13}$ C-NMR $\left(125 \mathrm{MHz}, \mathrm{CDCl}_{3}\right): \delta=150.2(\mathrm{CH}), 150.0\left(\mathrm{C}_{\mathrm{q}}\right), 148.2\left(\mathrm{C}_{\mathrm{q}}\right), 140.8(\mathrm{CH}), 137.2(\mathrm{CH})$, 124.8 (CH), $122.9(\mathrm{CH}), 113.4\left(\mathrm{C}_{\mathrm{q}}\right), 112.5\left(\mathrm{C}_{\mathrm{q}}\right), 112.2\left(\mathrm{C}_{\mathrm{q}}\right), 109.4\left(\mathrm{C}_{\mathrm{q}}\right)$.

IR (ATR): 3088, 2218, 1586, 1465, 993, $744 \mathrm{~cm}^{-1}$.

MS (EI) m/z (relative intensity) 211 (100) $\left[\mathrm{M}^{+}\right], 184$ (65), 167 (10), 158 (15), 139 (5), 78 (10).

HR-MS (EI) m/z calcd for $\mathrm{C}_{11} \mathrm{H}_{5} \mathrm{~N}_{3} \mathrm{~S}\left[\mathrm{M}^{+}\right]$211.0204, found 211.0205 .

$1 H$-Indole-2-carbonitrile (127j)<smiles>N#Cc1cc2ccccc2[nH]1</smiles>

A mixture of 127a (242 mg, $1.10 \mathrm{mmol})$, NaOEt (300 mg, $4.40 \mathrm{mmol})$ and DMSO (6.0 mL) was stirred at $110{ }^{\circ} \mathrm{C}$ under an $\mathrm{Ar}$ atmosphere for $24 \mathrm{~h}$. At ambient temperature, the reaction mixture was diluted with EtOAc $(30 \mathrm{~mL})$ and washed with brine $(30 \mathrm{~mL})$. The aqueous phase was extracted with EtOAc $(2 \times 30 \mathrm{~mL})$, and the combined organic phase was dried over $\mathrm{Na}_{2} \mathrm{SO}_{4}$. After filtration and evaporation of the solvents in vacuo, the crude product was purified by column chromatography on silica gel ( $n$-hexane/EtOAc 10:1) to yield 127j (153 mg, 98\%) as a colorless solid. M. p.: $97-98^{\circ} \mathrm{C}$.

${ }^{1} \mathbf{H}-\mathbf{N M R}\left(500 \mathrm{MHz}, \mathrm{CDCl}_{3}\right): \delta=8.64$ (br s, 1H), $7.66(\mathrm{dd}, J=8.1,0.9 \mathrm{~Hz}, 1 \mathrm{H}), 7.46-7.32$ (m, 2H), 7.23-7.18 (m, 2H).

${ }^{13}$ C-NMR $\left(125 \mathrm{MHz}, \mathrm{CDCl}_{3}\right): \delta=136.7\left(\mathrm{C}_{\mathrm{q}}\right), 126.2(\mathrm{CH}), 126.1\left(\mathrm{C}_{\mathrm{q}}\right), 122.0(\mathrm{CH}), 121.7$ $(\mathrm{CH}), 114.4(\mathrm{CH}), 114.1\left(\mathrm{C}_{\mathrm{q}}\right), 111.6(\mathrm{CH}), 106.2\left(\mathrm{C}_{\mathrm{q}}\right)$. IR (ATR): 3273, 2235, 1521, 1409, $1224,806,732,652 \mathrm{~cm}^{-1}$.

MS (EI) m/z (relative intensity) 142 (100) $\left[\mathrm{M}^{+}\right], 115$ (40), 98 (5), 89 (10), 74 (5), 63 (10).

HR-MS (EI) $\mathrm{m} / \mathrm{z}$ calcd for $\mathrm{C}_{9} \mathrm{H}_{6} \mathrm{~N}_{2}\left[\mathrm{M}^{+}\right]$142.0531, found 142.0528.

The spectral data were in accordance with those reported in the literature. ${ }^{161}$

Intermolecular Competition Experiment between Substrates 28b and 28c: 


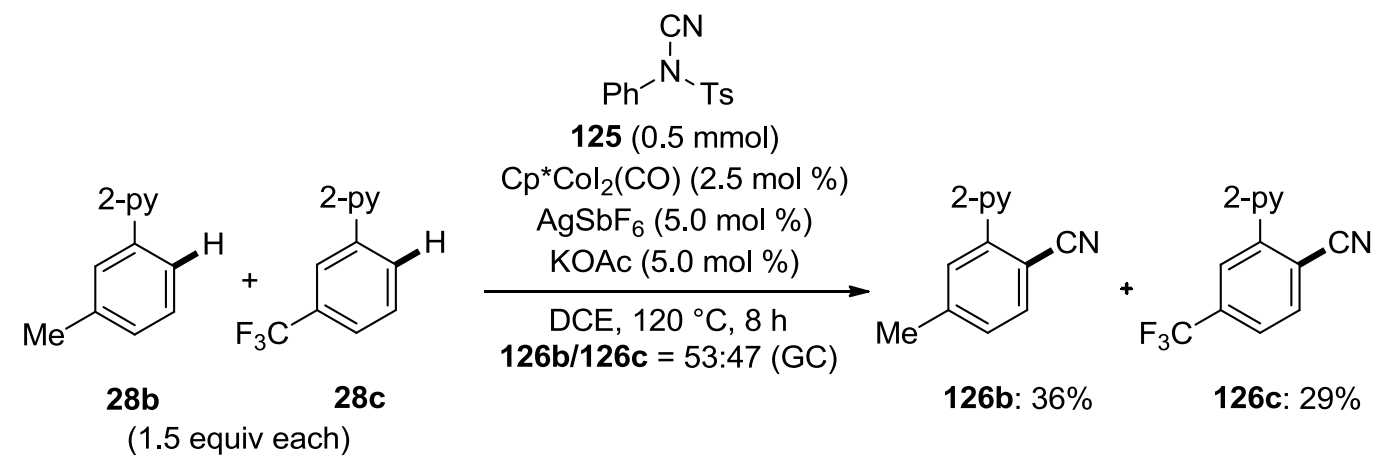

A suspension of NCTS (125) (136 mg, $0.50 \mathrm{mmol}), 2-(m$-tolyl)pyridine (28b) (127 mg, 0.75 mmol), 2-\{3-(trifluoromethyl)phenyl $\}$ pyridine (28c) $(167 \mathrm{mg}, 0.75 \mathrm{mmol}), \mathrm{Cp}^{*} \mathrm{CoI}_{2}(\mathrm{CO})$ (112, $6.0 \mathrm{mg}, 2.5 \mathrm{~mol} \%), \mathrm{AgSbF}_{6}(8.6 \mathrm{mg}, 5.0 \mathrm{~mol} \%)$ and KOAc (2.5 mg, $\left.5.0 \mathrm{~mol} \%\right)$ in DCE $(2.0 \mathrm{~mL})$ was stirred at $120{ }^{\circ} \mathrm{C}$ for $8 \mathrm{~h}$ under an atmosphere of Ar. At ambient temperature, the reaction mixture was evaporated in vacuo and the residue was purified by column chromatography on silica gel ( $n$-hexane $\left./ \mathrm{CH}_{2} \mathrm{Cl}_{2} / \mathrm{EtOAc} 1: 1: 0.1\right)$ to yield $\mathbf{1 2 6 b}(35 \mathrm{mg}$, $36 \%$ ) and 126c (36 mg, 29\%).

\section{Hammett plot of Reactions of 2-(4-R-Phenyl)pyridines 28 with NCTS (125):}

To an oven-dried $25 \mathrm{~mL}$ screw-capped vials, respective 2-(4-R-phenyl)pyridines ( $0.25 \mathrm{mmol})$, NCTS (125, $102 \mathrm{mg}, 0.38 \mathrm{mmol}), \mathrm{Cp}^{*} \mathrm{CoI}_{2}(\mathrm{CO})(\mathbf{1 1 2}, 3.0 \mathrm{mg}, 2.5 \mathrm{~mol} \%), \mathrm{AgSbF}_{6}(4.3 \mathrm{mg}$, $5.0 \mathrm{~mol} \%)$ and KOAc $(1.3 \mathrm{mg}, 5.0 \mathrm{~mol} \%)$ and DCE $(1.0 \mathrm{~mL})$ were added under a gentle stream of Ar. The reaction mixtures were stirred for $2 \mathrm{~h}$ at $120{ }^{\circ} \mathrm{C}$. After the reaction was finished, the screw-capped vials were cooled with an ice bath. The mixtures were filtered through a pad of Celite each and concentrated in vacuo. The residues were analyzed by ${ }^{1} \mathrm{H}-\mathrm{NMR}$ spectroscopy. Conversions of starting materials were determined using dibromomethane (43.5 $\mathrm{mg}, 0.25 \mathrm{mmol})$ as an internal standard.

\section{Table 10. 1.}

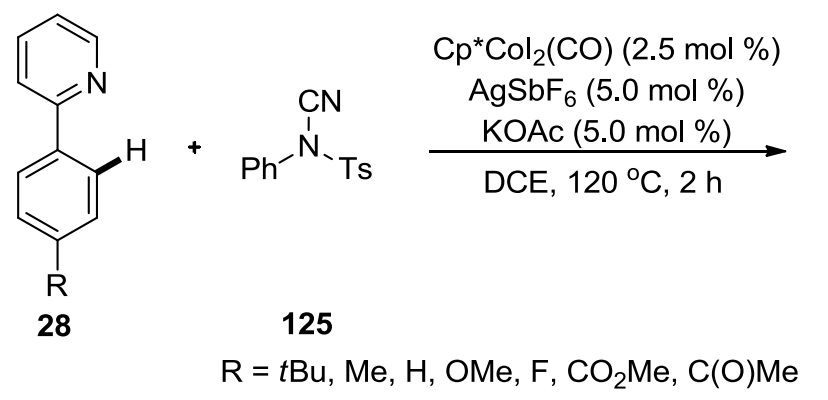<smiles>[R]c1ccc(-c2ccccn2)c(C#N)c1</smiles>

126 


\begin{tabular}{|l|c|c|c|c|c|}
\hline & $\mathbf{P}$ & $\mathbf{S M}$ & NMR Conv. & $\boldsymbol{\sigma}_{\mathbf{m}^{-}}$ & $\mathbf{L o g}(\operatorname{con} \mathbf{v .} \mathbf{X} /$ con v. H) \\
\hline $\mathrm{R}=t \mathrm{Bu}$ & $13 \%$ & $85 \%$ & $15 \%$ & -0.1 & -0.2863 \\
\hline $\mathrm{R}=\mathrm{Me}$ & $17 \%$ & $81 \%$ & $19 \%$ & -0.07 & -0.1836 \\
\hline $\mathrm{R}=\mathrm{H}$ & $29 \%$ & $71 \%$ & $29 \%$ & 0 & 0 \\
\hline $\mathrm{R}=\mathrm{OMe}$ & $25 \%$ & $74 \%$ & $26 \%$ & 0.12 & -0.0474 \\
\hline $\mathrm{R}=\mathrm{F}$ & $44 \%$ & $53 \%$ & $47 \%$ & 0.34 & 0.2097 \\
\hline $\mathrm{R}=\mathrm{CO}_{2} \mathrm{Me}$ & $29 \%$ & $69 \%$ & $31 \%$ & 0.37 & 0.0290 \\
\hline $\mathrm{R}=\mathrm{COMe}$ & $29 \%$ & $70 \%$ & $30 \%$ & 0.38 & 0.0147 \\
\hline
\end{tabular}

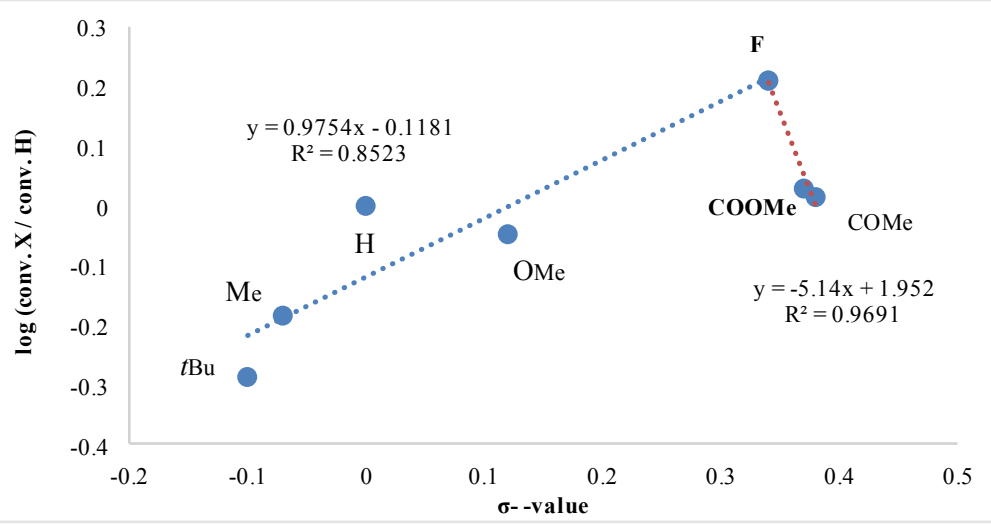

Cobalt-Catalyzed H/D Exchange in Substrate 28 a with $\mathrm{CD}_{3} \mathrm{OD}$ as the Cosolvent without NCTS:<smiles>c1ccc(-c2ccccn2)cc1</smiles>

$28 \mathrm{a}$

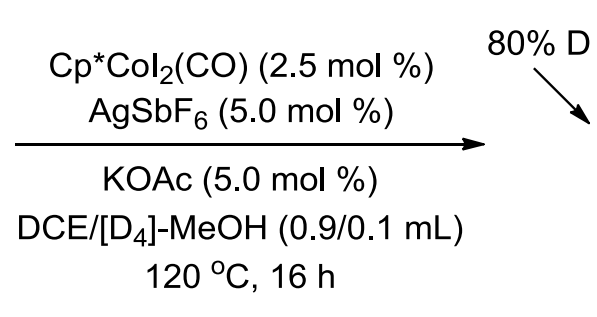

$\left[D_{n}\right]-28 a: 92 \%$

A suspension of 2-phenylpyridine (28a) (78 mg, $0.50 \mathrm{mmol}), \mathrm{Cp}^{*} \mathrm{CoI}_{2}(\mathrm{CO})(\mathbf{1 1 2}, 6.0 \mathrm{mg}, 2.5$ $\mathrm{mol} \%), \mathrm{AgSbF}_{6}(8.6 \mathrm{mg}, 5.0 \mathrm{~mol} \%)$ and $\mathrm{KOAc}(2.5 \mathrm{mg}, 5.0 \mathrm{~mol} \%)$ in a solvent mixture of DCE and $\mathrm{CD}_{3} \mathrm{OD}(0.9 / 0.1 \mathrm{~mL})$ was stirred at $120{ }^{\circ} \mathrm{C}$ for $16 \mathrm{~h}$ under an atmosphere of Ar. At ambient temperature, the reaction mixture was evaporated in vacuo and the residue was purified by column chromatography on silica gel ( $n$-hexane/EtOAc) to yield $\left[\mathrm{D}_{\mathrm{n}}\right]-\mathbf{2 8 a}(72 \mathrm{mg}$, $92 \%$ ) as a slightly yellow oil. The D-incorporation in $\left[D_{n}\right]-28$ a was estimated by ${ }^{1} \mathrm{H}-\mathrm{NMR}$ spectroscopy.

KIE experime nts with $\mathrm{V}-1$ a and $\left[D_{5}\right]-\mathrm{V}-1 \mathrm{a}$ as subs trates : 


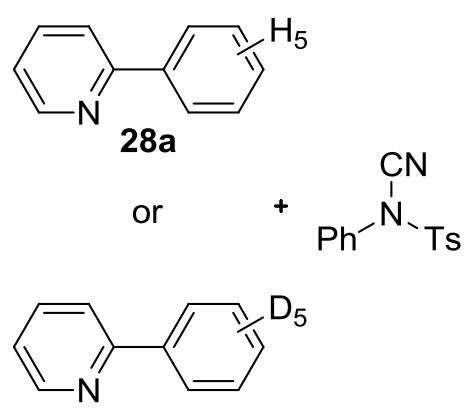

$\left[D_{5}\right]-28 a$

125

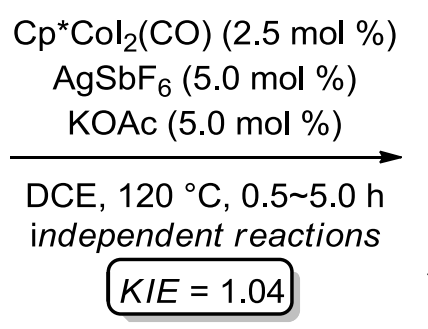

$K I E=1.04$

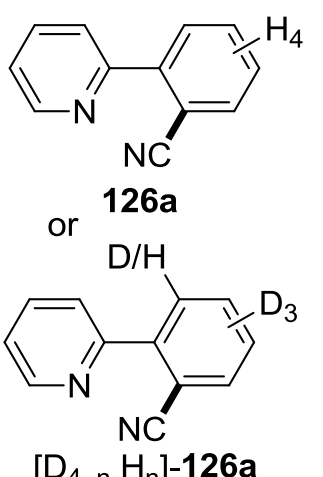

$\left[D_{4-n} H_{n}\right]-126 a$

Two parallel reactions with 28a and deuterated substrate $\left[\mathrm{D}_{5}\right] \mathbf{- 2 8 a}$ under the standard conditions were performed: A suspensions of NCTS (125) (102 mg, $0.38 \mathrm{mmol})$, substrates 28a (39.0 mg, $0.25 \mathrm{mmol})$ or $\left[\mathrm{D}_{5}\right]-\mathbf{2 8 a}(40.0 \mathrm{mg}, 0.25 \mathrm{mmol}), \mathrm{Cp} * \mathrm{CoI}_{2}(\mathrm{CO})(\mathbf{1 1 2}, 3.0 \mathrm{mg}, 2.5$ $\mathrm{mol} \%), \operatorname{AgSbF}_{6}(4.3 \mathrm{mg}, 5.0 \mathrm{~mol} \%)$ and $\mathrm{KOAc}(1.3 \mathrm{mg}, 5.0 \mathrm{~mol} \%)$ in DCE $(1.0 \mathrm{~mL})$ was stirred at $120^{\circ} \mathrm{C}$ under an atmosphere of Ar. The consumption of substrates V-1a or $\left[\mathrm{D}_{5}\right]-\mathbf{2 8 a}$ and the appearance of the products $\mathbf{1 2 6 a}$ or $\left[\mathrm{D}_{\mathrm{n}}\right] \mathbf{- 1 2 6 a}$ were monitored by GC analys is (with 0.25 mmol dodecane as an internal standard) after $0.5 \mathrm{~h}, 1.0 \mathrm{~h}, 1.5 \mathrm{~h}, 2.0 \mathrm{~h}, 2.5 \mathrm{~h}, 3.0 \mathrm{~h}, 3.5 \mathrm{~h}$, $4.0 \mathrm{~h}, 5.0 \mathrm{~h}$, respectively. The consumption of substrate $\mathbf{2 8 a}$ or $\left[\mathrm{D}_{5}\right]-\mathbf{2 8 a}$ and the appearance of the products 126a or $\left[D_{n}\right]-\mathbf{1 2 6 a}$ were monitored by GC analys is (with 0.25 mmol dodecane as an internal standard).

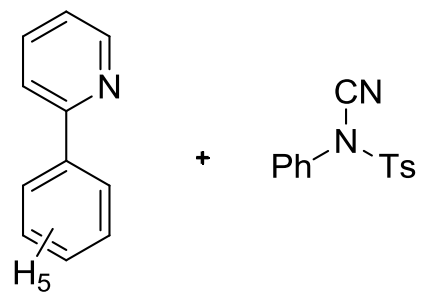

$\mathrm{Cp}^{*} \mathrm{Col}_{2}(\mathrm{CO})(2.5 \mathrm{~mol} \%)$

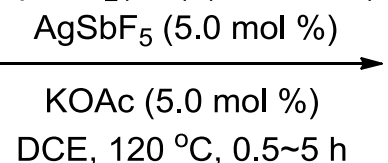

125<smiles>N#Cc1ccccc1-c1ccccn1</smiles>

126a

\begin{tabular}{|c|c|c|c|c|c|c|c|c|c|}
\hline $\mathbf{t} /(\mathbf{h})$ & $\mathbf{0 . 5}$ & $\mathbf{1 . 0}$ & $\mathbf{1 . 5}$ & $\mathbf{2 . 0}$ & $\mathbf{2 . 5}$ & $\mathbf{3 . 0}$ & $\mathbf{3 . 5}$ & $\mathbf{4 . 0}$ & $\mathbf{5 . 0}$ \\
\hline $\mathbf{1 2 6 a}$ & 0.13 & 0.29 & 0.39 & 0.43 & 0.48 & 0.50 & 0.53 & 0.55 & 0.58 \\
\hline $\mathbf{2 8 a}$ & 0.84 & 0.69 & 0.56 & 0.52 & 0.50 & 0.45 & 0.47 & 0.43 & 0.42 \\
\hline
\end{tabular}

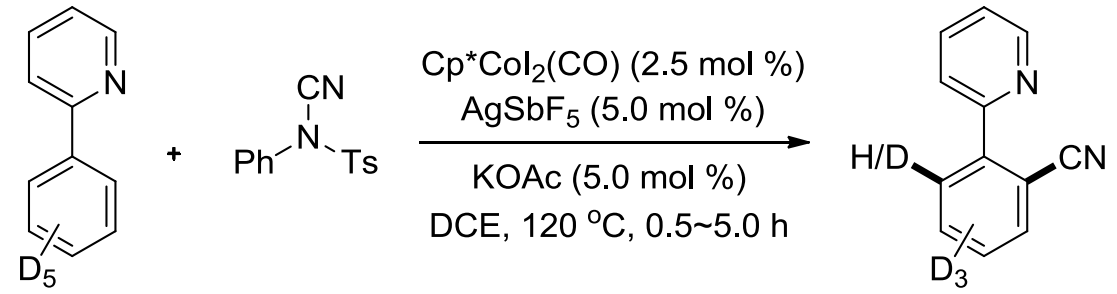

[D $\left.D_{5}\right]-28 a$

125

$\left[\mathrm{D}_{4-\mathrm{n}} \mathrm{H}_{\mathrm{n}}\right]-126 \mathrm{a}$

\begin{tabular}{|c|c|c|c|c|c|c|c|c|c|}
\hline $\mathbf{t} /(\mathbf{h})$ & $\mathbf{0 . 5}$ & $\mathbf{1 . 0}$ & $\mathbf{1 . 5}$ & $\mathbf{2 . 0}$ & $\mathbf{2 . 5}$ & $\mathbf{3 . 0}$ & $\mathbf{3 . 5}$ & $\mathbf{4 . 0}$ & $\mathbf{5 . 0}$ \\
\hline$\left[\mathrm{D}_{\mathrm{n}}\right]-\mathbf{1 2 6 a}$ & 0.13 & 0.30 & 0.35 & 0.37 & 0.46 & 0.48 & 0.50 & 0.54 & 0.55 \\
\hline
\end{tabular}




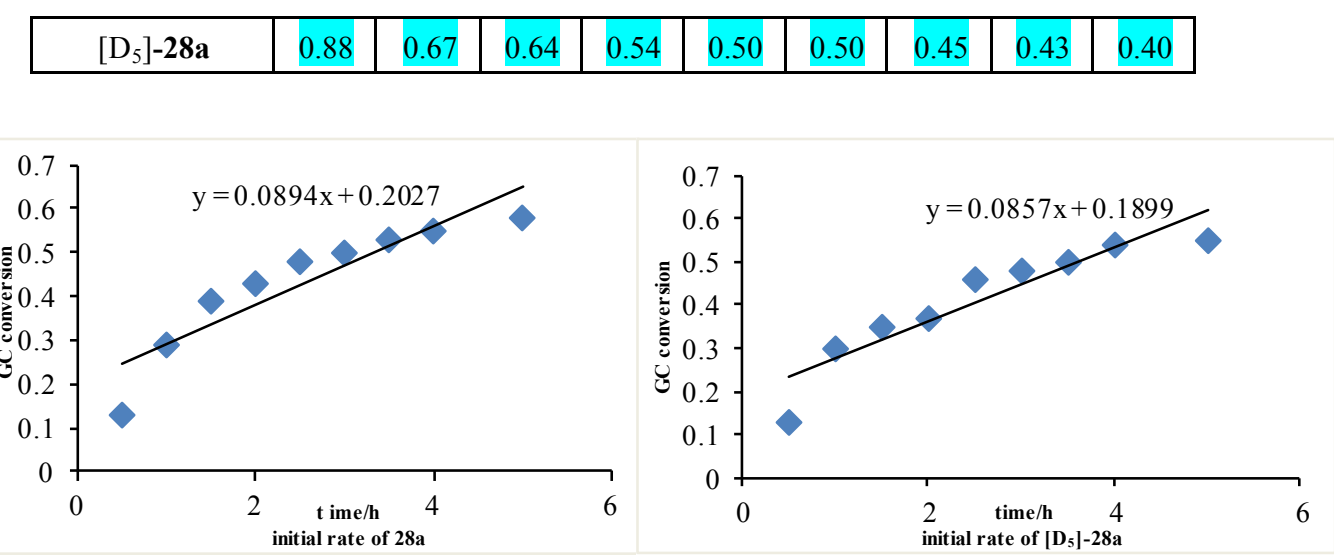

Intramolecular competition expe rime nt: Kinetic isotope effect (KIE):<smiles>[2H]c1cccc(-c2ccccn2)c1</smiles>

$\left[\mathrm{D}_{1}\right]-28 \mathrm{a}$ (2.0 equiv)<smiles>CN(C#N)c1ccccc1</smiles>

125

(0.5 mmol) $\mathrm{Cp}^{*} \mathrm{Col}_{2}(\mathrm{CO})(2.5 \mathrm{~mol} \%)$ $\mathrm{AgSbF}_{6}(5.0 \mathrm{~mol} \%)$ $\mathrm{KOAc}(5.0 \mathrm{~mol} \%)$

DCE, $120^{\circ} \mathrm{C}, 100 \mathrm{~min}$

$K I E=1.08$<smiles>N#Cc1cccc([18OH])c1-c1ccccn1</smiles>

$\left[D_{n}\right]-126 ': 18 \%$

A suspension of NCTS (125) (136 mg, $0.50 \mathrm{mmol}), 2-[\mathrm{D}]$-phenylpyridine ([D $]$-28a) (156 mg, $1.0 \mathrm{mmol}), \mathrm{Cp}^{*} \mathrm{CoI}_{2}(\mathrm{CO})(\mathbf{1 1 2}, 6.0 \mathrm{mg}, 2.5 \mathrm{~mol} \%), \mathrm{AgSbF}_{6}(8.6 \mathrm{mg}, 5.0 \mathrm{~mol} \%)$ and $\mathrm{KOAc}$ (2.5 mg, $5.0 \mathrm{~mol} \%)$ in DCE $(2.0 \mathrm{~mL})$ was stirred at $120^{\circ} \mathrm{C}$ for $100 \mathrm{~min}$ under an atmosphere of Ar. At ambient temperature, the reaction mixture was evaporated in vacuo and the residue was purified by column chromatography on silica gel (n-hexane/EtOAc 6:1) to yield $\left[D_{n}\right]-126 a^{\prime}(16 \mathrm{mg}, 18 \%)$ as a solid. The D-incorporation in $\left[\mathrm{D}_{\mathrm{n}}\right]-\mathbf{1 2 6 \mathbf { a } ^ { \prime }}$ was estimated by ${ }^{1}$ H-NMR spectroscopy.

\section{2-(Pyridin-2-yl)be nzoic acid (152)}<smiles>O=C(O)c1ccccc1-c1ccccn1</smiles>

A suspension of 2-(pyridin-2-yl)benzonitrile (126a) $(90 \mathrm{mg}, 0.5 \mathrm{mmol})$, which was prepared according to the general procedure $\mathbf{I}$ as indicated above, and $\mathrm{KOH}(180 \mathrm{mg}, 5.0 \mathrm{mmol}, 1.25$ $\mathrm{mL}$ of a $4 \mathrm{~N}$ aq. solution) was stirred at $105^{\circ} \mathrm{C}$ for $12 \mathrm{~h}$. After completion of the reaction, the 
reaction mixture was neutralized by $\mathrm{HCl}$ aq. $(2 \mathrm{~N}, 2.8 \mathrm{~mL})$. Then the reaction was extracted with $\mathrm{CH}_{2} \mathrm{Cl}_{2}(200 \mathrm{~mL})$. The combined organic layers were dried over $\mathrm{Na}_{2} \mathrm{SO}_{4}$ and filtered. The solvent was concentrated in vacuo and the solid was washed with $\mathrm{Et}_{2} \mathrm{O}(3 \times 10 \mathrm{~mL})$ to afford product 152 (92 mg, 83\%, calculated from 28a) as a colorless solid. M. p.: = 201-204 C .

${ }^{1} \mathbf{H}$-NMR (500 MHz, $\left.\mathrm{CD}_{3} \mathrm{OD}\right): \delta=8.53$ (ddd, $\left.J=5.1,1.8,1.0 \mathrm{~Hz}, 1 \mathrm{H}\right), 7.92$ (ddd, $J=7.7,1.4$, $0.5 \mathrm{~Hz}, 1 \mathrm{H}), 7.88(\mathrm{td}, J=7.7,1.8 \mathrm{~Hz}, 1 \mathrm{H}), 7.62(\mathrm{td}, J=7.5,1.4 \mathrm{~Hz}, 1 \mathrm{H}), 7.57-7.51(\mathrm{~m}, 2 \mathrm{H})$, 7.50 (ddd, $J=7.6,1.4,0.5 \mathrm{~Hz}, 1 \mathrm{H}), 7.39$ (ddd, $J=7.6,5.0,1.1 \mathrm{~Hz}, 1 \mathrm{H})$.

${ }^{13}$ C-NMR (125 MHz, $\left.\mathrm{CD}_{3} \mathrm{OD}\right): \delta=171.2\left(\mathrm{C}_{\mathrm{q}}\right), 160.3\left(\mathrm{C}_{\mathrm{q}}\right), 149.1(\mathrm{CH}), 142.0\left(\mathrm{C}_{\mathrm{q}}\right), 138.5\left(\mathrm{C}_{\mathrm{q}}\right)$, $133.1(\mathrm{CH}), 132.4(\mathrm{CH}), 131.3(\mathrm{CH}), 131.2(\mathrm{CH}), 129.7(\mathrm{CH}), 125.2(\mathrm{CH}), 123.7(\mathrm{CH})$.

IR (ATR): 2405, 1715, 1429, 1255, 1004, $797 \mathrm{~cm}^{-1}$.

MS (EI) m/z (relative intensity) $199(5)\left[\mathrm{M}^{+}\right], 182$ (10), 155 (100), 127 (20), 115 (5), 102 (5).

HR-MS (ESI) m/z calcd for $\mathrm{C}_{12} \mathrm{H}_{8} \mathrm{NO}_{2}\left[\mathrm{M}-\mathrm{H}^{+}\right]$198.0555, found 198.0555.

The spectral data were in accordance with those reported in the literature. ${ }^{164}$

\section{1-(Pyrimidin-2-yl)-1H-indole-2-carboxamide (153)}<smiles>NC(=O)c1cc2ccccc2n1-c1ncccn1</smiles>

To a solution of 1-(pyrimidin-2-yl)- $1 H$-indole-2-carbonitrile (127a) $(66 \mathrm{mg}, 0.3 \mathrm{mmol})$ in $t \mathrm{BuOH}(5.0 \mathrm{~mL})$ was added $\mathrm{KOH}(336 \mathrm{mg}, 6.0 \mathrm{mmol})$ in small portions. The reaction mixture was stirred at $60{ }^{\circ} \mathrm{C}$ for $4 \mathrm{~h}$. Then the reaction was cooled to ambient temperature and extracted with EtOAc $(3 \times 10 \mathrm{~mL})$. The combined organic layers were dried over $\mathrm{Na}_{2} \mathrm{SO}_{4}$, filtered, concentrated in vacuo and purified by column chromatography ( $n$-hexane/EtOAc $1: 1 \rightarrow 1: 2)$ yielded $153(52 \mathrm{mg}, 72 \%$, calculated from 7a) as a colorless solid. M. p.: = $254-256^{\circ} \mathrm{C}$.

${ }^{1}$ H-NMR (500 MHz, $\left.\mathrm{CDCl}_{3}\right): \delta=9.37$ (br s, 1H), $8.70($ br s, $1 \mathrm{H}), 8.68(\mathrm{~d}, J=4.8 \mathrm{~Hz}, 2 \mathrm{H})$, $7.69(\mathrm{dd}, J=8.1,1.0 \mathrm{~Hz}, 1 \mathrm{H}), 7.46$ (dd, $J=8.3,1.1 \mathrm{~Hz}, 1 \mathrm{H}), 7.33$ (ddd, $J=8.3,7.0,1.1 \mathrm{~Hz}$,

164 M. Chai tanya, D. Yada gi i, P. Anbarasan, Org. Lett. 2013, 15, 4960-4963. 
1H), 7.17 (ddd, $J=8.0,7.0,1.0 \mathrm{~Hz}, 1 \mathrm{H}), 7.12(\mathrm{dd}, J=2.2,0.9 \mathrm{~Hz}, 1 \mathrm{H}), 7.06$ (t, $J=4.8 \mathrm{~Hz}$, $1 \mathrm{H})$.

${ }^{13}$ C-NMR (125 MHz, $\left.\mathrm{CDCl}_{3}\right): \delta=158.4(\mathrm{CH}), 158.4\left(\mathrm{C}_{\mathrm{q}}\right), 157.3\left(\mathrm{C}_{\mathrm{q}}\right), 136.9\left(\mathrm{C}_{\mathrm{q}}\right), 130.1\left(\mathrm{C}_{\mathrm{q}}\right)$, $127.5\left(\mathrm{C}_{\mathrm{q}}\right), 125.5(\mathrm{CH}), 122.4(\mathrm{CH}), 121.0(\mathrm{CH}), 116.8(\mathrm{CH}), 112.0(\mathrm{CH}), 104.2(\mathrm{CH})$.

IR (ATR): 3388, 3238, 1688, 1573, 1428, $739 \mathrm{~cm}^{-1}$.

MS (EI) m/z (relative intensity) $238(90)\left[\mathrm{M}^{+}\right], 210$ (40), 143 (100), 115 (50), 89 (60).

HR-MS (EI) m/z calcd for $\mathrm{C}_{13} \mathrm{H}_{10} \mathrm{~N}_{4} \mathrm{O}\left[\mathrm{M}^{+}\right]$238.0855, found 238.0864 .

1-(1-Methyl-1 H-indol-2-yl)propan-1-one (154)<smiles>CCC(=O)c1cc2ccccc2n1C</smiles>

To the mixture of 1-methyl- $1 H$-indole-2-carbonitrile $(31.0 \mathrm{mg}, 0.20 \mathrm{mmol})$, prepared from $1 H$-indole-2-carbonitrile (127j) according to previously described methods in 99\% yield, and $\mathrm{CuI}(1.0 \mathrm{mg}, 5.2 \mu \mathrm{mol})$ in anhydrous THF $(5 \mathrm{~mL})$ was added $\mathrm{EtMgBr}(0.21 \mathrm{mmol}, 70 \mu \mathrm{L}$ of a $3 \mathrm{~N}$ solution in THF), and the reaction mixture was stirred at ambient temperature for $10 \mathrm{~h}$. Then $1 \mathrm{~N} \mathrm{HCl}$ aq. solution $(3.0 \mathrm{~mL})$ was added at $0{ }^{\circ} \mathrm{C}$, and the resulting mixture was stirred at room temperature for another $4 \mathrm{~h}$. The organic layer was separated, and the aqueous layer was extracted with EtOAc $(3 \times 10 \mathrm{~mL})$. The combined organic layers were washed with brine, dried over $\mathrm{Na}_{2} \mathrm{SO}_{4}$ and concentrated under reduced pressure. The crude product was purified by column chromatography ( $n$-hexane/EtOAc 20:1) yielded 154 (20 mg, 52\%, calculated from 7a) as a slightly yellow solid. M. p.: $=55-57^{\circ} \mathrm{C}$.

${ }^{1}$ H-NMR $\left(500 \mathrm{MHz}, \mathrm{CDCl}_{3}\right): \delta=7.71-7.65(\mathrm{~d}, J=8.0 \mathrm{~Hz}, 1 \mathrm{H}), 7.40-7.33(\mathrm{~m}, 2 \mathrm{H}), 7.28(\mathrm{~d}, J$ $=0.7 \mathrm{~Hz}, 1 \mathrm{H}), 7.14(\mathrm{ddd}, J=8.0,5.6,2.2 \mathrm{~Hz}, 1 \mathrm{H}), 4.07(\mathrm{~s}, 3 \mathrm{H}), 3.00(\mathrm{q}, J=7.4 \mathrm{~Hz}, 2 \mathrm{H}), 1.23$ (t, $J=7.4 \mathrm{~Hz}, 3 \mathrm{H})$.

${ }^{13}$ C-NMR $\left(125 \mathrm{MHz}, \mathrm{CDCl}_{3}\right): \delta=194.9\left(\mathrm{C}_{\mathrm{q}}\right), 139.9\left(\mathrm{C}_{\mathrm{q}}\right), 134.6\left(\mathrm{C}_{\mathrm{q}}\right), 125.7\left(\mathrm{C}_{\mathrm{q}}\right), 125.6(\mathrm{CH})$, $122.7(\mathrm{CH}), 120.6(\mathrm{CH}), 110.8(\mathrm{CH}), 110.3(\mathrm{CH}), 33.1\left(\mathrm{CH}_{2}\right), 32.2\left(\mathrm{CH}_{3}\right), 9.0\left(\mathrm{CH}_{3}\right)$.

IR (ATR): 2951, 1663, 1511, 1315, 1155, $731 \mathrm{~cm}^{-1}$.

MS (EI) m/z (relative intensity) 187 (50) $\left[\mathrm{M}^{+}\right], 158$ (100), 144 (5), 130 (25), 115 (5), 89 (50).

HR-MS (EI) m/z calcd for $\mathrm{C}_{12} \mathrm{H}_{13} \mathrm{NO}\left[\mathrm{M}^{+}\right]$187.0997, found 187.0995. 
The spectral data were in accordance with those reported in the literature. ${ }^{165}$

\section{2-(1 H-Te trazol-5-yl)-1H-indole (155)}<smiles>c1ccc2[nH]c(-c3nnn[nH]3)cc2c1</smiles>

To the vial equipped with a magnetic bar, TBAF $3 \mathrm{H}_{2} \mathrm{O}$ (32 $\mathrm{mg}, 0.1 \mathrm{mmol}$ ), $1 H$-indole-2-carbonitrile (29 mg, $0.2 \mathrm{mmol})$ and $\mathrm{TMSN}_{3}(34.5 \mathrm{mg}, 0.3 \mathrm{mmol})$ were added. The vial was sealed and heated under vigorous stirring at $120^{\circ} \mathrm{C}$ for $18 \mathrm{~h}$. The crude reaction mixture was diluted with EtOAc $(20 \mathrm{~mL})$ and TBAF was removed by washing the organic phase with $1 \mathrm{~N}$ aq. $\mathrm{HCl}$ aqueous solution $(3 \times 5 \mathrm{~mL})$. The organic layer was dried over $\mathrm{Na}_{2} \mathrm{SO}_{4}$ and concentrated under reduced pressure. The crude solid was washed with a 2:1 $n$-hexane/Et $2 \mathrm{O}$ mixture $(2 \times 10 \mathrm{~mL})$ to afford $155(36 \mathrm{mg}, 94 \%$, calculated from $7 \mathbf{a})$ as a color less solid. M. p.: $=232-234{ }^{\circ} \mathrm{C}$.

${ }^{1}$ H-NMR $\left(500 \mathrm{MHz}, \mathrm{CD}_{3} \mathrm{OD}\right): \delta=7.63(\mathrm{dt}, J=8.0,1.0 \mathrm{~Hz}, 1 \mathrm{H}), 7.49(\mathrm{dd}, J=8.3,0.9 \mathrm{~Hz}$, 1H), $7.23(\mathrm{ddd}, J=8.3,7.0,1.0 \mathrm{~Hz}, 1 \mathrm{H}), 7.12(\mathrm{~d}, J=0.9 \mathrm{~Hz}, 1 \mathrm{H}), 7.09$ (ddd, $J=8.0,7.0,1.0$ $\mathrm{Hz}, 1 \mathrm{H})$.

${ }^{13}$ C-NMR (125 MHz, $\left.\mathrm{CD}_{3} \mathrm{OD}\right): \delta=152.3\left(\mathrm{C}_{\mathrm{q}}\right), 139.1\left(\mathrm{C}_{\mathrm{q}}\right), 129.4\left(\mathrm{C}_{\mathrm{q}}\right), 125.1(\mathrm{CH}), 122.9\left(\mathrm{C}_{\mathrm{q}}\right)$, $122.3(\mathrm{CH}), 121.5(\mathrm{CH}), 113.0(\mathrm{CH}), 105.1(\mathrm{CH})$.

IR (ATR): 3226, 1618, 1339, 1084, 924, $744 \mathrm{~cm}^{-1}$.

MS (EI) m/z (relative intensity) 185 (70) $\left[\mathrm{M}^{+}\right], 157$ (100), 142 (35), 130 (40), 115 (20), 103 (70).

HR-MS (EI) m/z calcd for $\mathrm{C}_{9} \mathrm{H}_{7} \mathrm{~N}_{5}\left[\mathrm{M}^{+}\right]$185.0701, found 185.0705.

The spectral data were in accordance with those reported in the literature. ${ }^{161}$

\subsubsection{Analytical Data for the Products of Cobalt(III)-Catalyzed Aryl and Alkenyl C-H Bond Aminocarbonylation with Isocyanates and Acyl Azides} $N$-Phe nyl-2-(1H-pyrazol-1-yl)benzamide (130aa)

${ }^{165}$ Y.-T. Hong, A. Barchuk, M. J. Krische, Angew. Chem. Int. Ed. 2006, 45, 6885-6888. 
<smiles>O=C(Nc1ccccc1)c1ccccc1-n1cccn1</smiles>

The general procedure $\mathbf{J}$ was followed using $\mathbf{1 2 8 a}$ (72 $\mathrm{mg}, 0.5 \mathrm{mmol})$ and 129a (119 mg, 1.0 mmol). Purification by column chromatography ( $n$-hexane/ $\mathrm{CH}_{2} \mathrm{Cl}_{2} / \mathrm{EtOAc} / \mathrm{Et}_{3} \mathrm{~N}$ 10:1:1:1\%) yie lded 130aa ( $88 \mathrm{mg}, 67 \%$ ) as a colorless solid. M. p.: $=167-169^{\circ} \mathrm{C}$.

${ }^{1}$ H-NMR (300 MHz, $\left.\mathrm{CDCl}_{3}\right): \delta=8.47$ (br s, 1H), 7.93-7.86 (m, 1H), 7.79-7.74 (m, 1H), 7.69 $(\mathrm{d}, J=2.4 \mathrm{~Hz}, 1 \mathrm{H}), 7.57-7.48(\mathrm{~m}, 2 \mathrm{H}), 7.45-7.35(\mathrm{~m}, 3 \mathrm{H}), 7.25$ (t, $J=7.6 \mathrm{~Hz}, 2 \mathrm{H}), 7.06$ (ddt, $J$ $=7.8,6.9,1.2 \mathrm{~Hz}, 1 \mathrm{H}), 6.44(\mathrm{t}, J=2.2 \mathrm{~Hz}, 1 \mathrm{H})$.

${ }^{13}$ C-NMR (100 MHz, $\left.\mathrm{CDCl}_{3}\right): \delta=164.4\left(\mathrm{C}_{\mathrm{q}}\right), 141.4(\mathrm{CH}), 137.8\left(\mathrm{C}_{\mathrm{q}}\right), 137.2\left(\mathrm{C}_{\mathrm{q}}\right), 133.0\left(\mathrm{C}_{\mathrm{q}}\right)$, $131.9(\mathrm{CH}), 131.3(\mathrm{CH}), 130.6(\mathrm{CH}), 129.1(\mathrm{CH}), 128.8(\mathrm{CH}), 126.7(\mathrm{CH}), 124.4(\mathrm{CH}), 120.0$ (CH), $107.8(\mathrm{CH})$.

IR (ATR): 3245, 1650, 1541, 1326, 942, $755 \mathrm{~cm}^{-1}$.

MS (EI) m/z (relative intensity) $263(5)\left[\mathrm{M}^{+}\right], 171$ (100), 144 (5), 130 (5), 116 (20), 89 (5).

HR-MS (EI) m/z calcd for $\mathrm{C}_{16} \mathrm{H}_{13} \mathrm{~N}_{3} \mathrm{O}\left[\mathrm{M}^{+}\right] 263.1059$, found 263.1061 .

The spectral data were in accordance with those reported in the literature. ${ }^{166}$

\section{5-Methyl- $N$-phe nyl-2-(1 H-pyrazol-1-yl)benzamide (130ba)}<smiles>Cc1ccc(-n2cccn2)c(C(=O)Nc2ccccc2)c1</smiles>

The general procedure $\mathbf{J}$ was followed using $\mathbf{1 2 8 b}$ (79 $\mathrm{mg}, 0.5 \mathrm{mmol}$ ) and 129a (119 $\mathrm{mg}, 1.0$ mmol). Purification by column chromatography ( $n$-hexane/ $\mathrm{CH}_{2} \mathrm{Cl}_{2} / \mathrm{EtOAc}_{\mathrm{Et}} \mathrm{N}$ 10:1:1:1\%) yielded 130 ba ( $84 \mathrm{mg}, 61 \%$ ) as a colorless solid. M. p.: $=168-170{ }^{\circ} \mathrm{C}$.

${ }^{1} \mathbf{H}-\mathbf{N M R}\left(500 \mathrm{MHz}, \mathrm{CDCl}_{3}\right): \delta=8.33$ (br s, $\left.1 \mathrm{H}\right), 7.85(\mathrm{~d}, J=8.0 \mathrm{~Hz}, 1 \mathrm{H}), 7.80-7.75(\mathrm{~m}, 1 \mathrm{H})$, $7.67(\mathrm{~d}, J=2.4 \mathrm{~Hz}, 1 \mathrm{H}), 7.40(\mathrm{~d}, J=7.3 \mathrm{~Hz}, 2 \mathrm{H}), 7.36-7.31(\mathrm{~m}, 1 \mathrm{H}), 7.28-7.24(\mathrm{~m}, 2 \mathrm{H})$, 7.22-7.21 (m, 1H), 7.09-6.96 (m, 1H), $6.44(\mathrm{t}, J=2.1 \mathrm{~Hz}, 1 \mathrm{H}), 2.43(\mathrm{~s}, 3 \mathrm{H})$.

${ }^{166}$ K. Murali rajan, K. Parthasara thy, C.-H. Cheng, Org. Lett. 2012, 14, 4262-4265. 
${ }^{13}$ C-NMR $\left(125 \mathrm{MHz}, \mathrm{CDCl}_{3}\right): \delta=164.3\left(\mathrm{C}_{\mathrm{q}}\right), 142.2\left(\mathrm{C}_{\mathrm{q}}\right), 141.3(\mathrm{CH}), 138.0\left(\mathrm{C}_{\mathrm{q}}\right), 137.1\left(\mathrm{C}_{\mathrm{q}}\right)$, $132.1(\mathrm{CH}), 130.8(\mathrm{CH}), 130.2\left(\mathrm{C}_{\mathrm{q}}\right), 130.0(\mathrm{CH}), 128.8(\mathrm{CH}), 127.6(\mathrm{CH}), 124.3(\mathrm{CH}), 120.0$ (CH), $107.7(\mathrm{CH}), 21.1\left(\mathrm{CH}_{3}\right)$.

IR (ATR): 3244, 1650, 1543, 1325, 952, $757 \mathrm{~cm}^{-1}$.

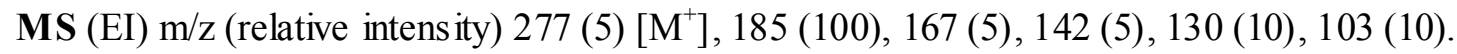

HR-MS (EI) m/z calcd for $\mathrm{C}_{17} \mathrm{H}_{15} \mathrm{~N}_{3} \mathrm{O}\left[\mathrm{M}^{+}\right]$277.1215, found 277.1216.

\section{5-Methoxy- $N$-phenyl-2-(1H-pyrazol-1-yl)benzamide (130ca)}<smiles>COc1ccc(-n2cccn2)c(C(=O)Nc2ccccc2)c1</smiles>

The general procedure $\mathbf{J}$ was followed using $\mathbf{1 2 8 c}(87 \mathrm{mg}, 0.5 \mathrm{mmol})$ and 129a (119 mg, 1.0 mmol). Purification by column chromatography ( $n$-hexane $/ \mathrm{CH}_{2} \mathrm{Cl}_{2} / \mathrm{EtOAc} / \mathrm{Et}_{3} \mathrm{~N}$ 8:1:1:1\%) yie lded 130ca (92 mg, 63\%) as a colorless solid. M. p.: $=122-124{ }^{\circ} \mathrm{C}$.

${ }^{1} \mathbf{H}-\mathbf{N M R}\left(500 \mathrm{MHz}, \mathrm{CDCl}_{3}\right): \delta=8.54($ br s, $1 \mathrm{H}), 7.78(\mathrm{dd}, J=1.9,0.7 \mathrm{~Hz}, 1 \mathrm{H}), 7.62(\mathrm{dd}, J=$ 2.4, $0.7 \mathrm{~Hz}, 1 \mathrm{H}), 7.45(\mathrm{~d}, J=3.0 \mathrm{~Hz}, 1 \mathrm{H}), 7.43-7.37(\mathrm{~m}, 2 \mathrm{H}), 7.28(\mathrm{~d}, J=8.7 \mathrm{~Hz}, 1 \mathrm{H})$, 7.27-7.23 (m, 2H), 7.08-7.05 (m, 1H), 7.05-7.02 (m, 1H), $6.42(\mathrm{t}, J=2.2 \mathrm{~Hz}, 1 \mathrm{H}), 3.88(\mathrm{~s}, 3 \mathrm{H})$.

${ }^{13}$ C-NMR $\left(125 \mathrm{MHz}, \mathrm{CDCl}_{3}\right): \delta=163.9\left(\mathrm{C}_{\mathrm{q}}\right), 160.0\left(\mathrm{C}_{\mathrm{q}}\right), 141.2(\mathrm{CH}), 137.8\left(\mathrm{C}_{\mathrm{q}}\right), 134.5\left(\mathrm{C}_{\mathrm{q}}\right)$, $132.6(\mathrm{CH}), 130.2\left(\mathrm{C}_{\mathrm{q}}\right), 128.8(\mathrm{CH}), 128.7(\mathrm{CH}), 124.4(\mathrm{CH}), 120.0(\mathrm{CH}), 117.7(\mathrm{CH}), 114.7$ (CH), $107.5(\mathrm{CH}), 55.8\left(\mathrm{CH}_{3}\right)$.

IR (ATR): 3244, 1653, 1555, 1444, 1037, $753 \mathrm{~cm}^{-1}$.

MS (EI) m/z (relative intensity) 293 (5) [M+], 201 (100), 186 (15), 174 (5), 158 (10), 146 (5).

HR-MS (ESI) $\mathrm{m} / \mathrm{z}$ calcd for $\mathrm{C}_{17} \mathrm{H}_{16} \mathrm{~N}_{3} \mathrm{O}_{2}\left[\mathrm{M}+\mathrm{H}^{+}\right]$294.1243, found 294.1237.

\section{5-Fluoro- $N$-phenyl-2-(1H-pyrazol-1-yl)be nzamide (130da)}<smiles>O=C(Nc1ccccc1)c1cc(F)ccc1-n1cccn1</smiles> 
The general procedure $\mathbf{J}$ was followed using $\mathbf{1 2 8 d}(81 \mathrm{mg}, 0.5 \mathrm{mmol})$ and 129a (119 mg, 1.0 mmol). Purification by column chromatography ( $n$-hexane/ $\mathrm{CH}_{2} \mathrm{Cl}_{2} / \mathrm{EtOAc}_{\mathrm{Et}} \mathrm{N}$ 10:1:1:1\%) yielded 130da (91 mg, 65\%) as a colorless solid. M. p.: $=142-144{ }^{\circ} \mathrm{C}$.

${ }^{1}$ H-NMR (400 MHz, $\mathrm{CDCl}_{3}$ ): $\delta=8.53$ (br s, $\left.1 \mathrm{H}\right), 7.80$ (dd, $\left.J=2.0,0.6 \mathrm{~Hz}, 1 \mathrm{H}\right), 7.71-7.57$ (m, 2H), 7.45-7.32 (m, 3H), 7.31-7.16 (m, 3H), 7.11-7.02 (m, 1H), 6.46 (dd, $J=2.4,1.9 \mathrm{~Hz}, 1 \mathrm{H})$. ${ }^{13}$ C-NMR $\left(100 \mathrm{MHz}, \mathrm{CDCl}_{3}\right): \delta=165.0\left(\mathrm{C}_{\mathrm{q}}, J_{\mathrm{C}-\mathrm{F}}=251.6 \mathrm{~Hz}\right), 162.7\left(\mathrm{C}_{\mathrm{q}}\right), 141.6(\mathrm{CH}), 137.6$ $\left(\mathrm{C}_{\mathrm{q}}\right), 135.3\left(\mathrm{C}_{\mathrm{q}}, J_{\mathrm{C}-\mathrm{F}}=7.8 \mathrm{~Hz}\right), 133.4\left(\mathrm{C}_{\mathrm{q}}, J=3.4 \mathrm{~Hz}\right), 132.4(\mathrm{CH}), 129.3\left(\mathrm{CH}, J_{\mathrm{C}-\mathrm{F}}=8.5 \mathrm{~Hz}\right)$, $128.9(\mathrm{CH}), 124.7(\mathrm{CH}), 120.1(\mathrm{CH}), 118.4\left(\mathrm{CH}, J_{\mathrm{C}-\mathrm{F}}=22.8 \mathrm{~Hz}\right), 117.9\left(\mathrm{CH}, J_{\mathrm{C}-\mathrm{F}}=24.6 \mathrm{~Hz}\right)$, $108.0(\mathrm{CH})$.

${ }^{19}$ F-NMR $\left(376 \mathrm{MHz}, \mathrm{CDCl}_{3}\right): \delta=-(110.2-110.3)(\mathrm{m})$.

IR (ATR): 3270, 1650, 1490, 1326, 1045, $732 \mathrm{~cm}^{-1}$.

MS (EI) m/z (relative intensity) 281 (5) [M+], 189 (100), 162 (5), 148 (5), 134 (10), 107 (10).

HR-MS (EI) m/z calcd for $\mathrm{C}_{16} \mathrm{H}_{12} \mathrm{~N}_{3} \mathrm{OF}\left[\mathrm{M}^{+}\right] 281.0964$, found 281.0972.

\section{5-Chloro- $N$-phe nyl-2-(1 H-pyrazol-1-yl)be nzamide (130ea)}<smiles>O=C(Nc1ccccc1)c1cc(Cl)ccc1-n1cccn1</smiles>

The general procedure $\mathbf{J}$ was followed using $\mathbf{1 2 8 e}(89 \mathrm{mg}, 0.5 \mathrm{mmol})$ and 129a (119 $\mathrm{mg}, 1.0$ mmol). Purification by column chromatography ( $n$-hexane $/ \mathrm{CH}_{2} \mathrm{Cl}_{2} / \mathrm{EtOAc} / \mathrm{Et}_{3} \mathrm{~N}$ 10:1:1:1\%) yie lded 130ea (106 mg, 71\%) as a colorless solid. M. p.: $=137-139{ }^{\circ} \mathrm{C}$.

${ }^{1} \mathbf{H}-\mathbf{N M R}\left(500 \mathrm{MHz}, \mathrm{CDCl}_{3}\right): \delta=8.63(\mathrm{br} \mathrm{s}, 1 \mathrm{H}), 7.85(\mathrm{~d}, J=2.2 \mathrm{~Hz}, 1 \mathrm{H}), 7.78-7.75(\mathrm{~m}, 1 \mathrm{H})$, $7.68(\mathrm{dd}, J=2.4,0.7 \mathrm{~Hz}, 1 \mathrm{H}), 7.47$ (dd, $J=8.6,2.5 \mathrm{~Hz}, 1 \mathrm{H}), 7.39$ (dd, $J=8.6,1.2 \mathrm{~Hz}, 2 \mathrm{H})$, $7.32(\mathrm{~d}, J=8.6 \mathrm{~Hz}, 2 \mathrm{H}), 7.28-7.21(\mathrm{~m}, 1 \mathrm{H}), 7.13-7.01(\mathrm{~m}, 1 \mathrm{H}), 6.45(\mathrm{dd}, J=2.2,1.8 \mathrm{~Hz}, 1 \mathrm{H})$. ${ }^{13}$ C-NMR $\left(125 \mathrm{MHz}, \mathrm{CDCl}_{3}\right): \delta=163.1\left(\mathrm{C}_{\mathrm{q}}\right), 141.7(\mathrm{CH}), 137.6\left(\mathrm{C}_{\mathrm{q}}\right), 135.7\left(\mathrm{C}_{\mathrm{q}}\right), 135.0\left(\mathrm{C}_{\mathrm{q}}\right)$, $134.2\left(\mathrm{C}_{\mathrm{q}}\right), 131.9(\mathrm{CH}), 131.3(\mathrm{CH}), 130.6(\mathrm{CH}), 128.9(\mathrm{CH}), 127.9(\mathrm{CH}), 124.7(\mathrm{CH}), 120.1$ (CH), $108.1(\mathrm{CH})$.

IR (ATR): $3115,1656,1552,1324,944,748 \mathrm{~cm}^{-1}$.

MS (EI) m/z (relative intensity) 297 (5) $\left[\mathrm{M}^{+}\right], 205$ (100), 178 (5), 150 (10), 142 (5), 123 (5). 
HR-MS (EI) m/z calcd for $\mathrm{C}_{16} \mathrm{H}_{13} \mathrm{~N}_{3} \mathrm{OCl}\left[\mathrm{M}+\mathrm{H}^{+}\right]$298.0747, found 298.0742.

\section{5-Ace tyl- $N$-phe nyl-2-(1H-pyrazol-1-yl)benzamide (130fa)}<smiles>CC(=O)c1ccc(-n2cccn2)c(C(=O)Nc2ccccc2)c1</smiles>

The general procedure $\mathbf{J}$ was followed using $\mathbf{1 2 8 f}$ (93 $\mathrm{mg}, 0.5 \mathrm{mmol})$ and 129a (119 $\mathrm{mg}, 1.0$ mmol). Purification by column chromatography ( $n$-hexane $/ \mathrm{CH}_{2} \mathrm{Cl}_{2} / \mathrm{EtOAc} 5: 1: 1$ ) yie lded $130 \mathrm{fa}(60 \mathrm{mg}, 39 \%)$ and 130 fa' (72 mg, 34\%) as off white solids. 130fa: M. p.: $=173-175{ }^{\circ} \mathrm{C}$.

${ }^{1} \mathbf{H}-\mathbf{N M R}\left(300 \mathrm{MHz}, \mathrm{CDCl}_{3}\right): \delta=8.68(\mathrm{br} \mathrm{s}, 1 \mathrm{H}), 8.34(\mathrm{~d}, J=1.9 \mathrm{~Hz}, 1 \mathrm{H}), 8.07$ (dd, $J=8.3,1.9$ $\mathrm{Hz}, 1 \mathrm{H}), 7.84-7.73(\mathrm{~m}, 2 \mathrm{H}), 7.57-7.40(\mathrm{~m}, 3 \mathrm{H}), 7.36-7.18(\mathrm{~m}, 2 \mathrm{H}), 7.08(\mathrm{~d}, J=7.3 \mathrm{~Hz}, 1 \mathrm{H})$, $6.48(\mathrm{dd}, J=1.9,1.8 \mathrm{~Hz}, 1 \mathrm{H}), 2.60(\mathrm{~s}, 3 \mathrm{H})$.

${ }^{13}$ C-NMR $\left(125 \mathrm{MHz}, \mathrm{CDCl}_{3}\right): \delta=196.2\left(\mathrm{C}_{\mathrm{q}}\right), 164.1\left(\mathrm{C}_{\mathrm{q}}\right), 142.0(\mathrm{CH}), 140.4\left(\mathrm{C}_{\mathrm{q}}\right), 137.6\left(\mathrm{C}_{\mathrm{q}}\right)$, $136.5\left(\mathrm{C}_{\mathrm{q}}\right), 132.2\left(\mathrm{C}_{\mathrm{q}}\right), 131.3(\mathrm{CH}), 130.9(\mathrm{CH}), 130.7(\mathrm{CH}), 128.9(\mathrm{CH}), 126.0(\mathrm{CH}), 124.7$ (CH), $120.1(\mathrm{CH}), 108.5(\mathrm{CH}), 26.7\left(\mathrm{CH}_{3}\right)$.

IR (ATR): 3289, 1677, 1651, 1594, 1243, 935, $750 \mathrm{~cm}^{-1}$.

MS (EI) m/z (relative intensity) 305 (5) $\left[\mathrm{M}^{+}\right], 213$ (100), 170 (10), 143 (5), 115 (5).

HR-MS (EI) m/z calcd for $\mathrm{C}_{18} \mathrm{H}_{15} \mathrm{~N}_{3} \mathrm{O}_{2}\left[\mathrm{M}^{+}\right]$305.1164, found 305.1172 .

\section{5-Ace tyl-N1,N3-diphe nyl-2-(1 H-pyrazol-1-yl)is ophthalamide (130fa')}<smiles>CC(=O)c1cc(C(=O)Nc2ccccc2)c(-n2cccn2)c(C(=O)Nc2ccccc2)c1</smiles>

M. p.: $=89-91^{\circ} \mathrm{C}$.

${ }^{1}$ H-NMR (300 MHz, $\left.\mathrm{CDCl}_{3}\right): \delta=11.29(\mathrm{~s}, 1 \mathrm{H}), 7.95(\mathrm{~d}, J=2.0 \mathrm{~Hz}, 1 \mathrm{H}), 7.86(\mathrm{dd}, J=8.5,2.0$ $\mathrm{Hz}, 1 \mathrm{H}), 7.82(\mathrm{dd}, J=1.8,0.6 \mathrm{~Hz}, 1 \mathrm{H}), 7.71(\mathrm{~d}, J=2.6 \mathrm{~Hz}, 1 \mathrm{H}), 7.63-7.56(\mathrm{~m}, 2 \mathrm{H}), 7.31(\mathrm{t}, J=$ $8.0 \mathrm{~Hz}, 2 \mathrm{H}), 7.27$ (d, $J=8.7 \mathrm{~Hz}, 1 \mathrm{H}), 7.14-6.99$ (m, 4H), $6.81(\mathrm{~s}, 2 \mathrm{H}), 6.55$ (dd, $J=2.6,1.7 \mathrm{~Hz}$, $1 \mathrm{H}), 2.55$ (s, 3H). 
${ }^{13}$ C-NMR $\left(125 \mathrm{MHz}, \mathrm{CDCl}_{3}\right): \delta=195.5\left(\mathrm{C}_{\mathrm{q}}\right), 151.6\left(\mathrm{C}_{\mathrm{q}}\right), 151.6\left(\mathrm{C}_{\mathrm{q}}\right), 142.4(\mathrm{CH}), 138.6\left(\mathrm{C}_{\mathrm{q}}\right)$, $137.7\left(\mathrm{C}_{\mathrm{q}}\right), 137.7\left(\mathrm{C}_{\mathrm{q}}\right), 137.1\left(\mathrm{C}_{\mathrm{q}}\right), 134.4\left(\mathrm{C}_{\mathrm{q}}\right), 130.2(\mathrm{CH}), 129.7(\mathrm{CH}), 129.5(\mathrm{CH}), 129.0(\mathrm{CH})$, 129.0 (CH), $128.6(\mathrm{CH}), 128.4(\mathrm{CH}), 124.2(\mathrm{CH}), 120.2(\mathrm{CH}), 109.1(\mathrm{CH}), 26.5\left(\mathrm{CH}_{3}\right)$.

IR (ATR): 3067, 1714, 1521, 1226, 935, $752 \mathrm{~cm}^{-1}$.

MS (EI) m/z (relative intensity) 425 (5) $\left[\mathrm{M}^{+}\right], 213$ (100), 170 (10), 143 (5), 119 (35).

HR-MS (ESI) m/z calcd for $\mathrm{C}_{25} \mathrm{H}_{21} \mathrm{~N}_{4} \mathrm{O}_{3}\left[\mathrm{M}+\mathrm{H}^{+}\right] 425.1614$ found 425.1608.

Methyl 3-(phe nylcarbamoyl)-4-(1H-pyrazol-1-yl)benzoate (130ga)<smiles>COC(=O)c1ccc(-n2cccn2)c(C(=O)Nc2ccccc2)c1</smiles>

The general procedure $\mathbf{J}$ was followed using $\mathbf{1 2 8 g}$ (101 mg, $0.5 \mathrm{mmol})$ and 129a (119 mg, 1.0 mmol). Purification by column chromatography ( $n$-hexane $/ \mathrm{CH}_{2} \mathrm{Cl}_{2} / \mathrm{EtOAc} / \mathrm{Et}_{3} \mathrm{~N}$ 8:1:1:1\%) yie lded 130ga (121 mg, 75\%) as a colorless solid. M. p.: $=163-165^{\circ} \mathrm{C}$.

${ }^{1} \mathbf{H}-\mathbf{N M R}\left(300 \mathrm{MHz}, \mathrm{CDCl}_{3}\right): \delta=8.50(\mathrm{~d}, J=2.0 \mathrm{~Hz}, 1 \mathrm{H}), 8.32(\mathrm{br} \mathrm{s}, 1 \mathrm{H}), 8.18(\mathrm{dd}, J=8.3,2.0$ $\mathrm{Hz}, 1 \mathrm{H}), 7.78$ (d, $J=2.1 \mathrm{~Hz}, 2 \mathrm{H}), 7.53$ (d, $J=8.3 \mathrm{~Hz}, 1 \mathrm{H}), 7.48-7.40$ (m, 2H), 7.28 (t, $J=7.9$ $\mathrm{Hz}, 2 \mathrm{H}), 7.16-7.03(\mathrm{~m}, 1 \mathrm{H}), 6.47$ (dd, $J=2.0,2.1 \mathrm{~Hz}, 1 \mathrm{H}), 3.95(\mathrm{~s}, 3 \mathrm{H})$.

${ }^{13}$ C-NMR $\left(125 \mathrm{MHz}, \mathrm{CDCl}_{3}\right): \delta=165.4\left(\mathrm{C}_{\mathrm{q}}\right), 164.0\left(\mathrm{C}_{\mathrm{q}}\right), 142.0(\mathrm{CH}), 140.5\left(\mathrm{C}_{\mathrm{q}}\right), 137.6\left(\mathrm{C}_{\mathrm{q}}\right)$, $132.3\left(\mathrm{C}_{\mathrm{q}}\right), 132.3(\mathrm{CH}), 131.9(\mathrm{CH}), 131.4(\mathrm{CH}), 130.2\left(\mathrm{C}_{\mathrm{q}}\right), 129.0(\mathrm{CH}), 126.0(\mathrm{CH}), 124.8$ (CH), $120.1(\mathrm{CH}), 108.4(\mathrm{CH}), 52.6\left(\mathrm{CH}_{3}\right)$.

IR (ATR): 3281, 1714, 1547, 1303, 1121, $742 \mathrm{~cm}^{-1}$.

MS (EI) m/z (relative intensity) 321 (5) [M+], 290 (5), 229 (100), 197 (5), 170 (5).

HR-MS (EI) m/z calcd for $\mathrm{C}_{18} \mathrm{H}_{15} \mathrm{~N}_{3} \mathrm{O}_{3}\left[\mathrm{M}^{+}\right]$321.1113, found 321.1122 .

3-Methyl- $N$-phe nyl-2-(1H-pyrazol-1-yl)benzamide (130ha)<smiles>Cc1cccc(C(=O)Nc2ccccc2)c1-n1cccn1</smiles> 
The general procedure $\mathbf{J}$ was followed using $\mathbf{1 2 8 h}$ (79 $\mathrm{mg}, 0.5 \mathrm{mmol})$ and 129a (119 mg, 1.0 mmol). Purification by column chromatography ( $n$-hexane/ $\mathrm{CH}_{2} \mathrm{Cl}_{2} / \mathrm{EtOAc} / \mathrm{Et}_{3} \mathrm{~N}$ 12:1:1:1\%) yie lded 130ha (112 mg, 81\%) as a colorless solid. M. p.: $=109-111^{\circ} \mathrm{C}$.

${ }^{1}$ H-NMR $\left(500 \mathrm{MHz}, \mathrm{CDCl}_{3}\right): \delta=7.85(\mathrm{dd}, J=2.0,0.6 \mathrm{~Hz}, 1 \mathrm{H}), 7.82($ br s, $1 \mathrm{H}), 7.80-7.76(\mathrm{~m}$, 1H), $7.56(\mathrm{dd}, J=2.4,0.6 \mathrm{~Hz}, 1 \mathrm{H}), 7.48(\mathrm{t}, J=7.6 \mathrm{~Hz}, 1 \mathrm{H}), 7.45-7.41(\mathrm{~m}, 1 \mathrm{H}), 7.35-7.30(\mathrm{~m}$, 2H), 7.25-7.21 (m, 2H), 7.09-6.98 (m, 1H), $6.46(\mathrm{dd}, J=2.0,2.2 \mathrm{~Hz}, 1 \mathrm{H}), 2.05(\mathrm{~s}, 3 \mathrm{H})$. ${ }^{13}$ C-NMR (75 MHz, $\left.\mathrm{CDCl}_{3}\right): \delta=164.3\left(\mathrm{C}_{\mathrm{q}}\right), 141.6(\mathrm{CH}), 137.8\left(\mathrm{C}_{\mathrm{q}}\right), 137.2\left(\mathrm{C}_{\mathrm{q}}\right), 136.1\left(\mathrm{C}_{\mathrm{q}}\right)$, $135.1\left(\mathrm{C}_{\mathrm{q}}\right), 133.1(\mathrm{CH}), 132.8(\mathrm{CH}), 129.9(\mathrm{CH}), 128.8(\mathrm{CH}), 128.2(\mathrm{CH}), 124.4(\mathrm{CH}), 119.9$ (CH), $107.6(\mathrm{CH}), 17.1\left(\mathrm{CH}_{3}\right)$.

IR (ATR): 3107, 1668, 1542, 1441, 1319, $752 \mathrm{~cm}^{-1}$.

MS (EI) m/z (relative intensity) $277(5)\left[\mathrm{M}^{+}\right], 185$ (100), 156 (5), 142 (5), 130 (10), 103 (10).

HR-MS (ESI) m/z calcd for $\mathrm{C}_{17} \mathrm{H}_{16} \mathrm{~N}_{3} \mathrm{O}\left[\mathrm{M}+\mathrm{H}^{+}\right]$278.1293, found 278.1288.

\section{3-Fluoro- $N$-phenyl-2-(1 H-pyrazol-1-yl)be nzamide (130ia)}<smiles>O=C(Nc1ccccc1)c1cccc(F)c1-n1cccn1</smiles>

The general procedure $\mathbf{J}$ was followed using $\mathbf{1 2 8 i}$ ( $81 \mathrm{mg}, 0.5 \mathrm{mmol})$ and 129a (119 $\mathrm{mg}, 1.0$ mmol). Purification by column chromatography ( $n$-hexane $/ \mathrm{CH}_{2} \mathrm{Cl}_{2} / \mathrm{EtOAc} / \mathrm{Et}_{3} \mathrm{~N}$ 10:1:1:1\%) yielded 130ia (112 mg, 80\%) as a colorless solid. M. p.: $=175-177^{\circ} \mathrm{C}$.

${ }^{1}$ H-NMR (300 MHz, $\left.\mathrm{CDCl}_{3}\right): \delta=8.64$ (br s, 1H), 7.89-7.86 (m, 1H), $7.76(\mathrm{~d}, J=7.9 \mathrm{~Hz}, 1 \mathrm{H})$, $7.68(\mathrm{td}, J=1.6,0.7 \mathrm{~Hz}, 1 \mathrm{H}), 7.58-7.48(\mathrm{~m}, 1 \mathrm{H}), 7.43-7.38(\mathrm{~m}, 2 \mathrm{H}), 7.34(\mathrm{ddd}, J=9.1,8.3,1.4$ $\mathrm{Hz}, 1 \mathrm{H}), 7.29-7.20(\mathrm{~m}, 2 \mathrm{H}), 7.12-6.97(\mathrm{~m}, 1 \mathrm{H}), 6.51(\mathrm{dd}, J=2.5,1.9 \mathrm{~Hz}, 1 \mathrm{H})$.

${ }^{13}$ C-NMR $\left(125 \mathrm{MHz}, \mathrm{CDCl}_{3}\right): \delta=162.8\left(\mathrm{C}_{\mathrm{q}}, J_{\mathrm{C}-\mathrm{F}}=3.2 \mathrm{~Hz}\right), 157.4\left(\mathrm{C}_{\mathrm{q}}, J_{\mathrm{C}-\mathrm{F}}=253.5 \mathrm{~Hz}\right), 142.0$ $(\mathrm{CH}), 137.7\left(\mathrm{C}_{\mathrm{q}}\right), 136.3\left(\mathrm{C}_{\mathrm{q}}\right), 133.7\left(\mathrm{CH}, J_{\mathrm{C}-\mathrm{F}}=1.9 \mathrm{~Hz}\right), 130.9\left(\mathrm{CH}, J_{\mathrm{C}-\mathrm{F}}=8.2 \mathrm{~Hz}\right), 128.9(\mathrm{CH})$, $126.2\left(\mathrm{CH}, J_{\mathrm{C}-\mathrm{F}}=3.7 \mathrm{~Hz}\right), 125.7\left(\mathrm{C}_{\mathrm{q}}, J_{\mathrm{C}-\mathrm{F}}=13.8 \mathrm{~Hz}\right), 124.6(\mathrm{CH}), 120.0(\mathrm{CH}), 118.7\left(\mathrm{CH}, J_{\mathrm{C}-\mathrm{F}}\right.$ $=20.6 \mathrm{~Hz}), 107.9(\mathrm{CH})$.

${ }^{19}$ F-NMR $\left(376 \mathrm{MHz}, \mathrm{CDCl}_{3}\right): \delta=-121.9(\mathrm{dd}, J=9.3,5.1 \mathrm{~Hz})$.

IR (ATR): 3243, 1651, 1543, 1308, 977, $752 \mathrm{~cm}^{-1}$. 


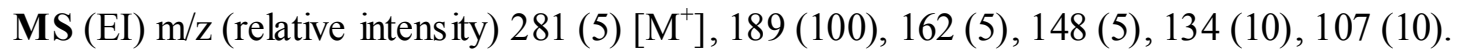

HR-MS (EI) m/z calcd for $\mathrm{C}_{16} \mathrm{H}_{13} \mathrm{~N}_{3} \mathrm{OF}\left[\mathrm{M}+\mathrm{H}^{+}\right]$282.1043, found 282.1037.

\section{3-Bromo- $N$-phe nyl-2-(1 H-pyrazol-1-yl)be nzamide (130ja)}<smiles>O=C(Nc1ccccc1)c1cccc(Br)c1-n1cccn1</smiles>

The general procedure $\mathbf{J}$ was followed using $\mathbf{1 2 8 j}$ (111 $\mathrm{mg}, 0.5 \mathrm{mmol})$ and $\mathbf{1 2 9} \mathbf{a}(119 \mathrm{mg}, 1.0$ mmol). Purification by column chromatography ( $n$-hexane $/ \mathrm{CH}_{2} \mathrm{Cl}_{2} / \mathrm{EtOAc} / \mathrm{Et}_{3} \mathrm{~N}$ 12:1:1:1\%) yie lded 130ja (116 mg, 68\%) as a colorless solid. M. p.: $=123-124{ }^{\circ} \mathrm{C}$.

${ }^{1} \mathbf{H}-\mathbf{N M R}\left(500 \mathrm{MHz}, \mathrm{CDCl}_{3}\right): \delta=8.44$ (br s, 1H), 7.89-7.83 (m, 2H), 7.77 (dd, $J=8.1,1.4$ Hz, 1H), 7.63 (dd, $J=2.4,0.6 \mathrm{~Hz}, 1 \mathrm{H}), 7.43$ (t, $J=7.9 \mathrm{~Hz}, 1 \mathrm{H}), 7.37-7.32$ (m, 2H), 7.20 (dd, $J=8.5,7.4 \mathrm{~Hz}, 2 \mathrm{H}), 7.12-6.98(\mathrm{~m}, 1 \mathrm{H}), 6.52-6.47(\mathrm{~m}, 1 \mathrm{H})$.

${ }^{13}$ C-NMR (125 MHz, $\left.\mathrm{CDCl}_{3}\right): \delta=163.0\left(\mathrm{C}_{\mathrm{q}}\right), 141.8(\mathrm{CH}), 137.6\left(\mathrm{C}_{\mathrm{q}}\right), 137.1\left(\mathrm{C}_{\mathrm{q}}\right), 136.2\left(\mathrm{C}_{\mathrm{q}}\right)$, $135.4(\mathrm{CH}), 133.3(\mathrm{CH}), 131.0(\mathrm{CH}), 129.6(\mathrm{CH}), 128.7(\mathrm{CH}), 124.5(\mathrm{CH}), 123.2\left(\mathrm{C}_{\mathrm{q}}\right), 120.0$ $(\mathrm{CH}), 107.7(\mathrm{CH})$.

IR (ATR): 3244, 1656, 1540, 1440, 1318, $749 \mathrm{~cm}^{-1}$.

MS (EI) m/z (relative intensity) 341 (5) $\left[\mathrm{M}^{+}\right], 249$ (100), 222 (5), 194 (5), 170 (10), 142 (10).

HR-MS (EI) m/z calcd for $\mathrm{C}_{16} \mathrm{H}_{14} \mathrm{~N}_{3} \mathrm{O}^{79} \mathrm{Br}\left[\mathrm{M}+\mathrm{H}^{+}\right]$342.0242, found 342.0237.

\section{2-(1 H-Indazol-1-yl)-5-methyl- $N$-phe nylbe nzamide (130ka)}<smiles>Cc1ccc(-n2ncc3ccccc32)c(C(=O)Nc2ccccc2)c1</smiles>

The general procedure $\mathbf{J}$ was followed using 128k (104 mg, $0.5 \mathrm{mmol})$ and 129a (119 mg, 1.0 mmol). Purification by column chromatography ( $n$-hexane $/ \mathrm{CH}_{2} \mathrm{Cl}_{2} / \mathrm{EtOAc} / \mathrm{Et}_{3} \mathrm{~N}$ 12:1:1:1\%) yie lded 130ka ( $87 \mathrm{mg}, 53 \%)$ as a colorless solid. M. p.: $=128-130{ }^{\circ} \mathrm{C}$. 
${ }^{1} \mathbf{H}-\mathbf{N M R}\left(500 \mathrm{MHz}, \mathrm{CDCl}_{3}\right): \delta=8.42($ br s, $1 \mathrm{H}), 8.27$ (d, $\left.J=1.0 \mathrm{~Hz}, 1 \mathrm{H}\right), 7.91-7.85(\mathrm{~m}, 1 \mathrm{H})$, $7.75(\mathrm{~d}, J=8.2 \mathrm{~Hz}, 1 \mathrm{H}), 7.42(\mathrm{ddd}, J=8.2,2.1,0.9 \mathrm{~Hz}, 1 \mathrm{H}), 7.36(\mathrm{ddd}, J=8.6,6.9,1.1 \mathrm{~Hz}, 1 \mathrm{H})$, $7.33(\mathrm{~d}, J=8.0 \mathrm{~Hz}, 1 \mathrm{H}), 7.28(\mathrm{dd}, J=8.5,1.0 \mathrm{~Hz}, 1 \mathrm{H}), 7.26-7.23(\mathrm{~m}, 2 \mathrm{H}), 7.18(\mathrm{~m}, 3 \mathrm{H})$, 7.07-6.94 (m, 1H), $2.49(\mathrm{~s}, 3 \mathrm{H})$.

${ }^{13}$ C-NMR (125 MHz, $\left.\mathrm{CDCl}_{3}\right): \delta=164.4\left(\mathrm{C}_{\mathrm{q}}\right), 141.4\left(\mathrm{C}_{\mathrm{q}}\right), 139.7\left(\mathrm{C}_{\mathrm{q}}\right), 137.7\left(\mathrm{C}_{\mathrm{q}}\right), 135.7(\mathrm{CH})$, $133.9\left(\mathrm{C}_{\mathrm{q}}\right), 133.3\left(\mathrm{C}_{\mathrm{q}}\right), 132.3(\mathrm{CH}), 131.9(\mathrm{CH}), 128.7(\mathrm{CH}), 128.0(\mathrm{CH}), 127.8(\mathrm{CH}), 124.3$ $\left(\mathrm{C}_{\mathrm{q}}\right), 124.3(\mathrm{CH}), 121.9(\mathrm{CH}), 121.2(\mathrm{CH}), 120.0(\mathrm{CH}), 110.2(\mathrm{CH}), 21.2\left(\mathrm{CH}_{3}\right)$.

IR (ATR): 3059, 1648, 1547, 1330, 831, $747 \mathrm{~cm}^{-1}$.

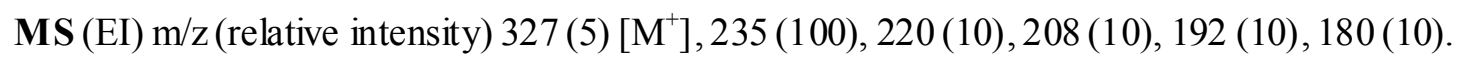

HR-MS (EI) m/z calcd for $\mathrm{C}_{21} \mathrm{H}_{17} \mathrm{~N}_{3} \mathrm{O}\left[\mathrm{M}^{+}\right]$327.1372, found 327.1368 .

\section{4-Methyl- $N$-phe nyl-2-(1H-pyrazol-1-yl)benzamide (130la)}<smiles>Cc1ccc(C(=O)Nc2ccccc2)c(-n2cccn2)c1</smiles>

The general procedure $\mathbf{J}$ was followed using 1281 (79 $\mathrm{mg}, 0.5 \mathrm{mmol})$ and 129a (119 $\mathrm{mg}, 1.0$ mmol). Purification by column chromatography ( $n$-hexane $/ \mathrm{CH}_{2} \mathrm{Cl}_{2} / \mathrm{EtOAc} / \mathrm{Et}_{3} \mathrm{~N}$ 12:1:1:1\%) yie lded 130la (91 mg, 66\%) as a colorless solid. M. p.: $=130-132{ }^{\circ} \mathrm{C}$.

${ }^{1} \mathbf{H}-\mathbf{N M R}\left(300 \mathrm{MHz}, \mathrm{CDCl}_{3}\right): \delta=8.52(\mathrm{br} \mathrm{s}, 1 \mathrm{H}), 7.76(\mathrm{~d}, J=1.9 \mathrm{~Hz}, 1 \mathrm{H}), 7.74-7.69(\mathrm{~m}, 1 \mathrm{H})$, $7.65(\mathrm{~d}, J=2.4 \mathrm{~Hz}, 1 \mathrm{H}), 7.43-7.38(\mathrm{~m}, 2 \mathrm{H}), 7.35-7.29$ (m, 1H), 7.28-7.20 (m, 3H), 7.09-7.01 (m, 1H), $6.42(\mathrm{~s}, 1 \mathrm{H}), 2.43(\mathrm{~s}, 3 \mathrm{H})$.

${ }^{13}$ C-NMR $\left(125 \mathrm{MHz}, \mathrm{CDCl}_{3}\right): \delta=164.5\left(\mathrm{C}_{\mathrm{q}}\right), 141.2(\mathrm{CH}), 139.5\left(\mathrm{C}_{\mathrm{q}}\right), 137.9\left(\mathrm{C}_{\mathrm{q}}\right), 134.8\left(\mathrm{C}_{\mathrm{q}}\right)$, $132.7\left(\mathrm{C}_{\mathrm{q}}\right), 132.1(\mathrm{CH}), 131.9(\mathrm{CH}), 131.1(\mathrm{CH}), 128.8(\mathrm{CH}), 126.8(\mathrm{CH}), 124.4(\mathrm{CH}), 120.0$ $(\mathrm{CH}), 107.6(\mathrm{CH}), 21.0\left(\mathrm{CH}_{3}\right)$.

IR (ATR): 3241, 1661, 1543, 1323, 1025, $748 \mathrm{~cm}^{-1}$.

MS (EI) m/z (relative intensity) $277(5)\left[\mathrm{M}^{+}\right], 185$ (100), 258 (5), 142 (5), 130 (10), 103 (10).

HR-MS (ESI) m/z calcd for $\mathrm{C}_{17} \mathrm{H}_{16} \mathrm{~N}_{3} \mathrm{O}\left[\mathrm{M}+\mathrm{H}^{+}\right]$278.1293, found 278.1288. 
<smiles>O=C(Nc1ccccc1)c1ccc(C(F)(F)F)cc1-n1cccn1</smiles>

The general procedure $\mathbf{J}$ was followed using $\mathbf{1 2 8} \mathbf{m}$ (106 mg, $0.5 \mathrm{mmol})$ and 129a (119 mg, 1.0 mmol), $\mathrm{Cp}^{*} \mathrm{CoI}_{2}(\mathrm{CO})(\mathbf{1 1 2}, 24 \mathrm{mg}, 10 \mathrm{~mol} \%), \operatorname{AgNTf}_{2}(39.0 \mathrm{mg}, 20 \mathrm{~mol} \%)$ and $\mathrm{AgOPiv}(20.8$ mg, $20 \mathrm{~mol} \%$ ). Purification by column chromatography ( $n$-hexane $/ \mathrm{CH}_{2} \mathrm{Cl}_{2} / \mathrm{EtOAc} / \mathrm{Et}_{3} \mathrm{~N}$ 8:1:1:1\%) yielded 130ma (78 mg, $42 \%$ ) as a colorless solid. M. p.: $=142-144{ }^{\circ} \mathrm{C}$.

${ }^{1}$ H-NMR $\left(300 \mathrm{MHz}, \mathrm{DMSO}-d_{6}\right): \delta=10.39(\mathrm{~s}, 1 \mathrm{H}), 8.26(\mathrm{~d}, J=2.5 \mathrm{~Hz}, 1 \mathrm{H}), 8.05(\mathrm{~s}, 1 \mathrm{H}), 7.86$ $(\mathrm{d}, J=1.1 \mathrm{~Hz}, 2 \mathrm{H}), 7.70(\mathrm{~d}, J=1.8 \mathrm{~Hz}, 1 \mathrm{H}), 7.62-7.53(\mathrm{~m}, 2 \mathrm{H}), 7.31(\mathrm{t}, J=7.9 \mathrm{~Hz}, 2 \mathrm{H})$, 7.16-7.04 (m, 1H), $6.51(\mathrm{dd}, J=2.5,1.8 \mathrm{~Hz}, 1 \mathrm{H})$.

${ }^{13}$ C-NMR (125 MHz, DMSO- $\left.d_{6}\right): \delta=164.5\left(\mathrm{C}_{\mathrm{q}}\right), 141.4(\mathrm{CH}), 138.7\left(\mathrm{C}_{\mathrm{q}}\right), 137.6\left(\mathrm{C}_{\mathrm{q}}\right), 135.0$ $\left(\mathrm{C}_{\mathrm{q}}\right), 130.7\left(\mathrm{C}_{\mathrm{q}}, J_{\mathrm{C}-\mathrm{F}}=32.6 \mathrm{~Hz}\right), 130.4(\mathrm{CH}), 130.2(\mathrm{CH}), 128.6(\mathrm{CH}), 123.3\left(\mathrm{C}_{\mathrm{q}}, J_{\mathrm{C}-\mathrm{F}}=273.1\right.$ $\mathrm{Hz}), 123.7(\mathrm{CH}), 120.2\left(\mathrm{CH}, J_{\mathrm{C}-\mathrm{F}}=3.9 \mathrm{~Hz}\right), 119.6(\mathrm{CH}), 107.8(\mathrm{CH})$.

IR (ATR): 3041, 1649, 1449, 1314, 1126, $758 \mathrm{~cm}^{-1}$.

MS (EI) m/z (relative intens ity) $331(5)\left[\mathrm{M}^{+}\right], 312$ (5), 239 (100), 212 (5), 198 (5), 184 (5).

HR-MS (EI) $\mathrm{m} / \mathrm{z}$ calcd for $\mathrm{C}_{17} \mathrm{H}_{12} \mathrm{~N}_{3} \mathrm{~F}_{3} \mathrm{O}\left[\mathrm{M}^{+}\right]$331.0932, found 331.0941.

\section{$N$-Phe nyl-2-(1H-pyrazol-1-yl)thiophe ne-3-carboxamide (130na)}<smiles>O=C(Nc1ccccc1)c1ccsc1-n1cccn1</smiles>

The general procedure $\mathbf{J}$ was followed using $\mathbf{1 2 8 n}$ (75 mg, $0.5 \mathrm{mmol})$ and 129a (119 mg, 1.0 mmol). Purification by column chromatography ( $n$-hexane $/ \mathrm{CH}_{2} \mathrm{Cl}_{2} / \mathrm{EtOAc} / \mathrm{Et}_{3} \mathrm{~N}$ 10:1:1:1\%) yie lded 130na (123 mg, 91\%) as a colorless solid. M. p.: $=129-131{ }^{\circ} \mathrm{C}$.

${ }^{1} \mathbf{H}-\mathbf{N M R}\left(500 \mathrm{MHz}, \mathrm{CDCl}_{3}\right): \delta=10.31$ (br s, $\left.1 \mathrm{H}\right), 7.88(\mathrm{dd}, J=2.0,0.6 \mathrm{~Hz}, 1 \mathrm{H}), 7.80(\mathrm{dd}, J=$ 2.5, $0.7 \mathrm{~Hz}, 1 \mathrm{H}), 7.61-7.57(\mathrm{~m}, 2 \mathrm{H}), 7.56(\mathrm{~d}, J=5.8 \mathrm{~Hz}, 1 \mathrm{H}), 7.30(\mathrm{~d}, J=7.6 \mathrm{~Hz}, 1 \mathrm{H}), 7.29(\mathrm{~d}$, $J=7.6 \mathrm{~Hz}, 1 \mathrm{H}), 7.14(\mathrm{~d}, J=5.8 \mathrm{~Hz}, 1 \mathrm{H}), 7.07(\mathrm{tt}, J=7.4,1.2 \mathrm{~Hz}, 1 \mathrm{H}), 6.52(\mathrm{dd}, J=2.5,2.0 \mathrm{~Hz}$, $1 \mathrm{H})$. 
${ }^{13}$ C-NMR $\left(125 \mathrm{MHz}, \mathrm{CDCl}_{3}\right): \delta=159.5\left(\mathrm{C}_{\mathrm{q}}\right), 142.0(\mathrm{CH}), 139.6\left(\mathrm{C}_{\mathrm{q}}\right), 138.3\left(\mathrm{C}_{\mathrm{q}}\right), 133.8(\mathrm{CH})$, $131.0\left(\mathrm{C}_{\mathrm{q}}\right), 130.1(\mathrm{CH}), 128.9(\mathrm{CH}), 124.2(\mathrm{CH}), 121.2(\mathrm{CH}), 119.8(\mathrm{CH}), 108.6(\mathrm{CH})$.

IR (ATR): 3225, 1660, 1534, 1312, 1057, $751 \mathrm{~cm}^{-1}$.

MS (EI) m/z (relative intensity) 269 (15) $\left[\mathrm{M}^{+}\right], 177$ (100), 150 (5), 122 (10), 105 (5).

HR-MS (EI) $\mathrm{m} / \mathrm{z}$ calcd for $\mathrm{C}_{14} \mathrm{H}_{11} \mathrm{~N}_{3} \mathrm{OS}\left[\mathrm{M}^{+}\right] 269.0623$, found 269.0612 .

\section{1-Methyl- $N$-phe nyl-2-(1 H-pyrazol-1-yl)-1 $H$-indole-3-carboxamide (130oa)}<smiles>Cn1c(-n2cccn2)c(C(=O)Nc2ccccc2)c2ccccc21</smiles>

The general procedure $\mathbf{J}$ was followed us ing $\mathbf{1 2 8 0}$ (106 mg, $0.5 \mathrm{mmol})$ and $\mathbf{1 2 9}$ a (119 mg, 1.0 $\mathrm{mmol}), \mathrm{Cp}^{*} \mathrm{CoI}_{2}(\mathrm{CO})(\mathbf{1 1 2}, 24 \mathrm{mg}, 10 \mathrm{~mol} \%), \operatorname{AgNTf}_{2}(39.0 \mathrm{mg}, 20 \mathrm{~mol} \%)$ and $\mathrm{AgOPiv}(20.8$ $\mathrm{mg}, 20 \mathrm{~mol} \%$ ). Purification by column chromatography ( $n$-hexane $/ \mathrm{CH}_{2} \mathrm{Cl}_{2} / \mathrm{EtOAc} / \mathrm{Et}_{3} \mathrm{~N}$ 8:1:1:1\%) yielded $\mathbf{1 3 0 0 a}(66 \mathrm{mg}, 42 \%)$ as a yellow oil.

${ }^{1}$ H-NMR (500 MHz, $\left.\mathrm{CDCl}_{3}\right): \delta=8.40$ (ddd, $\left.J=8.0,1.3,0.9 \mathrm{~Hz}, 1 \mathrm{H}\right), 8.08$ (br s, $\left.1 \mathrm{H}\right), 8.01$ (dd, $J=1.9,0.6 \mathrm{~Hz}, 1 \mathrm{H}), 7.78(\mathrm{dd}, J=2.5,0.6 \mathrm{~Hz}, 1 \mathrm{H}), 7.46-7.42(\mathrm{~m}, 2 \mathrm{H}), 7.40(\mathrm{ddd}, J=8.3,6.7$, $1.3 \mathrm{~Hz}, 1 \mathrm{H}), 7.37-7.31(\mathrm{~m}, 2 \mathrm{H}), 7.28-7.24(\mathrm{~m}, 2 \mathrm{H}), 6.63(\mathrm{dd}, J=2.5,1.9 \mathrm{~Hz}, 1 \mathrm{H}), 3.50(\mathrm{~s}, 3 \mathrm{H})$. ${ }^{13}$ C-NMR (125 MHz, $\left.\mathrm{CDCl}_{3}\right): \delta=161.3\left(\mathrm{C}_{\mathrm{q}}\right), 143.1(\mathrm{CH}), 138.5\left(\mathrm{C}_{\mathrm{q}}\right), 134.4\left(\mathrm{C}_{\mathrm{q}}\right), 134.3(\mathrm{CH})$, $133.3\left(\mathrm{C}_{\mathrm{q}}\right), 128.8(\mathrm{CH}), 125.3\left(\mathrm{C}_{\mathrm{q}}\right), 124.42(\mathrm{CH}), 123.6(\mathrm{CH}), 122.6(\mathrm{CH}), 122.5(\mathrm{CH}), 119.5$ $(\mathrm{CH}), 109.7(\mathrm{CH}), 108.5(\mathrm{CH}), 107.3\left(\mathrm{C}_{\mathrm{q}}\right), 29.6\left(\mathrm{CH}_{3}\right)$.

IR (ATR): 1658, 1528, 1449, 1245, 1103, $747 \mathrm{~cm}^{-1}$.

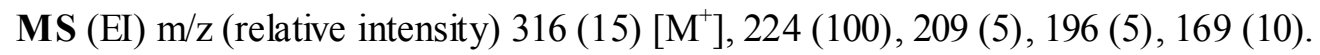

HR-MS (EI) m/z calcd for $\mathrm{C}_{19} \mathrm{H}_{16} \mathrm{~N}_{4} \mathrm{O}\left[\mathrm{M}^{+}\right]$316.1324, found 316.1333 .

\section{Methyl 4-(phe nylcarbamoyl)-5-(1H-pyrazol-1-yl)furan-2-carboxylate (130pa)}<smiles>COC(=O)c1cc(C(=O)Nc2ccccc2)c(-n2cccn2)o1</smiles> 
The general procedure $\mathbf{J}$ was followed using $\mathbf{1 2 8} \mathbf{p}$ (96 mg, $0.5 \mathrm{mmol}$ ) and 129a (119 mg, 1.0 $\mathrm{mmol}), \mathrm{Cp}^{*} \mathrm{CoI}_{2}(\mathrm{CO})(\mathbf{1 1 2}, 24 \mathrm{mg}, 10 \mathrm{~mol} \%), \mathrm{AgSbF}_{6}(34.4 \mathrm{mg}, 20 \mathrm{~mol} \%)$ and $\mathrm{AgOPiv}(20.8$ $\mathrm{mg}, 20 \mathrm{~mol} \%$ ). Purification by column chromatography ( $n$-hexane $/ \mathrm{CH}_{2} \mathrm{Cl}_{2} / \mathrm{EtOAc} / \mathrm{Et}_{3} \mathrm{~N}$ 8:1:1:1\%) yielded 130pa (78 mg, 50\%) as a colorless solid. M. p.: $=140-142{ }^{\circ} \mathrm{C}$.

${ }^{1} \mathbf{H}-\mathbf{N M R}\left(300 \mathrm{MHz}, \mathrm{CDCl}_{3}\right): \delta=12.3(\mathrm{br} \mathrm{s}, 1 \mathrm{H}), 8.26(\mathrm{dd}, J=2.8,0.6 \mathrm{~Hz}, 1 \mathrm{H}), 7.93(\mathrm{~d}, J=1.7$ Hz, 1H), 7.83 (s, 1H), 7.69 (d, $J=8.4 \mathrm{~Hz}, 2 \mathrm{H}), 7.34$ (dd, $J=8.4,7.5 \mathrm{~Hz}, 2 \mathrm{H}), 7.10$ (dd, $J=7.5$, $7.5 \mathrm{~Hz}, 1 \mathrm{H}), 6.60(\mathrm{dd}, J=2.8,1.7 \mathrm{~Hz}, 1 \mathrm{H}), 3.91(\mathrm{~s}, 3 \mathrm{H})$.

${ }^{13}$ C-NMR $\left(125 \mathrm{MHz}, \mathrm{CDCl}_{3}\right): \delta=158.1\left(\mathrm{C}_{\mathrm{q}}\right), 157.3\left(\mathrm{C}_{\mathrm{q}}\right), 144.2\left(\mathrm{C}_{\mathrm{q}}\right), 142.2(\mathrm{CH}), 138.5\left(\mathrm{C}_{\mathrm{q}}\right)$, $138.3\left(\mathrm{C}_{\mathrm{q}}\right), 130.2(\mathrm{CH}), 129.0(\mathrm{CH}), 124.2(\mathrm{CH}), 122.7(\mathrm{CH}), 120.0(\mathrm{CH}), 110.0\left(\mathrm{C}_{\mathrm{q}}\right), 108.9$ (CH), $52.3\left(\mathrm{CH}_{3}\right)$.

IR (ATR): 3027, 1739, 1557, 1317, 1147, $748 \mathrm{~cm}^{-1}$.

MS (EI) m/z (relative intensity) $311(25)\left[\mathrm{M}^{+}\right], 280$ (5), 243 (5), 219 (100), 187 (5), 151 (15).

HR-MS (EI) m/z calcd for $\mathrm{C}_{16} \mathrm{H}_{13} \mathrm{~N}_{3} \mathrm{O}_{4}\left[\mathrm{M}^{+}\right]$311.0906, found 311.0903 .

\section{3-Methyl-2-(1 H-pyrazol-1-yl)- $N$-(p-tolyl)be nzamide (130hb)}<smiles>Cc1ccc(NC(=O)c2cccc(C)c2-n2cccn2)cc1</smiles>

The general procedure $\mathbf{J}$ was followed us ing $\mathbf{1 2 8 h}$ (79 $\mathrm{mg}, 0.5 \mathrm{mmol})$ and $\mathbf{1 2 9 b}$ (133 $\mathrm{mg}, 1.0$ mmol). Purification by column chromatography ( $n$-hexane $/ \mathrm{CH}_{2} \mathrm{Cl}_{2} / \mathrm{EtOAc}_{\mathrm{Et}} \mathrm{N}$ 12:1:1:1\%) yie lded 130hb (74 mg, 51\%) as a colorless solid. M. p.: $=149-151^{\circ} \mathrm{C}$.

${ }^{1} \mathbf{H}-\mathbf{N M R}\left(500 \mathrm{MHz}, \mathrm{CDCl}_{3}\right): \delta=7.84(\mathrm{dd}, J=2.0,0.6 \mathrm{~Hz}, 1 \mathrm{H}), 7.79(\mathrm{dd}, J=7.6,1.6 \mathrm{~Hz}, 1 \mathrm{H})$, 7.73 (br s, 1H), 7.55 (dd, $J=2.4,0.6 \mathrm{~Hz}, 1 \mathrm{H}), 7.47$ (dd, $J=7.6,7.6 \mathrm{~Hz}, 1 \mathrm{H}), 7.42$ (ddd, $J=7.6$, $1.7,0.8 \mathrm{~Hz}, 1 \mathrm{H}), 7.21(\mathrm{~d}, J=8.4 \mathrm{~Hz}, 2 \mathrm{H}), 7.03(\mathrm{~d}, J=8.4 \mathrm{~Hz}, 2 \mathrm{H}), 6.45(\mathrm{dd}, J=2.2,1.6 \mathrm{~Hz}, 1 \mathrm{H})$, $2.26(\mathrm{~s}, 3 \mathrm{H}), 2.05(\mathrm{~s}, 3 \mathrm{H})$.

${ }^{13}$ C-NMR (125 MHz, $\left.\mathrm{CDCl}_{3}\right): \delta=164.2\left(\mathrm{C}_{\mathrm{q}}\right), 141.5(\mathrm{CH}), 137.1\left(\mathrm{C}_{\mathrm{q}}\right), 136.1\left(\mathrm{C}_{\mathrm{q}}\right), 135.2\left(\mathrm{C}_{\mathrm{q}}\right)$, $135.2\left(\mathrm{C}_{\mathrm{q}}\right), 134.0\left(\mathrm{C}_{\mathrm{q}}\right), 133.0(\mathrm{CH}), 132.7(\mathrm{CH}), 129.8(\mathrm{CH}), 129.3(\mathrm{CH}), 128.1(\mathrm{CH}), 120.0$ $(\mathrm{CH}), 107.5(\mathrm{CH}), 20.8\left(\mathrm{CH}_{3}\right), 17.1\left(\mathrm{CH}_{3}\right)$. 
IR (ATR): 3244, 1672, 1523, 1317, 1050, $751 \mathrm{~cm}^{-1}$.

MS (EI) m/z (relative intensity) 291 (5) [M+], 185 (100), 158 (5), 142 (5), 130 (10), 103 (5).

HR-MS (EI) m/z calcd for $\mathrm{C}_{18} \mathrm{H}_{17} \mathrm{~N}_{3} \mathrm{O}\left[\mathrm{M}^{+}\right]$291.1372, found 291.1381 .

\section{$N$-(4-Methoxyphe nyl)-3-me thyl-2-(1 H-pyrazol-1-yl)be nzamide (130hc)}<smiles>COc1ccc(NC(=O)c2cccc(C)c2-n2cccn2)cc1</smiles>

The general procedure $\mathbf{J}$ was followed using $\mathbf{1 2 8 h}$ (79 $\mathrm{mg}, 0.5 \mathrm{mmol})$ and $\mathbf{1 2 9}$ c (149 $\mathrm{mg}, 1.0$ mmol). Purification by column chromatography ( $n$-hexane $/ \mathrm{CH}_{2} \mathrm{Cl}_{2} / \mathrm{EtOAc} / \mathrm{Et}_{3} \mathrm{~N}$ 8:1:1:1\%) yielded $130 \mathrm{hc}$ (78 $\mathrm{mg}, 51 \%$ ) as colorless solids. M. p.: $=98-100{ }^{\circ} \mathrm{C}$.

${ }^{1} \mathbf{H}-\mathbf{N M R}\left(500 \mathrm{MHz}, \mathrm{CDCl}_{3}\right): \delta=7.83(\mathrm{dd}, J=2.0,0.6 \mathrm{~Hz}, 1 \mathrm{H}), 7.76(\mathrm{dd}, J=7.6,1.1 \mathrm{~Hz}, 1 \mathrm{H})$, 7.71 (br s, 1H), $7.55(\mathrm{dd}, J=2.4,0.6 \mathrm{~Hz}, 1 \mathrm{H}), 7.47$ (dd, $J=7.6,7.6 \mathrm{~Hz}, 1 \mathrm{H}), 7.43-7.39(\mathrm{~m}, 1 \mathrm{H})$, $7.22(\mathrm{~d}, J=9.0 \mathrm{~Hz}, 2 \mathrm{H}), 6.76(\mathrm{~d}, J=9.0 \mathrm{~Hz}, 2 \mathrm{H}), 6.46(\mathrm{t}, J=2.1 \mathrm{~Hz}, 1 \mathrm{H}), 3.74(\mathrm{~s}, 3 \mathrm{H}), 2.04(\mathrm{~s}$, $3 \mathrm{H})$.

${ }^{13}$ C-NMR (125 MHz, $\left.\mathrm{CDCl}_{3}\right): \delta=164.1\left(\mathrm{C}_{\mathrm{q}}\right), 156.4\left(\mathrm{C}_{\mathrm{q}}\right), 141.4(\mathrm{CH}), 137.1\left(\mathrm{C}_{\mathrm{q}}\right), 136.1\left(\mathrm{C}_{\mathrm{q}}\right)$, $135.2\left(\mathrm{C}_{\mathrm{q}}\right), 132.9(\mathrm{CH}), 132.8(\mathrm{CH}), 130.9\left(\mathrm{C}_{\mathrm{q}}\right), 129.8(\mathrm{CH}), 128.1(\mathrm{CH}), 121.7(\mathrm{CH}), 113.9$ $(\mathrm{CH}), 107.5(\mathrm{CH}), 55.4\left(\mathrm{CH}_{3}\right), 17.1\left(\mathrm{CH}_{3}\right)$.

IR (ATR): 3103, 1659, 1511, 1243, 1035, $759 \mathrm{~cm}^{-1}$.

MS (EI) m/z (relative intensity) 307 (5) $\left[\mathrm{M}^{+}\right], 185$ (100), 156 (5), 142 (5), 130 (5), 103 (5).

HR-MS (EI) m/z calcd for $\mathrm{C}_{18} \mathrm{H}_{17} \mathrm{~N}_{3} \mathrm{O}_{2}\left[\mathrm{M}^{+}\right]$307.1321, found 307.1327.

\section{$N$-(4-Fluorophe nyl)-3-me thyl-2-(1H-pyrazol-1-yl)be nzamide (130hd)}<smiles>Cc1cccc(C(=O)Nc2ccc(F)cc2)c1-n1cccn1</smiles> 
The general procedure $\mathbf{J}$ was followed using $\mathbf{1 2 8 h}$ (79 $\mathrm{mg}, 0.5 \mathrm{mmol})$ and $\mathbf{1 2 9 d}$ (114 mg, 1.0 mmol). Purification by column chromatography ( $n$-hexane $/ \mathrm{CH}_{2} \mathrm{Cl}_{2} / \mathrm{EtOAc} / \mathrm{Et}_{3} \mathrm{~N}$ 8:1:1:1\%) yie lded 130hd (143 mg, 97\%) as a colorless solid. M. p.: $=144-146{ }^{\circ} \mathrm{C}$.

${ }^{1}$ H-NMR (400 MHz, $\mathrm{CDCl}_{3}$ ): $\delta=7.94$ (br s, $\left.1 \mathrm{H}\right), 7.83$ (dd, $\left.J=2.0,0.6 \mathrm{~Hz}, 1 \mathrm{H}\right), 7.78-7.71$ (m, 1H), $7.55(\mathrm{dd}, J=2.4,0.6 \mathrm{~Hz}, 1 \mathrm{H}), 7.46(\mathrm{dd}, J=7.6,7.6 \mathrm{~Hz}, 1 \mathrm{H}), 7.43-7.39(\mathrm{~m}, 1 \mathrm{H}), 7.31-7.25$ (m, 2H), 7.01-6.77 (m, 2H), $6.46(\mathrm{dd}, J=2.4,1.9 \mathrm{~Hz}, 1 \mathrm{H}), 2.04$ (s, 3H).

${ }^{13}$ C-NMR (125 MHz, $\left.\mathrm{CDCl}_{3}\right): \delta=164.3\left(\mathrm{C}_{\mathrm{q}}\right), 159.3\left(\mathrm{C}_{\mathrm{q}}, J_{\mathrm{C}-\mathrm{F}}=243.7 \mathrm{~Hz}\right), 141.4(\mathrm{CH}), 137.1$ $\left(\mathrm{C}_{\mathrm{q}}\right), 136.1\left(\mathrm{C}_{\mathrm{q}}\right), 134.9\left(\mathrm{C}_{\mathrm{q}}\right), 133.8\left(\mathrm{C}_{\mathrm{q}}, J_{\mathrm{C}-\mathrm{F}}=2.9 \mathrm{~Hz}\right), 133.1(\mathrm{CH}), 132.8(\mathrm{CH}), 129.8(\mathrm{CH})$, $128.1(\mathrm{CH}), 121.7\left(\mathrm{CH}, J_{\mathrm{C}-\mathrm{F}}=7.9 \mathrm{~Hz}\right), 115.4\left(\mathrm{CH}, J_{\mathrm{C}-\mathrm{F}}=22.4 \mathrm{~Hz}\right), 107.5(\mathrm{CH}), 17.1\left(\mathrm{CH}_{3}\right)$. ${ }^{19}$ F-NMR $\left(376 \mathrm{MHz}, \mathrm{CDCl}_{3}\right): \delta=-117.8(\operatorname{td}, J=8.4,4.5 \mathrm{~Hz})$.

IR (ATR): 3056, 1673, 1508, 1211, 1055, $754 \mathrm{~cm}^{-1}$.

MS (EI) m/z (relative intensity) 295 (5) [M+], 185 (100), 156 (5), 142 (5), 130 (10), 103 (5).

HR-MS (EI) $\mathrm{m} / \mathrm{z}$ calcd for $\mathrm{C}_{17} \mathrm{H}_{14} \mathrm{~N}_{3} \mathrm{FO}\left[\mathrm{M}^{+}\right] 295.1121$, found 295.1123 .

\section{$N$-(4-Chlorophe nyl)-3-methyl-2-(1 H-pyrazol-1-yl)be nzamide (130he)}<smiles>Cc1cccc(C(=O)Nc2ccc(Cl)cc2)c1-n1cccn1</smiles>

The general procedure $\mathbf{J}$ was followed using $\mathbf{1 2 8 h}$ (79 $\mathrm{mg}, 0.5 \mathrm{mmol})$ and $\mathbf{1 2 9 e}$ (153 $\mathrm{mg}, 1.0$ mmol). Purification by column chromatography (n-hexane $/ \mathrm{CH}_{2} \mathrm{Cl}_{2} / \mathrm{EtOAc} / \mathrm{Et}_{3} \mathrm{~N}$ 10:1:1:1\% $\rightarrow 10: 2: 1: 0)$ yielded $\mathbf{1 3 0 h e}(128 \mathrm{mg}, 82 \%)$ as a colorless solid. M. p.: $=153-155{ }^{\circ} \mathrm{C}$. ${ }^{1}$ H-NMR (400 MHz, $\mathrm{CDCl}_{3}$ ): $\delta=7.97$ (br s, $\left.1 \mathrm{H}\right), 7.83(\mathrm{dd}, J=1.8,0.6 \mathrm{~Hz}, 1 \mathrm{H}), 7.75$ (ddd, $J=$ 7.6, 1.8, 0.6 Hz, 1H), 7.55 (dd, $J=2.4,0.6 \mathrm{~Hz}, 1 \mathrm{H}), 7.47$ (dd, $J=7.6,7.6 \mathrm{~Hz}, 1 \mathrm{H}), 7.44-7.41(\mathrm{~m}$, 1H), $7.28(\mathrm{~d}, J=8.9 \mathrm{~Hz}, 2 \mathrm{H}), 7.17(\mathrm{~d}, J=8.9 \mathrm{~Hz}, 2 \mathrm{H}), 6.46(\mathrm{dd}, J=2.4,1.9 \mathrm{~Hz}, 1 \mathrm{H}), 2.04(\mathrm{~s}$, $3 \mathrm{H})$.

${ }^{13}$ C-NMR (100 MHz, $\left.\mathrm{CDCl}_{3}\right): \delta=164.3\left(\mathrm{C}_{\mathrm{q}}\right), 141.5(\mathrm{CH}), 137.2\left(\mathrm{C}_{\mathrm{q}}\right), 136.4\left(\mathrm{C}_{\mathrm{q}}\right), 136.1\left(\mathrm{C}_{\mathrm{q}}\right)$, $134.8\left(\mathrm{C}_{\mathrm{q}}\right), 133.3(\mathrm{CH}), 132.9(\mathrm{CH}), 129.9(\mathrm{CH}), 129.2\left(\mathrm{C}_{\mathrm{q}}\right), 128.8(\mathrm{CH}), 128.1(\mathrm{CH}), 121.1$ $(\mathrm{CH}), 107.6(\mathrm{CH}), 17.1\left(\mathrm{CH}_{3}\right)$. 
IR (ATR): 3236, 1678, 1491, 1397, 1312, $753 \mathrm{~cm}^{-1}$.

MS (EI) m/z (relative intensity) 311 (5) [M+], 185 (100), 156 (5), 142 (5), 130 (10), 103 (5).

HR-MS (EI) $\mathrm{m} / \mathrm{z}$ calcd for $\mathrm{C}_{17} \mathrm{H}_{14} \mathrm{~N}_{3} \mathrm{OCl}\left[\mathrm{M}^{+}\right]$311.0825, found 311.0826.

\section{3-Methyl-2-(1 H-pyrazol-1-yl)- $N$-( $m$-tolyl)be nzamide (130hf)}<smiles>Cc1cccc(NC(=O)c2cccc(C)c2-n2cccn2)c1</smiles>

The general procedure $\mathbf{J}$ was followed using $\mathbf{1 2 8 h}$ (79 $\mathrm{mg}, 0.5 \mathrm{mmol})$ and $\mathbf{1 2 9 f}$ (133 $\mathrm{mg}, 1.0$ mmol). Purification by column chromatography ( $n$-hexane $/ \mathrm{CH}_{2} \mathrm{Cl}_{2} / \mathrm{EtOAc} / \mathrm{Et}_{3} \mathrm{~N}$ 10:1:1:1\%) yie lded 130hf (74 mg, 51\%) as a colorless solid. M. p.: =119-121 ${ }^{\circ} \mathrm{C}$.

${ }^{1}$ H-NMR (400 MHz, $\mathrm{CDCl}_{3}$ ): $\delta=7.84(\mathrm{dd}, J=2.0,0.6 \mathrm{~Hz}, 1 \mathrm{H}), 7.79$ (br s, 1H), 7.76 (ddd, $J=$ 7.7, 1.8, $0.7 \mathrm{~Hz}, 1 \mathrm{H}), 7.55(\mathrm{dd}, J=2.4,0.6 \mathrm{~Hz}, 1 \mathrm{H}), 7.47$ (dd, $J=7.6,7.6 \mathrm{~Hz}, 1 \mathrm{H}), 7.42$ (ddd, $J$ $=7.6,1.8,0.7 \mathrm{~Hz}, 1 \mathrm{H}), 7.25(\mathrm{dd}, J=1.3,0.7 \mathrm{~Hz}, 1 \mathrm{H}), 7.16-7.08(\mathrm{~m}, 1 \mathrm{H}), 7.05(\mathrm{~m}, 1 \mathrm{H})$, 6.91-6.75 (m, 1H), $6.46(\mathrm{dd}, J=2.4,1.9 \mathrm{~Hz}, 1 \mathrm{H}), 2.28(\mathrm{~s}, 3 \mathrm{H}), 2.05(\mathrm{~s}, 3 \mathrm{H})$.

${ }^{13}$ C-NMR (100 MHz, $\left.\mathrm{CDCl}_{3}\right): \delta=164.3\left(\mathrm{C}_{\mathrm{q}}\right), 141.5(\mathrm{CH}), 138.7\left(\mathrm{C}_{\mathrm{q}}\right), 137.7\left(\mathrm{C}_{\mathrm{q}}\right), 137.2\left(\mathrm{C}_{\mathrm{q}}\right)$, $136.1\left(\mathrm{C}_{\mathrm{q}}\right), 135.2\left(\mathrm{C}_{\mathrm{q}}\right), 133.0(\mathrm{CH}), 132.7(\mathrm{CH}), 129.8(\mathrm{CH}), 128.5(\mathrm{CH}), 128.1(\mathrm{CH}), 125.1$ $(\mathrm{CH}), 120.5(\mathrm{CH}), 117.0(\mathrm{CH}), 107.5(\mathrm{CH}), 21.4\left(\mathrm{CH}_{3}\right), 17.1\left(\mathrm{CH}_{3}\right)$.

IR (ATR): 3111, 1656, 1551, 1323, 944, $748 \mathrm{~cm}^{-1}$.

MS (EI) m/z (relative intensity) 291 (5) $\left[\mathrm{M}^{+}\right], 185$ (100), 156 (5), 142 (5), 130 (10), 103 (5).

HR-MS (EI) m/z calcd for $\mathrm{C}_{18} \mathrm{H}_{17} \mathrm{~N}_{3} \mathrm{O}\left[\mathrm{M}^{+}\right]$291.1372, found 291.1375.

\section{2-(1 H-pyrazol-1-yl)- $N$-(p-tolyl)be nzamide (130ab)}<smiles>Cc1ccc(NC(=O)c2ccccc2-n2cccn2)cc1</smiles> 
The general procedure $\mathbf{J}$ ( or K) was followed using 128a (72 mg, $0.5 \mathrm{mmol}$ ) and 129b (133 mg,

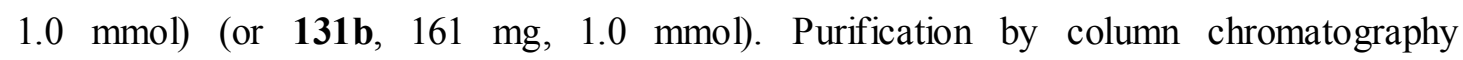
(n-hexane $/ \mathrm{CH}_{2} \mathrm{Cl}_{2} / \mathrm{EtOAc} / \mathrm{Et}_{3} \mathrm{~N}$ 10:1:1:1\%) yielded 130ab (83 mg, 60\%; or $72 \mathrm{mg}, 52 \%$ ) as a colorless solid. M. p.: $=167-169^{\circ} \mathrm{C}$.

${ }^{1} \mathbf{H}-\mathbf{N M R}\left(300 \mathrm{MHz}, \mathrm{CDCl}_{3}\right): \delta=8.22($ br s, 1H), 7.98-7.87 (m, 1H), 7.80-7.76 (m, 1H), 7.69 (dd, $J=2.5,0.6 \mathrm{~Hz}, 1 \mathrm{H}), 7.62-7.48(\mathrm{~m}, 2 \mathrm{H}), 7.46-7.38(\mathrm{~m}, 1 \mathrm{H}), 7.29$ (d, $J=8.4 \mathrm{~Hz}, 2 \mathrm{H}), 7.06$ (d, $J=8.3 \mathrm{~Hz}, 2 \mathrm{H}), 6.44$ (d, $J=2.5,2.2 \mathrm{~Hz}, 1 \mathrm{H}), 2.28$ (s, 3H).

${ }^{13}$ C-NMR $\left(125 \mathrm{MHz}, \mathrm{CDCl}_{3}\right): \delta=164.1\left(\mathrm{C}_{\mathrm{q}}\right), 141.3(\mathrm{CH}), 137.2\left(\mathrm{C}_{\mathrm{q}}\right), 135.2\left(\mathrm{C}_{\mathrm{q}}\right), 134.1\left(\mathrm{C}_{\mathrm{q}}\right)$, $133.0\left(\mathrm{C}_{\mathrm{q}}\right), 131.9(\mathrm{CH}), 131.2(\mathrm{CH}), 130.6(\mathrm{CH}), 129.3(\mathrm{CH}), 129.1(\mathrm{CH}), 126.8(\mathrm{CH}), 120.0$ (CH), $107.7(\mathrm{CH}), 20.9\left(\mathrm{CH}_{3}\right)$.

IR (ATR): 3245, 1650, 1541, 1325, 1040, $753 \mathrm{~cm}^{-1}$.

MS (EI) m/z (relative intensity) 277 (5) $\left[\mathrm{M}^{+}\right], 171$ (100), 144 (5), 130 (5), 116 (15), 89 (10).

HR-MS (EI) m/z calcd for $\mathrm{C}_{17} \mathrm{H}_{15} \mathrm{~N}_{3} \mathrm{O}\left[\mathrm{M}^{+}\right]$277.1215, found 277.1208.

\section{$N$-(4-Methoxyphe nyl)-2-(1H-pyrazol-1-yl)be nzamide (130ac)}<smiles>COc1ccc(NC(=O)c2ccccc2-n2cccn2)cc1</smiles>

The general procedure $\mathbf{K}$ was followed using $128 \mathrm{a}$ (72 $\mathrm{mg}, 0.5 \mathrm{mmol})$ and 131c (177 $\mathrm{mg}, 1.0$ mmol). Purification by column chromatography ( $n$-hexane $/ \mathrm{CH}_{2} \mathrm{Cl}_{2} / \mathrm{EtOAc} / \mathrm{Et}_{3} \mathrm{~N}$ 8:1:1:1\%) yie lded 130ac (88 mg, 60\%) as a colorless solid. M. p.: $=130-132{ }^{\circ} \mathrm{C}$.

${ }^{1}$ H-NMR (500 MHz, $\left.\mathrm{CDCl}_{3}\right): \delta=8.22$ (br s, 1H), 7.94-7.88 (m, 1H), 7.77 (dd, $J=1.9,0.7$ $\mathrm{Hz}, 1 \mathrm{H}), 7.70$ (dd, $J=2.4,0.7 \mathrm{~Hz}, 1 \mathrm{H}), 7.60-7.46$ (m, 2H), 7.43-7.38 (m, 1H), 7.30 (d, $J=$ $9.0 \mathrm{~Hz}, 2 \mathrm{H}), 6.80(\mathrm{~d}, J=9.0 \mathrm{~Hz}, 2 \mathrm{H}), 6.45(\mathrm{dd}, J=2.4,1.9 \mathrm{~Hz}, 1 \mathrm{H}), 3.75(\mathrm{~s}, 3 \mathrm{H})$.

${ }^{13}$ C-NMR (125 MHz, $\left.\mathrm{CDCl}_{3}\right): \delta=164.2\left(\mathrm{C}_{\mathrm{q}}\right), 156.5\left(\mathrm{C}_{\mathrm{q}}\right), 141.3(\mathrm{CH}), 137.2\left(\mathrm{C}_{\mathrm{q}}\right), 133.1\left(\mathrm{C}_{\mathrm{q}}\right)$, $132.0(\mathrm{CH}), 131.2(\mathrm{CH}), 130.9\left(\mathrm{C}_{\mathrm{q}}\right), 130.6(\mathrm{CH}), 129.1(\mathrm{CH}), 126.8(\mathrm{CH}), 121.8(\mathrm{CH}), 114.0$ $(\mathrm{CH}), 107.7(\mathrm{CH}), 55.4\left(\mathrm{CH}_{3}\right)$.

IR (ATR): 3049, 1665, 1510, 1243, 1031, $764 \mathrm{~cm}^{-1}$. 
MS (EI) m/z (relative intensity) 293 (10) $\left[\mathrm{M}^{+}\right], 171$ (100), 144 (5), 116 (15).

HR-MS (EI) m/z calcd for $\mathrm{C}_{17} \mathrm{H}_{15} \mathrm{~N}_{3} \mathrm{O}_{2}\left[\mathrm{M}^{+}\right]$293.1164, found 293.1167.

\section{$N$-([1,1'-Biphe nyl]-4-yl)-2-(1 H-pyrazol-1-yl)be nzamide (130ag)}<smiles>O=C(Nc1ccc(-c2ccccc2)cc1)c1ccccc1-n1cccn1</smiles>

The general procedure $\mathbf{K}$ was followed using $128 \mathbf{a}$ (72 $\mathrm{mg}, 0.5 \mathrm{mmol})$ and $\mathbf{1 3 1 g}$ (223 $\mathrm{mg}, 1.0$ mmol). Purification by column chromatography ( $n$-hexane $/ \mathrm{CH}_{2} \mathrm{Cl}_{2} / \mathrm{EtOAc} / \mathrm{Et}_{3} \mathrm{~N}$ 8:1:1:1\%) yie lded 130ag (97 mg, 57\%) as a colorless solid. M. p.: $=168-169{ }^{\circ} \mathrm{C}$.

${ }^{1} \mathbf{H}-\mathbf{N M R}\left(500 \mathrm{MHz}, \mathrm{CDCl}_{3}\right): \delta=8.63(\mathrm{br} \mathrm{s}, 1 \mathrm{H}), 7.91(\mathrm{dd}, J=7.2,2.1 \mathrm{~Hz}, 1 \mathrm{H}), 7.80(\mathrm{~d}, J=1.9$ $\mathrm{Hz}, 1 \mathrm{H}), 7.71(\mathrm{~d}, J=2.4 \mathrm{~Hz}, 1 \mathrm{H}), 7.61-7.45(\mathrm{~m}, 8 \mathrm{H}), 7.44-7.35(\mathrm{~m}, 3 \mathrm{H}), 7.33-7.25(\mathrm{~m}, 1 \mathrm{H})$, $6.46(\mathrm{t}, J=2.1 \mathrm{~Hz}, 1 \mathrm{H})$.

${ }^{13}$ C-NMR (125 MHz, $\left.\mathrm{CDCl}_{3}\right): \delta=164.4\left(\mathrm{C}_{\mathrm{q}}\right), 141.4(\mathrm{CH}), 140.5\left(\mathrm{C}_{\mathrm{q}}\right), 137.3\left(\mathrm{C}_{\mathrm{q}}\right), 137.2\left(\mathrm{C}_{\mathrm{q}}\right)$, $137.2\left(\mathrm{C}_{\mathrm{q}}\right), 132.9\left(\mathrm{C}_{\mathrm{q}}\right), 132.0(\mathrm{CH}), 131.4(\mathrm{CH}), 130.7(\mathrm{CH}), 129.1(\mathrm{CH}), 128.7(\mathrm{CH}), 127.5$ (CH), $127.0(\mathrm{CH}), 126.8(\mathrm{CH}), 126.8(\mathrm{CH}), 120.3(\mathrm{CH}), 107.8(\mathrm{CH})$.

IR (ATR): 3243, 1656, 1518, 1303, 840, $734 \mathrm{~cm}^{-1}$.

MS (EI) m/z (relative intensity) $339(5)\left[\mathrm{M}^{+}\right], 171$ (100), 144 (5), 130 (5), 116 (10).

HR-MS (ESI) m/z calcd for $\mathrm{C}_{22} \mathrm{H}_{17} \mathrm{~N}_{3} \mathrm{O}\left[\mathrm{M}+\mathrm{H}^{+}\right]$339.1372, found 339.1376 .

\section{N-(4-B romophe nyl)-2-(1 H-pyrazol-1-yl)be nzamide (130ah)}<smiles>O=C(Nc1ccc(Br)cc1)c1ccccc1-n1cccn1</smiles>

The general procedure $\mathbf{K}$ was followed us ing $\mathbf{1 2 8 a}$ ( $72 \mathrm{mg}, 0.5 \mathrm{mmol})$ and $\mathbf{1 3 1 h}$ (225 $\mathrm{mg}, 1.0$ mmol). Purification by column chromatography ( $n$-hexane/ $\mathrm{CH}_{2} \mathrm{Cl}_{2} / \mathrm{EtOAc} / \mathrm{Et}_{3} \mathrm{~N}$ 8:1:1:1\%) yie lded 130ah (107 mg, 63\%) as a colorless solid. M. p.: $=148-150{ }^{\circ} \mathrm{C}$. 
${ }^{1} \mathbf{H}-\mathbf{N M R}\left(500 \mathrm{MHz}, \mathrm{CDCl}_{3}\right): \delta=8.64(\mathrm{br} \mathrm{s}, 1 \mathrm{H}), 7.90(\mathrm{dd}, J=7.5,1.9 \mathrm{~Hz}, 1 \mathrm{H}), 7.77(\mathrm{~d}, J=1.9$ $\mathrm{Hz}, 1 \mathrm{H}), 7.68(\mathrm{~d}, J=2.4 \mathrm{~Hz}, 1 \mathrm{H}), 7.61-7.47$ (m, 2H), 7.40-7.28 (m, 5H), 6.45 (t, $J=2.2 \mathrm{~Hz}$, $1 \mathrm{H})$.

${ }^{13}$ C-NMR $\left(125 \mathrm{MHz}, \mathrm{CDCl}_{3}\right): \delta=164.3\left(\mathrm{C}_{\mathrm{q}}\right), 141.4(\mathrm{CH}), 137.2\left(\mathrm{C}_{\mathrm{q}}\right), 137.0\left(\mathrm{C}_{\mathrm{q}}\right), 132.8\left(\mathrm{C}_{\mathrm{q}}\right)$, $132.1(\mathrm{CH}), 131.8(\mathrm{CH}), 131.5(\mathrm{CH}), 130.8(\mathrm{CH}), 129.2(\mathrm{CH}), 126.9(\mathrm{CH}), 121.5(\mathrm{CH}), 117.0$ $\left(\mathrm{C}_{\mathrm{q}}\right), 107.9(\mathrm{CH})$.

IR (ATR): 3249, 1662, 1518, 1318, 1073, $750 \mathrm{~cm}^{-1}$.

MS (EI) m/z (relative intensity) $341(5)\left[\mathrm{M}^{+}\right], 171$ (100), 144 (5), 116 (10), 89 (5).

HR-MS (EI) m/z calcd for $\mathrm{C}_{16} \mathrm{H}_{12} \mathrm{~N}_{3} \mathrm{O}^{79} \mathrm{Br}\left[\mathrm{M}^{+}\right]$341.0164, found 341.0164.

2-(1H-Pyrazol-1-yl)- $N$-\{4-(trifluorome thyl)phe nyl\}be nzamide (130ai)<smiles>O=C(Nc1ccc(C(F)(F)F)cc1)c1ccccc1-n1cccn1</smiles>

The general procedure K was followed using 128a (72 mg, $0.5 \mathrm{mmol})$ and 131i (215 mg, 1.0 mmol). Purification by column chromatography ( $n$-hexane $/ \mathrm{CH}_{2} \mathrm{Cl}_{2} / \mathrm{EtOAc} / \mathrm{Et}_{3} \mathrm{~N}$ 8:1:1:1\%) yielded 130ai (110 mg, $66 \%)$ as a colorless solid. M. p.: $=168-170{ }^{\circ} \mathrm{C}$.

${ }^{1}$ H-NMR $\left(300 \mathrm{MHz}, \mathrm{CDCl}_{3}\right): \delta=9.03(\mathrm{br} \mathrm{s}, 1 \mathrm{H}), 7.88(\mathrm{dd}, J=6.9,2.5 \mathrm{~Hz}, 1 \mathrm{H}), 7.79(\mathrm{~d}, J=1.9$ $\mathrm{Hz}, 1 \mathrm{H}), 7.69(\mathrm{~d}, J=2.5 \mathrm{~Hz}, 1 \mathrm{H}), 7.62-7.41(\mathrm{~m}, 6 \mathrm{H}), 7.35(\mathrm{dd}, J=7.0,2.2 \mathrm{~Hz}, 1 \mathrm{H}), 6.47(\mathrm{dd}, J$ $=2.2,1.9 \mathrm{~Hz}, 1 \mathrm{H})$.

${ }^{13}$ C-NMR $\left(125 \mathrm{MHz}, \mathrm{CDCl}_{3}\right): \delta=164.7\left(\mathrm{C}_{\mathrm{q}}\right), 141.4(\mathrm{CH}), 141.0\left(\mathrm{C}_{\mathrm{q}}\right), 137.2\left(\mathrm{C}_{\mathrm{q}}\right), 132.6\left(\mathrm{C}_{\mathrm{q}}\right)$, $132.1(\mathrm{CH}), 131.7(\mathrm{CH}), 130.9(\mathrm{CH}), 129.2(\mathrm{CH}), 126.9(\mathrm{CH}), 126.1\left(\mathrm{CH}, J_{\mathrm{C}-\mathrm{F}}=7.5 \mathrm{~Hz}\right), 126.1$ $\left(\mathrm{C}_{\mathrm{q}}, J_{\mathrm{C}-\mathrm{F}}=32.9 \mathrm{~Hz}\right) 124.0\left(\mathrm{C}_{\mathrm{q}}, J_{\mathrm{C}-\mathrm{F}}=271.5 \mathrm{~Hz}\right), 119.5(\mathrm{CH}), 108.0(\mathrm{CH})$.

${ }^{19}$ F-NMR $\left(\mathrm{CDCl}_{3}, 282 \mathrm{MHz}\right): \delta=-62.2(\mathrm{~s})$.

IR (ATR): 3255, 1668, 1538, 1318, 1109, $749 \mathrm{~cm}^{-1}$.

MS (EI) m/z (relative intensity) 331 (5) [M+], 171 (100), 144 (5), 130 (5), 116 (15), 89 (10).

HR-MS (EI) $\mathrm{m} / \mathrm{z}$ calcd for $\mathrm{C}_{17} \mathrm{H}_{12} \mathrm{~N}_{3} \mathrm{OF}_{3}\left[\mathrm{M}^{+}\right]$331.0932, found 331.0936 . 


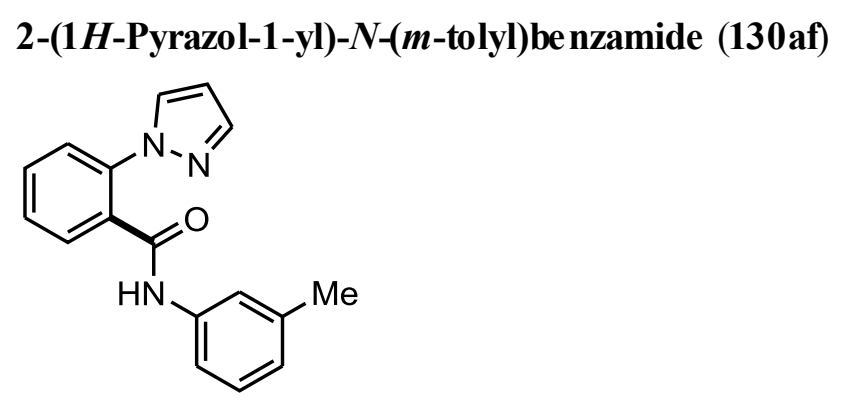

The general procedure $\mathbf{K}$ was followed using 128a (72 $\mathrm{mg}, 0.5 \mathrm{mmol})$ and $\mathbf{1 3 1 f}$ (161 $\mathrm{mg}, 1.0$ mmol). Purification by column chromatography ( $n$-hexane $/ \mathrm{CH}_{2} \mathrm{Cl}_{2} / \mathrm{EtOAc} / \mathrm{Et}_{3} \mathrm{~N}$ 8:1:1:1\%) yie lded 130af ( $87 \mathrm{mg}, 63 \%)$ as a colorless solid. M. p.: $=127-129^{\circ} \mathrm{C}$.

${ }^{1} \mathbf{H}-\mathbf{N M R}\left(500 \mathrm{MHz}, \mathrm{CDCl}_{3}\right): \delta=8.29(\mathrm{br} \mathrm{s}, 1 \mathrm{H}), 7.94-7.86(\mathrm{~m}, 1 \mathrm{H}), 7.77(\mathrm{~d}, J=1.9 \mathrm{~Hz}, 1 \mathrm{H})$, $7.70(\mathrm{~d}, J=2.4 \mathrm{~Hz}, 1 \mathrm{H}), 7.53(\mathrm{~m}, 2 \mathrm{H}), 7.41(\mathrm{dd}, J=7.5,1.7 \mathrm{~Hz}, 1 \mathrm{H}), 7.31$ (s, 1H), 7.16-7.12 (m, 2H), $6.88(\mathrm{ddd}, J=4.5,3.0,1.1 \mathrm{~Hz}, 1 \mathrm{H}), 6.44(\mathrm{t}, J=2.2 \mathrm{~Hz}, 1 \mathrm{H}), 2.29$ (s, 3H).

${ }^{13}$ C-NMR (125 MHz, $\left.\mathrm{CDCl}_{3}\right): \delta=164.4\left(\mathrm{C}_{\mathrm{q}}\right), 141.4(\mathrm{CH}), 138.8\left(\mathrm{C}_{\mathrm{q}}\right), 137.7\left(\mathrm{C}_{\mathrm{q}}\right), 137.2\left(\mathrm{C}_{\mathrm{q}}\right)$, $133.1\left(\mathrm{C}_{\mathrm{q}}\right), 131.9(\mathrm{CH}), 131.3(\mathrm{CH}), 130.6(\mathrm{CH}), 129.1(\mathrm{CH}), 128.6(\mathrm{CH}), 126.8(\mathrm{CH}), 125.3$ $(\mathrm{CH}), 120.7(\mathrm{CH}), 117.1(\mathrm{CH}), 107.8(\mathrm{CH}), 21.5\left(\mathrm{CH}_{3}\right)$.

IR (ATR): 3240, 1663, 1548, 1311, 1042, $742 \mathrm{~cm}^{-1}$.

MS (EI) m/z (relative intensity) 277 (5) $\left[\mathrm{M}^{+}\right], 171$ (100), 144 (5), 116 (15).

HR-MS (ESI) m/z calcd for $\mathrm{C}_{17} \mathrm{H}_{16} \mathrm{~N}_{3} \mathrm{O}\left[\mathrm{M}+\mathrm{H}^{+}\right]$278.1293, found 278.1286.

\section{$N$-(2-Fluorophe nyl)-2-(1 H-pyrazol-1-yl)be nzamide (130aj)}<smiles>O=C(Nc1ccccc1F)c1ccccc1-n1cccn1</smiles>

The general procedure $\mathbf{K}$ was followed using 128a (72 mg, $0.5 \mathrm{mmol})$ and $\mathbf{1 3 1} \mathbf{j}$ (165 mg, 1.0 mmol). Purification by column chromatography ( $n$-hexane $/ \mathrm{CH}_{2} \mathrm{Cl}_{2} / \mathrm{EtOAc} / \mathrm{Et}_{3} \mathrm{~N}$ 10:1:1:1\%) yie lded 130aj (110 mg, 78\%) as a colorless solid. M. p.: $=135-137^{\circ} \mathrm{C}$.

${ }^{1} \mathbf{H}-\mathbf{N M R}\left(300 \mathrm{MHz}, \mathrm{CDCl}_{3}\right): \delta=8.42($ br s, $1 \mathrm{H}), 8.36-8.26(\mathrm{~m}, 1 \mathrm{H}), 7.90(\mathrm{dd}, J=7.5,1.7 \mathrm{~Hz}$, 1H), $7.74(\mathrm{~d}, J=1.9 \mathrm{~Hz}, 1 \mathrm{H}), 7.71(\mathrm{~d}, J=2.4 \mathrm{~Hz}, 1 \mathrm{H}), 7.62-7.48(\mathrm{~m}, 2 \mathrm{H}), 7.43$ (dd, $J=7.3,1.9$ Hz, 1H), 7.09 (ddd, $J=8.2,5.6,3.1 \mathrm{~Hz}, 1 \mathrm{H}), 7.05-6.97$ (m, 2H), 6.46-6.36 (m, 1H). 
${ }^{13}$ C-NMR $\left(125 \mathrm{MHz}, \mathrm{CDCl}_{3}\right): \delta=164.8\left(\mathrm{C}_{\mathrm{q}}\right), 152.6\left(\mathrm{C}_{\mathrm{q}}, J_{\mathrm{C}-\mathrm{F}}=245.3 \mathrm{~Hz}\right), 141.6(\mathrm{CH}), 137.5$ $\left(\mathrm{C}_{\mathrm{q}}\right), 132.3\left(\mathrm{C}_{\mathrm{q}}\right), 131.5(\mathrm{CH}), 131.2(\mathrm{CH}), 130.6(\mathrm{CH}), 128.9(\mathrm{CH}), 126.4(\mathrm{CH}), 126.3\left(\mathrm{C}_{\mathrm{q}}, J_{\mathrm{C}-\mathrm{F}}\right.$ $=10.5 \mathrm{~Hz}), 124.7\left(\mathrm{CH}, J_{\mathrm{C}-\mathrm{F}}=7.5 \mathrm{~Hz}\right), 124.4\left(\mathrm{CH}, J_{\mathrm{C}-\mathrm{F}}=3.7 \mathrm{~Hz}\right), 122.0(\mathrm{CH}), 114.9\left(\mathrm{CH}, J_{\mathrm{C}-\mathrm{F}}=\right.$ 19.1 Hz), $107.8(\mathrm{CH})$.

${ }^{19}$ F-NMR $\left(282 \mathrm{MHz}, \mathrm{CDCl}_{3}\right): \delta=-129.0(\mathrm{ddd}, J=8.2,2.8 \mathrm{~Hz})$.

IR (ATR): 3254, 1657, 1450, 1317, 1039, $749 \mathrm{~cm}^{-1}$.

MS (EI) m/z (relative intensity) 281 (5) $\left[\mathrm{M}^{+}\right], 171$ (100), 144 (5), 130 (5), 116 (10).

HR-MS (EI) m/z calcd for $\mathrm{C}_{16} \mathrm{H}_{12} \mathrm{~N}_{3} \mathrm{OF}\left[\mathrm{M}^{+}\right]$281.0964, found 281.0976.

(Z)-N,2-Diphenyl-3-(1H-pyrazol-1-yl)acrylamide (133aa)<smiles>O=C(Nc1ccccc1)/C(=C/c1ccccc1)n1cccn1</smiles>

The general procedure $\mathbf{J}$ was followed using 132a ( $85 \mathrm{mg}, 0.5 \mathrm{mmol})$ and 129a (119 mg, 1.0 $\mathrm{mmol}), \mathrm{Cp}^{*} \mathrm{CoI}_{2}(\mathrm{CO})(\mathbf{1 1 2}, 24 \mathrm{mg}, 10 \mathrm{~mol} \%), \operatorname{AgNTf}_{2}(39.8 \mathrm{mg}, 20 \mathrm{~mol} \%)$ and $\mathrm{AgOPiv}(20.8$ mg, $20 \mathrm{~mol} \%$ ). Purification by column chromatography ( $n$-hexane $/ \mathrm{CH}_{2} \mathrm{Cl}_{2} / \mathrm{EtOAc} / \mathrm{Et}_{3} \mathrm{~N}$ 10:1:1:1\%) yielded 133aa (80 mg, 55\%) as a colorless solid. M. p.: $=180-181^{\circ} \mathrm{C}$.

${ }^{1} \mathbf{H}-\mathbf{N M R}\left(500 \mathrm{MHz}, \mathrm{CDCl}_{3}\right): \delta=7.87(\mathrm{~s}, 1 \mathrm{H}), 7.87(\mathrm{~d}, J=2.6 \mathrm{~Hz}, 1 \mathrm{H}), 7.60(\mathrm{~d}, J=1.8 \mathrm{~Hz}, 1 \mathrm{H})$, 7.54 (dd, $J=8.6,1.2 \mathrm{~Hz}, 2 \mathrm{H}), 7.52-7.48(\mathrm{~m}, 2 \mathrm{H}), 7.43-7.28$ (m, 6H), 7.18-7.07 (m, 1H), 6.33 (dd, $J=2.6,1.8 \mathrm{~Hz}, 1 \mathrm{H})$.

${ }^{13}$ C-NMR $\left(125 \mathrm{MHz}, \mathrm{CDCl}_{3}\right): \delta=165.4\left(\mathrm{C}_{\mathrm{q}}\right), 141.8(\mathrm{CH}), 137.5\left(\mathrm{C}_{\mathrm{q}}\right), 134.9\left(\mathrm{C}_{\mathrm{q}}\right), 130.0(\mathrm{CH})$, $129.1(\mathrm{CH}), 129.0(\mathrm{CH}), 128.6(\mathrm{CH}), 126.6(\mathrm{CH}), 126.5\left(\mathrm{C}_{\mathrm{q}}\right), 126.2(\mathrm{CH}), 124.9(\mathrm{CH}), 120.2$ (CH), $108.1(\mathrm{CH})$.

IR (ATR): 3250, 1646, 1540, 1439, 953, $750 \mathrm{~cm}^{-1}$.

MS (EI) m/z (relative intensity) 289 (5) [M+], 221 (5), 197 (100), 169 (40), 115 (10).

HR-MS (ESI) $\mathrm{m} / \mathrm{z}$ calcd for $\mathrm{C}_{18} \mathrm{H}_{15} \mathrm{~N}_{3} \mathrm{O}\left[\mathrm{M}^{+}\right] 289.1215$, found 289.1216. 
<smiles>O=C(Nc1ccc(F)cc1)/C(=C/c1ccccc1)n1cccn1</smiles>

The general procedure $\mathbf{J}$ was followed using 132a ( $85 \mathrm{mg}, 0.5 \mathrm{mmol})$ and 129d (137 mg, 1.0 mmol), $\mathrm{Cp}^{*} \mathrm{CoI}_{2}(\mathrm{CO})(\mathbf{1 1 2}, \mathrm{mg}, 10 \mathrm{~mol} \%), \operatorname{AgNTf}_{2}(39.8 \mathrm{mg}, 20 \mathrm{~mol} \%$ ) and AgOPiv (20.8 $\mathrm{mg}, 20 \mathrm{~mol} \%$ ). Purification by column chromatography ( $n$-hexane $/ \mathrm{CH}_{2} \mathrm{Cl}_{2} / \mathrm{EtOAc} / \mathrm{Et}_{3} \mathrm{~N}$ $8: 1: 1: 1 \% \rightarrow 5: 1: 0: 0)$ yielded 133ad $(81 \mathrm{mg}, 53 \%)$ as a colorless solid. M. p.: $=184-185{ }^{\circ} \mathrm{C}$. ${ }^{1} \mathbf{H}-\mathrm{NMR}\left(300 \mathrm{MHz}, \mathrm{CDCl}_{3}\right): \delta=7.99($ br s, $1 \mathrm{H}), 7.81(\mathrm{~d}, J=2.6 \mathrm{~Hz}, 1 \mathrm{H}), 7.60(\mathrm{~d}, J=1.8 \mathrm{~Hz}$, 1H), 7.55-7.43 (m, 4H), 7.40-7.34 (m, 3H), $7.33(\mathrm{~s}, 1 \mathrm{H}), 7.01(\mathrm{t}, J=8.7 \mathrm{~Hz}, 2 \mathrm{H}), 6.34(\mathrm{t}, J=$ $2.2 \mathrm{~Hz}, 1 \mathrm{H})$.

${ }^{13}$ C-NMR $\left(125 \mathrm{MHz}, \mathrm{CDCl}_{3}\right): \delta=165.4\left(\mathrm{C}_{\mathrm{q}}\right), 159.7\left(\mathrm{C}_{\mathrm{q}}, J_{\mathrm{C}-\mathrm{F}}=244.3 \mathrm{~Hz}\right), 141.8(\mathrm{CH}), 134.9$ $\left(\mathrm{C}_{\mathrm{q}}\right), 133.5\left(\mathrm{C}_{\mathrm{q}}, J_{\mathrm{C}-\mathrm{F}}=2.9 \mathrm{~Hz}\right), 130.2(\mathrm{CH}), 129.0(\mathrm{CH}), 128.6(\mathrm{CH}), 126.6(\mathrm{CH}), 126.5\left(\mathrm{C}_{\mathrm{q}}\right)$, $126.1(\mathrm{CH}), 122.1\left(\mathrm{CH}, J_{\mathrm{C}-\mathrm{F}}=8.0 \mathrm{~Hz}\right), 115.7\left(\mathrm{CH}, J_{\mathrm{C}-\mathrm{F}}=22.6 \mathrm{~Hz}\right), 108.0(\mathrm{CH})$.

${ }^{19}$ F-NMR $\left(282 \mathrm{MHz}, \mathrm{CDCl}_{3}\right): \delta=-117.2(\mathrm{tt}, J=8.2,4.8 \mathrm{~Hz})$.

IR (ATR): 3240, 1636, 1505, 1391, 1211, $747 \mathrm{~cm}^{-1}$.

MS (EI) m/z (relative intensity) 307 (5) [M $], 239$ (5), 197 (100), 169 (45), 142 (5), 115 (10).

HR-MS (EI) m/z calcd for $\mathrm{C}_{18} \mathrm{H}_{14} \mathrm{FN}_{3} \mathrm{O}\left[\mathrm{M}^{+}\right] 307.1121$, found 307.1125 .

\section{(Z)-N,3-Diphenyl-3-(1H-pyrazol-1-yl)acrylamide (133ba)}<smiles>O=C(Nc1ccccc1)C(=[Pt]c1ccccc1)n1cccn1</smiles>

The general procedure $\mathbf{J}$ was followed using $\mathbf{1 3 2} \mathbf{b}$ ( $85 \mathrm{mg}, 0.5 \mathrm{mmol})$ and 129a (119 $\mathrm{mg}, 1.0$ $\mathrm{mmol}), \mathrm{Cp}^{*} \mathrm{CoI}_{2}(\mathrm{CO})(\mathbf{1 1 2}, 24 \mathrm{mg}, 10 \mathrm{~mol} \%), \operatorname{AgNTf}_{2}(39.8 \mathrm{mg}, 20 \mathrm{~mol} \%)$ and $\mathrm{AgOPiv}(20.8$ $\mathrm{mg}, 20 \mathrm{~mol} \%$ ). Purification by column chromatography ( $n$-hexane $/ \mathrm{CH}_{2} \mathrm{Cl}_{2} / \mathrm{EtOAc} / \mathrm{Et}_{3} \mathrm{~N}$ 8:1:1:1\%) yielded $133 \mathrm{ba}(78 \mathrm{mg}, 54 \%)$ as a colorless solid. M. p.: $=159-161{ }^{\circ} \mathrm{C}$. 
${ }^{1} \mathbf{H}-\mathbf{N M R}\left(300 \mathrm{MHz}, \mathrm{CDCl}_{3}\right): \delta=9.69$ (br s, 1H), $7.85(\mathrm{~d}, J=2.0 \mathrm{~Hz}, 1 \mathrm{H}), 7.51-7.45$ (m, 3H), 7.43-7.31 (m, 3H), 7.30-7.18 (m, 4H), 7.12-7.01 (t, $J=2.2 \mathrm{~Hz}, 1 \mathrm{H}), 6.45(\mathrm{t}, J=2.2 \mathrm{~Hz}, 1 \mathrm{H})$, $6.37(\mathrm{~s}, 1 \mathrm{H})$.

${ }^{13}$ C-NMR $\left(125 \mathrm{MHz}, \mathrm{CDCl}_{3}\right): \delta=162.0\left(\mathrm{C}_{\mathrm{q}}\right), 142.8\left(\mathrm{C}_{\mathrm{q}}\right), 141.2(\mathrm{CH}), 138.0\left(\mathrm{C}_{\mathrm{q}}\right), 136.4\left(\mathrm{C}_{\mathrm{q}}\right)$, $133.0(\mathrm{CH}), 130.4(\mathrm{CH}), 128.8(\mathrm{CH}), 128.8(\mathrm{CH}), 127.6(\mathrm{CH}), 124.2(\mathrm{CH}), 119.8(\mathrm{CH}), 119.2$ (CH), $107.7(\mathrm{CH})$.

IR (ATR): 3054, 1658, 1539, 1440, 1085, $755 \mathrm{~cm}^{-1}$.

MS (EI) m/z (relative intensity) $289(5)\left[\mathrm{M}^{+}\right], 197$ (65), 169 (5), 129 (100), 102 (10), 77 (15).

HR-MS (EI) m/z calcd for $\mathrm{C}_{18} \mathrm{H}_{15} \mathrm{~N}_{3} \mathrm{O}\left[\mathrm{M}^{+}\right]$289.1215, found 289.1214 .

(Z)- $N$-(4-Fluorophe nyl)-3-phe nyl-3-(1 H-pyrazol-1-yl)acrylamide (133bd)<smiles>O=C(/C=C(\c1ccccc1)n1cccn1)Nc1ccc(F)cc1</smiles>

The general procedure $\mathbf{J}$ was followed us ing $\mathbf{1 3 2 b}$ ( $85 \mathrm{mg}, 0.5 \mathrm{mmol})$ and 129d (137 $\mathrm{mg}, 1.0$ $\mathrm{mmol}), \mathrm{Cp}^{*} \mathrm{CoI}_{2}(\mathrm{CO})(\mathbf{1 1 2}, 24 \mathrm{mg}, 10 \mathrm{~mol} \%), \operatorname{AgNTf}_{2}(39.8 \mathrm{mg}, 20 \mathrm{~mol} \%)$ and $\mathrm{AgOPiv}(20.8$ $\mathrm{mg}, 20 \mathrm{~mol} \%$ ). Purification by column chromatography ( $n$-hexane $/ \mathrm{CH}_{2} \mathrm{Cl}_{2} / \mathrm{EtOAc} / \mathrm{Et}_{3} \mathrm{~N}$ $8: 1: 1: 1 \% \rightarrow 5: 1: 0: 0)$ yielded $133 \mathrm{bd}\left(97 \mathrm{mg}, 63 \%\right.$ ) as a colorless solid. M. p.: $=150-152{ }^{\circ} \mathrm{C}$. ${ }^{1} \mathbf{H}-\mathbf{N M R}\left(500 \mathrm{MHz}, \mathrm{CDCl}_{3}\right): \delta=9.92($ br s, $1 \mathrm{H}), 7.94-7.82(\mathrm{~m}, 1 \mathrm{H}), 7.53(\mathrm{dd}, J=2.5,0.6 \mathrm{~Hz}$, 1H), 7.52-7.48 (m, 2H), 7.48-7.45 (m, 1H), 7.44-7.38 (m, 2H), 7.30-7.25 (m, 2H), 7.01 (dd, $J$ $=9.1,8.3 \mathrm{~Hz}, 2 \mathrm{H}), 6.52(\mathrm{dd}, J=2.5,1.9 \mathrm{~Hz}, 1 \mathrm{H}), 6.40(\mathrm{~s}, 1 \mathrm{H})$.

${ }^{13}$ C-NMR (125 MHz, $\left.\mathrm{CDCl}_{3}\right): \delta=162.0\left(\mathrm{C}_{\mathrm{q}}\right), 159.2\left(\mathrm{C}_{\mathrm{q}}, J_{\mathrm{C}-\mathrm{F}}=243.4 \mathrm{~Hz}\right), 143.0\left(\mathrm{C}_{\mathrm{q}}\right), 141.1$ $(\mathrm{CH}), 136.3\left(\mathrm{C}_{\mathrm{q}}\right), 134.1\left(\mathrm{C}_{\mathrm{q}}, J_{\mathrm{C}-\mathrm{F}}=2.9 \mathrm{~Hz}\right), 133.0(\mathrm{CH}), 130.5(\mathrm{CH}), 128.8(\mathrm{CH}), 127.6(\mathrm{CH})$, $121.5\left(\mathrm{CH}, J_{\mathrm{C}-\mathrm{F}}=7.8 \mathrm{~Hz}\right), 118.8(\mathrm{CH}), 115.4\left(\mathrm{CH}, J_{\mathrm{C}-\mathrm{F}}=22.5 \mathrm{~Hz}\right), 107.7(\mathrm{CH})$.

${ }^{19}$ F-NMR $\left(282 \mathrm{MHz}, \mathrm{CDCl}_{3}\right): \delta=-118.0(\mathrm{tt}, J=8.3,4.8 \mathrm{~Hz})$.

IR (ATR): 3006, 1659, 1508, 1210, 921, $761 \mathrm{~cm}^{-1}$.

MS (EI) m/z (relative intensity) 307 (5) $\left[\mathrm{M}^{+}\right], 197$ (80), 169 (5), 129 (100), 102 (10).

HR-MS (ESI) $\mathrm{m} / \mathrm{z}$ calcd for $\mathrm{C}_{18} \mathrm{H}_{14} \mathrm{~N}_{3} \mathrm{FO}\left[\mathrm{M}+\mathrm{H}^{+}\right]$307.1121, found 307.1118. 


\section{H/D Exchange Experiments:}

Cobalt-Catalyzed H/D Exchange in Substrate 128a with $\left[\mathrm{D}_{4}\right]-\mathrm{MeOH}$ as the Cosolvent without Is ocyanate 129:<smiles>c1ccc(-n2cccn2)cc1</smiles>

$128 a$
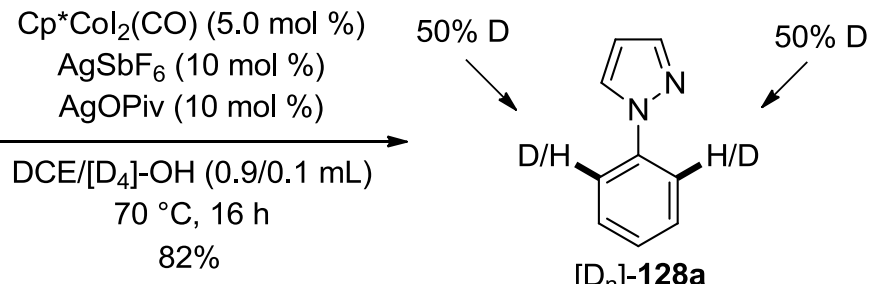

$\left[D_{n}\right]-128 a$

A suspension of 1-phenyl-1H-pyrazole (128a) $(72 \mathrm{mg}, 0.50 \mathrm{mmol}), \mathrm{Cp}^{*} \mathrm{CoI}_{2}(\mathrm{CO})(\mathbf{1 1 2}, 12$ mg, $5.0 \mathrm{~mol} \%), \operatorname{AgSbF}_{6}(17.2 \mathrm{mg}, 10.0 \mathrm{~mol} \%$ ) and AgOPiv (10.4 mg, $10.0 \mathrm{~mol} \%$ ) in a solvent mixture of $\mathrm{DCE}$ and $\mathrm{CD}_{3} \mathrm{OD}(0.9 / 0.1 \mathrm{~mL})$ was stirred at $70{ }^{\circ} \mathrm{C}$ for $16 \mathrm{~h}$ under an atmosphere of Ar. At ambient temperature, the reaction mixture was evaporated in vacuo and the residue was purified by column chromatography on silica gel ( $n$-hexane/EtOAc) to yield $\left[D_{n}\right]-128 a(59 \mathrm{mg}, 82 \%)$ as a slightly yellow oil. The D-incorporation in $\left[D_{n}\right]-128 a$ was estimated by ${ }^{1} \mathrm{H}-\mathrm{NMR}$ spectroscopy.

Scheme S-2. Cobalt-Catalyzed H/D Exchange in Substrate $\left[D_{5}\right]-128$ a with Isocyanate 129a:

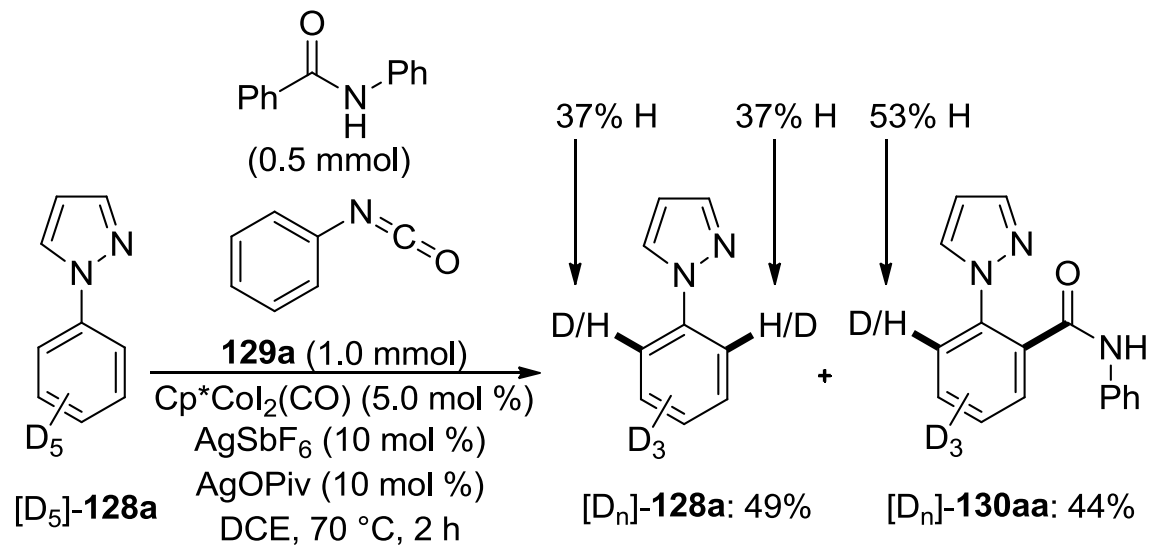

A suspension of $\left[\mathrm{D}_{5}\right]$-128a (74 mg, $\left.0.5 \mathrm{mmol}\right), \mathbf{1 2 9 a}(119 \mathrm{mg}, 1.0 \mathrm{mmol}), N$-phenylbenzamide (99 mg, $0.5 \mathrm{mmol}), \mathrm{Cp}^{*} \mathrm{CoI}_{2}(\mathrm{CO})(\mathbf{1 1 2}, 12 \mathrm{mg}, 5.0 \mathrm{mmol} \%), \mathrm{AgSbF}_{6}(17.2 \mathrm{mg}, 10 \mathrm{~mol} \%)$ and AgOPiv (10.4 mg, $10 \mathrm{mmol} \%)$ in DCE $(2.0 \mathrm{~mL})$ was stirred at $70{ }^{\circ} \mathrm{C}$ for $2 \mathrm{~h}$ under an atmosphere of Ar. At ambient temperature, the solvent was evaporated in vacuo and the 
residue was purified by column chromatography on silica gel ( $n$-hexane $/ \mathrm{CH}_{2} \mathrm{Cl}_{2} / \mathrm{EtOAc} / \mathrm{Et}_{3} \mathrm{~N}$ 10:1:1:1\%) to recover starting material $\left[\mathrm{D}_{\mathrm{n}}\right]-\mathbf{1 2 8 a}(36 \mathrm{mg}, 49 \%)$ as a slight yellow oil and yield product $\left[D_{n}\right]-\mathbf{1 3 0} \mathbf{a a}(58 \mathrm{mg}, 44 \%)$ as a colorless solid. The D-incorporation in $\left[D_{n}\right]-\mathbf{1 2 8 a}$ and $\left[D_{n}\right]-130$ aa was estimated by ${ }^{1} \mathrm{H}-\mathrm{NMR}$ spectroscopy.

\section{KIE De termination Experiments:}

KIE De termination Expe riments with $128 \mathrm{a}$ and $\left[\mathrm{D}_{5}\right]-128 \mathrm{a}$ as Subs trates :<smiles>c1ccc(-n2cccn2)cc1</smiles>

$128 \mathrm{a}$

or<smiles>[2H][13c]1ccc(-n2cccn2)cc1</smiles>

$\left[\mathrm{D}_{5}\right]-128 \mathrm{a}$

(1.0 mmol each)

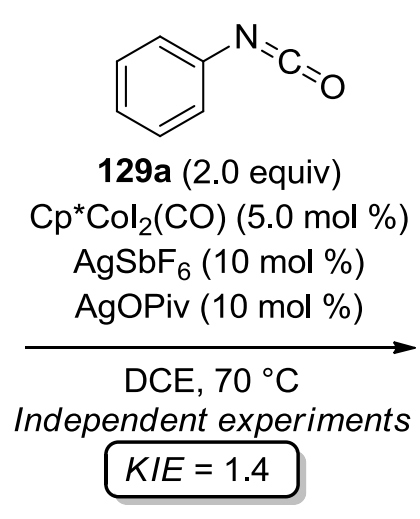<smiles>O=C(Nc1ccccc1)c1ccccc1O</smiles>

$\left[D_{n}\right]-130 a a$

1). Two independent reactions with $\mathbf{1 2 8 a}$ or deuterated substrate $\left[D_{5}\right] \mathbf{- 1 2 8} \mathbf{a}$ under the standard conditions were performed: Two reaction mixtures each containing phenyl isocyanate (129a) (338 mg, $2.0 \mathrm{mmol})$, substrates $\mathbf{1 2 8 a}(144 \mathrm{mg}, 1.00 \mathrm{mmol})$ or $\left[\mathrm{D}_{5}\right]-\mathbf{1 2 8 a}(149 \mathrm{mg}, 1.00 \mathrm{mmol})$, $\mathrm{Cp}^{*} \mathrm{CoI}_{2}(\mathrm{CO})(\mathbf{1 1 2}, 24 \mathrm{mg}, 5.0 \mathrm{~mol} \%), \mathrm{AgSbF}_{6}(34.4 \mathrm{mg}, 10.0 \mathrm{~mol} \%)$ and AgOPiv (20.8 mg, $10.0 \mathrm{~mol} \%)$ in DCE $(4.0 \mathrm{~mL})$ were stirred at $70{ }^{\circ} \mathrm{C}$ under an atmosphere of Ar. The consumption of substrates $128 \mathrm{a}$ or $\left[\mathrm{D}_{5}\right]-\mathbf{1 2 8 a}$ and the appearance of the products $130 \mathrm{a}$ or $\left[D_{n}\right]-130 a$ were monitored by GC analysis after 0.5 h, 1.0 h, 1.25 h, 1.5 h, 1.75 h, 2.0 h, 2.25 h, $2.5 \mathrm{~h}, 2.75 \mathrm{~h}, 3.0 \mathrm{~h}$, respectively. These experiments indicated that the $\mathrm{C}-\mathrm{H}$ bond activation is not the rate-limiting step of the cobalt-catalyzed $\mathrm{C}-\mathrm{H}$ bond aminocarbonylation reaction. 


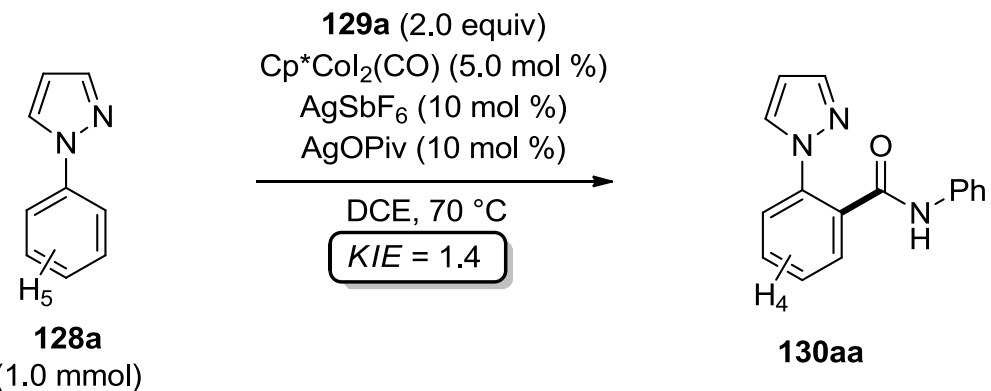

\begin{tabular}{|c|c|c|c|c|c|c|c|c|c|c|}
\hline t/(h) & $\mathbf{0 . 5}$ & $\mathbf{1 . 0}$ & $\mathbf{1 . 2 5}$ & $\mathbf{1 . 5}$ & $\mathbf{1 . 7 5}$ & $\mathbf{2 . 0}$ & $\mathbf{2 . 2 5}$ & $\mathbf{2 . 5}$ & $\mathbf{2 . 7 5}$ & $\mathbf{3 . 0}$ \\
\hline $\mathbf{1 3 0 a a}$ & 0.06 & 0.16 & 0.22 & 0.28 & 0.35 & 0.39 & 0.47 & 0.56 & 0.56 & 0.61 \\
\hline $\mathbf{1 2 8 a}$ & 0.94 & 0.84 & 0.78 & 0.72 & 0.65 & 0.61 & 0.53 & 0.44 & 0.44 & 0.49 \\
\hline
\end{tabular}

129 a ( 2.0 equiv)

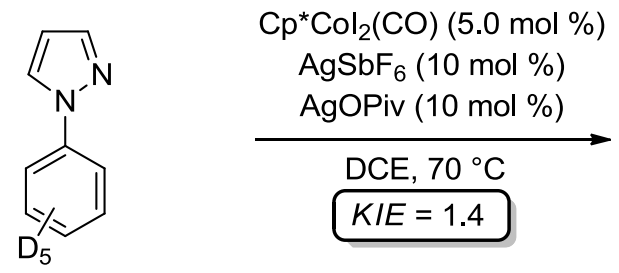

$\left[D_{5}\right]-128 a$

$(1.0 \mathrm{mmol})$<smiles>O=C(Nc1ccccc1)c1ccccc1-c1ccccc1</smiles>

$\left[D_{n}\right]-130 a a$

\begin{tabular}{|c|c|c|c|c|c|c|c|c|c|c|}
\hline $\mathbf{t} /(\mathbf{h})$ & $\mathbf{0 . 5}$ & $\mathbf{1 . 0}$ & $\mathbf{1 . 2 5}$ & $\mathbf{1 . 5}$ & $\mathbf{1 . 7 5}$ & $\mathbf{2 . 0}$ & $\mathbf{2 . 2 5}$ & $\mathbf{2 . 5}$ & $\mathbf{2 . 7 5}$ & $\mathbf{3 . 0}$ \\
\hline$\left[\mathrm{D}_{\mathrm{n}}\right]-\mathbf{1 3 0 a a}$ & 0.01 & 0.04 & 0.06 & 0.11 & 0.16 & 0.20 & 0.27 & 0.32 & 0.32 & 0.39 \\
\hline$\left[\mathrm{D}_{5}\right]-\mathbf{- 1 2 8 a}$ & 0.99 & 0.96 & 0.94 & 0.89 & 0.84 & 0.80 & 0.73 & 0.68 & 0.68 & 0.61 \\
\hline
\end{tabular}
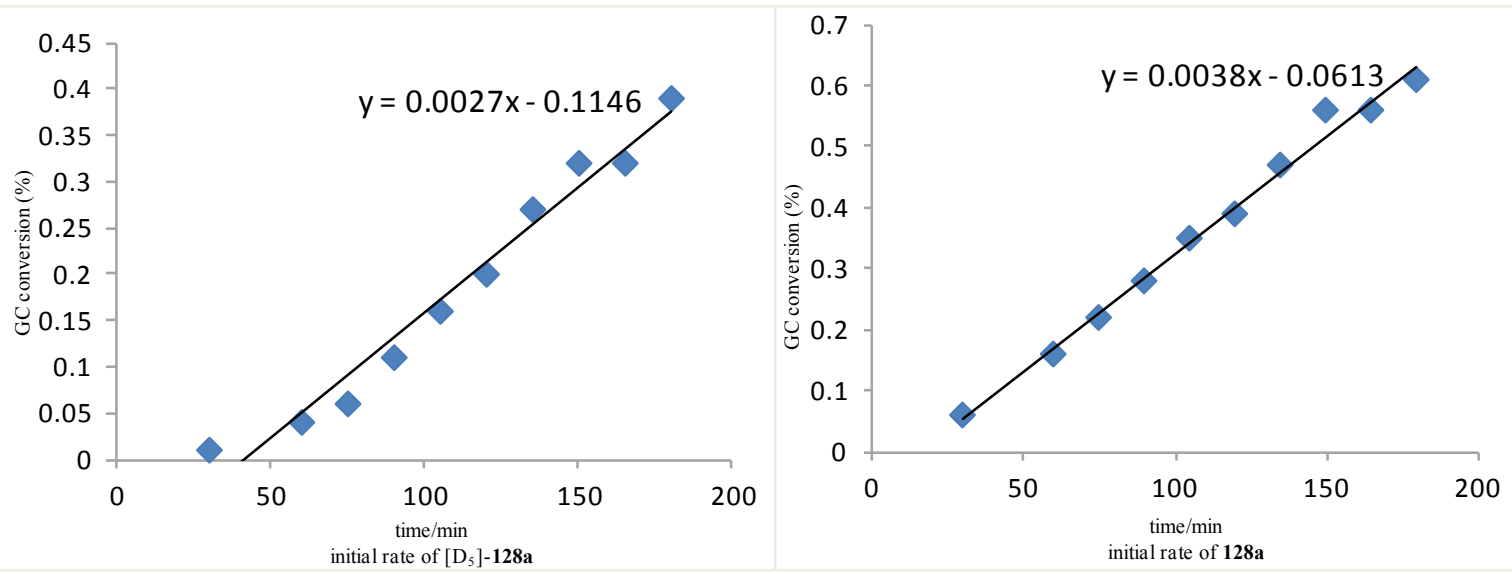

Intramolecular Competition Expe rime nt: Kinetic Isotope Effect (KIE): 


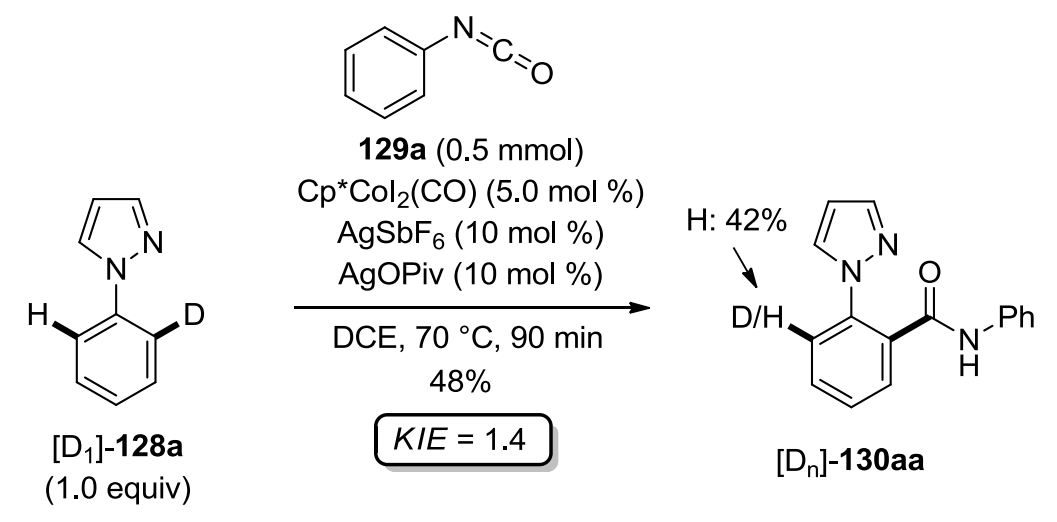

2). 1-(Phenyl-2- $d)$-1H-pyrazole ([D $\left.\left.\mathrm{D}_{1}\right]-\mathbf{1 2 8 a}\right)(73.0 \mathrm{mg}, 0.50 \mathrm{mmol})$, phenyl is ocyanate (129a) (60.0 mg, $0.50 \mathrm{mmol}), \quad \mathrm{Cp}^{*} \mathrm{CoI}_{2}(\mathrm{CO})(\mathbf{1 1 2}, 12.0 \mathrm{mg}, 5.0 \mathrm{~mol} \%), \operatorname{AgSbF}_{6}(17.2 \mathrm{mg}, 10.0$ $\mathrm{mol} \%)$ and AgOPiv (10.4 mg, $10.0 \mathrm{~mol} \%)$ in DCE $(2.0 \mathrm{~mL})$ was stirred at $70{ }^{\circ} \mathrm{C}$ for $90 \mathrm{~min}$ under an atmosphere of Ar. At ambient temperature, the reaction mixture was evaporated in vacuo, and the residue was purified by column chromatography on silica gel (n-hexane $/ \mathrm{CH}_{2} \mathrm{Cl}_{2} / \mathrm{EtOAc} / \mathrm{Et}_{3} \mathrm{~N}$ 10:1:1:0.01) to yield $\left[\mathrm{D}_{\mathrm{n}}\right]-130 \mathrm{aa}(63.0 \mathrm{mg}, 48 \%)$ as a colorless solid. The degree of deuterium incorporation in $\left[D_{n}\right]-130$ aa was estimated by ${ }^{1}$ H-NMR spectroscopy.

1-(Phe nyl-2- $d)-1 H$-pyrazole $\left(\left[\mathrm{D}_{1}\right]-128 a\right)$<smiles></smiles>

${ }^{1} \mathbf{H}-\mathbf{N M R}\left(500 \mathrm{MHz}, \mathrm{CDCl}_{3}\right): \delta=7.91(\mathrm{dd}, J=2.5,0.6 \mathrm{~Hz}, 1 \mathrm{H}), 7.71(\mathrm{dd}, J=1.8,0.7 \mathrm{~Hz}, 1 \mathrm{H})$, 7.70-7.67 (m, 1H), 7.51-7.36 (m, 2H), 7.27 (td, $J=7.5,1.1 \mathrm{~Hz}, 1 \mathrm{H}), 6.45$ (dd, $J=2.5,1.8 \mathrm{~Hz}$, 1H).

${ }^{13}$ C-NMR (125 MHz, $\left.\mathrm{CDCl}_{3}\right): \delta=141.0(\mathrm{CH}), 140.2\left(\mathrm{C}_{\mathrm{q}}\right), 129.4(\mathrm{CH}), 129.4(\mathrm{CH}), 126.7(\mathrm{CH})$, $126.4(\mathrm{CH}, J=1.6 \mathrm{~Hz}), 119.2(\mathrm{CH}), 119.2(\mathrm{CH}), 107.6(\mathrm{CH})$.

IR (ATR): 3066, 1593, 1391, 1045, 936, $742 \mathrm{~cm}^{-1}$.

MS (EI) m/z (relative intensity) 145 (100) $\left[\mathrm{M}^{+}\right], 118$ (30), 91 (25), 78 (45).

HR-MS (EI) m/z calcd for $\mathrm{C}_{9} \mathrm{H}_{7} \mathrm{DN}_{2}\left[\mathrm{M}^{+}\right]$145.0750, found 145.0752 .

\section{Competition Experiments:}


Inte rmolecular Competition Experime nt between Substrates VI-1b and VI-1d:

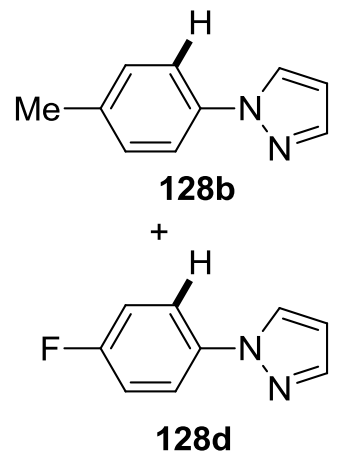

(2.0 equiv each)

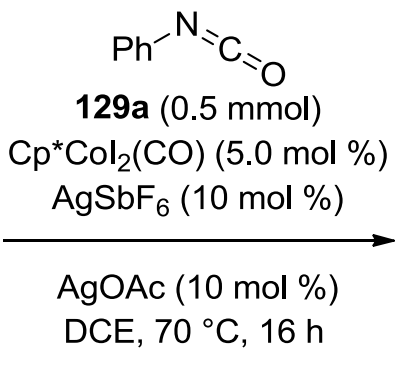<smiles>Cc1ccc(-n2cccn2)c(C(=O)Nc2ccccc2)c1</smiles>

130ba: $18 \%$<smiles>O=C(Nc1ccccc1)c1cc(F)ccc1-n1cccn1</smiles>

130da: $0 \%$

A suspension of phenyl isocyanate (129a) $(60 \mathrm{mg}, 0.5 \mathrm{mmol}), 1-p$-tolyl-1H-pyrazole (128b) (158 mg, $1.00 \mathrm{mmol}), 1-(4-f l u o r o p h e n y l)-1 H$-pyrazole (128d) (162 mg, $1.00 \mathrm{mmol})$, $\mathrm{Cp}^{*} \mathrm{CoI}_{2}(\mathrm{CO})(\mathbf{1 1 2}, 12 \mathrm{mg}, 5.0 \mathrm{~mol} \%), \mathrm{AgSbF}_{6}(17.2 \mathrm{mg}, 10.0 \mathrm{~mol} \%)$ and AgOPiv (10.8 mg, $10.0 \mathrm{~mol} \%)$ in DCE $(2.0 \mathrm{~mL})$ was stirred at $70{ }^{\circ} \mathrm{C}$ for $16 \mathrm{~h}$ under an atmosphere of Ar. At ambient temperature, the reaction mixture was evaporated in vacuo and the residue was purified by column chromatography on silica gel ( $n$-hexane $/ \mathrm{CH}_{2} \mathrm{Cl}_{2} / \mathrm{EtOAc} / \mathrm{Et}_{3} \mathrm{~N}$ 10:1:1:0.01) to yield $130 \mathrm{ba}(25 \mathrm{mg}, 18 \%)$ as a colorless solid.

Intermolecular Competition Experiment between Substrates 129b and 129d:

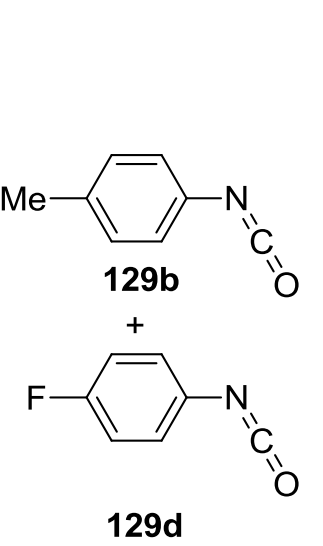

(2.0 equiv each)

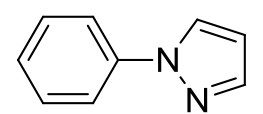

$$
128 \mathrm{a}(0.5 \mathrm{mmol})
$$
$\mathrm{Cp}^{*} \mathrm{Col}_{2}(\mathrm{CO})(5.0 \mathrm{~mol} \%)$ $\mathrm{AgSbF}_{6}(10 \mathrm{~mol} \%)$ $\operatorname{AgOAc}(10 \mathrm{~mol} \%)$

DCE, $70^{\circ} \mathrm{C}, 16 \mathrm{~h}$<smiles>Cc1ccc(NC(=O)c2ccccc2-n2cccn2)cc1</smiles>

130ab: $12 \%$<smiles>O=C(Nc1ccc(F)cc1)c1ccccc1-n1cccn1</smiles>

130ad: $55 \%$

A suspension of 1-phenyl-1H-pyrazole (128a) (72 $\mathrm{mg}, \quad 0.5 \quad \mathrm{mmol})$, 1-isocyanato-4-methylbenzene (129b) (133 mg, $1.00 \mathrm{mmol})$, 1-fluoro-4-isocyanatobenzene (129d) (137 mg, $1.00 \mathrm{mmol}), \mathrm{Cp}^{*} \mathrm{CoI}_{2}(\mathrm{CO})(\mathbf{1 1 2}, 12 \mathrm{mg}, 5.0 \mathrm{~mol} \%), \mathrm{AgSbF}_{6}(17.2 \mathrm{mg}, 10.0$ mol \%) and AgOPiv (10.8 mg, $10.0 \mathrm{~mol} \%)$ in DCE $(2.0 \mathrm{~mL})$ was stirred at $70{ }^{\circ} \mathrm{C}$ for $16 \mathrm{~h}$ under an atmosphere of Ar. At ambient temperature, the reaction mixture was evaporated in vacuo and the residue was purified by column chromatography on silica gel 
( $n$-hexane $/ \mathrm{CH}_{2} \mathrm{Cl}_{2} / \mathrm{EtOAc} / \mathrm{Et}_{3} \mathrm{~N}$ 10:1:1:1\%) to yield 130ab (17 mg, 12\%) and 130ad (77 mg, $55 \%)$ as color less solids.

N-(4-Fluorophe nyl)-2-(1 H-pyrazol-1-yl)be nzamide (130ad)<smiles>O=C(Nc1ccc(F)cc1)c1ccccc1-n1cccn1</smiles>

M. p.: $=130-132^{\circ} \mathrm{C}$.

${ }^{1}$ H-NMR (300 MHz, $\left.\mathrm{CDCl}_{3}\right): \delta=8.58$ (br s, $\left.1 \mathrm{H}\right), 7.96-7.84(\mathrm{~m}, 1 \mathrm{H}), 7.77(\mathrm{~d}, J=1.9 \mathrm{~Hz}, 1 \mathrm{H})$, $7.69(\mathrm{~d}, J=2.4 \mathrm{~Hz}, 1 \mathrm{H}), 7.62-7.46(\mathrm{~m}, 2 \mathrm{H}), 7.43-7.30$ (m, 3H), $6.94(\mathrm{dd}, J=9.1,8.3 \mathrm{~Hz}, 2 \mathrm{H})$, $6.46(\mathrm{t}, J=2.2 \mathrm{~Hz}, 1 \mathrm{H})$.

${ }^{13}$ C-NMR (125 MHz, $\left.\mathrm{CDCl}_{3}\right): \delta=164.4\left(\mathrm{C}_{\mathrm{q}}\right), 159.4\left(\mathrm{C}_{\mathrm{q}}, J_{\mathrm{C}-\mathrm{F}}=243.9 \mathrm{~Hz}\right), 141.3(\mathrm{CH}), 137.2$ $\left(\mathrm{C}_{\mathrm{q}}\right), 133.9\left(\mathrm{C}_{\mathrm{q}}, J_{\mathrm{C}-\mathrm{F}}=2.9 \mathrm{~Hz}\right), 132.8\left(\mathrm{C}_{\mathrm{q}}\right), 132.0(\mathrm{CH}), 131.4(\mathrm{CH}), 130.7(\mathrm{CH}), 129.2(\mathrm{CH})$, $126.8(\mathrm{CH}), 121.8\left(\mathrm{CH}, J_{\mathrm{C}-\mathrm{F}}=7.9 \mathrm{~Hz}\right), 115.5\left(\mathrm{CH}, J_{\mathrm{C}-\mathrm{F}}=22.4 \mathrm{~Hz}\right), 107.8(\mathrm{CH})$.

${ }^{19}$ F-NMR $\left(282 \mathrm{MHz}, \mathrm{CDCl}_{3}\right): \delta=-117.8(\mathrm{tt}, J=8.2,4.8 \mathrm{~Hz})$.

IR (ATR): 3258, 1652, 1507, 1209, 832, $754 \mathrm{~cm}^{-1}$.

MS (EI) m/z (relative intensity) 281 (5) $\left[\mathrm{M}^{+}\right], 171$ (100), 144 (5), 130 (10), 116 (15).

HR-MS (EI) m/z calcd for $\mathrm{C}_{16} \mathrm{H}_{12} \mathrm{~N}_{3} \mathrm{OF}\left[\mathrm{M}^{+}\right] 281.0964$, found 281.0961.

\section{4-Phenylpyrazolo[1,5-a]quinazolin-5(4H)-one (160)}<smiles>O=c1c2ccccc2n2nccc2n1-c1ccccc1</smiles>

A suspension of $N$-phenyl-2-(1H-pyrazol-1-yl)benzamide (130aa) $(53 \mathrm{mg}, 0.2 \mathrm{mmol})$ and $\mathrm{K}_{2} \mathrm{~S}_{2} \mathrm{O}_{8}(81 \mathrm{mg}, 0.3 \mathrm{mmol})$ in $\mathrm{H}_{2} \mathrm{O}(5.0 \mathrm{~mL})$ was stirred at $105^{\circ} \mathrm{C}$ for $1.0 \mathrm{~h}$. After completion of the reaction, at ambient temperature the reaction mixture was extracted with MTBE $(3 \times 10 \mathrm{~mL})$ at ambient temperature. The combined organic layers were dried over $\mathrm{Na}_{2} \mathrm{SO}_{4}$ and filtered. The solvent was concentrated in vacuo and purification by column chromatography 
( $n$-Hexane/EtOAc 4:1) yielded 160 (45 mg, 85\%) as a colorless solid. M. p.: $=160-162{ }^{\circ} \mathrm{C}$. ${ }^{1} \mathbf{H}-\mathbf{N M R}\left(500 \mathrm{MHz}, \mathrm{CDCl}_{3}\right): \delta=8.36-8.32(\mathrm{~m}, 1 \mathrm{H}), 8.23-8.18(\mathrm{~m}, 1 \mathrm{H}), 7.81$ (ddd, $J=8.3,7.3$, $1.5 \mathrm{~Hz}, 1 \mathrm{H}), 7.64(\mathrm{~d}, J=2.0 \mathrm{~Hz}, 1 \mathrm{H}), 7.59-7.54(\mathrm{~m}, 2 \mathrm{H}), 7.52-7.47(\mathrm{~m}, 1 \mathrm{H}), 7.47-7.40(\mathrm{~m}, 3 \mathrm{H})$, $5.47(\mathrm{~d}, J=2.0 \mathrm{~Hz}, 1 \mathrm{H})$.

${ }^{13}$ C-NMR (125 MHz, $\left.\mathrm{CDCl}_{3}\right): \delta=158.6\left(\mathrm{C}_{\mathrm{q}}\right), 141.7(\mathrm{CH}), 141.5\left(\mathrm{C}_{\mathrm{q}}\right), 137.7\left(\mathrm{C}_{\mathrm{q}}\right), 136.8\left(\mathrm{C}_{\mathrm{q}}\right)$, $135.0(\mathrm{CH}), 130.0(\mathrm{CH}), 129.4(\mathrm{CH}), 129.3(\mathrm{CH}), 127.8(\mathrm{CH}), 125.5(\mathrm{CH}), 116.3(\mathrm{CH}), 114.7$ $\left(\mathrm{C}_{\mathrm{q}}\right), 90.9(\mathrm{CH})$.

IR (ATR): 1668, 1553, 1482, 1127, 757, $684 \mathrm{~cm}^{-1}$.

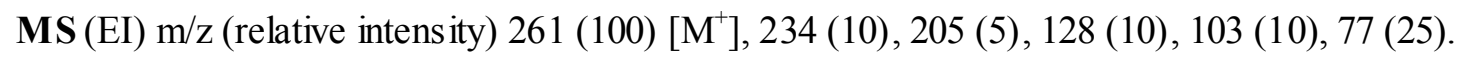

HR-MS (EI) m/z calcd for $\mathrm{C}_{16} \mathrm{H}_{11} \mathrm{~N}_{3} \mathrm{O}\left[\mathrm{M}^{+}\right]$261.0902, found 261.0903.

\section{3-(4-Methoxybe nzyl)- $N$-phe nyl-2-(1H-pyrazol-1-yl)be nzamide (161)}<smiles>COc1ccc(Cc2cccc(C(=O)Nc3ccccc3)c2-n2cccn2)cc1</smiles>

According to the established protocol, a suspension of $N$-phenyl-2-(1H-pyrazol-1-yl)benzamide (130aa) $\quad(66 \quad \mathrm{mg}, \quad 0.25 \quad \mathrm{mmol})$, 1-(chloromethyl)-4-methoxybenzene $(\mathbf{1 6 3}, 78 \mathrm{mg}, 0.5 \mathrm{mmol}),\left[\mathrm{RuCl}_{2}(p \text {-cymene })\right]_{2}(7.8 \mathrm{mg}$, $5.0 \mathrm{~mol} \%), 1-\mathrm{AdCO}_{2} \mathrm{H}(13.5 \mathrm{mg}, 30 \mathrm{~mol} \%)$ and $\mathrm{K}_{2} \mathrm{CO}_{3}(69 \mathrm{mg}, 0.5 \mathrm{mmol})$ in $\mathrm{PhMe}(1.0 \mathrm{~mL})$ was stirred at $100{ }^{\circ} \mathrm{C}$ for $20 \mathrm{~h} .{ }^{167}$ At ambient temperature, the reaction mixture was extracted with MTBE $(3 \times 10 \mathrm{~mL})$. The combined organic layers were dried over $\mathrm{Na}_{2} \mathrm{SO}_{4}$ and filtered. The solvent was concentrated in vacuo and purification by column chromatography ( $n$-Hexane/EtOAc 5:1) yielded 161 (45 mg, 47\%) as a colorless solid. M. p.: $=133-135{ }^{\circ} \mathrm{C}$. ${ }^{1} \mathbf{H}-\mathbf{N M R}\left(300 \mathrm{MHz}, \mathrm{CDCl}_{3}\right): \delta=7.85(\mathrm{~d}, J=1.9 \mathrm{~Hz}, 1 \mathrm{H}), 7.80(\mathrm{dd}, J=7.7,1.6 \mathrm{~Hz}, 1 \mathrm{H}), 7.73$ (br s, 1H), 7.51 (t, J=7.7 Hz, 1H), 7.42-7.35 (m, 2H), 7.35-7.29 (m, 2H), 7.25 (m, 1H), 7.22 (d, $J=8.3 \mathrm{~Hz}, 1 \mathrm{H}), 7.11-6.97$ (m, 1H), 6.88 (d, $J=8.6 \mathrm{~Hz}, 2 \mathrm{H}), 6.75(\mathrm{~d}, J=8.6 \mathrm{~Hz}, 2 \mathrm{H}), 6.38$ (t, $J$ $=2.2 \mathrm{~Hz}, 1 \mathrm{H}), 3.75(\mathrm{~s}, 3 \mathrm{H}), 3.64(\mathrm{~s}, 2 \mathrm{H})$.

${ }_{167}$ L. Ackermann, P. Novák, R. Vicente, N. Hofmann, Angew. Chem. Int. Ed. 2009, 48, 6045-6048. 
${ }^{13}$ C-NMR $\left(125 \mathrm{MHz}, \mathrm{CDCl}_{3}\right): \delta=164.3\left(\mathrm{C}_{\mathrm{q}}\right), 158.2\left(\mathrm{C}_{\mathrm{q}}\right), 141.6(\mathrm{CH}), 140.6\left(\mathrm{C}_{\mathrm{q}}\right), 137.7\left(\mathrm{C}_{\mathrm{q}}\right)$, $135.8\left(\mathrm{C}_{\mathrm{q}}\right), 135.5\left(\mathrm{C}_{\mathrm{q}}\right), 133.4(\mathrm{CH}), 133.0(\mathrm{CH}), 131.3\left(\mathrm{C}_{\mathrm{q}}\right), 130.1(\mathrm{CH}), 129.7(\mathrm{CH}), 128.8$ (CH), $128.5(\mathrm{CH}), 124.4(\mathrm{CH}), 119.9(\mathrm{CH}), 113.9(\mathrm{CH}), 107.5(\mathrm{CH}), 55.2\left(\mathrm{CH}_{3}\right), 35.8\left(\mathrm{CH}_{2}\right)$. IR (ATR): 3274, 1650, 1508, 1245, 1025, $750 \mathrm{~cm}^{-1}$.

MS (EI) m/z (relative intensity) 383 (15) $\left[\mathrm{M}^{+}\right], 290$ (80), 262 (100), 247 (25), 234 (5), 219 (15).

HR-MS (EI) m/z calcd for $\mathrm{C}_{24} \mathrm{H}_{21} \mathrm{~N}_{3} \mathrm{O}_{2}\left[\mathrm{M}^{+}\right] 383.1634$, found 383.1629 .

\section{Ethyl 3 '-(Phenylcarbamoyl)-2'-(1 H-pyrazol-1-yl)-[1,1'-biphe nyl]-4-carboxylate (162)}<smiles>CCOC(=O)c1ccc(-c2cccc(C(=O)Nc3ccccc3)c2-n2cccn2)cc1</smiles>

According to the established protocol, a suspension of $\mathrm{N}$-phenyl-2-(1H-pyrazol-1-yl)benzamide (130aa) (63 mg, $0.24 \mathrm{mmol})$, which was prepared according the general procedure $\mathbf{J}$, ethyl 4-chlorobenzoate (88k) (37 $\mathrm{mg}, 0.2 \mathrm{mmol})$, $\left[\mathrm{Ru}\left(\mathrm{MesCO}_{2}\right)_{2}(p\right.$-cymene) $](5.6 \mathrm{mg}, 5.0 \mathrm{~mol} \%)$ and $\mathrm{K}_{2} \mathrm{CO}_{3}(55 \mathrm{mg}, 0.4 \mathrm{mmol})$ in PhMe (1.0 $\mathrm{mL}$ ) was stirred at $120^{\circ} \mathrm{C}$ for $20 \mathrm{~h}$ under an atmosphere of $\mathrm{Ar} .{ }^{168}$ At ambient temperature, the reaction mixture was extracted with MTBE $(3 \times 10 \mathrm{~mL})$. The combined organic layers were dried over $\mathrm{Na}_{2} \mathrm{SO}_{4}$ and filtered. The solvent was concentrated in vacuo and purification by column chromatography ( $n$-hexane/EtOAc 5:1) and HPLC yielded product 162 (62 mg, 75\%) as a colorless solid. M. p.: $=147-149^{\circ} \mathrm{C}$.

${ }^{1}$ H-NMR (300 MHz, $\left.\mathrm{CDCl}_{3}\right): \delta=8.55$ (br s, $\left.1 \mathrm{H}\right), 8.04(\mathrm{dd}, J=7.6,1.8 \mathrm{~Hz}, 1 \mathrm{H}), 7.95(\mathrm{~d}, J=$ $8.7 \mathrm{~Hz}, 2 \mathrm{H}), 7.76-7.71(\mathrm{~m}, 1 \mathrm{H}), 7.69(\mathrm{~d}, J=7.7 \mathrm{~Hz}, 1 \mathrm{H}), 7.63(\mathrm{dd}, J=7.7,1.8 \mathrm{~Hz}, 1 \mathrm{H})$, 7.49-7.38 (m, 2H), 7.34-7.25 (m, 3H), 7.20 (d, $J=8.7 \mathrm{~Hz}, 2 \mathrm{H}), 7.14-7.03$ (m, 1H), 6.25 (dd, $J=2.4,1.9 \mathrm{~Hz}, 1 \mathrm{H}), 4.39$ (q, $J=7.1 \mathrm{~Hz}, 2 \mathrm{H}), 1.41$ (t, $J=7.1 \mathrm{~Hz}, 3 \mathrm{H})$.

${ }^{13}$ C-NMR (75 MHz, $\left.\mathrm{CDCl}_{3}\right): \delta=166.2\left(\mathrm{C}_{\mathrm{q}}\right), 164.0\left(\mathrm{C}_{\mathrm{q}}\right), 142.1\left(\mathrm{C}_{\mathrm{q}}\right), 141.0(\mathrm{CH}), 140.0\left(\mathrm{C}_{\mathrm{q}}\right)$, $137.9\left(\mathrm{C}_{\mathrm{q}}\right), 135.8\left(\mathrm{C}_{\mathrm{q}}\right), 134.9\left(\mathrm{C}_{\mathrm{q}}\right), 133.7(\mathrm{CH}), 132.8(\mathrm{CH}), 130.6(\mathrm{CH}), 130.1(\mathrm{CH}), 129.8$ $\left(\mathrm{C}_{\mathrm{q}}\right), 129.4(\mathrm{CH}), 128.9(\mathrm{CH}), 128.3(\mathrm{CH}), 124.5(\mathrm{CH}), 120.0(\mathrm{CH}), 107.8(\mathrm{CH}), 61.1\left(\mathrm{CH}_{2}\right)$,

${ }^{168}$ L. Ackermann, R. Vicente, H. K. Potukuchi, V. Pirova no, Org. Lett. 2010, 12, 5032-5035. 
$14.3\left(\mathrm{CH}_{3}\right)$.

IR (ATR): 3279, 1708, 1551, 1278, 1106, $756 \mathrm{~cm}^{-1}$,

MS (ESI) m/z (relative intensity) 412 (10) $\left[\mathrm{M}+\mathrm{H}^{+}\right], 434\left[\mathrm{M}+\mathrm{Na}^{+}\right], 450\left[\mathrm{M}+\mathrm{K}^{+}\right], 845$ $\left[2 \mathrm{M}+\mathrm{Na}^{+}\right]$.

HR-MS (EI) $\mathrm{m} / \mathrm{z}$ calcd for $\mathrm{C}_{25} \mathrm{H}_{22} \mathrm{~N}_{3} \mathrm{O}_{3}\left[\mathrm{M}+\mathrm{H}^{+}\right]$412.1661, found 412.1656. 


\section{Acknowledge ments}

Herein, I would like to extend my sincere gratitude to all my supervisors during my graduate study. I will not have the opportunity to become a science chaser without their instructions, especially, my advisor, Prof. Dr. Lutz Ackermann. Thanks for his professional advice and suggestions on my research.

I gratefully acknowledge China Scholarship Council (CSC) for the financial support during my research stay in Germany.

High tribute shall be paid to Prof. Dr. Konrad Koszinowski for kindly accepting to be my second referee of this thesis, and for the work as second supervisor. I deeply thank Dr. Sergei Kozhushov, Darko Santrač, Svenja Warratz and Marc Moselage, for their patience to correct this manuscript.

I am also deeply indebted to all the other people in the last four years during my chemistry studies, for their direct and indirect help to me. They are Mrs. Gabriele Keil-Knepel, Dr. Weifeng Song, Dr. Lianhui Wang, Dr. Qing Gu, Dr. Yingjun Zhu, Dr. Nora Hofmann, Dr. Marvin Schinkel, Dr. Xu Tian, Wenbo Ma, Jie Li, Fangzhi Yang, Weiping Liu, Sebastian Lackner, Darko Santrač, Svenja Warratz, Zhixiong Ruan, Ruhuai Mei, Hui Wang, Qingqing $\mathrm{Bu}$, Marc Moselage, Carina Tirler, Sachiyo Nakanowatari...

Special thanks should give to my girlfriend, Che Tang, my parents and family for their continuous support and encouragement, including all my friends.

In short, I would like to give my sincere appreciation to all the people who have nonetheless contributed to this thesis.

Jie (Jack) Li 


\section{Curriculum Vitae}

Name: $\quad$ Jie Li (Jack Li)

Sex: $\quad$ Male

Nationality: Chinese

Date of Birth: $\quad$ 26/04/1986

\section{Education Background:}

Nov. 2011-Present

Institute of Organic and Biomolecular Chemistry. University of Goettingen. Germany. PhD Thesis (Prof. Dr. Lutz Ackermann), Organic Chemistry.

Aug. 2009-Oct. 2011

Dept. of Synthetic Medicinal Chemisry. Institute of Materia Medica, Chinese Academy of Medical Sciences \& Peking Union Medical College. Exchange student. Master Thesis (Prof. Dr. Zhiyan Xiao). Pharmaceutical Chemistry

Sept. 2008-July. 2009 Dept. of Medicinal Chemistry, Graduate School of Shenyang Pharmaceutical University. Master Thesis (Prof. Dr. Huiming Hua) Pharmaceutical Chemistry.

Sept. 2004-Jun. 2008 Dept. of Pharmaceutical Engineering. Tianjin University of Commerce. Bachelor of Pharmaceutical Engineering.

Aug. 2012-Feb. 2015 Teaching Assistant (Institute of Organic and Biomolecular Chemistry. University of Goettingen)

\section{Publication:}

1. L. Wang*, J. Li, Y. Zhang, Q. Wang, The Study of Chinese Yam Against the 
Reproducibility Dysmnesia in Mice. Food Science, 2010, 31, 243-245.

2. J. Li, H.-M. Hua, Y. B. Tang, Z. Y. Xiao*, Synthesis and stereochemical characterization of novel podophyllotoxin analogs, Chin. J. Med. Chem. 2011, 21, $31-37$.

3. J. Li, H.-M. Hua, Y-B. Tang, S. Zhang, E. Ohkoshi, K.-H Lee, Z. Xiao*, Synthes is and evaluation of novel podophyllotoxin analogs. Bioorg. Med. Chem. Lett. 2012, 22, 4293-4295.

4. J. Li, L. Ackermann*, Ruthenium-Catalyzed Oxidative Alkyne Annulation by $\mathrm{C}-\mathrm{H}$ Activation on Ketimines. Tetrahedron 2014, 70, 3342-3348.

5. J. Li, M. John, L. Ackermann*, Amidines for Versatile Ruthenium(II)-Catalyzed Oxidative C-H Activations with Internal Alkynes and Acrylates. Chem. Eur. J. 2014, 20 , $5403-5408$.

6. J. Li, L. Ackermann*, Cobalt-Catalyzed C-H Cyanation of Arenes and Heteroarenes. Angew. Chem. Int. Ed. 2015, 54, 3635-3638. (Selected as hot paper).

7. J. Li, L. Ackermann*, Cobalt-Catalyzed C-H Arylations with Weakly-Coordinating Amindes and Tetrazoles: Expedient Route to Angiotens in-II-Receptor Blockers. Chem. Eur. J. 2015, 21, 5718-5722.

8. J. Li, L. Ackermann*, Cobalt(III)-Catalyzed Aryl and Alkenyl C-H Aminocarbonylation w ith Isocyanates and Acyl Azides. Angew. Chem. Int. Ed. 2015, 54, (DOI: 10.1002/anie.201501926).

9. J. Li, L. Ackermann*, Carboxylate-Assisted Ruthenium(II)-Catalyzed C-H Activations of Monodentate Amides with Alkenes. Org. Chem. Fronts. 2015, (submitted).

10. J. Li, L. Ackermann*, Ruthenium-Catalyzed Direct Arylation via C-C Bonds Cleavage. (unpublished) 